\title{
LIMITED USE ONLY \\ HOW CAN THE DESIGN OF AN \\ EVERYDAY OBJECT EVOKE AN INITIATION \\ OF USE IN CHRONIC STROKE SURVIVORS
}

BY

\section{MAILIN LEMKE}

\begin{abstract}
A thesis
submitted to the Victoria University of Wellington in fulfilment of the requirements for the degree of Doctor of Philosophy
\end{abstract}

Victoria University of Wellington

2018 



\begin{abstract}
Stroke causes significant damage to the brain and affects 15 million people annually worldwide. Symptoms commonly affect one or both limbs on one side of the body, limiting ability to perform daily activities. The preferential use of the less affected limb for performing everyday activities in the form of compensatory movement is a common phenomenon after a stroke and can lead to a "learned nonuse" of the affected arm and hand. This learned behaviour can be overcome by applying a physical restraint on the less affected arm to initiate use of the affected one. Stroke interventions that use physical restraint are criticised for being labour intensive and expensive and having a limited focus on the home environment of the stroke survivor. This study aimed to design everyday objects that restrain movement to initiate the use of the affected arm and hand. It was undertaken from a pragmatist theoretical perspective, using a human-centred design approach to develop an understanding of the users' needs and create design solutions that addressed the observed problem. A qualitative multimethod approach helped understanding of how the restraining effect needs to be delivered to initiate use of the affected arm, and which everyday objects are key in daily activities post-stroke. The research through design methodology was employed for developing expansive and serial design prototypes to test how the restraint could be incorporated into a design prototype. The prototypes were evaluated with health professionals and chronic stroke survivors to validate the intended initiation of use. Findings of this study indicate that the development of learned nonuse is multifactorial and occurs over time. The current use of restraint in clinical practice focuses on reminding the survivor to use the affected arm and hand rather than physically restraining its use. It was emphasised by the therapists that a behaviour change is a crucial element in overcoming learned nonuse in the long-term. The evaluation of the design prototypes indicated that the design of the object needs to take into consideration the conceptual model the user has of the object and the interaction needs to be feasible to perform for the stroke survivor. Five different design
\end{abstract}


strategies were developed to restrain movement and elicit an initiation of use. The restraining effect that is evoked by the design strategies can vary between the different users. Additionally, the object needs to provide sufficient feedforward to initiate the use of the affected arm and hand, increase self-efficacy beliefs, provide a repetitive and increasingly challenging movement, provide feedback and sensory input to secure engagement in the process. A behaviour change is an essential element to overcome the learned nonuse in the long-term. A behaviour contract was, therefore, incorporated in the form of the design components to facilitate such a change. At this stage it is unclear which strategy offers the greatest potential to evoke an initiation of use and if the behaviour contract contributes to overcoming the learned nonuse. Further studies are needed to increase the restraining effect and usability of the design prototypes and validate the long-term impact.

Keywords: stroke, stroke rehabilitation, chronic stroke, stroke survivors, human-centred design, iterative design, research through design, everyday object 


\section{Acknowledgements}




\section{Edgar Rodríguez Ramírez}

Edgar, I still remember that I refused to call you by your first name in my first week as a PhD student since from my German perspective, it seemed quite inappropriate - from a Kiwi perspective, it's a regular thing. You left an 'LOL' as a comment under my written summary of our first meeting, which pretty much summarises the last three years. You had introduced me to research in the field of design so many years ago when I was still studying Design in Germany and took part in an exchange programme in New Zealand to broaden my horizons. I remember my fascination that you invited lecturers from other disciplines to talk about their work so we would understand that there is more to design than just being 'pretty'. My German directness and (occasional) stubbornness are not always easy to work with, but I value that you let me follow some of my crazy ideas and brought me back on track when I seemed to get lost in my cloud of thoughts. Thank you so much! I couldn't have wished for a better supervisor.

\section{Brian Robinson}

Brian, I honestly wouldn't have made it without your support. The medical field is so interesting but as well so scary from a designer's perspective. Your support and constant feedback on my work made it possible for me to work in medical device design. I value so much that you took way more interest in my work and supported me more than any other secondary supervisor would have done. Whenever I talk to a PhD student who mentions how little input they receive, I feel so fortunate that you seem to be an exception to the rule. From my perspective, you and Edgar are the only reason this research was even possible. Thank you!

\section{Gillian McCarthy}

Gillian, my partner in crime during those last few years. The fact that you started a year before me and had already figured out all the obstacles that turned up regarding the different ethics applications, funding, and conferences was such a great help. The PhD can be a lonely place, thanks for all the chats and laughs that always sweetened my day. 


\section{Birgit Bachler}

You made me laugh with your stories of working with participants that reassured me that it's not just me who finds this work challenging yet extremely inspiring.

\section{Phillip Fay}

Phillip, you showed me how constraint-induced movement therapy looks in practice, and you put me in touch with some fascinating people who share your vision of increasing healthcare outcomes for stroke survivors.

\section{Dr Nada Signal}

Nada, I know it is not easy to work with a designer since our approach can look and be entirely different from your research discipline. Thank you so much for your input and thoughts on this project.

\section{Therapists}

I would like to thank all of you for taking part in this project. I know that you have already quite full schedules and I appreciate the time that all of you took to talk to me about your work and challenges on the way.

\section{Participants who have experienced a stroke}

Your input has been so crucial for this project, and it was always such significant input when I talked to you about my project and how design could address barriers that you experience after a stroke. Thank you all so much for your support and time

\section{Design students}

Whenever New Zealand Design is described the word 'quirky' is mentioned. I think you have a really interesting way of seeing the world. So, thank you all so much for sharing your viewpoint with me over the last few years.

\section{School of Design}

There are so many people at the School of Design who helped me on the way and their work is so often taken for granted. I would like to thank the former head of school Margaret Maile Petty and the current head of school Douglas Easterly. I 
would further like to thank the library staff members, the technical experts, Mark Shaw, Graeme Crawley, Arthur Mahon, Phil Jarret, Frances Matheson, Michael McKinnon, Stewart Milne, Terry Moore, Phil Nelson and Garry Sammons. Special thanks to the good souls who keep the heart of the school beating: Mailyn Webb, Bronwyn Knight, Eli Feth, Kim Victoria. Other special thanks to the staff members of the industrial design department, to Nan O'Sullivan and particular thanks to Simon Fraser!

My friends in Europe

I miss you guys. Three years sounded like an eternity when I started but looking back now it feels like the time passed way too quickly. Thank you for all your Skype calls for which you had to stay up late, and I had to get up way too early.

\section{My friends down under}

Thank you all so much for your help and support in the process. I know I could never contribute the most interesting work stories since my life just rotated around books, papers, and conferences. But I always enjoyed listening to your latest gossip.

\section{My parents}

No words can describe how much your support means to me. Love you to pieces!

\section{My brother and his lovely girlfriend}

Jörn, who is so much like me and at the same time the complete opposite. Friends who know both of us always describe us as having the same mad obsession with our work. (Must run in the family -that love for design :) Thank you and Adriane for everything. I hope you know that you mean the world to me.

\section{Alex}

Alex, you had to deal with me during those last months of the PhD where my mood started to depend greatly on my perceived progress. I think if it hadn't been for you I would have tried to sleep under my desk so that I could be even 
closer to the PhD. Thank you for your support and for letting me use you as my proof-reader. 
LIMITED USE ONLY

\section{Table of Contents}


Abstract 1

Acknowledgements $\quad 3$

Table of Contents $\quad \mathbf{8}$

List of Tables $\quad \mathbf{2 6}$

List of Figures $\quad 28$

List of Abbreviations $\quad \mathbf{3 7}$ 
Introduction $\quad 39$

1.1 Motivation $\quad 40$

1.2 Situating the Research $\quad \mathbf{4 1}$

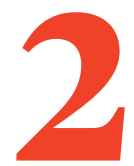

Background $\quad 46$

$\begin{array}{ll}\text { 2.1 Stroke } & 47\end{array}$

2.1.1 Definition of a stroke $\quad 47$

2.1.2 Impact of a stroke $\quad 47$

2.1.2.1 Changes of body structure and function $\quad 48$

2.1.2.2 Activities and participation in $\quad 50$ everyday activities

2.1.2.3 Environmental factors $\quad 51$

2.1.3 Stroke population

2.1.4 Recovery process

2.1.4.1 Compensatory movement $\quad \mathbf{5 6}$

2.1.4.2 Learned nonuse and initiation of use $\quad 57$

2.1.5 Stroke rehabilitation $\quad 59$

2.1.6 Motor learning in neurorehabilitation $\quad 60$

2.1.6.1 Constraint-induced movement therapy $\quad 61$

2.1.6.2 Interactive robotic therapy 63

2.1.6.3 Virtual reality-based therapy $\quad 64$ 
2.1.7 The everyday object 66

2.1.7.1 Interaction $\quad 66$

2.1.7.2 First encounter and affordance $\quad 66$

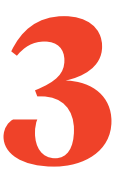

Methodology $\quad 70$

3.1 Research Aims, Objectives, Questions and Methods $\quad \mathbf{7 1}$

3.2 Theoretical Framework $\quad 73$

3.2.1 Pragmatism

3.3 Human-Centred Design $\quad \mathbf{7 5}$

3.3.1 The context of use $\quad 76$

3.3.2 Production of design solutions $\quad \mathbf{7 6}$

3.3.2.1 Research through design $\quad 76$

$\begin{array}{ll}\text { 3.3.2.2 Construction and communication } & 79\end{array}$ of knowledge

3.4 Evaluation with Stakeholders $\quad \mathbf{8 1}$

3.4.1 Formative usability testing 82

3.5 Chronology of the Research Process 84

3.6 Ethics $\quad \mathbf{8 6}$

3.6.1 Ethics granted by the university 86

3.6.2 Ethics granted by the Health and 86

Disability Ethics Committee

3.7 Summary

90 


\section{4}

\section{Interviews with Stroke}

\section{Therapists}

4.1 Introduction $\quad 92$

4.2 Background $\quad 92$

4.2.1 Professional health care provider 92

$\begin{array}{ll}4.3 \text { Method } & 93\end{array}$

4.3.1 Participants $\quad 93$

4.3.2 Semi-structured interview content 94

4.3.3 Thematic analysis $\quad 95$

4.4 Results $\quad 96$

$\begin{array}{ll}\text { 4.4.1 Themes } & 96\end{array}$

4.4.1.1 Factors contributing to learned nonuse $\quad 96$

4.4.1.2 Initiating use of the affected arm 99

and hand

4.4.1.3 General structure of the $\quad 100$

rehabilitation process

4.4.1.4 Addressing learned nonuse $\quad 103$

$\begin{array}{ll}\text { 4.4.1.5 Restraining the movement } & 107\end{array}$

$\begin{array}{ll}4.5 \text { Discussion } & 109\end{array}$

4.5.1 Factors contributing to learned nonuse 109

4.5.2 Addressing learned nonuse 110

4.5.3 Restraining the movement 112

4.5.4 Design criteria 113

4.6 Conclusion 114

4.7 Limitations 


\section{Interface to Facilitate Initiation} of Use

5.1 Introduction $\quad 116$

5.2 Background 116

5.2.1 Persuasive technology 116

5.2.2 Ethical considerations 118

5.2.3 Tangible user interfaces 119

5.2.4 Design evolution of radios $\mathbf{1 2 1}$

5.2.5 Reach to grasp movement 122

5.3 Methods $\quad \mathbf{1 2 3}$

5.3.1 Participants $\quad 123$

5.3.2 Research through design $\mathbf{1 2 3}$

5.3.3 Cognitive walkthrough $\mathbf{1 2 5}$

5.3.4 Thematic analysis $\quad \mathbf{1 2 5}$

5.4 Results $\quad \mathbf{1 2 5}$

5.4.1 Research through design results $\mathbf{1 2 5}$

5.4.1.1 Use of the radio 126

5.4.1.2 Restraining the movement $\quad 127$

5.4.1.3 Design prototypes $\quad 128$

5.4.2 Evaluation $\mathbf{1 4 0}$

5.4.2.1 Evaluation against design criteria $\quad \mathbf{1 4 0}$

5.4.2.2 Cognitive walkthrough with $\mathbf{1 4 2}$

health professionals

5.5 Discussion $\quad \mathbf{1 4 4}$

5.5.1 The design process $\quad \mathbf{1 4 4}$ 
6.2 Background $\quad 149$

6.2.1 Feedback and feedforward elements 149

6.2.2 Refinement of design criteria $\mathbf{1 5 2}$

6.3 Methods $\quad \mathbf{1 5 4}$

6.3.1 Participants

6.3.2 Research through design

6.3.3 Pluralistic walkthrough

6.3.4 Wizard of $O Z \quad 157$

6.3.5 Thematic analysis $\quad \mathbf{1 5 7}$

6.4 Results $\quad 157$

6.4.1 Research through design results 158

6.4.1.1 Prototype 07

6.4.1.2 Prototype $08 \quad 159$

6.4.1.3 Prototype $09 \quad 160$

6.4.1.3 Final design prototype 10

$\begin{array}{ll}6.4 .2 \text { Evaluation } & 169\end{array}$ 
6.4.2.1 Design criteria $\quad 169$

6.4.2.2 Pluralistic walkthrough 169

$\begin{array}{ll}\text { 6.5 Discussion } & \mathbf{1 7 5}\end{array}$

6.5.1 The design process $\quad \mathbf{1 7 5}$

6.5.2 Usability issues $\quad \mathbf{1 7 6}$

6.5.3 Feedforward and feedback $\quad \mathbf{1 7 6}$

6.5.4 Initiation of use $\quad \mathbf{1 7 7}$

6.6 Conclusion $\quad 178$

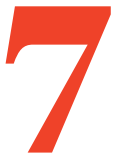

Designs of Everyday Objects 179 that Can Restrain Movement to Initiate the Use of the Affected Arm

7.1 Introduction $\quad \mathbf{1 8 0}$

$\begin{array}{ll}7.2 \text { Background } & 181\end{array}$

$\begin{array}{ll}\text { 7.2.1 Shaping } & 181\end{array}$

7.2.2 Design criteria 182

7.3 Methods 183

7.3.1 Participants 183

7.3.2 Research through design 183

7.3.2.1 Workshop outline 183

7.3.3 Analysis $\mathbf{1 8 6}$

$\begin{array}{ll}7.4 \text { Results } & \mathbf{1 8 7}\end{array}$

$\begin{array}{ll}\text { 7.4.1 Research through design results } & 187\end{array}$

$\begin{array}{ll}\text { 7.4.1.1 Restraining the movement } & 187\end{array}$ 
7.4.1.2 Enhanced repetition

7.4.1.3 Increased challenge

7.4.1.4 Feedback

7.4.1.5 Behaviour contract

7.5 Evaluation

7.5.1 Fluve

195

7.5.2 Lottiel

196

7.5.3 Smart Sticks

196

7.5.4 Coffee mugs

196

7.5.5 Cirgo knives

196

7.5.6 Water bottle

197

7.6 Discussion

197

7.6.1 The design process

197

7.6.2 The research through design results

194

199

\section{Digital Design Method Cards}

8.1 Introduction

8.2 Background

201

8.2.1 Design method cards

201

8.2.2 Context-specific cards

202

8.2.2.1 Empathy with the user

8.2.2.2 Inspiration

204

8.3 Methods

206

8.3.1 Participants

207 
8.3.2 Design method cards 207

8.3.3 Evaluation 208

8.3.4 Analysis 209

8.4 Results

8.4.1 Digital design method cards 210

8.4.1.1 Structure of the website 210

8.4.1.2 Colour code 211

8.4.1.3 Empathy 211

8.4.1.4 Inspiration 214

8.4.2 Evaluation 216

8.4.2.1 Design concepts 216

8.4.3 Themes 221

8.4.3.1 Website information 222

8.4.3.2 Use of the strategies 223

8.4.3.3 Perceived challenges 224

8.4.3.4 The use of strategies in daily practice 226

8.5 Discussion 226

8.5.1 Digital design method cards 226

8.5.1.1 Empathy 226

8.5.1.2 Inspiration $\quad 227$

8.5.2 Design fixation 227

8.5.3 Design strategies $\quad 228$

8.5.4 Further iterations 228

8.6 Conclusion $\mathbf{2 3 0}$

8.7 Limitations $\quad 231$ 


\section{Everyday Objects Used in}

\section{Daily Tasks Post Stroke}

9.1 Introduction

9.2 Background

9.3 Methods

9.3.1 Participants

236

9.3.2 Online survey

238

9.3.3 Analysis

238

9.4 Results

9.4.1 Survey results

240

9.4.2 ADL

240

9.4.3 ADL that have become difficult

9.4.4 Hobbies

9.4.5 Objects that play an essential role in $A D L$ and hobbies

9.5 Discussion

9.5.1 ADL

251

9.5.2 Most important tasks

9.5.3 Hobbies

9.5.4 Low demand tasks

252

9.5.5 Terminated tasks

253

9.5.6 Essential objects

253

9.6 Conclusion

9.7 Limitations

255 


\section{0}

\section{Everyday Objects Used in}

\section{Daily Tasks Post Stroke}

II

10.1 Introduction $\quad \mathbf{2 5 8}$

10.2 Methods $\quad 258$

10.2.1 Participants 258

10.2.2 Semi-structured interview content 259

10.2.3 Analysis 259

10.2.4 Triangulation $\quad 260$

10.3 Results 261

10.3.1 Interview results 261

$\begin{array}{ll}\text { 10.3.1.1 ADL } & 261\end{array}$

10.3.1.2 Objects used in the context of 263

daily activities and rehabilitation

$\begin{array}{ll}\text { 10.3.1.3 Themes } & \mathbf{2 6 7}\end{array}$

10.3.1.4 Triangulation $\quad 276$

10.4 Discussion $\quad 281$

10.4.1 Interviews 281

10.4.1.1 Goal setting $\quad 281$

10.4.1.2 ADL 282

10.4.1.3 Limitations of current rehabilitation 283

setting

10.4.1.4 Contextual factors $\quad 284$

10.4.1.5 Everyday objects $\quad 284$

10.4.2 Triangulation 285

10.4.2.1 Convergence assessment 285 


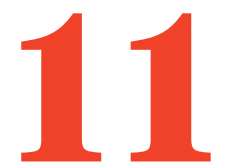

\section{Information Communication}

\section{Technology Post Stroke}

11.1 Introduction 291

11.2 Background $\quad 291$

11.3 Methods 293

11.3.1 Participants 293

11.3.2 Semi-structured interviews 294

11.3.3 Analysis $\quad 295$

11.4 Results $\quad 295$

11.4.1 Themes 295

11.4.1.1 Motivators $\quad 295$

$\begin{array}{ll}\text { 11.4.1.2 Barriers } & 301\end{array}$

$\begin{array}{ll}11.5 & \text { Discussion } \\ 306\end{array}$

11.5.1 Motivation 306

11.5.1.1 Connecting with others 306

11.5.1.2 Reintegration $\quad 307$

11.5.1.3 Technology adoption $\quad 307$

11.5.1.4 Leisure $\quad 308$

11.5.1.5 Rehabilitation purposes $\quad 308$

$\begin{array}{ll}\text { 11.5.2 Barriers } & 309\end{array}$

11.5.2.1 Physical and cognitive limitations $\quad 309$

11.5.2.2 Device specific limitations $\quad 309$ 
11.5.2.3 Context of use and

compensatory movement

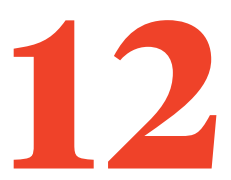

\section{Design Workshop}

12.1 Introduction

12.2 Background

12.2.1 Classification of influence

12.2.2 Design precedents

12.3.1 Participants

315

12.3.2 Research through design 316

12.3.2.1 Design workshop 316

12.3.3 Analysis $\quad 319$

12.4 Results $\quad 320$

12.4.1 Research through design results $\quad 320$

12.4.1.1 Brainstorming and sketching activities $\quad 320$

12.4.1.2 Crazy eight 322

12.4.1.3 Refined design concepts 327

12.4.2 Analysis 332

12.4.2.1 Decisive design concepts 332

12.4.2.2 Coercive design concepts 332

12.4.2.3 Seductive design concepts 333

12.4.2.4 Persuasive design concepts 333 

concepts

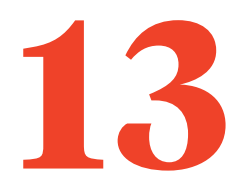

13.3.1 Participants

13.3.2 Research through design

13.3.3 Pluralistic walkthroughs

13.3.4 Analysis

13.4.1 RTD results

13.4.1.1 Smartphone

13.4.1.2 Persuasive design concept

13.4.1.3 Coercive design concept

13.4.2 Design criteria evaluation

13.4.3 Pluralistic walkthrough

351

13.4.3.1 Usability evaluation

351

13.4.3.2 Analysis 
13.5 Discussion 358

13.5.1 Persuasive influence 358

13.5.1.1 Initiation of use and restraint 358

13.5.1.2 General usability issues $\quad 359$

13.5.2 Coercive influence 359

13.5.2.1 Initiation of use and restraint 359

13.5.2.2 General usability issues $\quad 360$

13.5.3 Behaviour change contract $\mathbf{3 6 0}$

13.6 Further Design Iterations 361

13.6.1 Design iteration CO1 361

13.6.2 Design iteration CO2 $\mathbf{3 6 2}$

13.7 Conclusion 364

13.8 Limitations $\quad \mathbf{3 6 4}$

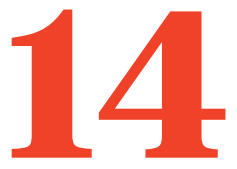

General Discussion $\quad 365$

14.1 Learned Nonuse and Initiation of Use $\quad 367$

14.1.1 Development of learned nonuse $\quad \mathbf{3 6 7}$

14.1.2 Initiation of use and the use of restraint 370

14.1.2.1 Use of restraint $\quad 370$

14.1.2.2 Self-efficacy 372

14.1.2.3 Initial design criteria $\quad 372$

14.2 Daily Activities Post Stroke $\quad 374$

$\begin{array}{lll}\text { 14.2.1 ADL } & 374\end{array}$

14.2.2 Everyday objects used as part of $A D L$

14.3 Design Prototypes and Theorising of Results $\quad \mathbf{3 7 8}$ 
14.3.1 Limited use only 379

14.3.1.1 Identify the developed learned nonuse $\quad 381$

14.3.1.2 Define $\quad 381$

14.3.1.3 Feedforward $\quad 382$

14.3.1.4 Restrain the movement 383

14.3.1.5 Action $\quad 386$

$\begin{array}{ll}\text { 14.3.1.6 Feedback } & 387\end{array}$

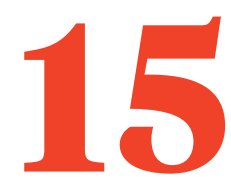

Limitations $\quad 389$

15.1 Focus on Qualitative Methods 390

15.2 Design Prototypes $\quad 390$

15.2.1 The object as a form of inquiry 390

15.2.2 Objects with a rehabilitative purpose 391

15.2.3 Use of design criteria 392

15.2.4 Evaluation of design solutions 392

15.2.5 Design for stroke survivors 393

15.3 Stroke Rehabilitation $\quad 393$

15.3.1 Literature vs clinical practice 393

15.3.2 Study sample of health professionals 394

15.3.3 Study sample of stroke survivors 395

15.4 User Generated Ideas 395 


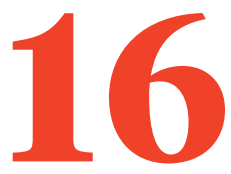

Conclusion

17

References

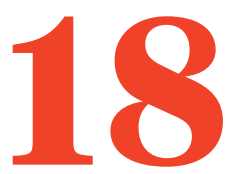

Appendices

Appendix A

Appendix B

Appendix C

545

Appendix D

593 
LIMITED USE ONLY

\section{List of Tables}


Table 1: Overview of research aims, objectives and questions

Table 2: Overview of formative usability evaluation methods employed in this study.

Table 3: List of DHBs which were contacted for this

study.

Table 4: Design elements mapped against elements of stroke rehabilitation and psychology.

Table 5: Overview of design strategies.

Table 6: Website content and hierarchy of the empathy section.

Table 7: Website content of the inspiration section.

Table 8: Employed design strategies.

Table 9: Online support groups.

Table 10: Coding example.

Table 11: Convergence coding based on the online

survey results with stroke survivors.

Table 12: Convergence coding based on the interviews

with stroke therapists.

Table 13: Convergence assessment.

Table 14: Study sample.

Table 15: Study sample.

Table 16: Formative usability evaluation. 
LIMITED USE ONLY

\section{List of Figures}


Figure 1: ICF model $\quad 48$

Figure 2: Assistive technology in the form of a chopping $\mathbf{5 3}$ board that can be used with just one hand. The board allows vegetables to be held down by the spikes on the right-hand side.

Figure 3: The handle on the secateurs work as signifiers, indicating how the hand needs to hold it.

Figure 4: The handles on the wheelbarrow require both hands $\mathbf{6 8}$ for interaction.

Figure 5: Overview of the different methods involved in this 85 research.

Figure 6: Initial design criteria.

Figure 7: The three factors in the Fogg Behaviour Model. 119

Figure 8: The red signal on the traffic lights indicates that it is $\mathbf{1 2 0}$ not safe to cross and that the driver should stop in front of it. Figure 9: Reach to grasp movement. 122

Figure 10: Design criteria set 02 which was used to evaluate $\mathbf{1 2 4}$ design concepts and prototypes during the development process.

Figure 11: Roly-poly or round bottom doll, a children's toy that rights itself when pushed over.

Figure 12: Bracelet with full functional NFC tag that is worn on the affected arm so the system recognises which arm is used.

Figure 13: The NFC bracelet worn by the user on the affected arm unlocks the functionality of the radio.

Figure 14: 3D printed radio prototype 01 with a felt sleeve to 129 muffle the sound. 
Figure 15: Prototype 02 with a wooden top part and ceramic base.

Figure 16: Prototype 03 contains a textured surface to address 131 tactile recognition deficits.

Figure 17: Prototype 04 with a changeable interaction area. 132

Figure 18: Prototype 05 has a wooden core element. 133

Figure 19: Functional elements of the final design prototype. $\mathbf{1 3 4}$

Figure 20: The user must move the affected hand close to the $\mathbf{1 3 5}$ white front area in order to turn the radio on. The radio starts bouncing in case the user does not have full control over their arm movement.

Figure 21: Back of the radio: the user can change the radio station, training interval and volume on this side. To change the training interval setting from 30s, 60s or 90 s the user pulls on the black string, which is connected to a soft potentiometer.

Figure 22: The user can detach the top component to add weight inside the bottom component to increase the challenge in the interaction.

Figure 23: The texture of the interaction area can be replaced. 137 Figure 24: Schematic overview of the technical components. 138 Figure 25: Radio board. 139 Figure 26: Arduino UNO and NFC board. 139

Figure 27: Evaluation against the design criteria set 02 . $\mathbf{1 4 1}$

Figure 28: Forms of feedback caused by an action. $\mathbf{1 5 0}$

Figure 29: Design criteria set 03.

Figure 30: Prototype 07.

Figure 31: Prototype 08 that required a downward motion. $\quad 159$ 
Figure 32: Prototype 09 which has the volume button situated $\mathbf{1 6 0}$ in the centre.

Figure 33: Components of the final design prototype.

Figure 34: Initial stage of the radio does not require a stand to $\mathbf{1 6 2}$ decrease the required wrist extension during use.

Figure 35: Advanced stage of the radio with the stand attached to the highest level. The user is required to extend the wrist to use the radio.

Figure 36: The design of the speakers.

Figure 37: The icon on the lower front component indicates that the user has to touch the radio here.

Figure 38: Interface of the app that presents the quantitative 165 use of the prototype.

Figure 39: Form and material experiments for the volume button.

Figure 40: There is no support for the fingers when the texture is solely on top of the button, which complicates use.

Figure 41: Four volume buttons that can be used with the final design prototype.

Figure 42: Technical components of the final design iteration.

Figure 43: Evaluation against the design criteria set 03. shaping.

Figure 45: Persona description of Susan.

Figure 46: Eating equipment.

Figure 47: Storyboard based on the observations. 
Figure 49: "Smart Sticks” chopsticks by Xavier Strom. 188

Figure 50: "Fluve" decanter by Nicole Hone. 189

Figure 51: Knives by Evangeline Martin named "Cirgo" that 189

rely on the intuitive use of a knife with the right hand.

Figure 52: Design strategies used to elicit a repetitive 190

movement.

Figure 53: Pouring mechanism inside of Nicole Hone's

191

"Fluve",

Figure 54: Design strategies used to offer progression in the interaction.

Figure 55: Water bottle by Jazz Klinkert that challenges the user by offering less water when the lid is rotated fully.

Figure 56: Coffee mugs and lids by Katie O’Brien.

Figure 57: "Lottiel” by Ashleigh Kennedy.

Figure 58: Design strategies used to provide feedback.

Figure 59: Design strategies used to include a behaviour contract.

Figure 60: Purpose of the design method cards.

Figure 61: Focus of the digital design method cards.

Figure 62: Structure of the website.

Figure 63: Structure of an information unit. Each page contained multiple units.

Figure 64: Information unit that describes common motor impairments of the shoulder joint.

Figure 65: First layer of information and on-demand examples as a source of inspiration.

Figure 66: Participant 01's concept for a watering can.

Figure 67: Details of the design concept. 
Figure 69: Sketches that break down the movement needed to 219 cut a bonsai tree.

Figure 70: Participant 02's concept for shears used to cut 219 bonsai trees.

Figure 71: Participants 03's concept for a tool to remove weeds from cracks.

Figure 72: Participant 04's concept for a toothbrush.

Figure 73: Refined use of the design method cards.

Figure 74: Exercises based on the design strategies.

Figure 75: ADL before and after the stroke based on the categories of the ICF.

Figure 76: Categories of tasks that participants would like to take part in again after the stroke.

Figure 77: Specific tasks that participants would like to take part in again after the stroke.

Figure 78: Tasks that participants liked doing before the stroke and would like to be able to take part in again after the stroke.

Figure 79: Tasks that have become more difficult after the stroke.

Figure 80: Categories of hobbies that participants liked to take part in before and after the stroke.

Figure 81: Hobbies that participants liked to take part in after the stroke.

Figure 82: Categories of tasks in which objects play an important role.

Figure 83: Specific objects that were named as playing an important task in daily activities after the stroke. 
Figure 84: Categories of tasks that were named by therapists as being often important to stroke survivors.

Figure 85: Tasks that are often important to stroke survivors. 262

Figure 86: Everyday objects named by therapists.

Figure 87: Assistive devices named by therapists.

Figure 88: Rehabilitation devices named by therapists.

Figure 89: Most frequently named devices.

Figure 90: Four types of influence based on the dimensions of 314 force and salience.

Figure 91: Design workshop overview.

Figure 92: Each card contained a further description and summary of the main themes on the back.

Figure 93: Overview of the three topics of the physical design method cards.

Figure 94: Crazy eight sketching activity.

Figure 95: Brainstorming outcomes and sketches during the

workshop.

Figure 96: Participant 01 crazy eight design concepts.

Figure 97: Participant 02 crazy eight design concepts.

Figure 98: Participant 03 crazy eight design concepts.

Figure 99: Participant 04 crazy eight design concepts.

Figure 100: Participant 05 crazy eight design concepts.

Figure 101: Design concept for the interface of a smartphone.

Figure 102: Design concept for an ergonomic restriction.

Figure 103: Design concept for an ergonomic restriction.

Figure 104: Details outlining the use of the app. 
Figure 105: The use of the phone as part of a balancing activity.

Figure 106: Design concept for a holding device.

Figure 107: Design concept for a handle at the back of the phone.

Figure 108: Design concept for a phone cover that restrains use.

Figure 109: Workshop outcomes.

Figure 110: Steps and employed methods.

Figure 111: Set of design criteria.

Figure 112: Standard smartphone used for testing, P00.

Figure 113: Non-functional interface.

Figure 114: Three grip elements of “Manta”, prototype P01.

Figure 115: "Manta” for left-handed use.

Figure 116: The design elements are decreasing in size.

Figure 117: The design incorporates an uneven back to limit compensatory movement patterns.

Figure 118: The two sets of packaging for the persuasive design prototype.

Figure 119: Packaging and behaviour note for P01.

Figure 120: “Cactacea”, prototype P02.

Figure 121: Packaging and behaviour note for P02.

Figure 122: Evaluation of the design against the design

criteria set.

Figure 123: Prototype P00, P01, and P02.

Figure 124: Rating of preference for the design prototypes. 
Figure 126: Design iteration of C01 that uses the bilateral

restraint in combination with ergonomic restrictions. The behaviour contract is included as a sticker.

Figure 127: Design iteration C1 can be gripped with the

affected hand and the likelihood of dropping the phone decreases.

Figure 128: Design prototype C02, which uses a handle to initiate use of the affected hand.

Figure 129: Design prototype C02 can be used as a handle or support for the affected side.

Figure 130: Factors influencing the development of learned nonuse adapted from Taub et al. (1994, p. 284). The contributions of this study are highlighted in black italic. Red \& red italic highlights refer to previous studies that outline factors contributing to learned nonuse.

Figure 131: Overcoming learned nonuse adapted from Taub et 371 al. (1994, p. 285). Initiation of use is outlined as part of 'B'. Figure 132: Initial design criteria.

Figure 133: Motivation for using information communication 376 devices (ICT) such as a smartphone after the stroke. The factors "Tracking recovery" and "Access to rehabilitation services" were named in previous studies.

Figure 134: Barriers that impact on the use of information communication devices (ICT) after a stroke.

Figure 135: Overview of design elements. 


\section{List of}

Abbreviations 
$\mathrm{ADL}=$ activities of daily living

ANZTR $=$ Australian \& New Zealand trial registration database

$\mathrm{BADL}=$ basic activities of daily living

CIMT $=$ constraint-induced movement therapy

$\mathrm{DHB}=$ district health board

FES $=$ functional electrical stimulation

HDEC $=$ Health and Disability Ethics Committee

HSA = home skill assignment

IADL = instrumental activities of daily living

ICF = international classification of functioning and disability

ICT $=$ information communication technology

MAL $=$ Motor Activity Log

MedTech CoRE $=$ Medical Technology Centre of Research Excellence

PIS $=$ participant information sheet

$\mathrm{PD}=$ persuasive design

$\mathrm{PT}=$ persuasive technology

RTD $=$ Research through Design

$\mathrm{SDM}=$ shared decision-making process

TUI $=$ tangible user interface

$\mathrm{UE}=$ upper extremity

$\mathrm{UEM}=$ usability evaluation methods 


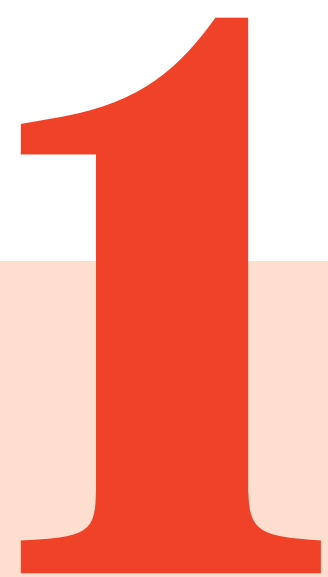

\section{Introduction}




\subsection{Motivation}

When I was doing my Master's degree, I read an article in which the author referred to Weiser's vision of ubiquitous computing. I was intrigued by his statement that technology of the future will vanish into the background as a natural consequence of its improvements. My first question was, "how would we know how to use this new kind technology?" The term "intuitive use" is something I would constantly hear as part of my job, but I rarely felt that the use of a new kind of technology had anything intuitive to it. My experience was that when I was working on a new type of interface, the user would often struggle to use it. I started to become fascinated by tangible user interfaces (TUI) and how they could be used to communicate the intended interaction in a much more precise way; for example, could a die as a tangible interface indicate multiple levels of information and interaction.

The opportunity to focus on stroke rehabilitation was made possible through the ambition of the Centre of Research Excellence in Medical Technologies (MedTech CoRE), which is an amalgamation of different healthfocused institutes in New Zealand. The MedTech CoRE brings together researchers from six institutions - the University of Auckland, the Auckland University of Technology, the University of Canterbury, Otago University, Victoria University of Wellington, and Callaghan Innovation. It builds on the collaborations established through the Consortium for Medical Device Technologies (CMDT), which was formed by these six institutions in 2012 to act as a coordinating entity for research in medical device technologies. The CMDT, funded by Callaghan Innovation, is a business-facing organisation and is the industry engagement vehicle of the MedTech CoRE.

I had the opportunity to collaborate with MedTech CoRE partners who are experts in the field of stroke rehabilitation. My research contributes to the "Flagship 5: Augmentation of upper arm stroke rehabilitation" of the "MedTech CoRE 3-year plan 2015-2017”. The goal of the Flagship is to provide personalised 
rehabilitation strategies for patients recovering from stroke using inexpensive sensor technology that can be deployed in home care settings (MedTech CoRE \& CMDT, 2018).

This close collaboration was helpful in the context of this work that includes a variety of stakeholders. I will introduce the context of this research in the following section.

\subsection{Situating the Research}

Stroke affects 15 million people worldwide and is the third most likely cause of death (McKay \& Mensah, 2004, p.18). Although the worldwide rate of stroke is declining, this rate of decline is four times slower in New Zealand where stroke is a leading factor for long-term disability (Feigin, McPherson, BarkerCollo, \& Krishnamurthi, 2014, p. 1). The risk of dying instantly from a stroke has decreased while the prevalence rate of experiencing a stroke has increased. This means that people survive this traumatic brain injury and the overall burden of stroke increases (Feigin, Forouzanfar et al., 2014, p. 245). If these observed trends continue, there will be almost 70 million stroke survivors by 2030 (Feigin, Forouzanfar et al., 2014, p. 253).

The impact that the stroke has on the survivor can be devastating, and impairments can affect physical and cognitive abilities. Around $20 \%$ of all new stroke survivors are limited in their mobility and require institutional care, while $30 \%$ of stroke patients can return to their homes (McNaughton, McRae, Green, Abernethy, \& Gommans, 2014, p. 10). Approximately 70\% of all stroke survivors experience altered arm function after a stroke, with $40 \%$ of stroke patients suffering persistent motor impairments in the affected arm (Intercollegiate Working Party for Stroke, 2012, p. 83).

I focus in this research on the functional improvement of the affected arm and hand that enables participation in daily tasks again. Stroke management guidelines recommend 60 minutes of therapy (Stroke Foundation of New Zealand \& New Zealand Guidelines Group, 2011, p. 164), but studies indicate that stroke 
survivors spend only a limited amount of time with their therapists (Bernhardt, Chan, Nicola, \& Collier, 2007, p. 43), and spend most of their day inactive and alone (Bernhardt, 2004, p. 1007; Fini, Holland, Keating, Simek, \& Bernhardt, 2017; Huijben-Schoenmakers, Gamel, \& Hafsteinsdóttir, 2009, p. 1148).

One approach that aims to address stroke-specific impairments to increase ability to perform daily activities is task-specific training. Task-specific training often uses real-world tasks or everyday tasks as the therapeutic medium in functional recovery (Hubbard, Parsons, Neilson, \& Carey, 2009, p. 180). Patients practise, for example, how to lift a cup to drink or use cutlery to eat independently. However, there is little focus is on the objects involved in the tasks that could be beneficial in the process. This might be because the task needs to be relevant to the patient to be both an extrinsic and intrinsic motivator (Hubbard et al., 2009, p. 181).

Task-specific training can help to induce neuroplastic changes at several levels in the brain using regions that have not been affected by the stroke (Arya et al., 2012, p. 193; Hubbard et al., 2009, p. 175). Essential elements of this form of rehabilitation are that the tasks are specific to the patient, randomly assigned, positively reinforced, and lead to the relearning of the whole task (Hubbard et al., 2009, pp. 181-185). Stroke survivors are required to perform hundreds of repetitions of a specific task to be able to accomplish it (Bütefisch, Hummelsheim, Denzler, \& Mauritz, 1995, p. 67; Lang et al., 2009, p. 1693). Relevant goals for the therapy can be determined with outcome measurements such as the Canadian Occupation Performance Measure (Law et al., 1990). However, such scales have been developed by therapists rather than stroke survivors.

The focus of the current research is on chronic stroke survivors with mild signs of impairment. The stroke itself is an event and the impairments are the result of this event. I will refer to the term 'mild stroke' as slight motor impairments that enable a high level of independence after the stroke (Tellier \& Rochette, 2009, p. 454). The terminology used to describe acute, subacute 
and chronic stroke survivors is not universally defined and often depends on the clinical context. It is generally agreed that the chronic stage commences six months after the stroke (Krakauer, Carmichael, Corbett, \& Wittenberg, 2012, p. 927).

I will use the term stroke survivor in this study to describe an individual who has experienced a stroke. The stroke survivors who participated in this study provided insights and feedback and were not enrolled in an active rehabilitation as part of this research. A patient implies that the individual is part of a therapistpatient relationship. I will use the term stroke patient to describe an individual who receives active rehabilitation after a stroke provided by a therapist.

Mild stroke survivors are most likely to return to their homes after being discharged from the hospital (Reynolds et al., 2001, p. 233), often without referral to rehabilitation services (Rochette, Desrosiers, Bravo, St-Cyr-Tribble, \& Bourget, 2007, p. 59; Tellier \& Rochette, 2009, p. 454). However, they are rarely focused on in the literature (Hodson, Gustafsson, Cornwell, \& Love, 2017, p. 296; Tellier \& Rochette, 2009, p. 454) and are often excluded from studies because they are expected to be almost recovered (Tellier \& Rochette, 2009, pp. 460-461), despite reports of persistent stroke-related symptoms even months later (Adamit et al., 2015, p. 667; Edwards, Hahn, Baum, \& Dromerick, 2006, pp. 155-156; Rochette et al., 2007, p. 65).

The limited functionality of the affected arm and hand can lead to compensating for the lost functionality with an increased use of the less affected arm, which can lead to a so-called learned nonuse (Sterr, Freivogel, \& Schmalohr, 2002, p. 1792; Taub, Uswatte, Mark, \& Morris, 2006). For instance, a right-handed person with an affected left hand is likely to stop using the left hand after the stroke. The person ends up eating using only the right hand and avoids food that needs both hands for cutting. Even mild impairments can lead to this suppression of movement (Han et al., 2013, p. 443) and significantly affect the performance of daily tasks. 
Telerehabilitation could be an alternative approach to address the shortage of resources and access to services for mild stroke survivors. Telerehabilitation services are "rehabilitation services [delivered] at a distance by means of electronic information and communication technology” (Rosen, 1999, p. 11). One example is the use of mobile phones to stay in touch with health professionals and remind the owner of the phone to perform rehabilitation activities. This is a relatively new approach, which was introduced in the literature in the late 1990s (Laver et al., 2013, p. 3). It comprises the provision of education and training to stroke survivors, and family members; helps monitor health consequences; and provides interventions by using different strategies and devices (Rosen, 1999, p. 11).

Telerehabilitation offers access to services for stroke survivors who might experience mobility issues and limited access to specialised services, especially in rural and remote areas. These areas often struggle to attract experienced health providers due to the perceived isolation from professional colleagues, lower remuneration, and lower associated prestige (Laver et al., 2013, p. 3; Rosen, 1999, p. 14). Telerehabilitation could further help to address mobility issues, for example travel issues caused by neurological and other impairments, long travel distances, and not having access to reliable transportation (Rosen, 1999, p. 14).

Aspects of telerehabilitation seem to be included in clinical practice, but evidence is still missing about how home-based therapy programmes that contribute to a functional improvement of the upper limb need to be structured (Coupar, Pollock, Legg, Sackley, \& van Vliet, 2012, p. 2).

Some research opportunities presented in the literature could be addressed from a design perspective. Firstly, studies on upper limb stroke rehabilitation that focus on task performance see the use of the objects as a given component in the process. There is little focus on the design of the object that could contribute to initiating use of the affected arm and hand through its form and required usage. Secondly, the concept of learned nonuse states that the neglect of the affected arm and hand in daily tasks can be overcome. However, survivors can feel reluctant 
to do so. Little focus seems to be placed on everyday tasks and involved objects that are meaningful to the survivor, and that they are motivated to be able to do again; for example, being able to cook again or work in the garden. Thirdly, the concept of learned nonuse originated from studies on animals. Findings that could contribute to the development of new treatment protocols for humans to promote the use of the affected arm have been scarce in recent years (Jones, 2017, p. 277), and the use of design objects to address compensatory movement patterns has not been explored in this context. 


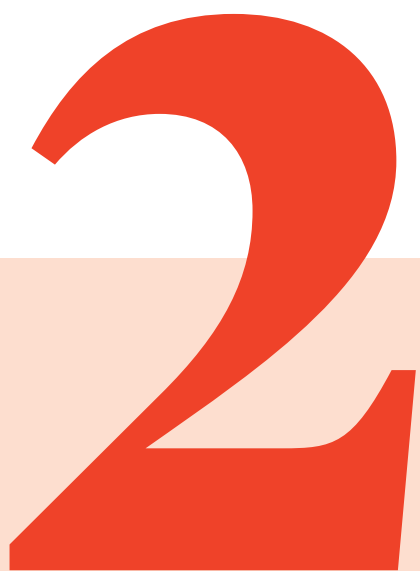

\section{Background}


This research is situated in multiple domains. Therefore, I did not carry out a systematic literature review but undertook an evaluative literature review, which reports on literature related to this study. The review will cover the areas of stroke, neurorehabilitation, psychology, motor learning principles, and design principles to form the theoretical base for my research.

\subsection{Stroke}

\subsubsection{Definition of a stroke}

A stroke will be referred to according to the definition by Sacco et al. (2013, p. 264):

A stroke is classically characterized as a neurological deficit attributed to an acute focal injury of the central nervous system (CNS) by a vascular cause, including cerebral infarction, intracerebral hemorrhage (ICH), and subarachnoid hemorrhage (SAH).

The ischaemic stroke, a focal collection of blood, accounts for most incidents, approximately $88 \%$, while the hemorrhagic stroke, the interruption of blood supply to the brain, is less common. Both forms lead to persistent brain, spinal cord, or retina cell death (Mallory, 2006, p. 34; Sacco et al., 2013, p. 266). Excluded from this definition is the transient ischaemic attack which resolves within 24 hours.

\subsubsection{Impact of a stroke}

The impact on the individual depends on the location and severity of the stroke (Mallory, 2006. p. 33). For the following section, the categories from the International Classification of Functionality and Health, the ICF (World Health Organization, 2002), will be used to describe the spectrum of problems regarding body structure, body function, activities and participation and environmental factors, see Figure 1.

The variety of symptoms and impairments after a stroke can have a significant impact on body function and the personal and social role of the survivor. The description of possible impairments is by no means extensive, but 
illustrates the complexity of this medical condition. In the following section, the focus will be on impairments of the upper limb that impact on the ability to participate in daily activities after the brain injury. Even though personal factors are part of the ICF model, there is no classification of these factors.

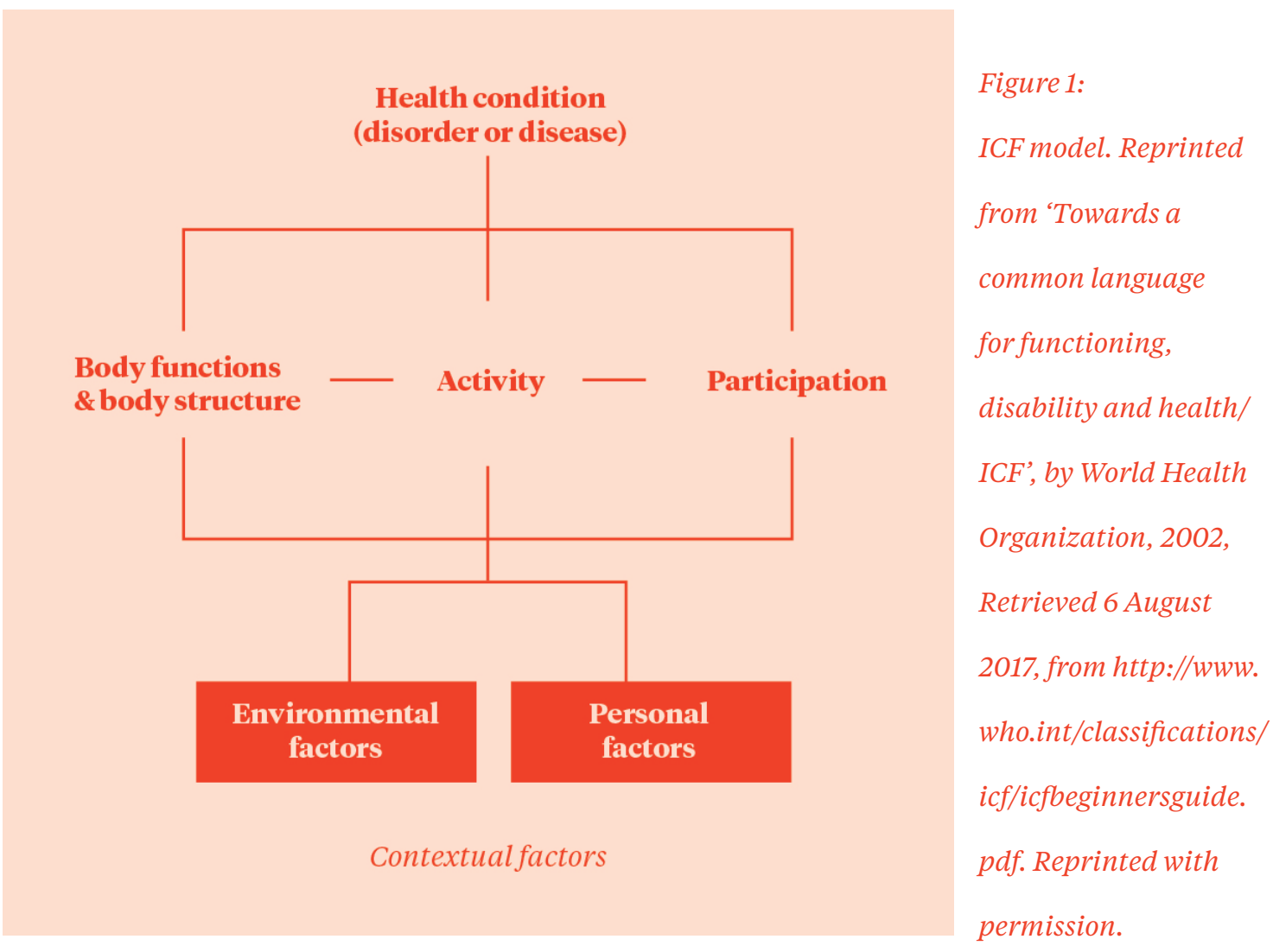

\subsubsection{Changes of body structure and function}

The changes of the anatomy of the body such as organs, limbs and their components are referred to as changes in the body structure. They can affect areas of cardiovascular, immunological and respiratory systems. Further impairments can impact the movement and structure of the upper extremity (i.e. arm), the shoulder region and the lower extremity (i.e. leg), as well as the structure of the brain and structure of the upper extremity (Geyh et al., 2004, pp. 138-139).

Changes to body function relate to physiological and psychological changes in the body (World Health Organization, 2002, p. 10) and can limit mental, 
neuromusculoskeletal, movement, and sensory abilities (Geyh et al., 2004, pp. 138139).

A focus in this section is on neuromusculoskeletal, movement-related functions and muscle strength that impacts on the performance of activities of daily living (ADL) (Pollock et al., 2014, p. 4). It needs to be considered, though, that impaired mental functioning, attention and memory function, (Stroke Foundation of New Zealand \& New Zealand Guidelines Group, 2011, p. 189) as well as emotional functions (ICF Research Branch, 2013, p. 1) can limit the participation in ADL.

Motor impairments are the most widely recognised impairment after the stroke (Langhorne, Bernhardt, \& Kwakkel, 2011, p. 1695) and cause significant limitations. The performance of an everyday task and return to life before the stroke are often severely limited (Doolittle, 1992, p. 124; Eriksson, Lindstrom, \& Ekenberg, 2010, p. 836; Tyson \& Turner, 2000, pp. 386-387; Wallenbert \& Jonsson, 2005, pp. 222-223). When the stroke affects one side of the body, it can manifest in decreased muscle functionality (hemiparesis), or paralysed motor function (hemiplegia) (DePiero, 2011a, 2011b). Common impairments of the upper limb affect the shoulder's external rotation, a partial and temporary shoulder dislocation, elbow extension, forearm supination, and wrist and finger extension (Pollock et al., 2014, p. 4; Stroke Foundation of New Zealand \& New Zealand Guidelines Group, 2011, p. 2000). The inability to use the arm inevitably leads to a swelling of the hand (Stroke Foundation of New Zealand \& New Zealand Guidelines Group, 2011, pp. 202-205).

Up to $85 \%$ of all survivors experience hemiparesis (Levin, Klein, \& Wolf, 2009, p. 314) and up to $70 \%$ of survivors experience altered arm function after a stroke. Forty percent of stroke survivors are left with a persistent lack of function in the affected arm (Intercollegiate Working Party for Stroke \& Royal College of Physicians of London, 2012, p. 83), which eventually affects their quality of life (Clarke, Lawrence, \& Black, 2000, p. 124).

Another factor that plays an essential role in everyday tasks is the ability 
to feel textures and sense the relative orientation of one's body parts. Damage to the sensory motor cortex, subcortical areas, and cerebellum sensory functions, as well as proprioceptive functions, can be altered (Pollock et al., 2014, p. 4). Common impairments are a decreased or enhanced sensation of touch, a numbness of tactile and vibratory sensations (Doyle, Bennett, Fasoli, \& McKenna, 2010, pp. 2-3), shaking, or a burning sensation (ICF Research Branch, 2013, p. 2).

\subsubsection{Activities and participation in everyday activities}

The outlined limitations and impairments significantly influence the performance of daily activities, and the consequences on the individual can be manifold. Survivors often feel a loss of control after a stroke that turns their life upside down (Salter, Hellings, Foley, \& Teasell, 2008, pp. 597-598). Common daily activities that are affected comprise mobility, communication, learning and applying knowledge, self-care, general tasks and demands, domestic life, interpersonal interactions and relationships, major life areas, community, social and civic life (Geyh et al., 2004, p. 138). Daily activities that involve self-care and tasks that relate to domestic life, for example being able to dress oneself, are often the primary focus during the early stage of the rehabilitation process (Brown et al., 2014, pp. 1022-1023; Duncan et al., 2005, p. e104).

The use of public transport and cars can become a major issue (ICF Research Branch, 2013, p. 8) that consequently affects professional work life as well as community, social, and civic life. Acquiring and keeping a job is therefore often difficult after the event, especially when the survivor is not allowed to drive again. Younger stroke survivors in particular can suffer under this limitation (Lister, 1999; McKevitt, Redfern, Mold, \& Wolfe, 2004, p. 1501).

Participation in daily activities and especially participating in society can be compromised by the limited ability to communicate. It is important to understand that cognitive and emotional limitations potentially increase withdrawal from tasks involving social participation; for example, resuming hobbies. Emotional difficulties commonly involve depression, anxiety, lack of 
trust in one's body, fatigue, and emotional changes such as swiftly getting angry.

Due to emotional changes caused by the stroke individuals can experience symptoms that contribute to the feeling of loss that vary from severe mood changes, fatigue as well as depression post-stroke and a limited memory and attention ability, a lack to self-evaluation of the condition, attention or memory dysfunction and an altered perception of time. They all have a significant impact on the individual's ability to participate in activities and impact on family as well as intimate relationships (Bogousslavsky, 2003, p. 1046; Choi-Kwon \& Kim, 2011, p. 334; Hackett, Yapa, Parag, \& Anderson, 2005, p. 8; Stroke Foundation of New Zealand \& New Zealand Guidelines Group, 2011, p. 212). Social activities like going to events such as movies or sports event are often affected by factors like fatigability, stress sensitivity, sensitivity to sound and light and emotional liability (Carlsson, Möller, \& Blomstrand, 2004, p. 1378). All these factors can furthermore contribute to the risk of social isolation and decrease the level of active participation in daily activities (Mukherjee, Levin, \& Heller, 2006, pp. 27-29; Taule \& Råheim, 2014, pp. 2112-2116).

Daily life after stroke is experienced by stroke survivors as a constant struggle to manage limitations. Survivors with "just" mild impairments often start to hide their impairments, even though they are still detectable months after the stroke (Taule \& Råheim, 2014, p. 2116). The reasons for this are manifold and vary from preventing feeling stigmatised to the feeling of not being able to fulfil the expectation of being fully recovered (Carlsson, et al., 2009, p. 780). It also appears that self-affirmation is created among mild stroke survivors by following the mantra "this is good enough" (Carlsson, et al., 2009, pp. 777-779). This attitude potentially contributes to the development of learned nonuse (Carlsson, et al., 2009, p. 778).

\subsubsection{Environmental factors}

The influence of environmental factors can arise from different areas such as service systems and policies, support and relationships, attitudes, natural 
environment, and human-made changes to the environment, interactions with immediate family members, as well as health services (Geyh et al., 2004, pp. 138139). They can be barriers as well as facilitators that contribute to the recovery process.

Research that focuses on the influence of services, system and policies is scarce, even though they can have a significant influence on outcomes after the stroke (Geyh et al., 2004, p. 140). One example is access to rehabilitation services, which can differ significantly between countries. They have been mentioned as facilitators as well as barriers in the process (Algurén, Lundgren-Nilsson, \& Sunnerhagen, 2009, p. 1589; Rochette, Desrosiers, \& Noreau, 2001, p. 2001; Sumathipala, Radcliffe, Sadler, Wolfe, \& McKevitt, 2012, p. 39). Therapists, in their role as healthcare providers, are seen as experts in the process who increase rehabilitation outcomes (Plant, Tyson, Kirk, \& Parsons, 2016, p. 924). However, their role seems to be more critical during the immediate period following the stroke which indicates a lack of support for stroke survivors in the community due to the fact that therapists might be less available later in the recovery process (Algurén, et al., 2009, pp. 1586-1589).

The reduction of physical barriers such as architectural obstacles in people's homes seems to be a traditional approach in the context of minimising environmental barriers. However, little attention has been given to how the environment can be used actively to facilitate the recovery process (Rochette et al., 2001, p. 559).

Assistive technology (AT) is used after a stroke to compensate for the loss of functionality of the upper and lower limb and enable a safe discharge into the community. AT is used as part of activities involving employment, mobility or communication (Algurén, et al., 2009, pp. 1586-1587; ICF Research Branch, 2013, p. 11). Commonly provided products are used for bathing, walking, and home adaptations such as modification of stairs (de Barros, Duarte, \& Cruz, 2009, p. 105; Gosman-Hedström, Claesson, \& Blomstrand, 2002, pp. 114-115; Sumathipala 


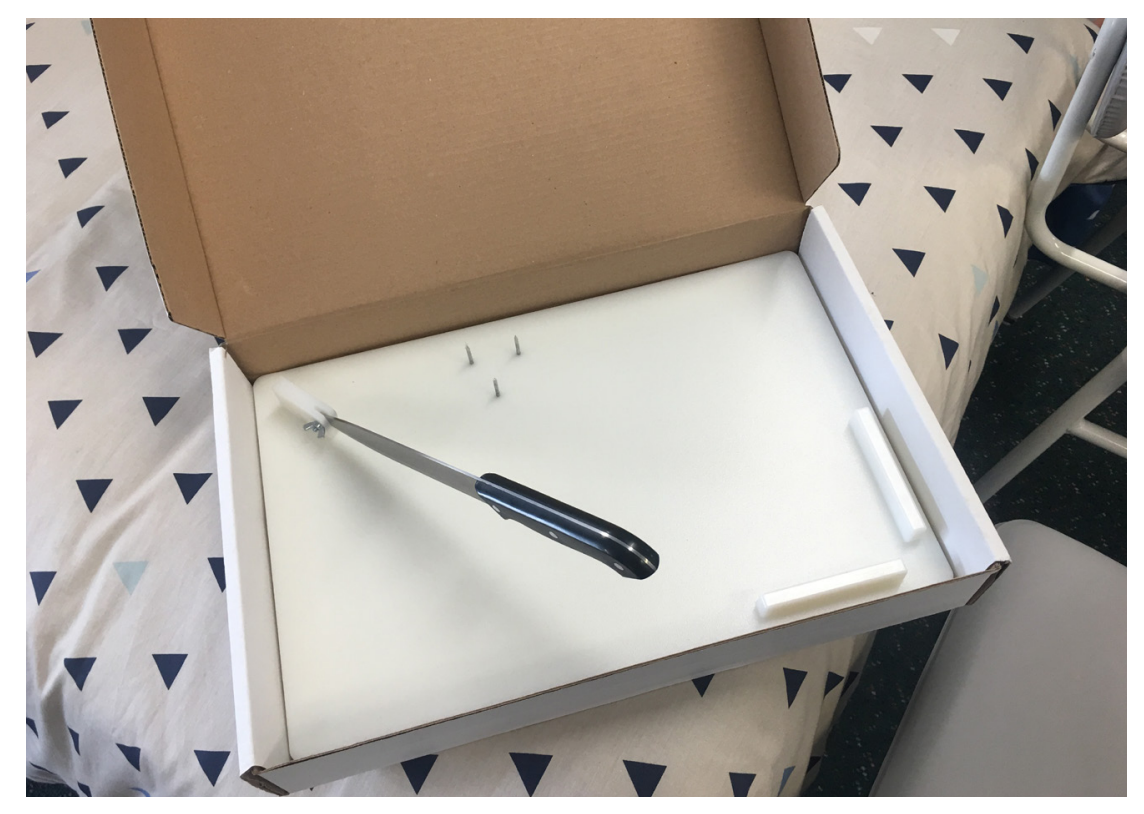

Figure 2:

Assistive technology in the form of a chopping board that can be used with just one hand. The board allows vegetables to be held down by the spikes on the right-hand side.

et al., 2012, p. 37). Products and technology that fall into the category of assistive products help compensate for the loss of functionality rather than contribute to the rehabilitation process. Figure 2 shows an example of a chopping board that can be used with just one hand. While these objects may help stroke survivors carry out everyday activities again, I argue that they also contribute to increased learned nonuse, as they help avoid the use of the affected limb. Everyday objects could be designed to be easier for stroke survivors to use and help them use their affected limbs as well, therefore contributing to their recovery.

Social support and positive attitudes after a stroke can be beneficial in the recovery process. However, immediate family members often must take over the role of the primary caregiver, providing extensive and comprehensive care after the stroke, which can be physically and emotionally draining (Andrew, Kilkenny, Naylor, Purvis, \& Cadilhac, 2015, pp. 1070-1072; Bugge, Alexander, \& Hagen, 1999, p. 1519; Murray, Ashworth, Forster, \& Young, 2003, p. 805). Overprotective family members or negative attitudes of neighbours contribute to a fear of stigmatisation and feelings of vulnerability and dependency after the stroke, consequently evoking an aversion to using assistive devices (Sumathipala et al. 2012, p. 40). 


\subsubsection{Stroke population}

Annually, 15 million people worldwide experience a stroke causing death in a third of them. Another third is left permanently impaired, disabled and experiencing handicaps (Wolfe, 2000, pp. 280-283, WHO, 2017). In New Zealand, it is the third most likely cause of death (Ministry of Health, 2009, p. 13), with 70,000 people having experienced a stroke in 2012/13 (Ministry of Health, 2013, p. 17). Three million women and 2.5 million men die from stroke each year worldwide (McKay \& Mensah, 2004, pp. 46-47) and because of their longer life expectancy, women are more likely to experience a stroke compared to men (Seshadri et al., 2006, p. 348). However, in New Zealand there is slightly increased risk for men compared to women (Ministry of Health, 2013, p. 17). In this country, the highest risk group are people aged 75 years and over (Ministry of Health, 2013, p. 17), which reflects the global risk for people over 55 years to experience stroke (McKay \& Mensah, 2004, p. 25).

Increased risk has been associated with some ethnicities (McKay \& Mensah, 2004, p. 52). This might offer a potential explanation for why in New Zealand the age-sex-standardised death rates from stroke are higher for Māori than nonMāori (Robson, Harris, \& Te Rōpū Rangahau Hauora a Eru Pōmare, 2007, p. 147), and why there is an increased risk for Pacific peoples (Carter et al., 2006, p. 59). Furthermore, Māori and Pacific people are often younger when they experience a stroke compared to those of European and Asian descent (Carter et al., 2006, p. 58). This causes additional issues for the survivor regarding loss of employment and access to funding (Daniel, Wolfe, Busch, \& McKevitt, 2009, pp. e433-e435). Often access to appropriate services is more restricted for people under 65 years (Fink, 2006, p. 1). Māori and Pacific people are more likely to live at home after a stroke and are more likely to be dependant compared to stroke survivors of European descent. Those differences in functional outcomes and living situations among Māori and Pacific descendants after a stroke have been explained by differing attitudes about caring for dependent relatives (McNaughton et al., 2011, 
p. 964) and a stronger sense of family compared to those of European descent (Kiata \& Kerse, 2004).

\subsubsection{Recovery process}

The recovery process after a stroke is complex but is likely influenced by two factors: the learning-dependent process and spontaneous neurologic recovery (Langhorne, Bernhardt \& Kwakkel, 2011, p. 1693). The concept of spontaneous neurological recovery (Gresham, 1986, p. 359) is often used to explain why improvements of motor impairments seem to plateau after the first weeks in the lower limb (Kwakkel, Kollen, \& Lindeman, 2004, p. 283); in the upper limbs, even within the first three months (Zarahn et al., 2011, p. 2716).

This study focuses on improvements to motor impairments of the upper limb that can take place in two different forms: The recovery of impairment or true recovery and the recovery of function (Zarahn et al., 2011, p. 2712). A number of concepts try to explain how true recovery takes place and why the most significant gains seem to occur early on after the stroke. The concepts comprise the restitution of non-infarcted penumbral area, the resolution of diaschisis and tissue repair (Kwakkel et al., 2004, pp. 280-290).

The concept of tissue repair outlines that the brain can induce functional and anatomical reorganisation of the central nervous system. This capacity of the brain to modify its structure or function based on gains made through learning is called plasticity (Lebeer, 1998, p. 352). The different factors that contribute to plasticity and the exact time frame in which it occurs, however, are still poorly understood (Kwakkel et al., 2004, p. 290).

The second form of improvement of motor impairments is the recovery of function (Zarahn et al., 2011, p. 2712) which can be caused by compensatory movement. Compensatory movement is the increased use of the less affected side to compensate for the loss of functionality of the affected arm and hand (Cirstea, \& Levin, 2007, p. 398). This can lead to a learned nonuse of the affected arm and hand (Taub, Uswatte, Mark, \& Morris, 2006). This research focuses on an initiation 
of use to overcome the learned nonuse of the affected arm and hand.

\subsubsection{Compensatory movement}

The following section will outline the principles of motor learning to describe the development of compensatory movement. The principles of motor learning used within stroke rehabilitation are based on the hypothesis that the stroke has caused a loss of skilled motor behaviour. Motor learning that aims to reestablish movement patterns similar to how they were before the stroke is situated under the concept of true recovery (Levin et al., 2009, p. 315).

Motor learning in functional recovery, on the other hand, can be due to compensation of movement that uses new movement patterns and alternative motor elements (Levin et al., 2009, p. 315). This current research focuses on compensatory movement, which is the increased use of the less affected side to compensate for the loss of motor abilities. Compensatory movement patterns are different compared to movement patterns before the stroke. Examples include the extension of the hand via trunk rotation rather than elbow extensions, or relying on the less affected side to perform a task rather than using both arms (Jones, 2017, p. 270).

Compensatory movement patterns are a learned behaviour of the stroke survivor, which can be overcome to initiate the use of the affected arm and hand (Adkins, Voorhies, \& Jones, 2004, p. 473; Kwakkel et al., 2004, p. 291). However, this definition of compensatory movement disregards the different dimensions in which compensation can take place. Levin et al. (2009, pp. 135-316) proposed a definition based on the ICF model, which distinguishes between a neuronal, performance, and functional level. This current research focuses on functional compensation; for example, using just one hand instead of two to open a packet of chips (Levin et al., 2009, pp. 316-317).

Compensatory movement patterns of the upper limb can take place in the form of an extensive trunk and shoulder abduction. They seem to offer advantages in the short term; for example, enabling the stroke survivor to lift a glass by relying 
on an extensive trunk movement using internal rotation and scapular elevation to assist arm and hand transport (Levin, Michaelsen, Cirstea, \& Roby-Brami, 2002, p. 178; Michaelsen, Jacobs, Roby-Brami, \& Levin, 2004, pp. 170-171).

Nonetheless, this kind of movement is based on atypical movement patterns, causing problems in the long-term (Cirstea \& Levin 2007, pp. 398-399) such as joint fractures, pain, and discomfort and can eventually lead to a learned nonuse of the affected side (Ada, Canning, Carr, Kilbreath \& Shepherd, 1994, pp. 246-249). They further impact on possible gains that could be made in the rehabilitation process (Allred, Maldonado, Hsu and, \& Jones, 2005, p. 301; Levin et al., 2009, p. 315; Taub, Uswatte, Mark, et al., 2006).

There is an expectation that most stroke survivors can recover approximately 70\% of their initial impairment (Prabhakaran et al., 2007, pp. 68-69). It is difficult, though, to narrow down how functional improvement occurs (Buma, Kwakkel, \& Ramsey, 2013, pp. 709-710; Krakauer, 2006, p. 87) and how much compensatory movement an individual has developed. Assessment scales commonly used in human and animal studies are often unable to distinguish between real recovery and compensation (Murphy \& Corbett, 2009, p. 861). Therefore, often distinctions are not made regarding improvements made after the stroke between true recovery, compensatory movement, or a combination of both. The onset of compensatory movement is therefore unclear, and further research is needed to determine factors contributing to it. This current research focuses on compensatory movement patterns and ways to address it using design objects. To design such objects, it is important to understand how learned nonuse starts to develop and which factors contribute to it.

\subsubsection{Learned nonuse and initiation of use}

The concepts of learned nonuse and the consequent initiation of use are based on studies on primates. Studies showed that after a neurological insult causing nerve damage to the upper limb, the animals stopped using the affected limb in free situations and neglected the affected limb even though they were 
able to use it. It was argued, therefore, that nonuse has a learned component to it (Knapp, Taub, \& Berman, 1963; Mott \& Sherrington, 1894; Taub et al., 1994, p. 282; Taub, 2012, p. 156).

The initial studies showed that the primates started to neglect the affected forelimb, which had received a surgical nerve interruption. Failed attempts to use the arm, pain, and incoordination led to falls and dropping food contributing to a behaviour suppression and masked ability to use the affected forelimb. At the same time compensatory movement patterns were positively reinforced causing that the less effective behaviour was strengthened. This caused a neglect of the affected limb and learned nonuse within two to six weeks after the surgery (Taub et al., 1994, p. 284).

The studies further indicated that the self-taught behaviour could be overcome by applying a physical restraint on the less affected arm to increase motivation to use the affected one. The physical restraint is part of the increased motivation outlined as part of the schematic model for overcoming learned nonuse (Taub et al., 1994, p. 285):

- Learned nonuse,

- masked recovery of limb use,

- increased motivation access function

- affected limb used,

- positive reinforcement,

- further practice,

- more reinforcement,

- limb used in life situations permanently.

It seems, though, that once the restraint was removed, the primate started compensating for the affected side again. Further studies (Taub et al., 1994, p. 282) showed that persistent results need to be based on the three elements of:

- restraining the intact arm for an extended amount of time,

- training specific movements such as grasping food, and 
- extending the limb and training the movement through approaching the desired movement in small steps, a technique referred to as shaping.

Studies on humans confirmed that the concept applied to stroke survivors as well (Ostendorf \& Wolf, 1981, p. 1027; Taub et al., 1994, p. 284; Taub, 2013, p. 127). The intervention named constraint-induced movement therapy (CIMT) was based on these observations and is a promising intervention for upper limb arm function (Langhorne et al., 2011, p. 1699; Langhorne, Coupar, \& Pollock, 2009, p. 759; Rensink, Schuurmans, Lindeman, \& Hafsteinsdottir, 2009, p. 747). It combines task-specific training with high intensity in the form of many repetitions of a particular movement, to contribute to the process of functional recovery (Kwakkel et al., 2004, p. 295).

There seems to be a clear correlation between the inability to perform tasks and a functional degradation (Dancause, Nadeau, \& Rossignol, 2015, pp. 339-341) such as muscle contractures due to a reduced level of movement (Pollock et al., 2014, p. 4). Nevertheless, there is little focus in studies on the concept of learned nonuse (Sunderland \& Tuke, 2005, p. 89) and contributing factors in humans. It is further unclear what the relationship is between the ability to perform a movement and spontaneous use (Andrews \& Steward, 1979, p. 47; Sterr, et al., 2002, pp. 1729-1730; Stewart \& Cramer, 2013, pp. 1115-1116). Andrews \& Steward (1979, p. 45), for example, reported that participants chose not to use the affected arm at home even though they could use it in the hospital environment.

\subsubsection{Stroke rehabilitation}

Stroke rehabilitation comprises different approaches such as biomedical, psychological, social, educational, and vocational interventions (Kwakkel et al., 2004 , p. 81) that aim to enable each stroke survivor to reach their highest physical and psychological potential after the stroke (Mallory, 2006, p. 51). An effective rehabilitation should be tailored to the individual needs and impairments (Stroke Foundation of New Zealand \& New Zealand Guidelines Group, 2011, p. 97). It should aim to support a level of functional independence that allows 
a safe discharge into the community (Kwakkel et al., 2004, p. 81) while taking measures to prevent a stroke recurring, as well as secondary disabilities caused by physical and psychological impairments (Gresham et al., 1997, p. 1522). Motor impairments are commonly treated with physiotherapy interventions including neurophysiological, motor learning, and orthopaedic approaches (Pollock, Baer, Pomeroy, \& Langhorne, 2007, pp. 5-10; Pollock, Baer, et al., 2014, pp. 2-3).

It needs to be emphasised at this stage that each recovery process is unique and depends on different factors such as infarct size and location, prestroke comorbidities, education and experience, age, socioeconomic status, and caregiver status (Cramer, 2008, p. 278). Even though the concept of spontaneous recovery suggests a specific time frame where improvements can occur, gains and improvements can be achieved months or even years after the stroke (Cramer, 2008, p. 278; Teasell et al., 2012, p. 461). This current research focuses on stroke survivors who have reached the chronic stage of their stroke recovery and live at home. It has been shown that they still have the potential to improve and benefit from appropriate stroke interventions.

\subsubsection{Motor learning in neurorehabilitation}

The following section will focus on rehabilitation interventions based on motor learning principles. Those principles have been chosen because even though there are apparent differences between true recovery and compensation, both principles rely on the process of learning a particular movement pattern (Krakauer, 2006, p. 86).

Motor learning principles offer a conceptual model for the difference between real recovery and compensatory movement. Motor learning principles are incorporated into rehabilitation interventions to initiate the use of the affected arm and hand. Principles of effective interventions are based on a number of steps, see below. The last principle is a crucial element in the context of motor learning (Langhorne et al., 2011, p. 1695).

- The involvement of a multidisciplinary team; 
- a high engagement in the process by the stroke survivor and family members;

- goal setting;

- high-intensity practice; and

- context and task-specific training.

A number of neurorehabilitation approaches are based on principles of motor learning (Krakauer, 2006, pp. 87-89; Krakauer, 2015, p. 59):

- constraint induced movement therapy (CIMT);

- interactive robotic therapy;

- arm ability training;

- electromyogram-triggered neuromuscular stimulation;

- virtual reality-based therapy;

- action observation; and

- bilateral priming.

CIMT, interactive robotics therapy, and virtual reality-based therapies use objects as part of the intervention. The three interventions are outlined in the following section.

\subsubsection{Constraint-induced movement therapy}

Constraint-induced movement therapy (CIMT) is based on the concept that a portion of the functional deficits is caused by the development of compensatory movement patterns (Taub, Uswatte, Mark, et al., 2006). The stroke survivor has latent motor abilities that have become neglected due to compensatory movements.

The first studies primarily focused on chronic stroke survivors with mild to moderate impairments using a restraint to overcome the learned nonuse (Taub, 2013, p. 132). In studies on acute and subacute stroke survivors, the restraint is more likely to prevent compensatory movement patterns (Krakauer, 2006, pp. 87-88; MacKenzie \& Viana, 2016, pp. 217-216).

The intervention comprises four main components that evoke an initiation 
of use (Taub, Uswatte, Mark, et al., 2006, p. 259; Taub, 2012, p. 161; 2013, pp.129131):

- shaping, which is a training technique;

- a repetitive, task-oriented training in the form task practice;

- a physical constraint or restraint such as a mitt or a sling that is worn on the less affected arm;

- a transfer package which includes a set of behavioural techniques.

Shaping is a training method that prompts task performance to initiate use of the affected hand. It involves some standard techniques such as approaching the task in small steps, immediate and frequent feedback about improvements, coaching the correct movement and cueing to avoid interruptions of the movement (Taub, 2013, pp. 129-130). The task-oriented training focuses on contextualising the movement and is performed continuously for 15-20 minutes (Morris, Taub, \& Mark, 2006, p. 259). The use of the restraint in the form of a mitt or cast aims to reduce compensatory movement patterns (Morris et al., 2006, p. 264). The transfer package includes a set of techniques that aim to evoke a feeling of responsibility and help transfer gains from the rehabilitation environment to the real world (Taub, 2013, p. 130). In the context of motor learning, CIMT is based on the principles of reinforcement through verbal instruction and rewarded practice, which is included in all four components (Krakauer, 2015, p. 60).

It is unclear at this stage which of the CIMT components contributes most to the positive outcome of the intervention and how it can be implemented into clinical practice (Krakauer, 2006, p. 88; Viana \& Teasell, 2012, p. 110). Alternative methods of delivering the intervention include a partially self-directed CIMT protocol, reduced training hours, home based interventions (MacKenzie \& Viana, 2015, p. 221), and automated working stations to train shaping tasks (Taub, Lum, Hardin, Mark, \& Uswatte, 2005).

CIMT remains highly controversial due to a number of limitations. Firstly, its primary focus is on the chronic stroke population where recovery is expected 
to have plateaued. It is outside clinical practice of stroke therapists such as occupational therapists to provide rehabilitation at this stage. Historically, interventions for chronic stroke survivors would focus on compensation for lost motor abilities rather than a rehabilitation of such. CIMT employs a methodology based on principles of behaviour analysis, which is unfamiliar to most therapists (Taub, 2013, p. 133).

Secondly, CIMT is criticised for its limited generalisability due to its focus on mild stroke survivors and embedded inclusion criteria that require highly mobile participants. According to estimates, just $6 \%-10 \%$ of all stroke survivors meet these criteria (Dromerick et al., 2009, p. 196; Viana \& Teasell, 2012, p. 2012; Wolf et al., 2006, p. 2098).

Thirdly, therapists and stroke survivors are often hesitant to use CIMT because of the intensity of training needed and the applied constraint (Page, Levine, Sisto, Bond, \& Johnston, 2002, pp. 57-59). The original CIMT protocol outlines 6 hours of intense training with the therapist (MacKenzie \& Viana, 2015, p. 220), which seems to be unfeasible to include in clinical practice due to financial constraints. The use of the physical restraint can also be a safety concern from the therapist's perspective as it impacts on the balance of the stroke survivor (Page et al., 2002, p. 59). Stroke survivors, on the other hand, can feel reluctant to wear the restraint for an extended period (Page et al., 2002, p. 57).

CIMT uses specific products in the form of physical restraints, specifically the cast or mitt and everyday objects, to train shaping tasks. However, there is no focus on the use of the object to restrain movement. This current research investigates how the design of an everyday object can facilitate an initiation of use of the affected arm without the need for a physical restraining device. It also further investigates how the restraining effect can be provided outside a clinical setting to reduce associated costs.

\subsubsection{Interactive robotic therapy}

The use of interactive robots offers an opportunity to control and measure 
therapeutic efficacy objectively. They can be used to intensify practice, operating for an unlimited amount of time. They train complex movements and offer assistance in active as well as passive forms at different levels of complexity (Krakauer, 2006, pp. 88-89; Xie, 2016, pp. 48-51). However, a limitation of these end-effector robots is that they train just certain movements and cannot independently control multiple joints of the upper limb. Exoskeleton robots, on the other hand, can mimic the kinematic structure of the human limb and operate alongside them (Xie, 2016, p. 32).

Different end-effector robots are used in the context of stroke research, such as MIT Manus (Krebs et al., 2004), the Mirror Image Movement Enhancer (MIME) (Lum et al., 2006), as well as passive, non-motorised exoskeletons such as the Dampace (Stienen et al., 2007), T-WREX (Sanchez et al., 2006, pp. 379-382) and active, motorised exoskeletons including the EXO-UL7 (Kim et al., 2013, pp. 154-155), and the ARMin III (Nef, Guidali, Klamroth-Marganska, \& Riener, 2009). Commercially available end-effector robots include products such as the InMotion Arm $^{\mathrm{TM}}$, InMotion Wrist ${ }^{\mathrm{TM}}$ and InMotion Hand ${ }^{\mathrm{TM}}$ (Bionik, 2017), the ArmeoPower, ArmeoSpring and ArmeBoom (Hocoma, 2017).

Similar to CIMT are robotics based on motor learning principles of reinforcement as a core learning strategy. The exact learning principles used as part of robotic therapy are rarely described (Krakauer, 2015, pp. 60-61) which leaves it unclear how an initiation of use is evoked.

\subsubsection{Virtual reality-based therapy}

Virtual reality-based therapies use computer-generated virtual environments or virtual realities that the user can experience through different human-machine interfaces (Holden, 2005, pp. 187-188). Different levels and forms of immersion provide a motivating environment to conduct repetitive training, which can be augmented by different forms of feedback (Holden, 2005, p. 189; Krakauer, 2006, p. 89).

The virtual environment can be experienced through different interfaces 
such as head mounted displays, projections, liquid crystal display (LCD); flicker glasses; multi-person, room-sized, high-resolution 3D audio; and video systems such as the CAVE ${ }^{\mathrm{TM}}$ (Holden, 2005, p. 188). Motion can be tracked with devices such as commercial gaming consoles like the Microsoft Kinect (Lee et al., 2016, p. 740) or the Nintendo Wii (Givon, Zeilig, Weingarden, \& Rand, 2016, p. 386). Other forms of tracking systems include magnetic tracking devices, gloves like the RAPAEL Smart Glove ${ }^{\mathrm{TM}}$ (Shin et al., 2016, p. 3) or the CyberGlove (Merians, Poizner, Boian, Burdea, \& Adamovich, 2006, p. 258). In New Zealand, the ableM and ableX (ableX Healthcare Limited, 2017) is available to stroke survivors for purchase or rent. Feedback can be provided during the training in a tactile or auditory form. One example is the Hand Dance Pro ${ }^{\mathrm{TM}}$ that uses auditory cues and feedback in the form of music and visuals (Combs et al., 2012, p. 1293).

Virtual reality uses instructions and principles of learning by imitation as motor learning techniques. It is unclear whether any adverse effects are associated with this kind of stroke rehabilitation (Crosbie et al., 2007, p. 1144) - for example, motion sickness. It also remains unclear whether the gains made in the virtual world are transferable into the real world (Krakauer, 2006, p. 89) and how an initiation of use of the affected arm and hand can be systematically evoked as part of the intervention.

In summary, it can be said that specific equipment, as well as everyday objects, has been mentioned as part of stroke interventions that focus on motor learning approaches. However, it remains unclear how the objects could facilitate an initiation of use of the affected arm and hand. In fact, some everyday objects targeted at helping stroke survivors may be counterproductive to their rehabilitation. For instance, the chopping board shown in Figure 2 helps people avoid using the affected arm, therefore reinforcing learned nonuse even in people who may have enough mobility to perform some everyday tasks. Consequently, it is unclear which kind of everyday objects offer the greatest potential to evoke an initiation of use for chronic stroke survivors. 


\subsubsection{The everyday object}

This last part of the background review focuses on the characteristics of everyday objects and how the design of the form of an object could be used to facilitate an initiation of use of the affected arm.

Csikszentmihalyi and Halton (1981, p. 53) assign objects an aura of action opportunities that can expand or restrict the scope of action possibilities to its owner. The authors describe the relationship between objects and people to be founded by the object's role to be used as part of action activities or for contemplation purposes. They further see an object to be meaningful to the self or to have a meaning when it is used in the context to connect with other people (Csikszentmihalyi \& Halton, 1981, p. 112). A bike is, for example, an object for action purposes that relates to the self, while a photo is used for contemplation relating to others. This current study focuses on everyday objects used in the home environment for action purposes; for example, a toothbrush used to brush one's teeth or a pan used for cooking food. However, the central question is how could such an object be used to evoke use with one specific side of the body? In the following section, interaction with an object and inherent qualities of it will be elaborated on.

\subsubsection{Interaction}

To use a three-dimensional object, some sort of interaction is necessary. Interaction can be translated literally as "action-in-between”. It indicates what is going on between a human being, on the one hand, and an object, on the other. Both entities have a relationship to each other and act upon each other (Verbeek, 2015, p. 26). Interaction consists of several layers that take a person from an aim to a goal. The actual interaction is physical (Verbeek, 2015, p. 28).

\subsubsection{First encounter and affordance}

Before the actual interaction takes place, the user validates the different elements of the object. This first visual encounter with the object is taking place on the visceral level (Norman, 2005, p. 36) and does not require interaction with 
the product. During this first visual encounter, the perceived different qualities of an object elicit different behaviours in the user, referred to as affordance. The user needs information from the product to guide intuitive interaction with the object. The information is provided both in the form of feedforward and feedback elements (Locher, Overbeeke, \& Wensveen, 2010, p. 73). The feedforward elements are evident in the artefacts' appearance and offer information about its use. Preexisting knowledge, experience and context of use all influence the perception of the feedforward elements. A focus of the current research is on feedforward design elements that can be used to restrain movement to evoke initiation of use. An efficient design solution would indicate and require the use of the affected hand, while a non-efficient design solution would allow the user to compensate for the affected side.

Gibson (1977) introduced the term affordance in 1977, to describe action possibilities offered by the environment. He derived this term from the concepts of valence, invitation, and demand used in gestalt psychology (Gibson, 2011, p. 138). The term has been used in the field of neuroscience and biobehavioural research; however, with a multitude of meanings and conceptual cognitive foundations (Osiurak, Rossetti, \& Badets, 2017, p. 409).

Norman (1990), influenced by Gibson's concept of affordance, introduced it to the context of human-computer interaction (Norman, 1990, pp. 8-11). Later, he distinguished between real affordance and perceived affordance. Real affordance comprises static elements inherent in the material, for example a door that can open. Perceived affordance is based on our pre-existing knowledge. One example is the knowledge to rotate the door knob to open a door. Kannengiesser and Gero (2012, pp. 53-55) base their definition on Norman's definition of perceived and real affordance and distinguish between reflexive, reactive, and reflective affordance. Similar to this is Akrich and Latour's (1992, pp. 259-264) definition. Affordance communicates action possibilities, while signifiers indicate where the action should take place - such as door handles that communicate that the door needs to 
be pulled instead of pushed (Norman, 2013, p. 14). Figures 3 and 4 show examples of garden equipment that afford a certain kind of interaction.

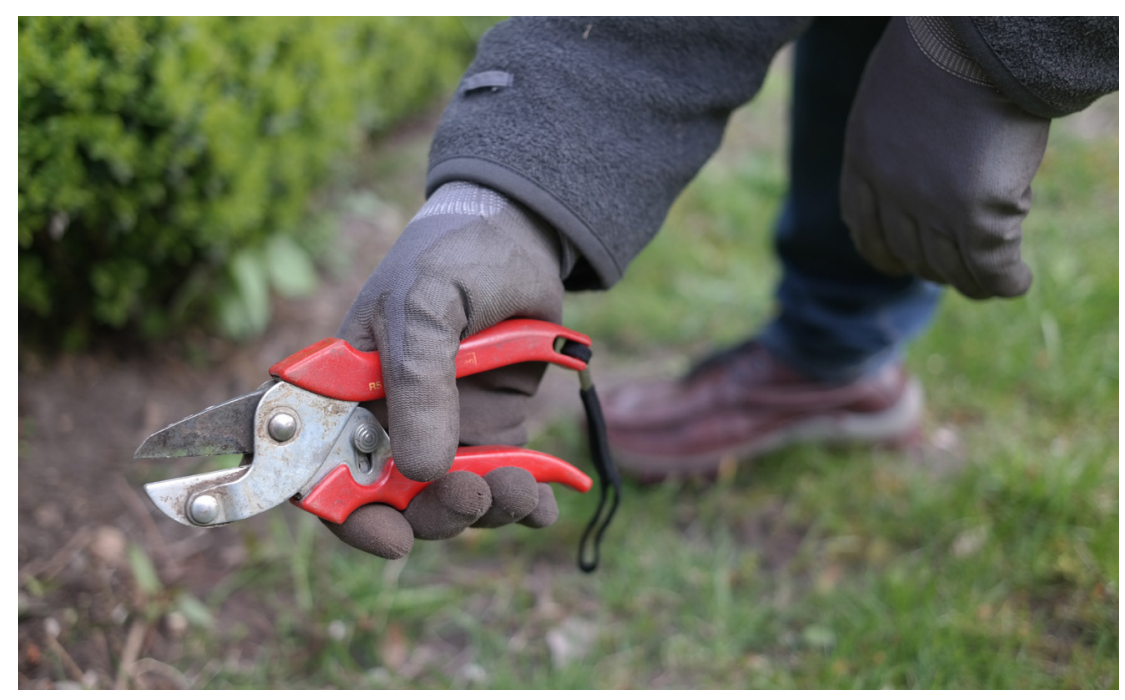

Figure 3:

The handle on the

secateurs work as

signifiers, indicating

how the hand needs to hold it.

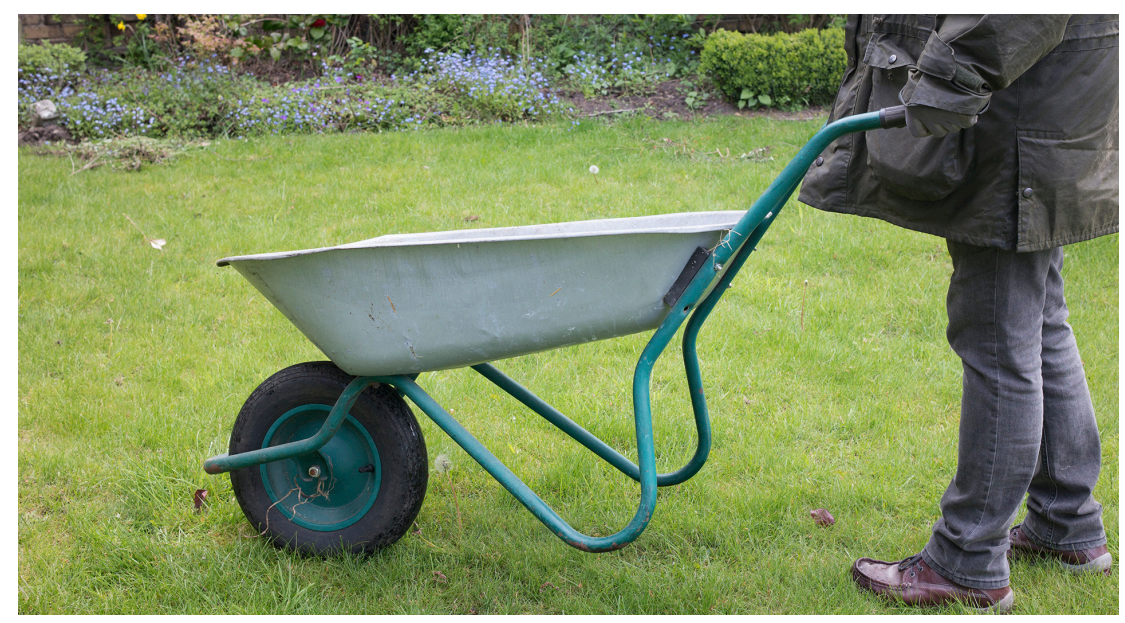

Figure 4:

The handles on the wheelbarrow require both hands for interaction.

Norman (2013) emphasises the role of affordance and feedback; the concepts of visibility; the use of physical; semantical; and cultural constraints; mapping, and consistency in product-human interaction. Affordance refers to action possibilities, while the actual design elements that indicate how to use the object are named signifiers (Norman 2013, p. 12). 
When a user interacts with an object, feedforward and feedback allow a coupling between action and function (Locher, et al., 2010). Further elements that help the user to interact with everyday objects and develop a conceptual model of the object are constraints and mapping elements embedded in the physical form of the object (Norman, 1990, pp. 12-13).

In the context of this study Norman's concept of physical constraints needs to be mentioned. Physical constraints are closely connected to real affordance (Norman, 1999, pp. 40-41). Physical constraints allow, like CIMT, certain movements while limiting others. The design of appropriate physical constraints, as well as conventions that rely on learned actions, offers the possibility of creating everyday objects that initiate the use of the affected arm and hand. A focus on affordance and the design of appropriate signifiers offers an opportunity to design everyday objects that evoke an initiation of use of the affected arm and hand by restraining or encouraging a specific use. A possible example could be a cup that can be used with just one side of the body.

I visited a shop which sells everyday objects to stroke survivors to identify possible design precedents. The focus of these commercial devices is to compensate the lost functionality rather than evoking an initiation of use. The use of a web search engine did not identify any relevant products either. The focus of my research is on rehabilitative objects rather than on assistive design solutions which enable compensatory movement patterns. Currently, there are no objects available to chronic stroke survivors that could be used in a self-directed way in the home environment.

The following chapter will outline the research questions, aims and objects that were defined based on the evaluative literature review, as well as the theoretical perspective, methodology and methods that have been used to address them. 


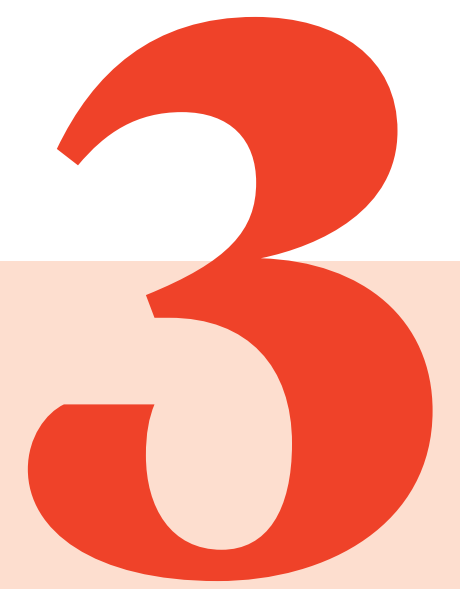

\section{Methodology}




\subsection{Research Aims, Objectives, Questions and Methods}

The literature review indicated that learned nonuse is a common phenomenon after a stroke, affecting particularly chronic stroke survivors. It can be addressed by restraining movement; for example, using a mitt to initiate the use of the affected arm again. The main research question has been defined as:

1. How can an everyday object that chronic stroke survivors use in activities of daily living restrain movement of the less affected upper limb to initiate use of the affected arm and hand?

To address this main question three further research questions were used to address specific areas.

1.1.1 How is movement of the upper limb restrained during rehabilitation to initiate use of the affected arm and hand in stroke survivor?

1.2.1 Which everyday objects are used by stroke survivors during activities of daily living that they like to take part in, and therefore may assist in facilitating use of the affected arm?

1.3.1 How can we design an everyday object that restrains movement of the less affected arm to facilitate and initiate the use of the affected arm?

Please refer to Table 1 for an overview of research aims, objectives and questions for this thesis. 
Table 1: Overview of research aims, objectives and questions

\begin{tabular}{|c|c|c|c|}
\hline RESEARCH AIM & RESEARCH OBJECTIVE & RESEARCH QUESTION & RESEARCH METHOD \\
\hline \multirow[t]{5}{*}{$\begin{array}{l}\text { 1. To design everyday } \\
\text { objects for chronic stroke } \\
\text { survivors living at home } \\
\text { that restrain movement } \\
\text { of the less affected arm to } \\
\text { evoke an initiation of use } \\
\text { of the affected arm and } \\
\text { hand }\end{array}$} & $\begin{array}{l}\text { 1.1 Identify and map } \\
\text { elements used in upper } \\
\text { limb stroke rehabilitation } \\
\text { interventions to restrain } \\
\text { the movement of the less } \\
\text { affected arm in order to } \\
\text { evoke an initiation of use } \\
\text { of the affected arm and } \\
\text { hand }\end{array}$ & $\begin{array}{l}\text { 1.1.1 How is movement of } \\
\text { the upper limb restrained } \\
\text { during rehabilitation to } \\
\text { initiate use of the affected } \\
\text { arm and hand in stroke } \\
\text { survivor? }\end{array}$ & $\begin{array}{l}\text { 1.1.1.a Semi structured } \\
\text { interviews with health } \\
\text { professionals } \\
\text { 1.1.1.b Thematic analysis }\end{array}$ \\
\hline & $\begin{array}{l}1.2 \text { Identify and map } \\
\text { everyday objects used } \\
\text { by stroke survivors in } \\
\text { activities of daily living } \\
\text { after a stroke }\end{array}$ & $\begin{array}{l}1.2 .1 \text { Which everyday } \\
\text { objects are used by stroke } \\
\text { survivors during activ- } \\
\text { ities of daily living that } \\
\text { they like to take part in, } \\
\text { and therefore may assist } \\
\text { in facilitating use of the } \\
\text { affected arm? }\end{array}$ & $\begin{array}{l}\text { 1.2.1.a Online } \\
\text { survey with } \\
\text { stroke survivors } \\
\text { 1.2.1.b Semi-structured } \\
\text { interviews with health } \\
\text { professionals } \\
\text { 1.2.1.c Semi-structured } \\
\text { interviews with stroke } \\
\text { survivors }\end{array}$ \\
\hline & $\begin{array}{l}1.3 \text { Design an everyday } \\
\text { object that restrains } \\
\text { movement of the less } \\
\text { affected arm to evoke an } \\
\text { initiation of use of the } \\
\text { affected arm and hand of } \\
\text { a chronic stroke survivor }\end{array}$ & $\begin{array}{l}\text { 1.3.1 How can we design } \\
\text { an everyday object that } \\
\text { restrains movement of } \\
\text { the less affected arm to } \\
\text { facilitate and initiate the } \\
\text { use of the affected arm? }\end{array}$ & $\begin{array}{l}\text { 1.3.1.a Research through } \\
\text { design: Radio prototypes } \\
\text { 1+2, Phone prototype } \\
\text { 1.3.1.b Usability } \\
\text { evaluation: Cognitive } \\
\text { walkthroughs with health } \\
\text { professionals } \\
\text { 1.3.1.c Usability } \\
\text { evaluation: Pluralistic } \\
\text { walkthroughs with stroke } \\
\text { survivors }\end{array}$ \\
\hline & $\begin{array}{l}\text { 1.4 Identify and map } \\
\text { strategies that can be } \\
\text { incorporated into the } \\
\text { design of an everyday } \\
\text { object to restrain } \\
\text { movement and evoke an } \\
\text { initiation of use of the } \\
\text { affected arm }\end{array}$ & & $\begin{array}{l}\text { 1.4.1a Design } \\
\text { workshops with design } \\
\text { students following a } \\
\text { research-through-design } \\
\text { approach } \\
\text { 1.4.1.b Usability } \\
\text { evaluation: Cognitive } \\
\text { walkthroughs with health } \\
\text { professionals }\end{array}$ \\
\hline & & & $\begin{array}{l}\text { 1.4.1.c Research through } \\
\text { design: Digital design } \\
\text { method cards } \\
\text { 1.4.1.d Usability } \\
\text { evaluation: Post-test } \\
\text { interviews with designers }\end{array}$ \\
\hline
\end{tabular}




\subsection{Theoretical Framework}

This research aims to develop everyday objects that can be used by chronic stroke survivors in tasks that are meaningful to them. This focus requires an epistemology that takes account of the subjective nature of those meaningful tasks. No design artefacts were available that facilitate initiation and restrain movement that could be referred to and evaluated. The practice of design was a necessary part of the research process to develop design prototypes that addresses the observed problem and contributed to the rehabilitation process.

Objectivism and subjectivism have been divided into qualitative and quantitative research approaches with quantitative being assigned to objectivism and qualitative to subjectivism (Pernecky, 2016, pp. 14 -22). In the context of this research, the positivist and postpositivist lenses that are based on the objectivist epistemology (Creswell, 2014, p. 7) are not appropriate. According to the view of these two approaches, the research should aim to mirror natural science as close as possible (Pernecky, 2016, p. 17). However, the context of this research focuses on human interactions which do not share the same characteristics as classic sciences such as chemistry. Human and social interactions are influenced by factors such as politics and values which are impossible to isolate from the data collection process. These two approaches neglect my subjective role in the research.

Theoretical perspectives under a subjective epistemology that could be used as a theoretical framework in the context of this research are structuralism, poststructuralism and constructivism. Structuralism focuses on the real or essential underlying structure of social interactions. The view of structuralism outlines that a rational inquiry can reveal those underlying structures and consequently reveal the truth. A view which has been criticised due to its assumption that is possible to remove any form of bias the data collection process (Given, 2008, pp. 837-839). Poststructuralism on the other hand has been regarded as being more wide-ranging and theoretical nuanced with a focus on identifying meaning that is 
context specific (Given, 2008, p. 667). This view has been subject to considerable critique since its claims are relative without a focus of claiming validity (Given, 2008, p. 669). Under the subjective epistemology, the object's meaning is not connected to the interaction with that object (Crotty et al., 1998, p. 9). This view is not applicable to this research either.

The pragmatist epistemology allows a designerly inquiry, which was necessary to address the main research question. I refer to designerly inquiry as an explorative and transformative process through which designers draw upon their repertoire of knowledge and competences as well as resources in the situation, including instruments, in order to create something novel and appropriate that changes an incoherent or undesirable situation for the better (Dalsgaard, 2017, p. 24).

The designerly inquiry involves the process of creating a design which helps develop a deeper understanding for the observed problem and validate possible design solutions (Dalsgaard, 2014, p. 150; Dalsgaard, 2017, p. 24). The pragmatic approach correlates with design practice to address "wicked problems" within the design field. It commonly addresses ill-defined challenges with confusing information and multiple stakeholders involved in the process (Buchanan, 1992, p. 15). It aligns with the methodology of research through design (RTD), where researchers participate in the process of making to develop a new understanding (Dalsgaard, 2014, p. 153). Therefore, the pragmatist perspective was chosen for this current research. A detailed description of this theoretical perspective, methodology and methods involved will follow in the next section.

\subsubsection{Pragmatism}

Pragmatism does not see the world as one unit and cannot be restricted to just one philosophy or reality. It offers a freedom of choice regarding the methods that are used to address a research question (Creswell, 2015, pp. 10-11). Methods used under this theoretical framework can be chosen based on the intended consequences. Although truth in pragmatism is considered as what "works at the 
time" (Creswell, 2015, p. 11) it is influenced by the social, historical, and political factors in which it occurs. However, its aim is not to investigate reality or laws of nature (Creswell, 2015, pp. 10-11): instead, it focuses on the temporary stability in a given situation (Dalsgaard, 2014, p. 146). Pragmatism has a strong focus on the situations in which human activity occurs. It is based on the foundation that objects and events need to be evaluated in the context of the given situation (Dalsgaard, 2014, p. 146; Biskjaer \& Dalsgaard, 2012, p. 68). Pragmatism has been criticised for its lack of rigour and explicitness. However, the development of theories is an important part of pragmatism that is inherently explicit, rigorous, specific, and structured and addresses criticism in regards to its scientific rigour. Theories developed under the pragmatist view are constantly tested in practice to validate their use (Biesenthal, 2014, p. 650).

The criticism of pragmatism has been addressed in this current research by involving multiple qualitative methods and explicitly outlining the generated insights and knowledge. The methods for this research were chosen based on a human-centred design approach that focuses on the user and intended use. Insights that were based on the process of making were theorised in the form of bridging concepts and validated in practice (Dalsgaard, 2014, p. 153).

\subsection{Human-Centred Design}

A user-centred design or so-called human-centred design approach (Säde, 2001, p. 26) was chosen to develop an understanding of the intended user and context of use. Methods used as part of human-centred design help to understand the needs and requirements of the user and context of use to create suitable design solutions that go beyond the "hard" factors of user requirements needed for the engineering process. Companies such as IDEO or Smart Design following this approach have proven to develop commercially successful designs (Coleman, Lebbon, \& Myerson, 2003, p. 481).

User involvement and evaluation takes place throughout the process to test and redefine assumptions about the user and context of use, to validate design 
solutions, and redefine them (Coleman, et al., 2003, p. 483). The following sections will outline the methods that were used to develop an understanding for the user, how design was used as a systematic form of inquiry to address the observed problem, and the evaluation methods that were used throughout the process to address my subjective role within the research and evaluate the different design prototypes.

\subsubsection{The context of use}

A multimethod approach was chosen, using different qualitative methods to gain a deeper understanding of the diverse needs of chronic stroke survivors, as well as requirements that rehabilitation devices need to fulfil. Semi-structured interviews with health professionals and stroke survivors and an online survey distributed through online platforms for stroke survivors helped develop an understanding of meaningful everyday activities, daily structure, and effective rehabilitation interventions.

\subsubsection{Production of design solutions}

Under the pragmatism perspective, a research-through-design (RTD) methodology was chosen to address the main research question. It needs to be emphasised that under pragmatic maxim, theory, and practice must merge. Theory needs to be developed out of practice, and its value is assessed in the way it helps to understand the world (Dalsgaard, 2014, p. 146). The different design prototypes developed as part of this study were used to address the main research question.

\subsubsection{Research through design}

Frayling’s political pamphlet (Frayling \& Royal College of Art, 1993) and Bruce Archer's article "the nature of research" (1995) marked the beginning of an ongoing debate on the use of design to conduct research. The designerly way of knowing (Cross, 1982, p. 223), which addresses so-called wicked problems is done in the form producing artefacts and developing theories (Zimmerman, Stolterman, \& Forlizzi, 2010, p. 311). 
The term has evolved over time and is now been referred to in different forms such as "design research", "research into design", "research through design”, "research for design”, "research by design”, “design-oriented research", "research-oriented design" (Sevaldson, 2010, pp. 11-12) as well as "constructive design research” (Bang, Krogh, Ludvigsen, \& Markussen, 2012, p. 2) that tries to move away from the expanded use of prepositions. I will use the term research through design (RTD) in this thesis as a form of "conceptualizing research done by means of the skilful practice of design activity revealing research insights." (Krogh, Markussen, \& Bang, 2015, p. 41).

Despite the ongoing discussions and publications focusing on RTD, there is still a need to develop it into "a proper research methodology that can produce relevant and rigorous theory" (Zimmerman, et al., 2010, p. 316). A set of standards and processes would potentially provide a foundation for the legitimacy of the discipline and might manage internal and external communications and expectations (Zimmerman, et al., 2010, pp. 316-317). However, it has been argued that those rules and standards could limit research results (Gaver, 2012, pp. 937938).

\subsection{Design experiments}

Experiments that lead to design artefacts are crucial to RTD. They manifest the choices and decisions made by the designer with a level of detail that would be impossible to display in a written form (Gaver, 2012, p. 944). A designed object can, therefore, contain the designer's perspective that can vary from philosophical, functional, and aesthetic elements and can embody possible futures (Zimmerman et al., 2008, p. 42). The object does not analytically represent those components but instead functions as a representation of the designer's judgement about how to best address the issues associated with the research question (Gaver, 2012, p. 944). The designerly inquiry in the form of design experiments can be conducted in the form of prototypes, products, or models (Zimmerman et al., 2008, p. 42) and can take place in different temporal sequences. 
This study has developed a number of design prototypes to address the research question. Each design prototype of this current study was influenced by the generated knowledge, and outcomes of the previous ones (Krogh et al., 2015, p. 46). The prototypes developed as part of this current research take over the role of a "research archetype - a physical embodiment of research concepts or perspectives that have broad application, but also that require specific examples to demonstrate their potential and justify that they constitute a contribution" (Wensveen \& Matthews, 2015, p. 270). Krogh et al. (2015, p. 44) describe five distinct methods of knowledge production through design experimentation: Accumulative, comparative, serial, expansive and probing. This research employs serial as well as expansive design experiments in different stages, please refer to 3.5 for an overview of the different steps.

\subsection{Criteria based model}

In order to address my subjective role in the research the "Postgraduate thesis model for research through design based on criteria” has been used to follow a systematic approach. The model outlines four steps that structure the design process as a systematic form of inquiry (Rodríguez Ramírez, 2017, p. 14), and was used to assess the developed prototypes during the design process. A set of criteria was developed based on the literature and interviews conducted with health professionals and stroke survivors. Serial design experiments and expansive design experiments (Krogh et al., 2015, pp. 46-47) were then carried out in an iterative way and assessed based on the developed criteria. Three refined prototypes were evaluated by health professionals and stroke survivors. The employed evaluation method will be outlined at a later stage in this chapter.

\subsection{Theorising results}

The designed object is the manifestation of a theory and at the same time the instrument of conducting the research. By testing the prototype, the theory is tested in practice, which aligns with the pragmatic epistemology. The objects are used to test and evoke discussion about how existing knowledge can be 
incorporated in the object (Haynes \& Carroll 2007, p. 162) and evoke conversation between stakeholders and the designer (Dalsgaard, 2017). However, it has been emphasised that learning outcomes of each step need to be articulated to justify decisions that were made during the process (Ehn \& Ullmark, 2017, p. 82) to explain how the design addresses the observed problem (Haynes \& Carroll, 2007, p. 168). This notion is incorporated by summarising the different design iterations, findings, and conclusions in regards to the criteria based thesis model (Rodríguez Ramírez, 2017, p. 14). The detailed design process was documented as design workbooks (Gaver, 2011), see Appendix B, C, \& D.

A set of design strategies was developed, as part of this study can be used by other designers to guide the design process and as a source of inspiration. The role of theory development and process is outlined in the next section.

\subsubsection{Construction and communication of knowledge}

The theories developed as part of the RTD process differ from those used in natural sciences and human and social sciences. Theories in those disciplines are evaluated according to their ability to explain an existing reality in a meaningful way. Gaver (2012, p. 938) argues that theories in RTD are more than mere descriptions and explanations of existing realities "instead of being extensible and verifiable, theory produced by research through design tends to be provisional, contingent and aspirational” (Gaver 2012, p. 938).

Theories of design are unfalsifiable in other words: the standards used in scientific research programmes of controlled experiments do not take account of the nature of design where too many factors are in place (Gaver, 2012, p. 940). The theories developed through means of design are too vague and depend on a number of different decisions which are made in the design process. This causes an underspecification which is necessary for falsification. The implication of this underspecification is that the theories are not falsifiable in principle. Theories such as 'Follow process A to design for X' can lead to successful outcomes but there is no guarantee that it will. Designers furthermore aim to use design theory 
to create good designs rather than showing that the theory can lead to bad ones (Gaver, 2012, pp. 940 - 941). Instead of trying to aim for falsifiability, which would contradict the nature of RTD, it is argued that theories in RTD should deliver creative propositions or statements for investigating a potential future. Zimmerman \& Forlizzi (2008, p. 44) call those theories nascent theory of the near future that make propositions of essential relationships between phenomena.

It has been emphasised in the context of practice-oriented research in the field of human-computer interaction (HCI) that research based on practice leads to constructs of intermediate level knowledge situated between the instances, the artefacts, and theories (Höök \& Löwgren, 2012, pp. 1-2). While a theory presents the condensed knowledge and is the foundation of understanding reality within the discipline (Stolterman \& Wiberg, 2010, p. 99), is the knowledge generated as part of this study specific and situated in a certain context (Höök \& Löwgren, 2012, p. 3).

Whenever a practitioner of a conceptual work explains and discusses the rationales leading to the final artefact "they engage in a form of implicit conceptual work” (Gaver 2012, p. 938) by elaborating on issues, similarities to other artefacts, and applied criteria that were used to assess the object. Those constructions of knowledge have been introduced in different forms. Examples are annotated portfolios (Gaver \& Bowers, 2012), strong concepts (Höök \& Löwgren, 2012), patterns (Borchers, 2001), conceptual constructs (Stolterman \& Wiberg, 2010), heuristics (Nielsen \& Moloch, 1990), bridging concepts (Dalsgaard \& Dindler, 2014), guiding philosophies, conceptual frameworks, manifestos, and design implications (Markussen 2017, p. 90)

Three models are applicable to explain the knowledge produced in the context of this research: Strong concepts, conceptual constructs and bridging concepts. While strong concepts are based on design cases and have the primary goal of informing practice, conceptual constructs are influenced by theory and focus on the theoretical advancement. The generated knowledge aligns with the 
bridging concepts definition. Bridging concepts are influenced by both theory and practice, and their purpose is to bridge the gap between the two (Dalsgaard \& Dindler, 2014, p. 1637). This form of intermediate knowledge aligns with the pragmatism perspective of this study (Dalsgaard \& Dindler, 2014, pp. 1638-1639).

\subsection{Evaluation with Stakeholders}

The evaluation of a system or artefact was developed in response to the demands of system analysis and human factors. The use of usability evaluation methods (UEM) that assessed the usability of a design or system go back more than three decades (Hartson, Andre, \& Williges, 2001, pp. 373-374; Nielsen \& Molich, 1990). Since then, the term usability has been defined in multiple varying ways. According to the ISO 9241-11 definition, the term usability comprises the three factors effectiveness, efficiency, and satisfaction (International Organization for Standardization, 1998).

There is often a focus on the quantification of usability issues to secure the effectiveness, efficiency, and satisfaction of a product (ISO, 1998) rather than qualitative terms such as quality of use, ease of use, or ergonomic design (Green \& Jordan, 1999, p. 5). A quantified focus does not take account of the wider role that objects can play in people’s lives in the form of "living objects" (Green \& Jordan, 1999, p. 6) that the owner attaches meaning to (Csikszentmihalyi \& Halton, 1981, p. 112). The visual aesthetic of the product, its technical specification, performance against user expectation, as well as emotions and values that are associated with the product should be considered during the usability evaluation. The focus on such qualitative elements outside the traditional usability testing has been sparse in the past (Jordan, 1998, p. 26), but recent studies emphasise qualitative elements and the involvement of stakeholders in the process of designing rehabilitative and assistive technology solutions (Balaam et al. 2011, p. 3075; Scherer, 2008; Tetteroo, Timmermans, Seelen, \& Markopoulos, 2014). 
The design iterations were assessed according to the criteria based thesis model (Rodríguez Ramírez, 2017, p. 14). However, from the pragmatist perspective the design solutions need to be assessed in practice. Multiple prototypes were evaluated by health professionals and stroke survivors to evaluate the intended initiation of use and gather feedback for further refinements. I will focus in this current research on qualitative evaluation methods involving health professionals and stroke survivors.

\subsubsection{Formative usability testing}

The iterative development process of an interactive system involves different steps such as the design, evaluation and redesign process (Hartson et al., 2001, p. 374). Kies, Williges, \& Rosson (1998, p. 782) looked at different iterative models used in HCI and stated that all the procedures employed the three characteristics of initial, prototype and final design stage. User testing and feedback is included throughout the design cycle. In the context of this current research the focus of the usability evaluation is on the initial and prototype stages.

The formative usability evaluation takes place during the prototype design stage before the final design is accepted to be released. Different usability evaluation methods such as user evaluation, expert evaluation, model-mismatch evaluation, and evaluation location can provide a better understanding of usability issues. The aim of the formative evaluation process is not the collection of quantitative data for comparison, but instead to refine the design (Hartson et al., 2001, p. 375; Kies et al., 1998, p. 785).

For this current research, a formative usability evaluation has been included in different stages of the research to evaluate and increase the intended initiation of use. I refer to the term usability evaluation method (UEM) as "any method or technique used to perform formative usability evaluation (i.e., usability evaluation or testing to improve usability) of an interaction design at any stage of its development.” (Hartson et al., 2001, p. 377). 
Cognitive walkthroughs (Wilson 2014, p. 65-79) with health experts, as well as pluralistic walkthroughs (Wilson 2014, p. 81-97) with stroke survivors were employed to evaluate the different design iterations, see Table 2 . The cognitive walkthrough with experts is a task-driven approach and aims to encounter usability problems in much the same way as the end-user would do (Hartson et al., 2001, p. 379). The design method cards which were developed as part of this study have been evaluated by designers, followed by a post-test semi structured interview.

Table 2: Overview of formative usability evaluation methods employed in this study.

\begin{tabular}{|c|c|}
\hline Prototype & Formative usability evaluation \\
\hline $\begin{array}{l}\text { Design experiment 01: } \\
\text { Radio I }\end{array}$ & Cognitive walkthroughs with health professionals \\
\hline $\begin{array}{l}\text { Design experiment } 02 \text { : } \\
\text { Radio II }\end{array}$ & $\begin{array}{l}\text { Pluralistic cognitive walkthroughs with stroke } \\
\text { survivors }\end{array}$ \\
\hline $\begin{array}{l}\text { Design workshop 01: } \\
\text { Design prototypes developed } \\
\text { with industrial design students }\end{array}$ & Cognitive walkthroughs with health professionals \\
\hline $\begin{array}{l}\text { Digital design method cards: } \\
\text { Digital design method cards }\end{array}$ & Post-test semi structured interviews with designers \\
\hline $\begin{array}{l}\text { Design experiment 03: } \\
\text { Design to be used with a smartphone }\end{array}$ & $\begin{array}{l}\text { Pluralistic cognitive walkthroughs with stroke } \\
\text { survivors }\end{array}$ \\
\hline
\end{tabular}




\subsection{Chronology of the Research Process}

In this chapter, I have outlined that this study involves a number of qualitative methods which have been used in different stages of the research to address my research questions. Figure 5 shows an overview of the process that this research has followed.

So far there are no everyday objects available to chronic stroke survivors which can be used in a self-directed way in the home environment. The designerly inquiry was an essential element in the process to address my main research question. The human-centred design approach that this research has followed focused on the involvement of stakeholders. Participants were recruited based on their clinical experience in the context of upper limb stroke rehabilitation or their experience of a stroke. The methods used to include them in the process helped to understand the context of use, as well as producing and evaluating suitable design solutions.

There were six phases of prototype development involved in this research. Prototypes developed during phase 1, 2 and 6 were developed by myself. The design prototypes were used to assess the feasibility to restrain movement and helped to start conversations with health professionals and stroke survivors. A third prototype was developed in phase 6 based on responses collected through an online survey, interviews and observations with chronic stroke survivors. This quite late involvement of stroke survivors was due to the ethics process and recruitment of suitable participants, please see Chapter 3.6 for a detailed description. The prototypes were evaluated by stakeholders to address my subjective role in the process.

Phase 3 and 5 involved design workshops with designers and design students to create additional design concepts for the restraining and rehabilitative effect of the prototypes. Phase 4 focused on the theorised results of the design process which were tested with designers. 


\section{START OF THE PROJECT}

\begin{tabular}{|l|}
\hline $\begin{array}{l}\text { Radio prototype } \\
\text { Development stage 01 } \\
\text { 1. Serial design experiments \& critena } \\
\text { based thesis model } \\
\text { 2. Formative usability evaluation with } \\
\text { health professionals } \\
\text { 3. Thematic analysis }\end{array}$ \\
\hline $\begin{array}{l}\text { Radio prototype } \\
\text { Development stage } \mathbf{0 2} \\
\text { 1. Serial design experiments \& criteria } \\
\text { based thesis model } \\
\text { 2. Formative usability evaluation with } \\
\text { health professionals } \\
\text { 3. Thematic analysis }\end{array}$ \\
\hline
\end{tabular}

Online survey

Chronic stroke survivors

1. Online survey

2. Thematic analysis

Interviews and observation of ICT use Chronic stroke survivors

1. Semi-structured interviews and observations

2. Thematic analysis

\section{ICT design prototype}

1. Serial design experiments \& criteria based thesis model

2. Formative usability evaluation with chronic stroke survivors

3. Thematic analysis
ETHIC APPLICATION STARTED

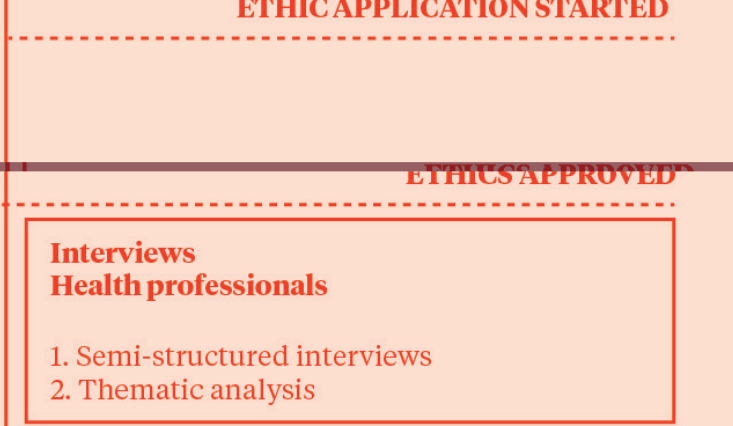

Design workshop 1

Undergraduate design students

1. Serial design experiments

2. Formative usability evaluation with health professionals

3. Thematic analysis

Digital design method cards

Graduated designers

1. Development of digital design method cards

2. Formative usability evaluation with

designers

3. Thematic analysis

Design workshop 2

Design students and graduate designers

1. Design workshop involving brainstorming and sketching of design concepts

2. Thematic analysis

Figure 5:

Overview of design methods. 


\subsection{Ethics}

Medical research and work with stroke survivors require strict ethical guidelines and procedures. The next section outlines the different steps that needed to be followed to obtain permission to invite stroke survivors to participate in this study.

\subsubsection{Ethics granted by the university}

Part of this research involves professional health care providers, such as occupational therapists and physiotherapists, to take part in a semi-structured interview, as well as working with designers. Ethics approval was sought and granted by the Victoria University of Wellington Human Ethics Committee (0000022410). Please refer to Appendix A for the participant information sheets and the consent form that were used as part of this study.

\subsubsection{Ethics granted by the Health and Disability Ethics}

\section{Committee}

A second ethics approval was sought to include participants who have experienced a stroke. The application was approved for this research by the Health and Disability Ethics Committee (HDEC), approval number: 16/CEN/37.

The Health and Disability Ethics Committees (HDECs) are Ministerial committees (established under section 11 of the New Zealand Public Health and Disability Act), whose function is to secure the benefits of health and disability research by checking that it meets or exceeds established ethical standards. (Ministry of Health - Manatū Hauora, 2017).

The required material and steps needed to apply to HDEC for an interventional study are outlined below. Please refer to Appendix A for the participant information sheets and the consent form that were used as part of this research.

- The HDEC online form, which states the different risks, reasoning for this study, cultural appropriateness, financial involvement, and 
background of the investigators involved.

- The research protocol outlining the clinical reasoning, methods used, forms of assessment, recruitment and analysis.

- The participant information sheet (PIS) needs to be checked by an expert in the field prior to submission and needs to score a predetermined readability value.

- The relevant Māori Tikanga training, which takes place at the hospital.

The training makes sure that culturally relevant and sensitive components are involved in the research

- The recruitment flyer and anticipated recruitment process.

The application is then assessed by a group of researchers and issues are clarified during a meeting with the leading researchers. Once permission was granted the trial had to be registered at the Australian \& New Zealand trial registration database (ANZTR) where further documents need to be provided despite the PIS, study protocol, and HDEC confirmation letter. The information on the ANZTR (Trial Id: ACTRN12616001014448) further needs to be updated once a year.

Once the trial is registered each district health board (DHB) needs to be contacted to apply for permission to conduct the study in their area. This permission is referred to as locality approval. After this approval was given, therapists operating in a particular DHB who worked with stroke survivors were contacted to determine if they could hand out the recruitment flyer to potential participants. Interested stroke survivors could then get in contact with the primary investigator.

The inclusion criteria for this study were: experience of a stroke at least 6 months prior to the interview, living at home, having an active wrist and finger, the ability to walk independently, an age of 40-75 years, and good general health. 
Exclusion criteria were signs of severely impaired verbal communication, inability to give consent, severe neurocognitive deficits, excessive pain in any joint of the paretic arm, terminal illness, or life-threatening comorbidities. It is unclear which percentage of the stroke population meets those inclusion criteria. Studies suggest that just $6 \%$ to $11 \%$ of all stroke survivors meet the inclusion and exclusion criteria of this research (Dromerick et al., 2009, p. 196; Viana \& Teasel 2012, p. 105; Wolf et al., 2006, p. 2098).

The locality approval process varied significantly between DHBs. Some DHBs had designated contact people who provided information and support for this kind of research project, while others supported only internal research projects within their organisation. I started the process by recruiting through a private clinic in Auckland and applied in the following months for locality approval to every DHB in New Zealand. Most of the participants of this study were recruited through a research facility in Auckland. Please refer to Table 3 for an overview of DHBs that granted locality approval and handed out recruitment flyers.

A professional recruitment agency was contacted to discuss how recruitment could be optimised using their services. The recruitment agency, unfortunately, did not offer the possibility of assessment to validate that participants met the inclusion criteria and was therefore not further pursued. The overall application process took 17 months from the start of the process to finding the first participants. 
Table 3: List of DHBs which were contacted for this study.

\begin{tabular}{|c|c|c|c|}
\hline DHB & Contacted DHB & $\begin{array}{l}\text { Locality approval } \\
\text { granted }\end{array}$ & Recruitment started \\
\hline Auckland DHB & $\mathrm{x}$ & & \\
\hline Private clinic in Auckland & $\mathrm{x}$ & $\mathrm{x}$ & $\mathrm{x}$ \\
\hline Research facility in Auckland & $\mathrm{x}$ & $\mathrm{x}$ & $\mathrm{x}$ \\
\hline Bay of plenty DHB & $\mathrm{x}$ & & \\
\hline Canterbury DHB & $\mathrm{x}$ & & \\
\hline Capital \& Coast DHB & $\mathrm{x}$ & $\mathrm{x}$ & \\
\hline Counties Manukau DHB & $\mathrm{x}$ & & \\
\hline Hawke’s Bay DHB & $\mathrm{x}$ & $\mathrm{x}$ & $\mathrm{X}$ \\
\hline Hutt Valley DHB & $\mathrm{x}$ & & \\
\hline Lakes DHB & $\mathrm{x}$ & $\mathrm{x}$ & \\
\hline MidCentral DHB & $\mathrm{x}$ & $\mathrm{x}$ & \\
\hline Nelson Marlborough DHB & $\mathrm{x}$ & & \\
\hline Northland DHB & $\mathrm{x}$ & $\mathrm{x}$ & \\
\hline South Canterbury DHB & $\mathrm{x}$ & & \\
\hline Southern DHB & $\mathrm{x}$ & & \\
\hline Tairāwhiti DHB & $\mathrm{x}$ & & \\
\hline Taranaki DHB & $\mathrm{x}$ & & \\
\hline Waikato DHB & $\mathrm{x}$ & & \\
\hline Wairarapa DHB & $\mathrm{x}$ & & \\
\hline Waitematā DHB & $\mathrm{x}$ & $\mathrm{x}$ & $\mathrm{x}$ \\
\hline West Coast DHB & $\mathrm{x}$ & & \\
\hline Whanganui DHB & $\mathrm{x}$ & & \\
\hline
\end{tabular}


Further requirements of the HDEC approval stated that usability testing should be restricted to 30 minutes. This led to a decision to focus on a formative usability evaluation as a summative evaluation was outside the scope of this research.

\subsection{Summary}

This research is based on a pragmatic epistemology focusing on a designerly inquiry to address the main research question. The prototypes are developed from a human-centred design perspective and take account of specific user needs. They are manifestations of possible solutions that address the observed problem area. The prototypes are tested under the pragmatist perspective and knowledge is incorporated into bridging concepts. The testing of design prototypes and bridging concepts aims to publicise knowledge that can be used by other researchers and design practitioners to structure and inspire the design process. 


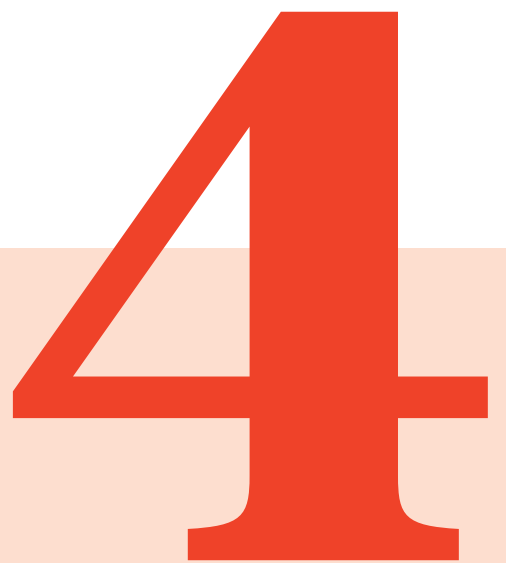

\section{Interviews with}

\section{Stroke Therapists}




\subsection{Introduction}

This chapter addresses the research question:

1.1 How is movement of the upper limb restrained during rehabilitation to initiate use of the affected arm and hand in stroke survivors?

I interviewed 13 health professionals to investigate how an initiation of use of the affected arm and hand is evoked, and how the less affected side is restrained to maximise rehabilitation outcomes. The knowledge generated was used to produce a set of design criteria that was used in the design process to assess the design prototypes. The focus of this research is on stroke survivors who live independently at home, have an impaired upper limb, and have reached the chronic stage where it is likely that they have developed symptoms of learned nonuse.

\subsection{Background}

\subsubsection{Professional health care provider}

Professionals who focus on the delivery of upper limb stroke rehabilitation are in most cases physiotherapists and occupational therapists. However, doctors, nurses, speech-language therapists, recreation therapists, psychologists, caregivers, and family members significantly contribute to the process (Meyer, Foley, Pereira, Salter, \& Teasell, 2012, p. 150; Pollock et al., 2014, p. 4).

The therapy process often starts while the person is still at the hospital in the form of inpatient rehabilitation and is continued during early supported discharge at home or in an outpatient setting as individual therapy or classes (Pollock et al., 2014, p. 4). Once officially discharged, stroke survivors can enrol in health service groups, charity collaborations or fitness centres (Best et al., 2012, p. 403). 
Chronic stroke survivors and their caregivers are often frustrated by the focus of resources on inpatient rehabilitation, where most of the recovery is expected to occur, and that is also the focus of most clinical studies (Teasell et al., 2012, p. 457). Long-term outcome studies that focus on chronic stroke survivors tend to investigate mortality rates rather than opportunities for and mechanisms of stroke recovery (Hardie, Hankey, Jamrozik, Broadhurst, \& Anderson, 2003).

Once a stroke survivor is discharged into the community, access to rehabilitation can be quite limited. They often wait for up to two weeks until the first therapy session starts, which tends to be an assessment. In New Zealand, there seems to be heterogenetic service delivery of specialised stroke rehabilitation (Gommans et al., 2003, p. 5; McNaughton et al., 2014, p. 17) and just a few services offer two to three days per week of community-based therapy (McNaughton et al., 2014, p. 17).

The use of CIMT and restraint in clinical practice has been the focus of a number of reviews indicating a negative perception of the intervention, especially restrictive devices (Daniel, Howard, Braun, \& Page, 2012; Page et al., 2002). A lack of education about CIMT and the different components used to address learned nonuse and facilitate an initiation of use has been reported to potentially lead to a misconception about the intervention (Fleet et al., 2014, pp. 65-67). This might be due to the limited CIMT-specific training in the UK, United States, and Australia (Pedlow, Lennon, \& Wilson, 2014, p. 257). Previous studies have employed surveys to determine how CIMT is used in clinical practice. Semi-structured interviews with health professionals have been chosen as part of this study as they offer a richer description of the perceived advantages and barriers to using a restrictive device and factors contributing to an initiation of use. 


\subsection{Method}

\subsubsection{Participants}

Given the heterogeneity of stroke services in New Zealand, participants with different roles and experiences were recruited. For this qualitative study, I used nonprobability sampling in the form of snowball sampling (Atkinson \& Flint, 2011). Interview partners recommended further possible interviewees.

Health professionals were eligible for this study if they had worked with acute, subacute, or chronic stroke survivors in clinical practice or a research context. Each participant provided written informed consent. The interviews took between 30 and 120 minutes, with an average of 60 minutes. Each interview took place at a location convenient for the participant. The interviews were audio recorded, transcribed in full and then analysed.

Thirteen interviews were conducted with stroke therapists and researchers. Clinical and research experience varied from 2 months to 10 years. Nine interview partners were female $(n=9)$, and four were male $(n=4)$; Three were occupational therapists $(n=3)$; two worked as physiotherapists $(n=2)$; one worked as a neuro physiotherapist $(\mathrm{n}=1)$; one worked as a professional therapist focusing on assessment post-stroke ( $\mathrm{n}=1)$; one was a physiotherapist student $(\mathrm{n}=1)$; one worked in the tertiary sector and had a background in musculoskeletal therapy $(n=1)$; two worked as researchers focusing on stroke therapy $(n=2)$; one worked as a scientist focusing on the recruitment of acute stroke survivors for studies $(n=1)$; and one was a supervisor of a clinic $(n=1)$. Two therapists $(n=2)$ worked in Australia; and 11 ( $\mathrm{n}=11)$ worked in New Zealand.

\subsubsection{Semi-structured interview content}

I used the semi-structured interviews for this study to gain a deeper understanding of the rehabilitation process and strategies that can help to overcome learned nonuse. Potential interview partners were informed about the purpose, the expected length of the interview and the topic under discussion. The primary questions of the interview guide were kept broad and open. Prompts that 
repeated the key concept were used during the interview to gain more in-depth information (Whiting, 2008, p. 37). The interview script outlined the main topics for the interview and started with questions about the participant's expertise. This included prompts focusing on their role in the rehabilitation process. The flow of the interview influenced the order in which the remaining primary interview questions were asked. If participants did not elaborate on the concept of learned nonuse or had little involvement with chronic stroke survivors, additional follow up questions were asked if they were aware of the concept. Each interview was transcribed in full.

The primary questions during the interview were:

1. Please tell me about your role in the rehabilitation process.

2. How do you structure the rehabilitation process?

3. What can you tell me about constraint-induced movement therapy?

4. Which components of the CIMT protocol do you use?

5. Is the movement restrained during the rehabilitation? If yes, in which form?

6. Which kinds of emotions are influencing the rehabilitation process?

7. What kind of resources can stroke survivors access?

8. How would the ideal rehabilitation system look?

9. How is feedback provided during the rehabilitation?

10. What are the steps to set up goals for the rehabilitation?

11. How are home-based assignments implemented in the process?

12. What kind of activities of daily living are perceived as being important to a stroke survivor?

13. What kind of objects play an important role in those activities?

\subsubsection{Thematic analysis}

The transcripts were analysed using thematic analysis in a theoretical 
or deductive form (Braun \& Clarke, 2006, p. 83) to identify, report, and analyse patterns and themes within the data (Braun \& Clarke, 2006, p. 79). The deductive approach was followed to find themes relevant to the research question. Deductive data analysis provides less of a rich description of the data and focuses more on analysing aspects of it. I took the following steps (Braun \& Clarke, 2006, p. 87):

1. familiarising myself with the data;

2. generating initial codes;

3. searching for themes;

4. reviewing themes;

5. defining and naming themes;

6. producing the report.

The software NVivo (version 11.2.2, qualitative data analysis software; QSR International Pty Ltd, Docaster, VIC, Australia) was used to structure initial codes, to name and redefine themes.

\subsection{Results}

\subsubsection{Themes}

The next section describes which factors, in the view of clinicians, contributed to the learned nonuse of the affected arm and hand, how an initiation of use was evoked in rehabilitation, and how the movement was restrained during the rehabilitation.

\subsubsection{Factors contributing to learned nonuse}

During the interviews four different factors were mentioned that contribute to the development of learned nonuse after a stroke. The four factors are physiological factors, the structure of current rehabilitation, individual behaviour, and social factors.

Physiological factors included factors such as cognitive impairments, apraxia that causes difficulties in understanding instructions and the purpose of the rehabilitation, spasticity of the affected arm and hand, fatigue that decreases 
the overall level of activity of the person, visual neglect, a shift of body awareness, and sensory deficits that can cause issues with proprioception and light touch, which is essential when using the hands. Once a person compensates the less affected side, the muscles of the affected side start to decondition, which causes a decline in muscle strength and muscle bulk.

What it tells us is that even a moderate level of impairment will promote nonuse. You have to be really, really good before you routinely use that hand and arm for everyday tasks and that is shocking so the impairment scale is 66 out of 66 and we have found, and others have found, that before that if your score falls below around 55 you won't use that hand and arm. Your MAL [Motor Activity Log] score will drop below three for both amount and quality of use.

Scientist 01 (Participant 05)

The structure of the current rehabilitation provision was mentioned as a further factor contributing to learned nonuse. A focus in rehabilitation often seems to be on the lower limb because of the wish to be able to walk again. This is reinforced by the ambition of the health services to minimise inpatient stays. One result is that the upper limb is focused on relatively late or not at all.

It was further mentioned that people with a mild stroke and a rather high level of motor functionality are often confronted with statements telling them that they are not expected to get much better and that the focus is on more severe and acute survivors. Another factor that was pointed out is that historically the focus has been on compensating for the loss of functionality once a person has reached the chronic stage. The focus on compensation and use of assistive devices, which would often be provided by occupational therapists, contribute to the development of learned nonuse by facilitating the use of the less affected side. 
They basically tell people when you are on a higher level. There is nothing you can do, 'you are pretty good, so just be glad that you are as good as you are'; 'go home and don't worry about the things that you would like to do', 'just thank god that you are alive and not too bad'. That is bad, I reckon. I think because I never ever met any stroke patient who told me that they are not too bad.

Occupational therapist (Participant 02)

Individual behaviours that contribute to the phenomenon include that stroke survivors tend to neglect the affected side after failed attempts to use it during the acute stage. The self-taught compensatory movement is reinforced through the easier and faster performance of the less affected side and forgetting to use the affected arm and hand. Unsupervised training can be regarded as reinforcing compensatory movement even further. There seems to be an increased risk of developing learned nonuse when the non-dominant hand is affected by the stroke.

People quite often quickly become one-handed especially when it is their non-dominant hand that is affected by the stroke. They just find it easier to do something with their dominant hand rather than trying to take the time to do it with their non-dominant and affected hand, which is going to be slower and you know it is just harder to do and more frustrating. Physiotherapist (Participant 06)

Social factors contribute to the learned nonuse phenomenon as well. Relatives and occasionally hospital staff would try to make the life of the stroke survivors as comfortable as possible.

So in some cultures, if an older family member becomes ill, then the expectation is on the rest of the family that they will now do everything for 
that person. That person has to be completely supported. They have to be hugged up to dress; they are not allowed to do any housework. They are not allowed to do any cooking or their meals are made for them and it is like they're a baby again, you know. Everything, everything is done for them. Scientist (Participant 05)

\subsubsection{Initiating use of the affected arm and hand}

During the interviews, there were two different ways participants referred to the initiation of use. Therapists who used CIMT as part of their daily routine specifically talked about the learned nonuse of the upper limb in chronic stroke survivors and how they would target it, as it is a specific element of CIMT. Participants who worked in the acute or subacute setting mainly talked about the general structure of the rehabilitation. This might be due to the structure of funding for stroke rehabilitation in New Zealand.

[CIMT] is not a therapy approach that is commonly available in New Zealand even though we know there is quite some evidence behind it, to prove that it does make people better. We don't have a funding mechanism in New Zealand to really make that available to people so it is five days a week for two weeks coming in for between three and six hours a day. If you had to pay an hourly rate for a therapist that would be cost prohibitive as one might say.

Senior research officer (Participant 11)

The expected plateau effect after the acute stage and resource limitations restrict access to rehabilitation to a limited time frame. A common focus in the process appears to be on the lower limb first, often leaving it unclear whether people in the chronic stage have reached their full recovery potential. 
You don't know if you haven't seen the patients early after stroke and you don't know if they met their most potential after stroke and got worse because they don't use their arm very much and you just bring them up to the same level or whether you are taking them even beyond that point where they got with their original recovery. I think it is a tricky time you know a lot of that I think is they are getting a little bit better because they are doing some form of exercise and it doesn't matter what approach you take they are probably going to get better with some sort of exercise as opposed to making some really significant neurological changes.

Physiotherapist (Participant 13)

\subsubsection{General structure of the rehabilitation process}

In the next section the initial assessment, goal setting, feedback, emotions, and elements of an effective rehabilitation intervention are outlined.

An initial assessment of the survivor is the first step in the process. The assessment helps determine motor deficits and helps the therapist understand motivations, daily routine, and attitude of the survivor to set appropriate goals.

So there are lots of things to think about: What's good, what's bad, what's missing? Then think okay in terms of physiology the physiological process, what is happening inside the brain, what is happening down to the fingers? Musculoskeletal therapist working in tertiary sector (Participant 01)

The assessment is the baseline for outcome measurements that can be reflected to the survivor. In the process, different assessment scales would be used depending on the apparent deficits of the survivor. Based on the assessment outcomes, appropriate goals are determined in close collaboration with the stroke survivor. The purpose of the rehabilitation is to restore appropriate function based on the baseline that the person is presenting. Goals that are focused on during 
rehabilitation need to be relevant to the survivor, specific, achievable, purposeful, and part of daily activities and potentially competitive. The goals should be task based rather than abstract ones such as "I want to use my arm again”. It was further mentioned that goals need to be contextualised. Once the goals are determined, milestones are defined that help during the process to validate improvements. Complex movements can be broken down into components during training.

So kind of like finding out what is important to them and what they want to get out of therapy. I mean they might come to us, and we can clearly see that their upper limb is affected, but they might not want to look at that at all. They might want to focus on their lower limb, so that is kind of ... their guide where they want to go in their therapy. Physiotherapist student (Participant 10)

During the rehabilitation, feedback is provided in the form of oral feedback as well as in a "hands-on" way. It should be provided instantly and be appropriate for the person and task. Feedback is used to improve movement patterns as well as being a motivational aid.

So you want people to remain as positive and optimistic as possible but you have to be realistic so [...]. I don't think you ever need to limit expectations because nobody ever really knows how much somebody may achieve. [...] So most of us actually exceed our own expectations in one way or another, and that is the same for people who just had a significant injury. [...] it just needs to be realistic so saying something like 'Okay... I really see that you want to get back to work, but you are still in the hospital right now'. So what steps do we need to take to get you there? You need to do $\mathrm{x}, \mathrm{y}$, and $\mathrm{z}$ but right now you just need to focus on the first part. To be able to get back to work you 
might need to be able to walk, or you might need to be able to concentrate to complete a task for an hour or so. So [...] let's work on what you can do now and go to the next step.

Professional therapist (Participant 04)

The emotions that the stroke survivor goes through can have a significant impact on the process. Therapists mentioned mainly negative emotions such as sadness, fear, depression, grief, anger, or frustration about the rehabilitation process and funding system. Survivors have to go through the process of accepting the loss of their previous roles such as being the main provider for the family, and coming to terms with their new role and limitations post-stroke. Rarely would someone see the stroke as a life-changing event that transforms their life positively. Positive emotions were mainly associated with positive rehabilitation outcomes.

We even have patients crying in the gym out of frustration because they are disappointed.

Supervisor of a student-run clinic (Participant 09)

Effective rehabilitation was described as being founded on the three elements of information, motivation, and strategy. Information is provided in the form of education about the stroke; the process and purpose of the rehabilitation; the concept of neuroplasticity; and a discussion about attainable goals for the survivor. This educational element offers transparency about the process by allowing the survivor to ask questions and help them develop realistic expectations. It was pointed out that the family and caregivers should ideally be included in the process to maximise the rehabilitation outcomes.

The use of feasible, meaningful, and specific goals for the process motivates during the often-frustrating rehabilitation process. The survivor needs to develop 
a feeling of responsibility for their process. The role of the therapist was described as a form of personal trainer helping reach the rehabilitation goals. They would provide motivational input in the form of feedback during the rehabilitation.

Strategies for effective rehabilitation would involve using the family in the process, incorporating self-directed training elements in a daily routine, and taking account of the environment such as the survivor 's home. The rehabilitation should aim to drive neuroplasticity and include an increased repetition of the movement.

The other half of the people, their families come here, and then they watch the treatment, but we also involve them. We also ask them in the first interview how much they want to be involved. Some actually give guidelines and say, I would do that, but I wouldn't do that so, 'I will supervise the programme, but I will not put hands on or help'. Whereas other families are really in there and put the electrodes on.

Supervisor of a student-run clinic (Participant 09)

\subsubsection{Addressing learned nonuse}

Learned nonuse was addressed with the steps of determining the amount of learned nonuse, determining appropriate goals, demonstrating the correct movement, evoking an initiation of use and providing feedback to keep the survivor motivated. It was further mentioned that there is a need to elicit a behaviour change in the person to overcome the self-taught compensation of the affected arm. The amount of use and associated learned nonuse is determined using assessment scales. The Motor Activity Log (MAL), which is a set of 30 questions used in combination with an "amount" and a "how well" scale, was recognised as giving a good indication of how much a person would still try to use the affected arm. The therapists' experience provided a further estimate of the kind of progress that can be expected from the survivor. 
The other assessment that I would obviously use as part of the CI therapy protocol is the 'Motor Activity Log' and it is important in two ways. 'A' it tells the patient how much they are currently doing and 'B' [...] it has been criticised as being more of an intervention tool than an assessment tool but I still think it is really important, particularly as it allows the person to see what they could be doing with their arm and that is the beauty of it I think. Occupational therapist (Participant 03)

Survivors would often provide goals such as "I just want to use my arm again" which is too vague to use as a therapeutic goal. To initiate use of the affected arm specific goals need to be determined in close collaboration with the survivor. The ideal goals were described as creating a feeling of enthusiasm in the survivor, being specific, meaningful and either achievable or made achievable by adjusting the level of difficulty. Using the MAL during the initial assessment process would indicate some opportunities of ADL where the arm could be used.

So he started off with 'So I just want to use my arm'. So I was 'Good, but I really want you to tell me some things that you can't do rather than just tell me that'. It is quite nebulous 'I can't use my arm'. Yeah, we know that but ... and then he was like 'Oh, I would like to do this or that' and so then I usually got them to find out two or three hobbies or you know just ADL type of... you know 'Activities of Daily Living'... so things that they really want to be able to do and then I get those couple of things and then I think about the motor stuff and how I get them to be able to do that.

Occupational therapist (Participant 02)

The demonstration of the correct movement helps the survivor understand how to perform the movement correctly. Documenting the movement in a video recording would be used to provide visual documentation and offer room for 
discussion as to how to improve the movement.

Motivational input from the therapist encourages an initiation of use and convinces the survivor that they should be trying to use the arm and that it is just the learned nonuse that impacts on their functional performance.

You can't start these guys off trying to do more complicated things because they are doing nothing. So even if you think that they have some capacity [...]. The first couple of days with those people are always focused on trying to get them to just initiate the use of it and that can be anything really. Occupational therapist (Participant 02)

Motivation is an essential element in encouraging use of the affected arm. Survivors who are not able to use the affected arm require a considerable amount of it. For more severe stroke survivor the task that is focused on during the rehabilitation would not play a significant role. The task just to has to keep the survivor motivated to try using the affected arm and hand.

Sometimes the initiation is just not there, and then they are completely relying on someone starting that whole process for them - yeah that is tricky. I think often fatigue or forgetting is a limitation. Occupational therapist (Participant 07)

Feedback is provided in the form of correcting the movement and motivational input. It was emphasised that it is important to allow the survivor to perform the movement before correcting it. Feedback would be reinforced through video recordings to illustrate and document the process. Constant motivational input encourages the survivor to keep on trying. Survivor starting rehabilitation would often be depressed and had given up trying to use the arm at all and to work hard during the therapy session can be experienced as an extremely confronting 
situation. However, there seem to be some positive emotions involved in the process as well. The feeling of surprise after being able to perform movements evoked the desire to continue trying to use the affected arm in more activities once positive results set in.

Success breeds success. And as a therapist, if I set up an activity that is not too difficult and then they are succeeding at [completing the activity]. How are they going to get that positive neurochemical interaction to say 'I can do this.'? So as a therapist setting the activity bar at the right height is really important. It can't be too easy, but it can't be unachievable either. Occupational therapist (Participant 03)

It was emphasised that the person needs to go through a behaviour change process to overcome the learned nonuse. Carers and family members are educated about the process and involved in it to secure an effective treatment. The use of a behaviour contract signed by the therapist and stroke survivors seems to be an effective tool to evoke a feeling of responsibility in the survivor and create the necessary behaviour change. There are different techniques such as diaries and home practice sheets that remind the survivor to use the affected arm as often as possible.

I am thinking about anything which promotes behavioural change so that might be something which reminds them to use that limb or makes it harder to use the other limb or easier to use the affected limb. I think once you are at home the thing to push is that activity and not getting too fussy about how "pretty" it looks in terms of the quality of movement and things like that. The bottom line is the more the person can do with their affected limb the more likely they are to improve, and I think anything you can design that supports them to use their affected limb more than they would 
otherwise, is a good thing.

Clinical researcher (Participant 08)

\subsubsection{Restraining the movement}

Therapists and researchers referring to CIMT mentioned the use of a restraint as part of a rehabilitation intervention. In the following section the themes of functional purpose, the form of the restraint, as well as the associated limitations will be outlined.

The functional purpose of the restraint is not primarily to restrain the movement but to remind the survivor to use the affected arm and limit the possibility of compensating with an enhanced use of the less affected side. It was emphasised that CIMT is a behaviour change intervention and that the physical restraint is just one element of the intervention.

So you refer to the constraint, but it is more about the behavioural constraints. They are the ones that are important, and I put far more weight on the behavioural than on the physical. So it is not about the mitt. Occupational therapist (Participant 03)

The form of the restraint is mainly a soft mitt. One therapist mentioned using a hard cast just in the context of paediatrics to remind the child not to use the less affected side. Some therapists in referring to CIMT tried to incorporate the intervention by not allowing the survivor to use the less affected hand during the therapy session, but to sit on their hand while performing movements with the affected arm.

There were some associated limitations mentioned regarding the use of the physical restraint as part of an intervention, such as the context of use and the limited number of suitable stroke survivors. The restraint needs to be used in combination with therapeutic exercises, behavioural strategies, and educational 
elements that explain the role and purpose of the restraint to the survivor. Further criticism was that the focus on unilateral training contradicts how most everyday tasks are performed. Another risk that was frequently named is that the restraint might cause balance issues and increase the risk of falling. Some participants mentioned that its use appeared not to be feasible in clinical practice.

For some people they didn’t use any kind of restraint because restraining their unaffected limb affected their safety and they were much more mindful of the fact that you use your upper limbs bi-manually. Clinical scientist (Participant 08)

The full constraint is not really feasible and takes a huge amount of dedication of the people. Occupational therapist (Participant 07)

Survivor that are suitable to receive the CIMT intervention need to be able to perform movements with the less affected side, which requires the absence of cognitive limitations and large neurological insults, and possession of sufficient gross and fine motor skills. Patients who are already reminding themselves to use the affected side as much as possible would not benefit from the restraint.

If you want the mitt on one hand then it can only be used for people who can use the other hand quite well, so you are talking about a fairly mild [stroke]... you are probably generous to call it moderate.

Physiotherapist (Participant 12) 


\subsection{Discussion}

\subsubsection{Factors contributing to learned nonuse}

The concept of learned nonuse was described by therapist who worked with chronic stroke survivors and by therapists who referred to the concept of CIMT. The descriptions of physiological factors and individual behaviour correlate with the description of the development of learned nonuse in the literature. Learned nonuse is described as a self-taught behaviour that develops over time and is reinforced through unsuccessful motor attempts, failure, punishment, and pain (Taub \& Morris, 2001, pp. 279-280). CIMT is used in the context of chronic stroke as well as acute and subacute stroke populations (MacKenzie \& Viana, 2016, p. 219), which was confirmed by some participants. In the acute and subacute setting, it prevents the development of learned nonuse rather than remedying it (Krakauer, 2006, pp. 87-88).

Additional personal and physiological factors that were mentioned in previous studies are the extensive time required to plan a movement; initiate and complete a task; fatigue; a lack of confidence and control over the situation; pain; fear of negative consequences; and fear of becoming dependent on others (Taule \& Råheim, 2014, pp. 2113-2114; Wallenbert \& Jonsson, 2005, pp. 220-223).

A study by Wallenbert and Jonsson (2005) explored habits in daily living after a stroke. The study focused on habit development rather than learned nonuse, but findings identified the inner struggle of survivors in adapting to their new situation or waiting for improvements. The change in habits was experienced as being necessary but at the same time associated with abandoning possible improvements by relying on technical aids, environmental improvements, and other people (Wallenbert \& Jonsson, 2005, p. 222).

Another factor that potentially contributes to the development of learned nonuse is the gender of the stroke survivor. A study by Taub, Uswatte, King et al. (2006, p. 1048) showed that female participants made greater gains on the MAL than males, with the possible explanation that the females faced a stronger 
expectation to use the affected arm to perform daily tasks again for example to be able to cook again for the family. This was not confirmed in this study.

The findings of this study regarding social factors correlate with previous findings (Lynch et al., 2008, p. 520). Social isolation caused by losing contact with friends and colleagues, the feeling of not belonging to the work community anymore, and attempts to hide the condition in order to appear normal to others reinforce compensatory movements (Lynch et al. 2008, pp. 519-520, Salter et al., 2008, p. 600; Taule \& Råheim, 2014, p. 2115).

The current structure of rehabilitation seems to limit access to stroke rehabilitation services to a certain timeframe. Further factors that could contribute to the learned nonuse mentioned in previous studies are the role of caregivers and early supported discharge systems that offer an early discharge from the hospital to the home environment. Patients often wait several months to access community-based rehabilitation despite being in the acute stage of their stroke. During this time, they are likely to start compensating for the affected side. Caregivers often fail to receive adequate training, which potentially contributes to the development of learned nonuse (Cobley, Fisher, Chouliara, Kerr, \& Walker, 2013, p. 754; Lynch et al., 2008, p. 520; Wood, Connelly, \& Maly, 2010, p. 1052). The caregiver's intention of helping the stroke survivor could potentially decrease the motivation of the stroke survivor to keep attempting to use the affected arm and hand. Further studies are needed to investigate the development of learned nonuse over time and investigate factors that contribute to its development.

\subsubsection{Addressing learned nonuse}

In the context of neuroplasticity, learned nonuse has been connected to the principle of "use it or lose it” (Dancause et al., 2015, p. 339; Kleim \& Jones, 2008, p. 227). Its impact influences motor learning and neurorehabilitation outcomes, including long-term engagement and self-efficacy of the survivor. Some factors were mentioned that seem to be unique in addressing learned nonuse:

- The assessment of the amount of learned nonuse; 
- Strategies to evoke, and the initiation of use, based on assessed motor capabilities and motivational input; and

- The need to elicit a behaviour change.

Crucial to the success of addressing learned nonuse seems to be that it is based on achievable goals and the belief of the stroke survivors that they can perform the movement. This perceived competence to attain a specific level of performance in a given environment is known as self-efficacy (Dancause et al., 2015, p. 341).

Self-efficacy is the subjective cognition that one can carry out a particular behaviour and does not necessarily relate to actual competence (Clark \& Zimmerman, 2014, p. 485). Efficacy beliefs are constructed through a complex process of self-persuasion influenced by diverse sources of efficacy information (Bandura, 1997, p. 115). It seems to be essential for the stroke survivor in developing self-efficacy to choose to use the paretic limb. However, little is known about it in the context of upper limb stroke rehabilitation, due to a focus on the lower limb in the majority of studies (Dancause et al., 2015, pp. 341-342).

The elements of the rehabilitation that were mentioned in the current study can be mapped to the elements of self-efficacy of:

- Mastery experiences;

- Vicarious experiences; and

- Verbal persuasion.

The fourth source of self-efficacy, the physiological and emotional state (Bandura, 1997, p. 79) was not mentioned by participants as part of the current study.

Motivational input and trying to keep the survivor engaged in the process of initiating use of the affected arm and hand was pointed out by the therapists of the current study as being a core element of evoking an initiation of use. This motivational input can be mapped to verbal persuasion that is part of self-efficacy, specifically regarding the degree of appraisal disparity (Bandura, 1997, p. 105). 
Motivational input in the form of appraisal disparity highlights the fact that the participant is able to use the affected arm and hand even though the survivor might doubt it. Motivational input has the overall goal to convince the stroke survivor that they have the potential to use the arm and that it is just the learned nonuse that is stopping them from doing so.

To increase self-efficacy beliefs is a complex process involving multiple factors. Self-efficacy beliefs rely on acquirable skills, increasing the belief of the individual that they can gain these skills, modelling the requisite skills, and refining activities in a way that makes them achievable, as well as providing explicit feedback during the process (Bandura, 1997, p. 105).

Self-efficacy in the stroke rehabilitation literature has mainly been investigated in the form of goal setting, follow up, and individualised approaches with structured information and professional support through self-management programmes (Warner, Packer, Villeneuve, Audulv, \& Versnel, 2015, p. 2154). There is no clear distinction between evoking a behaviour change through the use of self-efficacy or through providing an education programme to the stroke survivor (Jones \& Riazi, 2011, p. 808).

\subsubsection{Restraining the movement}

The participants who used CIMT, involving the use of a restraint, mentioned that the protective safety mitt was preferred over the sling or cast. The mitt limits the ability to use the finger and hand in ADL. It retains the person's ability to extend the arm in case of a fall or to compensate for balance problems (Taub, Crago, \& Uswatte, 1998, p. 159; Uswatte, Taub, Morris, Barman, \& Crago, 2006, p. 149). Therapists using CIMT emphasised that the intervention aims to evoke a behaviour change rather than being repetitive task training and the restraint is not the central part of it, even though the name might suggest it is (Taub, 2012, p. 162). Findings of the current study confirm that the physical restraint has to act as an extrinsic motivator in reminding the survivor not to use the unaffected limb (Dancause et al., 2015, p. 339). 


\subsubsection{Design criteria}

Based on the findings of the interviews, a set of design criteria (Rodríguez Ramírez, 2017, p. 14) was defined, see Figure 6. The design criteria were used as a checklist during the iterative design process, which investigated the design of an exemplary everyday object to evoke initiation of use of the affected arm and hand.

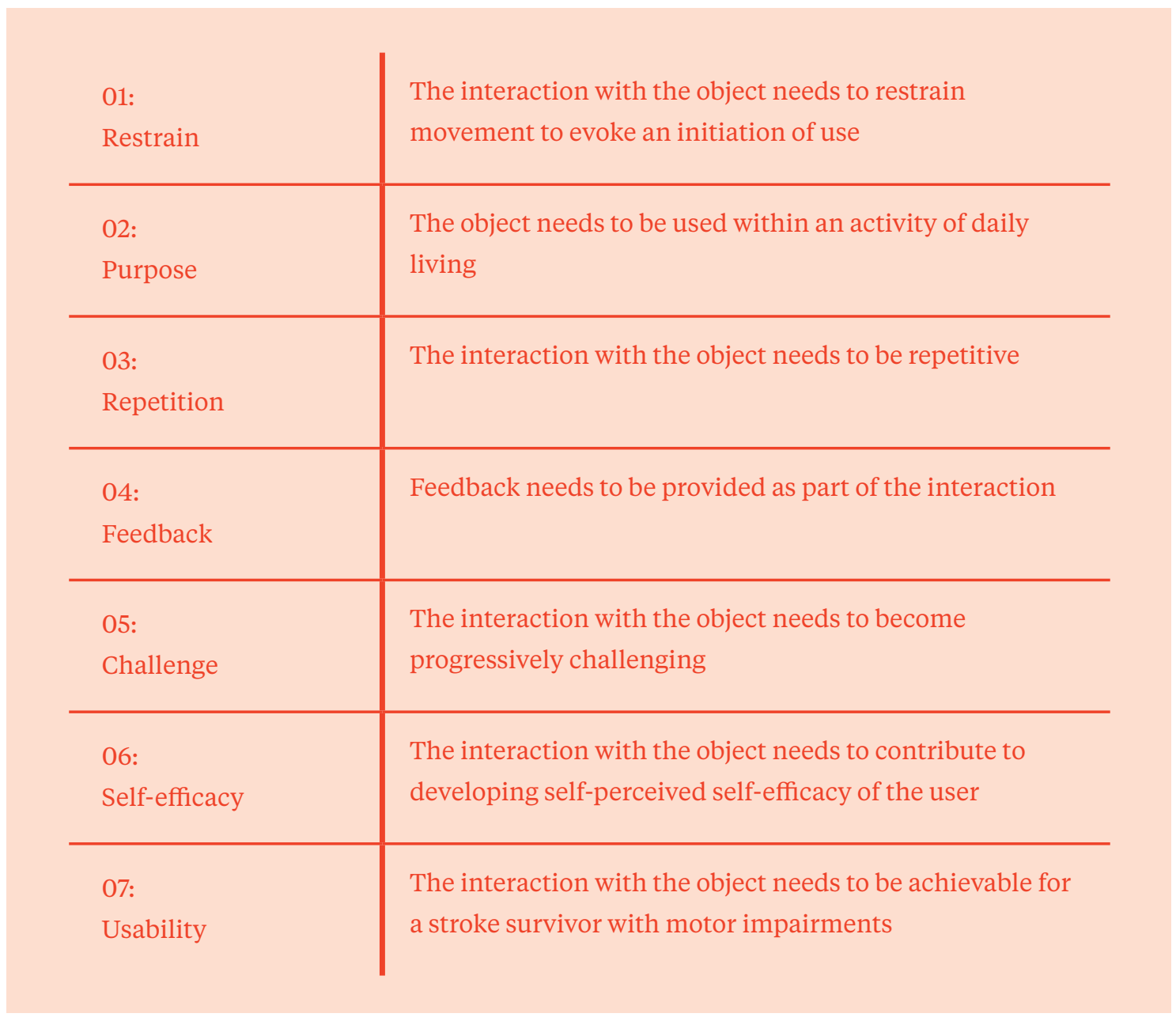

Figure 6:

Initial design criteria. 


\subsection{Conclusion}

The movement of the upper limb is restrained during rehabilitation by applying a mitt on the less affected side to initiate use of the affected one. The restraint was referred to only in the context of CIMT and functions as a reminder, rather than physically restraining the movement. An initiation of use in chronic stroke survivors with signs of learned nonuse is evoked through addressing selfefficacy beliefs in the individual and emphasising the potential to use the affected arm and hand.

\subsection{Limitations}

The majority of participants worked in private clinics and research contexts where stroke survivors might be more encouraged to work on their motor deficits. The use of CIMT and restraint was identified by one participant as belonging to the domain of physiotherapy. Physiotherapists would traditionally focus on rehabilitation rather than compensation, which is the field of occupational therapy. However, participants from both professions described using a restraint as part of their rehabilitation therapies. 


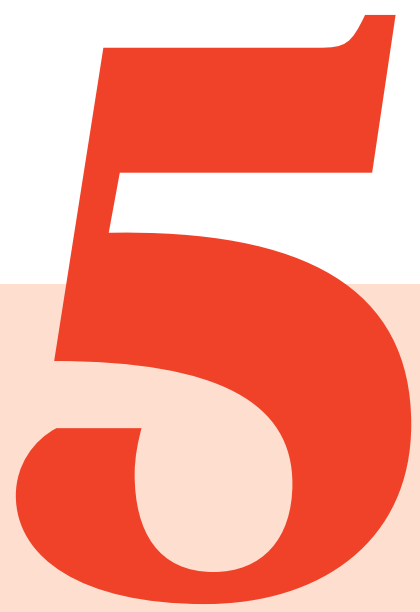

\section{The Design of a}

\section{Tangible Interface}

\section{to Facilitate}

\section{Initiation of Use}




\subsection{Introduction}

The focus of this study is to investigate how the design of everyday objects may facilitate initiation of use of the upper limb after a stroke. I used a radio as the medium of exploration for this study. The radio was chosen based on the home skill assignment (HSA) used as part of CIMT. It offers furthermore the possibility to collect data about the use of the object in order to provide feedback to the user. This chapter reports on the first phase of the prototype development process. Design outcomes were evaluated by stroke therapists. Chapter 6 will outline the second phase of the process which led to the design of a radio which was evaluated by stroke survivors.

The results of this chapter were used to address the research question:

1.3.1 How can we design an everyday object that restrains movement of the less affected arm to facilitate and initiate the use of the affected arm?

This study has been published in the Taylor and Francis Design Journal (Lemke, Rodríguez Ramírez, \& Robinson, 2017b)

\subsection{Background}

The design of the prototype investigates how an everyday object may facilitate initiation of use of the affected arm and hand. There are no precedents available in the context of this study. Therefore, the next section outlines examples of persuasive design solutions, which have a similar focus of encouraging a specific behaviour in the user.

\subsubsection{Persuasive technology}

This chapter reports on the design of a radio that aims to restrain movement to initiate use of the affected arm and hand. Persuasive technology (PT) and persuasive design (PD) have a similar focus and aim to influence the behaviour of the user. PT comprises three dimensional objects as well as digital applications, 
portable devices, websites and games (Fogg, 2009b, p. 1) that "attempt to change attitudes or behaviours or both without using coercion or deception” (Fogg, 2003, p. 15) and requires that a person voluntarily changes a specific behaviour (IJsselsteijn, de Kort, Midden, Eggen, \& van den Hoven, 2006, p. 1).

PD has various applications. Its aim can be to promote sustainable user behaviour (Laschke, Hassenzahl, Diefenbach, \& Tippkämper, 2011, pp. 637-638), influence energy consumption (Gustafsson \& Gyllenswärd, 2005, pp. 1424-1425), aims to decrease distractions in the work environment (Pereira et al., 2016, p. 1), and promotes face to face interaction by limiting the time spent online (Ho, 2016). PD even includes health-promoting concepts for example eating healthy or avoiding unhealthy sitting postures (Chi, Chen, Chu, \& Lo, 2008, pp. 118-121; Obermair, Reitberger, Meschtscherjakov, Lankes, \& Tscheligi, 2008, pp. 131-133) as well as health management for example using wearable sensors to manage diabetes type 1 (Chatterjee et al., 2012, pp. 32-36). Health topics in particular have attracted the attention of researchers (Chatterjee \& Price, 2009, p. 176; Torning \& Oinas-Kukkonen 2009, p. 7). It has been pointed out that designs using PT could potentially help to treat and manage health conditions such as obesity, alcoholism, or internet addiction (Torning \& Oinas-Kukkonen 2009, p. 7).

Theoretical design models can help designers in the process of creating effective persuasive technology. However, a review of four commonly used models which are the "Design with Intent (DWI) Method" (Lockton, Harrison, \& Stanton, 2010), the "Persuasive System Design (PSD)" (Oinas-Kukkonen \& Harjumaa, 2009) the "Eight-Step Design Process" (Fogg, 2009b) and the "Behaviour Wizard model” (Fogg \& Hreha, 2010) showed limitations. The models were deficient in implementing research methods systematically, analysing the collected data with established methods, and refining designs accordingly (Torning, 2013, pp. 23-24).

Persuasive design studies still lack a set of established research methods and are often situated on the intersection between human-computer interaction, psychology, and rhetoric, further complicating the ways data need to be collected 
and analysed (Torning, 2013, p. 24). It has been pointed out that assessment of the claimed behaviour change is not always carried out. Particularly, long-term, large user acceptance studies are still missing (IJsselsteijn et al., 2006, p. 4).

I took account of this criticism in the current PD studies. The focus of the evaluation process in this study is on the initiation of use of the affected arm and hand. The initiation of use can be validated within the 30-minute testing timeframe allowed by the ethics committee with each stroke participant. Learned nonuse can be overcome but requires a behaviour change in the user that needs time to develop. Validation that the object successfully contributed to overcoming learned nonuse over the long-term is therefore outside the scope of this study. This chapter presents the formative usability evaluation with stroke experts. The experts gave feedback on the usability of the design and anticipated initiation of use. The refined prototype has been tested by a chronic stroke survivor, see Chapter 6.

\subsubsection{Ethical considerations}

"The line between persuasion and coercion can be a fine one" (Fogg, 2003, p. 21) and needs to be included in ethical considerations of the design of PT. PT depends on the user's voluntarily changing, which is often regarded as an "all-ornothing concept, whereas voluntariness comes of course in degrees” (Smids, 2012, p. 131).

Fogg's (2009a, p. 1) behaviour model outlines three factors that need to be considered in the context of persuasive design and that align with the description of factors necessary to initiate use of the affected hand in chronic stroke survivors. The three factors are motivation, ability, and prompts, see Figure 7. All three factors need to be present to elicit the intended behaviour.

The described prototype works as a signal that reminds the user to perform a certain behaviour, see Figure 7. An everyday example of such a trigger is a traffic light (Figure 8), which indicates when a certain behaviour needs to be performed, in this case when the car can cross the intersection (Fogg, 2009a, p. 6). 


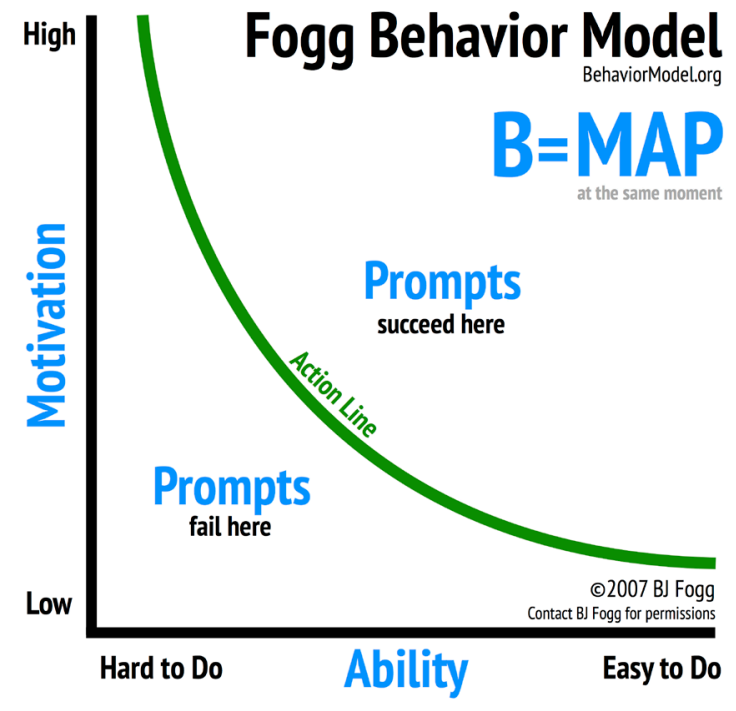

Figure 7:

The three factors in the

Fogg Behaviour Model.

The three factors in

the Fogg Behaviour

Model. Reprinted from

The Behaviour Model

by B. J. Fogg, 2018,

retrieved September

28, 2018, from https://

www.behaviormodel.

org/. Copyright 2018 by

Fogg. Reprinted with

permission.

The therapists described the use of restraint in clinical practice as a behavioural restraint that reminds the user to use the affected arm. The persuasive influence of the design prototype depends on the willingness as well as the ability of the user to use the affected arm. The object then prompts and reminds the user to use the affected arm and hand. Users who do not have motivation or ability would experience the design as coercive. Restraint has been pointed out by stroke survivors as being too restrictive and the restrictive nature of the design prototype could potentially be experienced in a similar way.

\subsubsection{Tangible user interfaces}

A stroke can affect the left as well as the right side of the body. The use of digital technology allows creation of an everyday object that can take account of the varying needs of the users. Therefore, a tangible user interface (TUI) was chosen as a starting point for the development of a prototype that could be used 


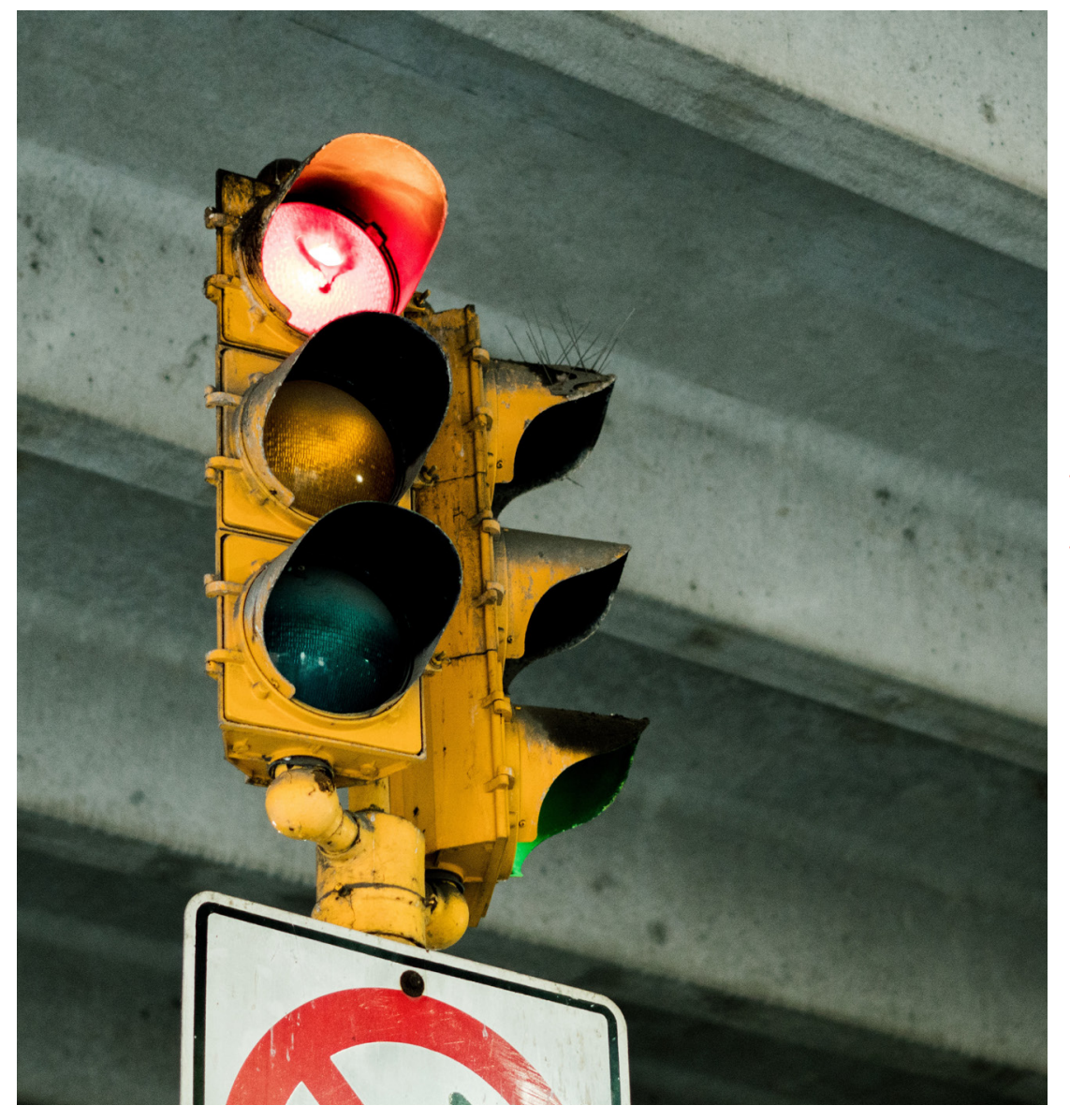

Figure 8:

The red signal on the

traffic lights indicates

that it is not safe to

cross and that the

driver should stop in

front of it. Reprinted

from unsplash.com, $H$.

Rivera, 2018, Retrieved

January 1, 2018, from

https://unsplash.com/

photos/CiUiXZZYQGI.

Copyright 2018 by

Hermes Rivera.

Reprinted with

permission.

by left-handed and right-handed stroke survivors. The TUI incorporates digital technology in an object and was used to investigate methods of restraining the upper limb in more socially acceptable ways, given the negative attitude that a physical restraint such as a mitt may elicit in the survivor. Restraints have also been criticised by therapists as being potentially dangerous in case the user experiences balance issues. The design prototype described in this study recognises the affected hand through the use of digital technology and so-called tags that are worn on the affected arm. This means the object can be used by participants affected on the right as well as the left side of the body.

The term TUI was introduced as an alternative concept to graphic user interfaces (GUI) with the goal of providing a richer interaction with digital systems by focusing on three-dimensional objects. Fitzmaurice, Ishii, and Buxton (1995, pp. 
442-443) introduced it as "graspable user interfaces"; however, most studies refer to the definition by Ishii and Ullmer (1997, p. 234) of a physical artefact named TUI as a materialisation of digital information by either representing data or as a means of controlling computational functions. I refer to tangible user interfaces as a three-dimensional object incorporating a computational function based on this definition (Ishii \& Ullmer, 1997, p. 234).

\subsubsection{Design evolution of radios}

The everyday object that I focus on in this study is the radio, based on the home skill assignment (HSA) process that is used as part of CIMT to encourage the use of the affected hand outside of the clinic. The HSA outlines a list of everyday tasks that stroke survivors can choose from to train the affected arm in the home environment. The radio was chosen because it offered the possibility to adjust the electrical functionality in a way necessary to restrain movement. The radio furthermore offered the opportunity to collect data about its use and reflect it back to the user to increase engagement in the process.

The radio was introduced as a new piece of wireless broadcasting in 1922 (Forty, 1992, p. 200) and its design evolved rapidly, leading to three different design solutions. One solution was to integrate it into furniture that was already used in people's homes, such as cabinets. A second solution disguised it by combining it with an object that had an entirely different purpose, such as an armchair. The third approach was to give it a completely new form - to use it as a manifestation of the future through its unfamiliar design (Forty, 1992, pp. 11-12).

This variety of designs can be explained by its functionality. Wireless broadcasting brought the outside world, its sounds and information, into the home. The radio soon became a symbol of potential changes that technology would bring to everyday life and possibilities that the twentieth century could offer (Forty, 1992, p. 200). Technical improvements have been minimal since the 1950s, but the design of the radio changed continuously to convey the aura of "breaking the frontiers of science" (Forty, 1992, p. 205). 
This evolution of the design of the radio was considered in the design process and influenced design decisions by focusing on futuristic forms rather than established ones. This was done to increase the area on the object that can be used to evoke an initiation of use and decrease potential product-related stigma.

\subsubsection{Reach to grasp movement}

Interaction with the radio focuses on initiating use of the affected arm and hand to carry out a reach and grasp movement, see Figure 9. This movement is essential in ADL and is focused on during the rehabilitation process with therapists. The movement requires a combination of gross and fine motor skills involving shoulder rotation, forearm flexion and extension, wrist tangential velocity, global hand rotation, as well as hand pronation, supination, adduction and abduction (Fan, He, \& Tillery, 2006).

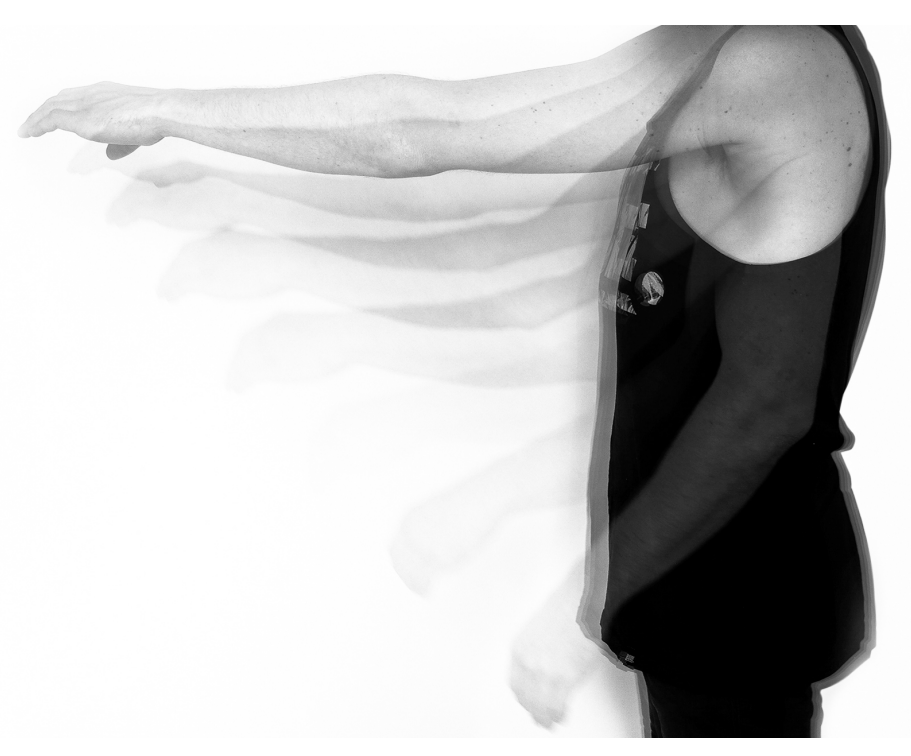

Figure 9:

Reach to grasp

movement. 


\subsection{Methods}

Three methods were used to address the research question. A RTD process led to the development of a number of prototypes intended to facilitate initiation of use, as currently none existed. The design iterations were assessed using the criteria based thesis model. During a cognitive walkthrough, experts gave feedback on the usability, safety, and anticipated initiation of use of those prototypes. Feedback was analysed with thematic analysis.

\subsubsection{Participants}

Health professionals with experience in using CIMT in clinical practice who had participated in the interviews were invited to participate in this part of the study and provide feedback on the design prototypes.

Two $(n=2)$ were occupational therapists and one $(n=1)$ worked as a clinical researcher and had previously worked as a physiotherapist. One was female $(n=1)$ and two male $(\mathrm{n}=2)$. Two worked with chronic stroke survivors $(\mathrm{n}=2)$, and one worked in the context of research with stroke survivors $(n=1)$. One used CIMT as the primary treatment, one used it on a regular basis and one had used it frequently in the past but was not working actively in a clinic anymore.

\subsubsection{Research through design}

A series of prototypes based on a trial and error basis were developed to address the research question under the pragmatist perspective, validate ideas, and guide further development. They were developed following an iterative design process in the form of serial design experiments (Krogh et al., 2015, p. 46) and evaluated during according to the defined criteria (Rodríguez Ramírez, 2017, p. 14).

The various design prototypes are based on the set of design criteria that was used in the process, see Figure 10. The design criteria are based on analysis of the interviews with stroke therapists, see Chapter 4. Additional design criteria were added based on the context of the intended movement and tangible interface requirements, see criteria 08 and 09. The insights and conclusions are summarised for each prototype in the results section. 


\begin{tabular}{|c|c|}
\hline $\begin{array}{l}\text { 01: } \\
\text { Restrain }\end{array}$ & $\begin{array}{l}\text { The interaction with the object needs to } \\
\text { restrain movement to evoke an initiation of use }\end{array}$ \\
\hline $\begin{array}{l}\text { 02: } \\
\text { Purpose }\end{array}$ & $\begin{array}{l}\text { The object needs to be used within an activity of } \\
\text { daily living }\end{array}$ \\
\hline $\begin{array}{l}\text { 03: } \\
\text { Repetition }\end{array}$ & $\begin{array}{l}\text { The interaction with the object needs to be } \\
\text { repetitive }\end{array}$ \\
\hline $\begin{array}{l}\text { 04: } \\
\text { Feedback }\end{array}$ & $\begin{array}{l}\text { Feedback needs to be provided as part of the } \\
\text { interaction }\end{array}$ \\
\hline $\begin{array}{l}\text { 05: } \\
\text { Challenge }\end{array}$ & $\begin{array}{l}\text { The interaction with the object needs to } \\
\text { become progressively challenging }\end{array}$ \\
\hline $\begin{array}{l}\text { 06: } \\
\text { Self-efficacy }\end{array}$ & $\begin{array}{l}\text { The interaction with the object needs to } \\
\text { contribute to developing self-perceived } \\
\text { self-efficacy of the user }\end{array}$ \\
\hline $\begin{array}{l}\text { 07: } \\
\text { Usability }\end{array}$ & $\begin{array}{l}\text { The interaction with the object needs to be } \\
\text { achievable for a stroke survivor with motor } \\
\text { impairments }\end{array}$ \\
\hline $\begin{array}{l}\text { 08: } \\
\text { Movement }\end{array}$ & $\begin{array}{l}\text { The interaction with the object needs to evoke a } \\
\text { "reach to grasp" movement }\end{array}$ \\
\hline $\begin{array}{l}\text { 09: } \\
\text { Technical } \\
\text { requirements }\end{array}$ & $\begin{array}{l}\text { The object needs to incorporate the NFC board + } \\
\text { Arduino Uno in a way that the user can interact } \\
\text { with the NFC tag attached to the } \\
\text { user's affected arm in order to turn the radio on }\end{array}$ \\
\hline
\end{tabular}

Figure 10:

Design criteria set 02 which was used to evaluate design concepts and prototypes during the development process 
Please refer to Appendix B, Workbook 01, for a full description of the different design iterations and reflections on the design elements.

\subsubsection{Cognitive walkthrough}

A formative usability evaluation during the design process was used to secure safe use of the object and validate the anticipated initiation of use. Experts can help in the process of evaluating the design and uncover usability problems in the same way the user would (Hartson et al., 2001, p. 379). Cognitive walkthroughs (Wilson, 2014, p. 66) were initially designed for "walk-up-and-use" interfaces. The method is based on the concept of a hypothetical user and does not require any actual users. Stroke survivors were involved in the second stage of this research, see Chapter 6. The task that has been focused on in the evaluation process with stroke therapists is that of performing a reach to grasp movement to turn on the radio. The focus of the cognitive walkthrough was on the intended initiation of use aimed to evoke by the object.

\subsubsection{Thematic analysis}

A thematic analysis in a deductive form (Braun \& Clarke, 2006, p. 83) was used to identify themes within the feedback that stroke therapists provided (Braun \& Clarke, 2006, p. 79). NVivo (version 11.2.2, qualitative data analysis software; QSR International Pty Ltd, Docaster, VIC, Australia) helped to structure themes mentioned during the feedback in regards to initiation of use and usability.

\subsection{Results}

\subsubsection{Research through design results}

Please refer to Appendix B, Workbook 1, for the full description of the different design prototypes. The following section outlines intended use, the different design iterations and the final design prototype. The reflections on each design prototype are outlined in the form of findings and conclusions that influenced the next design prototype. The reflections are based on the design criteria that were used as part of this study. 


\subsubsection{Use of the radio}

The object has the functionality of a radio, which is part of an activity of daily living: listening to music or conversations on radio stations. The user wears a bracelet on the affected side that lets the system recognise which arm is being used and which arm needs to be trained. The user can stroke, touch, and hit the top of the object to turn it on. Force applied depends on the type of touch and makes the radio bounce, similar to roly-poly children's toys, see Figure 11.

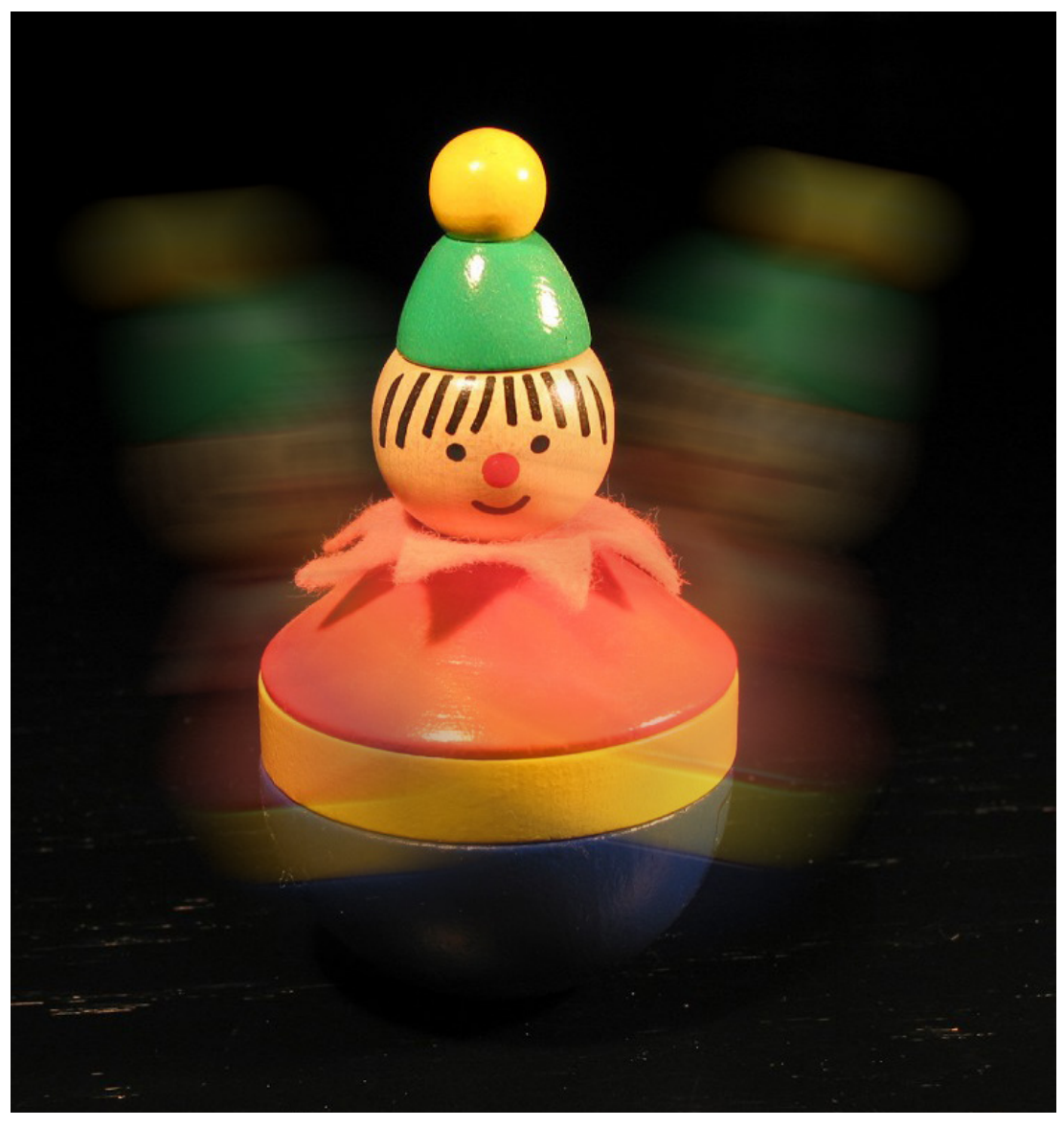

Figure 11:

Roly-poly or round bottom doll, a children's toy that rights itself when pushed over. Adapted from Stehaufmann in Wikipedia, B. D.

Wongx, 2006, retrieved January 1, 2018, from https://commons. wikimedia.org/wiki/ File:Stehaufmann. Printed with permission.

This bouncing gives immediate feedback that the movement has been completed and that the radio should start playing music. However, the radio only plays music when the user uses the affected arm to reach towards the object to touch it. The bracelet worn on the affected arm activates the digital components inside the radio and turns it on. 


\subsubsection{Restraining the movement}

To restrain movement, the radio uses digital technology that recognises when the affected arm and hand has been used. This recognition is based on near field communication (NFC) technology. For interaction with the final design prototype, a NFC bracelet carries the NFC tag that unlocks the full functionality of the radio, see Figure 12 and 13. A bracelet can be worn on either wrist, making it an ideal option as a stroke can affect either the left or the right side. Its appearance is similar to a watch to prevent stigmatising the user.

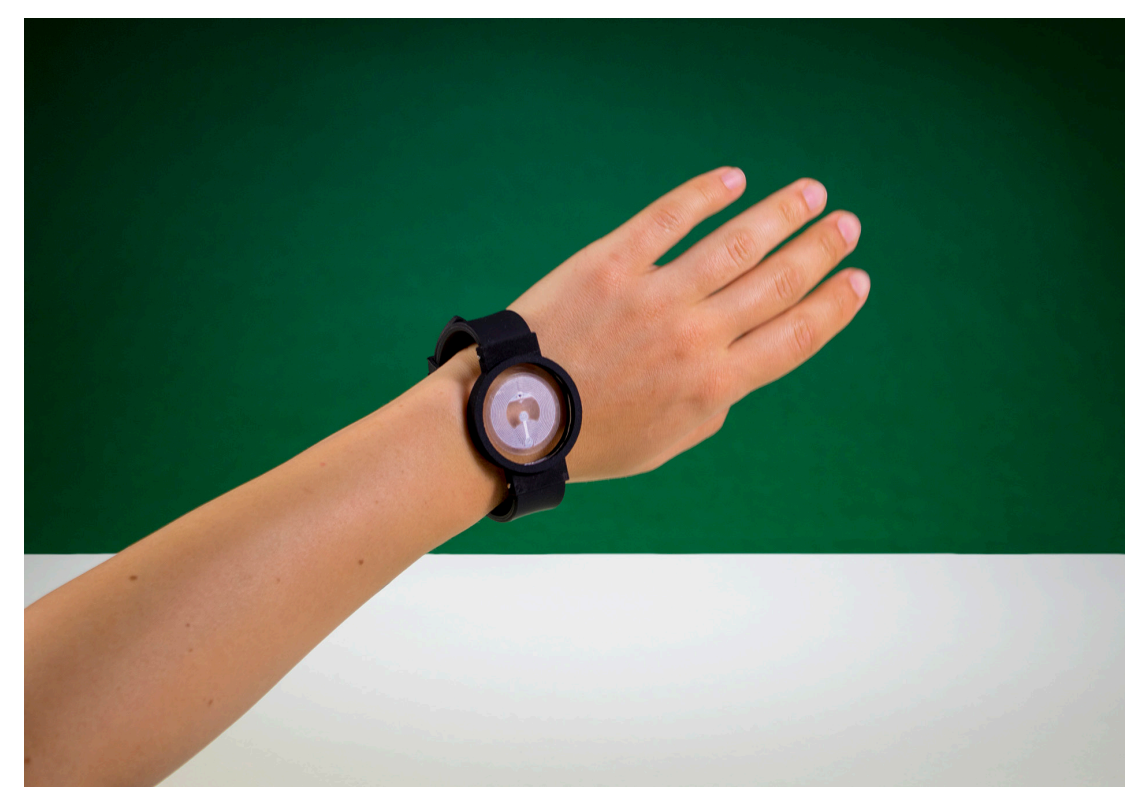

Figure 12:

Bracelet with full

functional NFC tag that is worn on the affected arm so the system recognises which arm is used.

The design is based on a persuasive influence which requires that the user is motivated and has the ability to use the affected arm and hand. There might be a potential for cheating by wearing the NFC rtag on the less affected hand. However, the design relies on the motivation of the user to use the affected arm and hand as much as possible and works as a reminder rather than forcing the user to use that side of the body.

The user is asked to interact with the radio repeatedly to have the music 


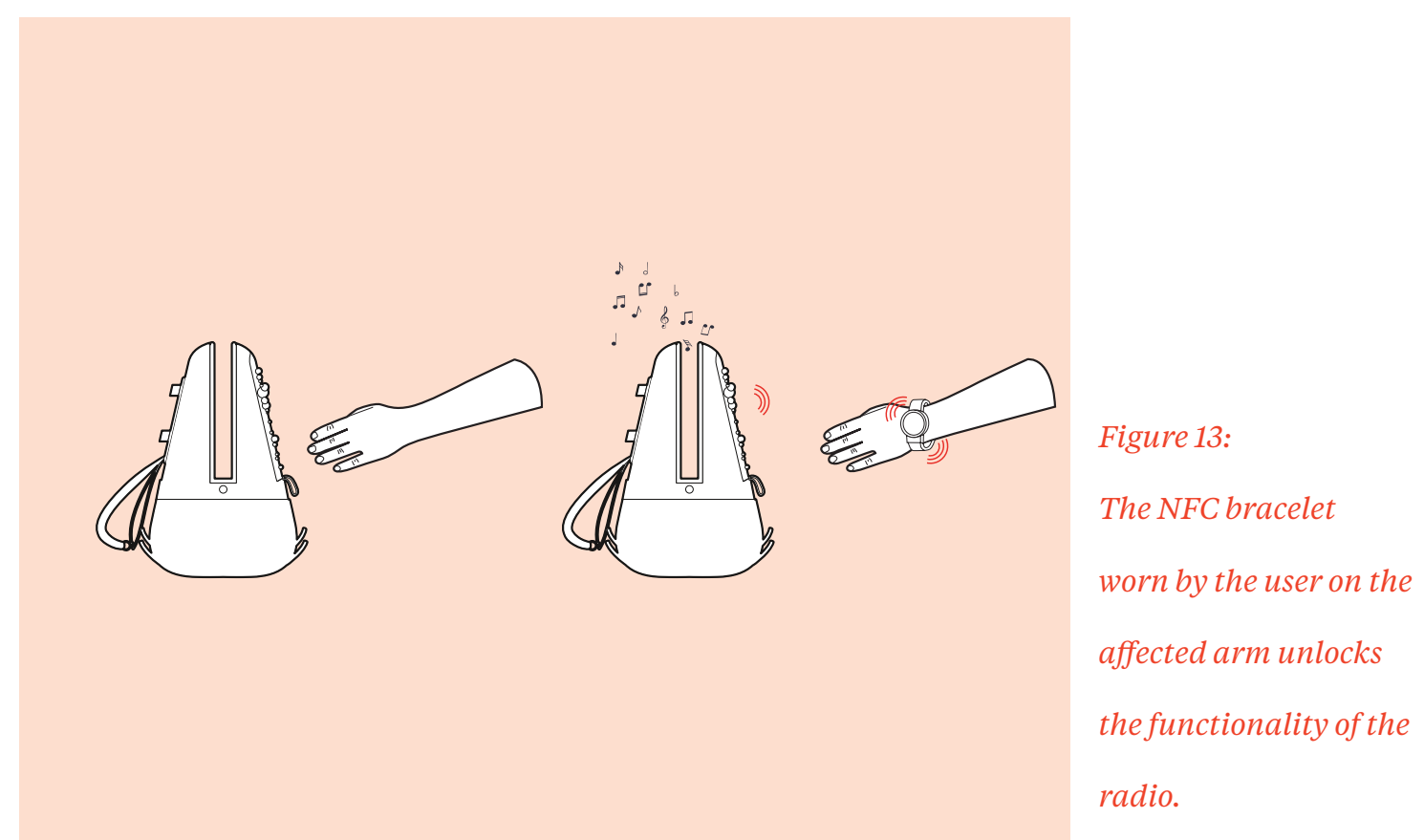

continually playing. Repetition of a movement is an essential element in the rehabilitation process. A repetitive movement is necessary to contribute to the development of plasticity in the brain. However, the user can change the time interval during which the radio plays music by pulling on a strip on its back. This strip is a soft potentiometer that controls the time setting.

\subsubsection{Design prototypes}

The following sections describe the different design iterations. Findings and conclusions are summarised according to the criteria based thesis model that was used during the design development process. 


\subsection{Prototype 01}

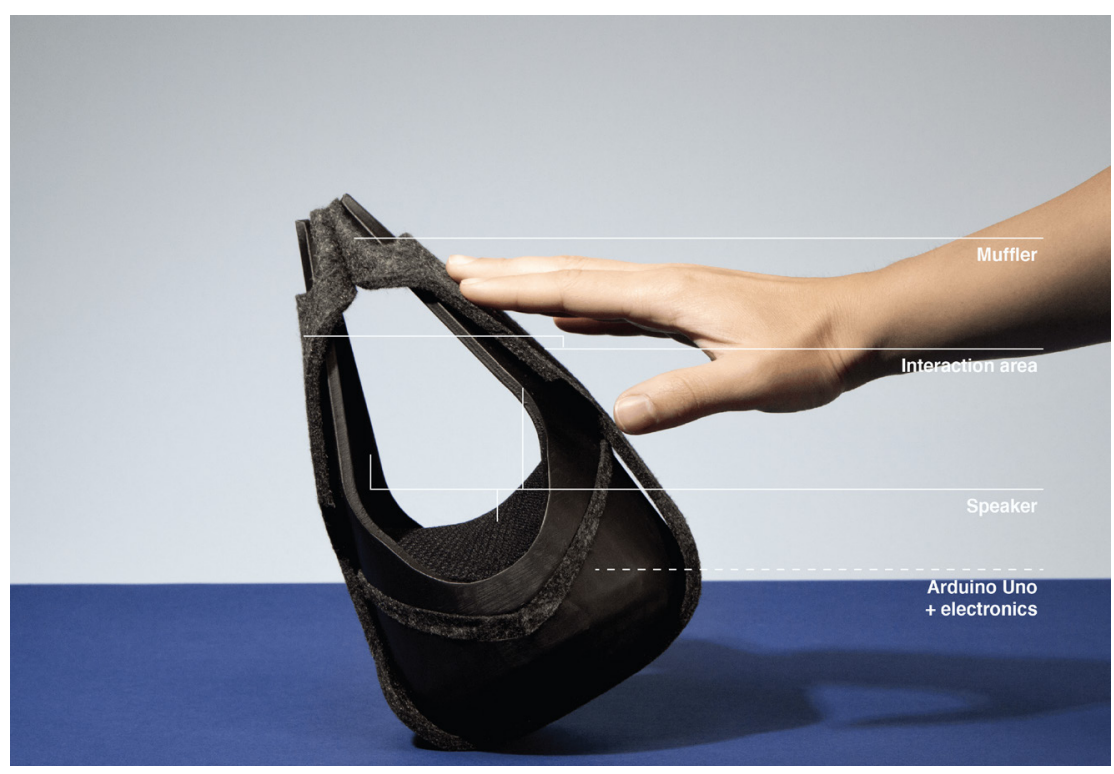

Figure 14:

3 printed radio prototype 01 with a felt sleeve to muffle the sound.

The concept of the first design prototype is that it only plays music when the user makes it bounce (see Figure 14). The movement gives the user an immediate indication about the movement quality; for example, whether too much force was applied. The object is elliptical to increase the bouncing movement. The user can touch the entire top part of the radio to turn it on and make it move.

Findings: Because of the ellipse form, the radio started rotating around its own centre after being hit the first time. The prototype was printed using a tabletop UP 3D printer and ABS printing material, which resulted in it making a lot of noise during movement. A sleeve of $5 \mathrm{~mm}$ felt was added to muffle the noise created by movement. Situating the NFC reader in the top rather than the bottom component increased the connectivity of the device.

Conclusion: The radio should be a circular shape to secure smooth movement. The weight is required to be as low as possible to ensure a smooth movement and to prevent the object from falling over. The electronics need to be carried in the top component, which is the activation zone to start the radio. 


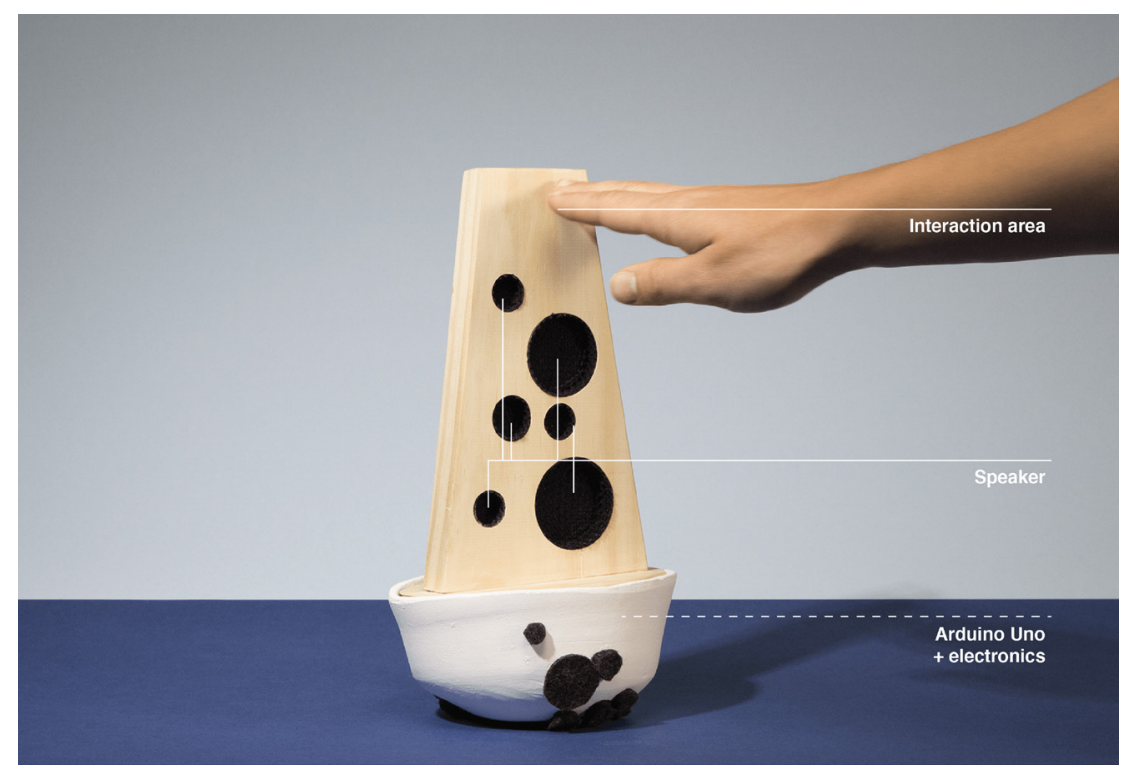

Figure 15:

Prototype 02 with a wooden top part and ceramic base.

This iteration of the radio shows a slimmer design, while the materials of the object relate more to objects that are used in the home environment: wood and ceramics (see Figure 15).

Findings: The design showed stable movement due to the heavy weight at the top. The top component did not provide sufficient space to house the NFC board, Arduino Uno and radio board (130mm x $55 \mathrm{~mm} \times 20 \mathrm{~mm})$. When bouncing, the radio could potentially fall to the ground, and the ceramic material could easily break.

Conclusion: The heavy weight of the base component provides a stable and smooth movement. The top component needs to be adjusted to house the electronics, requiring increased space inside the radio. 


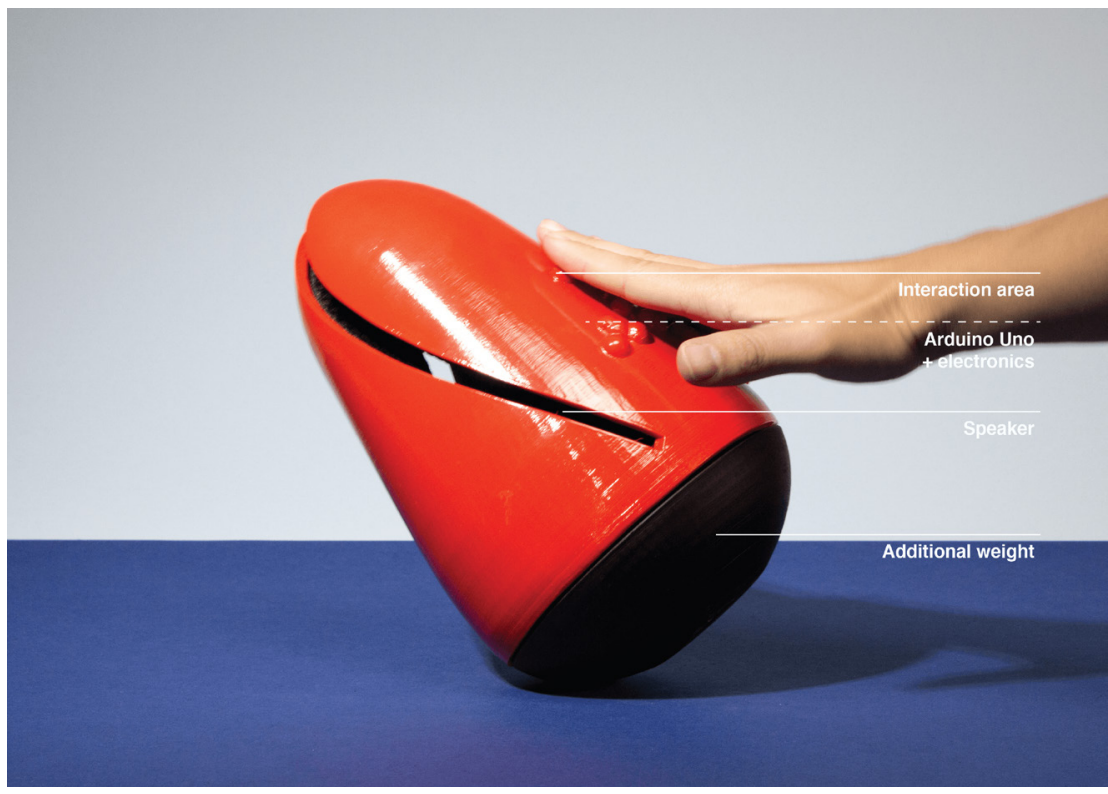

Figure 16:

Prototype 03 contains a textured surface to address tactile recognition deficits.

For the third iteration, the top component was changed to accommodate all the required electronics. A tactile surface visible on the top of the radio was designed to indicate and direct the user's interaction with the object, see Figure 16. The gap in the middle of the top component allows that the music can be clearly heard.

Findings: The fabric in the centre was not visible, and the gap was too small, potentially trapping fingers. The top part of the radio should be replaceable to allow people with tactile recognition deficits in their hands to choose a pattern that they can feel.

Conclusion: The pattern indicating where to interact with the object needs to be adjustable to allow different sensory experiences. Due to the increased height of the object, to accommodate all the electronics, the centre of gravity is situated too high. 


\subsection{Prototype 04}

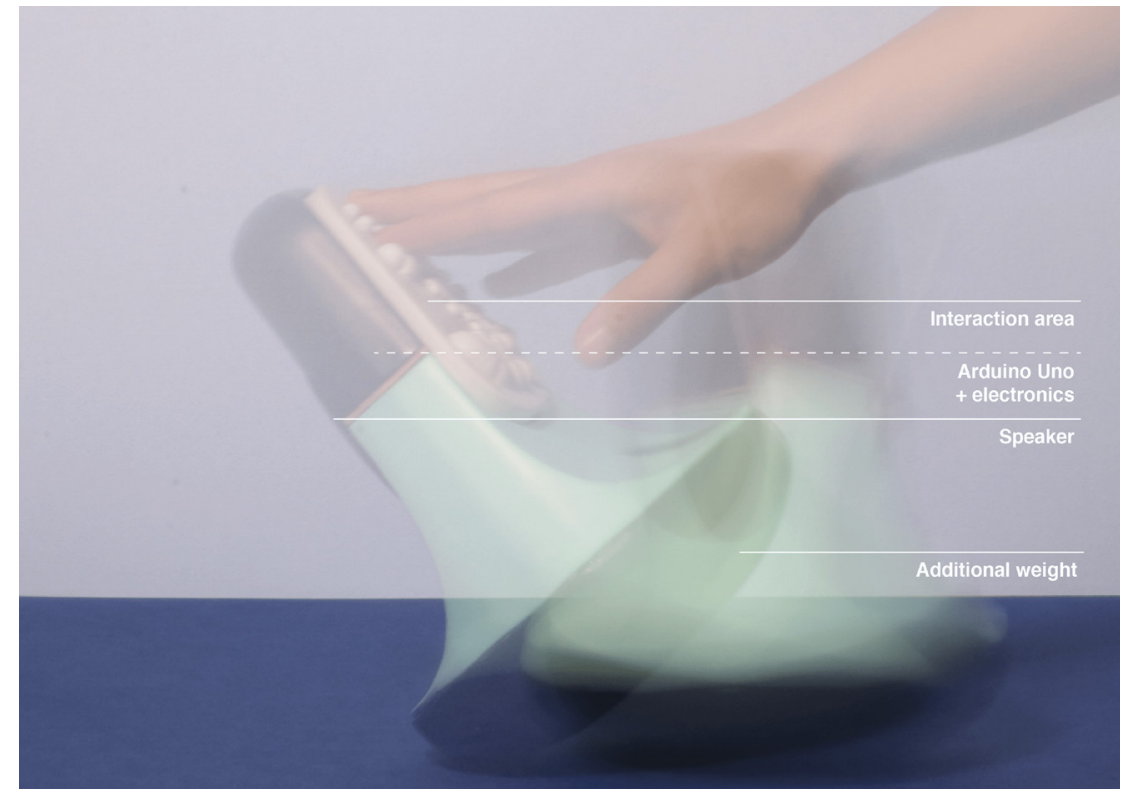

Figure 17:

Prototype 04 with a changeable interaction area.

The fourth iteration of the radio demonstrates a replaceable interaction area at the front with a round speaker at the back, see Figure 17. The base contains additional weight.

Findings: The movement was very stable due to the low centre of gravity. The top and bottom parts were permanently glued together, limiting the ability to change the weight inside the radio.

Conclusion: Elements in and on the radio that can be changed by the user offer progression and challenge, which are beneficial to the rehabilitation process. There should be different textured areas available to the user, and the user should be able to increase the weight of the radio. 


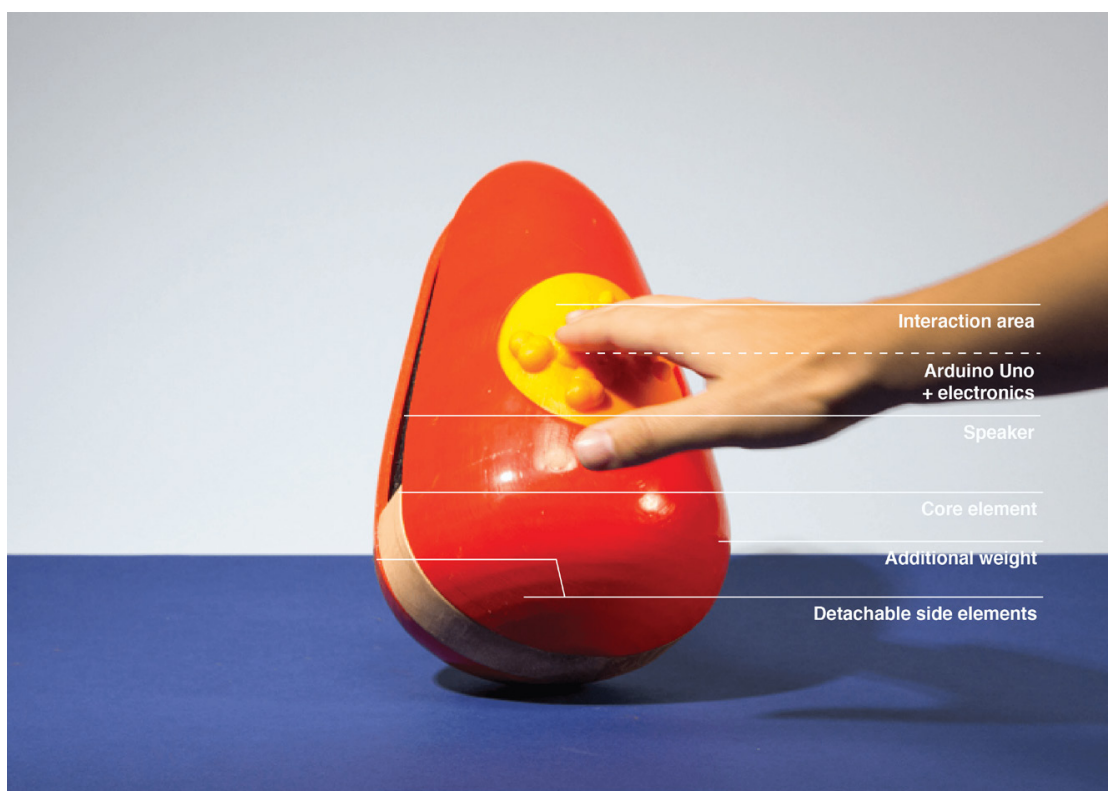

Figure 18:

Prototype 05 has a wooden core element.

For the fifth iteration, the radio was divided into two main sections situated around a wooden core, see Figure 18. The interaction area for the initiation of use is evident from a colour change and its tactile qualities.

Findings: The weight was too light, and the radio always fell over after the first touch. The concept was that the side elements could be detached from the core to offer the opportunity to adjust the internal weights. This was somewhat difficult to realise, and the shells should be permanently attached to the centre.

Conclusion: The top should contain all electronics and the base should provide the weight. The user should have the opportunity to progress in training and so should have a chance to face new challenges. The ability to change the training intervals should be incorporated. 


\subsection{Final design prototype 06}

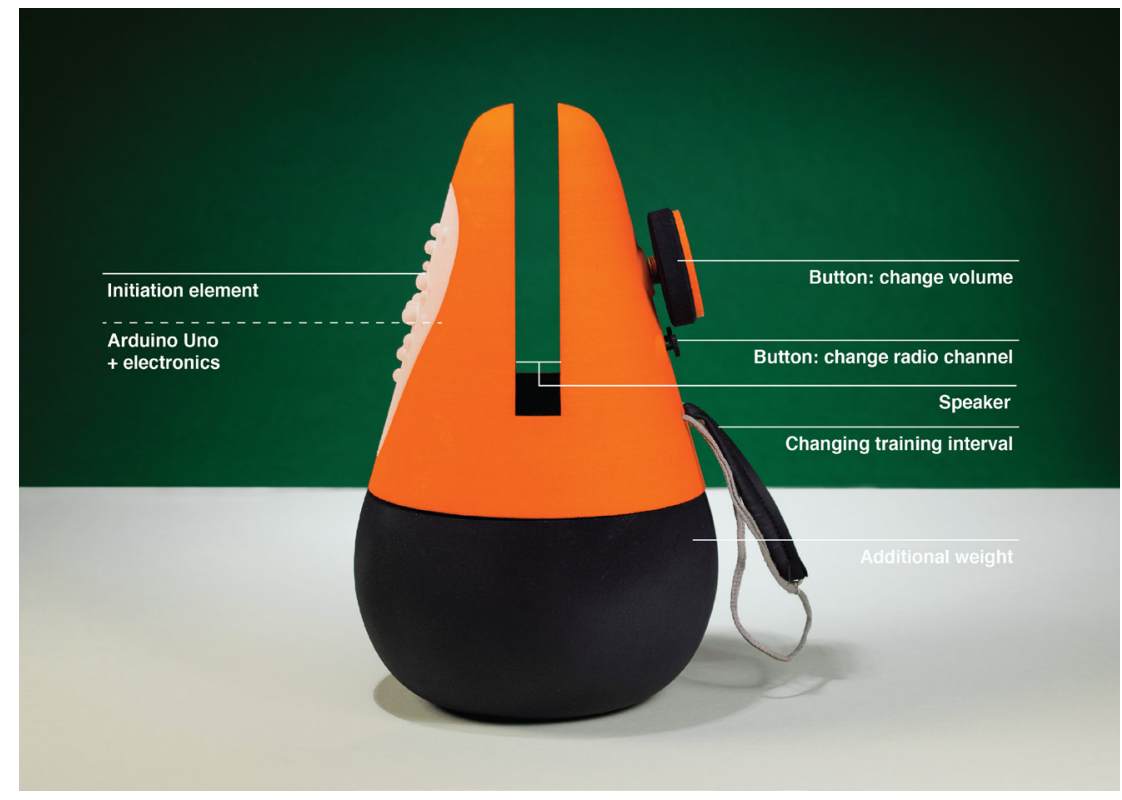

Figure 19:

Functional elements

of the final design

prototype.

The final design prototype contains two main components, see Figure 19. The top component is visually divided into two main interaction areas. The front, top area has an embedded, textured initiation area that can be detached and replaced by the user. The texture of this area addresses tactile discrimination deficits in the hands of the user and gives an indication of where to touch the radio. To ensure the NFC tag in the bracelet is recognised in a timely manner the user needs to touch the object on the front area, see Figure 20. This is because the location of the NFC board, which is right behind the initiation area. 


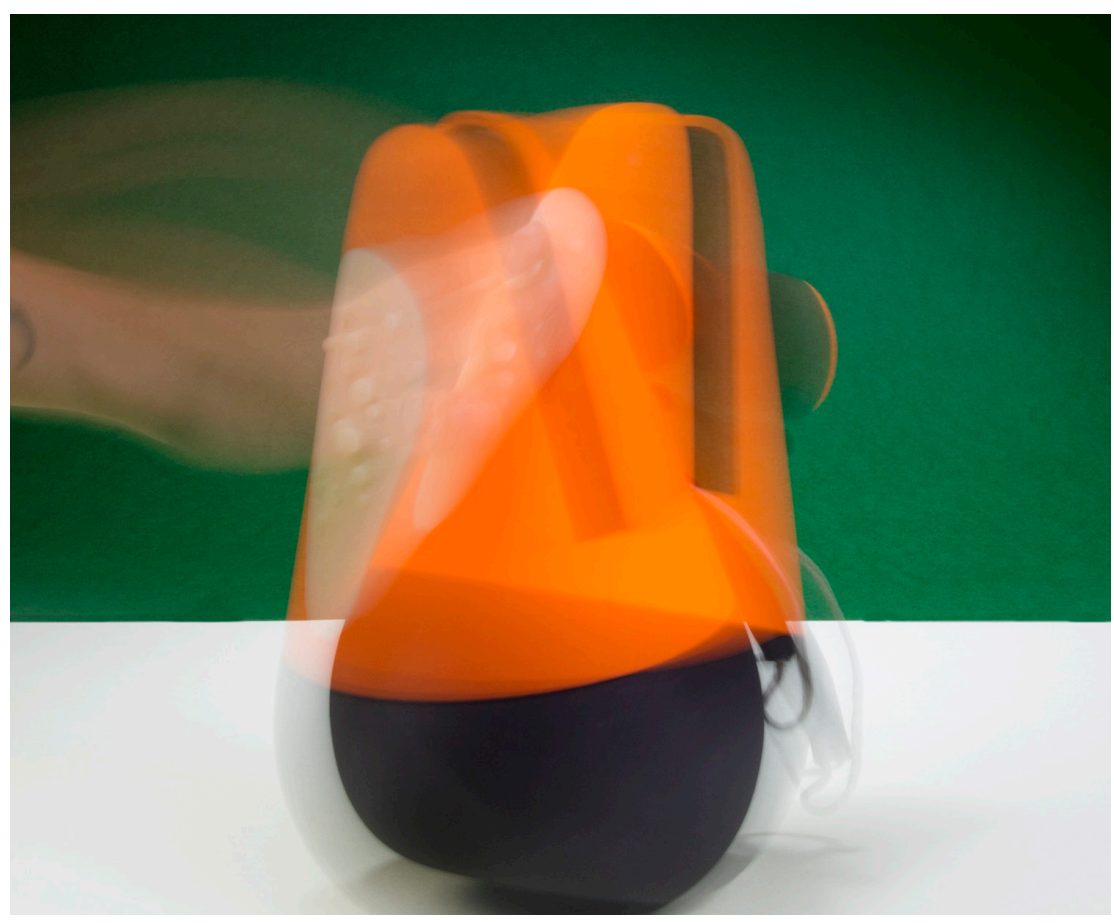

Figure 20:

The user must move the affected hand close to the white front area in order to turn the radio on. The radio starts bouncing in case the user does not have full control over their arm movement.

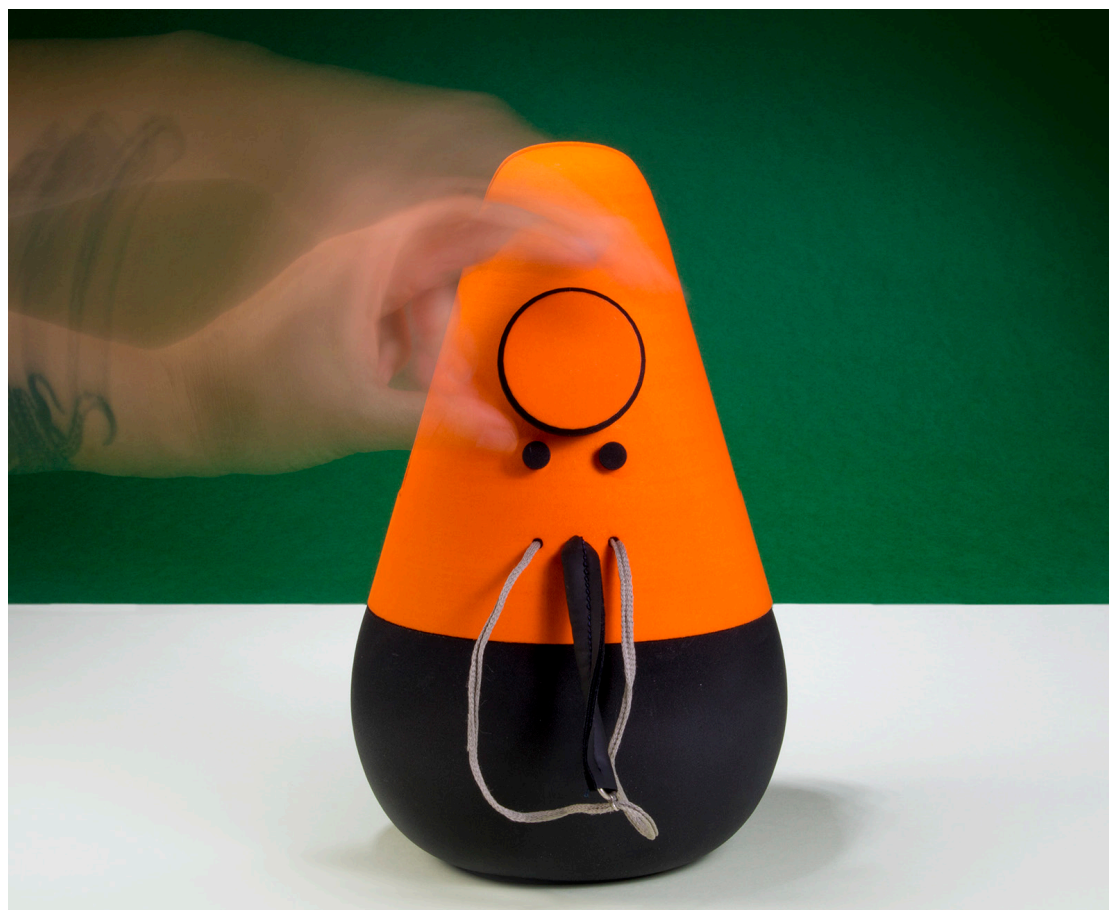

Figure 21:

Back of the radio: the user can change the radio station, training interval and volume on this side. To change the training interval setting from 30 s, $60 \mathrm{~s}$ or 90 s the user pulls on the black string, which is connected to a soft potentiometer. 
A second interaction area is located on the back of the radio. Here the user can change the training interval by pulling on the black strip of the soft potentiometer, or change the radio station and volume by pushing and turning the buttons on the back of the radio; see Figure 21. Once the user interacts with the radio by pushing the top area, it is activated and starts playing music for a pre-set time interval before turning off. It was emphasised by the stroke therapists during the interviews, see Chapter 4 , that increased repetitions of a movement are necessary for the development of plasticity in the brain. The user is therefore required to interact with the radio again once it has stopped playing. This training interval is based on the three different time settings of 30 seconds, 60 seconds, and 90 seconds that can be adjusted with a soft potentiometer at the back of the radio (see Figure 21).

Progression is implemented within the interaction in two forms. The object has some additional weight at the bottom that can be increased to make it more challenging, see Figure 22. Secondly, the interaction area is made from 3D printed material with a textured surface that can be changed according to the user's deficits in the hands, see Figure 23. 


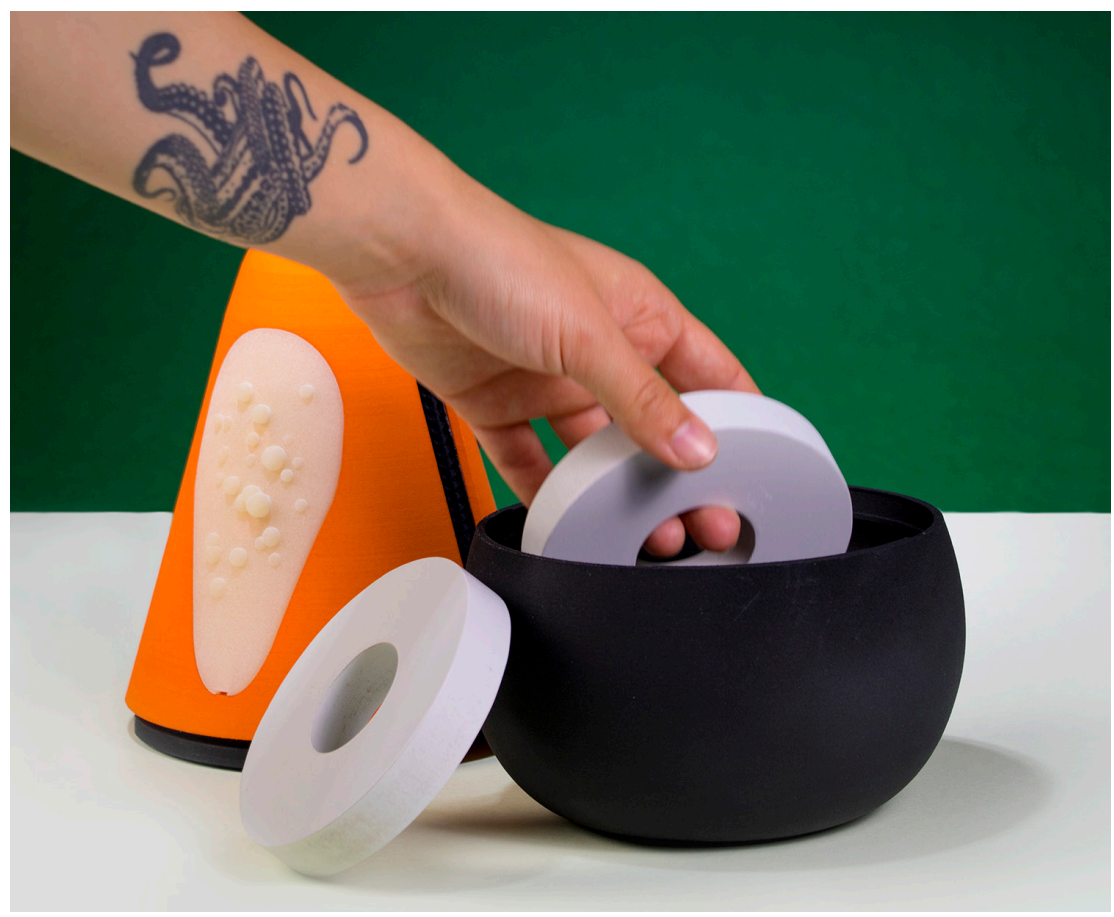

Figure 22:

The user can detach the top component to add weight inside the bottom component to increase the challenge in the interaction.

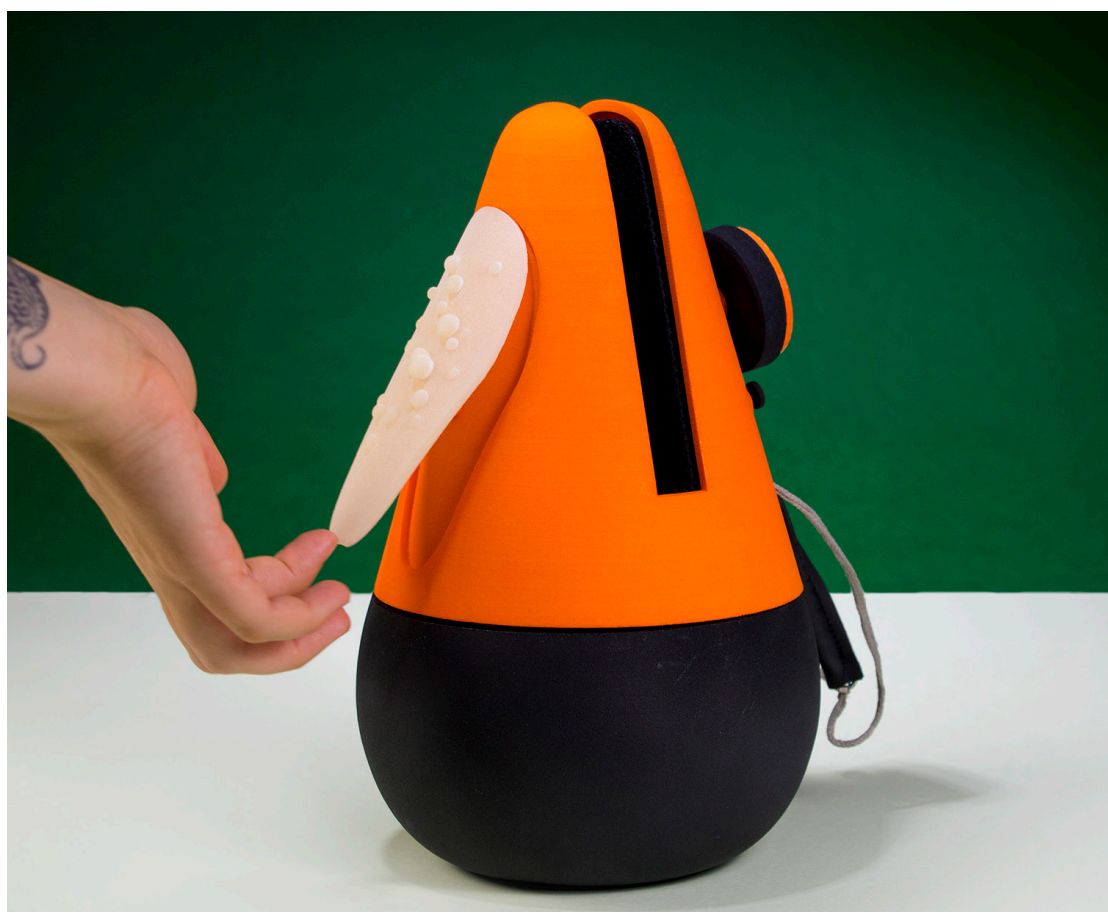

Figure 23:

The texture of the

interaction area can be replaced. 


\subsection{Technology to restrain the movement}

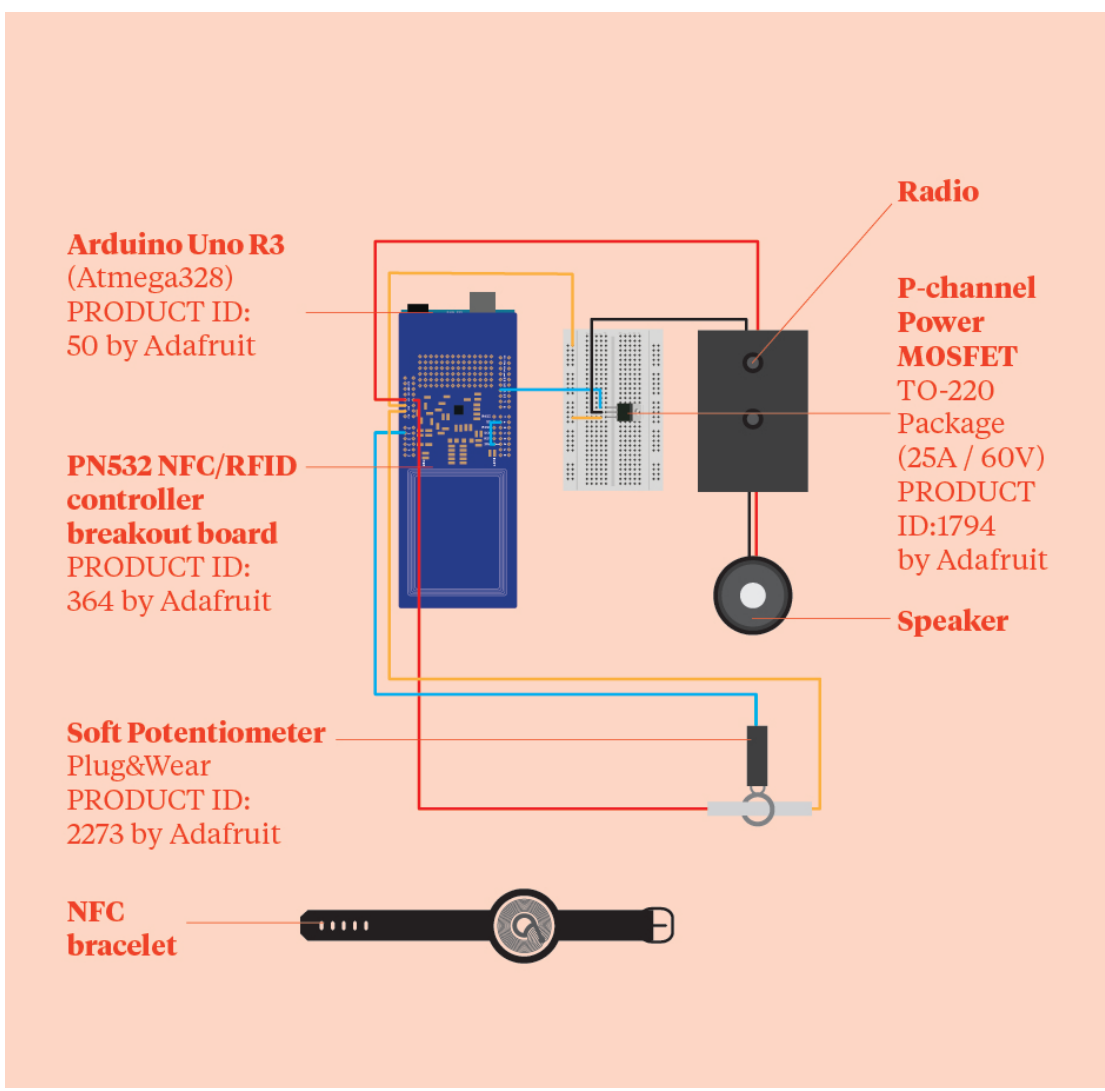

Figure 24:

Schematic overview

of the technical

components.

Arduino, a rapid prototyping tool, was chosen for the technical development. The Arduino Uno needs to recognise which arm is being used to interact with the object to start the restraining effect appropriately. Near field communication (NFC) offers this recognition in the form of an NFC board that is attached to the Arduino UNO board and NFC tags that the user can wear on the affected arm and hand. See Figure 24 for a schematic overview of the different components. The Arduino UNO and NFC board regulate the electricity for the radio for a pre-set time before turning it off again.

The final setup has the NFC board located in the top right of the radio, see Figure 25, and the radio and speaker are on the left side, see Figure 26 . The radio board is based on a commercially available COBLAT PLAU00335 shower radio and was chosen after numerous tests because of its small size. 


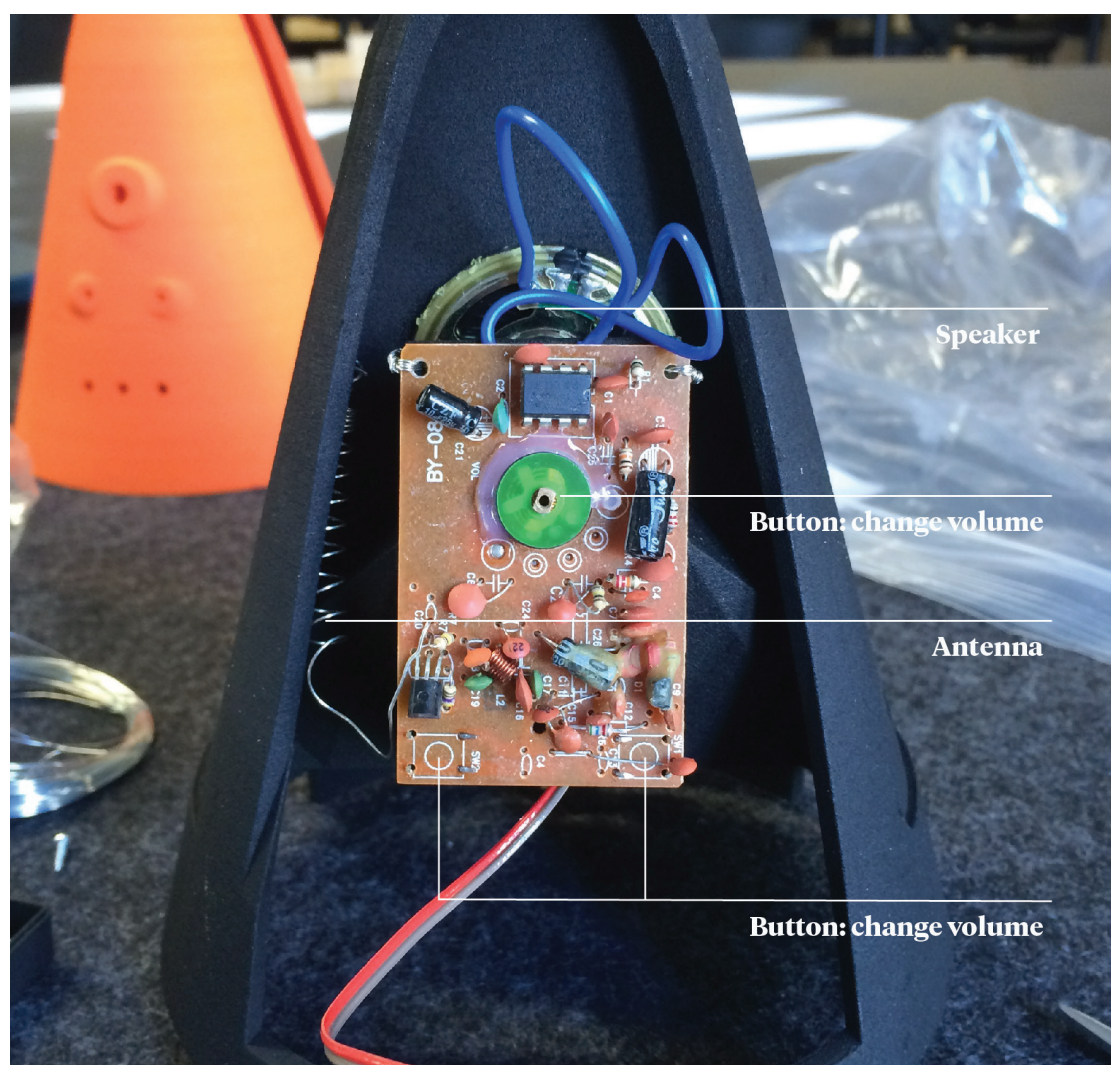

Figure 25:

Radio board.

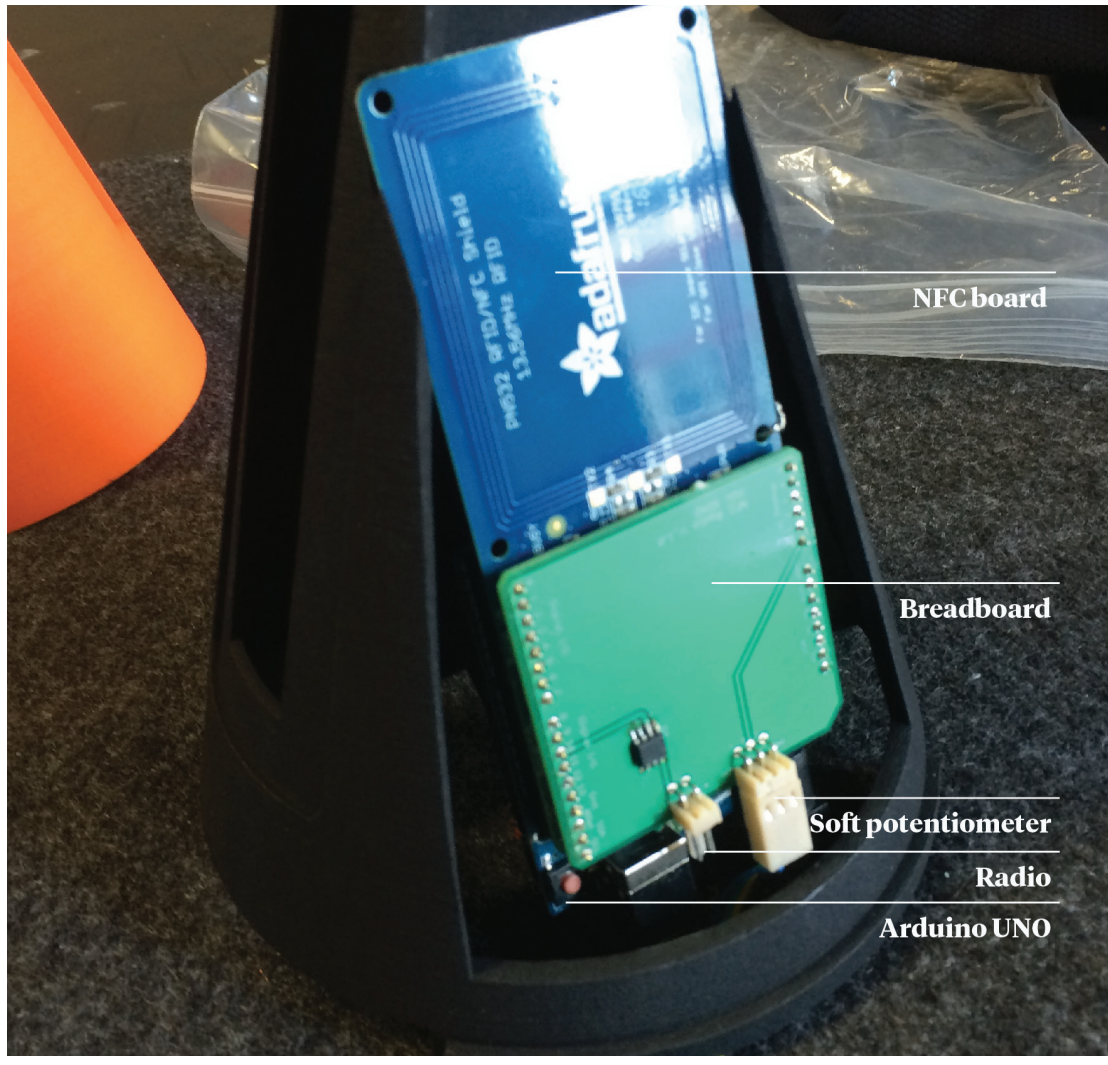

Figure 26:

Arduino UNO and

NFC board. 


\subsubsection{Evaluation}

\subsubsection{Evaluation against design criteria}

The prototypes were evaluated during the design process against the set of design criteria, see Figure 27. Health professionals evaluated the design prototype with a cognitive walkthrough focusing on initiation of use and usability issues. The results will be outlined in the following section.

Criteria 06, 07 and 08 could not be evaluated during the design process because they require stroke survivors. The criteria were tested with a pluralistic walkthrough, see Chapter 6. Criteria 06 as a design criterion is too complex due to the lack of information concerning effective ways to evoke self-efficacy in the context of upper limb stroke rehabilitation. The development of self-efficacy needs to be evaluated with long-term studies, which is outside the scope of this research. 


\begin{tabular}{|c|c|c|c|c|c|c|c|}
\hline & & 1 & 2 & 3 & 4 & 5 & 6 \\
\hline $\begin{array}{l}\text { 01: } \\
\text { Restrain }\end{array}$ & $\begin{array}{l}\text { The interaction with the object needs to } \\
\text { restrain movement to evoke an initiation } \\
\text { of use }\end{array}$ & & & & & & $\mathbf{x}$ \\
\hline $\begin{array}{l}\text { 02: } \\
\text { Purpose }\end{array}$ & $\begin{array}{l}\text { The object needs to be used within an } \\
\text { activity of daily living }\end{array}$ & $\mathbf{x}$ & $\mathbf{x}$ & $\mathbf{x}$ & $\mathbf{x}$ & $\mathbf{x}$ & $\mathbf{x}$ \\
\hline $\begin{array}{l}\text { 03: } \\
\text { Repetition }\end{array}$ & $\begin{array}{l}\text { The interaction with the object needs to } \\
\text { be repetitive }\end{array}$ & $\mathbf{x}$ & $\mathbf{x}$ & $\mathbf{x}$ & $\mathbf{x}$ & $\mathbf{x}$ & $\mathbf{x}$ \\
\hline $\begin{array}{l}\text { 04: } \\
\text { Feedback }\end{array}$ & $\begin{array}{l}\text { Feedback needs to be provided as part of } \\
\text { the interaction }\end{array}$ & $\mathbf{x}$ & $\mathbf{x}$ & $\mathbf{x}$ & $\mathbf{x}$ & $\mathbf{x}$ & $\mathbf{x}$ \\
\hline $\begin{array}{l}\text { 05: } \\
\text { Challenge }\end{array}$ & $\begin{array}{l}\text { The interaction with the object needs to } \\
\text { become progressively challenging }\end{array}$ & & & $\mathbf{x}$ & $\mathbf{x}$ & $\mathbf{x}$ & $\mathbf{x}$ \\
\hline $\begin{array}{l}\text { 06: } \\
\text { Self-efficacy }\end{array}$ & $\begin{array}{l}\text { The interaction with the object needs to } \\
\text { contribute to developing self-perceived } \\
\text { self-efficacy of the user }\end{array}$ & & & & & & \\
\hline $\begin{array}{l}\text { 07: } \\
\text { Usability }\end{array}$ & $\begin{array}{l}\text { The interaction with the object needs to } \\
\text { be achievable for a stroke survivor with } \\
\text { motor impairments }\end{array}$ & & & & & & \\
\hline $\begin{array}{l}\text { 08: } \\
\text { Movement }\end{array}$ & $\begin{array}{l}\text { The interaction with the object needs to } \\
\text { evoke a "reach to grasp" movement }\end{array}$ & & & & & & \\
\hline $\begin{array}{l}\text { 09: } \\
\text { Technical } \\
\text { require- } \\
\text { ments }\end{array}$ & $\begin{array}{l}\text { The object needs to incorporate the NFC } \\
\text { board + Arduino Uno in a way that the } \\
\text { user can interact with the NFC tag at- } \\
\text { tached to the user's affected arm in order } \\
\text { to turn the radio on }\end{array}$ & & & & $\mathbf{x}$ & & $\mathbf{x}$ \\
\hline
\end{tabular}

Figure 27:

Evaluation against the design criteria set 02. 


\subsubsection{Cognitive walkthrough with health professionals}

\subsection{Themes}

The next section describes the outcomes of the cognitive walkthrough that was carried out with health professionals. Feedback was analysed in a deductive form according to the themes of initiation of use and usability.

\section{Initiation of use}

The therapists stated that the interaction with the radio requires a movement that would be too difficult for most stroke survivors because of the wrist extension necessary to make the object move.

Bending the hand at 90 degrees won't work. Clinical researcher (Participant 08)

The object in its current form was too difficult to use to evoke initiation of use. The fine motor skills for this type of movement require a high level of accuracy to use the small buttons at the rear of the radio.

[...] because most people don't go from there to there they go there [makes a downwards movement with the finger]. So you need some of these buttons either resisting or coming out so you can do still do that task so gradually as they get better they can put it in and fold it back up or something like that. Occupational therapist (Participant 02)

The therapists recommended an initial focus on a gross movement and increasing the difficulty and accuracy required for the interaction over time. This was implemented in later design iterations. This approach would be less frustrating for the user and more feasible for self-directed use in the home environment. It was further pointed out that the object needs to focus more on the 
movement that is needed to interact with the object. The reach to grasp movement is just one movement component that is needed to interact with the radio.

That is like going to the gym and doing curls that is never going to teach you how to pick that up.

Occupational therapist (Participant 03)

One of the therapists appreciated the bouncing of the radio as an additional form of feedback while another therapist mentioned that the bouncing increases the risk of the object dropping to the floor. The decreased balance ability of stroke survivors makes it difficult for them to pick up objects from the ground. They recommended attaching the radio to a surface to prevent it from falling.

So it has to be things that are either easy to set back up or harder to knock over because they keep knocking them over. They knock them on the floor, then the balance is not that good and the try to get it off the floor and they are falling over.

Occupational therapist (Participant 02)

It also became clear that interaction with the object depends on a lot of explanation. Neither the function nor intended use of the object seemed to be obvious at this stage.

\section{Usability}

The therapists recommended focusing on other tasks involving everyday objects, for example light switches or mobile phones. These tasks are potentially more beneficial in developing plasticity because people interact with them more frequently on a day-to-day basis. 
Yes, for people who use a radio that might be great. Maybe for some it is not the radio, maybe it is the computer or an iPad or whatever stuff on the wall. Occupational therapist (Participant 02)

The base component contains weights that the participant can increase over time to keep the interaction challenging. One therapist identified that the weights in the bottom should be decreased instead of increased over time to challenge the user more by requiring a higher level of coordination skills.

It could involve coordination training if you have to tip it at a specific distance and this could help with controlling.

Clinical researcher (Participant 08)

The therapists mentioned that interaction with the object would be mastered by the user at some stage. They recommended that once this stage is reached, the object should have a purpose and be able to be used in the home environment.

\subsection{Discussion}

This section discusses the design process and evaluation with experts.

\subsubsection{The design process}

Stroke can produce a variety of symptoms, and feedback from the therapists indicated that no one design solution will fit this diverse user group. A rehabilitation session was observed at the beginning of this research to gain an understanding of how motor impairments of the arm and hand look after a stroke. However, ensuring the usability of the design for someone with motor impairments was extremely challenging because of the variety of symptoms that are possible after a stroke. I participated in a workshop with a focus on CIMT that provided some important insights into how motor impairments look and how they 
affect movement. That workshop was extremely beneficial in understanding the impact of motor impairments after a stroke.

Conclusion: Motor impairments and the correct movement need to be visually presented to develop understanding of and empathy for the user.

\subsubsection{Technology to facilitate the initiation of use}

The technology used to restrict interaction to one side of the body required the NFC tag to be in proximity for a relatively long time interval. The potential user would have to interact rather slowly so the radio could recognise the tag on the bracelet. A ring with an NFC tag could potentially increase the connection between the board and the tag to make sure that the tag is recognised immediately.

Conclusion: An NFC ring could potentially replace the NFC bracelet.

\subsubsection{Initiation of use}

The object requires a high level of wrist extension and fine motor skills as part of the initiation of use. The reaching movement depends on the coordination of shoulder flexors, elbow extensors, and posture supporting muscles. This coordination is often affected in stroke survivors (Kisiel-Sajewicz et al., 2011, p. 359). Further factors contributing to poor performance that need to be considered are abnormal muscle activation patterns (Dewald, Pope, Given, Buchanan, \& Rymer, 1995), impaired sensation, joint individuation deficits, sensory impairments (Zackowski, 2004, p. 1045), muscle weakness especially in the handgrip (Mercierand \& Bourbonnais, 2004, p. 220) and a weakened functional coupling between the brain and muscles or among the synergists (Fang et al., 2009).

Conclusion: The amount of wrist extension needs to be reduced, and buttons need to be situated horizontally. Weakened muscles, impaired sensation, and abnormal muscle synergies could further impact on the movement and need to be considered. 
Further points of improvement mentioned by the therapists are the balancing issues caused by the bouncing movement of the object and the constant challenge that the object needs to provide during the interaction. A lack of balance is a common symptom post-stroke, affecting up to $83 \%$ of all stroke survivors (Tyson, Hanley, Chillala, Selley, \& Tallis, 2006, p. 30).

Conclusion: The interaction with the object should start with a gross movement and progressively get more challenging. The object needs to be stable and situated on a surface, for example a kitchen counter.

\subsubsection{Therapeutic value}

It was pointed out that the object evokes a repetitive movement which is referred to as blocked practice. Changes in the brain as a result of blocked practice are not permanent and do not necessarily lead to the acquisition of skilful motor performance (Carey, Bhatt, \& Nagpal, 2005, p. 27). Complex tasks, on the other hand, that demand a cognitive reflection process contribute to improved skill acquisition. One concept explaining why more complex tasks are beneficial for plasticity is contextual interferences (Carey et al., 2005, p. 27). These have been shown to produce better recall and increased adaptability when compared to blocked practice (Magill \& Hall, 1990, p. 247). Factors such as the task itself, the practice schedule, or feedback are seen as potential sources of interference that can increase learning outcomes (Magill \& Hall, 1990, p. 244) and contribute to long-term changes in the brain.

Conclusion: The interaction with the object should focus on random practice rather than blocked practice.

Feedback that influences the engagement of the participant is an essential part of the rehabilitation process (Morris et al., 2006, p. 260). The use of digital technology offers the opportunity to collect data about the amount and intensity of use. The data then can be reflected back to the user to show progress over time and influence long-term engagement. 
Conclusion: The object should include feedback in the form of a summary of performance after each session. Feedback frequency should decrease over time so that the person relies on intrinsic learning. The radio was assessed as being suitable for the intended target group, but a focus on activities of daily living such as turning on the lights might be more beneficial.

Conclusion: Further investigations are needed of everyday objects used in activities of daily living that can be used to evoke initiation of use. This was done in an online survey with chronic stroke survivors; see Chapter 9.

The usability evaluation started with a description of the intended use of the object. Some of the design elements had to be explained in detail during the evaluation process.

Conclusion: The object needs to provide clear feedforward and feedback elements to ensure intuitive use. Feedforward elements need to indicate clearly how to interact with the object to make sure that the affected arm and hand is used.

\subsection{Conclusion}

The design prototypes aim to restrict movement to one side of the body. Experts evaluated the design and recommended changes to improve the intended initiation of use and usability of the design. The design needs to provide clear feedforward and feedback elements to be intuitively usable and needs to consider the users' decreased level of fine motor skills. These recommendations will be implemented in the second stage of the design iterations reported in Chapter 6. 


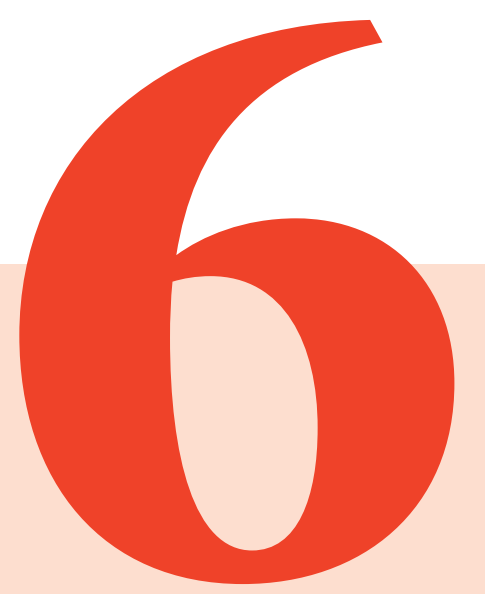

\section{The Design of a} Tangible Interface to Facilitate an Initiation of Use II 


\subsection{Introduction}

This chapter is based on the initial findings of the previous chapter and presents the refinement of the initial design prototype. The final design prototype was evaluated by chronic stroke survivors using a pluralistic walkthrough. The results of this chapter were used to address the research question:

1.3.1 How can we design an everyday object that restrains movement of the less affected arm to facilitate and initiate the use of the affected arm?

\subsection{Background}

The cognitive walkthrough with health professionals revealed that the object's feedback and feedforward elements needed to be refined.

\subsubsection{Feedback and feedforward elements}

The theoretical framework of Crilly, Moultrie, \& Clarkson (2004) describes cognition and affect as the main factors influencing how a user perceives and uses an object. The framework distinguishes between the three design elements that influence the cognition of an object: aesthetic attributes, semantic interpretation, and symbolic association (Crilly et al., 2004, p. 555).

Although the theoretical framework by Crilly et al. (2004) offers a rich description of elements that influence the use and perception of an object, the framework ends with the performed action and lacks explanations of the feedback that arises as part of the action. In this study I will, therefore, refer to the Interaction Frogger by Wensveen, Djajadiningrat, \& Overbeeke (2004) (Figure 28) for feedforward and feedback elements. The Interaction Frogger does not take account of symbolic and aesthetic elements of design but defines feedforward and feedback elements in a greater detail. 
LIMITED USE ONLY

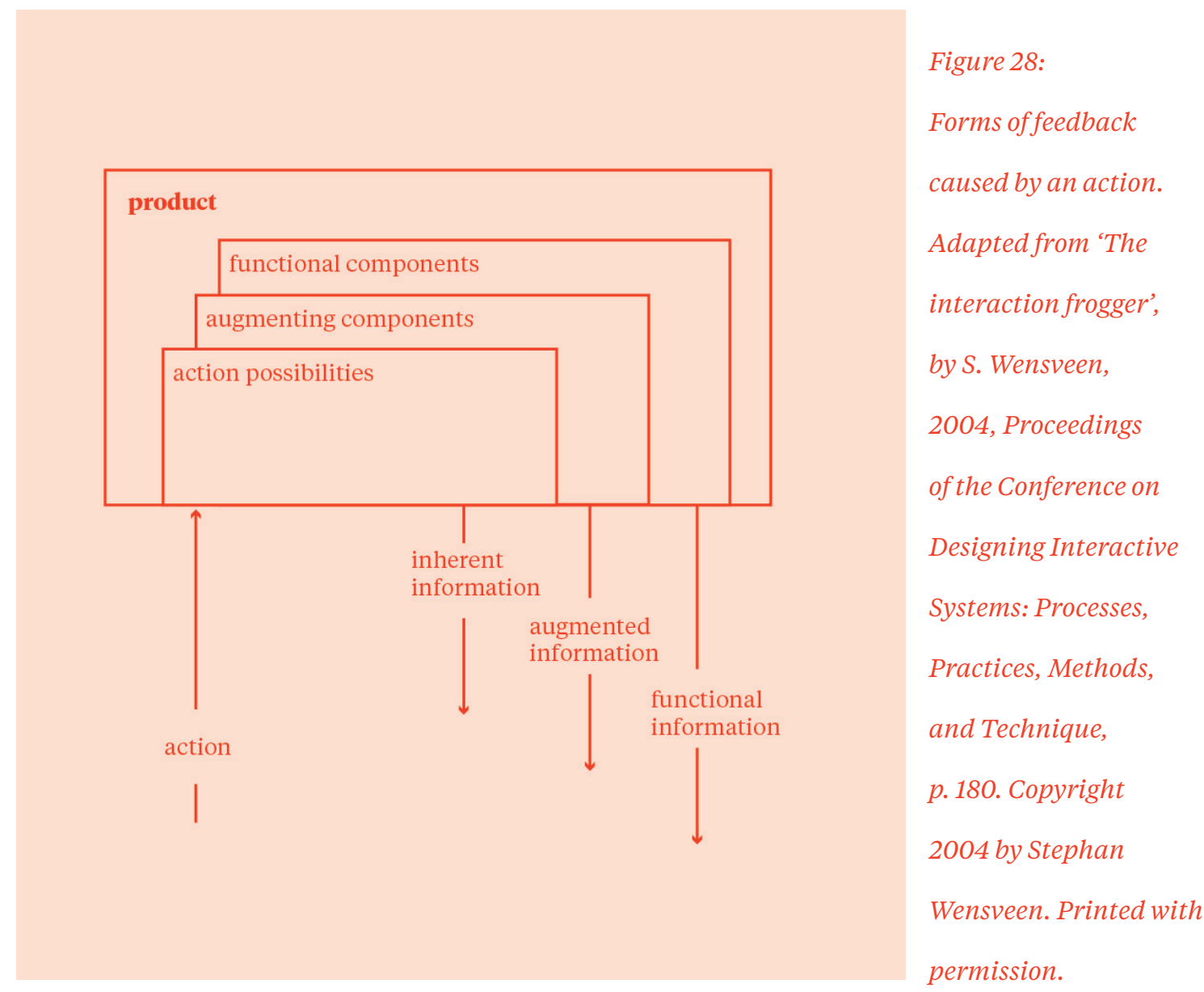




\begin{tabular}{|c|c|c|c|}
\hline $\begin{array}{l}\text { Design: } \\
\text { Coupling action and } \\
\text { function } \\
\text { (Wensveen et al., 2004) }\end{array}$ & $\begin{array}{l}\text { Stroke rehabilitation: } \\
\text { Initiation of use } \\
\text { (Semi-structured } \\
\text { interview content) }\end{array}$ & $\begin{array}{l}\text { Psychology: } \\
\text { Self-efficacy } \\
\text { (Bandura, 1997) }\end{array}$ & $\begin{array}{l}\text { Formative usability } \\
\text { evaluation }\end{array}$ \\
\hline $\begin{array}{l}\text { Functional feedforward } \\
\text { Form: The information } \\
\text { about the general } \\
\text { purpose of a product and } \\
\text { its features. }\end{array}$ & $\begin{array}{l}\text { Task oriented training of an } \\
\text { activity of daily living } \\
\text { Form: Use of an everyday } \\
\text { object }\end{array}$ & & $\begin{array}{l}\text { Analytical evaluation is the } \\
\text { analysis of the characteris- } \\
\text { tics of the design through } \\
\text { examination of the design } \\
\text { representation, prototype, } \\
\text { or implementation }\end{array}$ \\
\hline \multirow{3}{*}{$\begin{array}{l}\text { Augmented feedforward } \\
\text { Form: The information } \\
\text { received from an additional } \\
\text { source about the action } \\
\text { possibilities or the purpose } \\
\text { of the action possibilities }\end{array}$} & $\begin{array}{l}\text { Coaching } \\
\text { Form: Demonstration of the } \\
\text { right movement }\end{array}$ & $\begin{array}{l}\text { Vicarious experience } \\
\text { Form: Different variations } \\
\text { of modelling such as actual } \\
\text { and symbolic modelling }\end{array}$ & \\
\hline & $\begin{array}{l}\text { Use of the MAL } \\
\text { Form: Semi-structured } \\
\text { interview determining } \\
\text { goals for rehabilitation }\end{array}$ & $\begin{array}{l}\text { Verbal persuasion } \\
\text { Form: Degree of appraisal } \\
\text { disparity }\end{array}$ & \\
\hline & $\begin{array}{l}\text { Restraining the movement } \\
\text { to initiate use of the affect- } \\
\text { ed upper extremity (UE) } \\
\text { Form: physical restraint on } \\
\text { the less affected side as a } \\
\text { reminder }\end{array}$ & & \\
\hline $\begin{array}{l}\text { Inherent feedforward } \\
\text { Form: The information that } \\
\text { communicates what kind of } \\
\text { action is possible and how } \\
\text { the action can be carried } \\
\text { out. }\end{array}$ & $\begin{array}{l}\text { Restraining the movement } \\
\text { to initiate use of the } \\
\text { affected UE } \\
\text { Form: physical restraint on } \\
\text { the less affected side as a } \\
\text { reminder }\end{array}$ & & \\
\hline $\begin{array}{l}\text { Functional feedback } \\
\text { Form: The information } \\
\text { received about the actual } \\
\text { purpose of the action. }\end{array}$ & $\begin{array}{l}\text { Success } \\
\text { Form: Being able to carry } \\
\text { out the movement }\end{array}$ & $\begin{array}{l}\text { Mastery experience } \\
\text { Form: Indicators of } \\
\text { capabilities through } \\
\text { mastery of a difficult task }\end{array}$ & \multirow[t]{5}{*}{$\begin{array}{l}\text { Empirical evaluation that } \\
\text { is based on observations of } \\
\text { performance of the design } \\
\text { in use }\end{array}$} \\
\hline \multirow[t]{3}{*}{$\begin{array}{l}\text { Augmented feedback } \\
\text { Form: The information } \\
\text { received from an additional } \\
\text { source once the action has } \\
\text { been performed }\end{array}$} & $\begin{array}{l}\text { Quantitative feedback: } \\
\text { Form: Number of } \\
\text { successful attempts to } \\
\text { carry out the movement }\end{array}$ & \multirow[t]{2}{*}{$\begin{array}{l}\text { Verbal persuasion } \\
\text { Form: Framing of } \\
\text { performance feedback }\end{array}$} & \\
\hline & $\begin{array}{l}\text { Qualitative feedback: } \\
\text { Form: Movement } \\
\text { quality is assessed \& } \\
\text { possible improvements } \\
\text { are recommended }\end{array}$ & & \\
\hline & $\begin{array}{l}\text { Behaviour change } \\
\text { techniques } \\
\text { Form: Home diary, } \\
\text { behaviour contract, } \\
\text { home training sheets }\end{array}$ & & \\
\hline $\begin{array}{l}\text { Inherent feedback } \\
\text { Form: Information returned } \\
\text { from the action itself }\end{array}$ & & $\begin{array}{l}\text { Physiological and affective } \\
\text { states } \\
\text { Form: Level of activation }\end{array}$ & \\
\hline
\end{tabular}




\subsubsection{Refinement of design criteria}

I took into consideration how feedforward and feedback elements of an object can be used to guide the user and mapped those elements against requirements of upper limb stroke rehabilitation and elements of self-efficacy in a table (Table 4). The elements were incorporated as additional design criteria and referred to during the design process. The column on the right shows how the elements were evaluated during the cognitive walkthroughs.

Based on the elements of Table 4, functional, augmented, inherent feedback and feedforward elements of the design should be incorporated in the following way:

Functional feedforward: The semantic interpretation should communicate the general purpose of the object and how it should be used in a specific ADL. There were no elements outlined in the description of self-efficacy that could be mapped to functional feedforward.

Augmented feedforward: The element of "coaching" within stroke rehabilitation teaches the stroke survivor how to perform the correct movement. It contributes to vicarious experience as part of self-efficacy through actual and symbolic modelling (Bandura, 1997, p. 93); for example, seeing someone else perform the movement to understand what it should look like. The MAL as part of CIMT helps assess the degree of learned nonuse that the chronic stroke survivor has developed. Its use correlates with the description of verbal persuasion by using a credible persuader and indicating degrees of appraisal disparities (Bandura, 1997, pp. 105 106); for example, persuading the stroke survivor that the use of the affected arm is possible. The augmented feedback of the object should reassure the user that they can perform the movement.

Inherent feedforward: The inherent feedforward should remind the user to use the affected arm. However, just telling someone that they are capable of it might not be enough. Efficacy beliefs can be increased by presenting the pursuit as relying on acquirable skills, raising performer's beliefs in their abilities to acquire 
the skills, modelling the requisite skills, structuring activities in achievable steps that ensure a high level of initial success, and providing explicit feedback of continuous progress (Bandura, 1997, p. 105). The use of the restraint in clinical practice correlates more with augmented feedforward. Nonetheless, including it in the inherent feedforward potentially could be beneficial. Inherent feedforward seems to offer the greatest potential to initiate the use of the affected arm and hand from a design point of view since it communicates the kind of actions that are possible to perform with the object.

Functional feedback: Successful completion of a difficult task contributes to mastery of experience, which is part of self-efficacy and increases the individual's belief in their capabilities (Bandura, 1997, p. 82). This description concerns more the actual task than the functional feedback that is provided. The functional feedback, however, needs to reflect that the task has been successfully completed.

Augmented feedback: Quantitative and qualitative feedback is provided as part of the rehabilitation process. Evaluative feedback within self-efficacy is used to increase self-efficacy beliefs by highlighting personal capabilities (Bandura, 1997, p. 102). Stroke therapists indicated that assessing the quality of movement and providing qualitative feedback is an extremely complex process. The augmented feedback should, therefore, focus on quantitative feedback in the form of knowledge of result and contribute to a behaviour change.

Inherent feedback: Inherent feedback is an essential element of the performance of a movement, but it remains unclear how it should be delivered. For the inherent feedback of the object, the focus will be on physiological and affective states, and the delivery of a moderate level of arousal that heightens attentiveness and facilitates deployment of skills (Bandura, 1997, p. 108).

The outlined feedforward and feedback elements were included in the set of design criteria and referred to in the design process. 


\subsection{Methods}

The methods for this study included RTD, which led to a series of design prototypes, an evaluation in the form of a pluralistic walkthrough with chronic stroke survivors, and analysing their responses with thematic analysis.

\subsubsection{Participants}

Participants were recruited through a therapist in Sydney, Australia, and a therapist in Napier, New Zealand. Please refer to section 3.5.2 for a description of the inclusion and exclusion criteria of this study.

The first testing was a group testing with three participants $(n=3)$ with their therapist present. The second testing was individual testing with a family member present. Three participants were male $(n=3)$, one was female $(n=1)$. Their age varied between 60 and 75 years. Time since stroke was six years, two years, eight months and two months. The participant who had experienced the stroke two months before the testing did not meet the inclusion criteria of the study, which required that the stroke take place at least six months before the testing. This only became apparent after the group testing was finished. All participants had signs of a mild stroke on one side of their upper limb. The participant who did not meet the inclusion criteria took part in the group testing, which made it impossible to filter out the comments in the audio recording.

\subsubsection{Research through design}

Multiple design prototypes were developed and refined in a serial design process (Krogh et al., 2015, p. 46) based on the initial design concept of the radio. Please refer to the methodology chapter, section 3.3.2 for a detailed description. The set of initial design criteria (Rodríguez Ramírez, 2017, p. 14) was redefined and extended according to the conclusions drawn from the therapists' feedback as well as from feedforward and feedback elements outlined in the previous section, see Figure 29. 


\begin{tabular}{|c|c|}
\hline 01: Restrain & $\begin{array}{l}\text { The interaction with the object needs to restrain movement to } \\
\text { evoke an initiation of use }\end{array}$ \\
\hline 02: Purpose & The object needs to be used within an activity of daily living \\
\hline 03: Repetition & $\begin{array}{l}\text { The interaction with the object needs to be repetitive in form of } \\
\text { random practice }\end{array}$ \\
\hline 04: Feedback & Feedback needs to be provided as part of the interaction \\
\hline 05: Challenge & $\begin{array}{l}\text { The interaction with the object needs to become progressively } \\
\text { challenging and offer different levels of complexity }\end{array}$ \\
\hline 06: Usability & $\begin{array}{l}\text { The interaction with the object needs to be achievable for a } \\
\text { stroke patient with motor impairments }\end{array}$ \\
\hline 07: Movement & $\begin{array}{l}\text { The interaction with the object needs to evoke a "reach to grasp" } \\
\text { movement }\end{array}$ \\
\hline 08: Movement II & $\begin{array}{l}\text { The interaction with the object should avoid wrist extension and } \\
\text { fine motor skills }\end{array}$ \\
\hline $\begin{array}{l}\text { 09: Movement } \\
\text { III }\end{array}$ & $\begin{array}{l}\text { The interaction with the object should take account of sensory } \\
\text { deficits and weakened muscles }\end{array}$ \\
\hline $\begin{array}{l}10: \text { Technical } \\
\text { requirements }\end{array}$ & $\begin{array}{l}\text { The object needs to secure the connectivity of the NFC board + } \\
\text { Arduino Uno in a way that the user can interact with the NFC tag } \\
\text { attached to the user's affected arm in order to turn the radio on }\end{array}$ \\
\hline $\begin{array}{l}\text { 11: Functional } \\
\text { feedforward }\end{array}$ & $\begin{array}{l}\text { The design of the object should be based on a familiar object } \\
\text { such as the stereotype of a radio to indicate its function }\end{array}$ \\
\hline $\begin{array}{l}\text { 12: Augmented } \\
\text { feedforward }\end{array}$ & $\begin{array}{l}\text { Augmented feedforward should contribute to reminding the user } \\
\text { to use the affected side of the body }\end{array}$ \\
\hline $\begin{array}{l}\text { 13: Inherent } \\
\text { feedforward }\end{array}$ & $\begin{array}{l}\text { Inherent feedforward should indicate how the user can interact } \\
\text { with the object and that the movement needs to be carried out } \\
\text { with one particular side of the body }\end{array}$ \\
\hline $\begin{array}{l}\text { 14: Functional } \\
\text { feedback }\end{array}$ & $\begin{array}{l}\text { Functional feedback should indicate the successful completion } \\
\text { of a task }\end{array}$ \\
\hline $\begin{array}{l}\text { 15: Augmented } \\
\text { feedback }\end{array}$ & $\begin{array}{l}\text { Augmented feedback should provide quantitative feedback } \\
\text { about the movement }\end{array}$ \\
\hline $\begin{array}{l}\text { 16: Inherent } \\
\text { feedback }\end{array}$ & Inherent feedback should contribute to a moderate level of effort \\
\hline
\end{tabular}

Figure 29:

Design criteria set 03. 


\subsubsection{Pluralistic walkthrough}

Pluralistic walkthroughs are a formative usability evaluation method following a predefined set of task scenarios (Wilson, 2014, p. 82). This method was chosen because it allows actual users to test the system and point out usability issues while offering the opportunity to discuss possible design solutions (Wilson, 2014, p. 83). In the context of this study it allowed evaluation of the anticipated initiation of use of the affected hand.

I conducted group and individual pluralistic walkthroughs for this study. The method usually involves the participant writing down the experience and limitations of the tested prototype. This step was left out because of the common limitation of fine motor skills in stroke survivors that makes writing a timeconsuming and cognitively demanding task. The testing was audio recorded and partially transcribed. The use of the object was video recorded if the situation allowed it. The video recordings showed the hands of the participant using the object and were used to validate initiation of use. If video recordings could not be made, observations of the movement were recorded in the form of sketches and notes.

Each participant was informed about the study and provided written consent. The task that was focused on was turning on the radio prototype to evaluate an initiation of use. The testing started with describing the testing session and emphasising that the feedback provides valuable insights for further design iterations.

The first part of the testing contained an analytical evaluation of the object and the second part was based on an empirical evaluation, see Table 4. First, the participants were asked to describe how they would interact with the object and what they believed its functionality was. Then the participants were asked to interact with it and provide feedback. The participants could ask questions about the intended use and design elements during the analytical evaluation. 
After testing the participants could point out one element that they would keep in a further design iteration and one element that should be left out.

\subsubsection{Wizard of $\mathrm{OZ}$}

The test of the final object before the pluralistic walkthrough showed that the technical components did not function reliably. The radio board occasionally had problems finding a frequency. The IR sensor had some issues detecting people in dim lighting situations. A Wizard of Oz technique was chosen to make sure that the objects could function during the testing and did not affect results. With this technique the user interacts with the interactive system but unknown to them the researcher is operating the system behind the scenes simulating the system responses (Molin, 2004, p. 425). For the testing, a second Bluetooth radio was installed inside the final prototype which could be controlled via a smartphone to mimic the radio functionality and restraining effect of the NFC board.

\subsubsection{Thematic analysis}

The data have been analysed with thematic analysis in a deductive form (Braun \& Clarke, 2006, p. 83) according to themes of usability issues, general issues relating to the device, semantic interpretation and initiation of use of the affected arm and hand.

\subsection{Results}

The following section will present the outcomes of the RTD process, followed by the results of the evaluation process analysed using thematic analysis. Please refer to Appendix C, Workbook 2, for a detailed description of the design development. Findings and conclusions are summarised according to the criteria based thesis model that was used during the design development process. 


\subsubsection{Research through design results}

\subsubsection{Prototype 07}

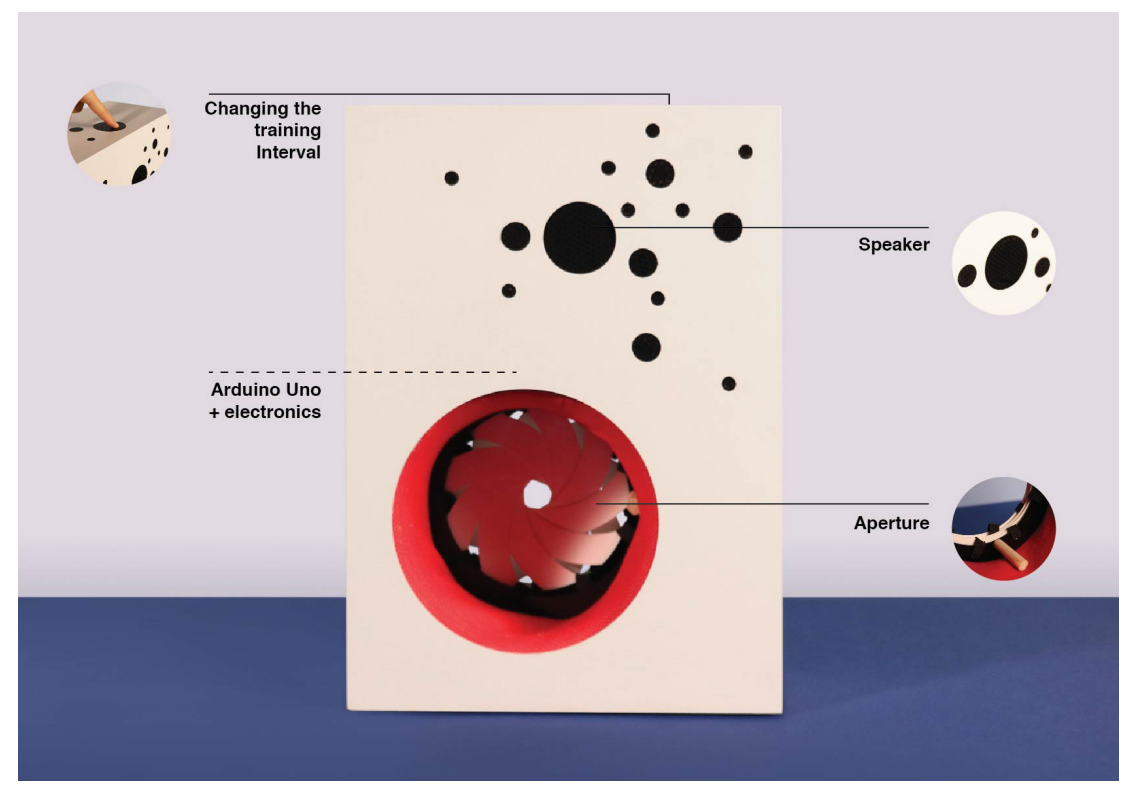

Figure 30:

Prototype 07.

The concept of this design iteration was to encourage the user to move the affected arm and hand inside the red area. Inside this area there is a mechanism like that of a camera aperture, see Figure 30. During the first stage, the aperture is wide open. This opening can progressively be made smaller to challenge the user. The training interval, which allows the radio to play music for a pre-set time, can be changed by touching different buttons on the top.

Findings: The radio turned out to be rather large with dimensions of $300 \mathrm{~mm} \times 450 \mathrm{~mm} \times 200 \mathrm{~mm}$. The buttons on the top looked like the ones at the front of the speaker, which might make it difficult to distinguish between them. The visual appearance of the object resembled a washing machine.

Conclusion: The next iteration should promote a reach and grasp movement in a downward orientation to decrease wrist extension. The functional feedforward elements should relate to the form of a stereotypical radio. 


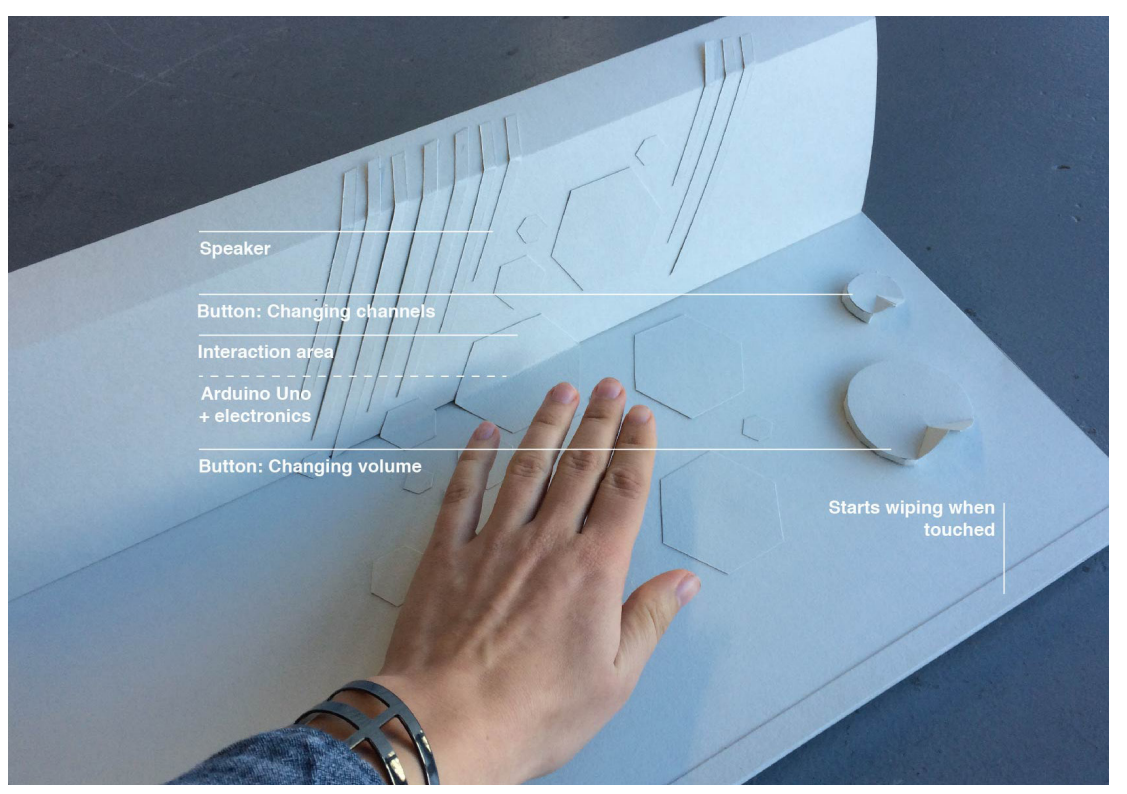

Figure 31:

Prototype 08 that required a downward motion.

For this design iteration, the primary interaction area was moved to a horizontal plane so that the user could interact with it in a downwards movement. The radio would turn on when the faceted area was touched, see Figure 31. It would gently start rocking as an immediate indication of successful movement. The two buttons on the right can be used to change the radio station and volume.

Findings: The horizontal area where the user has to touch the radio needed to be rather large to offer enough room for the hand. In their current form the buttons to change the radio station and volume required a high degree of fine motor skills in the affected hand. The radio remained in the tipped position after the first interaction. The radio showed some resemblance to an electronic keyboard.

Conclusion: The radio should be smaller to be able to fit on a small table. The functional and inherent feedback needed to be redefined to communicate the intended use. The next iteration should investigate how the buttons needed to turn on the radio can be integrated into the centre of the object. 


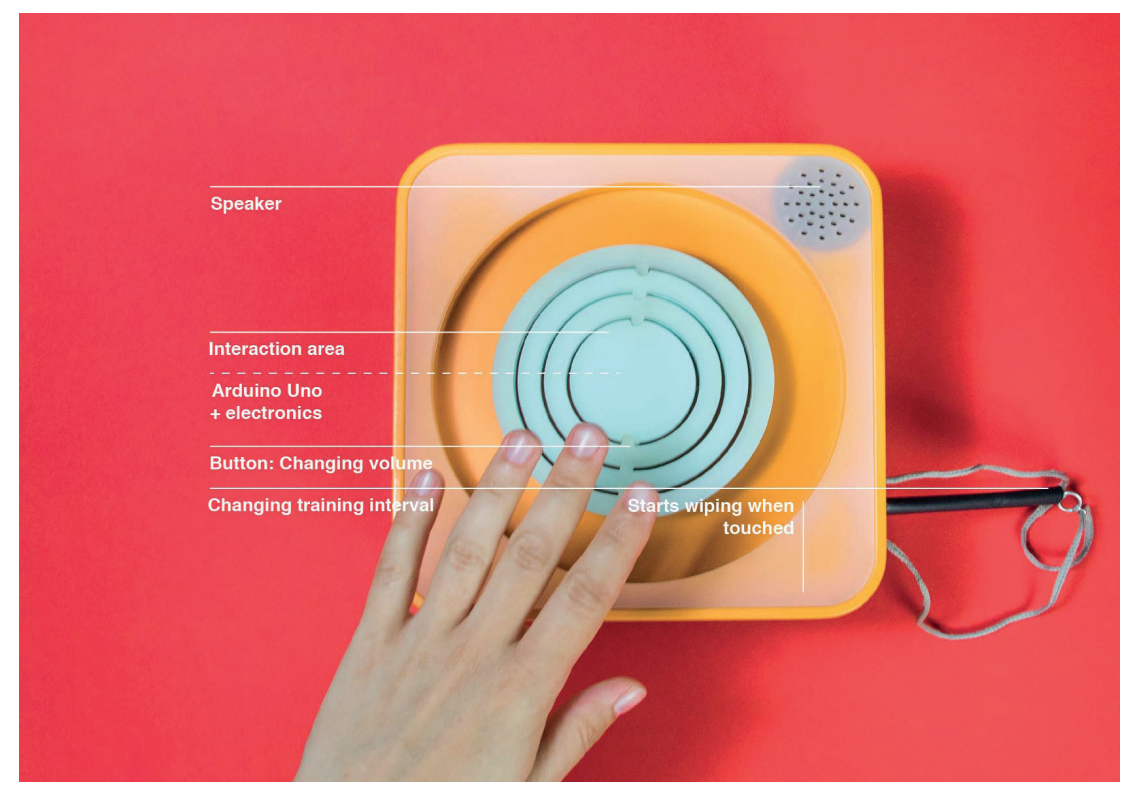

Figure 32:

Prototype 09 which has the volume button situated in the centre.

This iteration has the main interaction area situated in the centre, see Figure 32. When this area is touched the radio tips to the front and gives immediate feedback about the completion of the task. The user can change the volume by rotating the button in the centre. Progression is included in the form of multiple buttons in the centre decreasing in size. The user can add or remove rings to make the use more challenging.

Findings: The main goal of the object is to enable the user to practice wrist extension at some stage with it. This iteration did not address this. The biggest ring was too big to be used by someone with limited finger extension ability and limited muscle strength. Additional texture on the button could offer support during the movement.

Conclusion: The next iteration should include a stand that offers progression in terms of needed wrist extension. How different textures could be used to offer support on the main button in the middle, as well as progression, should be further investigated. 


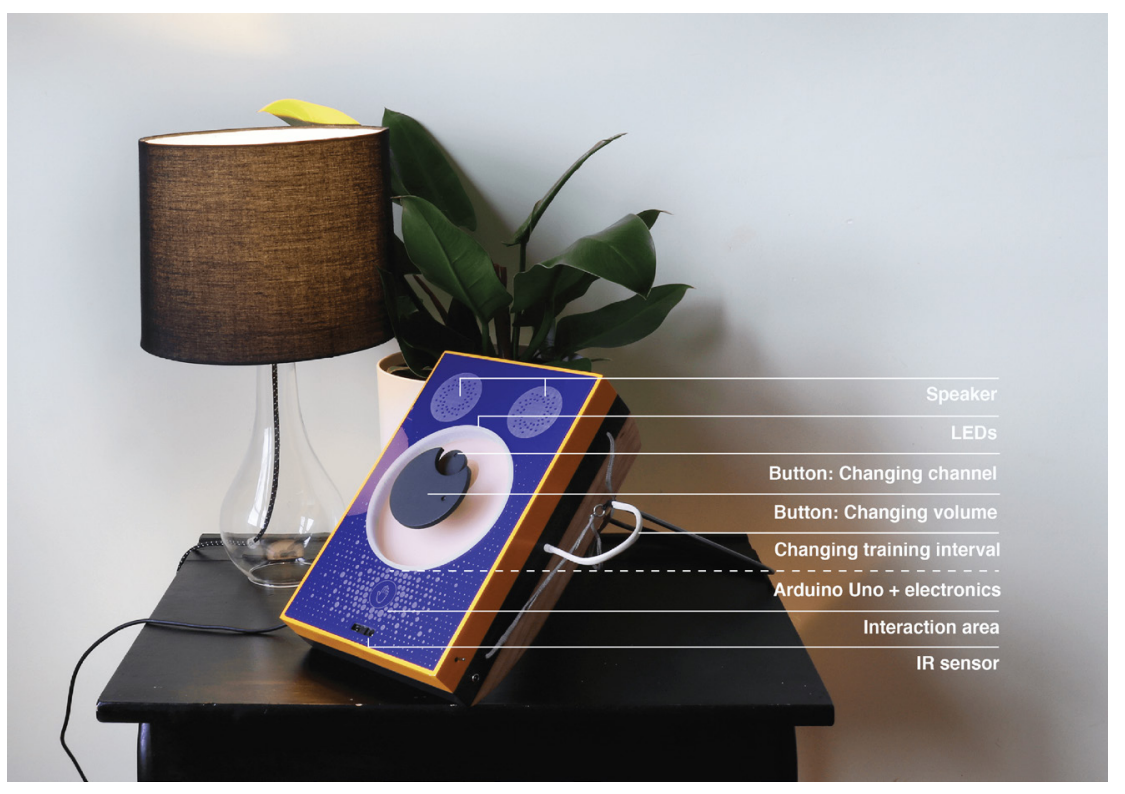

Figure 33:

Components of the final design prototype.

The final design iteration had a rectangular form and could be visually separated into a main blue front area and a wooden back area, see Figure 33. At the back, a metal stand can be attached to gradually increase the angle of the radio and the required wrist extension; see Figures 34 and 35. The user has to perform a reach and grasp movement, touching the front lower area that shows a hand icon to start the radio.

The white circle in the centre of the front area contains two buttons. The large one allows the user to change the volume and the small one regulates the radio channel. A soft potentiometer is situated on the right side of the radio, which controls the training interval of the radio. 


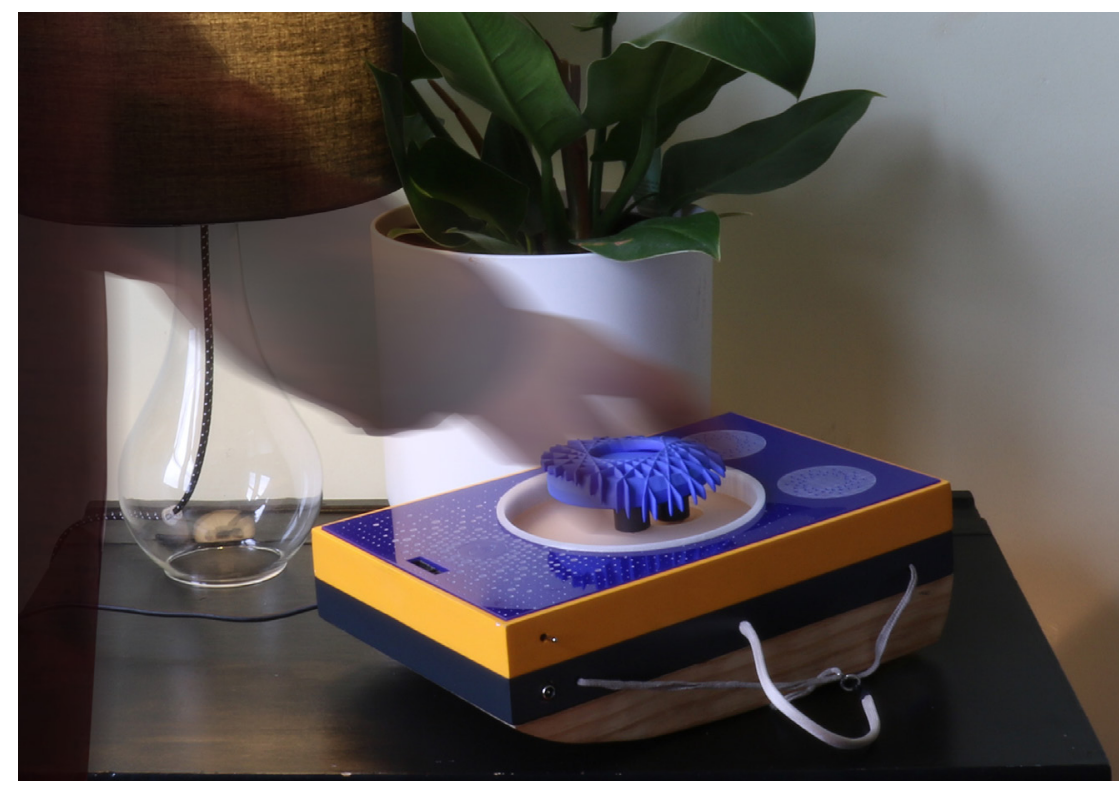

Figure 34:

Initial stage of the radio does not require a stand to decrease the required wrist extension during use.

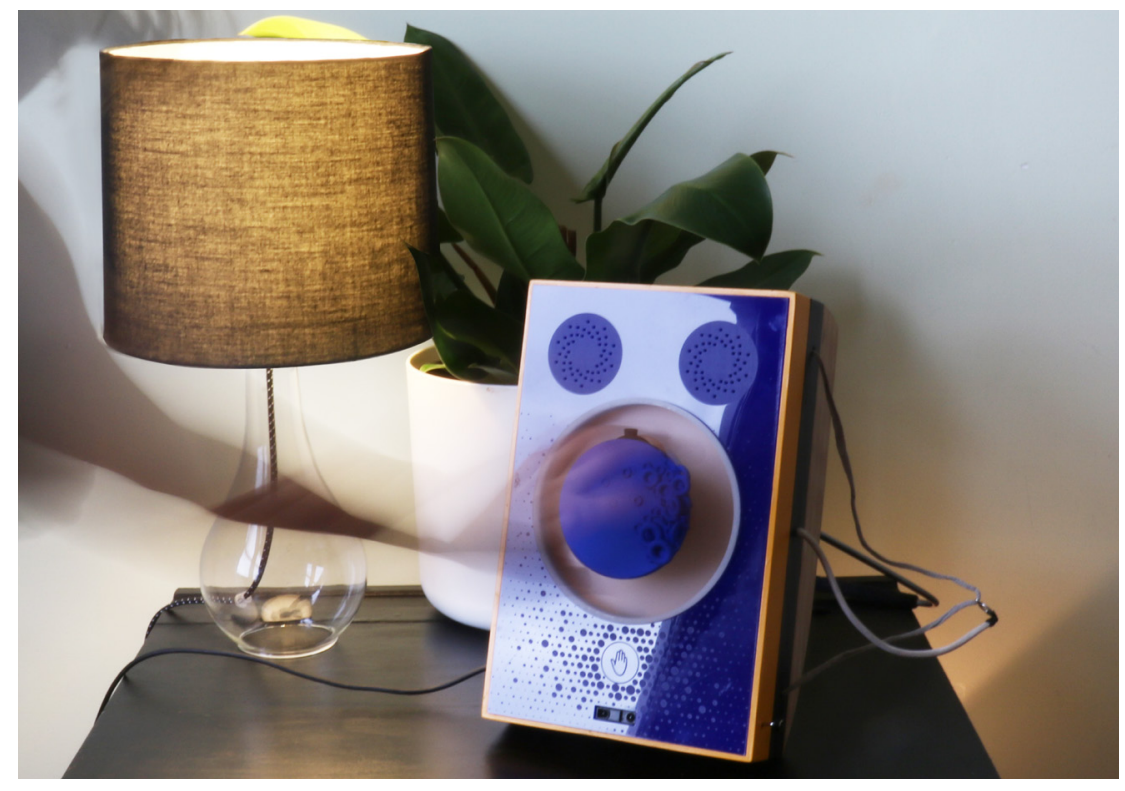

Figure 35:

Advanced stage of the radio with the stand attached to the highest level. The user is required to extend the wrist to use the radio. 
In the following section, the different feedforward and feedback that are incorporated into the design will be outlined.

\subsection{Functional feedforward}

The prototype was influenced by cultural norms and conventions (Norman, 2013, p.130) of stereotypical radios. The design includes some typical speakers in the top front that indicate the object produces sound, see Figure 36 . The location of the main button to change the volume resembled that of a vintage radio to provide a clearer indication about the functionality of the object and to relate it to the age of the intended audience.

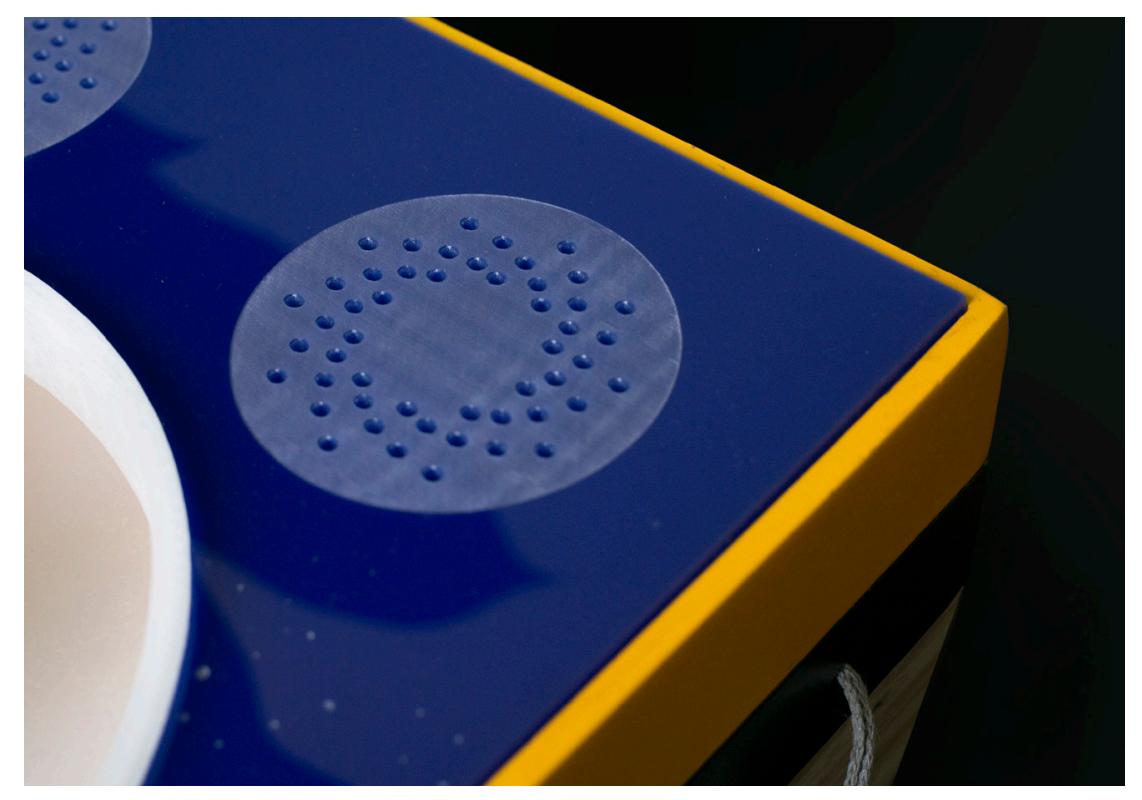

Figure 36:

The design of the speakers.

\subsection{Augmented feedforward}

Augmented feedforward is included in the form of two elements. An engraved icon on the front of the radio, see Figure 37, indicates where the hand needs to be placed. A second component in the form of an LED strip is located around the white centre. Every time the user passes the radio within a distance of $10 \mathrm{~cm}$ to $80 \mathrm{~cm}$ the LEDs give a small light impulse to function as a visual prompt to remind the user to interact with the object. 


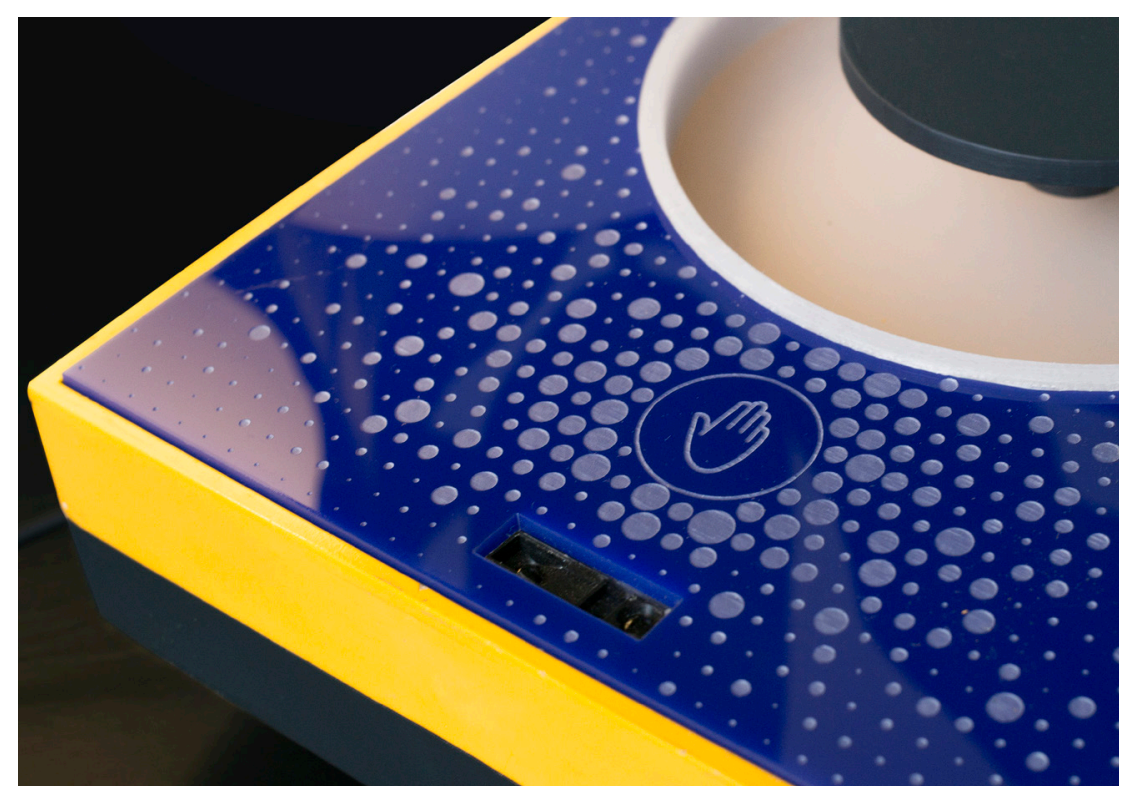

Figure 37:

The icon on the lower

front component

indicates that the user

has to touch the radio

here.

\subsection{Inherent feedforward}

Inherent feedback is included in the form of the NFC bracelet or NFC ring that is used to interact with the object, which reminds the user constantly which arm they must use. Further feedforward elements are the changeable volume buttons that can be attached to the front area of the radio.

\subsection{Functional feedback}

Functional feedback is included in the design by allowing the radio to play music only when the NFC tag is detected in order to evoke an initiation of use. The volume and channel buttons provide further functional feedback when used.

\subsection{Augmented feedback}

The radio provides augmented feedback quantitatively in the form of an app that takes account of the feedback provided by the health professionals. One therapist pointed out that it is important to provide a summary of performance after each session to make sure that the user stays engaged in the progress and keeps on trying to use the affected hand. The feedback provided should decrease over time. The app records how often the user has interacted with the radio and presents this data to the user as different circles representing use during the last seven days as well as the entire year; see Figure 38. The importance of 
involving family members in the rehabilitation process was highlighted during the interviews with health professionals, see Chapter 4 . Therefore, the user is able to share progress with family and friends by emailing the results.

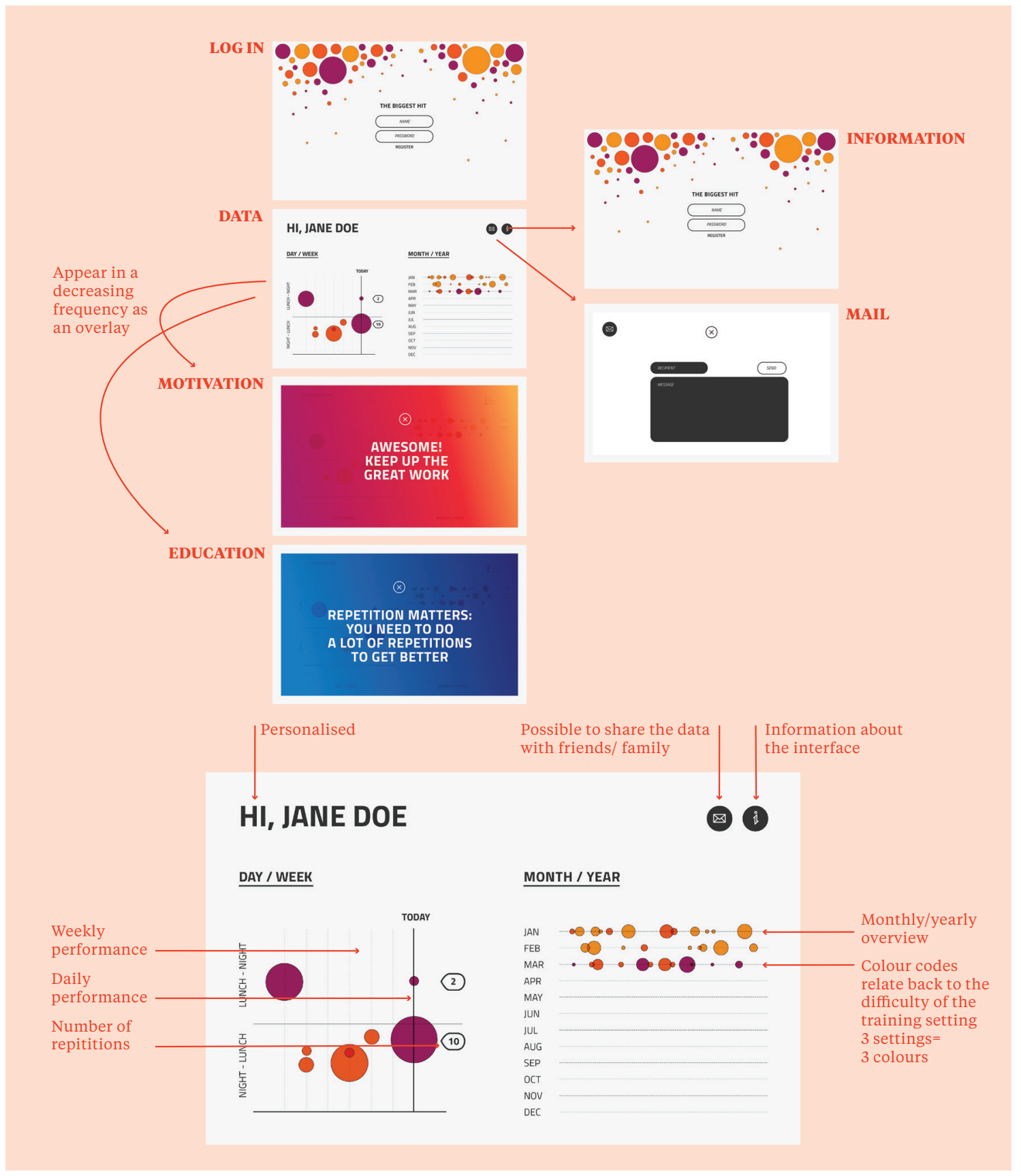

Figure 38:

Interface of the app that presents the quantitative use of the prototype. 
I conducted a series of material experiments investigating potential forms and textures for the volume button, situated in the front of the radio. The initial material experiments focused on a textured top surface, see Figures 39 and 40. However, further tests focused on material around the border to offer support for the fingers during the rotating movement.

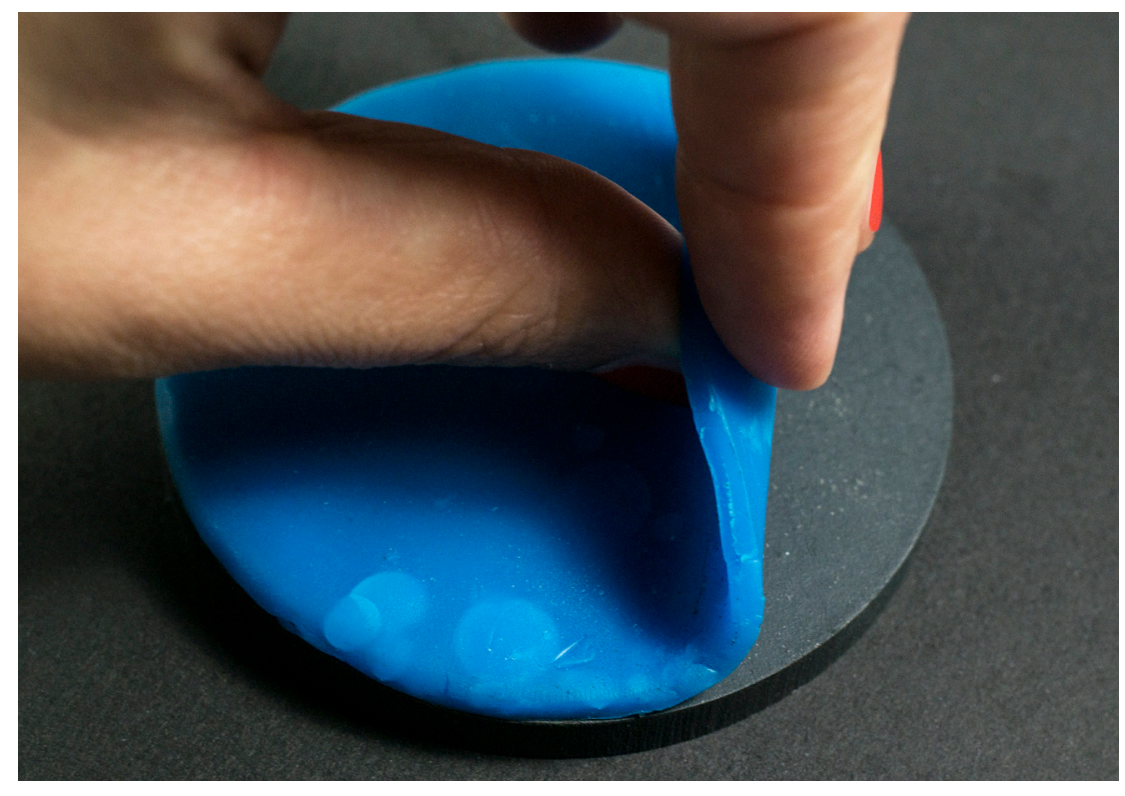

Figure 39:

Form and material experiments for the volume button.

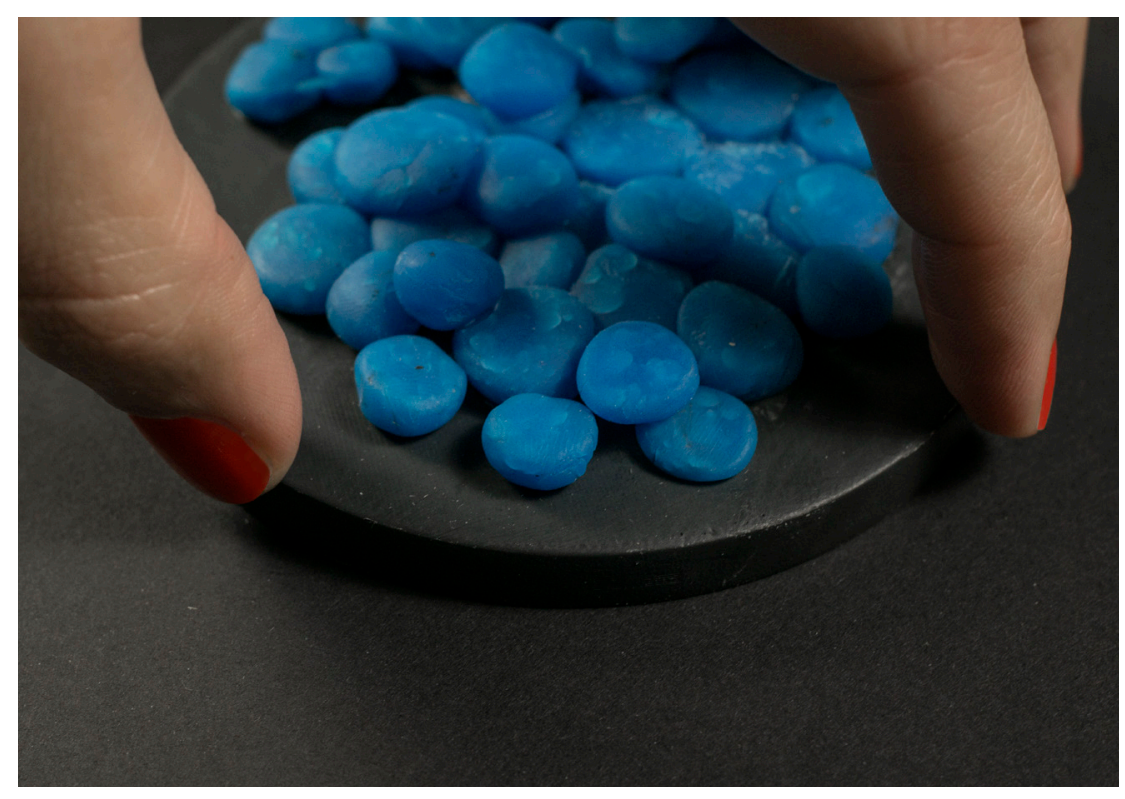

Figure 40:

There is no support for the fingers when the texture is solely on top of the button, which complicates use. 
The final design prototype offered four different buttons to the user. The texture progressively offers less support to carry out the rotation movement and makes the interaction more challenging. Each button can be simply clicked onto the main button in the centre of the radio. See Figure 41 for an overview of the four buttons.

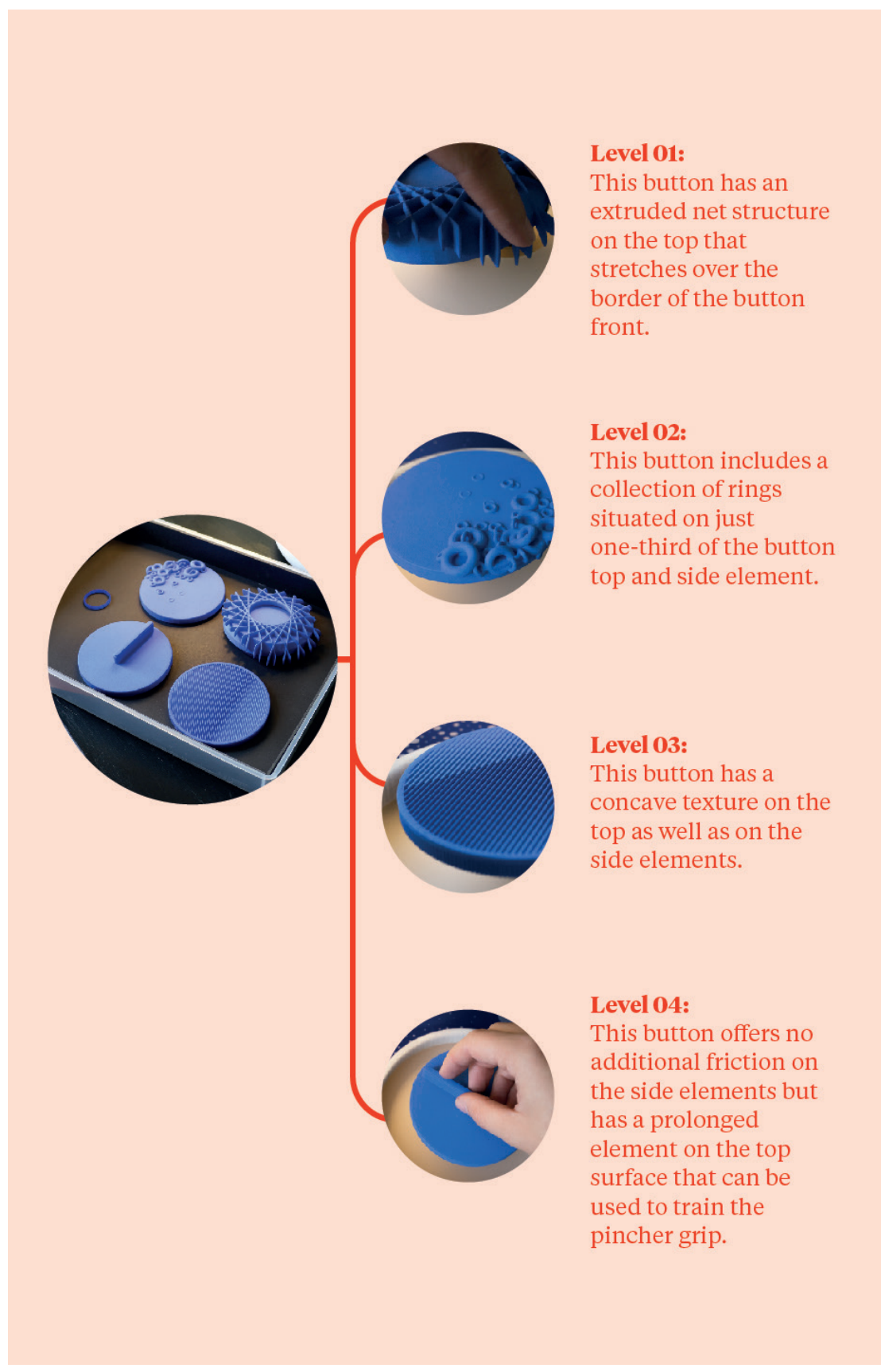

Figure 41:

Four volume buttons

that can be used

with the final design

prototype. 


\subsection{Technical components}

The circuit used for the design prototype included additional electronics to provide feedback and feedforward elements. See Figure 42 for a schematic overview. An infrared distance sensor (Adafruit, United States) recognises when the user is passing at a $10 \mathrm{~cm}$ to $80 \mathrm{~cm}$ distance in front of the object and turns on a set of LEDs in the white centre. A Bluetooth sensor connects to a Lenovo Tab 2 tablet and transfers the data about the use of the object to the connected app.

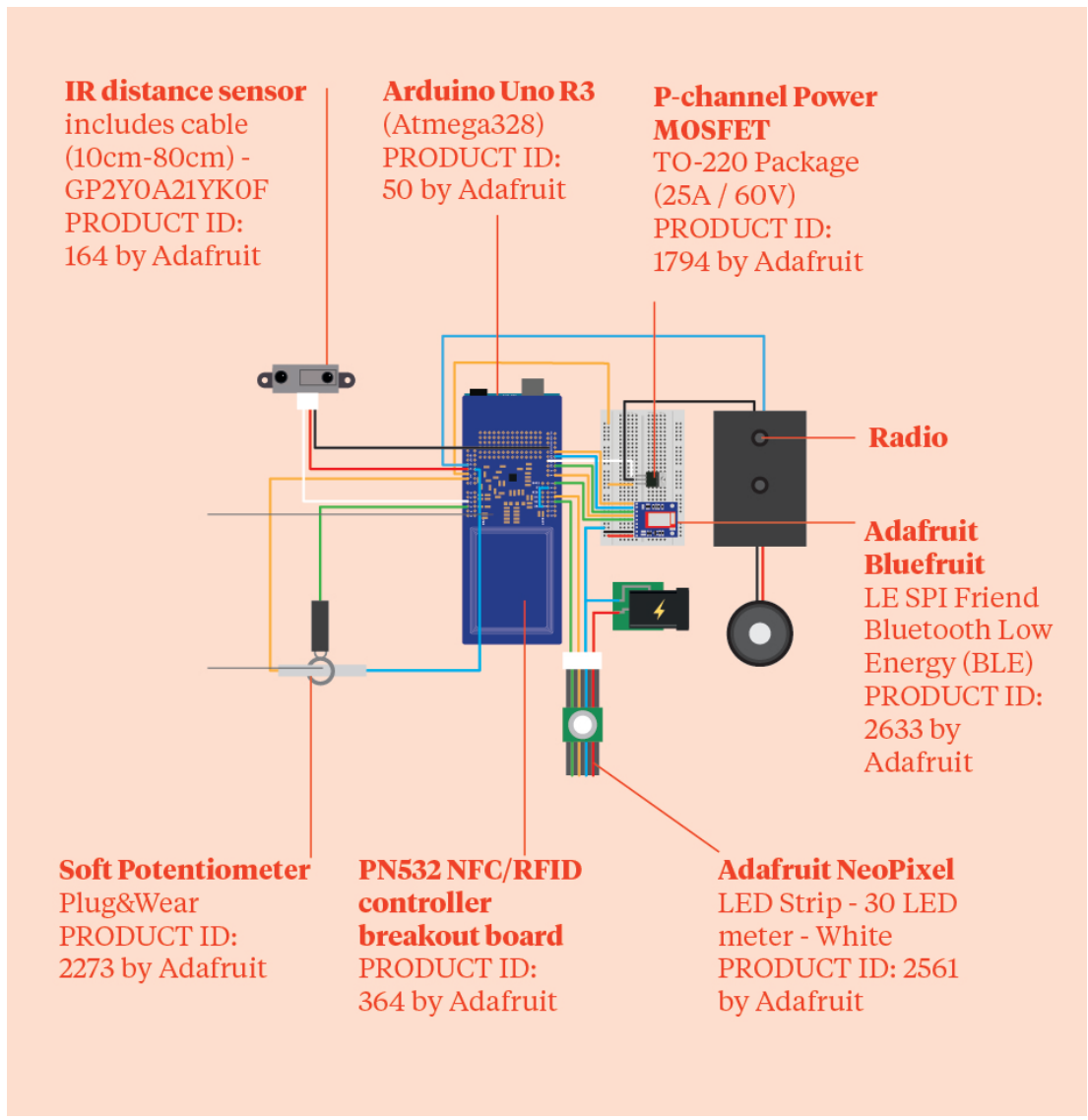

Figure 42:

Technical components of the final design iteration. 


\subsubsection{Evaluation}

The design prototypes were evaluated against the set of design criteria during the iterative process. The final design prototype was evaluated with chronic stroke survivors using a pluralistic walkthrough.

\subsubsection{Design criteria}

Some of the criteria could not be assessed during the design process, see Figure 43. Criteria 01, 06, 07, 08, 12, 16 were therefore evaluated during the pluralistic walkthrough. For Criteria 03: The object still delivers a blocked practice rather than randomised practice. However, using feedforward elements might contribute to random practice but could not be assessed within the scope of this research because the usability evaluation time is restricted to 30 minutes.

\subsubsection{Pluralistic walkthrough}

\subsection{Themes}

The feedback provided by the stroke survivors was analysed according to the themes of usability issues, feedforward and feedback elements and initiation of use of the affected arm and hand.

The bracelet was identified during the group evaluation as the preferred design element and the button to change the radio channel as the least preferred. During the individual evaluation, the aesthetic of the design was the least preferred component with the size of the volume button being the most preferred.

Usability issues

A number of usability and conceptual issues were mentioned during the testing. These centred around the use of the radio, the movement required, and the aesthetics of the prototype. It was observed that some of the design elements seemed to evoke compensatory movements. The buttons to change the radio channel and volume required some wrist extension that was quite difficult for most participants. During group testing, the participants stood in front of the radio to use it. This was circumstantial because of the way the group was set up. During individual testing, however, the participants preferred to sit down. This 


\begin{tabular}{|c|c|c|c|c|c|}
\hline & & No 07 & No 08 & No 09 & No 10 \\
\hline $\begin{array}{l}\text { 01: } \\
\text { Restrain }\end{array}$ & $\begin{array}{l}\text { The interaction with the object needs to } \\
\text { restrain movement to evoke an initiation of use }\end{array}$ & \multicolumn{4}{|c|}{ Evaluated with stroke survivors } \\
\hline $\begin{array}{l}\text { 02: } \\
\text { Purpose }\end{array}$ & $\begin{array}{l}\text { The object needs to be used within an activity of daily } \\
\text { living }\end{array}$ & $\mathrm{x}$ & $\mathrm{x}$ & $\mathrm{x}$ & $\mathrm{x}$ \\
\hline $\begin{array}{l}\text { 03: } \\
\text { Repetition }\end{array}$ & $\begin{array}{l}\text { The interaction with the object needs to be repetitive in } \\
\text { form of random practice }\end{array}$ & \multicolumn{4}{|c|}{$\begin{array}{l}\text { Outside the scope of this re- } \\
\text { search due to time restrictions } \\
\text { of the usability testing }\end{array}$} \\
\hline $\begin{array}{l}\text { 04: } \\
\text { Feedback }\end{array}$ & Feedback needs to be provided as part of the interaction & & & & $\mathrm{x}$ \\
\hline $\begin{array}{l}\text { 05: } \\
\text { Challenge }\end{array}$ & $\begin{array}{l}\text { The interaction with the object needs to become } \\
\text { progressively challenging and offer different levels of } \\
\text { complexity }\end{array}$ & $\mathrm{x}$ & & $\mathrm{x}$ & $\mathrm{x}$ \\
\hline $\begin{array}{l}\text { 06: } \\
\text { Usability }\end{array}$ & $\begin{array}{l}\text { The interaction with the object needs to be achievable } \\
\text { for a stroke patient with motor impairments }\end{array}$ & \multicolumn{4}{|c|}{ Evaluated with stroke survivors } \\
\hline $\begin{array}{l}\text { 07: } \\
\text { Movement }\end{array}$ & $\begin{array}{l}\text { The interaction with the object needs to evoke a } \\
\text { "reach to grasp" movement }\end{array}$ & \multicolumn{4}{|c|}{ Evaluated with stroke survivors } \\
\hline $\begin{array}{l}\text { 08: } \\
\text { Movement II }\end{array}$ & $\begin{array}{l}\text { The interaction with the object should avoid wrist } \\
\text { extension and fine motor skills }\end{array}$ & \multicolumn{4}{|c|}{ Evaluated with stroke survivors } \\
\hline $\begin{array}{l}\text { 09: } \\
\text { Movement } \\
\text { III }\end{array}$ & $\begin{array}{l}\text { The interaction with the object should take account of } \\
\text { sensory deficits and weakened muscles }\end{array}$ & $\mathrm{x}$ & & & $\mathrm{x}$ \\
\hline $\begin{array}{l}\text { 10: } \\
\text { Technical } \\
\text { require- } \\
\text { ments }\end{array}$ & $\begin{array}{l}\text { The object needs to secure the connectivity of the NFC } \\
\text { board + Arduino Uno in a way that the user can interact } \\
\text { with the NFC tag attached to the user's affected arm in } \\
\text { order to turn the radio on }\end{array}$ & $\mathrm{x}$ & $\mathrm{x}$ & $\mathrm{x}$ & $\mathrm{x}$ \\
\hline $\begin{array}{l}\text { 11: } \\
\text { Functional } \\
\text { feedforward }\end{array}$ & $\begin{array}{l}\text { The design of the object should be based on a familiar } \\
\text { object such as the stereotype of a radio to indicate its } \\
\text { function }\end{array}$ & & & & $\mathrm{x}$ \\
\hline $\begin{array}{l}\text { 12: } \\
\text { Augmented } \\
\text { feedforward }\end{array}$ & $\begin{array}{l}\text { Augmented feedforward should contribute to } \\
\text { reminding the user to use the affected side of the body }\end{array}$ & \multicolumn{4}{|c|}{ Evaluated with stroke survivors } \\
\hline $\begin{array}{l}\text { 13: } \\
\text { Inherent } \\
\text { feedforward }\end{array}$ & $\begin{array}{l}\text { Inherent feedforward should indicate how the user can } \\
\text { interact with the object and that the movement needs to } \\
\text { be carried out with one particular side of the body }\end{array}$ & $\mathrm{x}$ & $\mathrm{x}$ & $\mathrm{x}$ & $\mathrm{x}$ \\
\hline $\begin{array}{l}\text { 14: } \\
\text { Functional } \\
\text { feedback }\end{array}$ & $\begin{array}{l}\text { Functional feedback should indicate the successful } \\
\text { completion of a task }\end{array}$ & $\mathrm{x}$ & $\mathrm{x}$ & $\mathrm{x}$ & $\mathrm{x}$ \\
\hline $\begin{array}{l}\text { 15: } \\
\text { Augmented } \\
\text { feedback }\end{array}$ & $\begin{array}{l}\text { Augmented feedback should provide quantitative } \\
\text { feedback about the movement }\end{array}$ & & & & $\mathrm{x}$ \\
\hline $\begin{array}{l}\text { 16: } \\
\text { Inherent } \\
\text { feedback }\end{array}$ & $\begin{array}{l}\text { Inherent feedback should contribute to a moderate } \\
\text { level of effort }\end{array}$ & \multicolumn{4}{|c|}{ Evaluated with stroke survivors } \\
\hline
\end{tabular}


particular participant tried to use the object, but turning the button turned out to be too tricky while sitting down. Therefore, the participant stood up and leaned over the object, which was placed on a small table.

Yeah, I have to stand up to change the station

Participant 04.

To compensate for the lack of wrist extension, the participant started rotating her whole body instead of just the wrist. Another form of compensation by using the index finger to rotate the volume button was observed in both the group and the individual session. The structure of the button was used to "lock in" the finger while turning the button. Some participants had issues extending all their fingers around the volume button to grip it. However, one participant mentioned that it was a good size, despite having issues extending all the fingers on the affected hand. Observations indicated it was not clear that the purpose of the radio was to evoke a reach to grasp movement. All four participants continually altered the volume during the testing. This made it quite difficult to ascertain whether the radio had turned off because of the training interval or because the user had muted the volume. One participant questioned the rehabilitative purpose of the object.

If I would train that 50 times a day, would I be able to pick up a ball? Participant 04

Most of the participants had the impression that the object trained wrist rotation. They further questioned why they needed an additional object to train the movement when they could rely on objects they already used, for example a jar. One participant liked the functionality of it, while most of the participants questioned the focus on the radio. 
But the radio is kind of passé.

So unless you are in a car, you are not listening to it.

Participant 01

They suggested alternative everyday tasks that they enjoyed or that were important to them. One participant did not like the overall appearance of the object.

The microwave would be a good one. If you want to get it warm, you have to use the right hand. Otherwise, it won't turn itself on.

Participant 02

I don't like the box, and I don't like the size of it Participant 04

\section{Functionalfeedforward}

The function of the radio was not clear to the participants. However, they recognised what it was after they interacted with it and heard the music.

A radio? In this?

Participant 01

During group testing the speaker holes were identified as a form of voice control, but the participants in the individual testing identified them as target points where the volume button has to be moved towards. The button that changes the volume was pushed rather than turned and was identified as the main element to start the object. 
[I] presume that I put my hand on that.

Participant 04

The button to change the radio channel was criticised as being too small and difficult to use due to its size and location beneath the volume button. Its use and function were unclear to the participants, and it was the least liked element during the group testing.

So I can't understand how to change the station.

Participant 01

The function and use of the soft potentiometer were not recognised. One participant tried to pull on it to interact with it. Another one mentioned that it looked like something that needed to be put on the arm.

Augmented feedforward

The hand icon was described as a form of stop sign. The LEDs situated at the front of the radio were supposed to give a visual prompt to remind the user to interact with it. However, the lighting conditions impacted on the sensors for the LEDs. The augmented feedforward could not be tested.

\section{Inherent feedforward}

The inherent feedforward of the bracelet that reminds the user to interact with the radio was unclear to the users. The participants received an explanation after the analytical evaluation of how to interact with the object and it was explained that they should wear the bracelet on the affected side. A commercially available product would provide a similar explanation. All participants used their affected arm to interact with the object during the testing session. 


\section{Functionalfeedback}

Once the music started playing, it was obvious to the participants that the object had the functionality of a radio. However, the purpose of the volume button and the button to change the channel were not completely understood.

\section{Augmented feedback}

During the individual evaluation, the participants mentioned that it would be appropriate to use an app but the function to share the results with family members would be unnecessary. The app was criticised during the group testing as being too difficult to use.

I think the app is not all that practical because as you can see we are too limited to go on things like apps. Later on, when everything is settled you don't need things like this.

Participant 02

\section{Inherentfeedback}

The different textures of the buttons did not seem to increase the difficulty of the task. The first level button with the extruded surface pattern was used by interlocking the index finger into the pattern to compensate for the required wrist rotation.

\section{Initiation of use}

The intention to restrain movement to initiate the use of the affected side evoked different responses. One participant expressed concerns about the restraining function of the object.

I don't know if I would get one

Participant 01 
Others mentioned that they would tolerate the restraining effect. However, they pointed out that it might be annoying if the radio stopped playing in the middle of a favourite song. They were asked if they would prefer the restraining effect in its current form or as a reminder. It was mentioned in the group evaluation that the reminder might be the better option. However, a reminding rather than restraining effect was not tested.

\subsection{Discussion}

\subsubsection{The design process}

The design process was influenced by an attempt to implement feedback provided by the therapists and include feedback and feedforward elements.

Firstly, a focus was on creating an interaction that would not require any wrist extension. However, it was emphasised by one therapist that the object should start with low movement requirements and then gradually become more challenging. Difficult and challenging movement patterns should not be avoided but incorporated in the form of manageable steps.

Secondly, the overall form of the design prototypes changed significantly in the process. Prototype 07 and 08 seemed to share elements of stereotypical objects such as a washing machine or an electronic keyboard. The aesthetic attributes play a significant role and influence acceptance of the object. One participant mentioned that she liked the functionality of the object but not its form. However, even though there are several aesthetic principles (Baxter, 1995, pp. 46-48) there is still no consensus on what is beautiful or what comprises beautiful artefacts (Crilly et al., 2004, pp. 555-565). There is furthermore no guidance in regard to the aesthetic of an everyday object that can be used as part of a self-directed rehabilitation.

A further element that potentially influences the use of the object is its symbolic meaning. Symbolic meaning is a culturally agreed meaning of an object that allows the user to communicate her or his identity through it (Crilly, 2004, 
p. 562). For example, a throne is a particular kind of chair for a king. Design is affected by and affects society and the modern economy (Forty, 1986, p. 8). It is influenced by design precedents and is a way for the consumer to express his or her individuality (Forty, 1992, p. 12). The symbolic meaning has not been evaluated as part of the current research.

Everyday objects in the form of assistive devices can contribute to a feeling of independence. Nonetheless, they are not valued like other things in the home and can even contribute to the feeling of stigma (Pape, Kim, \& Weiner, 2002, p. 17; Pettersson, Berndtsson, Appelros, \& Ahlström, 2005, p. 163; Renda, Jackson, Kuys, \& Whitfield, 2015). This is caused by unwanted attention which creates a sense of not fitting in (Pape et al., 2002, p. 13).

\subsubsection{Usability issues}

Some usability issues were observed during testing. The participants believed that the volume button was the centre point of interaction with the object and the intended reach to grasp movement was not performed repetitively. The constant use of the volume button might have been because of the conceptual model that they have of a radio. Here the button is often used to turn the radio on. In the context of this research, the object needs to take account of the already established conceptual model.

Another usability issue that needs to be considered is the context of use. During the group evaluation, the radio was placed on a table, and the participants were standing in front of it. During the individual testing, the participants preferred to stay seated, which made using the object tricky. The participants had to stand up to test the radio, which was on a small table. This contributed to a medial hip rotation to compensate the wrist rotation, which could potentially cause atypical movement patterns.

\subsubsection{Feedforward and feedback}

To decipher an object, the user refers to stereotypes, similar products, metaphors, characters, conventions, and clichés (Crilly et al., 2004, pp. 565-567). 
For example, "a stereotypical chair may be thought of as having four legs, a flat base and a straight back” (Crilly et al., 2004, p. 566). Stereotypical elements of a radio were included in the prototype's form. For example, having the volume button as a centre point and the perforations for the speakers. However, those elements need further refinement.

The evaluation showed that the feedforward elements were not effective and the intended interaction was not clear to the participant. The functional feedback did, however, contribute to the understanding of functionality. Once the music started to play all participants understood the object to be a radio. Augmented feedback in the form of quantified results, on the other hand, evoked mixed responses. One participant mentioned that she would use the app but the participants during group testing pointed out that using the tablet would be too challenging.

Some stroke therapists mentioned that stroke survivors can experience tactile discrimination deficits in their hands and that additional textures might be able to address this. Rehabilitation interventions that focus on recovery of sensory discrimination are scarce. The SENSe discrimination training (Carey, Macdonell, \& Matyas, 2011) relies on texture discrimination in the form of different graded textures. However, the training uses other elements such as limb position sense training and tactile object recognition (Carey et al., 2011, p. 306). The SENSe training could be used to guide further design iterations.

\subsubsection{Initiation of use}

Results that could indicate whether the object contributes to an initiation of use are inconclusive due to potential bias caused by the elements of the design. Participants used the affected arm to interact with the object during the $30 \mathrm{~min}$ testing. They were told that they needed to put on the bracelet to test the system. This might have increased the awareness to use the affected arm and hand which might have biased the results. However, it is expected that if the system were available, clinicians would recommend it and mention the use of the bracelet. 
A further limitation is that the participants constantly interacted with the volume button by rotating it. The functionality of the radio that turns itself off did not have any effect because of this constant movement. The participants identified the central training element to be the volume button rather than the reach to grasp movement.

One participant expressed clear concerns about the restrictive nature of the radio which requires the use of the affected arm to start the music. The other three expressed willingness to accept such a limited use. However, it was mentioned that the limited functionality might be annoying in the long term.

\subsection{Conclusion}

The results of this study show inconclusive results concerning the intended initiation of use. Even though the affected arm and hand were used during the evaluation, there is a potential bias caused by the design elements. The study indicates that taking account of the established conceptual model that the user has of the object can increase the usability of the design and enhance initiation of use. Rather than training a reach to grasp movement, the radio could be used to train wrist rotation. The next design iteration should first unpack the movement that is needed to interact with the object, break down complex movement patterns as a starting point to make the use feasible, and take account of the conceptual model that the user has of the object. The study further indicates that the restrictive nature of the design was accepted by most participants but potentially can be experienced as being coercive. Further research should investigate different forms of influence varying from reminding to restrictive influences. 


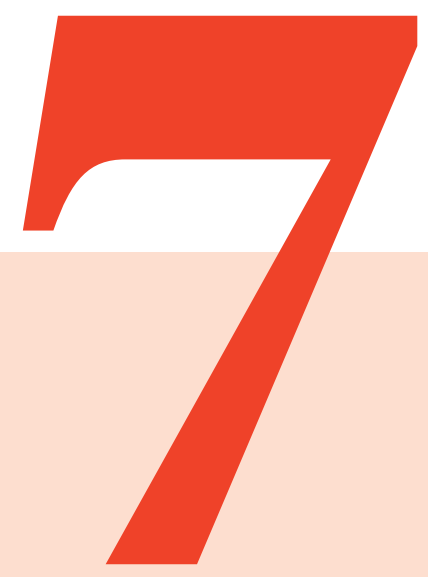

\section{Designs of}

\section{Everyday Objects \\ that Can Restrain} Movement to

\section{Initiate the Use of}

the Affected Arm 


\subsection{Introduction}

The focus of this study is on exploring further designs that can evoke an initiation of use of the affected arm and that go beyond the mitt, based on the results of Chapter 5 and 6 . The design prototypes presented in this study investigated possible ways of restraining the movement to initiate the use of the affected arm by taking account of the established conceptual model. This study presents the outcomes of an industrial design course that took place for five weeks with 31 undergraduate design students at the School of Design, Victoria University of Wellington, New Zealand. The task that was focused on for the project was eating, which therapists mentioned in previous interviews as being an important daily task. Shaping is used as part of CIMT and was used as a reference point to The results of this study contributed to the development of design strategies to address the research question:

1.3.1 How can we design an everyday object that restrains movement of the less affected arm to facilitate and initiate the use of the affected arm?

This study has been published in the Francis and Taylor Design Journal. (Lemke, Rodríguez Ramírez, Robinson, 2017a).

\subsection{Background}

Feedback indicated that the radio does not offer the greatest potential to facilitate an initiation of use. It was therefore decided to focus in this study on a task that therapists pointed out as being essential after a stroke: eating. CIMT uses a behavioural training technique named shaping to evoke an initiation of use of the affected arm and hand that even can be used to address complex movement patterns. The description of shaping offers a detailed description of its 
components and steps that are needed to initiate the use of the affected arm. The description of shaping was used to address the research question of this study. It helped determine movement components and was used to refine design criteria that could be used in the context of a design workshop.

\subsubsection{Shaping}

Shaping trains a task while restraining the movement and can be based on a simple movement or breaks more complex ones down into its components. It encourages the gradual development of a new behaviour by constantly reinforcing small improvements without necessarily waiting for the completion of the full behaviour (Michie, van Straken, \& West, 2011, p. 1491; Morgan, 1974; Panyan, 1980; Skinner, 1974, pp. 431-432). The within-level shaping is the conventional approach during which a task is gradually increasing in its difficulty. The between-level shaping replaces the task with a more challenging one after a moderate level of mastery has been reached (Taub, 2013, p. 129).

Taub (2012, p. 163) and colleagues have developed an extensive list of possible shaping tasks. The list contains 100 tasks with a preliminary task description for each of them. During CIMT, shaping involves a detailed outline of the elements of training elements, the intended movement, set up details such as the height of the table, possible progression and feedback elements.

The selection of tasks is based on the joint movements that show the most deficits but at the same time show the greatest potential for improvement and the participant's preference (Taub, 2012, p. 162). The tasks need to be difficult enough that the participant can accomplish them with a certain degree of effort. The difficulty needs to increase constantly to guarantee a constant challenge. The therapist provides coaching and modelling of the correct movement to improve specific elements of the action. This is done in the form of cuing and prompting. Feedback is provided immediately and in a positive form after the task is completed. If a shaping task was unsuccessful, it is ignored or adjusted in a way, so 
it becomes possible for the participant to perform (Morris et al., 2006, p. 260; Taub, 2012, p. 163). Verbal reinforcement is an essential part in the training and regularly provided in a way such as "that's excellent", "first class" or "keep on trying” (Taub, 2012, p. 163). The behaviour contract is used as part of CIMT to transfer gains made in the rehabilitation in the real world. It is a formal agreement signed by the therapist and stroke patient (Morris, 2006, p. 262).

The behaviour contract is not part of shaping but is an essential part of CIMT to evoke an initiation of use. The behavioural contract is used as part of the transfer package of CIMT and in the form of a formal written agreement between the therapist and the patient stating that the patient will use the affected arm as much as possible (Morris et al., 2006, pp. 262-263). It was therefore included as an additional design criterion.

\subsubsection{Design criteria}

Based on the literature review the following design criteria were defined, see Figure 44.

\begin{tabular}{l|l} 
01: Restrain & $\begin{array}{l}\text { The movement of the less affected arm } \\
\text { needs to be restrained to motivate the } \\
\text { user to use their affected arm } \\
\text { (initiation) }\end{array}$ \\
\hline 02: Repetition & $\begin{array}{l}\text { The movement of the affected arm } \\
\text { needs to be repetitive }\end{array}$ \\
\hline 03: Challenge & $\begin{array}{l}\text { The interaction with the object needs to } \\
\text { get more challenging over time }\end{array}$ \\
\hline 04: Feedback & $\begin{array}{l}\text { Feedback needs to be provided as part } \\
\text { of the use of the object }\end{array}$ \\
\hline $\begin{array}{l}\text { 05: Behaviour } \\
\text { change }\end{array}$ & $\begin{array}{l}\text { A behaviour contract needs to be } \\
\text { incorporated into the design }\end{array}$ \\
\hline
\end{tabular}

Figure 44:

Design criteria set 04 based on elements of shaping. 


\subsection{Methods}

For this study, a RTD method was used. Results of the design process were evaluated in form of a cognitive walkthrough with stroke therapists. The provided feedback was analysed with thematic analysis.

\subsubsection{Participants}

Two physiotherapists $(n=2)$ and one occupational therapist $(n=1)$ evaluated six of the student design concepts in a 30-minute usability evaluation. The intended use of the object was explained, followed by 30-second videos produced by the students showing the ideal use of the object. Each of the objects were afterwards presented to the participants to test. In the following part feedback and recommendation for changes are summarised.

\subsubsection{Research through design}

A design workshop that took part of an industrial design course was organised to develop design prototypes that evoke an initiation of use through different forms of restraint. The design workshop involving 31 undergraduate students took place at the School of Design, Victoria University of Wellington, New Zealand. Expansive design experiments were carried out to investigate restraining designs that evoke an initiation of use in the user

\subsubsection{Workshop outline}

The course took place over five weeks in four main stages:

1. Understanding the problem

2. The development of concepts

3. The development of prototypes

4. Formative usability evaluation

\subsection{Understanding the problem}

An introduction to the condition and stroke symptoms, and a detailed description of CIMT and the use of the restraint was given to the students to offer a better understanding of motor impairments, shaping tasks and the purpose of restraining the movement. Two videos were presented during the introduction 
showing the assessment process and shaping tasks (Blakey, 2014; University of Alabama at Birmingham, 2014). The students received the design brief based on the characteristics of shaping and a list of eating tasks described in the home skill assignment used as part of CIMT. The students further received two different persona descriptions (Canadian Partnership for Stroke Recovery, 2016) to guide the design decisions. One persona showed signs of a left arm impairment, see Figure 45, while the second persona showed signs of a right arm impairment.

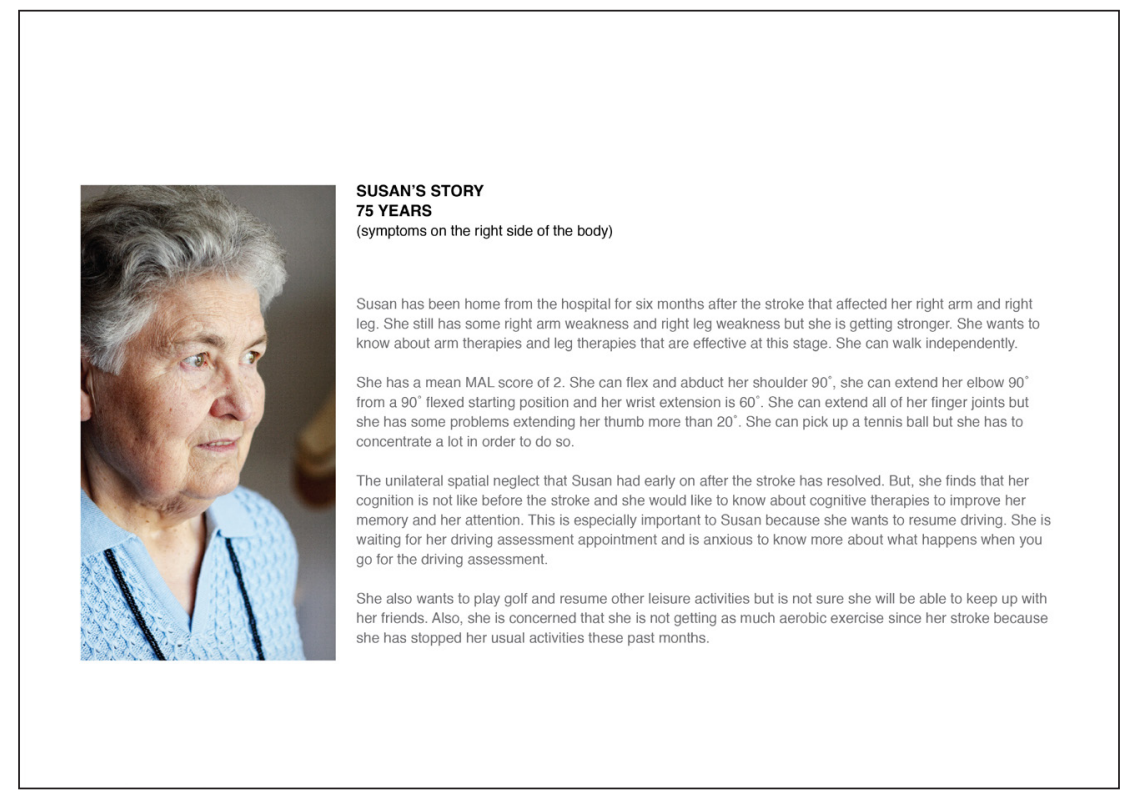

Figure 45: Persona description of Susan.

\subsection{Concept development}

The students were provided with eating equipment to carry out different eating tasks to break them down into their movement components, see Figure 46. While one student performed the task following the think-aloud protocol (Ericsson \& Simon, 1998), two students were videotaping the movement and taking notes. They developed a storyboard based on the observation and comments that showed the different movement components, see Figure 47. 


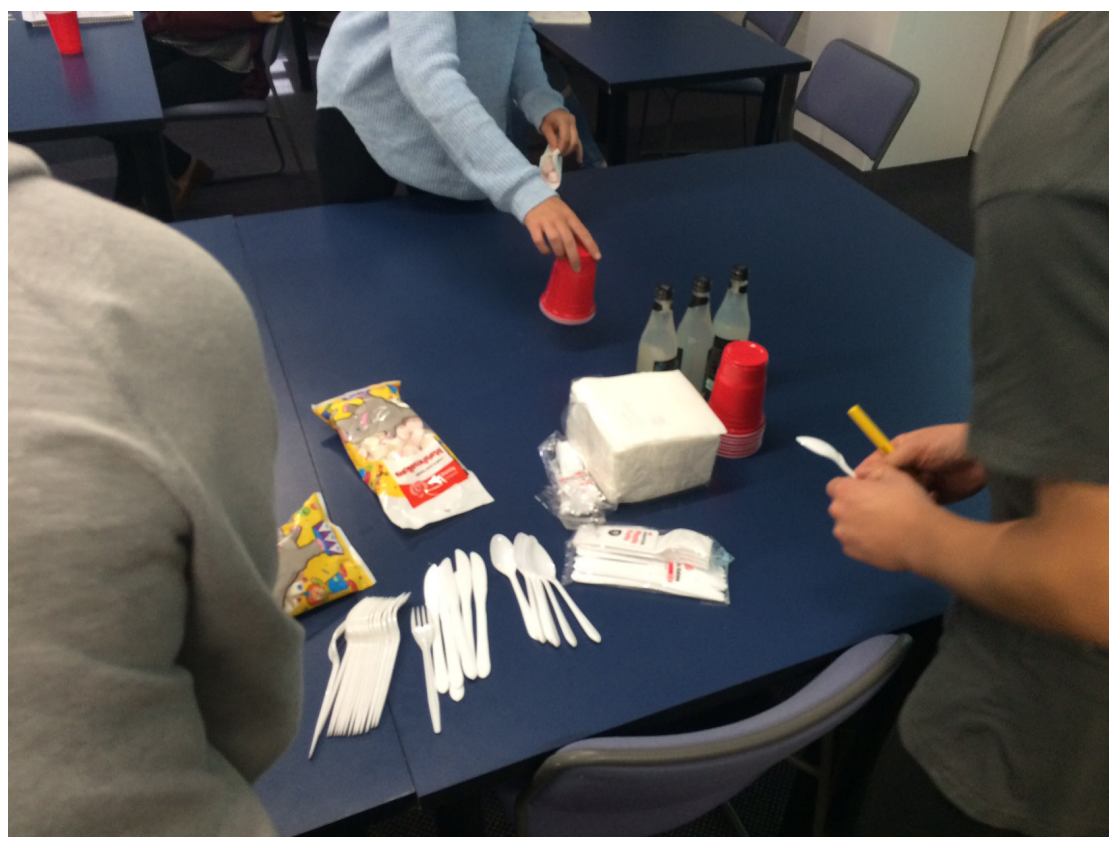

Figure 46:

Eating equipment.

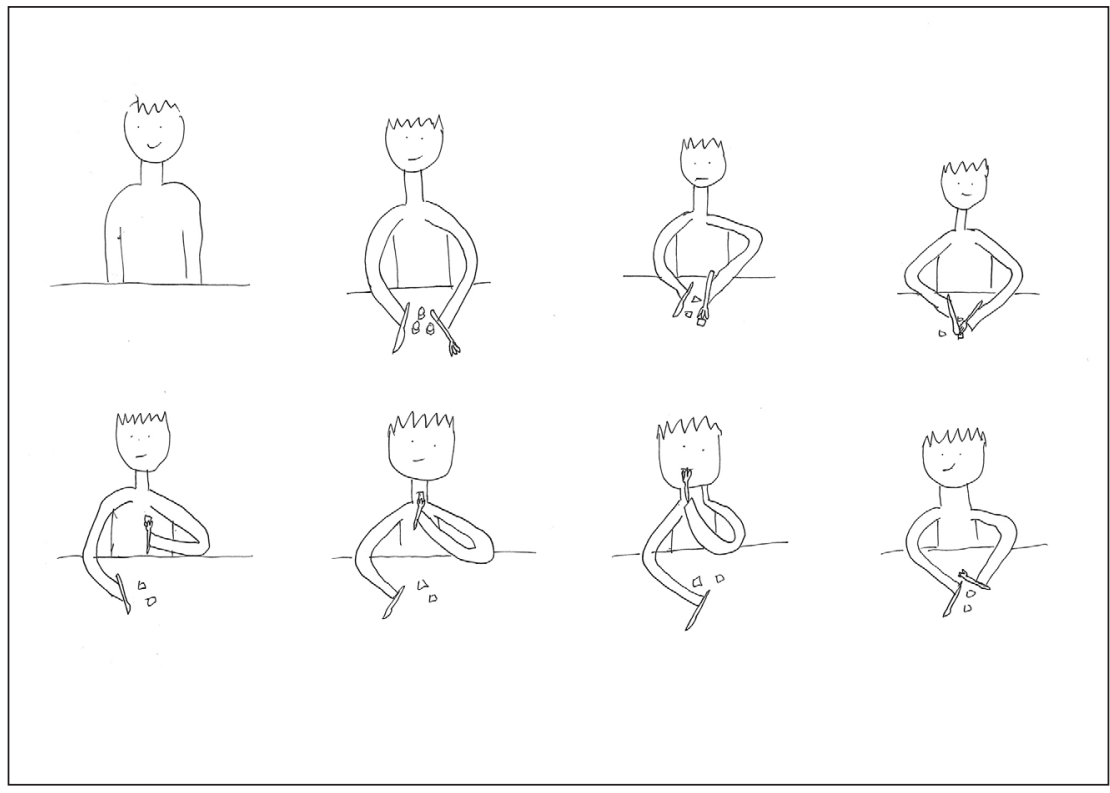

Figure 47:

Storyboard based on the observations.

\subsection{Prototype development}

The students worked individually in an iterative design process to develop concepts based on the observations that they made. Sketches led to different form prototypes using cardboard, foam, and a 3D printer generation to test and adjust the form of the object. For the final hand-in, the students submitted a working 
prototype and two videos: one showing the formative usability evaluation with peer students and one showing the intended use of the device.

\subsection{Evaluation}

A formative usability evaluation (Hartson et al., 2001) can be done at any stage of the design process in order to improve design outcomes. The working prototypes were used for two different usability evaluations in the form of cognitive walkthroughs. One was performed with peer students and one with therapists with vast experience in stroke rehabilitation. Six projects were chosen for assessment by two physiotherapists and one occupational therapist. The evaluation with experts explores the task performance of the designs and helps to identify usability problems the same way the user would (Hartson et al., 2001, p. 379; Wilson 2014, p. 66). It further assesses whether and how an initiation may be facilitated.

For the evaluation with peer students, a two-step protocol was used, focusing on the think-aloud protocol. The student started by presenting the design and asking the tester for a description of what they believed the intended use of the design was. In a second step, the tester could use the object and recommend changes.

The cognitive walkthrough with therapists started with the presentation of the video showing the intended use of the object. The therapist then had the opportunity to test the working prototypes and recommend changes.

\subsubsection{Analysis}

Thematic analysis (Braun \& Clarke, 2006, p. 83) in a deductive form was used to determine common design approaches in the design concepts. The design outcomes were analysed according to restraining the movement, enhanced repetition, increased challenge, feedback and behaviour change factors. The restraining effect included the anticipated initiation of use. Clustered bars have been used to present the main categories and visualise and ease the understanding of the data. 
The following sections will outline the five main elements, applied strategies and design outcomes that the students developed to address the design brief.

\subsection{Results}

\subsubsection{Research through design results}

\subsubsection{Restraining the movement}

The restraint is used as part of CIMT to remind the user to concentrate on the use of the more affected side and initiate the use of it. Three different strategies were employed by the students to encourage the participant to use a specific side without needing a physical restraint, see Figure 48.

The three approaches were:

1. Ergonomic restriction

2. Bilateral task to occupy the less affected arm

3. Intuitive use of an object

An example of an ergonomic restriction can be seen in Figure 49. "Smart sticks" are chopsticks designed so they are usable only with the right hand. The design looks similar to a pair of scissors, reducing the required wrist extension. The amount of required fine motor skills is reduced so the use of the chopsticks is feasible for the user. The focus on a bilateral task for the restraining effect is shown in Figure 50. "Fluve" is the concept for a decanter which requires the use of both arms. The less affected arm is essential in the task to unlock the object's full functionality. It either needs to balance the cup which has an uneven bottom or it can be used to hold the decanter. 


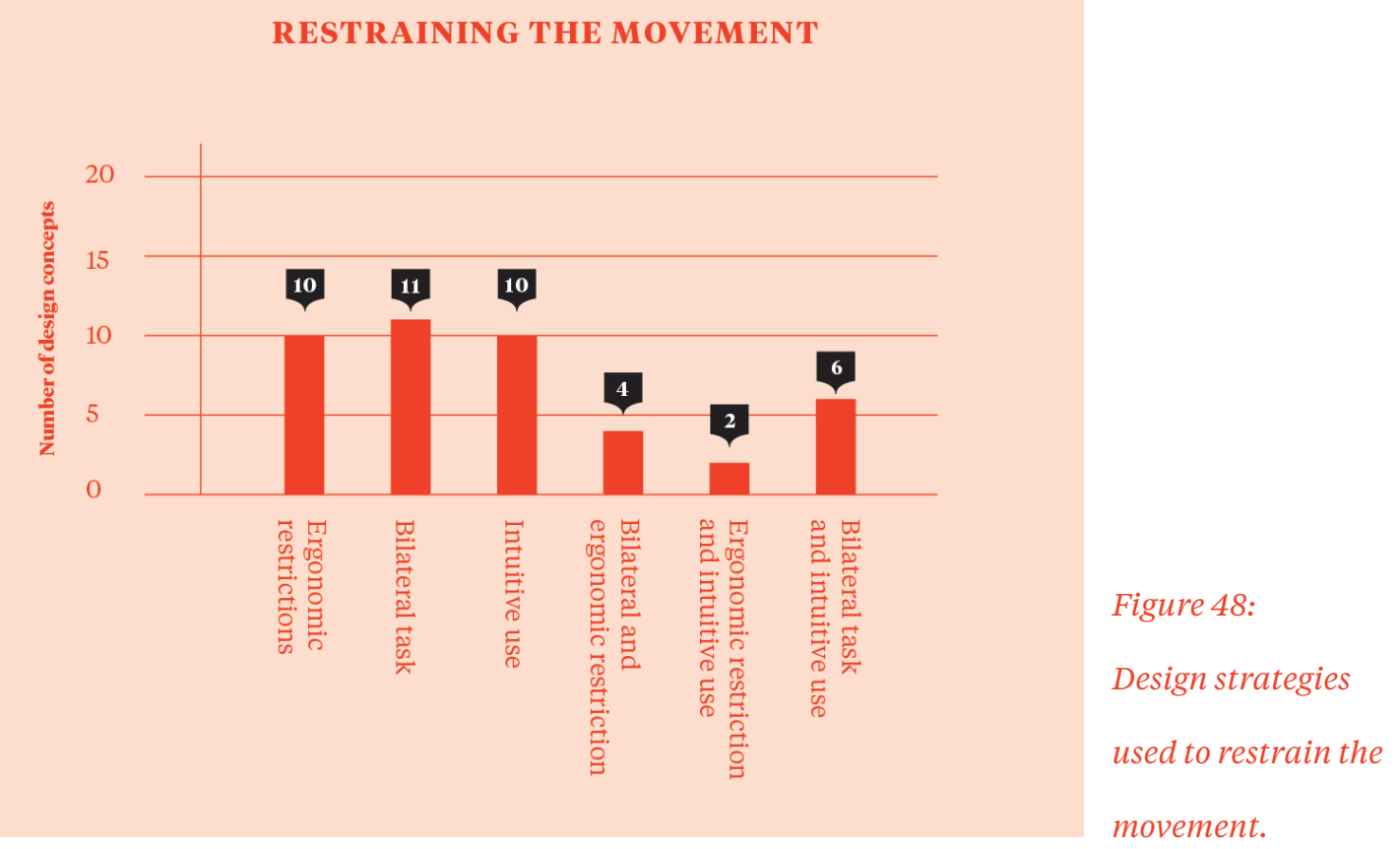

Figure 49:

"Smart Sticks"

chopsticks by Xavier

Strom. 


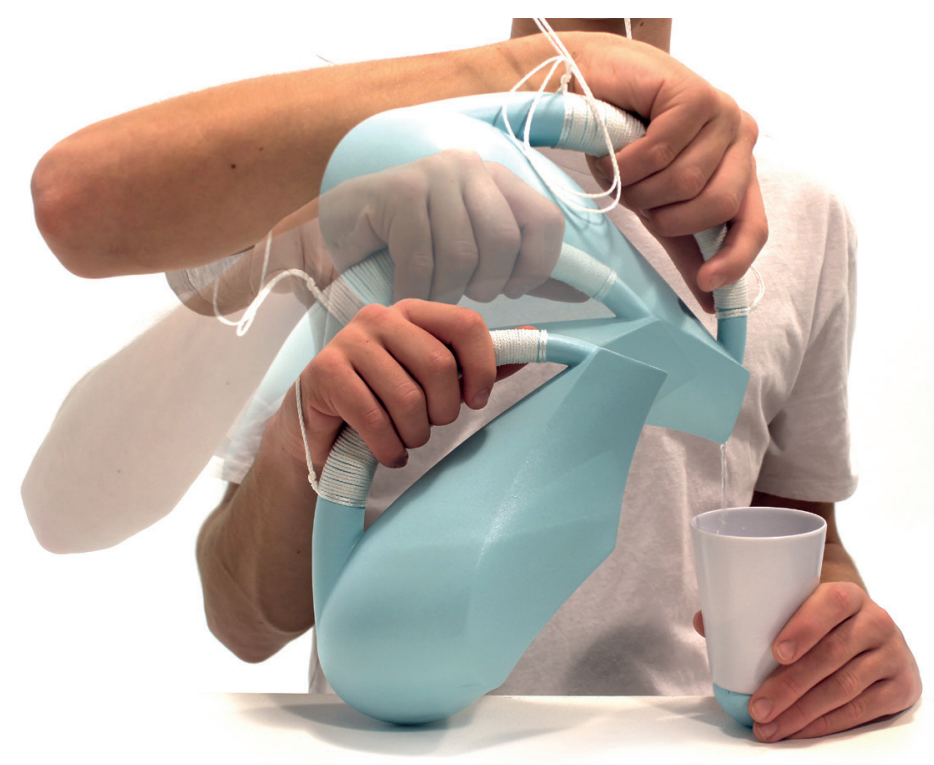

Figure 50:

"Fluve" decanter by

Nicole Hone.

The "Cirgo" knives are an example that rely on the intuitive use of an object with the right hand, Figure 51. The set of knives consists of four different variations which provide decreasing support during the cutting motion. The use of the knives becomes more challenging for the user the smaller the handle gets.

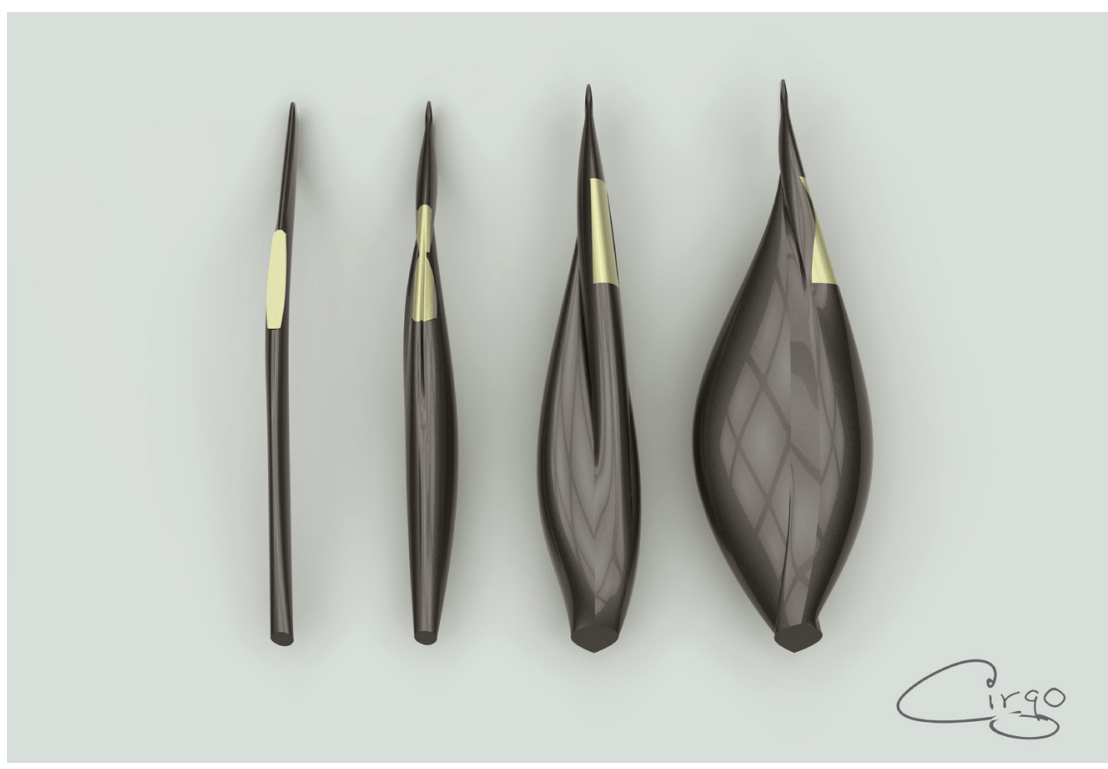

Figure 51:

Knives by Evangeline Martin named "Cirgo" that rely on the intuitive use of a knife with the right hand. 


\subsubsection{Enhanced repetition}

Enhanced repetition of a movement is essential to evoke plasticity in the brain. Two different design strategies were applied to deliver a repetitive movement, see Figure 52.

1. The design is part of a repetitive movement,

for example cutting food with a knife

2. The functionality of the design is reduced, leading to increased repetition

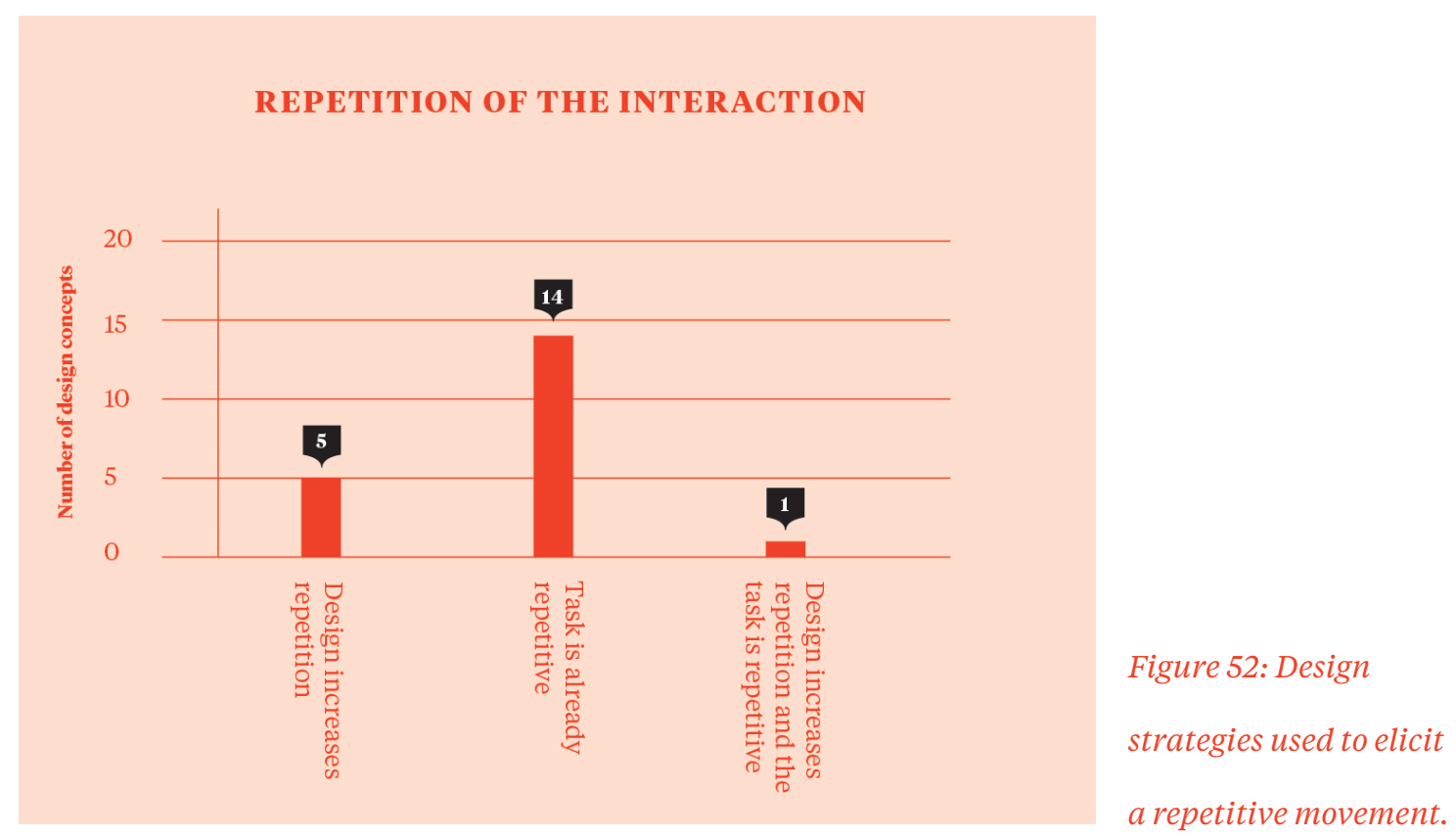

"Fluve" is a concept that reduces the functionality of the object to increase repetition efficiently. The amount of water that the user can pour into a cup is limited, requiring the user to repeat the movement multiple times to fill the cup. The vessel contains a mechanism in the handle that fills up with water each time it is rotated 90 degrees. The mechanism is invisible from the outside and just allows a certain amount of water to be poured out, see Figure 53. 


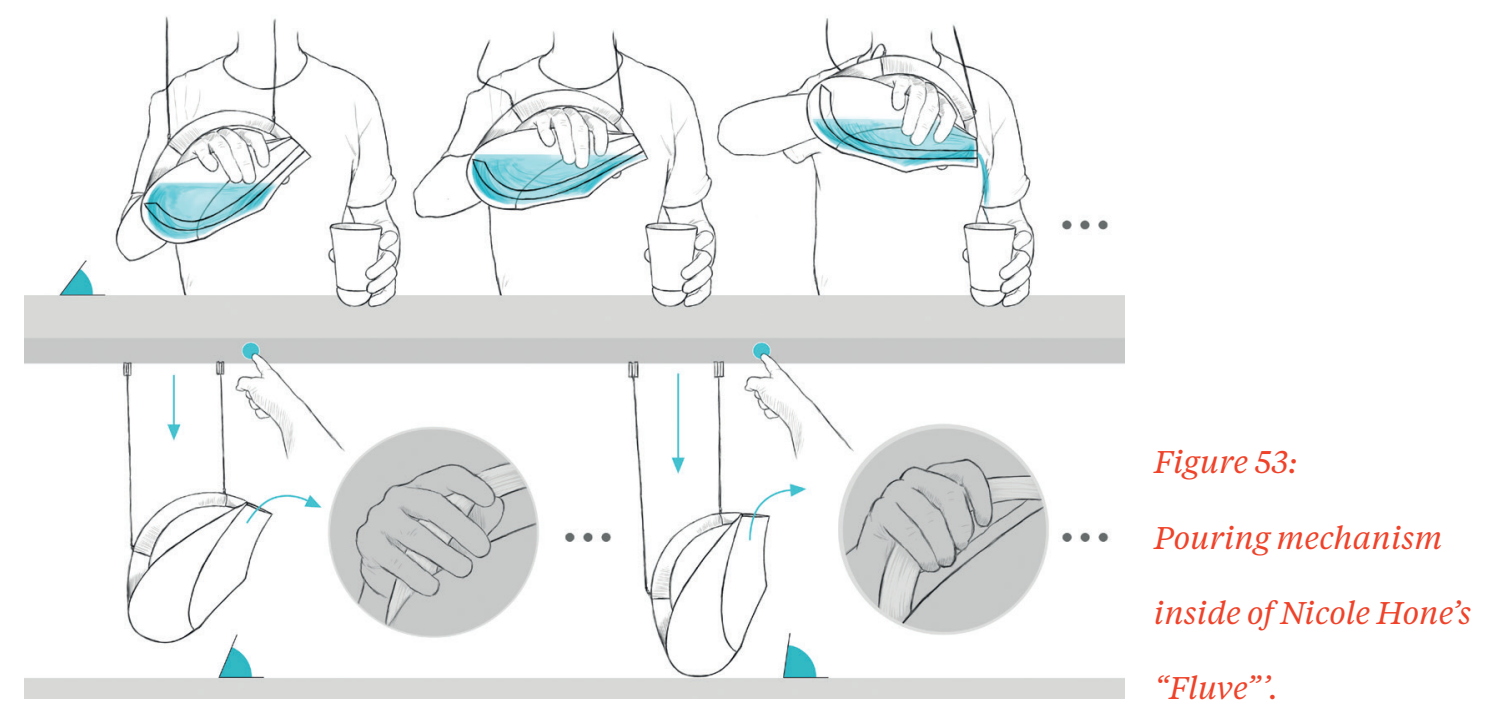

\subsubsection{Increased challenge}

The tasks that are trained during shaping steadily increase in difficulty to improve motor capabilities. Progress depends on the participant's ability to carry out the task to decrease frustration. The different design strategies based on the thematic analysis can be separated into four different strategies that partly overlapped, see Figure 54.

1. Progress is determined by the user

2. Progress is determined by the object

3. Progress is incorporated in one design object

4. Progress is incorporated in multiple design objects

Most students chose the form of progress that the user was able to selfdetermine. A design concept offering such a form of progression is a water bottle, see Figure 55. The bottle has a lid that limits the amount of water coming out of it to increase the difficulty of the interaction. 


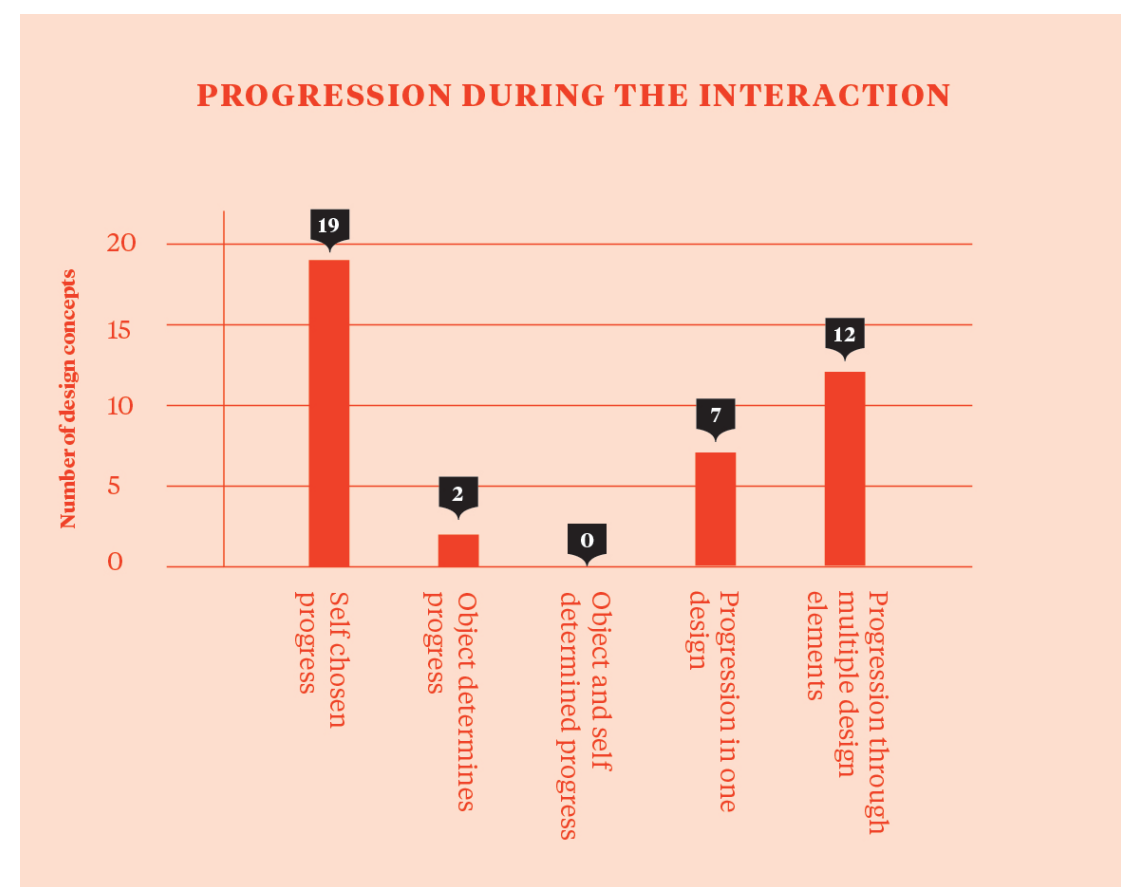

Figure 54:

Design strategies used to offer progression in the interaction.

Figure 55:

Water bottle by

Jazz Klinkert that

challenges the user

by offering less water

when the lid is rotated fully. 
Further designs that used a self-determined progress are shown in Figures 56 and 57. These designs focused on multiple design elements to offer progression in the interaction. The minority of concepts incorporated a predetermined progress by the object. Those design concepts used materials that changed over time. The longer the object was used, the smaller the surface area got to hold the object making it more challenging for the user to use it.

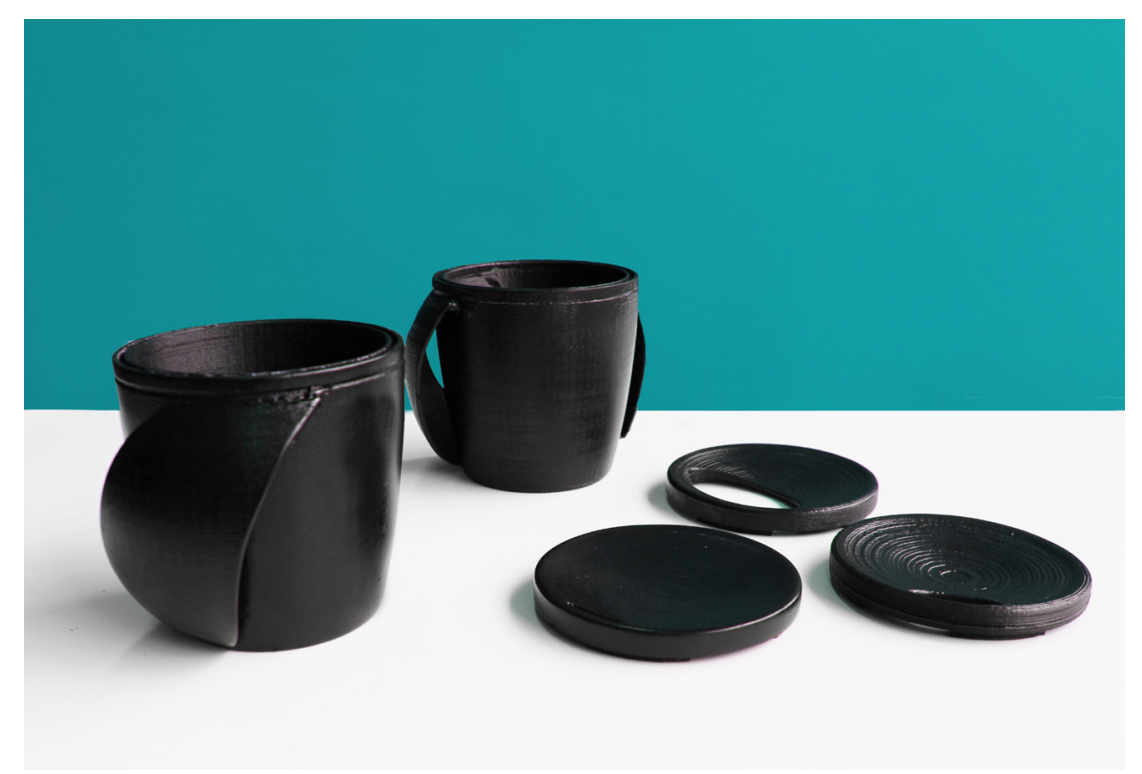

Figure 56:

Coffee mugs and lids by Katie O’Brien.
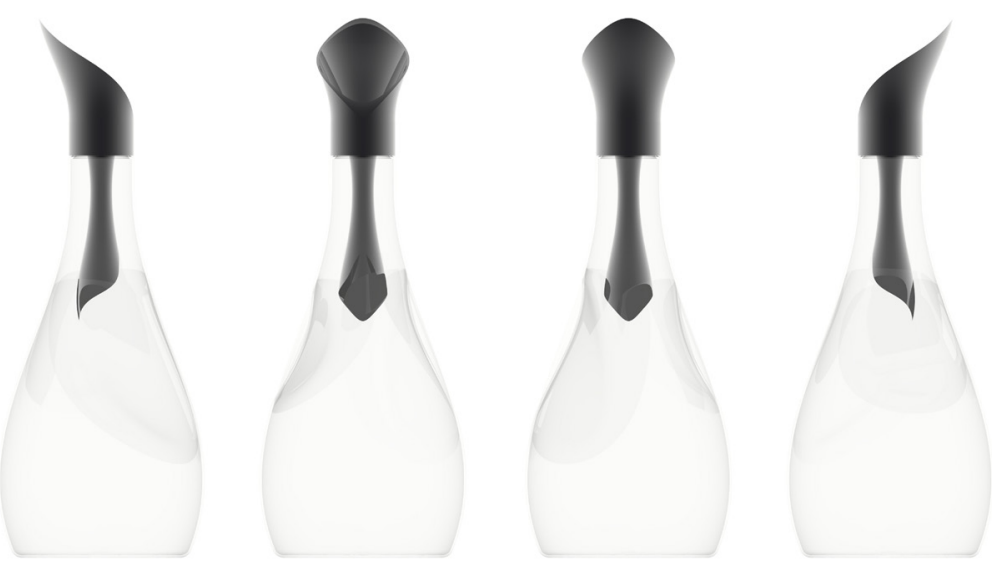

Figure 57:

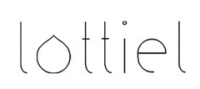

“Lottiel” by Ashleigh

Kennedy. 


\subsubsection{Feedback}

Feedback is an essential element in the rehabilitation process and is provided as part of shaping in an immediate, specific, quantitative, and positive form. The students were encouraged to include augmented feedback in the design concepts equivalent to that given by a therapist during the rehabilitation. However, none succeeded in implementing such a form of feedback, see Figure 58. The most common form of feedback was functional feedback, which is the actual function of the design; for example, that it pours water.

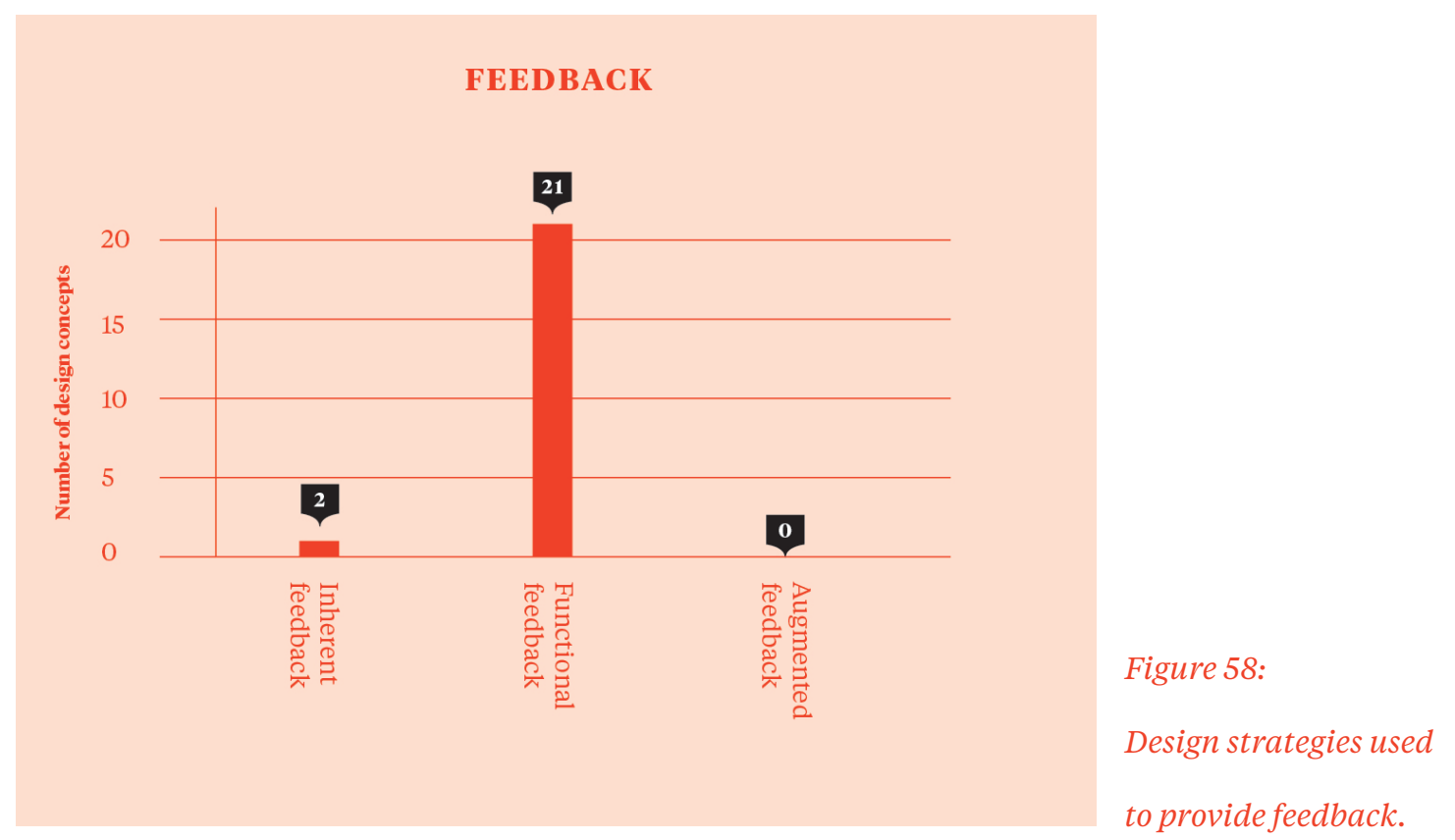

\subsubsection{Behaviour contract}

The behaviour contract is a component used within CIMT to evoke a feeling of responsibility in the participant and to translate gains made during the rehabilitation into the participant's real-world context. It was emphasised in the previously conducted interviews, see Chapter 3, that the stroke survivors have to go through a behaviour change to initiate the use of the affected arm and overcome the learned nonuse in the long-term. One therapist mentioned that patients would often refuse to sign the contract since they would underestimate 
its value in the process. The design workshop focused on alternative ways of incorporating the behaviour contract beyond the standard written form. The different design strategies see Figure 59, included:

1. Adding an additional, removable behaviour contract to the design

2. Including the behaviour contract in the design's packaging

3. Including the behaviour contract within the physical form of the design

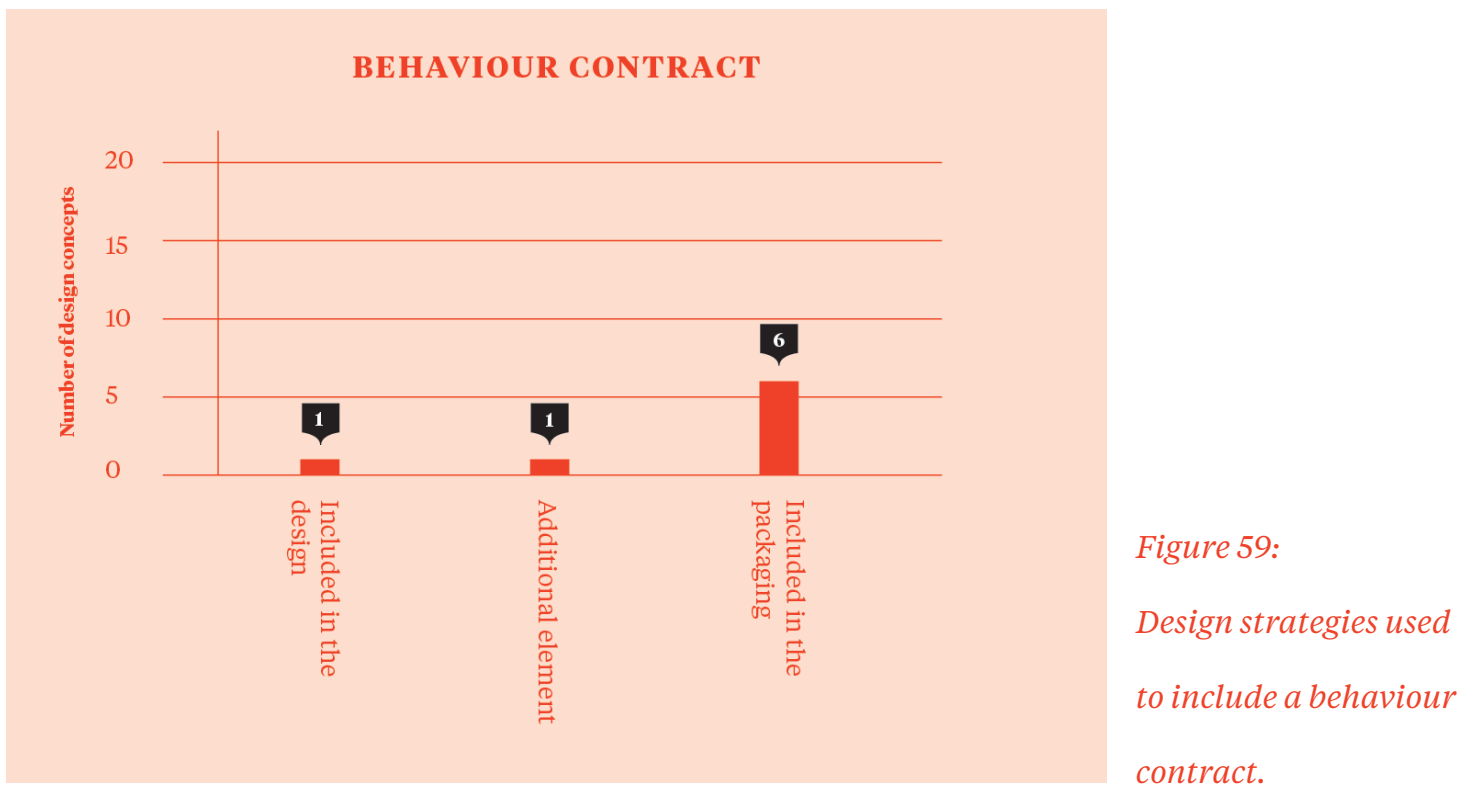

\subsection{Evaluation}

The following section outlines the results of the cognitive walkthrough with stroke therapists. Six design concepts were evaluated by the health experts.

\subsubsection{Fluve}

The therapist appreciated the aesthetic of the object but stated that it still appeared therapeutic. The design is supposed to hang on the wall to decrease shoulder pain during use but criticism was that its use would be difficult and frustrating over time. One therapist suggested using a stand might be more feasible to offset the weight. 


\subsubsection{Lottiel}

The therapists appreciated that the top part can be used on normal water bottles as well, but they indicated that stroke survivors often struggle with a normal water bottle because they squeeze it too hard. One therapist recommended adding more texture on the bottle and the top part to make the motion easier. Additional feedback in the form of an element that measures the water flow or letting the water appear coloured was suggested to be added.

\subsubsection{Smart Sticks}

The intended use of the object was not clear and the therapists identified it as a kind of scissors. They recommended a square pad at the end of the chopsticks to ease the picking up of items and using elastic bands to add resistance during use. They further suggested that it could be used for playing a board game or for picking up objects. One mentioned that tongs are already used in the rehabilitation process.

\subsubsection{Coffee mugs}

The mugs require an extension of the fingers, and especially the thumb, that would be too difficult for a stroke survivor. The purpose of the different lids was not understood and the handles were too small to be used as a support for the hand. The surface was criticised as being too smooth and it was recommended to add additional texture. Two of the therapists questioned the therapeutic value of the device and considered it impracticable.

\subsubsection{Cirgo knives}

The task of cutting is a huge challenge for a stroke survivor and a dangerous task to train. Adding more texture on the sides of the knife would offer more support during the task. Two therapists questioned how different this design is from what is currently available on the market, while one therapists stated this design solution offered a lot of potential. 


\subsubsection{Water bottle}

The size of the bottle was criticised as being too big to hold, and adding more texture was recommended. An additional strap that supports the hand would make the use of the bottle feasible for a stroke patient.

\subsection{Discussion}

\subsubsection{The design process}

The students expressed problems understanding medical terms and the description of physical limitations after the stroke. They preferred to design according to their preferences and liking and had issues designing for people with specific motor impairments. It was emphasised during the course that the initiation of use is not just based on restraining the movement but that augmented feedback and a behaviour contract were equally important in the process. The shortcomings in including those components might have been caused by the short course timeline and the students' focus on industrial design.

\subsubsection{The research through design results}

Different design strategies were used to address the design brief and incorporate shaping aspects. Some strategies such as ergonomic restrictions were frequently used, while other elements were seen to be rather challenging to include in the design, see Table 5.

Elements that are part of the therapeutic package of CIMT like the behaviour contract as well as augmented and specific feedback were included in the designs but in a modified way. The behaviour contract increases the participant's engagement in the process and evokes a feeling of responsibility. It is a formal written document signed by the therapist and participant (Morris et al., 2006, p. 262). The different behaviour contracts in this study took more of the form of a reminder that the user sees on the packaging or the object itself. Further studies need to evaluate whether those reminders have the same effect as the original contract, however these are outside the scope of this study. 
Table 5: Overview of design strategies.

\begin{tabular}{l|l|l} 
& Design strategy & Number of concepts \\
\hline \multirow{2}{*}{ Restraint } & Ergonomic restriction & 10 \\
\cline { 2 - 3 } & Bilateral task & 11 \\
\cline { 2 - 3 } & Intuitive use & 10 \\
\hline \multirow{2}{*}{ Pnhanced repetition } & Task is already repetitive & 14 \\
\cline { 2 - 3 } & Increased repetition & 5 \\
\cline { 2 - 3 } & Self-chosen progress & 19 \\
\cline { 2 - 3 } & Object determines progress & 2 \\
\cline { 2 - 3 } & Progress in one design element & 7 \\
\cline { 2 - 3 } & Progress in multiple design elements & 12 \\
\hline \multirow{2}{*}{ Feedback } & Inherent feedback & 2 \\
\cline { 2 - 3 } & Functional feedback & 21 \\
\hline \multirow{2}{*}{ Behaviour contract } & Included in the design & 1 \\
\cline { 2 - 3 } & Additional element & 6 \\
\cline { 2 - 3 } & Included in the packaging & 1 \\
\hline
\end{tabular}

One of the therapists pointed out that the use of those objects did not deliver a shaping task. Shaping is provided in a clinical environment and uses ten 30-second trials and explicit feedback (Morris et al., 2006, p. 259). The designs presented in this paper do incorporate principles of shaping but are intended to be used in a self-directed way in the participant's home environment without any time restrictions and without a therapist being present.

The purpose of design solutions that relied on one design element to provide progression and a constant challenge was understood, whereas design concepts with multiple elements caused some confusion about the intended use. Criticism of the design concepts often concerned the lack of texture and the size of the objects. Sensory deficits in the hands are a common symptom among stroke survivors and adding a rough texture would potentially offer more grip and allows to perform the movement. The extension of the hand, and especially 
the thumb, to the degree, that most designs required were stated as being too challenging. The reduction of the size and diameter was pointed out as a way to improve the designs. During the evaluation, questions were raised about how the designs made the task easier. Everyday objects used by stroke survivors are often assistive devices to enable them to interact with their environment. The focus of this workshop was not to design assistive devices, but rather to generate designs that challenge the user and evoke a behaviour change that initiates the use of the affected arm and hand. However, there seems to be an expectation that everyday objects are used for compensation purposes rather than rehabilitation purposes.

\subsection{Conclusion}

The findings of this study indicate that shaping aspects can be used to create everyday objects that encourage the initiation of use of the affected arm and hand in stroke survivors. Feedback provided by stroke therapists suggest that the designs potentially contain a therapeutic value. The design outcomes need to be further tested with stroke survivors to validate the usability and efficacy of the designs.

The design outcomes presented in this paper focus on industrial design solutions and do not employ any form of digital technology such as sensors or software applications. However, design solutions could potentially benefit from them. Further research focusing on these applications offers the potential to extend our proposed design strategies.

The strategies proposed in this paper were incorporated into a set of digital design method cards accessible under www.limited-use-only.com. The design method cards can work as an inspirational tool for other designers and offer guidance in designing everyday objects that restrain the movement to initiate the use of the affected arm and hand. The following chapter outlines the evaluation process of the design method cards with designers according to the pragmatist perspective. 


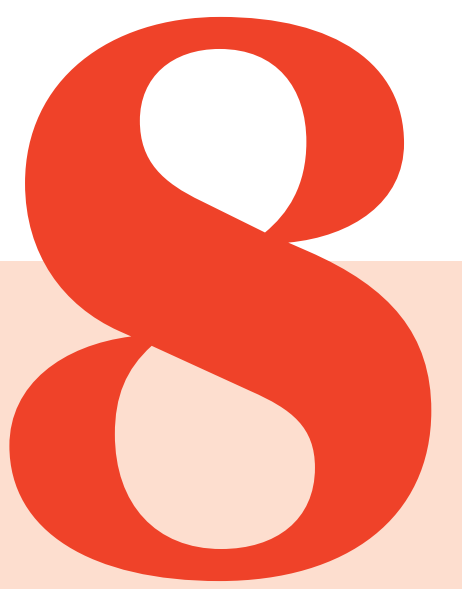

\section{Digital Design}

\section{Method Cards}




\subsection{Introduction}

From the pragmatist perspective, theories that are developed as part of the process need to continually validate their practical value and influence on practice (Dalsgaard, 2014, p. 153). This study presents a set of design strategies in the form of bridging concepts that bridge the gap between theory and practice (Dalsgaard \& Dindler, 2014, p. 1637). The design strategies are based on the results of the design workshop with industrial design students investigating design solutions that facilitate an initiation of use and restrain movement, see Chapter 7. The strategies were further refined and incorporated into digital design method cards that other designers can use in a self-directed way as a source of inspiration and guidance in the design process. Digital design method cards were chosen based on some informal feedback during the workshop indicating that audio-visual material was helpful in developing an understanding of the intended user. Under the pragmatist perspective, the results of this study are used to address the following research question:

1.3.1 How can we design an everyday object that restrains movement of the less affected arm to facilitate and initiate the use of the affected arm?

\subsection{Background}

\subsubsection{Design method cards}

Cards in the context of design are used to visualise the design process (Lafrenière, Dayton, \& Muller, 1999, pp. 151-152), work as inspirational tools in user-centred design approaches (IDEO, 2003; Lucero \& Arrasvuori, 2010, p. 30) and can help to explore ideas and methods (Golembewski \& Selby, 2010, p. 89; Rodríguez Ramírez, 2011, pp. 262-265). Cards have been used in the context of participatory design approaches to stimulate discussion between designer and participants and to evoke empathy and understanding for the user (Hudson, 2005, p. 56). In the context of this study, a focus is on the use of method cards 
that can function as a tool to disseminate research insights and provide a source of inspiration to other designers (Yoon, Desmet, \& Pohlmeyer, 2016, p. 6). The method cards that were developed as part of this study addressed a unique context and therefore needed to address two components: empathy and inspiration (see Figure 60).
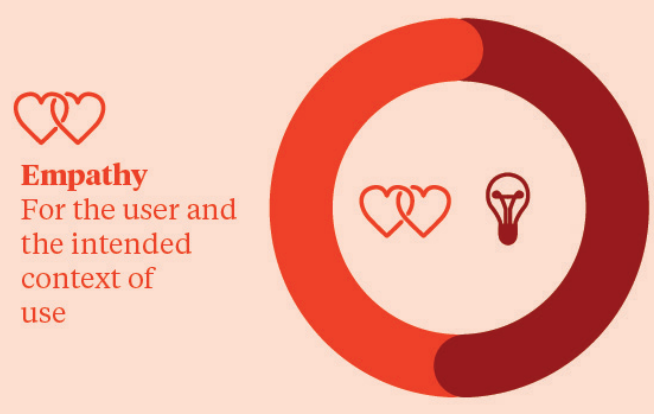

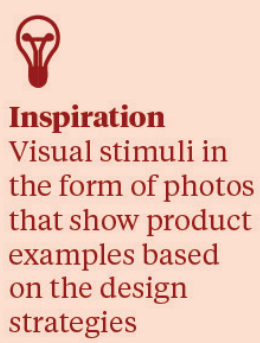

Figure 60:

Purpose of the design method cards.

\subsubsection{Context-specific cards}

Theory can be used as a basis for developing design method cards and has been employed in the context of design research. Examples include the "Design with intent toolkit” (Lockton et al., 2010), PLEX cards, which are based on a framework for designing playful experiences (Lucero \& Arrasvuori, 2010, pp. 28-29), or strategies to elicit surprise (Rodríguez-Ramírez, 2014). A review of method cards by Wölfel and Merritt (2013) analysed the intended purpose of the cards, duration of use and placement within the design process, methodology, the opportunity to customise the content of the cards, and formal qualities (Wölfel \& Merritt, 2013, pp. 480-482). The authors identified three archetypes of patterns of intended use: general purpose/repository cards, customisable cards, and contextspecific cards.

The design method cards developed as part of this study can be classified as context-specific cards. Context-specific cards are used as part of a specific context or agenda and provide instructions for a specific stage of the design process. 
Examples that follow a similar approach are PLEX Cards, the Sound Design Deck, and Design Play Cards (Alves \& Roque, 2011; Eco Innovators, 2013; Lucero \& Arrasvuori, 2010, pp. 30-34).

Despite recent attention in the literature, it is unclear which process needs to be followed to create design method cards or how they should be used in the context of conceptualising and designing (Yoon et al., 2016, p. 6). Some of the studies that focus on the use of card tools outline their intended use (Asheim, Divitini, Kjøllesdal, \& Mora, 2016, p. 21; Chung \& Liang, 2015, p. 20; Lenz, Diefenbach, \& Hassenzahl, 2013, p. 132). However, how the ideal setting was determined is often obscured (Yoon et al., 2016, p. 6).

The method cards developed as part of this study provide relevant information about the intended user before the design process begins. The cards also offer a source of inspiration that can be referred to before and during the design process.

\subsubsection{Empathy with the user}

Designing for stroke survivors requires an understanding of the intended user and the context of use. There is a lack of research that focuses on the use of method cards to design for a specific user group with motor impairments. Therefore, it is difficult to determine how much and what kind of information needs to be provided to the designer to evoke an understanding of the user. Designers are able to design for themselves but often struggle to create for others (Coleman, Clarkson, \& Cassim, 2007, p. 190).

Videos of stroke therapies and motor impairments were included in the development of the design strategies based on informal feedback provided by the design students during the design workshop (see Chapter 7).

\subsection{Information}

The optimal design method cards are a tool that is highly visual, reliable, interactive, intuitive, simple to use, provides inspirational elements, and has minimal costs attached (Nickpour \& Dong, 2009). Designers have access to 
information that describes specific user needs but often the information is presented in a way that is hard for the designer to understand because of the use of medical terms, dense language, and technical terms (Lebbon \& Coleman, 2003, p. $502)$.

The design method cards need to provide the "right amount" of information in the design process and use lay terms to describe medical terms. I took account of this by providing relevant information to the designers in small chapters. Each chapter includes a text component in combination with visual material to make the content easier to comprehend for the designers.

\subsection{Anthropometric data}

Anthropometric data aims to accommodate a range of end users from the 5th to the 95th percentile (McGinley \& Dong, 2011, p. 190) and was included in refining the design method cards. Different collections of ergonomic and anthropometric data sets that suit this purpose are available to designers. However, the data is often presented in a way that is not intuitive and can omit the human qualities of those it describes (McGinley \& Dong, 2011, p. 202).

Relevant information and visualisations were included in the design method cards of this study to take account of the intended focus on the upper limb.

\subsection{Non-expert tester}

Additional information needs to be provided for non-specialised participants. The influence of design method cards seems to vary between experts and junior designers. Usage guidelines can potentially increase the usability of the cards (Yoon et al., 2016, pp. 16-17).

I took this into account by focusing on one group of designers, namely junior designers with no experience in designing for stroke survivors. This group of designers has limited to no experience in designing for stroke survivors and so relies on additional information to develop empathy for the user. 


\subsubsection{Inspiration}

Inspiration can help to define the context of use, trigger ideas, and work as an anchor for structuring the mental idea of the design (Eckert \& Stacey, 2000, p. 525). Inspirational sources are valued by designers (Herring, Chang, Krantzler, \& Bailey, 2009, p. 87) but have received little attention in the literature (Gonçalves, Cardoso, \& Badke-Schaub, 2016, p. 4). Design method cards provide a structure for the design process and can be a source of inspiration. In this study, inspiration is defined as:

Any stimulus retrieved from one's memory or from the outside world, during (or beyond) a design process, that directly or indirectly influences the thinking process leading up to the framing of the problem or generation of a solution. This definition of inspiration can contain tangible entities but also digital artefacts (e.g., web pages) or even intangible entities (e.g., talk with a friend). (Gonçalves et al., 2016, p. 3).

Different kinds of stimulus are available that offer inspiration; for example, design precedents, art pieces, pictures, written documents, or non-related fields such as nature (Cheng, Mugge, \& Schoormans, 2014, p. 374; Gonçalves, Cardoso, \& Badke-Schaub, 2014, p. 29). Visual material seems to be the kind of stimuli preferred by designers (Gonçalves et al., 2014, p. 41; Mougenot, Bouchard, Aoussat, \& Westerman, 2008, p. 339). The details of the stimuli influence how the stimulus is perceived and potentially cause a design fixation.

\subsection{Form of stimulus}

Examples that are provided as line drawings (Cardoso \& Badke-Schaub, 2011, p. 141) help to create more original designs, while photographs are associated with the development of practicable designs (Cheng et al., 2014, p. 376). Designers have used design books and magazines as the primary source of inspiration in the past (Mougenot et al., 2008, p. 339), but the internet has increased in popularity as an inspirational source, especially among young designers (Gonçalves, BadkeSchaub, \& Cardoso, 2013, p. 6). Product designers favour examples that show the 
form and function of the design (Herring et al., 2009, pp. 90-91).

Photographs of the design concepts that were developed during the design workshop, see Chapter 7, were included in the design method cards as a source of inspiration. The photos show the physical form of the design and are presented in combination with a written explanation. The focus of this study is on the formative usability evaluation of the design method cards rather than an assessment of the originality of the designs.

\subsection{Design fixation}

There seems to be an agreement that the provided stimulus should not be closely related to the design topic, to trigger originality (Ozkan \& Dogan, 2013, p. 166) and to avoid design fixation. Recent studies, nonetheless, suggest otherwise (Chan, Dow \& Schuun, 2015, pp. 16-18). However, there is a potential risk that the provided context-specific examples will cause a design fixation. The designer starts to use parts or principles of the example unconsciously in their design (Jansson \& Smith, 1991, pp. 3-9; Linsey et al., 2010, p. 041003-9). This "path of least resistance" is based on our cognitive tendency to invest minimal effort in creative tasks, which allows the designer to refer to already known solutions (Cheng et al., 2014, p. 375). Siangliulue, Chan, Gajos, \& Dow (2015, p. 89) showed in their online experiment that not only is it important what kind of examples are shown, but it is also important when they are shown in the design process. Their results indicated that access to examples when needed instead of on a regular basis led to the highest ratings for novelty but did not necessarily increase productivity (Siangliulue et al., 2015, p. 88).

I incorporated this concept by providing on-demand design examples that just showed specific design solutions when required. 


\subsection{Methods}

Design method cards in a digital form were developed and evaluated by designers in a formative usability evaluation. Feedback was analysed with thematic analysis.

\subsubsection{Participants}

A number of graduate designers from my broader social connections were invited to participate in the study. Participants were eligible to take part in the study if they had a master's degree in a design area that focused on the development of product designs.

Seven design graduates from New Zealand and Germany were invited to participate in the study. One participant withdrew after two months, due to limited time to participate. Two participants $(n=2)$ were from New Zealand; four $(n=4)$ from Germany. Three $(n=3)$ were male, and three $(n=3)$ were female. Two $(n=2)$ had a degree in "master design innovation", three $(n=3)$ a master's degree in "communication in space" and one ( $\mathrm{n}=1)$ a master's degree in "design innovation". Each participant received $50 \mathrm{NZD} / 30 €$ after taking part in the study. The testing started at the beginning of June 2017 and the last of the data were collected at the end of October 2017.

\subsubsection{Design method cards}

Digital design method cards were developed based on the initial results of the design workshop with industrial design students. The method cards were included on the website www.limited use only.com and could be accessed remotely. The website contained information that helped develop empathy with the user and outlined specific design requirements and examples that could be referred to as a source of inspiration. Information to develop empathy was provided first, followed by the design strategies and design examples, see Figure 61. 


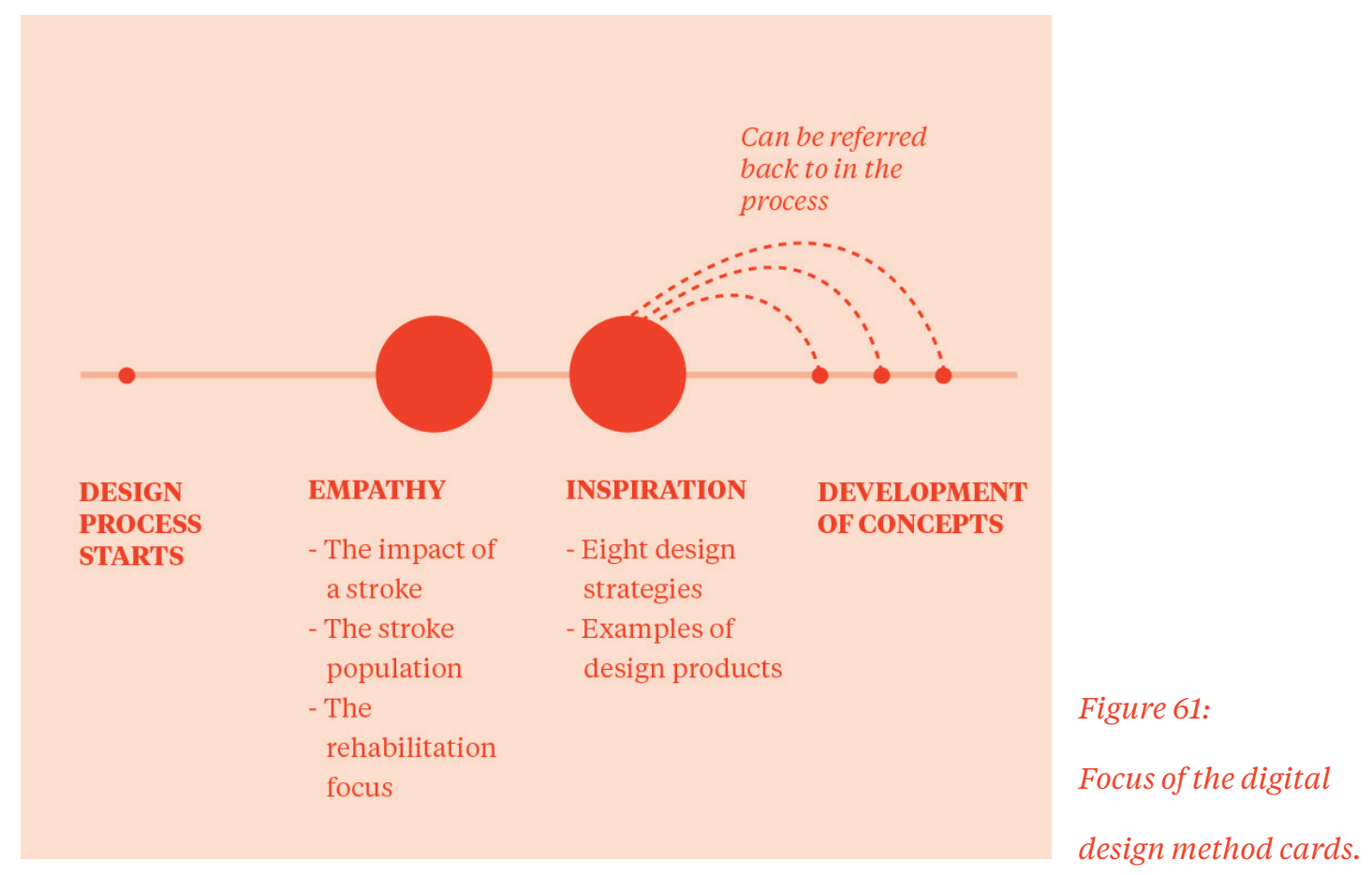

The eight design strategies were:

1. Task performance and movement components

2. Restraining movement

3. Repetition

4. Progress

5. Feedback

6. Engagement-increasing techniques

7. Texture

8. Anthropomorphic data

Each design strategy contained a brief description about its intended use. Further detailed information and visual stimuli were available as a second layer for on-demand inspiration. 


\subsubsection{Evaluation}

A formative usability evaluation of the digital design method cards focused on the user experience of the designers. The website was provided in English and tested with native and non-native speakers to evaluate the complexity of medical terms and information provided.

Each participant was asked to use the method cards to develop a concept for the design of an object that could be used as part of a gardening task. Gardening was chosen based on the initial review of the online survey with stroke survivors, see Chapter 9, and interviews with stroke therapists, see Chapter 10.

The participants were asked to develop a sketch for a design concept in a self-directed way influenced by the design method cards. They had up to four months to test the method cards. A post-test semi-structured interview was carried out at the end of the usability evaluation. Post-test interviews encourage more reflection on the design and user experience than questionnaires (Resnik, 2015, p. 263). Each participant provided informed consent.

The following interview guide was used:

1. Could you please explain the design concept that you have developed?

2. How would you expect the user to interact with it?

3. Did you read through the website and the strategies all at once or over a certain period?

4. Did you find any information difficult to understand?

5. What information did you find beneficial?

6. Did you watch any of the videos?

7. Did you click on the further information links on the website?

8. What kind of information was missing from your perspective?

9. Do you use design strategies as part of your work routine?

\subsubsection{Analysis}

The interviews were partially transcribed and analysed with thematic analysis in a deductive form (Braun \& Clarke, 2006, p. 83). 


\subsection{Results}

\subsubsection{Digital design method cards}

The digital design method cards were incorporated into a website that used multiple sub-pages as chapters. In the following section, the different types of content will be outlined.

\subsubsection{Structure of the website}

The website could be accessed via www.limited-use-only.com and contained seven pages: home, stroke, people, rehabilitation, strategies, about the project, and imprint, see Figure 62. The user could go through the content in chronological order starting with the "home" page and leading to the "strategies" page. A link at the end of each page transferred the user to the next chapter. It was possible to jump through chapters or read content a second time by clicking on the menu button at the top right corner.

\begin{tabular}{|c|c|}
\hline & \\
\hline Home screen & $\begin{array}{l}\text { Introduction and explanation of } \\
\text { what the website is about }\end{array}$ \\
\hline Stroke & Introduction to stroke \\
\hline People & \multirow{2}{*}{$\begin{array}{l}\text { Focus on different motor } \\
\text { impairments and abnormal } \\
\text { muscle synergies after a stroke }\end{array}$} \\
\hline Page 3 & \\
\hline Rehabilitation & \multirow{2}{*}{$\begin{array}{l}\text { General introduction to stroke } \\
\text { rehabilitation and a focus on } \\
\text { initiation of use as part of CIMT }\end{array}$} \\
\hline Page 4 & \\
\hline Strategies & \multirow{2}{*}{$\begin{array}{l}\text { Explanation of how to use the } \\
\text { strategies and outline of the } \\
\text { different steps }\end{array}$} \\
\hline Page 5 & \\
\hline About the project & \multirow{2}{*}{$\begin{array}{l}\text { Explanation of how the } \\
\text { strategies are part of a PhD } \\
\text { research project }\end{array}$} \\
\hline Page 6 & \\
\hline Imprint & \multirow[t]{2}{*}{ Contact information } \\
\hline Page 7 & \\
\hline
\end{tabular}




\subsubsection{Colour code}

Each page was colour coded and contained multiple small information units, see Figure 63. On the right-hand side of the website, an overview presented the overall content of the page.

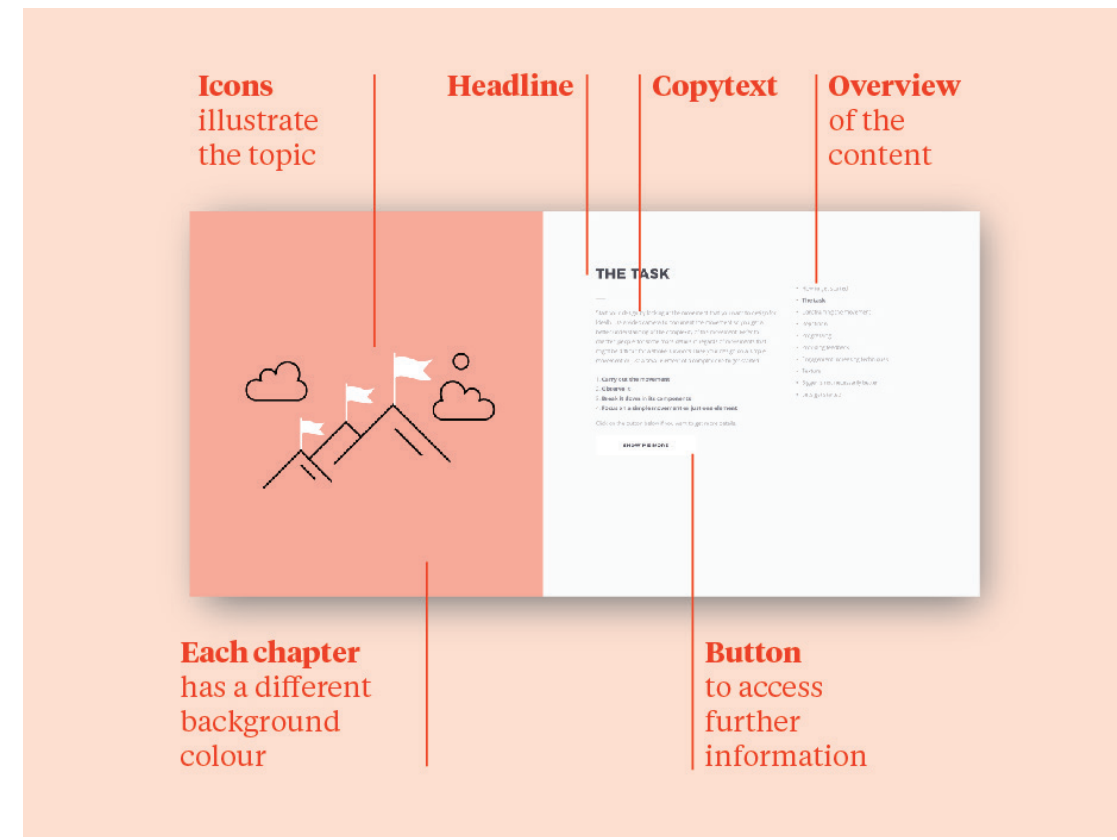

Figure 63:

Structure of an

information unit.

Each page contained multiple units.

\subsubsection{Empathy}

To develop an understanding for the user and common impairments, the second page focused on providing relevant information. It described the medical condition of a stroke, possible impairments and the effect it has on the survivor's life. The following pages focused on motor impairments of the upper limb with a focus on the shoulder, elbow, forearm, wrist, hand, and fingers. See Figure 64 for an example.

The following pages outlined how neurorehabilitation interventions are structured, what learned nonuse is and how restraint is used to initiate the use of the affected arm and hand (see Table 6). Additional information was provided in research articles and media content that the user could access on demand by clicking on a button. 


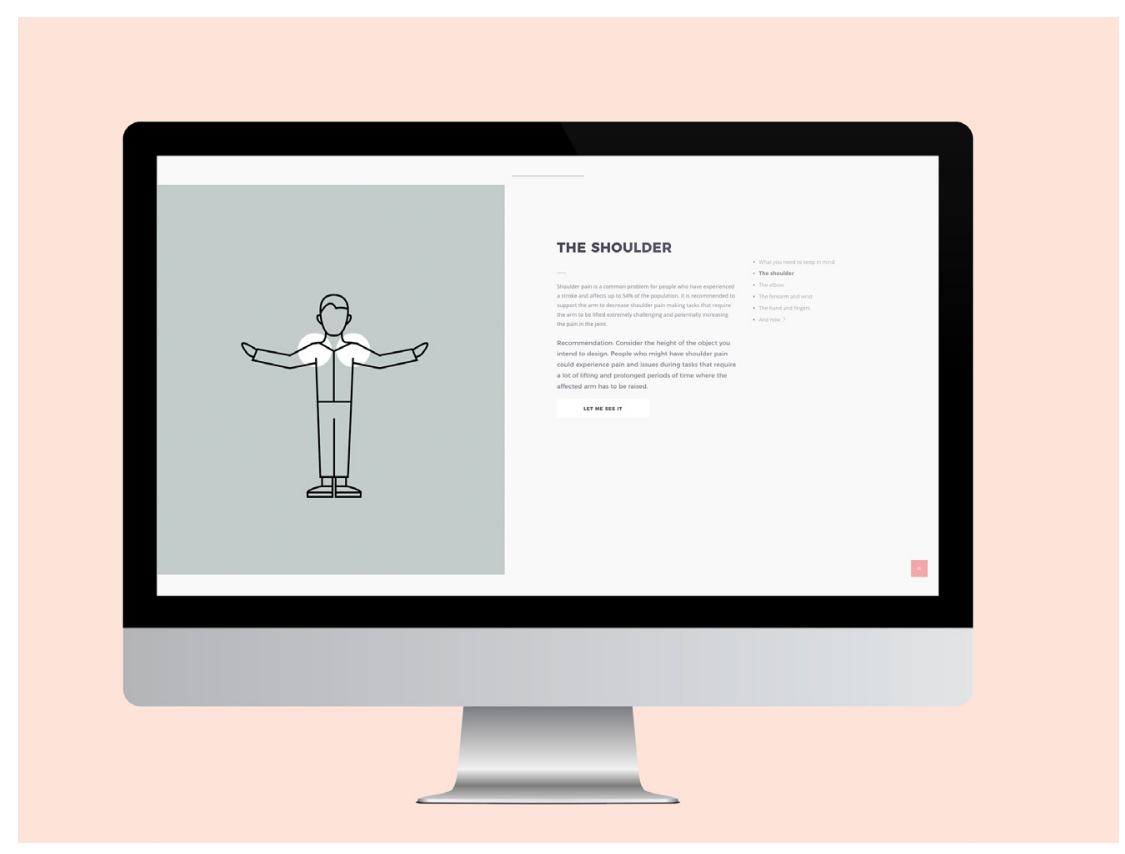

Figure 64:

Information unit that

describes common

motor impairments of

the shoulder joint.

Table 6: Website content and hierarchy of the empathy section.

\begin{tabular}{|c|c|c|c|}
\hline & $\begin{array}{l}\text { Page 02: } \\
\text { Stroke }\end{array}$ & $\begin{array}{l}\text { Page 03: } \\
\text { People }\end{array}$ & $\begin{array}{l}\text { Page 04: } \\
\text { Rehabilitation }\end{array}$ \\
\hline & Information unit 01 & Information unit 07 & Information unit 13 \\
\hline Headline & Welcome & $\begin{array}{l}\text { What you need to keep } \\
\text { in mind }\end{array}$ & Treatment \\
\hline $\begin{array}{l}\text { Content } \\
\text { details }\end{array}$ & $\begin{array}{l}\text { Brief explanation about } \\
\text { the strategies and the } \\
\text { different chapters }\end{array}$ & $\begin{array}{l}\text { Intended user group } \\
\text { experiences specific } \\
\text { movement impair- } \\
\text { ments }\end{array}$ & $\begin{array}{l}\text { Assessment of defi- } \\
\text { cits by stroke special- } \\
\text { ists and recommen- } \\
\text { dation for treatment }\end{array}$ \\
\hline \multirow[t]{2}{*}{$\begin{array}{l}\text { On-demand } \\
\text { information }\end{array}$} & & & $\begin{array}{l}\text { Link to the website } \\
\text { for stroke survivors } \\
\text { "enable me" }\end{array}$ \\
\hline & Information unit 02 & Information unit 08 & Information unit 14 \\
\hline Headline & What is a stroke & The shoulder & Timing \\
\hline $\begin{array}{l}\text { Content } \\
\text { details }\end{array}$ & $\begin{array}{l}\text { Medical definition of a } \\
\text { stroke }\end{array}$ & $\begin{array}{l}\text { Description of abnor- } \\
\text { mal muscle synergies }\end{array}$ & $\begin{array}{l}\text { Explanation of the } \\
\text { plateau effect in } \\
\text { the rehabilitation } \\
\text { process }\end{array}$ \\
\hline \multirow[t]{2}{*}{$\begin{array}{l}\text { On-demand } \\
\text { information }\end{array}$} & $\begin{array}{l}\text { Link to a video that } \\
\text { explains what a stroke } \\
\text { is }\end{array}$ & $\begin{array}{l}\text { Link to a video showing } \\
\text { compensatory } \\
\text { movement patterns of } \\
\text { the shoulder }\end{array}$ & \\
\hline & Information unit 03 & Information unit 09 & Information unit 15 \\
\hline
\end{tabular}




\begin{tabular}{|c|c|c|c|}
\hline Headline & $\begin{array}{l}\text { Who is likely to experi- } \\
\text { ence a stroke }\end{array}$ & The elbow & Neuroplasticity \\
\hline $\begin{array}{l}\text { Content } \\
\text { Details }\end{array}$ & $\begin{array}{l}\text { People who are affected } \\
\text { worldwide as well as NZ } \\
\text { statistics }\end{array}$ & $\begin{array}{l}\text { Abnormal muscle syn- } \\
\text { ergies and recommen- } \\
\text { dation to avoid certain } \\
\text { movements }\end{array}$ & $\begin{array}{l}\text { Strategies that } \\
\text { increase the develop- } \\
\text { ment of neuroplas- } \\
\text { ticity }\end{array}$ \\
\hline \multirow[t]{2}{*}{$\begin{array}{l}\text { On-demand } \\
\text { information }\end{array}$} & & $\begin{array}{l}\text { Link to a video showing } \\
\text { compensatory move- } \\
\text { ment patterns of elbow }\end{array}$ & $\begin{array}{l}\text { Link to an article that } \\
\text { describes neuroplas- } \\
\text { ticity }\end{array}$ \\
\hline & Information unit 04 & Information unit 10 & Information unit 16 \\
\hline Headline & $\begin{array}{l}\text { So what does this } \\
\text { mean? }\end{array}$ & The forearm and wrist & Learned nonuse \\
\hline $\begin{array}{l}\text { Content } \\
\text { details }\end{array}$ & $\begin{array}{l}\text { Symptoms of a stroke } \\
\text { can't be generalised }\end{array}$ & $\begin{array}{l}\text { Abnormal muscle syn- } \\
\text { ergies and movement } \\
\text { that might be problem- } \\
\text { atic }\end{array}$ & $\begin{array}{l}\text { Concept of learned } \\
\text { nonuse of the affect- } \\
\text { ed arm and hand }\end{array}$ \\
\hline $\begin{array}{l}\text { On-demand } \\
\text { information }\end{array}$ & $\begin{array}{l}\text { Link to a video in which } \\
\text { stroke survivors talk } \\
\text { about their life after the } \\
\text { stroke }\end{array}$ & & \\
\hline \multirow[b]{2}{*}{ Headline } & Information unit 05 & Information unit 11 & Information unit 17 \\
\hline & $\begin{array}{l}\text { The symptoms can be } \\
\text { broad }\end{array}$ & The hand and fingers & $\begin{array}{l}\text { Constraint-induced } \\
\text { movement therapy }\end{array}$ \\
\hline $\begin{array}{l}\text { Content } \\
\text { details }\end{array}$ & ICF model & $\begin{array}{l}\text { Abnormal muscle syn- } \\
\text { ergies and recommen- } \\
\text { dation to avoid certain } \\
\text { movements }\end{array}$ & $\begin{array}{l}\text { Elements that are } \\
\text { part of CIMT }\end{array}$ \\
\hline $\begin{array}{l}\text { On-demand } \\
\text { information }\end{array}$ & & $\begin{array}{l}\text { Link to a video showing } \\
\text { compensatory move- } \\
\text { ment patterns }\end{array}$ & $\begin{array}{l}\text { Link to a research } \\
\text { article about CIMT } \\
\text { and a YouTube video } \\
\text { about CIMT }\end{array}$ \\
\hline \multirow[b]{2}{*}{ Headline } & Information unit 06 & Information unit 12 & Information unit 18 \\
\hline & So many details & And now...? & Shaping \\
\hline $\begin{array}{l}\text { Content } \\
\text { details }\end{array}$ & & & $\begin{array}{l}\text { Description of the } \\
\text { role of shaping }\end{array}$ \\
\hline $\begin{array}{l}\text { On-demand } \\
\text { information }\end{array}$ & $\begin{array}{l}\text { Link to the people } \\
\text { subpage }\end{array}$ & $\begin{array}{l}\text { Link to the rehabilita- } \\
\text { tion subpage }\end{array}$ & $\begin{array}{l}\text { Link to a You Tube } \\
\text { video showing shap- } \\
\text { ing tasks }\end{array}$ \\
\hline \multirow[b]{2}{*}{ Headline } & & & Information unit 19 \\
\hline & & & Let's get started \\
\hline $\begin{array}{l}\text { Content } \\
\text { details }\end{array}$ & & & $\begin{array}{l}\text { Link to the design } \\
\text { strategies subpage }\end{array}$ \\
\hline
\end{tabular}




\subsubsection{Inspiration}

Page 5 presented the different design strategies, see Table 7. Each strategy was presented with a brief explanation and description of how it could be used as part of physical designs. Product examples could be accessed via a button that led to a further subpage, see Figure 65 . The subpage further outlined a more detailed description of the design strategy.

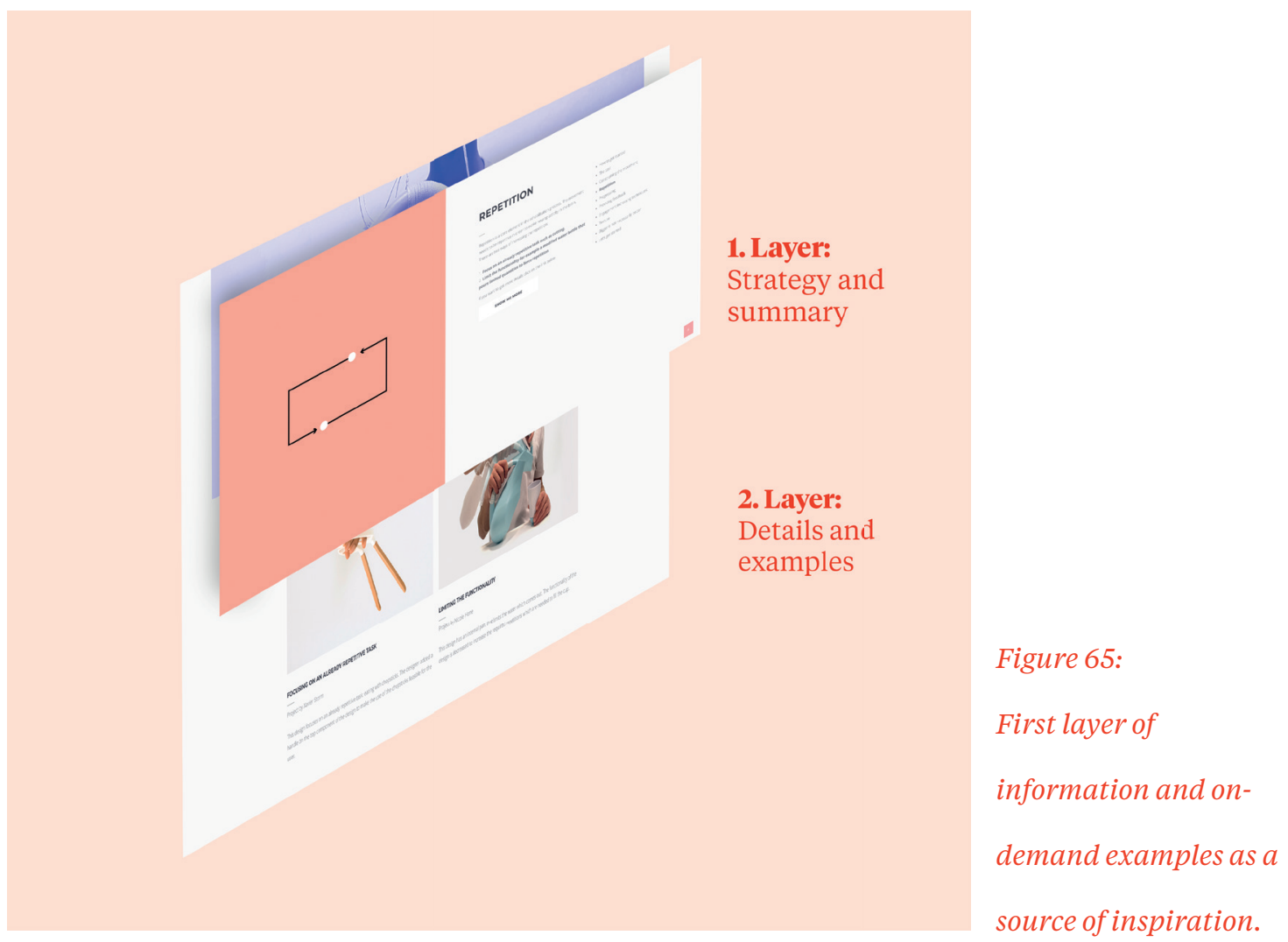


Table 7: Website content of the inspiration section.

\begin{tabular}{|c|c|}
\hline & $\begin{array}{l}\text { Subpage 04: } \\
\text { Strategy }\end{array}$ \\
\hline \multirow[b]{2}{*}{ Headline } & Information unit 20 \\
\hline & How to get started \\
\hline $\begin{array}{l}\text { Content de- } \\
\text { tails }\end{array}$ & Introduction to the use of the design strategies. \\
\hline \multicolumn{2}{|l|}{$\begin{array}{l}\text { On-demand } \\
\text { information }\end{array}$} \\
\hline \multirow[b]{2}{*}{ Headline } & Information unit 21 \\
\hline & The task \\
\hline $\begin{array}{l}\text { Content } \\
\text { details }\end{array}$ & $\begin{array}{l}\text { Instructions to focus on the movement necessary to use the object and } \\
\text { breaking it down into its components. } \\
\text { Sketching is advised to help in the design process. }\end{array}$ \\
\hline $\begin{array}{l}\text { On-demand } \\
\text { information }\end{array}$ & Link to design examples developed as part of the design workshop \\
\hline \multirow[b]{2}{*}{ Headline } & Information unit 22 \\
\hline & Restraining the movement \\
\hline $\begin{array}{l}\text { Content } \\
\text { details }\end{array}$ & $\begin{array}{l}\text { Instructions outline that the restraining effect is needed to evoke an } \\
\text { initiation of use. Three strategies are provided. }\end{array}$ \\
\hline $\begin{array}{l}\text { On-demand } \\
\text { information }\end{array}$ & Link to design examples developed as part of the design workshop \\
\hline \multirow[b]{2}{*}{ Headline } & Information unit 23 \\
\hline & Repetition \\
\hline $\begin{array}{l}\text { Content de- } \\
\text { tails }\end{array}$ & $\begin{array}{l}\text { Instructions outline that repetition is needed to evoke changes within } \\
\text { the brain. } \\
\text { Two strategies are provided. }\end{array}$ \\
\hline $\begin{array}{l}\text { On-demand } \\
\text { information }\end{array}$ & Link to design examples developed as part of the design workshop \\
\hline \multirow[b]{2}{*}{ Headline } & Information unit 24 \\
\hline & Progressing \\
\hline $\begin{array}{l}\text { Content } \\
\text { details }\end{array}$ & $\begin{array}{l}\text { Instructions outline that progress is needed to provide a challenging } \\
\text { experience. } \\
\text { Four strategies are provided. }\end{array}$ \\
\hline $\begin{array}{l}\text { On-demand } \\
\text { information }\end{array}$ & Link to design examples developed as part of the design workshop \\
\hline \multirow[b]{2}{*}{ Headline } & Information unit 25 \\
\hline & Providing feedback \\
\hline
\end{tabular}




\begin{tabular}{|c|c|}
\hline $\begin{array}{l}\text { Content } \\
\text { details }\end{array}$ & $\begin{array}{l}\text { Instructions outline how feedback is used as part of product designs. } \\
\text { Three strategies are provided. }\end{array}$ \\
\hline $\begin{array}{l}\text { On-demand } \\
\text { information }\end{array}$ & Link to design examples developed as part of the design workshop \\
\hline \multirow[b]{2}{*}{ Headline } & Information unit 25 \\
\hline & Engagement increasing techniques \\
\hline $\begin{array}{l}\text { Content } \\
\text { details }\end{array}$ & $\begin{array}{l}\text { A behaviour change needs to be elicited in the user to secure a long-term } \\
\text { use of the affected arm and hand. Three strategies are provided. }\end{array}$ \\
\hline $\begin{array}{l}\text { On-demand } \\
\text { information }\end{array}$ & Link to design examples developed as part of the design workshop \\
\hline \multirow[b]{2}{*}{ Headline } & Information unit 26 \\
\hline & Texture \\
\hline $\begin{array}{l}\text { Content } \\
\text { details }\end{array}$ & $\begin{array}{l}\text { Texture needs to be carefully considered in the design to increase the } \\
\text { usability of the object. Two recommendations are provided }\end{array}$ \\
\hline $\begin{array}{l}\text { On-demand } \\
\text { information }\end{array}$ & $\begin{array}{l}\text { Link to design examples developed as part of the design workshop and } \\
\text { stroke research that focuses on addressing sensory deficits. }\end{array}$ \\
\hline \multirow[b]{2}{*}{ Headline } & Information unit 27 \\
\hline & Bigger is not necessarily better \\
\hline $\begin{array}{l}\text { Content } \\
\text { details }\end{array}$ & $\begin{array}{l}\text { And increased size of an object does not necessarily mean that usability } \\
\text { is increased. The design should take account of anthropomorphic data. }\end{array}$ \\
\hline $\begin{array}{l}\text { On-demand } \\
\text { information }\end{array}$ & Link to relevant anthropomorphic data \\
\hline \multirow[b]{2}{*}{ Headline } & Information unit 28 \\
\hline & Let's get started \\
\hline $\begin{array}{l}\text { Content } \\
\text { details }\end{array}$ & Invitation to provide feedback on the use of the strategies \\
\hline $\begin{array}{l}\text { On-demand } \\
\text { information }\end{array}$ & Contact email address \\
\hline
\end{tabular}

\subsubsection{Evaluation}

\subsubsection{Design concepts}

\subsection{Process}

Two of the participants expressed significant difficulties with developing a concept in the time frame. I followed up on their process during that time. However, after four months the two participants did not have a concept idea. The four submitted design concepts are outlined in the following section. 


\subsection{Participant 01}

The design is intended to be used for the task of watering plants, see Figures 66 and 67. The "Watering Can" needs to be carried with both hands to make it work and initiate use of the affected arm. One hand holds the tank full of water, while the second holds the tube to water the plants. Both arms are necessary to make the water flow. The water flow can be regulated by pushing the mechanism at the end of the tube, see Figure 68.
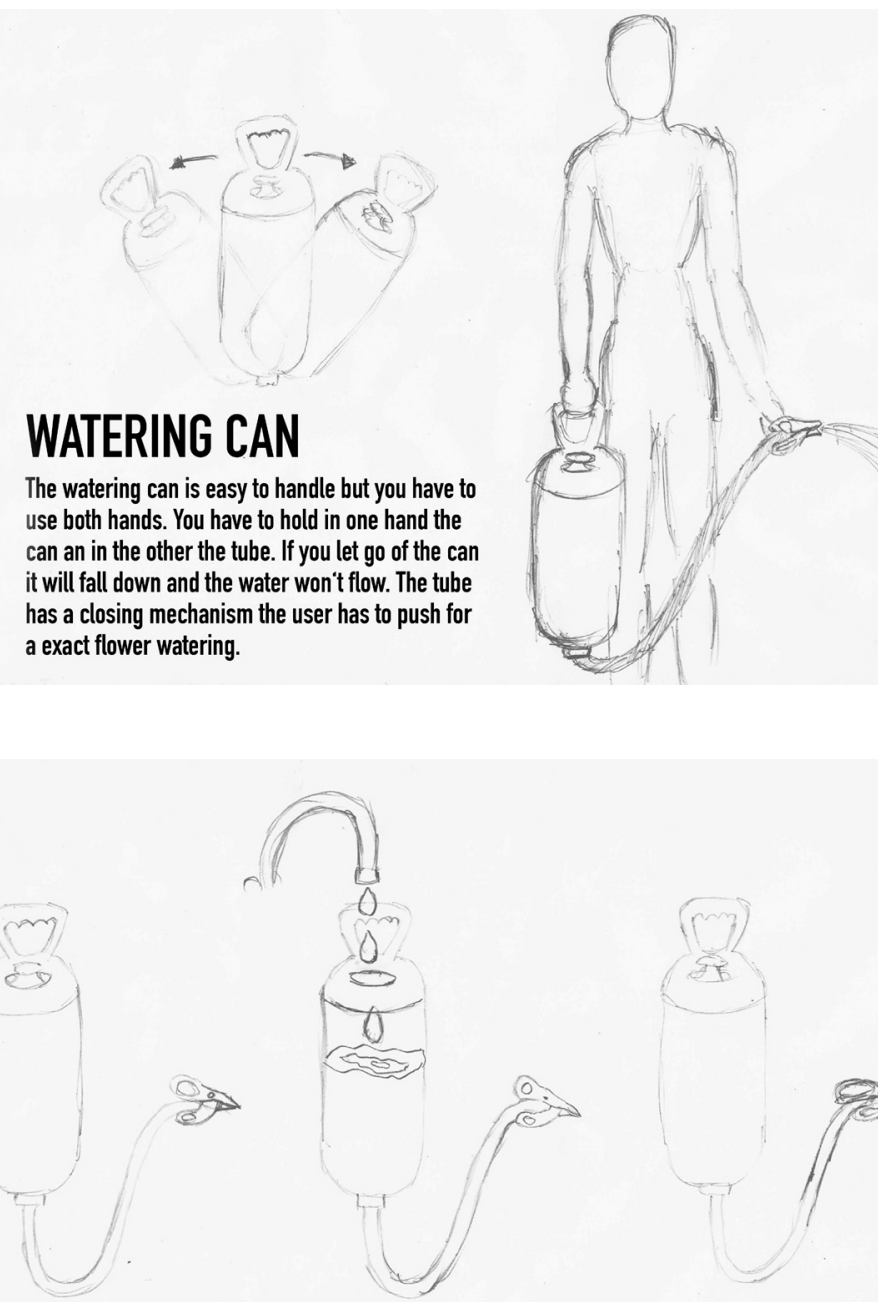

\section{THE CAN}

Figure 66:

Participant 01's concept for a watering can.
Figure 67:

Details of the design concept. 


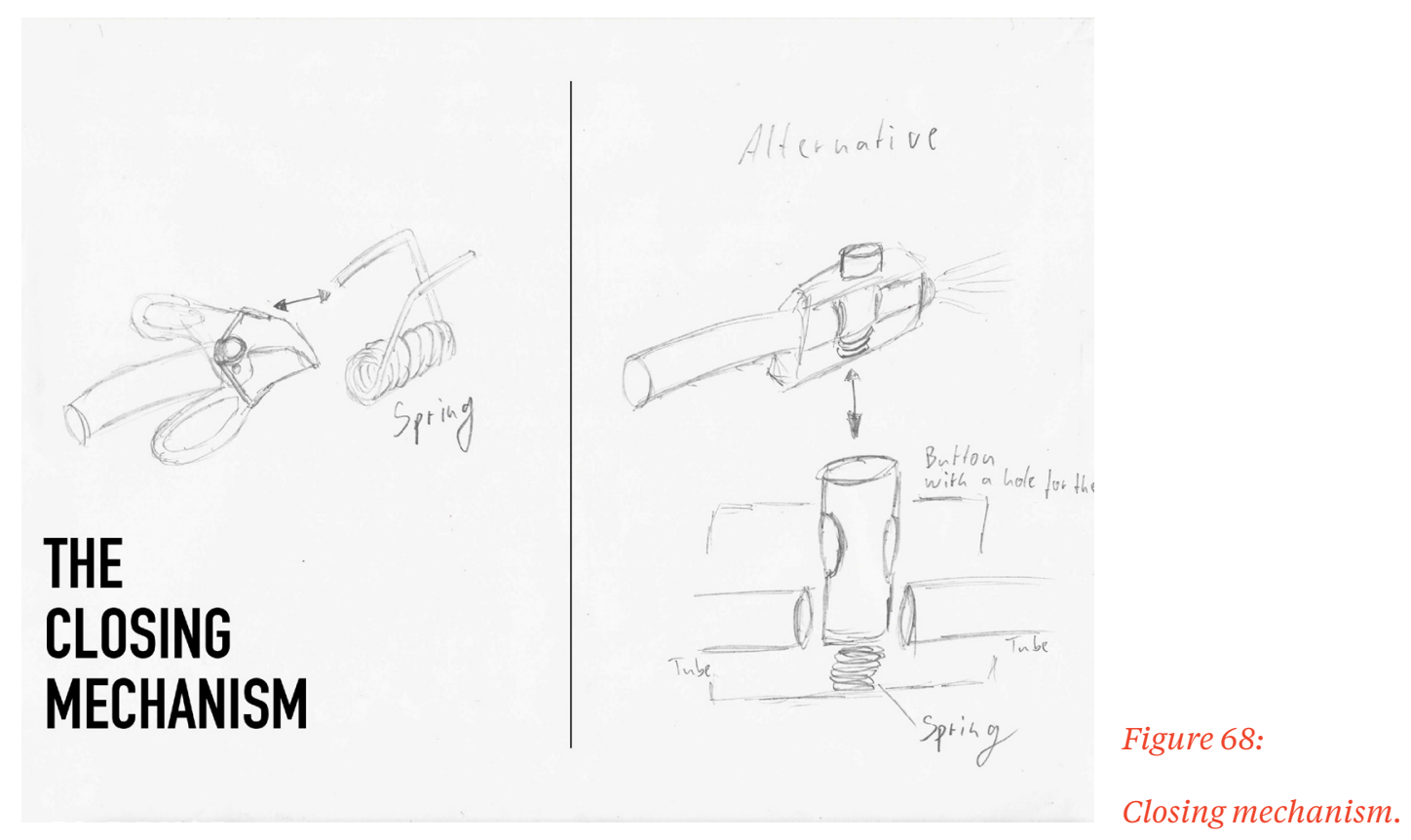

\subsection{Participant 02}

The participant started with a sketch that outlined the different steps needed to trim a bonsai tree, see Figure 69, and developed a concept for a design of shears, see Figure 70. This kind of shears needs unusually short blades to cut specific tree branches and leaves. The design uses an ergonomic restriction to restrain movement and provides additional textures on the handles. The participant envisaged incorporating an audible clicking noise every time the shears shut after cutting a branch. The participant described the sound as being a satisfying experience every time the it appeared, indicating successful completion of the task. 

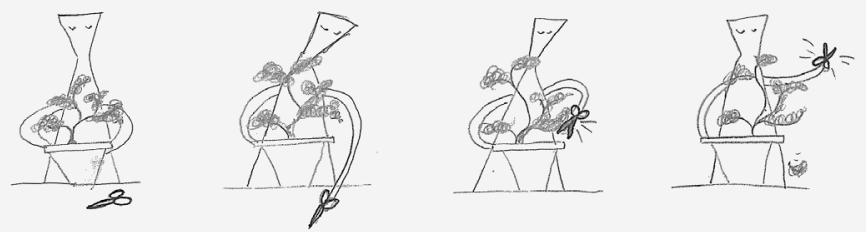

Figure 69:

Sketches that break

down the movement needed to cut a bonsai tree.

\section{Figure 70:}

Participant 02's concept for shears used to cut bonsai trees.

\subsection{Participant 03}

The third participant developed a design concept for a tool to remove weeds from cracks. The design uses an extended bar so the user does not need to bend down, see Figure 71. Different handles and hooks at the end of the stick offer progression and increase the challenge of using the object. The restraining effect is based on intuitive use of the object and ergonomic restrictions of the handles. Progression is provided in the form of different handles that can be attached to the object. 

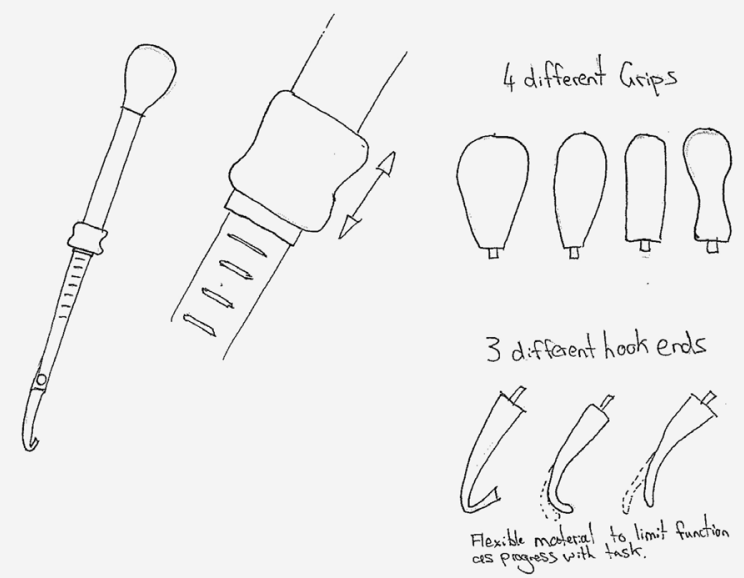

Figure 71:

Participants 03's

concept for a tool to

remove weeds from

cracks.

\subsection{Participant 04}

Participant 04 decided to focus on a different context for the design and developed an idea for a toothbrush that can be used by attaching it to the finger. The toothbrush can be worn on the index finger, see Figure 72. The participant based the concept on personal observation of a family member who had experienced a stroke. The task of brushing their teeth was the one the participant remembered as being extremely challenging for the person. The restraining effect relies on the intuitive use of the object. The object would further use vibration to clean the teeth in case the user is not strong enough to apply pressure.

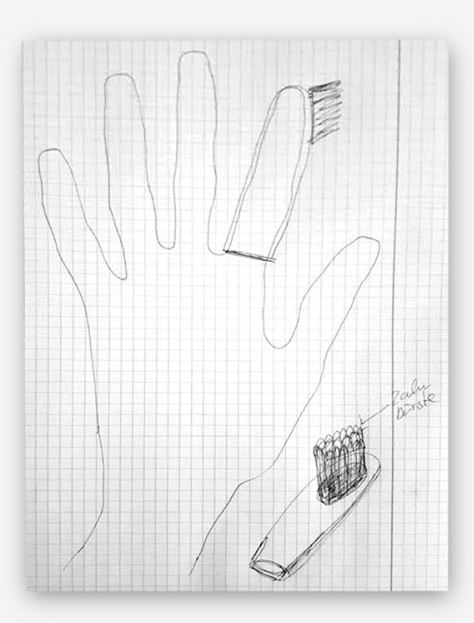

Figure 72:

Participant 04's

concept for a

toothbrush. 


\subsection{Design strategies employed}

Table 8 outlines the different strategies based on the description of the intended use that the participants mentioned.

Table 8: Employed design strategies.

\begin{tabular}{|c|c|c|c|c|}
\hline & Participant 01 & Participant 02 & Participant 03 & Participant 04 \\
\hline Intended use & Watering can & $\begin{array}{l}\text { Shears for bonsai } \\
\text { trees }\end{array}$ & Weed snatcher & Toothbrush \\
\hline $\begin{array}{l}\text { Breaking down } \\
\text { the task }\end{array}$ & Not defined & Yes & Not defined & Not defined \\
\hline Restrain & Bilateral task & $\begin{array}{l}\text { Ergonomic } \\
\text { restrictions / } \\
\text { Intuitive use }\end{array}$ & $\begin{array}{l}\text { Ergonomic } \\
\text { restrictions / } \\
\text { Intuitive use }\end{array}$ & Intuitive use \\
\hline Progress & Not defined & Not defined & $\begin{array}{l}\text { Self-determined } \\
\text { progress / multiple } \\
\text { objects }\end{array}$ & Not defined \\
\hline Repetition & $\begin{array}{l}\text { Already repetitive } \\
\text { task }\end{array}$ & $\begin{array}{l}\text { Already repetitive } \\
\text { task }\end{array}$ & $\begin{array}{l}\text { Limiting func- } \\
\text { tionality / already } \\
\text { repetitive task }\end{array}$ & $\begin{array}{l}\text { Already repetitive } \\
\text { task }\end{array}$ \\
\hline Texture & Not defined & Yes & Not defined & Not defined \\
\hline Feedback & $\begin{array}{l}\text { Functional } \\
\text { feedback }\end{array}$ & $\begin{array}{l}\text { Functional } \\
\text { feedback }+ \\
\text { inherent feedback }\end{array}$ & $\begin{array}{l}\text { Functional } \\
\text { feedback }\end{array}$ & $\begin{array}{l}\text { Functional } \\
\text { feedback }+ \\
\text { inherent feedback }\end{array}$ \\
\hline $\begin{array}{l}\text { Increasing } \\
\text { engagement }\end{array}$ & Not defined & Not defined & Not defined & Not defined \\
\hline $\begin{array}{l}\text { Anthropomor- } \\
\text { phic data }\end{array}$ & Not defined & Not defined & Not defined & Not defined \\
\hline $\begin{array}{l}\text { Takes account } \\
\text { of the } \\
\text { conceptual } \\
\text { model }\end{array}$ & No & Yes & Yes & No \\
\hline
\end{tabular}

\subsubsection{Themes}

Each participant took part in a post-task interview. Participants who did not submit an idea described the challenges that impacted on the process. The posttask interviews were audio recorded, partially transcribed and analysed according 
to the themes of information that is provided as part of the website, the use of the design strategies, perceived challenges, and the use of design strategies in daily practice.

\subsubsection{Website information}

\section{Comprehension}

The participants described the provided information as clear and understandable. Most participants just read parts of the provided content rather than the whole website. The participants who mentioned reading everything mainly did so because they felt that they had to do it as part of the evaluation process. One participant mentioned that most designers would likely "Not bother to know where the research is coming from” (Participant 02). It was suggested that a reduction of information would still make the website usable. One participant appreciated that each site guided the user to the following one but felt that it was hard to remember all the facts. Another participant mentioned that for someone who might not be familiar with a stroke, the information might be too much.

\section{Audio-visual material}

The videos helped to understand the context, provided visualised movement patterns, and explained clearly which kinds of movements might be challenging for a stroke survivor. Some of the participants mentioned that the videos helped to understand the user better and that the designed object needs to take account of a movement that might change over time. This understanding for the user facilitated the internal assessment process of refining initial design ideas. Nonetheless, not all the videos seemed to be helpful. In particular, some of the more general videos focusing on scientific explanations of a stroke were pointed out as being unhelpful. It was further noted that showing more videos of therapy sessions, general material about stroke therapy, and different exercises would be appreciated.

Recommended changes

It was recommended that the purpose of the website needs to be 
communicated more clearly. The information on the website should highlight the fact that the strategies are supposed to help in the design process to develop concept ideas that contribute to the initiation of use of the affected arm and hand in stroke survivors.

All the participants criticised the home page as being too difficult to read due to the background graphics. One participant mentioned that the links that provided on-demand content and led to other subpages were "Kind of annoying" (Participant 03). Another participant commented that the headings of the different subsections should be included in the main menu. This would make it possible to navigate to specific areas of the website. A bar graph could provide visual information on how much content is still to come, which could be helpful in the reading process.

\subsubsection{Use of the strategies}

\section{Inspiration}

The design examples provided as part of the design strategies were pointed out as helpful, but one participant mentioned that "A pool of ideas" (Participant 05), could help in the process. The participant did not submit a concept idea and commented that more examples of current devices might have contributed to developing a concept idea. It was further noticed that providing some videos that show the interaction with the described design prototypes would help to understand the intended use.

The initial step of breaking down the movement was seen as being "A bit strange” (Participant 03), but seemed to be helpful. Just one participant, however, used sketches as an initial step to assess movement components.

One participant used the strategy of providing feedback as a source of inspiration and included auditory feedback into the design of the bonsai clippers. Even though acoustic feedback is not explicitly outlined in the strategies, it is noteworthy that this is an interesting approach that could be included in the category of augmented feedback. 


\section{Timing}

Participants used the strategies in two distinct ways: either reading through them once and then starting the concept process, or reading through them and then referring back to them in the process. The strategies guided the process were used to add specific elements to the design brief and helped them to think about the usability of the product. Most of the participants went through all the strategies, with one participant mentioning having just chosen the strategies that seemed to be suitable for his design concept. One of the participants took notes while going through the strategies, which he referred to in the design process.

\section{Digital form}

The use of digital design method cards was seen as an advantage that allowed constant access to the strategies. One participant mentioned using strategy cards in a physical form occasionally for design work. The participant appreciated continuous access to the digital design method cards, but he preferred paper cards because of the tactile quality provided.

\subsubsection{Perceived challenges}

Some challenges were mentioned regarding comprehension, context, and use of the strategies as well as the development of empathy.

\section{Comprehension}

Two of the German participants expressed issues understanding the written explanations and videos, which were all provided in English. The perceived challenge was the level of complexity as well as the English language in general. A further difficulty was the amount of information provided, which was experienced as rather extensive and complicated to remember.

Context of use

The context of designing for stroke rehabilitation was unfamiliar to all participants. One participant mentioned that the video showing the "Wolf Motor Test” was interesting but she had to stop watching it after a couple of minutes because she found it quite upsetting. In the video you can see a stroke survivor 
taking part in a test that involves touching a box with the tips of the fingers. The movement requires a high level of motor control, which can often be difficult for stroke survivors.

Use of the strategies

The two participants who did not develop a concept sketch indicated that the intended context led to some high expectation of themselves to come up with a strong concept. Both participants had an initial idea but rejected it when they read more about stroke and the limitations that survivors experience. The fact that the intended interaction with the object needed to take account of an increased challenge and movement that changes over time was pointed out as being challenging

A further obstacle seems to be connected to the use of the strategies. One participant mentioned that it was not clear if the strategies were some kind of documentation of possible solutions or a form of exercise that is part of the design brief. The focus on breaking down the movement as a starting point was an unfamiliar approach that only one participant followed.

A further challenge seems to be associated with the level of detail of the provided design examples and possible design fixation on the examples. One participant mentioned that some of the design elements of the concept were borrowed from the examples that were visible on the website.

Empathy

One participant who had worked as a design researcher for a product design company expressed some difficulties with relying on the provided research without conducting field studies, observations, or interviews herself. The participant described the usual research process as a way to develop a deeper understanding of the user. She described the provided information on the website as being useful, but it seems that her traditional design process was interrupted and caused some issues for her. 


\subsubsection{The use of strategies in daily practice}

Design strategies are not always a given element in design education and design practice. The participants referred to design strategies in their daily routine to specific books such as the German “Kribbeln im Kopf”, the general humancentred design approach, or more specific methods such as persona development.

Design strategies were pointed out as often being too difficult to use in daily practice where most designers have to face significant pressure and time restrictions. However, the participant who mentioned using them occasionally appreciated the combination of examples and explanation of the underlying concept. While one participant pointed out that she started to develop her own strategies as part of her work, another one mentioned that rules and strategies had become so internalised that it would not be necessary for her to refer to a written document.

\subsection{Discussion}

In this section the use of the design method cards regarding empathy and inspiration will be discussed first, followed by a focus on further improvements of the strategies and method cards.

\subsubsection{Digital design method cards}

\subsubsection{Empathy}

The results of this study indicate that to design for a specific user group can be challenging from a designer's perspective. Two significant challenges seem to impact on the design process. Firstly, the movement impairments that the user might experience are often unfamiliar, can be experienced as confronting and limit the perceived freedom of the design development process. Secondly, with the aim in mind that initiation of movements needs to be followed by establishing movement patterns that improve over time. Multiple use scenarios and opportunities of interaction need to be incorporated within the design solution. Both challenges seemed to have influenced the internal assessment 
process of the design concepts and led to two incomplete evaluations in this study. The participants who expressed issues in coming up with a design concept had an expectation of themselves that the developed idea had to meet a high standard. Movement limitations and the progression of a challenging interaction were indicated as the primary factors that complicated the process. It also seems that the less the participant read about the condition and watched the provided images, the faster an idea was developed, which seems to be contradictory. However, information about motor impairments appeared to increase critical assessment of initial ideas.

\subsubsection{Inspiration}

None of the participants had worked in the context of designing for stroke survivors before and used the provided design examples as the sole source of inspiration. One participant asked for more examples in further refinements of the method cards to get a better understanding of what is currently provided to stroke survivors. It has been pointed out before that junior designers rely more strongly on inspirational resources than more senior designers (Mougenot et al., 2008, p. 338) and prefer different kinds of stimuli (Ozkan \& Dogan, 2013, pp. 187-188). The participants in this study were junior designers in the area of stroke designs. The use of the strategies according to their knowledge base was therefore not assessed as part of this study.

\subsubsection{Design fixation}

Participants used the design examples as a source of inspiration for the design process. One of the design concepts quite closely resembled a provided example. This is a phenomenon that has been mentioned in the context of cardbased tools in the past (Chung \& Liang, 2015, p. 22). The results of this study are in line with previous results (Siangliulue et al., 2015, p. 89) showing that participants tend to copy features of the provided examples rather than copying the design. It has been pointed out that participants develop more creative ideas with ondemand content (Siangliulue et al., 2015, p. 90). All the design examples provided 
as part of this study were on-demand examples; however, creativity was not assessed as part of this study.

\subsubsection{Design strategies}

The design workshops had already indicated that the provision of feedback in an augmented or inherent form seemed to be an unfamiliar challenge, see Chapter 7. The use of engagement increasing techniques that contributed to behaviour change in the user seemed to be another unfamiliar component. None of the submitted concepts used the engagement increasing strategies that were outlined on the website. This is noteworthy, as use of the designed objects needs to evoke a behaviour change in the user to initiate use of the affected arm and hand in order to overcome the learned nonuse.

\subsubsection{Further iterations}

The evaluation process revealed several limitations and recommendations for changes. Further iterations should address the following criticism:

Firstly, the information provided on the website was perceived as being helpful, but too extensive, complex, and difficult to remember. The amount of information should be limited to improve understanding of the provided information. The provided information should also be translated into different languages to help with understanding.

Secondly, the videos were perceived as being helpful for understanding the movement patterns of the user. However, it seems that participants who watched multiple videos struggled to come up with concepts. The videos should be included at a later stage of the process, once the first ideas have been developed. This might be helpful in generating initial concepts more freely; see Figure 73 for an updated overview of the workflow. It might also be helpful to include videos that show specific rehabilitation interventions and specific training examples that contribute to an initiation of use 


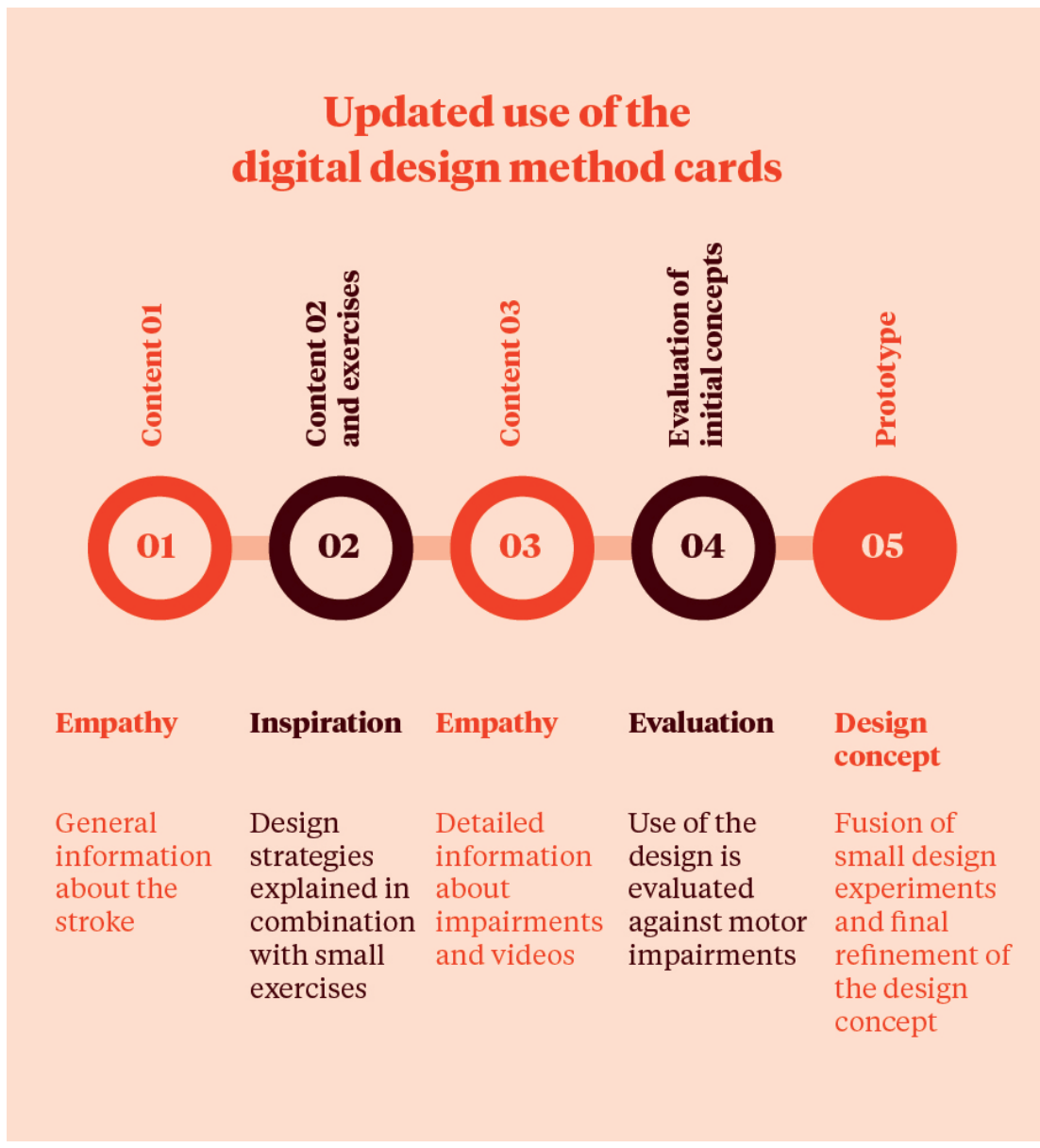

Figure 73:

Refined use of the design method cards.

\section{Development of design components according to the exercise}

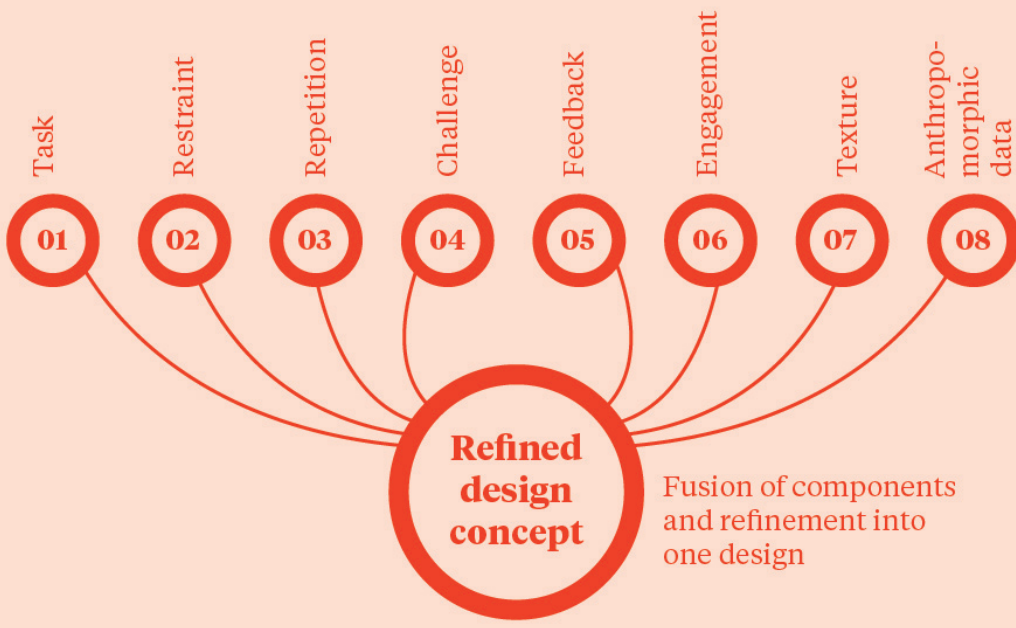

Figure 74:

Exercises based on the design strategies. 
Thirdly, the focus on a website offered the opportunity to include video material. However, including some additional cards outlining the design strategies would incorporate the current use of design method cards. A review of design method cards pointed out that digital apps often lose functionality compared to paper-based models (Wölfel \& Merrit, 2013, p. 485). The designer cannot add personal notes and can only look at one design card at a time (Wölfel \& Merrit, 2013, p. 485). Using a website to present the design strategies might, therefore, be too limiting. A paper-based summary of the cards could facilitate the sketching and concept process and should be incorporated into further refinements of the method cards. The participants would be able to take the physical cards, change the order of the strategies, and just pick the cards that seem to be appropriate.

Fourthly, a set of smaller exercises could help people to get started in the process. Rather than trying to include all the strategies at once, the exercises could help to develop components that can be included in a more complex concept; see Figure 74.

Fifthly, one participant mentioned that showing how to use the design examples in the form of videos might be helpful. A video could clarify the intended interaction with the object.

The design strategies were used in a self-directed way by the participants. This setting was chosen to be in line with the process of the design workshop where participants worked individually on the development of the concepts. However, the strategies could potentially be used in a group workshop. Designers who have worked with stroke survivors before could provide meaningful insights and extend the set of proposed design strategies.

\subsection{Conclusion}

The results of this study indicate that the developed digital method cards facilitated the design process of everyday objects that aim to initiate use of the affected arm and hand in stroke survivors. However, designers expressed problems 
in designing for others rather than for themselves, especially if the intended user experiences motor impairments. The use of videos showing motor impairments helped to develop an understanding of the user but can also impact significantly on the design process by increasing the expectation to develop an appropriate design concept. Further studies are needed to confirm the restraining effect of the designs and include recommended changes to increase the usability of the design method cards.

\subsection{Limitations}

None of the participants worked in the field of developing products for stroke survivors. Including experts in the field to test the digital design method cards could potentially offer further insights to increase the usability of the digital design method cards. A further limitation is that the design concepts were submitted in the form of sketches and the intended restraining effect was not physically tested or evaluated by health professionals or stroke survivors. It further needs to be considered that the chosen method of post-task interviews might have meant that some of the features and usability issues of the design method cards were not mentioned because participant might have forgotten to mention them during the interview. 


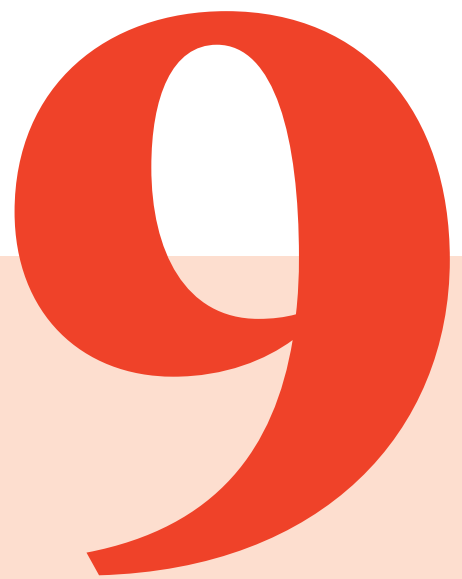

\section{Everyday Objects Used in Daily Tasks} Post Stroke 


\subsection{Introduction}

The focus of this study was to identify everyday objects that are used in daily activities that chronic stroke survivors like to take part in to address the research question:

1.2.1 Which everyday objects are used by stroke survivors during activities of daily living that they like to take part in, and therefore may assist in facilitating use of the affected arm?

The information was used to develop a new design prototype to initiate the use of the affected arm and hand. An online survey was distributed through online support groups to address the research question. Online support groups rather than direct observations were chosen because of the slow recruitment process required for direct observation studies. At this stage of the study, just one participant in New Zealand had come forward to take part in the study.

\subsection{Background}

The recovery process for stroke survivors is often not defined as the improvement of specific motor impairments, but as a way of getting back to their former selves, including being able to take part in activities that matter to them (Doolittle, 1992, p. 124; Eriksson et al., 2010, p. 836; Tyson \& Turner, 2000, pp. 386-387; Wallenbert \& Jonsson, 2005, pp. 222-223). Unfortunately, they often struggle to get back to their former lifestyle despite a good recovery and discharge into the community. Reasons for this are manifold. One explanation might be that a primary focus during the rehabilitation is often on mobility, independent selfcare, and performance of basic activities. This focus prepares the stroke survivor for discharge from the hospital rather than enabling them to return to their former life (Parker, Gladman, \& Drummond 1997, pp. 2-4; Tyson \& Turner, 2000, pp. 386-387). 
Findings highlight the fact that a focus on basic activities of daily living (BADL) in rehabilitation does not contribute to a quality of life (Samsa \& Matchar, 2004). Consequently, more complex activities of functioning that contribute to the subjective sense of wellbeing of an individual need to be taken into account during rehabilitation (Hartman-Maeir, Soroker, Ring, Avni, \& Katz, 2007, p. 564).

Stroke survivors often spend the majority of their day doing self-care and household activities, which are commonly affected by the stroke and take a longer time to complete (Wallenbert \& Jonsson, 2005, p. 222). It has been argued that stroke survivors should be trained to take part in BADLs as well as in instrumental activities of daily living (IADL), which are more complex tasks (Parker et al., 1997, p. 4) to increase their life satisfaction. A focus on activities that enable participation in personally relevant activities should therefore be considered in rehabilitation to decrease potential boredom, depression, and worsening of the condition (Mayo, Wood-Dauphinee, Côté, Durcan, \& Carlton, 2002, p. 1035). However, leisure and play activities that could contribute to quality of life (Wyller, Sveen, Sødring, Pettersen, \& Bautz-Holter, 1997, p. 143) in the form of satisfaction and happiness are often considered secondary goals in the rehabilitation process (Gillen, 2016, p. 298).

Rehabilitation aims to maximise participation in daily activities, but the clear majority of assessment scales used in the context of stroke still focus on body structure, function and activity (Salter, Foley, Jutai, \& Teasell, 2007, p. 341). A further limitation of current scales is that they are not routinely used in clinical practice: especially with stroke survivors who have reached an almost complete recovery or experienced mild symptoms of a stroke (Carod-Artal, GonzálezGutiérrez, Herrero, Horan, \& Seijas, 2002, p. 208). Despite it being named "mild stroke”, these stroke survivors can still experience significant deficits after discharge into the community (Wolf, Baum, \& Connor, 2009, pp. 3-4).

Assessment scales could be referred to as a source of inspiration in the design process. However, when an assessment scale is used, it is based on 
predefined lists of tasks, normally developed by health professionals. Scales have further been criticised for missing relevant daily tasks, for example the use of a phone (Carod-Artal et al., 2002, p. 215), being insensitive to measuring improvements in mild stroke and mild impairments (Carod-Artal et al., 2002, p. 213), and missing culture-specific activities for example visiting a temple as a social activity (Prakash, Ganesan, Vasanthan, \& Hariohm, 2017, pp. 203-205).

The perfect stroke scale does not exist (Quinn, Harrison, \& McArthur, 2013, p. 209), and scales that focus on participation in daily activities after a stroke are still scarce. There seems to be a lack of focus on more complex activities such as hobbies and IADL. Scales need to be validated more frequently to assess that the tasks that they contain represent relevant activities that take account of the cultural and social habits of the group under investigation.

Qualitative research in the context of stroke rehabilitation has been employed in the form of focus groups (Lynch et al., 2008, p. 518) and recording tasks in the rehabilitation setting (Logan, Gladman, Drummond, \& Radford, 2003, p. 251). The data have been used to determine and measure leisure activities, life satisfaction, and activity preference, but there has been no focus on the objects that play an important role in those tasks. Most studies seem to focus on identifying overarching themes such as changes of social relationships, coping strategies, physical functioning (Lynch et al., 2008, p. 519), and the perception of assistive devices (Pettersson et al., 2005, pp. 162-166).

An online survey with open-ended questions was developed to gain an understanding of objects that are commonly used in meaningful activities before and after stroke. Rather than using a predefined set of tasks in a way that assessment scales do, open-ended questions offer an opportunity to gather information about the broad spectrum of daily activities and objects that are important after a stroke. The online survey focused on ADL, IADL and hobbies before and after the stroke. The information has been used to define a list of objects and activities that might be useful when designing rehabilitation devices 


\subsection{Methods}

\subsubsection{Participants}

For this study, non-probability sampling in the form of convenience sampling was chosen, allowing potential participants to self-select into the sample. The results can consequently not be generalised to the entire population (Sue \& Ritter, 2012, p. 47). There are no formal restrictions involved in this form of sampling, and the survey is just posted on a website (Lewis-Beck, Bryman, \& Futing Liao, 2004; Sue and Ritter, 2012, pp. 44-45). I followed the rule of thumb stating that the sample size should be between 30 and 500 participants (Sue \& Ritter, 2012, p. 46). I contacted the following platforms and groups, see Table 9.

Data was collected from 1 February 2017 until 4 March 2017. One hundred and forty-two participants took part in the online questionnaire (male $n=66$, female $n=76$ ) with residency in Australia ( $n=6)$; Canada $(n=2)$; Chile $(n=1)$; Northern Ireland ( $n=1)$; the Netherlands $(n=1)$; New Zealand ( $n=6)$; Pakistan $(n=1)$; the United Kingdom ( $n=3)$; and the United States $(n=120)$. Their age ranged between 18 and 87 years, with an average age of 58 years. Most of the participants lived at home $(n=137)$; one lived in a skilled nursing facility $(n=1)$; one in an inpatient facility $(n=1)$ and three identified themselves under "other" $(n=3)$. Three participants had the stroke $0-3$ months ago $(n=3)$ ago, one $(n=1)$ had a stroke 6-9 months ago, six had it 9-12 months $(n=6)$ ago and the majority of participants $(n=132)$ more than 12 months.

One hundred and twenty-one participants ( $n=121)$ were right-handed and 21 were left-handed $(n=21)$. Forms of motor impairments were: an affected arm and leg on the left side $(n=50)$; an affected arm and leg on the right side $(n=38)$; just the right arm $(n=4)$; just the left arm $(n=6)$; and the lower limb on one side $(n=10)$. Further impairments were an affected right arm and left leg $(n=1)$; an affected left arm and right leg $(n=1)$; both arms and one leg affected $(n=2)$; both arms $(n=2)$; both arms and both legs $(n=3)$; and no effect on the upper limb was reported by $(n=25)$ participants. 
Table 9: Online support groups.

\begin{tabular}{|c|c|c|c|c|}
\hline Domain & Country & Contacted & $\begin{array}{l}\text { Posted } \\
\text { on the } \\
\text { website }\end{array}$ & $\begin{array}{l}\text { Included } \\
\text { into the } \\
\text { newsletter }\end{array}$ \\
\hline http://www.stroke.org.nz & $\begin{array}{l}\text { New } \\
\text { Zealand }\end{array}$ & $\mathrm{x}$ & & \\
\hline http://www.healthfulchat.org & Scotland & $\mathrm{x}$ & & \\
\hline $\begin{array}{l}\text { https://www.chss.org.uk/stroke-informa- } \\
\text { tion-and-support }\end{array}$ & Scotland & $\mathrm{x}$ & & \\
\hline http://brainfoundation.org.au/disorders/stroke & Australia & $\mathrm{x}$ & & \\
\hline https://nichs.org.uk & $\begin{array}{l}\text { Northern } \\
\text { Ireland }\end{array}$ & $\mathrm{x}$ & & \\
\hline http://www.heart.org & $\begin{array}{l}\text { United } \\
\text { States of } \\
\text { America }\end{array}$ & $\mathrm{x}$ & & \\
\hline http://www.stroke.org & $\begin{array}{l}\text { United } \\
\text { States of } \\
\text { America }\end{array}$ & $\mathrm{x}$ & & \\
\hline https://www.stroke.org.uk & $\begin{array}{l}\text { United } \\
\text { Kingdom }\end{array}$ & & $\mathrm{x}$ & \\
\hline http://www.strokenetwork.net & $\begin{array}{l}\text { United } \\
\text { States of } \\
\text { America }\end{array}$ & $\mathrm{x}$ & & $x$ \\
\hline https://enableme.org.au & Australia & $\mathrm{x}$ & $\mathrm{x}$ & \\
\hline http://www.stroke-rehab.com & $\begin{array}{l}\text { United } \\
\text { States of } \\
\text { America }\end{array}$ & $\mathrm{x}$ & & $\mathrm{x}$ \\
\hline $\begin{array}{l}\text { Stroke Foundation of New Zealand, Central } \\
\text { Region }\end{array}$ & $\begin{array}{l}\text { New } \\
\text { Zealand }\end{array}$ & $\mathrm{x}$ & $\mathrm{x}$ & \\
\hline
\end{tabular}

Most participants ( $n=129)$ had received some form of rehabilitation after the stroke while some $(n=13)$ reported having received no form of therapy. The most common forms of therapy were a combination of occupational and physiotherapy ( $n=29)$; occupational therapy with physiotherapy and speech therapy $(n=22)$; physiotherapy alone ( $n=14)$; occupational therapy $(n=6)$; and speech therapy $(n=6)$. Thirty-nine participants $(n=39)$ reported having received rehabilitation but did not specify its form. 


\subsubsection{Online survey}

Online surveys are a form of data collection that allows access to populations that might be otherwise difficult or impossible to reach, experience stigmatisation, or have specific attitudes and interests that are politically controversial (Wright, 2006). Surveys can be sent via email or smartphone, or be posted on a website (Sue \& Ritter, 2012, pp. 16-21). There are some risks associated with this form of data collection such as sampling issues, coverage, the lack of generalisable results, and nonresponse bias, that need to be taken into consideration once the results are evaluated (Lavrakas, 2008; Sue \& Ritter, 2012, pp. 19-20; Wright, 2006)

The online survey in this study contained 19 questions and started with demographic questions followed by questions concerning the kind and impact of the stroke. A second part that included open-ended questions about IADLs and ADLs before and after the stroke, which kind of activities participants would like to take part in again after the stroke, and objects that play an important role in those activities, see Appendix A. A third part asked about hobbies before and after the stroke. The survey included details about the study and contact information. Participants were able to leave their contact information to receive a summary of the study results. The online survey was set up using the www.qualtrics.com online platform. I sought permission from the website administrators to invite participants to take part in the study.

\subsubsection{Analysis}

Results of the survey were clustered using thematic analysis in a deductive form (Braun \& Clarke, 2006, p. 83) according to the themes of the ICF model. The ICF model outlines tasks that are part of the categories of:

- Learning and applying knowledge

- General tasks and demands

- Communication

- Mobility 
- Self-care

- Domestic life

- Interpersonal interactions and relations

- Major life areas and community

- Social and civic life

ADL and hobbies were clustered according to these themes and subthemes outlined as part of the ICF, see Table 10.

Table 10: Coding example.

Communication

d350 Conversation

D360 Using
telecommunication devices

Checking emails

Having a conversation

Talking

Texting a relative

Using social media for contact

Using a computer

Using a tablet

Using the internet

Using a laptop

Using a phone

I chose to distinguish between a general job and a specific job because some participants explicitly referred to their jobs, for example referring to being a doctor or lawyer and particular objects that are used as part of their profession. Other participants only mentioned that they would like to be able to work again. Jobs were clustered under the category d 845 "Acquiring, keeping, and terminating a job", even though it would fit into the category d850 "Remunerative employment". A precise definition outlining the difference between the two categories is missing in the ICF. 
The named tasks are presented in the form of descriptive statistics. Graphics are used as part of descriptive statistics as complementary presentations and for ease of understanding the data (Salkind, 2010, p. 3). Clustered bars present the main categories, while pie graphs are used to present subcategories and compare the changes in activities before and after the stroke. Participants could name more than one task of a particular activity. Due to overlapping results in the ADL section and hobby section the results will be presented separately.

\subsection{Results}

\subsubsection{Survey results}

The following section is divided into the results pertaining to ADL and hobbies. Some of the results and activities overlap because participants named hobbies as part of their daily routine and ADL, for example baking.

The tasks were clustered according to the main categories of the ICF. In the following section, the main categories will be referred to as well as specific tasks that were frequently mentioned.

\subsubsection{ADL}

Participants named 409 tasks that they liked to do before the stroke and 259 that they would like to after the stroke; see Figure 75. People named certain tasks more often to be important after the stroke. Those tasks were situated in the categories of communication and learning and applying knowledge. The categories of community, social and civic life, major life areas and mobility, see Figure 75, contained tasks that were less often named to be important after the stroke. It needs to be considered that the total number of named tasks decreased from 409 before the stroke to 245 designated tasks after the stroke. This might indicate an overall decline of activity.

The three most frequently named categories that participants indicated they would like to take part in again after the stroke were sports, walking and driving, see Figure 76. 


\title{
Categories of activities of daily living before and after the stroke
}
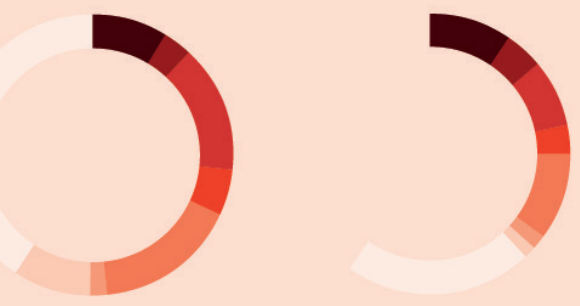

\begin{abstract}
Activities
I used to do

before the

stroke

409 tasks

named
\end{abstract}

Activities

Ilike to

carry out

after the

stroke

245 tasks

named

\section{Activities I}

would like to

do after the

stroke but I

don't

259 tasks

named

d1 Learning and applying

knowledge

- d6 Domestic life

- d3 Communication

- d7 Interpersonal Interactions and relationships

- d4 Mobility

d8 Major life areas

- d5 Self-care d9 Community, social and civic life

Figure 75:

ADL before and after

the stroke based on the

categories of the ICF.

\section{Categories of tasks that participants}

would like to take part in again after the stroke.

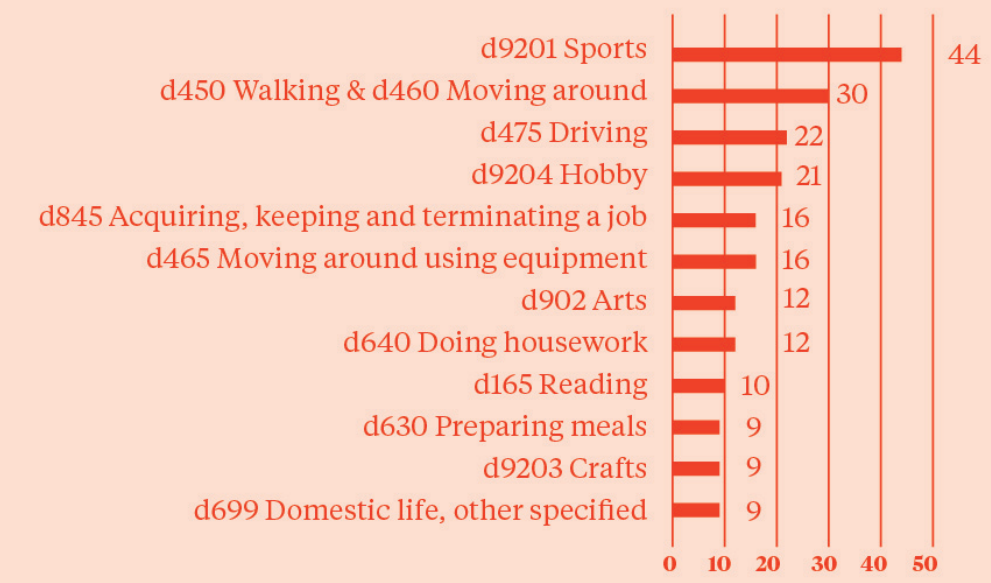

\section{4}

Figure 76:

Categories of tasks that

participants would like

to take part in again

after the stroke. 


\section{Tasks that participants would like to take part in again after the stroke}

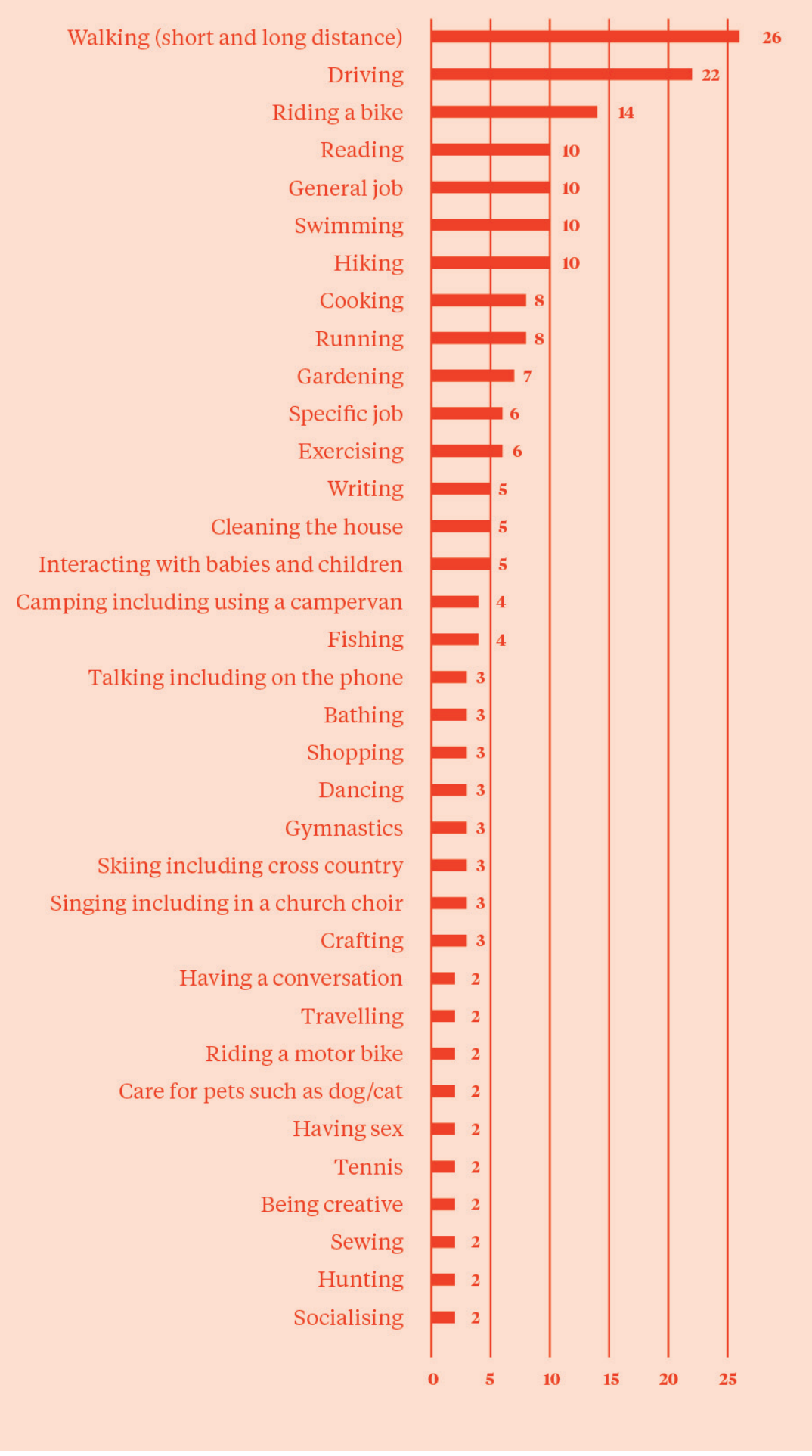

Figure 77:

Specific tasks that

participants would like

to take part in again

after the stroke. 


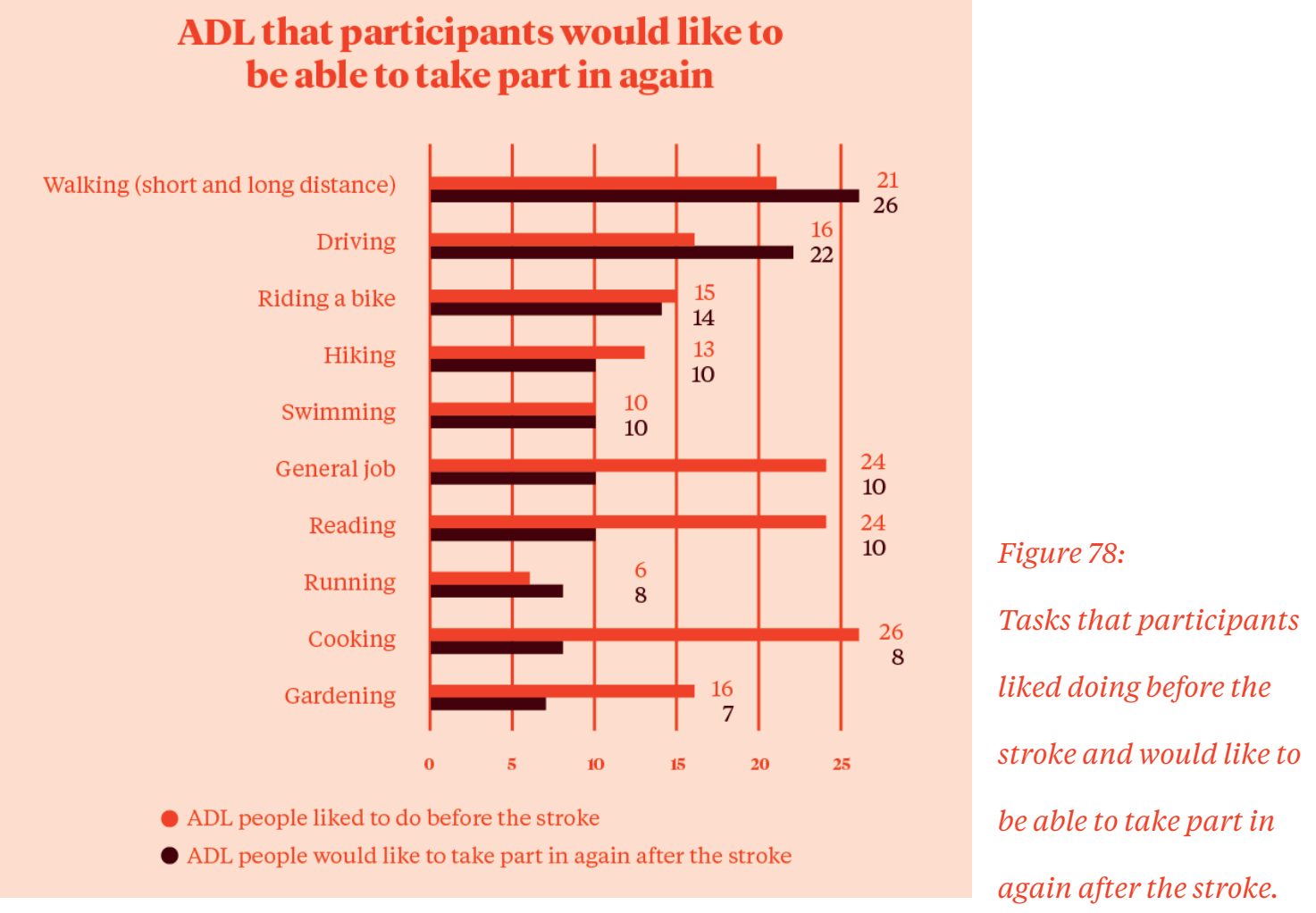

The three most commonly named tasks that people indicated they would like to take part in again were walking, driving and riding a bike, see Figure 77. Most tasks that were important to participants after the stroke seemed to have been more important before the stroke, see Figure 78. However, walking and driving, which were among the three most frequently named tasks after the stroke, seemed to have become more important, see Figure 78.

\subsubsection{ADL that have become difficult}

There were 68 ADL identified as being difficult after the stroke; see Figure 79. Walking and driving have been named as being difficult tasks after the stroke, but they are activities that participants named as wanting to be able to perform again. 


\section{Categories of ADL that are difficult to perform after the stroke}

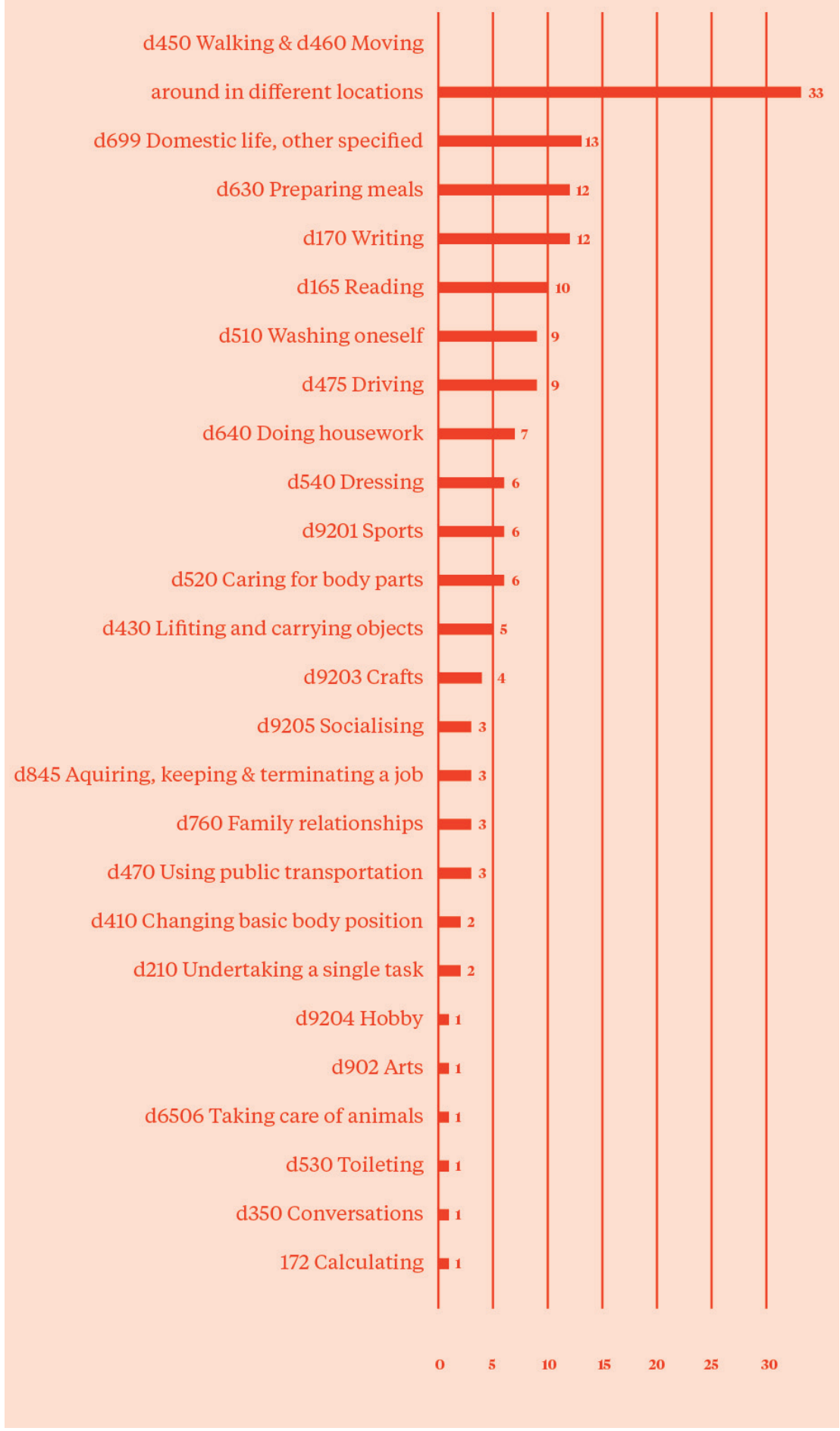

Figure 79:

Tasks that have become more difficult after the stroke. 


\subsubsection{Hobbies}

Participants reported on hobbies in the first part of the online survey as well as in the second part that specifically asked for hobbies. In the ADL section of the survey, 166 tasks were named that participants liked to do before the stroke, which align with the ICF category of community, social and civic life tasks. After the stroke they named just 89 tasks.

In the hobby section of the online survey, participants reported on 186 tasks that they liked to take part in before the stroke and 96 tasks that they enjoyed after the stroke that align with the ICF category of community, social and civic life.

Participants mentioned 298 tasks in total in the section of hobbies that they liked to take part in before the stroke, and 200 tasks that they liked to do after the stroke. The tasks were part of the categories of crafts, sports, arts, play, socialising, religion and spirituality. These are all part of the main category of community, social and civic life. However, a number of named tasks were based in categories of ADL rather than hobbies, for example reading; see Figure 80. 


\section{Categories of hobbies that participants liked to take part in before and after the stroke}

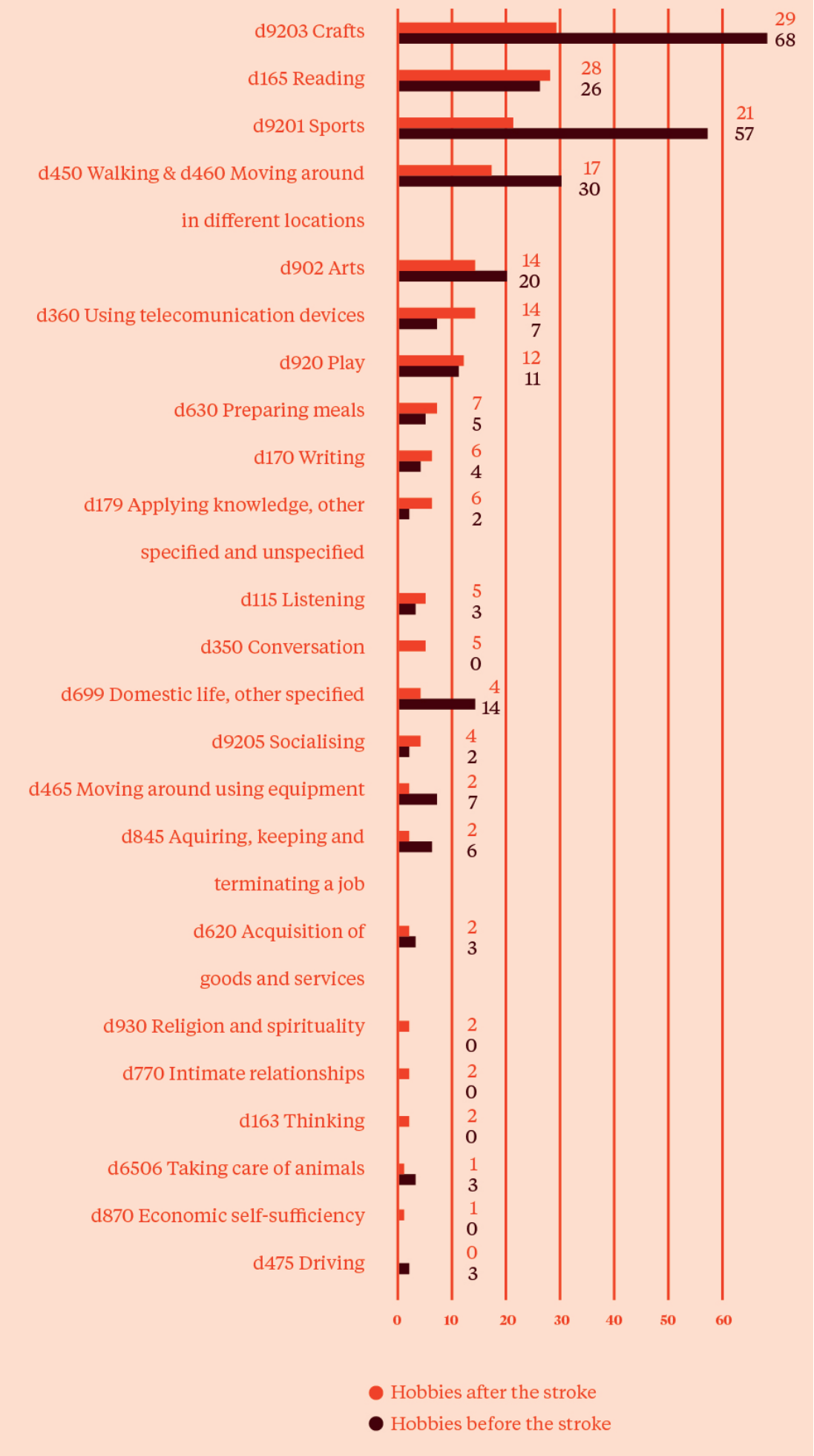

Figure 80:

Categories of hobbies

that participants liked

to take part in before

and after the stroke. 


\section{Hobbies that participants liked to take part in after the stroke}

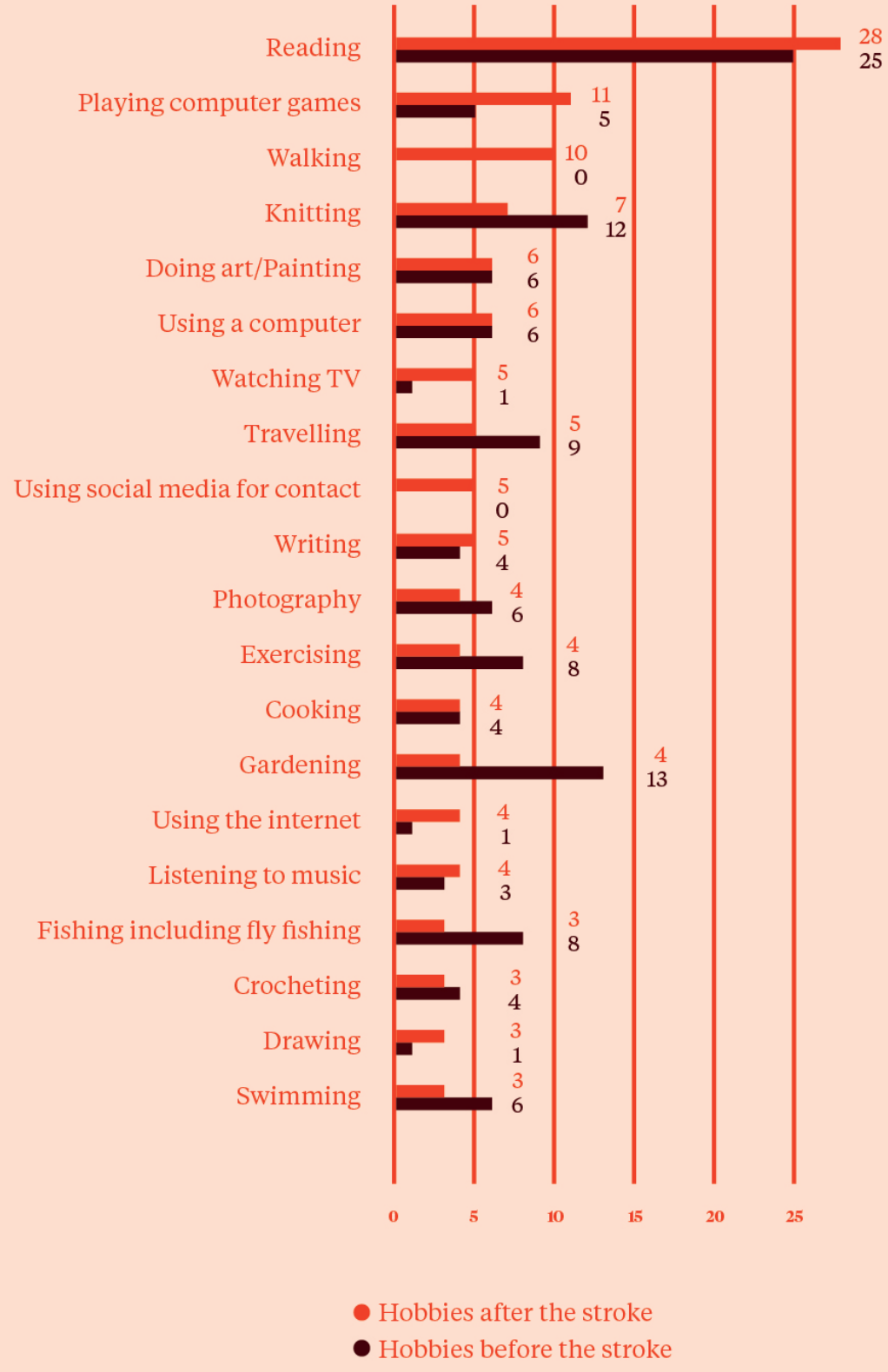

Figure 81:

Hobbies that

participants liked to take part in after the stroke. 
The activity of walking and using social media for contact were the only two hobbies named that people enjoyed after the stroke but not before the stroke, see Figure 81 .

\subsubsection{Objects that play an essential role in ADL and hobbies}

One hundred and twelve participants responded to the question about which kind of objects play an essential role in the named activities. Most objects that were named belonged to the category $\mathrm{d} 3600$ "Using telecommunication devices”, see Figure 82. Objects that were mentioned frequently $(n>3)$ were digital devices and mainly used for telecommunication purposes, for example the computer, the cell phone and the smartphone, see Figure 83. 


\section{Categories of objects that play a significant role in daily activities}

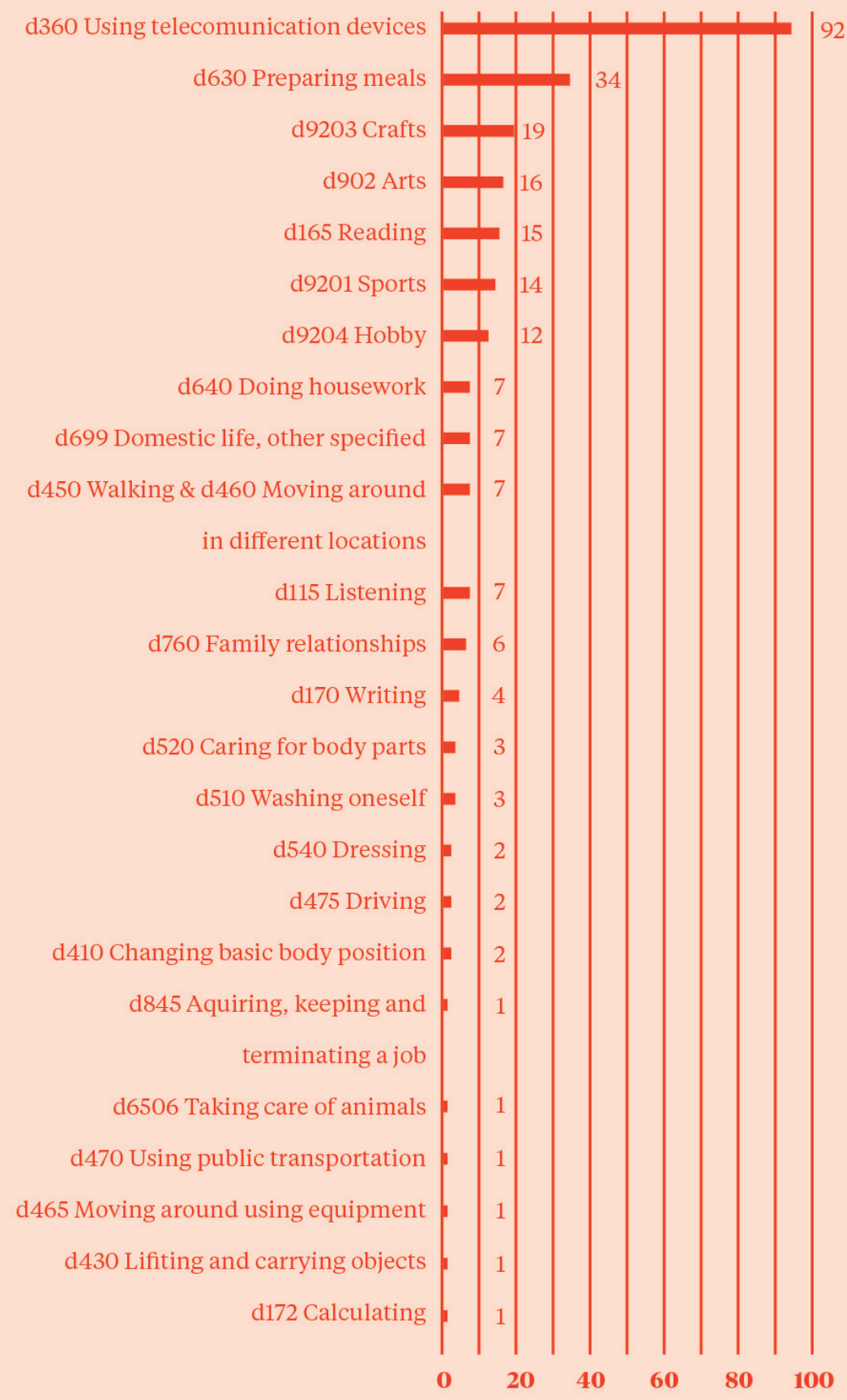

Figure 82:

Categories of tasks in which objects play an important role. 


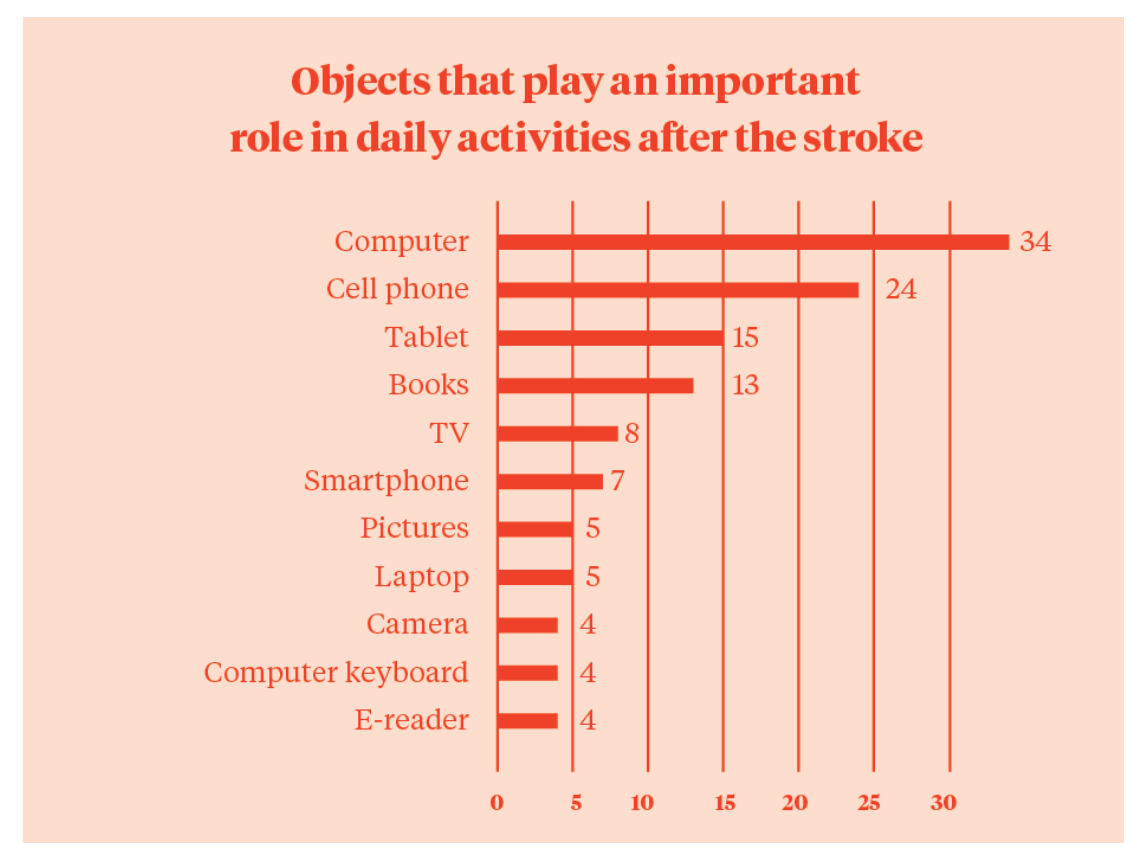

Figure 83:

Specific objects that were named as playing an important task in daily activities after the stroke.

\subsection{Discussion}

The results of this online survey are presented in line with categories of the ICF and indicate an overall decline of activities. While there were 409 tasks named as being relevant to the participants before the stroke, there were just 245 activities that they liked to do after the stroke.

Even though the ICF model is widely used, it has a significant lack of details outlining specific hobbies and leisure activities, which potentially impacted on the analysis in the present study. Nevertheless, the results indicate that leisure activities are an important factor in people's lives before and after stroke.

The survey was divided into two main areas of data collection focusing on ADL and leisure activities in the form of hobbies. Responses indicated those two areas might partially overlap. Multiple leisure activities were mentioned in the ADL section of the survey and vice versa. For example, the task of walking was named as an ADL as well as a hobby after the stroke. The ICF model needs to develop further details to take account of the motivation to participate in daily activities, for example, the task of eating. This task can be a necessity that needs to be performed, or it can be an enjoyable task in the company of friends. Further 
details are required in the ICF to mirror the nature of the tasks and environmental context.

For frequently named activities, it seems that most of the tasks focused on the lower limb or required a high level of function of the lower limb. Among the top 10 tasks that participants would have liked to be able to take part in again are seven tasks that involve the upper limb. To what extent remains up for speculation.

\subsubsection{ADL}

Participants named 245 ADL that they liked to take part in after the stroke and 259 that they would like to be able to do but currently could not. Involvement in ADL that is important and meaningful to the stroke survivor in the rehabilitation process has the potential to lead to greater gains compared to conventional rehabilitation methods (Narayan Arya et al., 2012, pp. 205-206).

\subsubsection{Most important tasks}

Two tasks were frequently named in the ADL section of the survey: Walking and driving. Among the 10 activities after the stroke that participants would have liked to be able to take part in again are five mobility tasks, with walking and moving around being number one, which confirms a review of qualitative studies on survivors' experience after stroke (Luker, Lynch, Bernhardsson, Bennett, \& Bernhardt, 2015, p. 1700). However, this activity has also been named as the most difficult. This correlates with results of previous studies stating that mobility is the task that most stroke survivors tend to focus on after the stroke (Logan et al., 2003, p. 252). This strong focus on a lower limb activity might be because in the study sample 50 participants reported both limbs on one side affected and a further 25 reported having no upper limb impairments at all.

The second ADL that was frequently named as a task that participants would like to be able to do again is driving. Driving was also the only task that participants did not like to undertake after the stroke. This might be because driving can be a necessity, so the ability to drive might not be seen as pleasurable 
per se, but can be seen as a key aspect of daily mobility: getting to work, shopping, and for access to leisure and social activities (Mauss \& Robinson, 2009, p. 1559).

The task of walking is focused mainly on the lower limbs, while driving is an extremely complex behaviour that needs to take account of a changing environment. To address such a task is outside the scope of this study.

\subsubsection{Hobbies}

Results of the current study show that leisure activities such as hobbies or sports are essential to chronic stroke survivors. They made up a significant proportion of tasks that participants mentioned in the survey. Leisure activities related to sport, hobbies, or social activities can be long-standing interests. Hobbies and social activities that contribute a sense of meaning to the self are vital aspects of a person's life and identity (Mauss \& Robinson, 2009, p. 1559). Participation in leisure activities can be an important way of coping with the stroke and can contribute to relaxation (Carlsson et al., 2009, p. 780). This might explain why the category related to hobbies and sports was the most frequently named category among ADL, with 195 named hobbies that participants would have liked to be able to take part in again after the stroke.

\subsubsection{Low demand tasks}

The hobbies that people liked to do after their stroke seemed to increase in terms of low demand activities. Hobbies that were mentioned more often after the stroke than before the stroke were watching TV, increased by $n=11$; followed by reading, increased by $n=7$; and playing computer games, increased by $n=5$. These results correspond with previous findings (Atler, 2016, p. 784), which indicated that stroke survivors mostly take part in low demand leisure activities and spend up to two hours per day watching TV, having the TV on while reading or using the computer; using the computer; or just reading. These low-level activities, rest, and social activities were perceived as being the most pleasurable. Work and highlevel activities were stated to be the least pleasurable. The feeling of pleasure was associated with being able to take part in activities again and avoiding tiring and 
confronting tasks (Atler, 2016, p. 784).

A focus on low demand activities that contribute to a feeling of pleasure might help facilitate and initiate use of the affected limb and participation in daily activities.

\subsubsection{Terminated tasks}

The decrease in tasks named by participants that they liked to do after the stroke compared to the number of activities named as liking to do before the stroke indicates that some activities are not pursued after brain injury. Robison et al. (2009, p. 1564) point out that not resuming activities after the stroke might be for multiple reasons, not just the physical impairments. Reasons can be social or psychological, for example, feeling uncomfortable in a public environment. Survivors might prefer to avoid an activity rather than taking part in it in a restricted way. Factors influencing leisure performance depend on the type of leisure task, the stage in the life cycle that the person is in, social and support systems, attitude to leisure, use of time, and barriers to leisure participation such as decreased skills, environmental and economic barriers, or transportation (Gillen, 2016, p. 301).

Mauss and Parker (1997, pp. 2-4) argue in their literature review that participating in leisure activities should be a goal in the rehabilitation process. Research in this area could take inspiration from other fields such as studies focusing on the elderly. Elderly people show a decline in participation in leisure activities that has been well studied and could provide meaningful insights into developing models for the more rapid decline seen in stroke survivors. Inspiration from able bodied participants might offer insights into activities that can contribute to initiation of use of the affected arm and hand.

\subsubsection{Essential objects}

Tasks that were frequently named as important after the stroke did not consequently correlate with the named objects that play an essential role in daily activities. The categories of mobility and community, social and civic life made 
up the majority of named tasks but did not contain the most frequently named objects, which were the computer, cell phone and tablet.

It needs to be considered that these objects are often used within a specific activity rather than in an isolated way. The use of telecommunication devices such as the cell phone could, therefore, be part of other activities such as reading or writing, or as part of a job activity. Further research is needed to identify tasks where telecommunication devices are essential to understand the motivation for using the devices.

This study indicates that telecommunication devices are frequently used in daily activities and could potentially contribute to an initiation of use. However, stroke-specific limitations can have an impact on the use of current devices. Therefore, the design should take account of stroke-specific impairments to evoke an initiation of use of the affected arm.

\subsection{Conclusion}

An online survey was distributed through online support groups to gain insights into ADL and hobbies that chronic stroke survivors enjoyed before the stroke and would like to take part in again after the traumatic event. The survey used open and closed questions, which provided an extensive list of daily tasks that can be used as a basis for designing everyday objects that contribute to an initiation of use. Results indicate that the number of ADL and leisure tasks decrease after the stroke, while low-level leisure activities seem to increase. The most frequently named objects that play an essential role in $\mathrm{ADL}$ and hobbies are digital devices used for telecommunication purposes. It is unclear in which context and for what purpose the objects are used. Further research is needed to determine ADL tasks and hobbies that those devices play a role in, and which barriers impact on their use. To secure the clinical value of the design prototypes that facilitate the initiation of use post-stroke, the clinical perspective of meaningful daily activities needs to be accounted for. 


\subsection{Limitations}

The results of this study do not claim to be representative of the situation of all stroke survivors. They are, however, demonstrative of the complexity of ADL and leisure activities that people do and would like to take part in after a traumatic brain injury.

The survey was distributed online and there is a potential for bias in the results. Furthermore, the named telecommunication devices are often used within a certain activity rather than in an isolated form. Tasks that involve telecommunication devices, for example using a cell phone, could therefore be considered part of an activity such as staying in touch with family members or as part of a job.

The results of this study are based on the ICF model that offers a comprehensive list of tasks and activities. Some tasks of the model can fulfil a number of different categories depending on the context of use, such as travelling, which is categorised under walking but could also be a hobby. The ICF also lacks details that would allow classification of activities according to their intensity.

A further limitation of this study is that the study sample reported high levels of upper and lower limb impairments. This might have influenced the reported activities and hobbies. The results of the present study were not weighted according to demographics because of the sampling strategy. It has been pointed out that there might be a discrepancy between genders (Drummond, Parker, Gladman, \& Logan, 2001, p. 653; Månsson, Fredriksson, \& Bränholm 1995, pp. 5253), and living situation (Mauss \& Robinson, 2009, p. 1557) as to what is considered a meaningful task. The study by Mauss \& Robinson (2009, p. 1557) further indicated that domestic activities are preferred by people living alone. This aspect has not been considered in the current study but could potentially influence the choice of ADL. There may also be a cultural component in regard to leisure activities. Prakash et al. (2017, pp. 203-204) found that recreational activities such as gardening or reading books were not important to their Indian participants, 
whereas taking part in social activities such as attending the temple or weddings were common social activities (Prakash et al., 2017, pp. 203-204).

The survey in the current study did not focus on the context in which the daily tasks and leisure activities are performed. Further studies are needed to determine the complex relationship between personal and environmental factors in developing an intervention for stroke survivors in the community that meets those needs and maximise participation outcomes (Hoyle, Gustafsson, Meredith, \& Ownsworth, 2012, p. 12). 


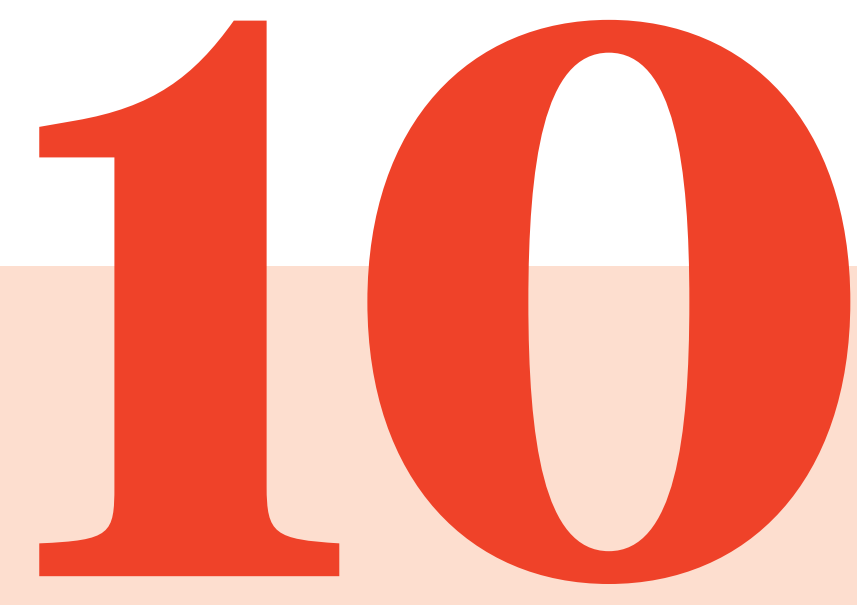

\section{Everyday Objects}

\section{Used in Daily}

\section{Tasks Post Stroke}

II 


\subsection{Introduction}

This study addresses the research question:

1.2.1 Which everyday objects are used by stroke survivors during activities of daily living that they like to take part in, and therefore may assist in facilitating use of the affected arm?

The previous chapter provided a number of daily activities and objects involved in them that are meaningful to stroke survivors. This chapter has the same focus but involves a therapeutic perspective. The interviews that were conducted with health professionals contained questions concerning ADL that are frequently focused on in the rehabilitation context. The results of this study present several tasks and associated objects that therapists mentioned during the interviews. Triangulation has been used to compare the results of the different forms of data collection to develop a comprehensive understanding of ADL and objects that can be used to facilitate an initiation of use. The results influenced the development of a new design prototype.

\subsection{Methods}

\subsubsection{Participants}

This study used non-probability sampling in the form of snowball sampling (Atkinson \& Flint, 2011). Participants had varying backgrounds and experience in stroke rehabilitation and worked with stroke patients at various stages of their recovery. Each participant provided written informed consent before taking part in the interviews. The interviews, which took 60 minutes on average, were audio recorded, transcribed in full, and then analysed.

Thirteen interviews were conducted with stroke therapists and researchers. Clinical and research experience varied from 2 months to 10 years. Nine interview partners were female $(n=9)$, and four were male $(n=4)$. Three were occupational 
therapists $(n=3)$; two worked as physiotherapists $(n=2)$; one worked as a neuro physiotherapist $(\mathrm{n}=1)$; one worked as a professional therapist focusing on assessment post-stroke $(n=1)$; one was a physiotherapist student $(n=1)$; one worked in the tertiary sector and had a background in musculoskeletal therapy $(n=1)$; two worked as researchers focusing on stroke therapy $(n=2)$; one worked as a scientist focusing on the recruitment of acute stroke patients for studies $(\mathrm{n}=1)$; and one was a supervisor of a clinic $(n=1)$. Two therapists $(n=2)$ worked in Australia, and 11 $(n=11)$ worked in New Zealand.

\subsubsection{Semi-structured interview content}

This study focused on ADL and associated objects that are used in rehabilitation. The findings are based on the responses to the interview questions 12 and 13 that were used as part of the interview guide; see chapter 3 for the results of the full interview guide.

12. What kind of activities of daily living are perceived as being important to a stroke survivor?

13. What kind of objects play an important role in those activities?

Follow up questions were asked depending on the field of expertise of the interview partner, for example physiotherapy or occupational therapy, to identify objects that are used with the aim of rehabilitating or compensating the affected side of the body.

\subsubsection{Analysis}

The transcripts were analysed using thematic analysis in a deductive form (Braun \& Clarke, 2006, p. 83) according to the themes of motivation to pursue ADL and use of everyday objects in the context of rehabilitation. Activities and objects named were categorised according to the ICF model (World Health Organization, 2017). Graphics in the form of clustered bar graphs and pie graphs present the results for ease of understanding the data (Salkind, 2010, p. 3). 


\subsubsection{Triangulation}

Triangulation is a methodological approach that contributes to the validity of the results by employing multiple methods, data sources, investigators, or theories to develop a comprehensive understanding of a specific phenomenon (Farmer, Robinson, Elliott, \& Eyles, 2006, p. 377; Patton, 1999, pp. 1192-1193). Triangulation is used to confirm or disconfirm previous research results (Erzberger \& Prein, 1997, pp. 143-144). It is a common misconception that triangulation is used purely to validate data. Triangulation can also be an opportunity to find different meaning within the data (Patton, 1999, p. 1193). It is a complementary approach for completeness purposes in the context of less explored or unexplored research areas (Farmer et al., 2006, p. 378; Erzberger \& Prein, 1997, pp. 1-4; Hussein 2009, p. 8).

Triangulation was used as part of this study to establish a comprehensive picture of essential daily activities and associated objects post-stroke. I chose a multiple triangulation (Kimchi, Polivka, \& Stevenson, 1991, p. 365) in the form of data triangulation (Carter, Bryant-Lukosius, DiCenso, Blythe, \& Neville, 2014, p. 545) for this study. Data triangulation involves collecting data from from a heterogeneous sample (Carter et al., 2014, p. 545) using a within-method triangulation (Kimchi, Polivka, \& Stevenson, 1991, p. 365) by employing two different forms of qualitative data collection. The use of various qualitative methods allows different perspectives that might otherwise be overlooked (Morse, 2009, p. 1524).

Limited literature is available describing the procedural steps that need to be undertaken to perform triangulation (Casey \& Murphy, 2009, p. 43; Farmer et al.2006, p. 379; Regmi, 2014, p. 5). It has been argued that the results of the different methods need to be analysed separately and then synthesized to identify similarities and differences. It furthermore needs to be taken account that using different methods might affect study results (Carter et al., 2014, p. 546; Morse, 2009, p. 1523). I have taken this into consideration and presented the results of the 
different methods in separate sections.

Farmer et al. 2006 (pp. 382-389) provide a description of the triangulation process that I will refer to in outlining the process of triangulation and presenting the results. Six steps need to be followed in the process:

1. Sorting the data;

2. Developing convergence coding;

3. Assessing convergence;

4. Comparing completeness;

5. Comparing assessment between researchers and as a final step;

6. Feedback of triangulation results.

Due to the nature of this study the research comparison between researchers, step 5, and feedback within the research team, step 6, will not be performed.

\subsection{Results}

The results of this study are divided into three different sections. The first section presents the activities and objects the therapists named. The second section displays the results of the thematic analysis that describes the meaning of objects and activities. The third section outlines the themes that were developed for the triangulation and the codes for the convergence assessment. These codes focus on ADL and objects that involve the upper limb.

\subsubsection{Interview results}

10.3.1.1 $A D L$

Most tasks that were mentioned during the interviews belonged to the category of self-care; for example, being able to wash oneself - see Figure 84.

While most of the tasks $(n>5)$ were part of the category of self-care, it seems the ability to drive and returning to work were crucial motivational drivers in the rehabilitation process, see Figure 85. 


\section{ADL that are often important} to stroke survivors

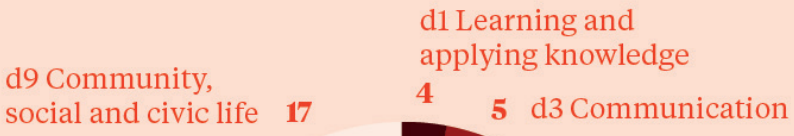

social and civic life 17

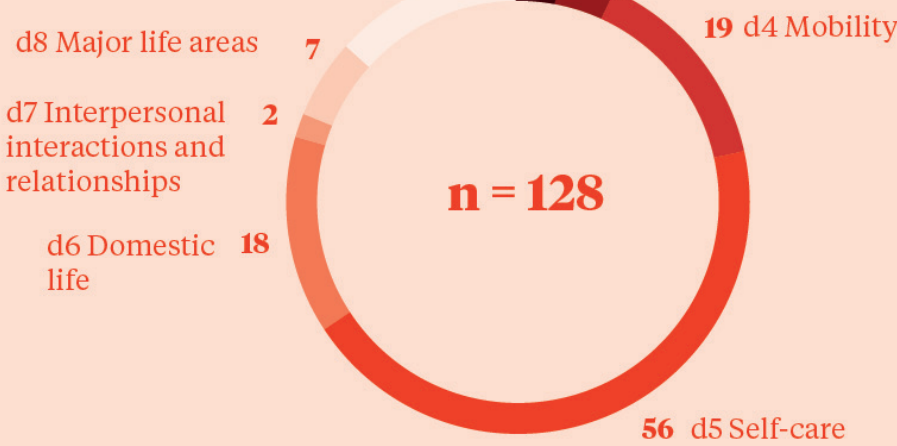

Figure 84:

Categories of tasks

that were named by

therapists as being often

important to stroke

survivors.

\section{Common tasks that participants would like to take part in again after the stroke}

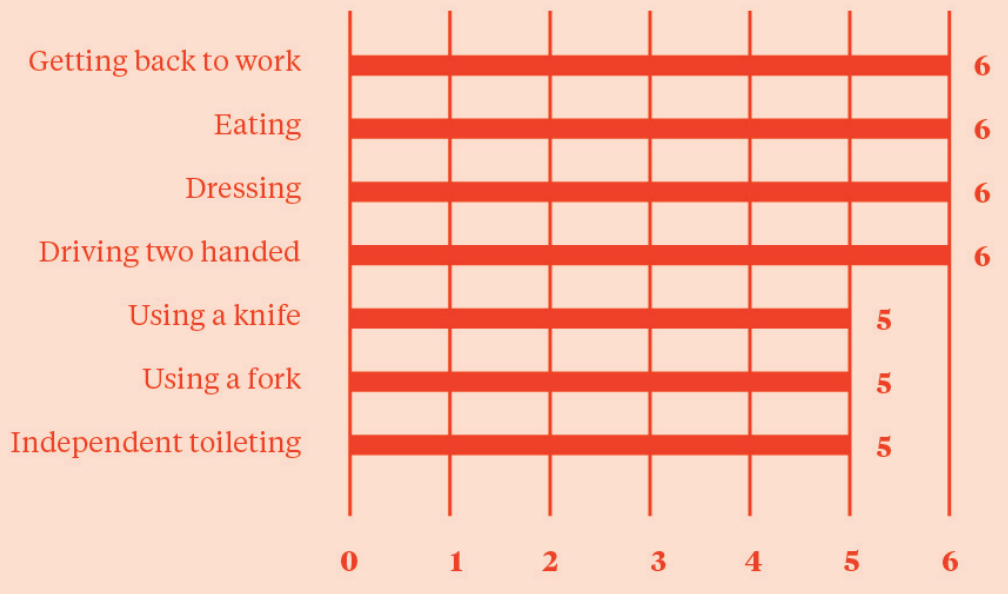

Figure 85:

Tasks that are often

important to stroke

survivors. 


\subsubsection{Objects used in the context of daily activities and} rehabilitation

The therapists emphasised that it is essential to examine the activity that a stroke patient wants to focus on in rehabilitation to determine the objects that play an important role in the activity. Objects that were named during the interviews were assistive devices, rehabilitation devices, and everyday objects; see Figures 86 to 88 .

No single object was pointed out as being essential in evoking an initiation of use of the affected arm and hand. It was emphasised, however, that objects are used in the rehabilitation process to train specific tasks for example using cutlery to train an eating task. 


\section{Everyday objects mentioned by therapists}
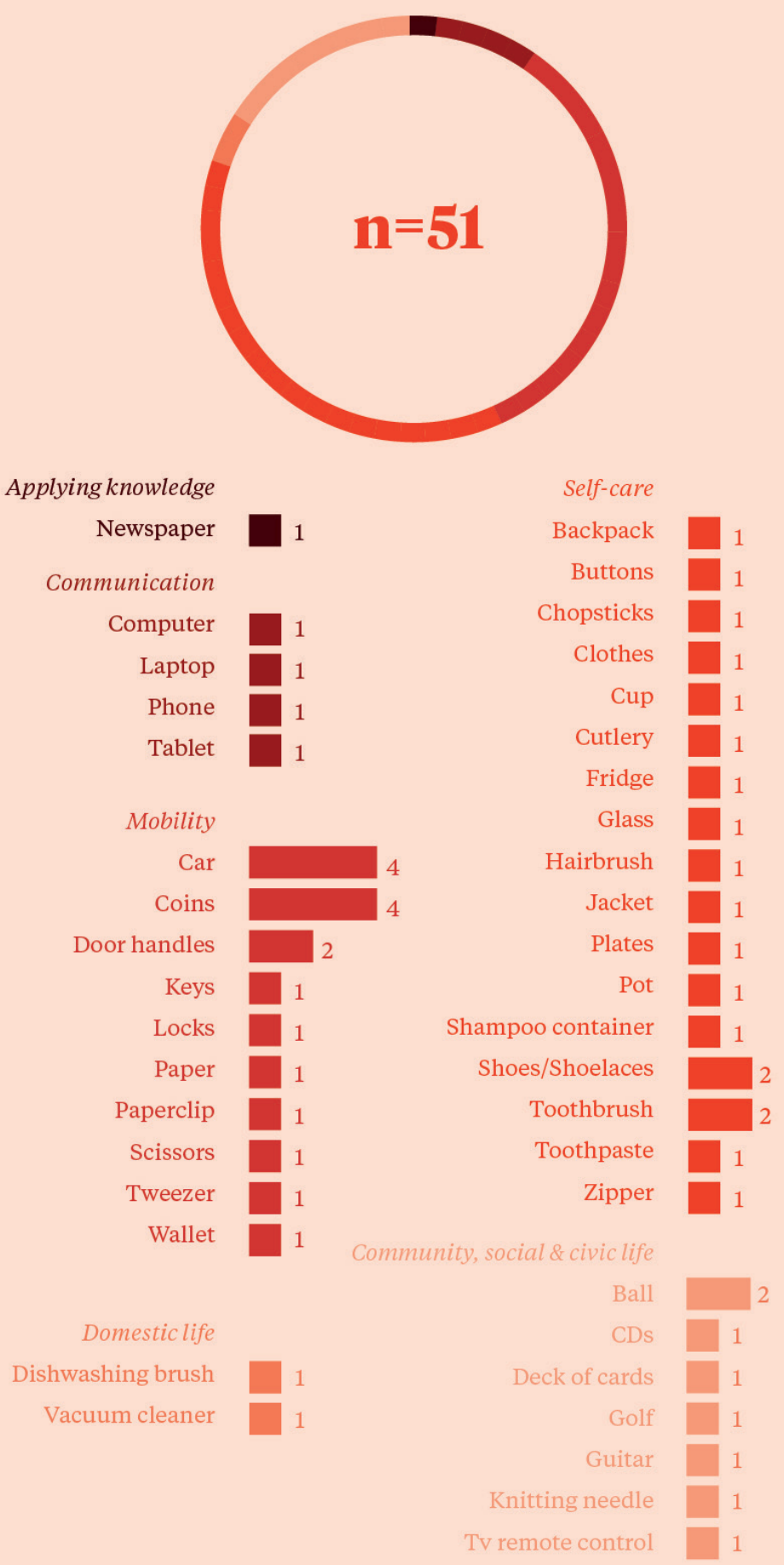

Figure 86:

Everyday objects named by therapists. 


\section{Assitive objects mentioned by therapists}

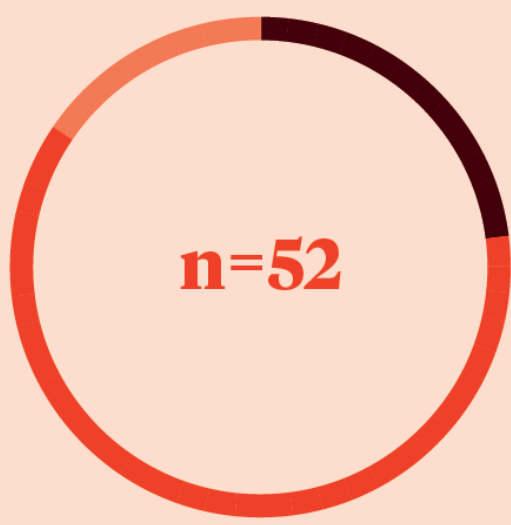

Lower limb

Specific ADLs

Ankle foot orthosis

1 Aid to help turning on the taps

Brace

Bathroom aids

Hoist

Bed aids

Four pronged stick

Standing frames

Splint for the foot

Walking aid

1

Bigger door knobs

Cup holder

Cutlery

Dressing aids

Grip hands

Walking frame

Walking stick

Wheelchair

12

Grip with a strap

Hairbrush

Hairdryer

Upper limb

Kettle tipper

Wrist splint

Brace

Sling

Velcro tape

Thera paddy

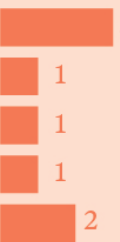

Lifts for chairs

Lifts to get out of bed

Modified tooth brush

Nonslip mats

One-handed chopping board

Pick up sticks

Pullies to get out of bed

Raised toilet seats

Shower chair

Spike board

Stair chair lift

System to get in and out of the shower

Trolleys to move things around
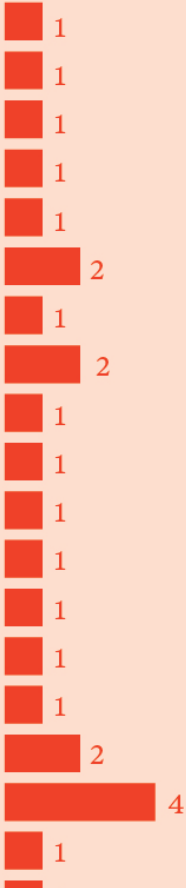

1

1

2

1

1

Figure 87:

Assistive devices named

by therapists. 


\section{Rehabilitation objects mentioned by therapists}

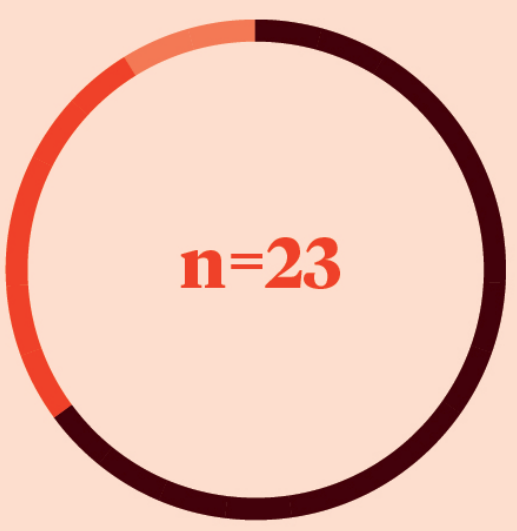

Devices

Biofeedback device BNS page 200 (splint + FES) Functional electrical stimulation (FES)

Kinect + Rehab software

Saebo Mas

Saebo splints

Smart Arm

'Stretchy' hand and finger trainer

Treadmill

Weights

$D I Y$

Self-made version of the smart arm Self-made sensory discrimination kit

Use cans as weights

Milk bottles with water or sand as weights

Rubber bands to train resistance

Robotics

1

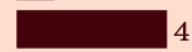

1

1

1

Figure 88:

Rehabilitation devices

named by therapists. 


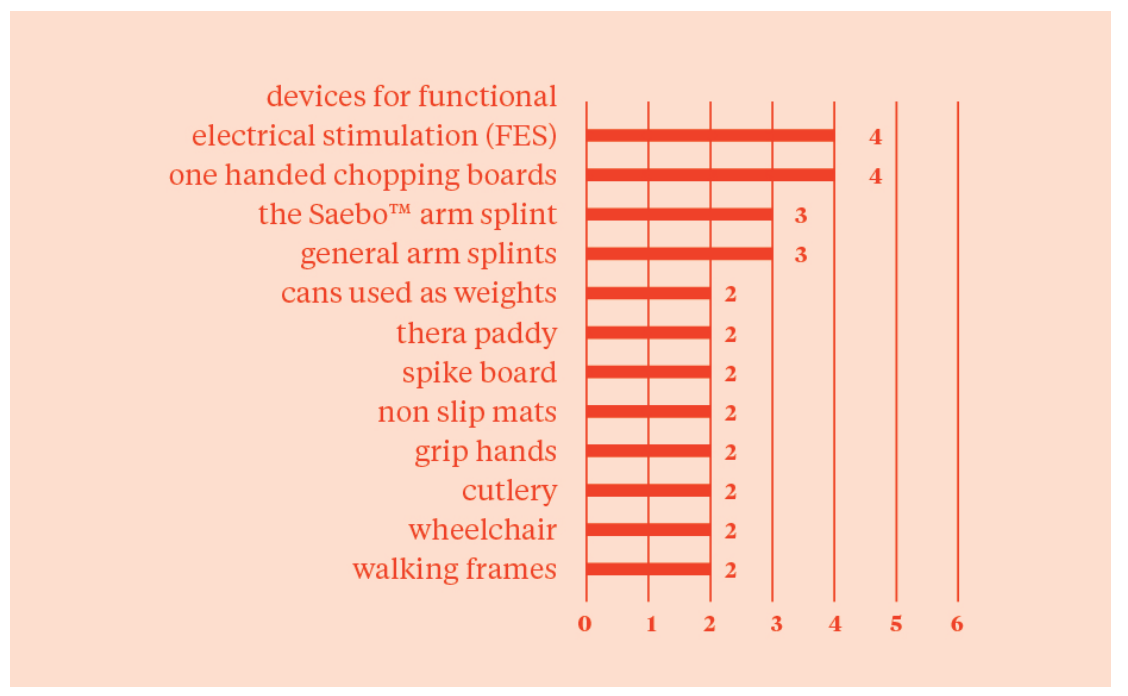

Figure 89:

Most frequently named devices.

The two most frequently named objects were FES devices, which are used in the rehabilitation process to stimulate the muscles to contract and one-handed chopping boards, see Figure 89. A one-handed chopping board is an assistive design that helps to compensate for the loss of functionality. It can be operated with just one hand to chop food and open cans. Other frequently named objects were splints such as the Saebo arm splint that stabilises the affected arm and prevents muscle contraction.

\subsubsection{Themes}

\subsection{Motivation to pursue ADL}

Two themes were mentioned in the context of ADL. The two themes were a sense of dignity and a sense of meaning. The therapists mentioned that it is not possible to generalise activities that everyone wants to relearn. However, a number of tasks seemed to come up frequently in the rehabilitation process. The kind of task would depend on personal preferences and level of disability of the stroke survivor.

I say it comes down to the person. Some people want to get back to the ironing. Some people want to get back to golf. Some people want to walk with the dogs, so there is not one size fits all.

Senior research officer (Participant 11) 
What was important to them before, so is that their pets or their kids or the golf that they did every weekend or going for lunch at the local pub. So you are trying to find out what were their normal routines before and what were some of their attitudes before and interests before and really try to find links to those so you can work it in and make the person see meaning. Often when they are not at home in their home environment, sometimes it is hard for them to see purpose or see the potential.

Occupational therapist (Participant 07)

\section{Sense of dignity}

The theme of dignity was mentioned in the context of tasks that might be experienced with a high degree of dependency. The ability to take part in tasks of self-care like getting dressed or washing oneself can contribute to the feeling of being independent.

The obvious thing to say would be the basic activities of daily living so anything that brings them dignity and supports them and maintains some pride if those things are really important to them. But as I said earlier that can vary from person to person. [...] Often with the males that I worked with they tend to pride... to maintain some dignity around some personal care, so things that they could do themselves.

Professional therapist (Participant 04)

Independent toileting, eating, independent showering and then independent transfers. So once you got those you can pretty much mostly look after yourself.

Musculoskeletal therapist working in tertiary sector (Participant 01) 
Stroke survivors who experience significant physical limitations especially seem to have an ambition to focus on daily tasks that secure independence rather than focusing on leisure activities. Stroke survivors with quite good recovery could potentially concentrate on more complex functions like hobbies. The perceived need to relearn specific tasks would often depend on which side of the body was affected.

I think patients are quite goal-oriented like they are quite interested in doing things that they know they need to be able to do. It is quite need driven. So if their stroke affected their left hand, but they write with the right hand they are not getting bothered to learn to write with their left hand it is just not something that they need to do.

Scientist 01 (Participant 05)

Apart from gender, age seems to be another factor that influences the choice of activities that contribute to the feeling of dignity. Younger stroke survivors would experience showering and getting dressed as quite a personal activity that they wanted to be able to perform by themselves. Some of the older stroke survivors would accept help because they could save their energy to do things that they would enjoy doing, such as spending time with the family.

An essential task after the stroke, especially during the inpatient stay, seems to be walking. The interview partners mentioned that stroke survivors associate walking with independence and the chance to be able to return home. However, they would often realise after a while that for most tasks they require a functioning upper limb. A therapist would, therefore, encourage a focus on upper limb tasks alongside working on the lower limb. 
When you are in the wards it is all about walking. Because walking gets you home.

Senior research officer (Participant 11)

The therapist would further guide the process of reaching the desired task by breaking it down in achievable steps.

I think it is a skill sometimes as well to have to guide people with that. Because people inside may be a bit blurry to what might be achievable at that point in time. So it is a part of taking the steps and be: okay so you want to be able to wash yourself completely in the morning and shower you know there might be a smaller goal like picking up the soap and putting it under your arm. So, it is really individual dependent.

Physiotherapist (Participant 12)

\section{Sense of meaning}

The second theme that was mentioned relates to re-establishing a sense of meaning. One participant mentioned that the grief stroke survivors experience is based on the loss of a specific role rather than the loss of certain motor functions.

People lose their roles... you know they feel they have lost the essence of who they are if they lose their primary role. Say someone was the breadwinner or they were the one in the relationship who made all decisions or dealt with the annoying person from Vodafone or whatever and they lose all of those roles. People grieve lost functions but I think more they grieve more lost roles. Clinical researcher (Participant 08) 
Another therapist stated that activities that contribute to a sense of meaning rather than to feeling happy are critical to stroke survivors. The feeling of helplessness and relying on others would influence decision making profoundly, especially during the early months after the stroke.

But ultimately it just depends what brings the person the most value and deliberately I use that word rather than happiness it is not necessarily about what activities make the person happy.

Professional therapist (Participant 04)

Two tasks were identified as being interlinked and essential to most people: driving and working.

Quite a lot of people want to be able to drive two-handed. That's another one that very often comes up, particularly for people who say they need to get back to work. They conceive that they need to be able to do that and that is a pretty feasible one.

Occupational therapist (Participant 02)

I guess they see work as part of their identity and you know you spent such a large part of your week at work and you know that can really be a key thing to feel like things are kind of coming back to some level of normality. Getting back to work and doing the things that you know that you can do and that kind of things so that can be really important as well. Physiotherapist (Participant 06)

Activities that incorporate a sense of meaning depend on multiple factors such as previous lifestyle, social expectations, gender, and location. The task of preparing a meal is part "self-care” but can be an essential part of people's life. 
So in the over 65s, women are much more into... it is not that they are interested, it is just that they are expected to learn how to do everything in the kitchen again because they were probably the ones that were doing all the cooking and the prep and you know all that kind of things. So we often get guys through who say 'Ah I don't need to know how to do that, my wife does it for me.' So yeah there is a really strong gender difference in that age group and probably in the younger age group to some extent but not as much.

Occupational therapist (Participant 13)

Especially depending on where you live but down south gardening was sort of the number one goal because everybody has a garden. But here [in the north] it is quite a big thing for people to relax.

Occupational therapist (Participant 13)

Leisure activities might be important, but they seem to be rarely focused on in the rehabilitation process because of the required movement abilities and limited resources. They also seem to become more important to people who have been discharged into the community.

So it really depends on where that person is at... and I have to say hobbies are so far down the list which is really sad but that is kind of the nature of the disability and how people prioritise self-care and the nature of our healthcare system where when you are good enough you are good enough and out the door.

Clinical researcher (Participant 08)

\subsection{Use of everyday objects}

The use of everyday objects in the rehabilitation process is dependent 
on limited financial resources and the intention to evoke plasticity in the brain. Everyday objects were either used in the form of unmodified objects or compensatory objects that an occupational therapist would provide. However, the participants emphasised that it is not possible to generalise ADL and associated objects that everyone wants to relearn after the stroke.

[It] depends on what their goal is. If it's typing on a computer, then they will use the keyboard. If it is using a phone, it will be the phone, and if it is your whiskey glass to your mouth then it is your whiskey glass like... that is how task-specific [...] So you know it is so contextual to the person, so it is not like there is equipment to go and be oh yeah you got this! Senior research officer (Participant 11)

\section{Compensatory objects}

Everyday objects in the home environment are used as compensatory design solutions or by adding design elements to pre-existing objects, for example bigger grips. Those design solutions help compensate for the loss of functionality post stroke and are often provided by an occupational therapist.

So, most things have some kind of manipulation because we use our hands for most things and having better upper limb dexterity control often means that probably we have a better sense of independence in most daily living tasks so most of the adaptive equipment usually is focusing around grips or pens, cutlery and knives and things to hold things in place, so hold grips, clothing and how we put them on and take them off. A lot of these seem to focus around making objects bigger and to be quite elastic. So obviously the smaller the object the [more] accelerator is required which often is a key problem.

Professional therapist (Participant 04) 
Two participants mentioned that they used assistive devices as part of the rehabilitation process. They would do this to make the task achievable for the stroke survivor rather than aiming to completely compensate for the loss of functionality.

A pen that has got a built-up handle. That is a classic example of modifying the equipment a little bit, and there are some examples of door handles. There are some door handles that I have asked patients to replace in their house so that they could practise the task.

Occupational therapist (Participant 03)

The ironical thing is that people use a lot of stuff in exactly the opposite way that I would use it. You know, giving somebody a one-handed chopping board or one of the spike boards. So I use those, but I make them use it with the affected hand.

Occupational therapist (Participant 02)

\section{Financial limitations}

Everyday objects that can be used in ADL to compensate for lost motor abilities were identified as belonging to the field of expertise of occupational therapists. Objects used to compensate and rehabilitate were criticised for often being quite expensive. Stroke survivors face financial limitations and would, therefore, use everyday objects or self-made design solutions as an alternative.

[...] but they are not routinely provided with equipment for ongoing therapy and so the home exercise programme that we have developed just uses household items like cups or a ball or pack of cards or paper and scissors. Things that people are likely to have around the house rather than specialist equipment and I think that is very practical because of costs. Patients are 
often older and no longer working, or if they are younger, they can't work. Scientist (Participant 05)

\section{Plasticity}

Another factor influencing the focus on everyday objects is that they are regarded as an essential part of specific tasks that contribute to changes in the brain.

What we know about relearning is that the more specific you can get to the task the person is having difficulties [with] and the context that they are going to use that skill, the better the learning is, so I would try and use that everyday object, and then if I couldn't use the everyday object I would go try and find other objects which make it simpler in some way.

Clinical researcher (Participant 08)

There seem to be some differences between countries, hospitals, and regions in providing equipment to stroke survivors. The kind of equipment that stroke survivors received upon discharge into the community would often depend on recommendations of the therapist. Another point of access seems to be the internet where survivors would look up solutions. However, one participant pointed out that bigger modifications to the home can be experienced as too much of an alteration to their previous living environment and evoke negative emotions.

Most of the time people stick to what they have got at home. That will depend quite a lot on what the therapist recommends. So I think if the therapist has something... and they find it really useful in a session with the therapist then they are more likely to go and buy it.

Occupational therapist (Participant 13) 


\subsubsection{Triangulation}

The results of this study underwent secondary analysis to develop convergence codes that could be used for the triangulation process. This first section outlines the codes of the online survey with stroke survivors. The second section will outline the convergence coding based on the interviews with health professionals. In the next step, the two sets were compared and assessed. Since the online survey provided no sample quotes (Farmer et al., 2006, pp. 382-389), quotes will only be included as part of the coding for the therapists' interviews.

10.3.1.4.1 Convergence coding of the online survey results

This study focuses on objects that can be used to facilitate use of the affected arm and hand. The convergence coding, therefore, contains additional details concerning specific tasks and objects that were named, see Table 11 . The column that contains the number of tasks shows two numbers. The first number refers to the ADL section, while the second one refers to the hobby section of the online survey. This is due to some overlaps in the results. The theme "social" refers to family members as well as friends. 
Table 11: Convergence coding based on the online survey results with stroke survivors.

\begin{tabular}{|c|c|c|c|c|}
\hline Theme & Tasks & Objects & $\begin{array}{l}\text { Number } \\
\text { of objects }\end{array}$ & $\begin{array}{l}\text { Number } \\
\text { of tasks }\end{array}$ \\
\hline \multirow{6}{*}{$\mathrm{ADL}$} & Doing housework & $\begin{array}{l}\text { Broom, dishwasher, dryer, dust rag, } \\
\text { vacuum cleaner, washing machine }\end{array}$ & 7 & $18 / 0$ \\
\hline & Dressing & Clothes, sock aid & 2 & $4 / 0$ \\
\hline & Driving & Car, bus & 3 & $23 / 2$ \\
\hline & Preparing meals & $\begin{array}{l}\text { Blender, bowls, chef knife, cook top, } \\
\text { cook book, cookware, cutting board, } \\
\text { food, fork, fridge, juicer, knives, lid, } \\
\text { microwave, mixer, oven, pan, pots, } \\
\text { press (kitchen), spatula, spoon, stove }\end{array}$ & 34 & $25 / 11$ \\
\hline & $\begin{array}{l}\text { Walking with and } \\
\text { without equipment }\end{array}$ & $\begin{array}{l}\text { Walking cane, hemi walker, ankle foot } \\
\text { orthosis }\end{array}$ & 7 & $77 / 56$ \\
\hline & $\begin{array}{l}\text { Washing oneself } \\
\& \text { caring for body } \\
\text { parts }\end{array}$ & $\begin{array}{l}\text { Floss, toothbrush, shower, shower bank, } \\
\text { tooth pick }\end{array}$ & 6 & $13 / 0$ \\
\hline \multirow{5}{*}{$\begin{array}{l}\text { Leisure } \\
\text { tasks }\end{array}$} & Art & $\begin{array}{l}\text { Brush, camera, camera lights, circular } \\
\text { knitting needles, diffuser for camera, } \\
\text { drums, feet controller, filters for } \\
\text { camera, guitar, iPod, music keyboard, } \\
\text { light box for camera }\end{array}$ & 16 & $23 / 34$ \\
\hline & Craft & $\begin{array}{l}\text { Chain saw, circular knitting needles, } \\
\text { crochet hook, hand tools, fishing pole, } \\
\text { knitting needle, needle, rotary cutter, } \\
\text { sewing machine, scissors, thread, tools, } \\
\text { yarn }\end{array}$ & 19 & 19 / 97 \\
\hline & $\begin{array}{l}\text { Garden and lawn } \\
\text { work }\end{array}$ & Gardening tools & 6 & $18 / 18$ \\
\hline & Reading & Books, magazines, newspaper & 15 & $41 / 54$ \\
\hline & Sport & $\begin{array}{l}2 \mathrm{lb} \text { barbells, baseball, basketball, } \\
\text { bowling ball, exercising bands, } \\
\text { exercising equipment, football, } \\
\text { treadmill, weights, golf club, ski boots, } \\
\text { snow boots }\end{array}$ & 14 & $73 / 78$ \\
\hline \multirow[t]{2}{*}{ Social } & Communication & $\begin{array}{l}\text { Laptop, phone, smartphone, tablet, } \\
\text { computer, computer mouse, keyboard, } \\
\text { printer }\end{array}$ & 94 & $25 / 26$ \\
\hline & Social & Photo album, pictures, vacation diary & 7 & $33 / 6$ \\
\hline Work & Work & Scalpel & 1 & $20 / 8$ \\
\hline
\end{tabular}




\subsection{Convergence coding of the interview results with stroke therapists}

\section{Convergence codes based on the interviews with therapists are outlined in}

\section{Table 12.}

Table 12: Convergence coding based on the interviews with stroke therapists.

\begin{tabular}{|c|c|c|c|c|}
\hline Theme & Tasks & Object & $\begin{array}{l}\text { Interview partner } \\
\text { mentioning each } \\
\text { factor }\end{array}$ & Sample quote \\
\hline \multirow{6}{*}{ Dignity } & Dressing tasks & $\begin{array}{l}\text { Clothes, shoes, } \\
\text { shoelaces, socks, } \\
\text { backpack, jacket, } \\
\text { buttons, zipper, } \\
\text { ribbon }\end{array}$ & 7 & $\begin{array}{l}\text { A lot of people try to put on the shoes } \\
\text { with one hand because they can't bend } \\
\text { forward enough and no one is really ... } \\
\text { so they end up either pushing that to } \\
\text { the end on one side and then somebody } \\
\text { helping with the other shoe. }\end{array}$ \\
\hline & $\begin{array}{l}\text { Eating and } \\
\text { drinking tasks }\end{array}$ & $\begin{array}{l}\text { Cutlery, } \\
\text { chopsticks, } \\
\text { glass, cups }\end{array}$ & 7 & $\begin{array}{l}\text { People want to be able to eat by } \\
\text { themselves and they want to be able to } \\
\text { go out and socially eat appropriately... } \\
\text { not having their food cut up for them }\end{array}$ \\
\hline & $\begin{array}{l}\text { High level fine } \\
\text { motor skills }\end{array}$ & $\begin{array}{l}\text { Keys, locks, } \\
\text { coins }\end{array}$ & 4 & $\begin{array}{l}\text { But I think keys and locks they are really } \\
\text { problematic for a lot of people }\end{array}$ \\
\hline & $\begin{array}{l}\text { Household } \\
\text { tasks }\end{array}$ & $\begin{array}{l}\text { Cooking } \\
\text { utensils, fridge, } \\
\text { pots, vacuum } \\
\text { cleaner, } \\
\text { dishwashing } \\
\text { brush, iron, } \\
\text { garden utensils }\end{array}$ & 4 & $\begin{array}{l}\text { Yeah, most coming up are household } \\
\text { activities so being able to do the dishes }\end{array}$ \\
\hline & Walking & $\begin{array}{l}\text { Walking aid, } \\
\text { walking stick, } \\
\text { four-pronged } \\
\text { stick, walking } \\
\text { frame, ankle foot } \\
\text { orthosis }\end{array}$ & 6 & $\begin{array}{l}\text { There are two goals that you can } \\
\text { guarantee: walking and driving }\end{array}$ \\
\hline & $\begin{array}{l}\text { Washing } \\
\text { oneself }\end{array}$ & $\begin{array}{l}\text { Shampoo } \\
\text { container, taps, } \\
\text { toothbrush, } \\
\text { toothpaste, } \\
\text { hairbrush }\end{array}$ & 6 & $\begin{array}{l}\text { Maybe for the younger ones doing } \\
\text { something like showering and dressing } \\
\text { because it is a quite personal thing being } \\
\text { able to do that even if that means using } \\
\text { equipment rather than having a person } \\
\text { to come in to help you. }\end{array}$ \\
\hline
\end{tabular}


LIMITED USE ONLY

\begin{tabular}{|c|c|c|c|c|}
\hline \multirow{5}{*}{$\begin{array}{l}\text { Sense of } \\
\text { meaning }\end{array}$} & $\begin{array}{l}\text { Communica- } \\
\text { tion }\end{array}$ & $\begin{array}{l}\text { phones/ } \\
\text { smartphones, } \\
\text { computer, } \\
\text { laptops }\end{array}$ & 3 & $\begin{array}{l}\text { I think to be honest the phone is a really } \\
\text { big one }\end{array}$ \\
\hline & $\begin{array}{l}\text { Leisure } \\
\text { activities }\end{array}$ & $\begin{array}{l}\text { Golf, knitting } \\
\text { needles, TV } \\
\text { remote control, } \\
\text { guitar, CD, ball, } \\
\text { cards }\end{array}$ & 3 & $\begin{array}{l}\text { What was important to them before so is } \\
\text { that their pets, or their kids, or the golf } \\
\text { that they did every weekend... or going } \\
\text { for lunch at the local pub or you know... }\end{array}$ \\
\hline & Mobility & Car & 4 & $\begin{array}{l}\text { Quite a lot of people want to be able to } \\
\text { drive two handed... that's another one } \\
\text { that very often comes up, particularly for } \\
\text { people who say they need to get back to } \\
\text { work }\end{array}$ \\
\hline & Reading & Newspaper & 1 & $\begin{array}{l}\text { Not that that matters but a lot of people } \\
\text { read newspapers and then drink cups } \\
\text { of tea }\end{array}$ \\
\hline & Work & $\begin{array}{l}\text { Work specific } \\
\text { objects such as } \\
\text { laptops or } \\
\text { doctor's } \\
\text { equipment }\end{array}$ & 5 & $\begin{array}{l}\text { Or you might have someone who tells } \\
\text { you straight away ,I am a writer, I need to } \\
\text { get back onto my laptop }\end{array}$ \\
\hline
\end{tabular}




\subsection{Convergence assessment}

In a second step, the initial convergence codes of the online survey were mapped to the codes of the interviews and assessed according to meaning and prominence of the theme, see Table 13.

Table 13: Convergence assessment.

\begin{tabular}{|c|c|c|c|c|c|}
\hline \multirow{3}{*}{ Themes } & \multirow{3}{*}{ Task } & \multicolumn{4}{|c|}{ Convergence code } \\
\hline & & \multicolumn{4}{|c|}{ Theme meaning and prominence } \\
\hline & & AG & PA & S & DA \\
\hline \multirow{6}{*}{ Dignity } & Dressing tasks & & & & $\mathrm{x}$ \\
\hline & Eating tasks & & & & $\mathrm{x}$ \\
\hline & High level fine motor skills & & & $\mathrm{x}$ & \\
\hline & Household tasks & & $\mathrm{x}$ & & \\
\hline & Walking & $\mathrm{x}$ & & & \\
\hline & Washing oneself & & $\mathrm{x}$ & & \\
\hline \multirow{8}{*}{ Sense of meaning } & Communication & & $\mathrm{x}$ & & \\
\hline & Art & & & $\mathrm{x}$ & \\
\hline & Craft & & & $\mathrm{x}$ & \\
\hline & Sport & & & & $\mathrm{x}$ \\
\hline & Social & & $\mathrm{x}$ & & \\
\hline & Mobility & $\mathrm{x}$ & & & \\
\hline & Reading & & & & $\mathrm{x}$ \\
\hline & Work & $\mathrm{x}$ & & & \\
\hline
\end{tabular}

$\mathrm{AG}=$ Agreement, $\mathrm{PA}=$ partial agreement, $\mathrm{S}=$ Silence $\mathrm{DA}=$ dissonance 


\subsection{Discussion}

In the following section, the themes of the interviews are discussed, followed by a review of the triangulation results.

\subsubsection{Interviews}

\subsubsection{Goal setting}

All interview partners emphasised that the ADL for the rehabilitation process are based on a shared decision-making (SDM) process with the stroke survivors. SDM is used to determine ADL and set goals for the process. This confirms results of a recent review that demonstrated the use of SDM in clinical practice (Rose, Rosewilliam, \& Soundy, 2017, p. 70) as a reflection of the biopsychosocial model of care that aims to maximise participation and activity (Plant et al., 2016, p. 927). Stroke survivors who are involved in the goal-setting process showed increased motivation (Brown et al., 2014, p. 1023) and satisfaction (Rose et al., 2017, p. 72).

Nonetheless, SDM can be a challenging experience from the survivor's perspective. They can feel unfamiliar with the process and are insecure about reachable goals (Rose et al., 2017, p. 72). They can also prefer to keep goals to themselves (Brown et al., 2014, p. 1025) and see the therapist as an expert "who will know best what to focus on" (Plant et al., 2016, p. 924). Findings of the current study support this. Interview partners pointed out that stroke survivors often try to focus on activities that are too complicated, too vague, or need to be approached in small steps.

Staff members have reported issues implementing SDM in clinical practice. They often have to spent more time to perform SDM in clinical practice (Rose et al., 2017, p. 71). The process of involving the patient efficiently and setting realistic goals and expectations for recovery seems to be a significant challenge (Plant et al., 2016, p. 925). Even though SDM appears to be beneficial, it is time-consuming and complex. 


\subsubsection{2 $A D L$}

The category that contained most of the named tasks was the one of selfcare; for example, being able to dress oneself or showering. These tasks contribute to a feeling of independence and dignity after the stroke. Tasks that contribute to a sense of meaning were also pointed out as being important. However, those tasks seem to be more complex and rarely focused on in clinical practice.

The interview focus was on upper limb stroke interventions, which might explain why interview partners referred to the task of walking less frequently even though they pointed out that it was the most important task during the inpatient stay.

\subsection{Sense of dignity}

Tasks that contribute to a sense of dignity help develop a feeling of independence after a stroke and are a primary focus of the rehabilitation process. The focus on physical functioning rather than leisure activities, especially during inpatient rehabilitation, has been reported in previous studies (Levack, Dean, Siegert, \& McPherson, 2011, p. 210; Plant et al., 2016, p. 927). There seems to be a focus on "survival needs" such as walking, toileting or dressing, or returning home. A focus on these tasks is based on common sense during the early phase to re-establish a sufficient level of independence. These ADL can be seen more as a necessity that assures a safe and quick discharge into the community rather than being a meaningful goal to the survivor (Brown et al., 2014, pp. 1022-1023; Levack et al., 2011, p. 210; Wood et al., p. 1049).

\subsection{Sense of meaning}

Stroke survivors often struggle to adjust to their new life after the stroke. They can feel helpless and threatened in their self-image (Erikson et al., 2010, p. 833; Wood et al., 2010, p. 1049). Tasks that contribute to a sense of meaning are therefore crucial to the individual. Meaningful in this context highly depends on the previous lifestyle of the participants. 
Nonetheless, two tasks were pointed out as being important:

- Getting back to work

- Driving

Driving is a key factor for everyday mobility, for shopping, being able to get back to work, and accessing leisure activities (Robison et al., 2009, p. 1559; White et al., 2012, p. 833). An inability to drive after a stroke impacts on the possibility of resuming work and can be experienced as a threat to the individual's identity. Money seems to be less of a motivation to return to work than other psychological factors such as increased self-esteem, independence and productivity (Hartke \& Trierweiler, 2015, p. 331). The ability to work again is often seen as proof of recovery and a way of moving forward (Brannigan et al., 2017, p. 220). The transition back into work life and driving both depend on an open discussion about limitations caused by the stroke and the provision of adequate information and adaptations (Brannigan et al., 2017, p. 221; White et al., 2012, p. 837). There is still a significant lack of knowledge about effective forms of assessment and vocational interventions that can help in the transition process (Baldwin \& Brusco, 2011, p. 571; Treger, Shames, Giaquinto, \& Ring, 2007, p. 1401).

\subsubsection{Limitations of current rehabilitation setting}

Tasks that contribute to a sense of dignity were pointed out as being a crucial element in the recovery process, but they require a high degree of complexity and movement requirements. A factor that limits the focus on these tasks might be the current rehabilitation setting.

The financial and organisational structure of health services can cause a tension between a clinician's goals and goals that the patient might have. Goals for the rehabilitation process must be SMART: specific, measurable, activity-related, realistic and time-limited (Wilson, 2008, p. 145). Stroke survivors, on the other hand, define the recovery process as a way to participate in meaningful activities again (Doolittle, 1992, p. 124; Plant et al., 2016, pp. 923-924). This view has no set time frames and end goals (Brown et al. 2014, p. 1025). Even though therapists 
might want to focus on tasks that contribute to a sense of meaning, it might often be difficult due to resource and institutional restrictions.

This raises the question of whether truly patient-centred goal setting is even possible in the inpatient setting (Levack et al., 2011, p. 212). Most of the interview participants worked with acute and subacute stroke survivors where impairments caused by the stroke are expected to improve. A focus on BADL seems to be more appropriate at this stage to secure a level of independence and safe discharge. It has been argued that the focus on participation goals such as hobbies is inappropriate during this period and that a clinician-led approach during the early phase might be more appropriate (Plant et al., 2016, p. 927).

The health professionals in the current study acknowledged that survivor strive for a sense of meaning and mentioned work, leisure activities, and social involvement as essential elements of that. Stroke support that addresses emotional and behavioural issues to secure participation in previous activities (Robison, 2009, pp. 1564-1565) seems to be necessary to achieve this. In New Zealand there seems to be lack of funding that would allow such a service.

\subsubsection{Contextual factors}

Results of the current study confirm previous results (Sveen, Bautz-Holter, Margrethe Sodring, Bruun Wyller, \& Laake, 1999, p. 376) that gender and the social context of culture influence choice of activity. It has been reported that interacting with others such as family members, friends, and colleagues can work as a form of inspiration, be a motivational driver (Erikson et al., 2010, p. 834) and evoke a sense of belonging. The context in which the design prototype would be used and possible gender-specific factors needed to be considered in its development.

\subsubsection{Everyday objects}

Everyday objects used by stroke survivors in the context of initiating the use of the affected arm and hand were used in two different ways:

- As a specifically designed assistive object, for example, a spike board 
- As a standard everyday object. Standard everyday objects can contain small adjustments to make the use feasible. An example of such an object would be cutlery that has some sticky tape attached to increase friction during use

The object named most frequently was the one-handed chopping board, which holds food in place while it is being cut. Rubber suction feet help secure the board to surfaces. While most participants referred to everyday objects as a way of compensating for lost functionality, two participants did point out that they would use those assistive devices to make a task "feasible to perform" for the survivor. Responses indicated a focus on the compensatory character of everyday objects rather than an opportunity to use them as an active form of rehabilitation.

10.4.1.5.1 Motivation to use everyday objects as training equipment

A stroke is associated with high costs (Demaerschalk, Hwang, \& Leung, 2010, p. 532) and stroke survivors often face financial limitations and struggle to buy specific equipment. This might explain using everyday objects as a replacement for specialised training equipment. Some of the interview partners mentioned that cans are often used as weight replacements.

The recovery process seems to be influenced by the socioeconomic status of the individual and limited financial resources have been associated with lower functional independence and recovery after stroke (Bettger et al., 2014, p. 7; Grube et al., 2012, p. 3328; van den Bos, 2002, p. 946). Design solutions should take account of this and offer solutions that are cost efficient to produce.

\subsubsection{Triangulation}

\subsubsection{Convergence assessment}

The convergence assessment considered the meaning and prominence of the mentioned themes. The following section outlines the results in terms of themes that reached an agreement, a partial agreement, a dissonance, and silence. 


\subsection{Agreement}

Three themes reached an agreement:

- Walking

- Mobility by being able to drive or using alternative forms of transportation,

- Working

Mobility, walking, and driving are essential tasks that contribute to feelings of self-identity and independence and were identified during the interviews with stroke therapists.

\subsection{Partial agreement}

Partial agreement was reached on the themes of:

- Social activities

- Communication

- Washing oneself

- Household tasks

Therapists highlighted the importance of tasks that contribute to feelings of dignity and independence, while the results of the online survey emphasised tasks that involved others, such as family members or friends. The named themes were mentioned in both forms of data collection but were prioritised slightly differently.

An explanation for this difference in preference might be that the stroke therapists who were interviewed mainly focused on the acute and sub-acute stage where self-care tasks are a priority. The participants of the online survey had reached the chronic stage when leisure tasks seem to become more relevant.

\subsection{Silence}

The level of silence was reached with themes of:

- High-level fine motor skills

- Art

- Craft

Leisure activities were mentioned by the therapists as being important, but 
there was a significant difference in the prominence of themes relating to leisure tasks. The difference in prominence for the task of high-level motor skills might be due to the perception of such tasks as being a necessity rather than an activity that contributes to a sense of meaning. However, further research needs to confirm contributing factors to the discrepancies in those themes.

\subsection{Dissonance}

Four themes showed a level of dissonance. Those themes were:

- Dressing tasks

- Eating

- Sport

- Reading

Tasks relating to those themes were mentioned in both studies but with a significant difference concerning their prominence. Therapists pointed out eating and self-care tasks as substantial aims in the rehabilitation and leisure tasks such as sport as secondary goals due to motor requirements. The online survey with stroke survivors, on the other hand, indicated that sport and hobbies such as reading are important after the stroke. However, just one therapist mentioned reading in the present study. The ability to read might be impacted by cognitive impairments as well as physical ones. The focus in the interviews with stroke therapists was on physical impairments, which might explain the difference regarding the prominence of this theme.

\subsubsection{Completeness comparison}

The purpose of this triangulation was to gain a deeper understanding of ADL post stroke and objects that play a crucial role in those activities. These objects can then be used to facilitate initiation of use of the affected arm and hand. The triangulation process produced a number of themes where both data sets reached a level of agreement. The interview data added factors that were not evident in the online survey, such as the overarching themes of a sense of meaning and dignity. 
Therapists emphasised the focus on dignity tasks while stroke survivors seemed to prioritise activities that contributed to a sense of meaning after the stroke. This discrepancy between the results of the online survey and the interview results might be caused by differing foci in the rehabilitation process. The interviewed therapists were mainly involved in the early stage after the stroke while the participants of the survey had reached the chronic phase of the stroke. This indicates that there is a time component involved in the process. A different explanation might be that ADL goals in the context of rehabilitation have to be SMART while recovery after a stroke is defined for a survivor as being able to return to a previous lifestyle. Further research is needed to determine reasons for this discrepancy.

This study focused on the objects that are part of essential ADL. Just three themes reached an agreement in the triangulation process. The named themes, unfortunately, take place in a highly complex environment, for example the street while driving; have a dominant focus on the lower limb, for example walking; or are too broad in focus, for example working.

The theme of communication had reached the level of partial agreement in the triangulation process. The category contains the objects that were named most frequently in the online survey - digital telecommunication devices. Communication can take place in different forms and the use of devices such as phones and computers is often a crucial element allowing communication with family members and friends who might not be living close by. During the interviews some of the therapists mentioned smartphones and tablets as being a particularly important element that people focus on in the rehabilitation process. The theme of communication was therefore chosen for the next design prototype. The focus on this theme can potentially contribute to a sense of meaning after a stroke, which seems to be prioritised in the chronic stage. 


\subsection{Conclusion}

The analysis of the interviews showed that stroke therapists mentioned two different themes regarding ADL that participants commonly focus on: Tasks that contribute to a sense of dignity and tasks that evoke a sense of meaning.

The use of everyday objects in the context of rehabilitation would depend on the ADL. Objects used in the context of stroke rehabilitation are rehabilitation devices, assistive devices, and common everyday objects. Objects in the home environment seemed to be used in the form of assistive devices that compensated for reduced motor functionality, and everyday objects.

The theme of communication reached a partial agreement in the triangulation process and was indicated by some of the therapists to be essential to a sense of meaning during the chronic stage. Telecommunication devices were the most frequently named objects in the online survey and seemed to offer great potential to contribute to an initiation of use due to their omnipresence in daily activities. Telecommunication devices are an essential element in the context of communication and social activities and have been chosen for the next phase of prototype development. Further research is needed to determine stroke-specific barriers that might impact on the use of the devices and specific activities in which they play a crucial role. 


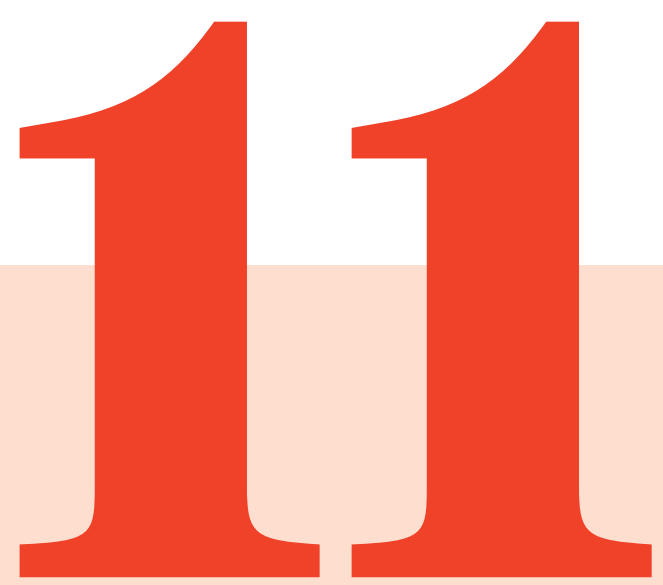

\section{Information}

\section{Communication}

\section{Technology}

\section{Post Stroke}




\subsection{Introduction}

The results of the triangulation process of the online survey results and interviews with stroke therapists indicated that information communication technology (ICT) devices are an essential element in everyday tasks after the stroke. It was important to understand which current barriers impact on the use of these devices in order to address the following research question and to design an everyday object which restrains movement:

1.2.1 Which everyday objects are used by stroke survivors during activities of daily living that they like to take part in, and therefore may assist in facilitating use of the affected arm?

Six semi-structured interviews with stroke survivors and observations of the use of ICT devices aimed to identify common barriers. The results have been used to influence the design of a device that contributes to an initiation of use and restrains movement which will be outlined in chapter 12 and 13.

\subsection{Background}

ICT includes feature phones, smartphones, tablets, and computers are an essential part of our everyday life. A preference seems to exist of using the smartphone on a daily basis over other devices such as the laptop (Research New Zealand, 2015, p. 3). In New Zealand, 91\% of smartphone owners report using the device every day (Research New Zealand, 2015, p. 9). The smartphone has been claimed to be omnipresent, but it seems that the feature phone or mobile phone is still a commonly used device, especially among older adults (Berenguer et al., 2017, p. 104).

It has been speculated that the smartphone will increase in popularity among older adults in the future for several reasons. It will become harder in the future to acquire a traditional feature phone while at the same time smartphones 
will reduce in cost (Berenguer et al. 2017, p. 107). Furthermore, the current generation who grows up with smartphones will become older (Kurniawan, 2008, p. 891) leading to an increased use of these devices among older adults. Predictions are that by 2023 the penetration of smartphones will reach $90 \%$ in developed countries with the highest rate of adoption rate among adults between 55 and 75 years, among which an $85 \%$ penetration is expected (Deloitte Touche Tohmatsu Limited, 2017, p. 7). It is further expected that by 2023, smartphone owners will use the device up to 65 times per day (Deloitte Touche Tohmatsu Limited, 2017, p. 7). In the context of this study, smartphones could contribute to an initiation of use due to their omnipresence and repeated use.

In the context of stroke, feature and smartphones can play an important role in activities of daily living. They are an affordable technology that allows access to health information and services. They promote confidence, organise daily tasks, evoke a feeling of safety, and create a sense of belonging and social relationships (Gustavsson, Ytterberg, Nabsen Marwaa, Tham, \& Guidetti, 2018, pp. 564-566; Kamwesiga, Tham, \& Guidetti, 2017, pp. 439-441). Devices like the feature phone can become an extension of the body that secures a socio-economic role, enables employment, and enables community integration. The degree of dependency on the device to ensure basic needs varies between users (Kamwesiga et al., 2017, p. 441).

It has been argued that these devices can facilitate the rehabilitation process and promote the use of the affected arm and hand (Gustavsson et al., 2018, p. 566; Kamwesiga et al., 2017, p. 448; Rand et al., 2013, p. 112). A number of design recommendations and checklists focus on design for feature and smartphones for the elderly population (Petrovčič, Taipale, Rogelj, \& Dolničar, 2017). However, there is little focus on the design of these devices that addresses stroke-specific limitations such as reduced fine motor skills, affected memory, cognition, perception, vision, speech, and decreased concentration (Gustavsson et al., 2018, pp. 565-566). 
There were just a few studies that investigated the use of smartphones and feature phones among stroke survivors in the home environment and none of them investigated which device specific features impact on the use of the devices. However, it is crucial to know which design elements currently impact on an initiation of use. The study by Kamwesiga et al. (2017) focused on the use of feature phones in Uganda, and the study by Gustavsson et al., (2018) took place in Sweden and Denmark and focused on the general use of information communication technology after stroke. Gustavsson et al. (2018) included the interview guide that has been used as a guide for this study. The study was missing questions concerning limitation and barriers that complicate the use of the devices. Therefore, two additional questions were included as well as the request to demonstrate how the device is used.

\subsection{Methods}

\subsubsection{Participants}

Participants were recruited through a physiotherapist in Auckland, New Zealand, and the leader of a stroke club in Wellington, New Zealand. Refer to 3.6.2 for a description of the inclusion and exclusion criteria.

Six participants were interviewed as part of the study. Each participant was informed about the study and provided written consent. All participants had experienced a stroke in the past and showed signs of mild to moderate motor impairments on one of the upper limbs. Two participants were female $(n=2)$, and four were male $(n=4)$. Time since the stroke varied between 8 months and 12 years, see Table 14. One participant did not meet the inclusion criteria in terms of his age. This just became apparent after the interview was completed. 
Table 14: Study sample.

\begin{tabular}{|c|c|c|c|c|c|c|}
\hline & Gender & Age & Effect of the stroke & $\begin{array}{l}\text { Time since } \\
\text { stroke }\end{array}$ & $\begin{array}{l}\text { Dominant } \\
\text { side }\end{array}$ & $\begin{array}{l}\text { Devices } \\
\text { used }\end{array}$ \\
\hline 01 & female & 60 & $\begin{array}{l}\text { Moderate impairments } \\
\text { of the upper and lower } \\
\text { limb on the left side }\end{array}$ & $\begin{array}{l}2 \text { years, } 7 \\
\text { months }\end{array}$ & $\begin{array}{l}\text { Right } \\
\text { handed }\end{array}$ & $\begin{array}{l}\text { Laptop, } \\
\text { iPad } \\
\text { Android } \\
\text { smartphone }\end{array}$ \\
\hline 02 & male & 82 & $\begin{array}{l}\text { Moderate impairments } \\
\text { of the upper and lower } \\
\text { limb on the left side }\end{array}$ & 12 years & $\begin{array}{l}\text { Right } \\
\text { handed }\end{array}$ & $\begin{array}{l}\text { Laptop, } \\
\text { feature } \\
\text { phone }\end{array}$ \\
\hline 03 & male & 74 & $\begin{array}{l}\text { Mild impairments of the } \\
\text { upper and lower limb } \\
\text { on the left side }\end{array}$ & 2 years & $\begin{array}{l}\text { Right } \\
\text { handed }\end{array}$ & $\begin{array}{l}\text { Laptop, } \\
\text { Android } \\
\text { smartphone }\end{array}$ \\
\hline 04 & male & 70 & $\begin{array}{l}\text { Moderate impairments } \\
\text { of the upper and lower } \\
\text { limb on the left side }\end{array}$ & 12 years & $\begin{array}{l}\text { Right } \\
\text { handed }\end{array}$ & $\begin{array}{l}\text { Feature } \\
\text { phone, } \\
\text { landline } \\
\end{array}$ \\
\hline 05 & male & 77 & $\begin{array}{l}\text { Moderate impairments } \\
\text { of the upper and lower } \\
\text { limb on the right side }\end{array}$ & 10 years & $\begin{array}{l}\text { Right } \\
\text { handed }\end{array}$ & \begin{tabular}{|l} 
Laptop, \\
feature \\
phone
\end{tabular} \\
\hline 06 & female & 72 & $\begin{array}{l}\text { Mild impairments of the } \\
\text { upper and lower limb on } \\
\text { the right side, reduced } \\
\text { sensation on the right } \\
\text { side }\end{array}$ & 8 months & $\begin{array}{l}\text { Left } \\
\text { handed }\end{array}$ & $\begin{array}{l}\text { Laptop, } \\
\text { iPad, Apple } \\
\text { smartphone }\end{array}$ \\
\hline
\end{tabular}

\subsubsection{Semi-structured interviews}

Six chronic stroke survivors were interviewed to determine the motivation for and barriers to using telecommunication devices in daily activities. The questions for the semi-structured interviews were based on Gustavsson et al. (2018, p. 563). Two additional questions, number 11 and 12 , were included in the interview guide. Each participant was asked to bring their feature phone or smartphone to the interview and demonstrate its use. The use of the device was video recorded and analysed later. Each interview was audio recorded and transcribed in full. The interview guide contained the following questions: 
1. Could you tell me about your stroke?

2. When did it happen and how has it affected you?

3. Could you tell me about the rehabilitation you have received?

4. What kinds of interventions have you been offered?

5. Can you tell me about your situation today?

6. What activities do you like to perform?

7. What kind of technology do you use in your everyday life, for example such as mobile phones, or tablets?

8. For what purpose do you use your smartphone/tablet?

9. Is there any difference from how you used the technology before your stroke?

10. Have you used any technology within the rehabilitation after stroke?

11. Is there something that makes the use of the device difficult for you?

12. Could you show me your device and how you use it?

\subsubsection{Analysis}

The transcribed interviews were analysed with thematic analysis in a deductive form (Braun \& Clarke, 2006, p. 83).

\subsection{Results}

\subsubsection{Themes}

The interviews were analysed according to the themes of motivation for use and barriers. In the following section, the motivation for use will be focused on first followed by the description of commonly experienced barriers. Participants mentioned using technology in the form of feature phones, smartphones, laptops, iPads, and computers.

\subsubsection{Motivators}

Motivators are safety, the role of the device as a facilitator of reintegration, reinforcement of technology adoption, leisure activities, connecting with others, 
and being contributors to the rehabilitation process.

\subsection{Safety}

The use of feature phones and smartphones was associated with an increased feeling of personal safety and preventing others from worrying. The devices conveyed the impression that it was possible to get in contact with others immediately in an emergency.

So I always have my phone with me in case I fall over.

\section{Participant 03}

But I think now, for other people's sake as well as my own, I take my phone.

\section{Participant 06}

This desire to be able to reach someone in case of a fall or emergency was mentioned by multiple participants as being stronger after the stroke.

So yeah that has become an issue now, whereas it wasn't one before. I didn't think I'd need to get hold of somebody.

\section{Participant 06}

But not just emergencies were mentioned in this context. One participant indicated that the device would offer the option to reach friends in situations where she felt exhausted and tired. One participant mentioned that his carer's phone number was the first contact number on his feature phone so he could reach him instantly. 
So we have an AA you see [shows the shortcut on the phone to call the carer]. And you just press the green button, and it rings and that sort of thing.

Participant 02

\subsection{Facilitator of reintegration}

The feature and smartphone but even more the laptop and the iPad were said to facilitate reintegration into daily activities. The fields of reintegration comprised social work and previous work occupations, organisational tasks such as writing official documents to public institutions, or managing everyday tasks, for example online banking. Most participants who used the devices in the context of work and writing preferred to use the laptop since it offered more comfortable writing and especially editing workflow.

I am using [the laptop] at the moment for... I am writing the wedding ceremony. So that's what I am doing at the moment, but normally I just use this [points at his smartphone].

Participant 03

The laptop was used in everyday tasks in terms of online banking and organisational matters such as "book a bach", a website to rent holiday homes. Smaller handheld devices help to structure daily tasks, for example what time the carer needed to come in the morning, what groceries needed to be bought from the supermarket, and to confirm appointments.

I find it very handy having my contacts in my phone book on here. I can just find them and ring them up and that's it.

Participant 05 
And also on my calendar. So my calendar, so I am always checking my calendar what am I doing today.

Participant 03

\subsection{Reinforcement of technology adoption}

Factors that reinforced technology adoption and encouraged the participant to use the devices or even upgrade to a newer version were mentioned in the form of family members and friends, economic factors, and the familiarity of the device. Friends and family members would often encourage the participants to use and explore the functionality of the devices.

So she gets me to do things I might not do otherwise. Look things up on google or when you want to know which movie is on or which time. Participant 06

[My friends] say ,If you got such a fancy phone. You should use it all the time’.

Participant 04

Familiarity with a device or software seems to encourage the participants to look for apps that meet their needs.

I don't know if that relates to the stroke, I think I am just more aware of the apps and stuff like that. So I found out there is another app that does calendar work that is brighter and better, so I am switching to that one. Participant 03

Two participants mentioned cost of devices. One participant preferred a feature phone over a smartphone because it was cheap to purchase and cost 
efficient to use. Another participant mentioned that the phone was provided because of his disability.

It came through Gold Card from the Work and Income people. It wasn't just for stroke; it was for disabled people.

Participant 02

\subsection{Leisure activities}

Just a few of the participants used the smartphone as part of leisure activities. The smartphone, iPad, and laptop allowed them to take photos, read the news and journals online or listen to Spotify, an app that plays music.

\subsection{Connecting with others}

The use of the devices seemed to be strongly associated with the possibility of connecting with others. The use of texts, emails, Facebook, and Skype was mentioned in this context. During the hospital stay, the phone and iPad enabled the owner to stay in touch with family members and friends. After the hospital, the phone helped them to be permanently reachable.

No, I did never have Facebook. So my sons set me up when I was in rehab so I could keep everyone up to date with my progress and keep in touch with everybody.

Participant 01

And when I was in the hospital I used my phone a lot because it was my main way of keeping in touch with people.

Participant 06

The phone and social platforms such as Facebook enabled people to share their experiences during the rehabilitation process as well as enabling 
involvement in research projects. Multiple participants mentioned that they started using Facebook after the stroke to stay in touch with family members and people they met through their social work. Some of the participants began to text and use Facebook more after the stroke due to having more spare time.

So she [the researcher of a different study] is up here, and it looks like she is stabbing me. So I put it up on FB with 'stabbing of a lab rat' or something like that.

\section{Participant 03}

Social connections were essential to all the participants. The use of Skype was referred to in the context of work and staying in touch with friends and family members who lived far away. Emails seemed to be a vital form of communication for most participants and were accessed through smartphones and laptops, but the laptop seemed the preferred way of responding to them.

We came back on Thursday... Friday night. And there were 53 emails for me. Most of it was rubbish of course, you know, supermarket information and that sort. But there were others too, and I rely on them.

\section{Participant 02}

\subsection{Contributors to the rehabilitation process}

Not many participants saw the use of the devices as an active part of the recovery process. Two participants had used apps in the past that trained fine motor skills as part of their rehabilitation. One of the participants mentioned using the computer keyboard and apps on the smartphone as part of her self-organised rehabilitation at home. 
But what I am trying to do with the iPad and the computer is... and it is hard, but I am trying to do bilateral typing. But it is difficult and laborious but I try to do it a bit.

Participant 06

\subsubsection{Barriers}

There were several barriers mentioned during the interviews that would make the use of the different devices challenging. In the following section the themes of physical and cognitive limitations, issues in the context of vision, device-specific limitations, the context of use, and possible compensatory movement patterns will be outlined.

\subsection{Physical and cognitive limitations}

Physical limitations after the stroke seemed to impact on the use of devices. All the participants mentioned issues with their fine motor skills regarding their dexterity and individual finger control, which made typing on smartphones and answering calls difficult.

I used to type with two hands, and now I just have to use the one finger which is frustrating because it takes very long.

Participant 01

Both hands are often needed to hold and interact with the smartphone or iPad. Participants mentioned that they found it difficult to use the affected hand for fine motor tasks. The affected hand was therefore primarily used to hold the phone while the less affected hand was used to interact with it. Devices that made the use of both arms necessary were experienced as being challenging, for example using a tablet to take a photo.

The reduced amount of sensation on the affected side and issues of proprioception caused a lack of trust in the ability to hold the phone with the 
affected hand while walking. Some of the participants mentioned that the affected hand would just open without them being aware of it. They would need to continually look at it to make sure that it would hold the device firmly.

I mean if I put it in there for a little while [points at the affected hand] it would stay in there, but then it would fall out.

Participant 01

Vision and cognitive limitations seem to further impact on the use of devices. One of the participants had an iPad where the font size had been increased while another always carried a pair of glasses to enable him to read the phone. Limited or even impaired vision can pose a significant barrier. However, cognitive limitations seem to have an even stronger influence. During the interviews, participants were asked as part of the follow-up questions if they had ever used the voice command function on their smartphone. One of the participants mentioned that his speech was quite affected by the stroke, which caused him to be extremely aware of the way he articulated words to minimise a potential slurring of his voice. He still had the impression that his speech meant he would not be able to use this software function.

I don't think my speech would be sufficiently clear to do it.

Participant 05

Another participant mentioned that the stroke had impacted on his ability to use a smartphone because of his cognitive abilities. 
Well I suppose bigger buttons would make the use of the phone a bit easier but I don't think that is really the problem. The problem is up here [tips on his forehead].

Participant 03

\subsection{Device specific limitations}

Apart from limitations caused by the stroke, there were some devicespecific limitations pointed out. One of the participants used an iPhone that her daughter had recommended to her. However, she felt the device was too big to hold comfortably. This was an issue reported by a number of participants

[It is] harder to hold! And... if I have got a call, it is actually much easier to put it on speaker phone.

Participant 6

Yeah, they [the smartphones] are too big, and I could use them if I put them down flat, but I am too clumsy. And anyway, I am not on Facebook or anything like that.

Participant 05

The size of smartphones such as the iPhone 6 caused difficulties in holding the device in the affected hand or storing it in a pocket while walking. One participant who relied on a walking stick had a pouch hanging around her neck where she would store her smartphone.

Yeah, so my aunty made this [the pouch] because if I wouldn't wear pockets, there is no way to wear my phone because I couldn't carry it, so she made me this which is really good.

Participant 01 
While all the participants were familiar with the use of a feature phone, just some of them had chosen to use a smartphone. The software on the smartphone seemed to pose a number of barriers, such as functions that seemed unfamiliar, functions which were too difficult to use, not needed, or were even regarded as a way of cheating and taking a shortcut in the process of training the affected arm and hand.

Ah yeah [the partner] has got one and she has got all sorts of other things on it which she can't really use, and I can't be bothered looking at it. Because if you put it down there and I have got to.... ah yeah... I don't know.

Participant 05

Most participants described the feature phones and smartphones as too challenging for writing texts or even emails. The difficulties comprised the actual writing as well as editing of the text.

When you wobble even just a little bit, it will type the same letter three times, so you have to cancel it and start again.

Participant 06

Two participants mentioned they would use the phone mainly to make and receive calls. One participant found the process of texting too tricky while the other one simply preferred to talk to people on the phone, which was from his point of view faster than exchanging multiple texts.

I don't text people! They text me saying something like ,Hey what are you up to'?. Only because... not because of lack of use. But I want to talk supplementary of that 'Are you coming tonight'?

Participant 05 
Participants who preferred the feature phone over a smartphone pointed out that the smartphones offer functions they would not need or that they would find too difficult to use. Multiple participants emphasised during the interview that they would not play online games anyway and would therefore not need a smartphone or a tablet.

Nah, I don’t play any games at any time. I am not a gaming person.

I am a doer.

Participant 05

One of the participants associated the use of the voice command on the smartphone as a way of cheating in the rehabilitation process.

Well, I always had this weakness that I like to challenge myself to do things the more complicated way. If there is a shortcut, that is a cheat.

Participant 04

\subsection{Context of use}

Another barrier that influences the use of technology, and especially phones, is the context of use. One participant mentioned that he used his feature phone before the stroke only in the context of his work. After the stroke, he preferred to talk to people on a landline phone because he preferred to be in his home environment while calling someone. He did mention that he liked elements of his feature phone such as the ability to store, manage, and access contacts. However, he would often leave the phone on his kitchen bench at home rather than taking it with him when he left the house. 


\subsection{Compensatory movement}

Some of the comments that were made during the interviews and even more so in the observations of use of feature and smartphones indicate that the devices potentially contribute to the development of compensatory movement patterns. One participant mentioned that he had to put a phone on a table to be able to use it with the less affected hand. Another participant said that he would train the affected hand in the context of his rehabilitation, but at home he would rely on the less affected side.

Very, very difficult. Because at home I exclusively use my right hand. Participant 04

\subsection{Discussion}

The smartphone and the feature phone were constant companions that provided a feeling of safety, enabled participation in daily activities and social roles, and were used by some participants as an active part of the rehabilitation process. Impairments caused by the stroke and device-specific limitations can pose barriers to using the devices. The themes of context of use, which impacts technology adoption, and potential encouragement of compensatory movement are ones that were not raised prior to this study.

\subsubsection{Motivation}

The themes relating to motivation to use telecommunication devices aligns with previous studies (Gustavsson et al., 2018, pp. 564-565; Kamwesiga et al., 2017, pp. 441-446). In the following section, the themes arising from the thematic analysis will be discussed and discrepancies and additional themes will be highlighted.

\subsubsection{Connecting with others}

The theme of being able to connect with others has been cited as a significant motivator to use feature phones and smartphones after a stroke 
(Gustavsson et al., 2018, p. 564; Kamwesiga et al., 2017, pp. 442-443). The devices seem to become a core element that allows maintenance of identity and the ability to continue with everyday life after the stroke, but can also evoke a feeling of being constantly controlled and checked on (Gustavsson et al., 2018, p. 564).

The use of feature phones and smartphones enables the stroke survivor to feel they are safe. It also reassures their family members. This has been mentioned in previous studies (Kamwesiga et al., 2017, pp. 445-446). It has been found that stroke survivors carry their phones regularly with them after their stroke (Gustavsson et al., 2018, p. 564), which is supported by the results of this study. The design of a phones that facilitates an initiation of use could easily be integrated into daily activities, enable survivors to stay in touch with people and be a constant companion that reminds the user to use the affected arm.

\subsubsection{Reintegration}

Devices enable and facilitate reintegration into previous social and professional roles (Gustavsson et al., 2018, p. 565). A recent study in Uganda (Kamwesiga et al., 2017, p. 444) that investigated the use of feature phones after a stroke reported that the feature phone became an essential tool to sustain the family's income. Most of the participants in the current study were already retired or had to stop working because of the stroke. This might offer an explanation of why the theme was not as prominent in this study. However, younger stroke survivors could potentially benefit from a device that addresses their physical limitations to be able to return to their work. This could encourage an initiation of use.

\subsubsection{Technology adoption}

Family members and friends, economic factors, and the familiarity of the device reinforced technology adoption among stroke survivors, which is in line with previous findings (Gustavsson et al., 2018, p. 566). It has been mentioned in studies involving participants with acquired brain injuries that therapists can be motivators who encourage an increased use of the phone in daily activities 
to compensate for cognitive limitations (Larsson Lund, Lövgren Engström, \& Lexell, 2011, p. 406). This study excluded participants with cognitive limitations. However, digital devices might enable participation in daily activities for survivors with cognitive limitations.

\subsubsection{Leisure}

In the current study, just three of the six participants used the devices for leisure purposes. The study by Gustavsson et al. (2018, p. 565), on the other hand, reported that all of their participants used the devices for entertainment purposes such as listening to the radio and music, watching TV, or playing games. The participants described the activities as a source of inspiration and motivation to interact with the devices. This was not confirmed in the current study. A possible explanation might be that the three participants who used the phone for leisure activities owned a smartphone which offers more applications, while the three participants who did not use the phone for leisure purposes owned a feature phone.

\subsubsection{Rehabilitation purposes}

The use of the devices for rehabilitation purposes is of particular interest in the context of this study. Participants in a previous study (Gustavsson et al., 2018, p. 565) practised their keyboard skills to be able to use the computer again. It has been further reported that stroke survivors tend to train their cognitive skills by playing games such as Sudoku on the devices. The devices would further offer the possibility of tracking recovery progress (Gustavsson et al., 2018, p. 565). This aspect was not confirmed in the present study, but potential to provide augmented feedback could be included in the design prototype. Just two of the participants used the device to play a game named "Crabs" which was recommended to them by a therapist to train fine motor skills. Instead of using an app, the form of the phone could train fine motor skills every time the user picks it up.

A slightly different use of the devices in the context of rehabilitation has been reported by Kamwesiga et al. (2017, p. 445). Participants faced limited 
access to rehabilitation resources and used the feature phone to reach health professionals. This was not a finding of the present study. However, the use of feature phones and smartphones in the context of stroke rehabilitation seems to have the potential to improve healthcare access and be an active part of training both gross and fine motor skills.

\subsubsection{Barriers}

\subsubsection{Physical and cognitive limitations}

The effects of physical and cognitive limitations such as limited fine motor skills correlate with previous findings (Gustavsson et al., 2018, p. 566). It has been reported that stroke survivors buy new hardware and try new functions to address those limitations (Gustavsson et al., 2018, p. 566). This is an aspect that has not been confirmed in the present study but could potentially offer insights into making use of the devices feasible.

\subsubsection{Device specific limitations}

Participants were hesitant to use devices, particularly the smartphone, when the functions were perceived as too difficult to use, not needed, or as a way of cheating. Other than the aspect of cheating in the recovery process, previous findings were confirmed in this study (Fager \& Burnfield 2014, p. 126; Gustavsson et al., 2018, p. 566). Stroke survivors might need support to learn and use unfamiliar technology. Participants in the current study who explored the capabilities of the devices were often encouraged by family members and friends.

In the context of able bodied older adults, similar factors applied. Subjective barriers that influence the use of the devices were mentioned in the form of a lack of knowledge that can be reinforced through decreased mental and physical abilities because of old age (Berenguer et al., 2017, p. 107).

The participants in the current study felt comfortable using the device that they owned; just one participant mentioned that she preferred a slightly smaller phone. Previous studies indicate differing preferences for the size of the phone among genders, but the reports on preference for bulkier or smaller phones 
contradict each other (Kim et al., 2007, p. 955; Kurniawan, 2008, p. 894).

\subsubsection{Context of use and compensatory movement}

Two themes that relate to possible barriers to using electronic devices are the context of use and compensatory movement patterns. The two themes have not been reported in previous studies.

If the devices had been mainly used in the work environment before the stroke, motivation to use them might be lacking after termination of employment following the stroke. Possible compensatory movement patterns, on the other hand, seem to be reinforced when the phone is difficult to hold. Most of the participants used the less affected side to pick up the phone, access the software, and hold it while calling someone. To dial a number, they put it on the table and then picked it up with the less affected hand once they had confirmed the call to hold it to their ear. Some of the participants reminded themselves to use the affected side as much as possible. However, use of the affected side was difficult due to decreased fine motor skills.

\subsection{Conclusion}

ICT is an essential part of everyday life after a stroke. Participants used the computer, laptop, smartphone, and feature phone daily to communicate with others, sustain their social roles, and manage daily tasks. There were several barriers pointed out that impact on the use of the devices, especially the smartphone. The next chapter will focus on the development of a design prototype that addresses common barriers to make use of the device feasible, while restraining movement in order to initiate use of the affected arm and hand. 


\subsection{Limitations}

Participants for the study were recruited through physiotherapists in Auckland and a leader of a stroke support group in Wellington. However, no formal assessment protocol was involved prior to the interviews. This might have meant that some of the participants did not meet the outlined minimal movement criteria of the wrist. A further limitation is that two of the interviews were conducted in the participants' home environment, while four took place at a research facility. The context of the home might have potentially contributed to memory barriers and context-based motivations. 


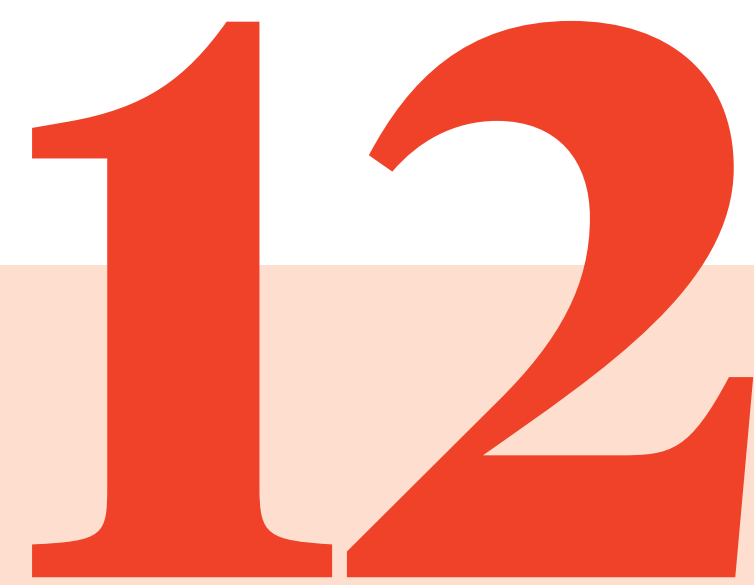

\section{Design Workshop}




\subsection{Introduction}

This chapter addresses the research question:

1.3.1 How can we design an everyday object that restrains movement of the less affected arm to facilitate and initiate the use of the affected arm?

To address this research question two objects were developed, using a RTD methodology, to investigate possible ways a design could restrain movement to initiate the use of the affected hand. The design process started with a design workshop that focused on the findings of the interviews with stroke survivors. The outcomes of the design workshop were further refined and will be outlined in Chapter 13.

\subsection{Background}

\subsubsection{Classification of influence}

The formative usability evaluation of the radio prototypes indicated that the persuasive design concept could be experienced as coercive. Fogg's Behaviour Model (Fogg, 2009a, p. 5), which was used for the radio prototype, focuses purely on persuasive design. The "Classification of Influence Based on Intended User Experience” (Tromp, Hekkert, \& Verbeek, 2011, p. 12), however, takes account of different forms of influence such as coercive, seductive, persuasive, and decisive, see Figure 86. The description of persuasive designs correlates with Fogg's description of a trigger, which was used as a basis for the development of the radio prototype.

The separation between decisive and persuasive design concepts can in some cases be challenging to narrow down. Decisive design concepts restrain movement but are experienced as being part of the design, for example ergonomic restrictions. Persuasive design solutions can be added to the design to evoke an 
initiation of use. However, in terms of the analysis of the design concepts, only the user of the design can categorise its influence (Tromp et al., 2011, p. 12).

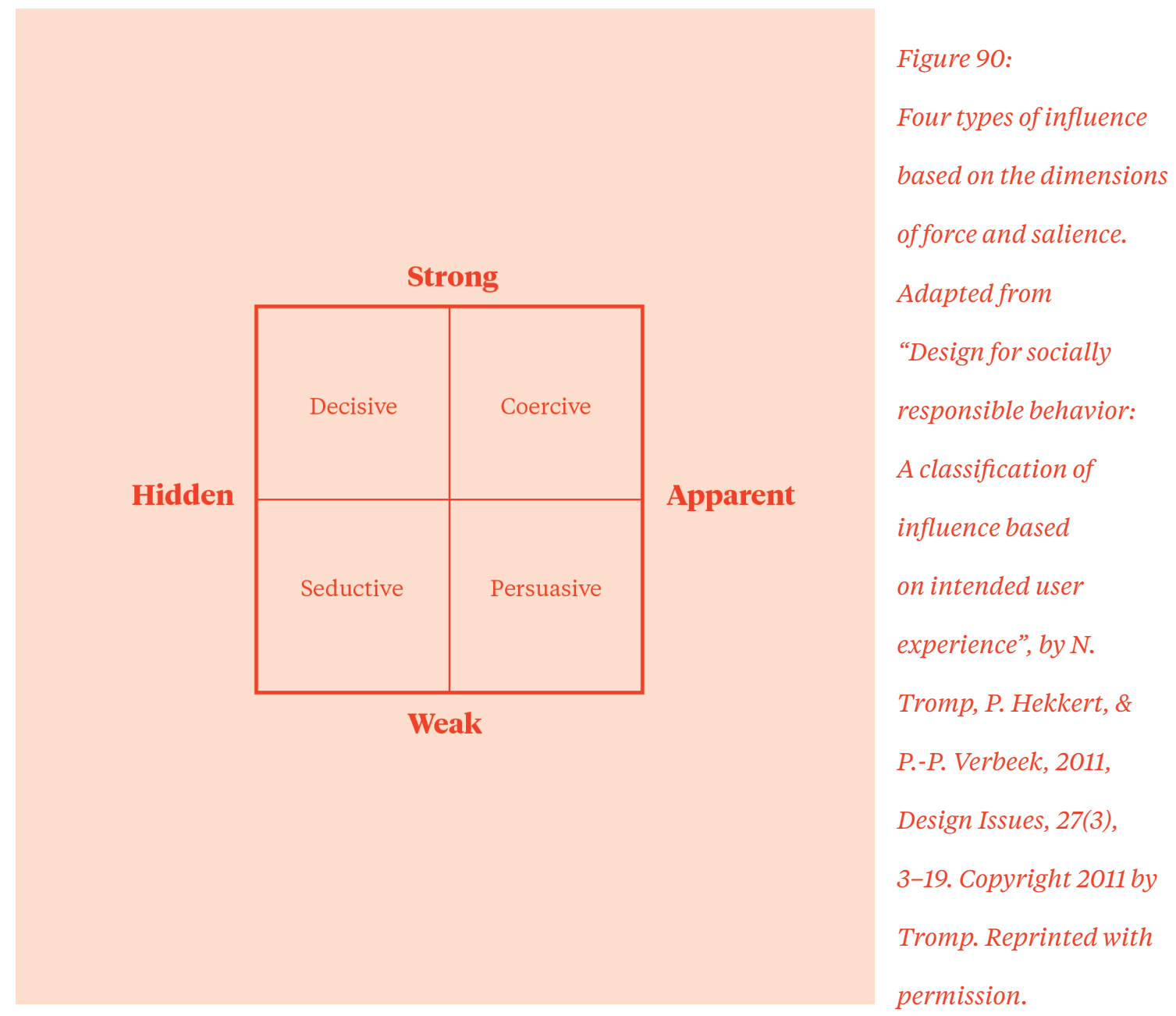

The different forms vary in their force of influence as well as the salience of influence, see Figure 90. The classification was chosen for the analysis of the design concepts to map influences that are outside of the persuasive design area. It needs to be emphasised that the classification and strategies outlined as part of the classification do not aim to guarantee a specific influence (Tromp et al. 2011, p. 13) but offer an indication of the anticipated influence on the user.

The design concepts developed as part of the design workshop were not evaluated by users, but two refined design concepts were tested by stroke survivors 
to evaluate the expected influence, see Chapter 13.

\subsubsection{Design precedents}

A search for design examples in the form of phones or phone attachments that stroke survivors can buy or that have been reported in the literature produced few results. The only commercial available phone is the OwnFone (2018) which is a mobile phone that uses reduced features and enables the user to personalise the screen design. The phone offers 12 contact buttons that appear on the screen as names or images. To make a call, the user simply presses the name or image of the person. The aim of the phone is to make its use feasible for stroke survivors but it does not restrain movement.

Devices such as the "Lazy-hand" (Lazy-hands grips, 2018) offer handles that can be attached to phones to make the holding of the phone feasible but are not specifically designed for stroke survivors. However, they could potentially address a lack of fine motor skills and enable to survivors to hold larger phones. It seems no devices or phone cases are available to stroke survivors that take account of common motor impairments and enable the user to hold bigger phones. There also seems to be a lack of devices or phone cases that restrain movement to evoke an initiation of use.

\subsection{Methods}

For this study, a RTD methodology was used. Results of the design were analysed with thematic analysis in a deductive form. Two design prototypes were further refined and will be outlined in Chapter 13.

\subsubsection{Participants}

Five participants took part in the design workshop. Four were female $(n=4)$, and one was male $(n=1)$. Four were enrolled as Master's students at the Victoria University of Wellington School of Design in the course "Design Innovation", and one worked as an associate professor at the School of Design. Two participants focused on media design $(n=2)$, while three participants had a background in 
industrial design $(n=3)$. All the participants were involved in research projects that focused on design for health interventions. Each participant provided written consent.

\subsubsection{Research through design}

12.3.2.1 Design workshop

The design method cards were used as part of a design workshop to investigate how handheld phones could evoke an initiation of use. The design workshop followed a RTD methodology and took place at the School of Design, Victoria University of Wellington, New Zealand. The workshop focused on expansive designs (Krogh et al., 2015, pp. 46-47). The outcomes of the design workshop were design concepts in the form of sketches and an explanation of how the designs would be used; see Figure 91 for an overview of the design workshop. The different steps of the workshop will be outlined in the following section. 


\begin{tabular}{|c|c|c|}
\hline Activity & Duration & Description and rationale \\
\hline $\begin{array}{l}\text { Background } \\
\text { - Intro } \\
\text { - Goal of } \\
\text { workshop } \\
\text { - Background } \\
\text { info } \\
\text { - Framing the } \\
\text { problem }\end{array}$ & $20 \mathrm{~min}$ & $\begin{array}{l}\text { Introduction to the topic and goal of the } \\
\text { workshop. The information was divided } \\
\text { into the parts of: } \\
\text { - Design strategies and focus on the } \\
\text { "restraint" strategy } \\
\text { - Barriers to using smartphones and } \\
\text { feature phones after a stroke } \\
\text { - Motivation for using smartphones and } \\
\text { feature phones after a stroke } \\
\text { In order to avoid a design fixation } \\
\text { specific examples or available design } \\
\text { solutions were not presented. }\end{array}$ \\
\hline $\begin{array}{l}\text { Brainstorm } \\
\text { activities } \\
\text { - General } \\
\text { introduction } \\
\text { - Power of ten } \\
\text { - Presentation of } \\
\text { concepts }\end{array}$ & $30 \mathrm{~min}$ & $\begin{array}{l}\text { Based on the testing of the digital } \\
\text { design method cards, it seems that a } \\
\text { focus on all the strategies at once can } \\
\text { slow down the creative process. For the } \\
\text { brainstorming activity, the topic of } \\
\text { "restraint" was chosen. The "Power of } \\
\text { Ten" was used as a creative technique } \\
\text { which is based on extremes to } \\
\text { encourage liberated thinking. }\end{array}$ \\
\hline $\begin{array}{l}\text { Sketching Part } \\
\text { 01 } \\
\text { - Introduction } \\
\text { - Crazy eight }\end{array}$ & $15 \mathrm{~min}$ & $\begin{array}{l}\text { In the first sketching exercise, the } \\
\text { participants had to sketch eight } \\
\text { design concepts in a limited amount } \\
\text { of time. Each participant had a } \\
\text { minute per idea before moving on to } \\
\text { sketching the next idea. This limited } \\
\text { amount of time allows participants } \\
\text { focus on the quantity of the ideas } \\
\text { rather than the quality. }\end{array}$ \\
\hline $\begin{array}{l}\text { Sharing ideas } \\
\text { - Share out } \\
\text { - Voting }\end{array}$ & $30 \mathrm{~min}$ & $\begin{array}{l}\text { Each participant was asked to present } \\
\text { their eight ideas and then vote for the } \\
\text { concepts with the most potential. }\end{array}$ \\
\hline $\begin{array}{l}\text { Sketching Part } \\
\text { O2 } \\
\text { - Refinement } \\
\text { - Presentation of } \\
\text { concepts }\end{array}$ & $30 \mathrm{~min}$ & $\begin{array}{l}\text { Each participant had to choose one } \\
\text { concept and produce a refined sketch } \\
\text { within } 25 \text { minutes and then present } \\
\text { the design concept. }\end{array}$ \\
\hline $\begin{array}{l}\text { Next steps } \\
\text { - Plan for next } \\
\text { step } \\
\text { - Feedback on } \\
\text { how session went }\end{array}$ & $5 \mathrm{~min}$ & $\begin{array}{l}\text { The workshop ended with an } \\
\text { explanation that some of the designs } \\
\text { would be chosen, refined and tested } \\
\text { with stroke survivors. }\end{array}$ \\
\hline
\end{tabular}

Figure 91:

Design workshop

overview. 


\subsection{Introduction}

The introduction covered the goal of the workshop and context of the study. For the workshop, the topic of "restraint" was chosen. Based on the feedback provided as part of the usability evaluation of the website www.limited-use-only. com, see Chapter 8, physical method cards were developed for the workshop. The front of each card showed the title of the main theme in combination with an abstract icon. On the back, an explanation was provided as well as a summary of the main strategies, see Figure 92. During the workshop, the cards were verbally presented, and specific examples were given outlining the content of the card. Three main topics were focused on in the introduction covering: design strategies (red), barriers (blue) and motivation (green) of using feature and smartphones, see Figure 93.

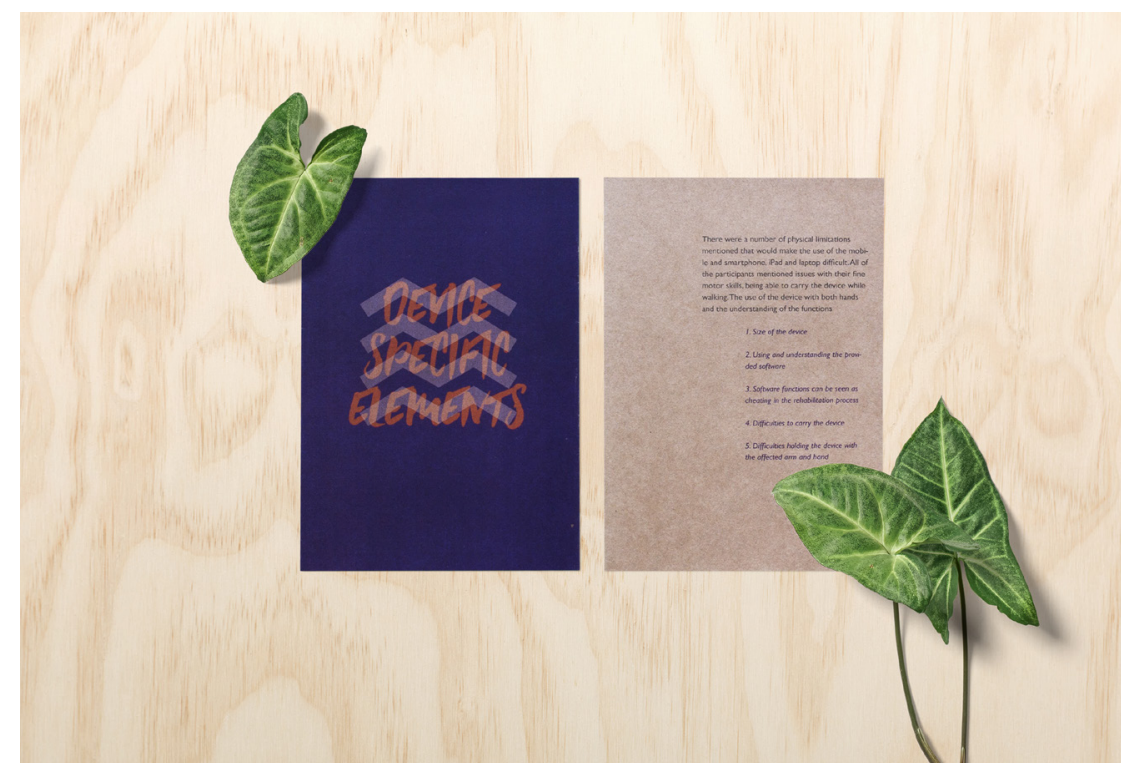

Figure 92:

Each card contained a further description and summary of the main themes on the back.

\subsection{Brainstorming}

After the introduction to the workshop, a brainstorm activity focused on the development of concepts that could facilitate initiation of use by using a feature phone or smartphone. Additional cards based on the creative design method "Powers of Ten" (Brown, 2017) were presented to challenge the creative process. 


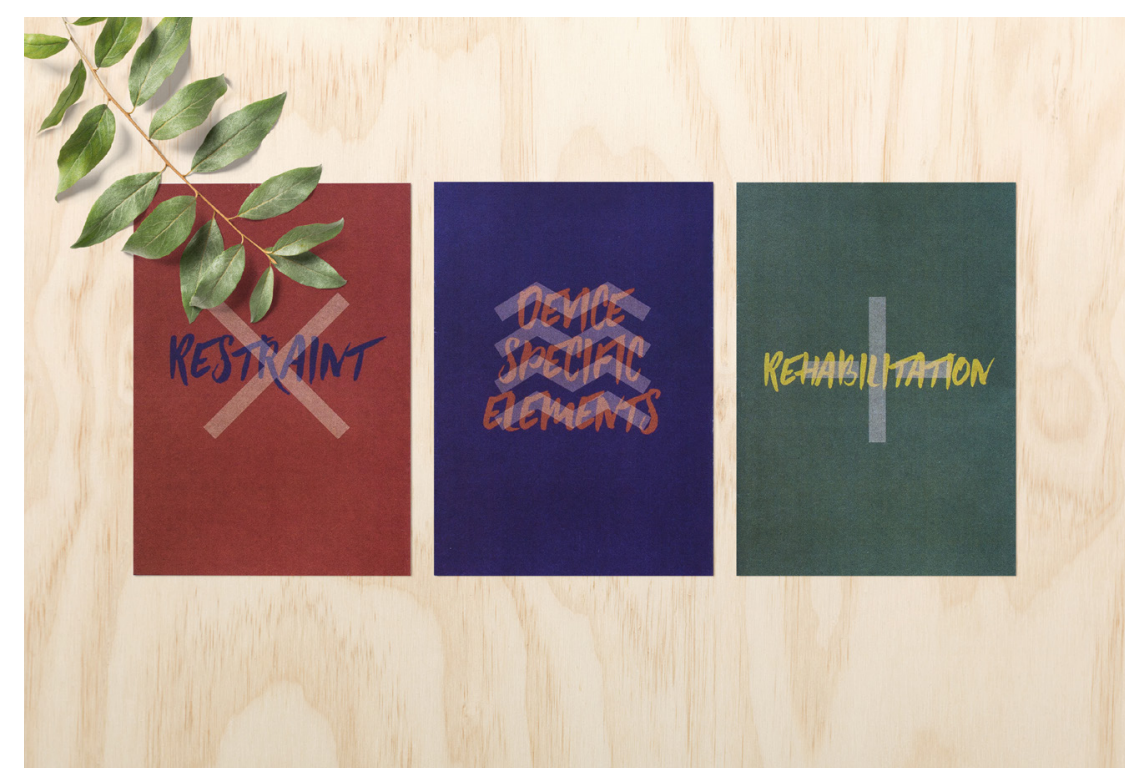

Figure 93:

Overview of the three topics of the physical design method cards.

The cards raised the questions:

1. How can the design remind the user?

2. How can the design force the user?

3. What if it had to cost more than a million dollars to implement?

4. What about under 25 cents?

5 . What if you could only use it when you are alone?

6 . What if you could only use it in collaboration with others

\subsection{Crazy eight and refinement of design concepts}

After the brainstorming activity, the participants were asked to develop design concepts in a short timeframe. Each participant had a minute to sketch an idea before moving on to the next one. After 8 minutes each participant was asked to present the eight developed design ideas. A sketch template was provided for the activity, see Figure 94. The participants were asked to vote for the concepts they believed would be beneficial in the intended context. Twenty-five minutes of sketching followed that focused on refinement of a design idea.

\subsubsection{Analysis}

The concepts developed as part of the design workshop were analysed using thematic analysis in a deductive form (Braun \& Clarke, 2006, p. 83) according 
to the description of the "Classification of Influence Based on Intended User Experience”, which is based on the expected influence the design has on the user. The thematic analysis of the developed design concepts of the design workshop focused on the themes of persuasive, coercive, decisive, and seductive.

\subsection{Results}

\subsubsection{Research through design results}

\subsubsection{Brainstorming and sketching activities}

The following section outlines the outcome of the design workshop. Thirtyone ideas were developed by the participants in the form of sketches and notes on post-its, see Figure 95. The design concepts developed as part of the crazy eight technique will be outlined first, followed by a description of the refined design concepts. 


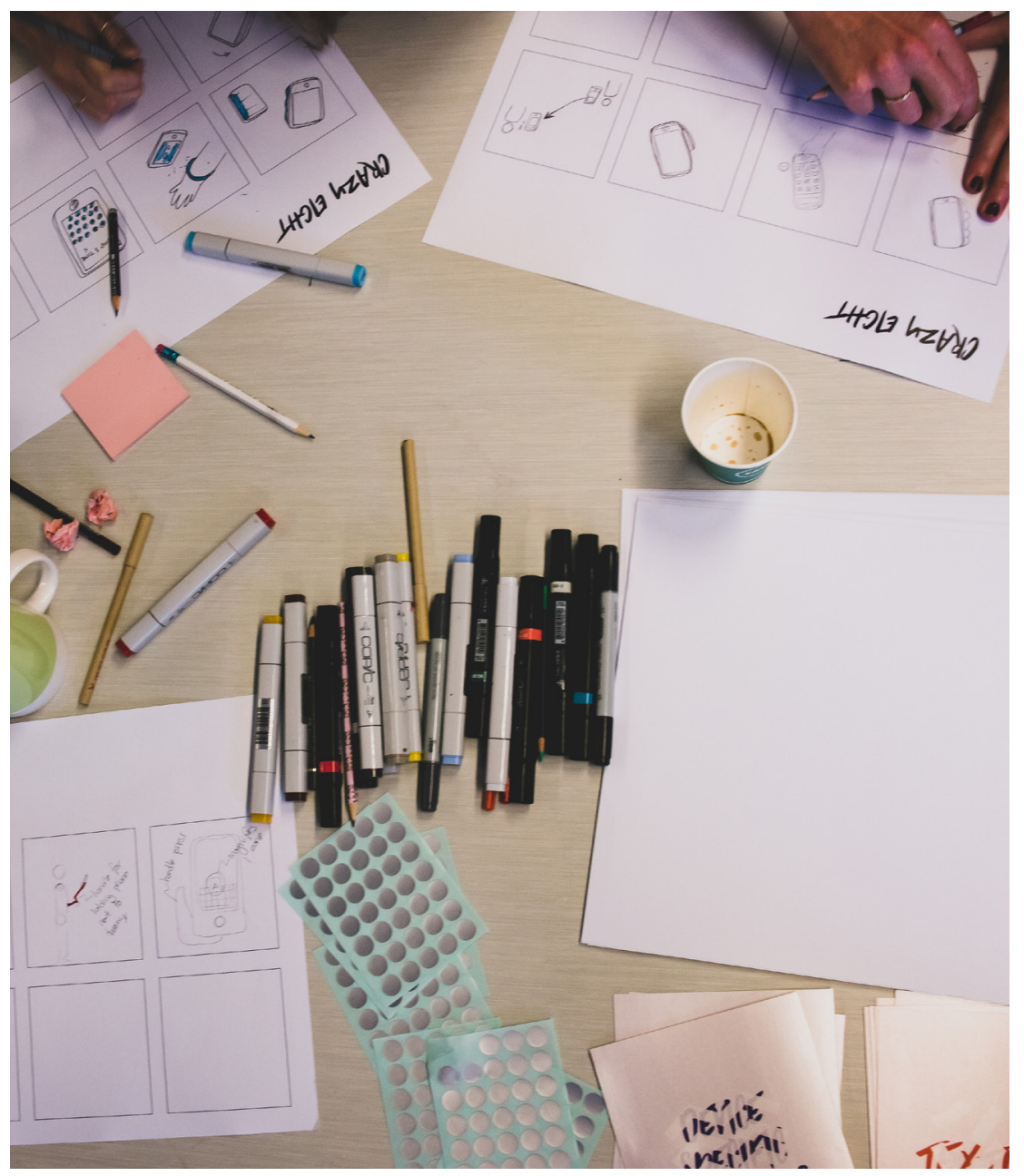

Figure 94:

Crazy eight sketching activity.

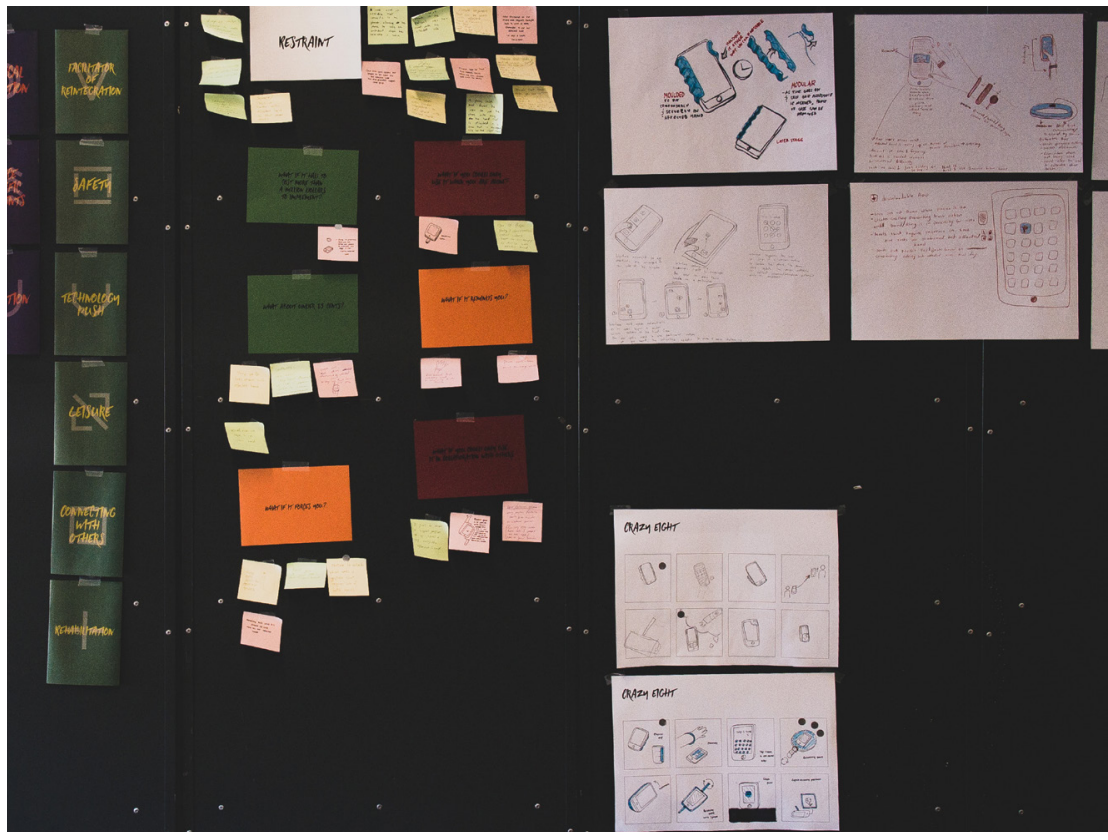

Figure 95:

Brainstorming

outcomes and sketches

during the workshop. 
12.4.1.2 Crazy eight

12.4.1.2.1 Participant 01

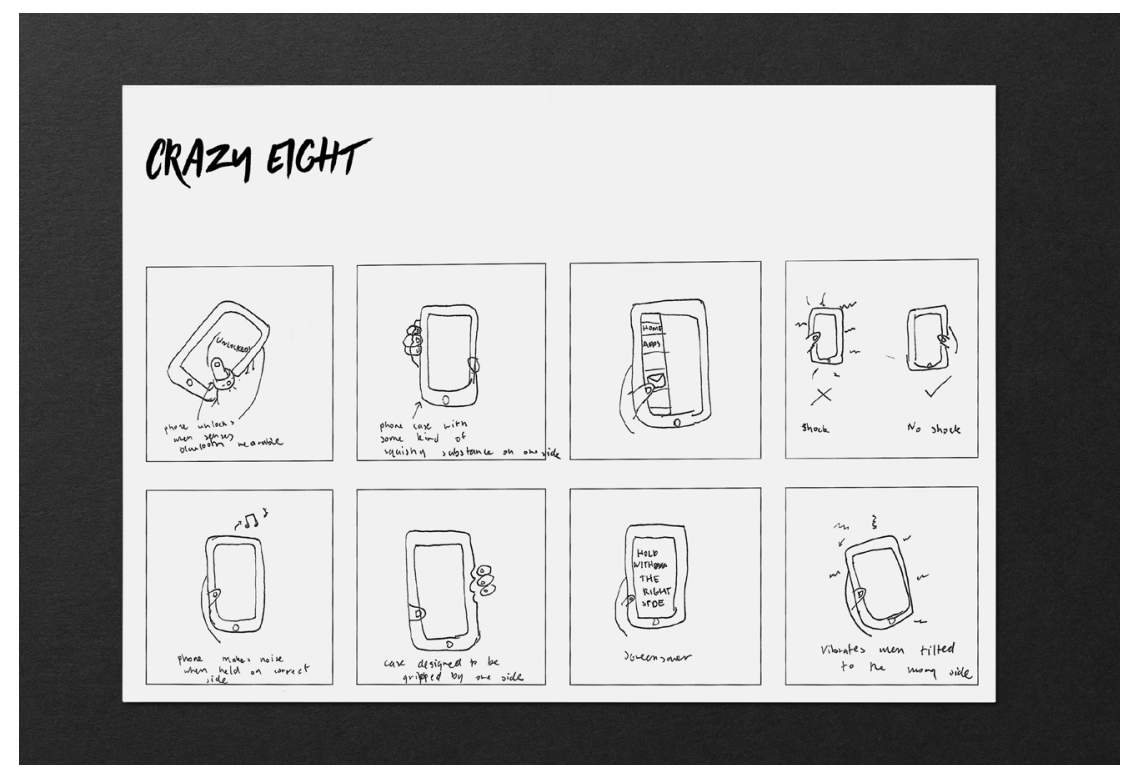

Figure 96:

Participant 01 crazy

eight design concepts.

Design concepts of participant 01 (Figure 96) from top left to bottom right:

1. A bluetooth ring is worn on the affected side and only unlocks the phone if that hand is used

2. Phone with some squishy elements on the side that support the grip of the user to allow an initiation of use

3. Interface that is situated on the side of the phone that can be reached more easily with the affected hand encourages in this way an initiation of use

4. The phone shocks the user if the less affected side is used to hold the phone to discourage compensatory movement

5. The phone makes a pleasant noise when it is held on the correct side as a positive reminder

6. A case that allows an easier grip with the affected hand and uses ergonomic restrictions 
7. Screensaver that reminds the user to use the affected side

8. Phone that vibrates when held with the wrong side

\subsection{Participant 02}

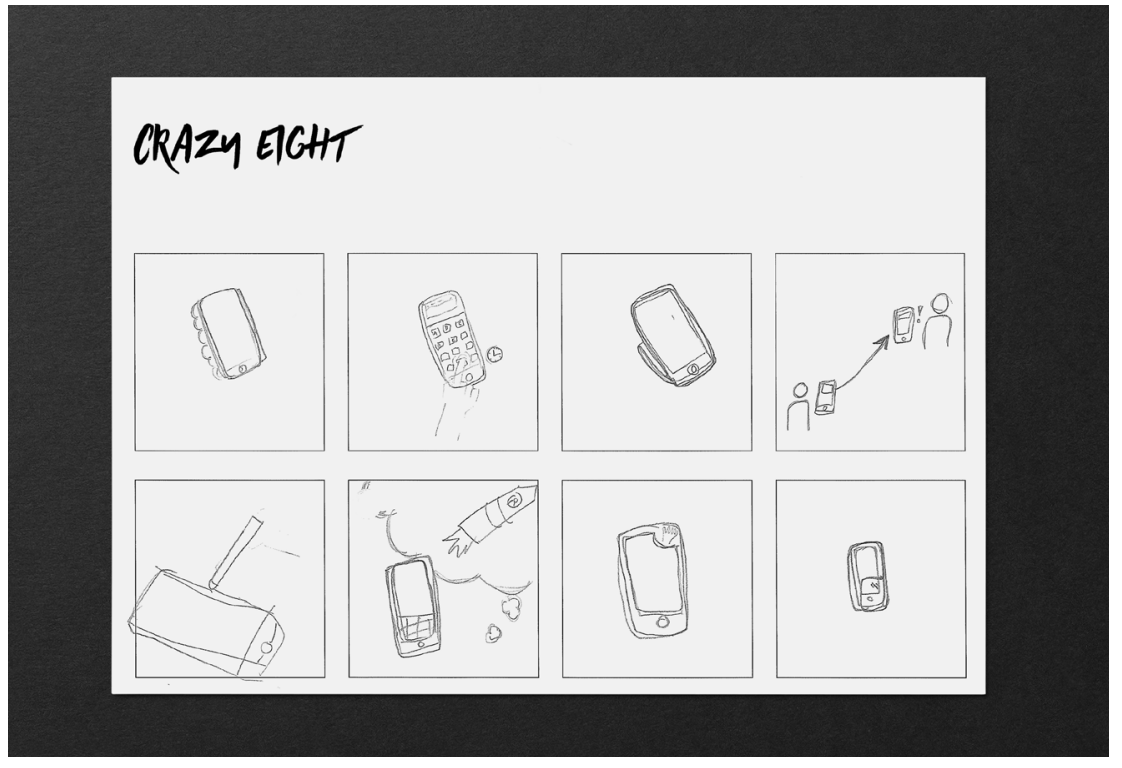

Figure 97:

Participant 02 crazy

eight design concepts.

Design concepts of participant 02, see Figure 97:

1. A phone case that supports the affected hand and has a sharp edge on the side of the less affected hand to discourage compensatory movement

2. Concept for an app with bigger buttons that allows use of the software and initiates use of the fingers

3. A handle at the back of the phone to support the grip and allows initiation of use of the affected hand

4. Texting a support person regularly with the affected hand as a reminder to initiate the use of the hand

5. Stylus pen input that reduces accidental double-clicking with the affected hand to minimise device specific problems

6. Game that can be played only with the affected hand 
7. Visual reminder on top of the phone to use the affected hand

8. One side of the phone is covered so only the affected hand can reach inside to use it

\subsection{Participant 03}

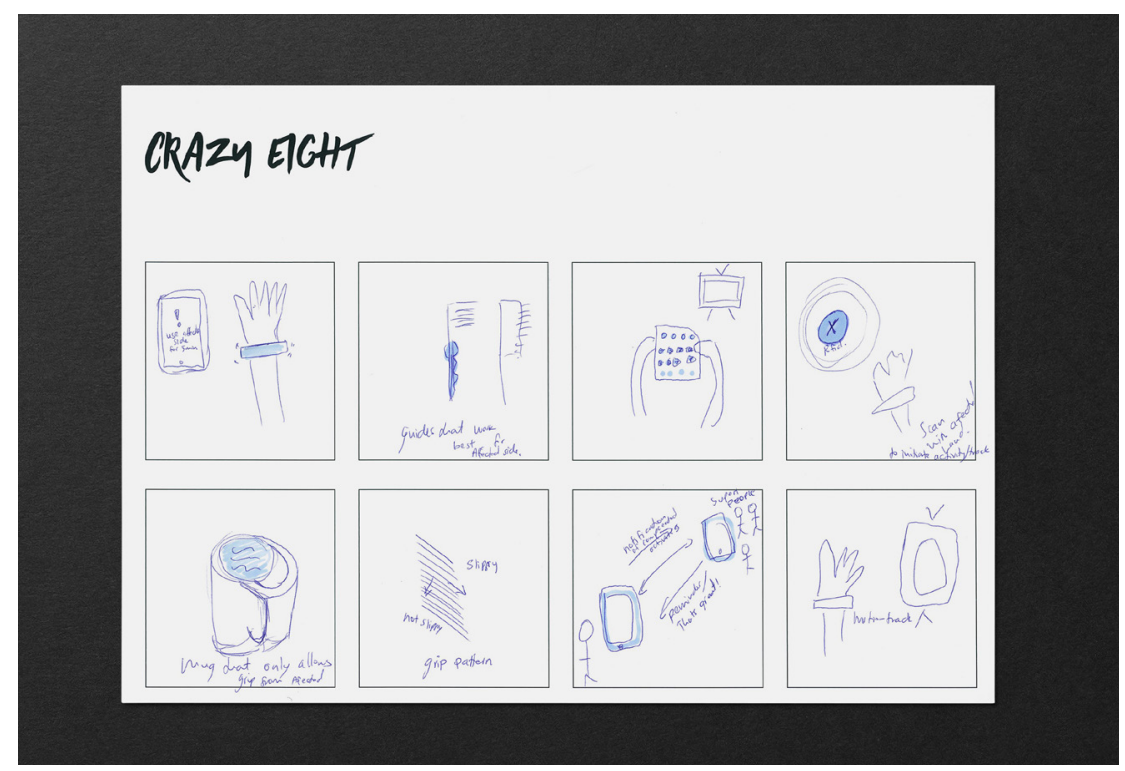

Figure 98:

Participant 03 crazy

eight design concepts.

Design concepts of participant 03, see Figure 98:

1. A bracelet that is worn on the affected side and unlocks the functionality of the phone

2. Ergonomic restrictions that allow the use of the phone only with the affected hand

3. Handles that allow the use of a TV remote control

4. A bracelet that is worn on the affected side and monitors the use of the affected arm in the home environment

5. Coffee mug that restricts use to just one side of the body

6. Grip pattern that has a slippery and a non-slippery side

7. Concept for texting a support person that reminds the user to use the affected arm 
8. A motion tracker system worn on the affected side to gather information on how often the affected arm is used.

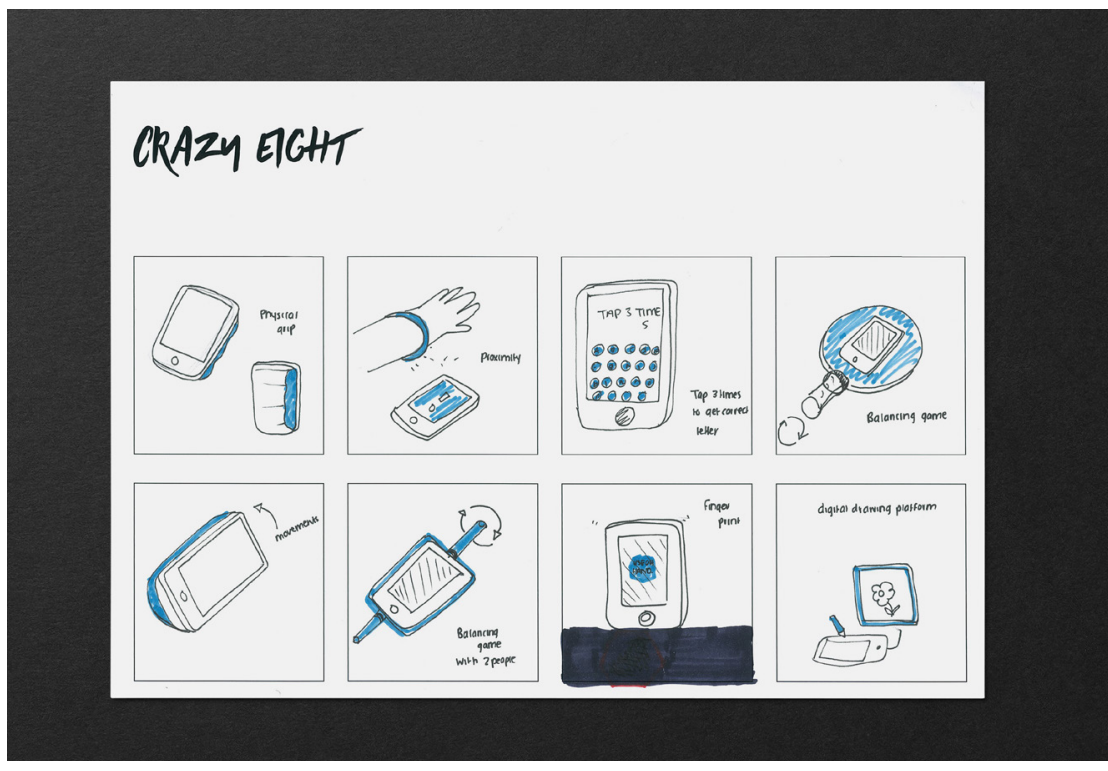

Figure 99:

Participant 04 crazy eight design concepts.

Design concepts of participant 04, see Figure 99:

1. A phone case with a grip on one side that makes it easier to hold with the affected hand

2. A bracelet that is worn on the affected side and activates the phone if it is in close proximity

3. An app that requires the user to tap three times. The app compensates for lack of fine motor skills to initiate use of the affected hand

4. A game that requires the user to balance the phone inside a handle

5. A phone that rolls to the side and requires both hands to hold it

6. A balancing game that uses the phone in the middle. Two people are required to play the game. The restraining effect is reinforced through the social interaction

7. An app that requires the affected finger to be scanned 
8. Use of the smartphone as a digital drawing platform as an alternative leisure activity

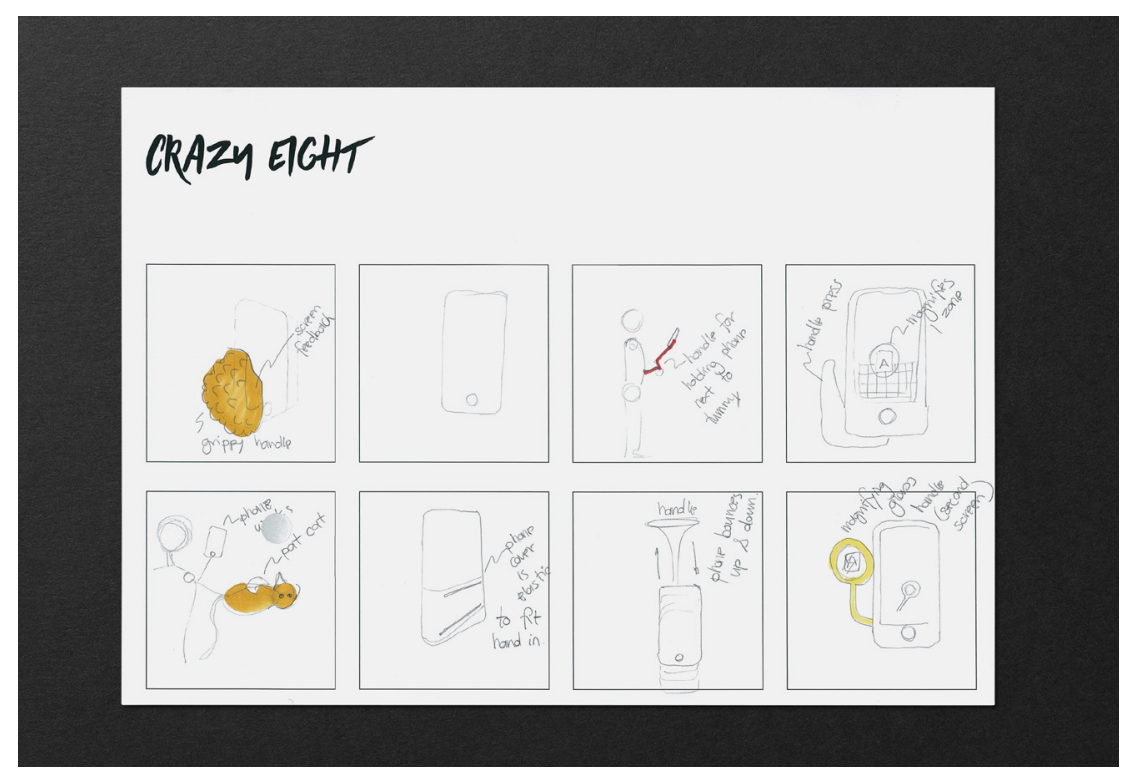

Figure 100:

Participant 05 crazy eight design concepts.

Design concepts of participant 05, see Figure 100:

1. A big handle on the side used by the affected hand that allows the phone to be held more easily. The interface in the corner provides feedback about the interaction

2. No idea submitted

3. A handle that allows the phone to be held closer to the stomach and compensates for a lack of fine motor skills

4. A handle that can be held with the affected hand. The handle can be pressed and magnifies areas on the phone

5. A phone that only works when you stroke an artificial pet cat

6. An elastic phone cover that enables the phone to be held with the affected hand 
7. A handle that lets the phone bounce up and down in case it is dropped by the affected hand

8. An additional screen that works as a magnifier to compensate for cognitive limitations

\subsubsection{Refined design concepts}

For the refinement of the design concepts, the participants were asked to make the object more challenging to use over time.

\subsection{Participant 01}

Participant 01 developed a concept for a smartphone interface that restrains movement using shortcuts on just one side of the screen. The interface would automatically rearrange itself to encourage the user to move the thumb in a specific motion, see Figure 101. The participant further envisioned that the interface could automatically update as the user begins to master the movement. The interface would require the user to swipe using a certain motion to unlock the phone.

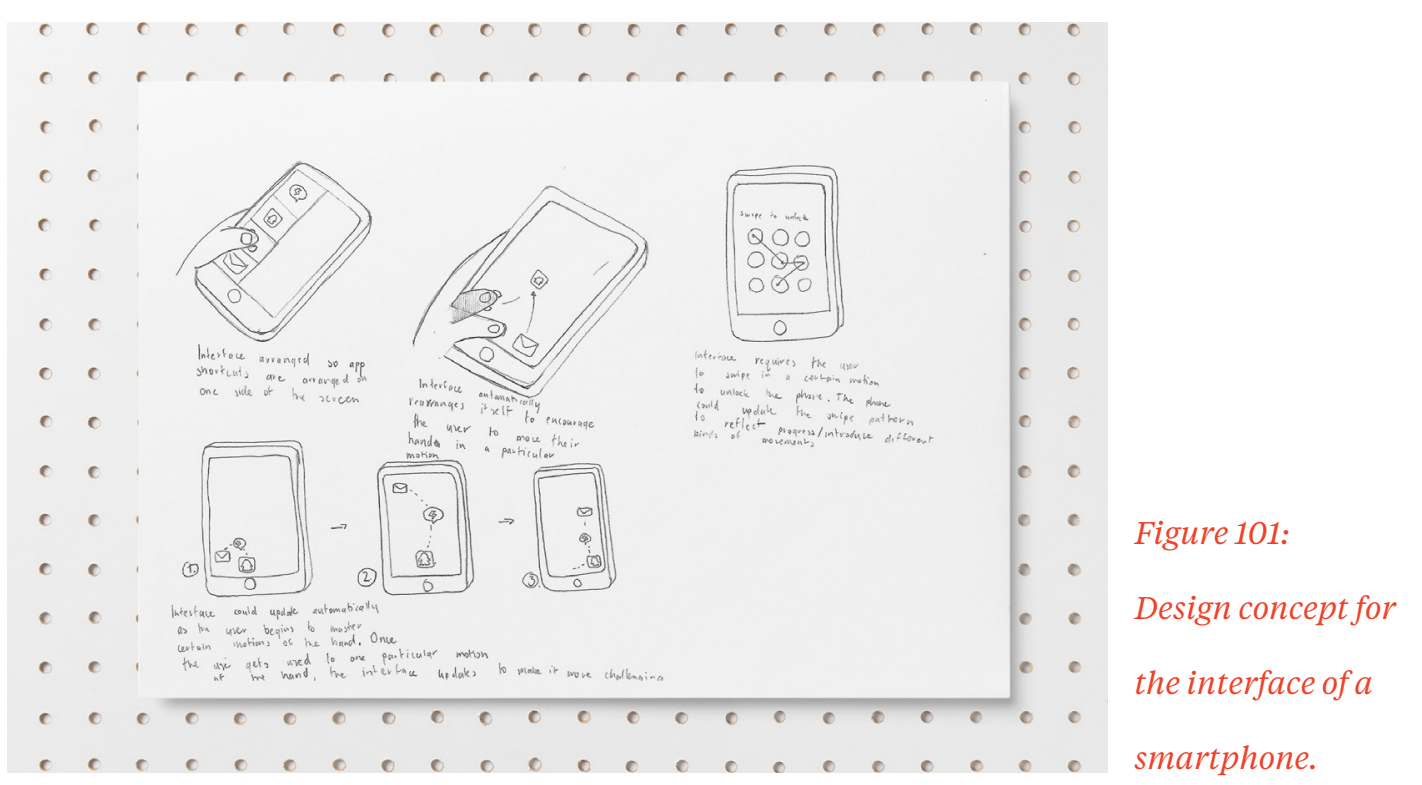




\subsection{Participant 02}

This concept focused on an ergonomic restriction that would allow the use of the phone with one hand and had a stable holding position. An attempt to compensate for the less affected side would make holding it slightly uncomfortable. Different handle elements allow a change of design to reduce assistance and have a restraining effect, see Figure 102.

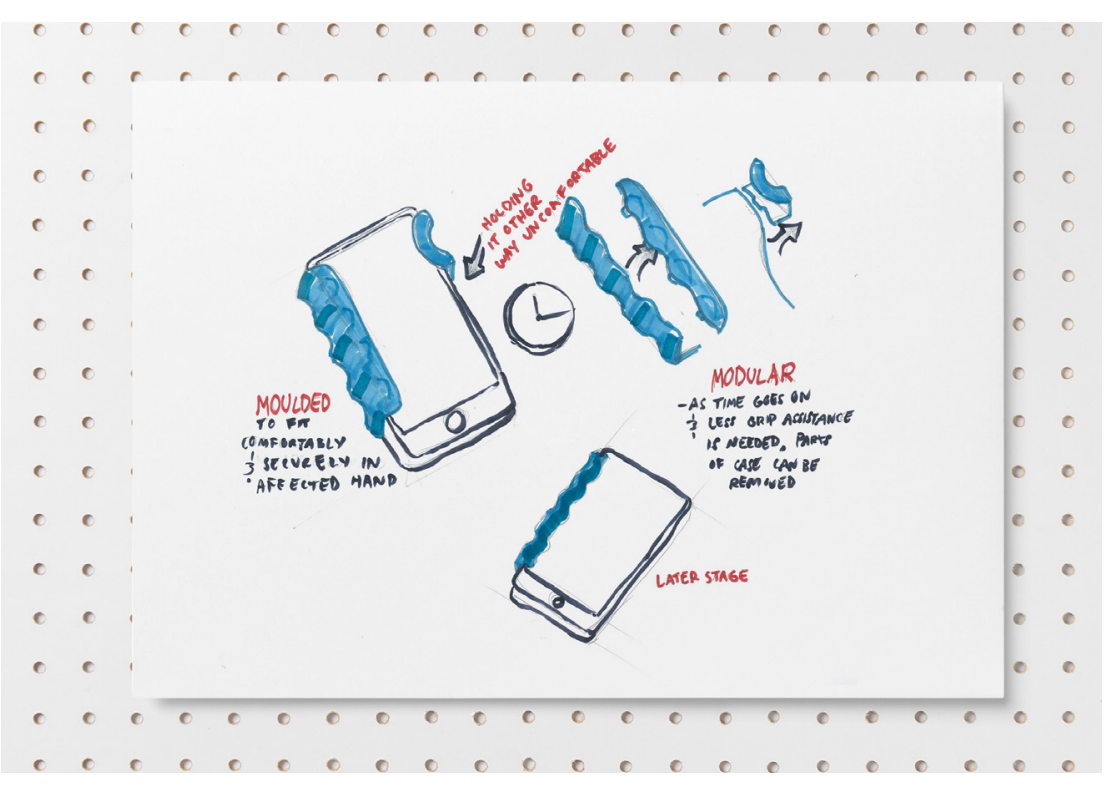

Figure 102:

Design concept for an ergonomic restriction.

\subsection{Participant 03}

The third concept focused on a wearable device such as a ring, bracelet or band, in combination with an app on the phone, see Figure 103. This wearable element has an embedded chip that communicates with the phone. The wearable device, worn on the affected arm, would unlock the phone's software when it is in proximity to the smartphone. The installed app would turn off the touch function after a while to evoke a repetitive movement, see Figure 104. The amount and frequency of this "locking function" increases over time to provide progress in the interaction. The app would further be able to send progress notifications, positive feedback, and reminders. 
The participant saw it as a possible way of activating other kinds of technology in the home environment.

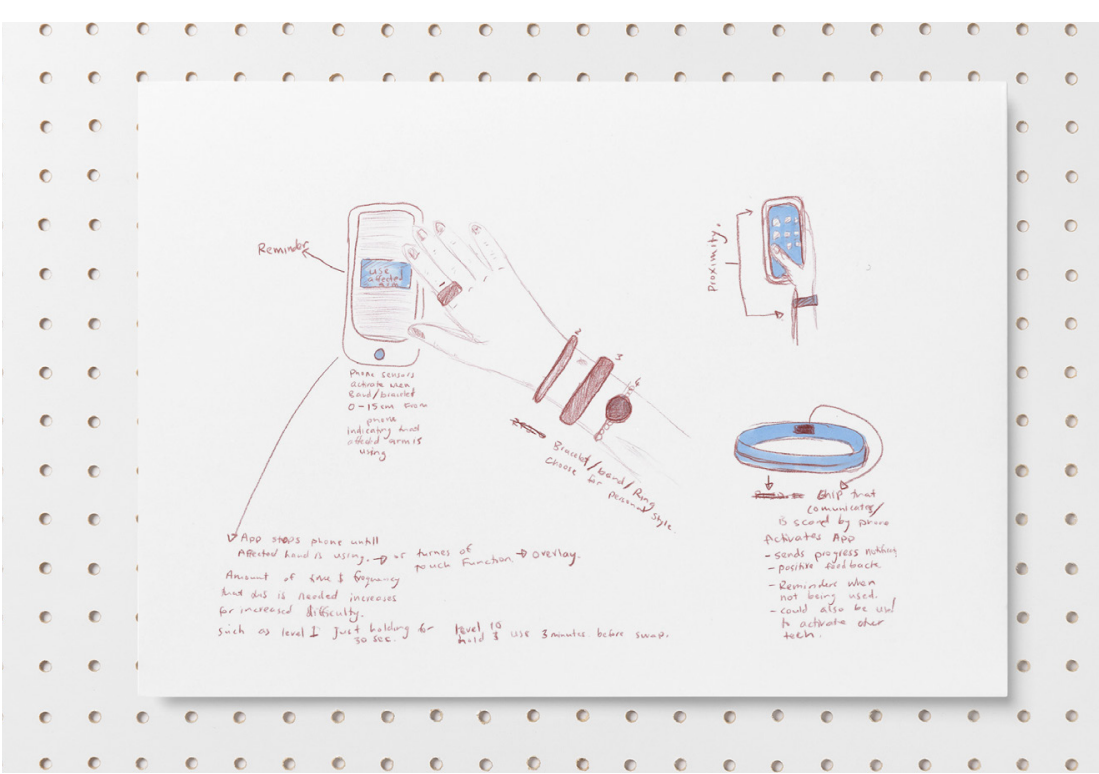

Figure 103:

Design concept for an ergonomic restriction.

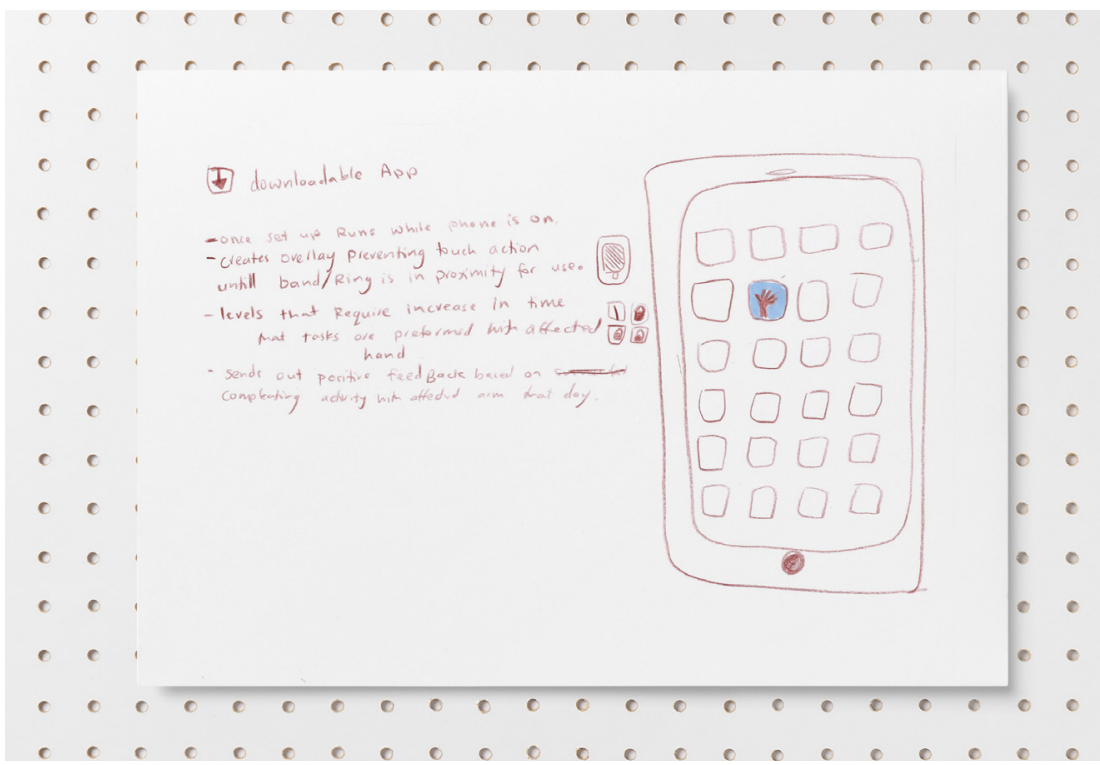

Figure 104:

Details outlining the use of the app. 


\subsection{Participant 04}

Participant 04 developed two concepts that aimed to create a playful interaction. Concept one used the phone as part of a game. The user receives different handles and must balance the phone inside the handle grips to train motor skills and improve the pinching grip; see Figure 105. Concept two situates the phone inside design elements that require the user to rotate the wrist to balance it.

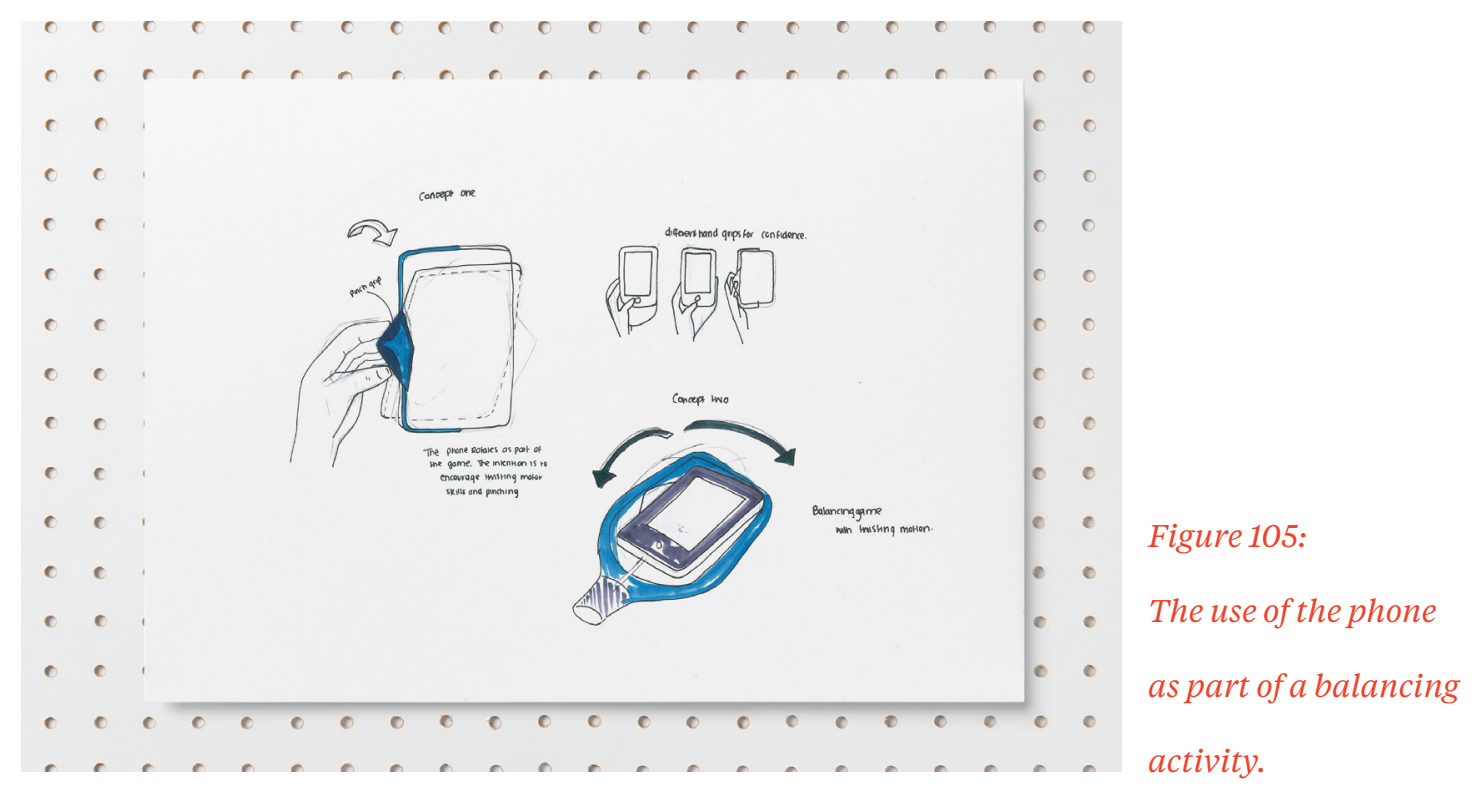

\subsection{Participant 05}

Participant 05 redefined three concepts. Concept one is a handling device that requires the user to hold the phone closer to the body with the affected hand. The device has a universal clipping system or magnet at the top that holds the device at eye level. The bottom element shows a texture that can stick to clothes, see Figure 106. Concept two is a handle that can be folded out from the back of the phone to support the affected hand in holding the device. The side of the design has a rough texture to decrease compensatory movement with the less affected side, see Figure 107. Concept three is a phone case with a handle on one side and an uncomfortable texture on the other side, see Figure 108. 
LIMITED USE ONLY

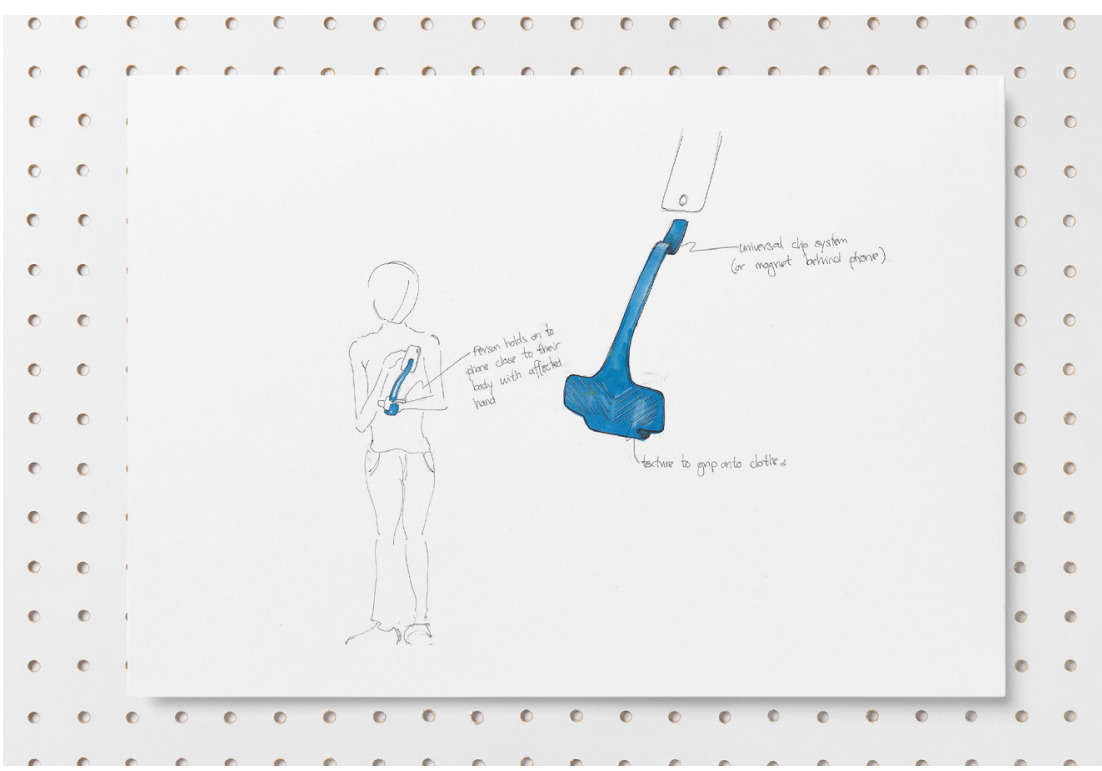

Figure 106:

Design concept for a holding device.

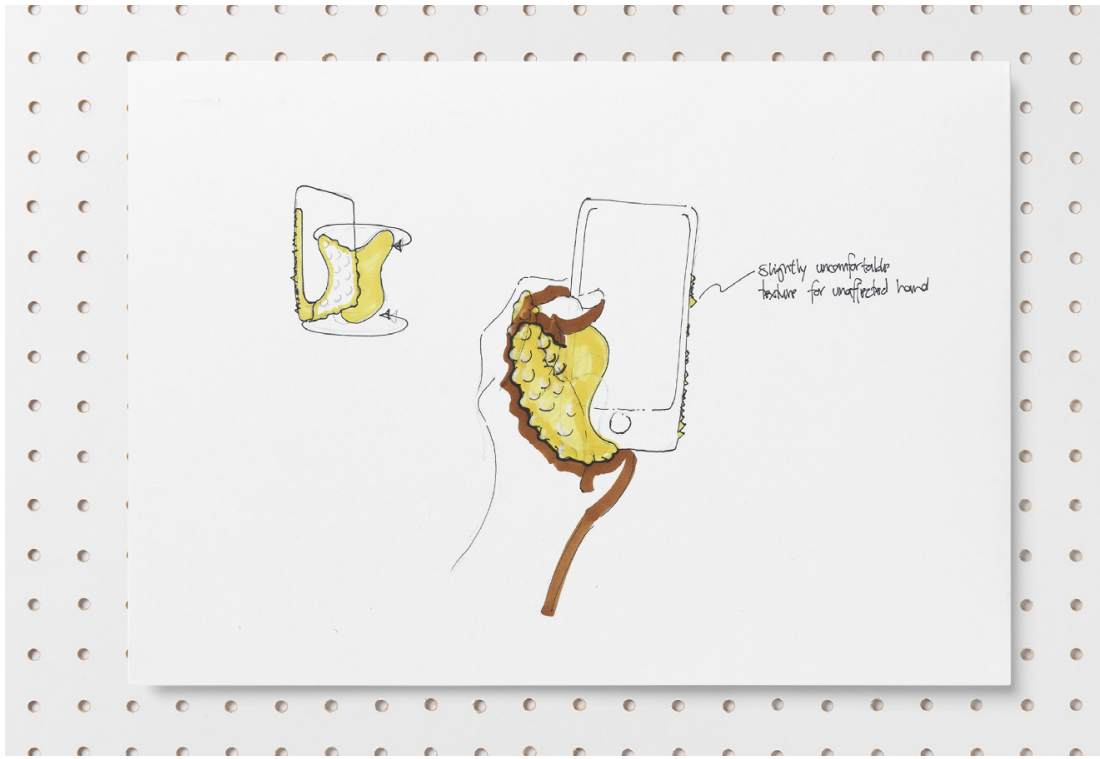

Figure 107:

Design concept for a handle at the back of the phone. 


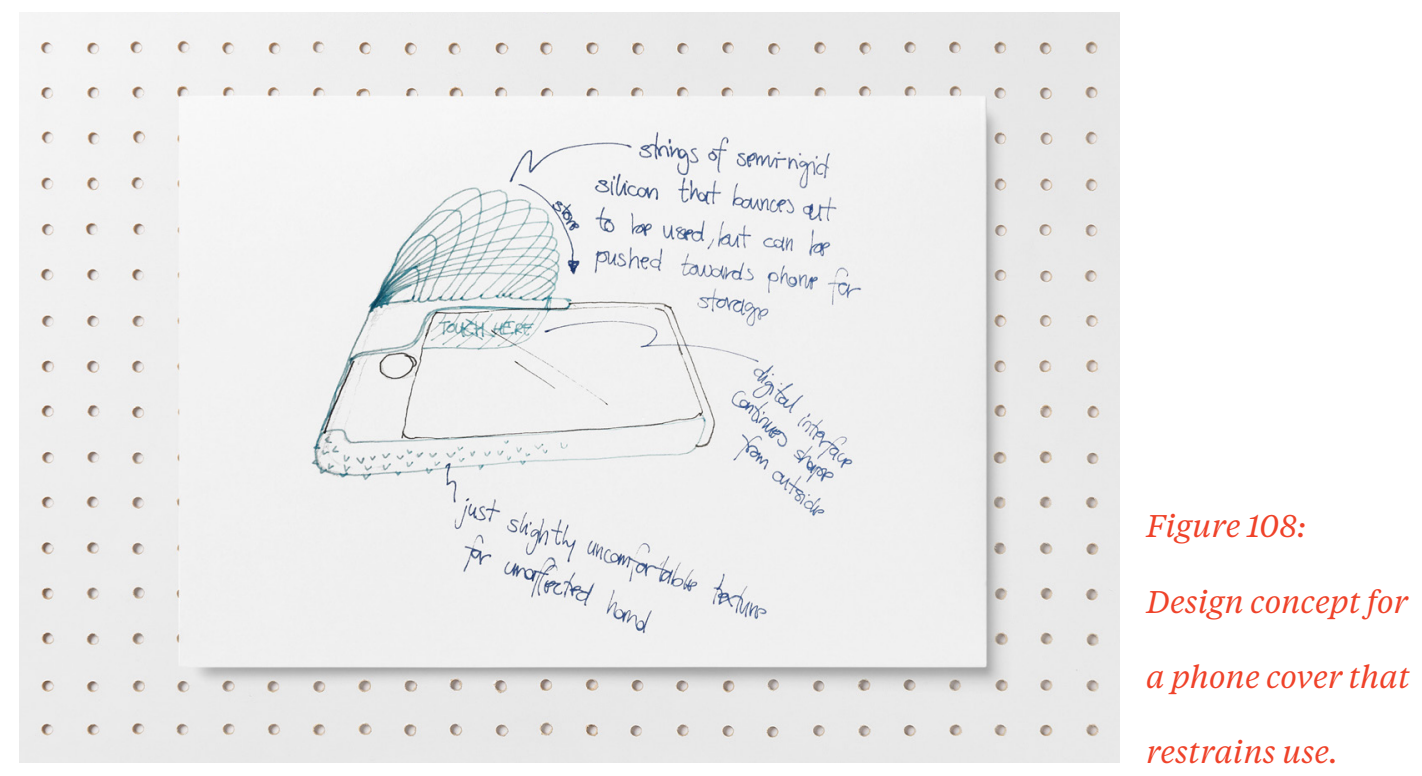

\subsubsection{Analysis}

The produced design concepts were clustered according to Tromp et al. (2011, p. 12) “Classification of Influence Based on Intended User Experience”. The analysis is based on the expected influence of the objects.

\subsubsection{Decisive design concepts}

Decisive design solutions are experienced by the user as external influences but the influences are not seen as being imposed by the designer (Tromp et al., 2011, p. 12). The design concepts in this study focused on ergonomic restrictions that make the desired behaviour the only possible one; phone case attachments to allow use with the affected hand; features on the phone that require the use of both hands; and wearable components that unlock the full functionality of the phone.

\subsubsection{Coercive design concepts}

Coercive design concepts have a strong and explicit influence and are experienced as such by the user (Tromp et al., 2011, p.12). Design concepts included an annoying buzz until the phone was picked up and used with the affected hand, a phone that shocks when used with the "wrong" hand, a phone cover that needs to be pumped every two minutes, and a squeeze and press handle 
that requires the use of the affected side to pump it up so that the phone works.

12.4.2.3 Seductive design concepts

Seductive design concepts evoke a behaviour that the user would probably experience as being internally motivated rather than caused by the design element (Tromp et al., 2011, p. 12). Design concepts included the development of simplified keyboards that can be used with the affected hand and have only a few keys, a new digital keyboard that responds better to stroke-specific impairments, and the habit formation of texting someone multiple times a day using the affected hand.

\subsubsection{Persuasive design concepts}

Persuasive design concepts are experienced as externally motivated but are experienced as weaker than a coercive design (Tromp et al., 2011, p.12). Concepts that evoke an initiation of use of the affected arm and hand included the design of a strap to decrease the likelihood of dropping the phone, a handle that tracks the phone's use and informs loved ones and clinicians of the amount of use, rewards if the affected side is used, visual cues such as a sticker on the case as a reminder to use the affected hand, phone notification about therapy activities, a wristband that would unlock the phone's software when it is in proximity to the phone and a phone case that vibrates lightly when the less affected hand holds the phone. Two games were mentioned - a balancing game using the phone as a controller, and a conversation game during which the user has to reply with the affected hand.

\subsubsection{Form and influence of the design concepts}

The different design concepts were grouped according to their expected influence on the user for force and salience, see Figure 109. Some of the decisive and seductive design concepts focused on enabling the use of the phone rather than restraining its use. The three elements of enabling the use of the software, enabling use of the hardware, and reducing the likelihood of dropping the phone were therefore situated under the theme of problem-solving. The design components that addressed this problem solving could be experienced as decisive and seductive, and were consequently grouped among the two areas. 


\section{Influence of the developed \\ design concepts based on the \\ dimensions of force and salience}

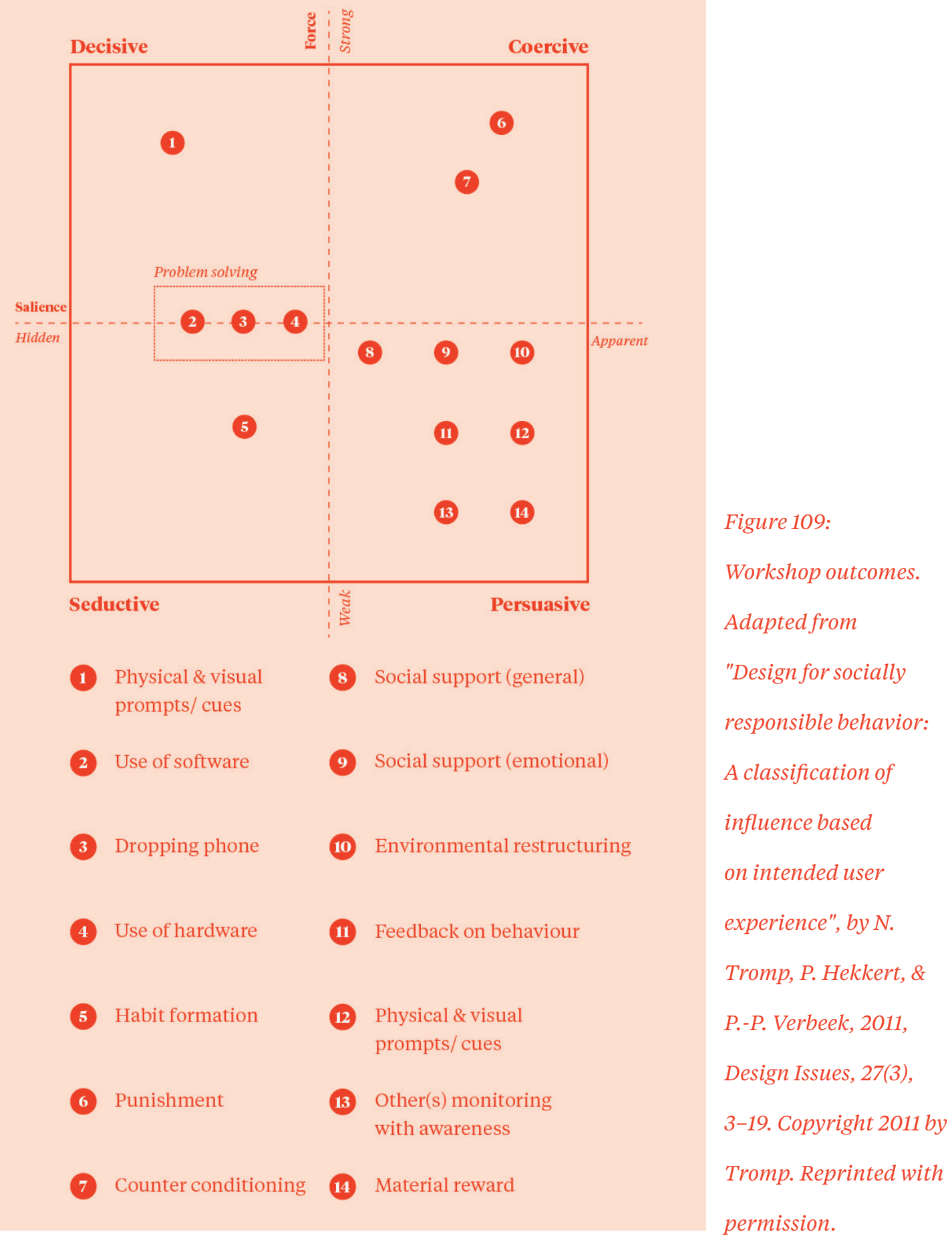




\subsection{Discussion}

The design workshop led to concepts that were persuasive, coercive, seductive, and decisive. Interestingly a number of concepts focused on the ability to use the phone; for example, by adding a strap or handle or changing the software so it took account of stroke-specific limitations. The ideal concept should combine those features to make use of the object feasible while evoking an initiation of use and restraining movement.

The designers who took part in the workshop often referred to anecdotes that were mentioned in the introduction as a point of reference. It seems that empathy in the design process can be more easily developed when personal stories are included in the design strategies. The focus on restraint rather than all seven design strategies was confirmed to be a manageable challenge and led to 31 design concepts in total.

Design concepts that depend on the physical form of the phone to restrain movement were situated under persuasive, decisive, and coercive designs. Decisive and persuasive design concepts both employed physical and visual prompts to evoke an initiation of use of the affected arm and hand. It was unclear at this stage which design features contribute to the perception of a decisive and a persuasive design influence. To distinguish between those two influences, users need to be involved in the assessment process.

A number of concepts focused on the involvement of a behaviour change reinforced through social interaction, for example, texting relatives who remind the user to use the affected hand. It is outside the scope of this study to test such concepts, but further studies could use them as a starting point to investigate restraining effects through social interaction. 


\subsection{Conclusion}

The design workshop led to several restraining design concepts for smartphones that are expected to have varying influences on the user, ranging from seductive, persuasive, coercive, and decisive. Concepts that have a strong focus on the physical form of the device should have a persuasive, decisive, and coercive influence. The persuasive and decisive design concepts were based on the use of visual and physical prompts to evoke an initiation of use. The influence that they have on the user depends on whether the user experiences the design element as an added component or part of the design. The design concepts further focused on ways to make the use of the phone feasible for the stroke survivor. The next design prototype needs to make using the object feasible while restraining movement.

\subsection{Limitation}

I invited stroke survivors to participate in the workshop. Unfortunately, none got in contact to take part in the discussion and only designers were involved in the workshop. 


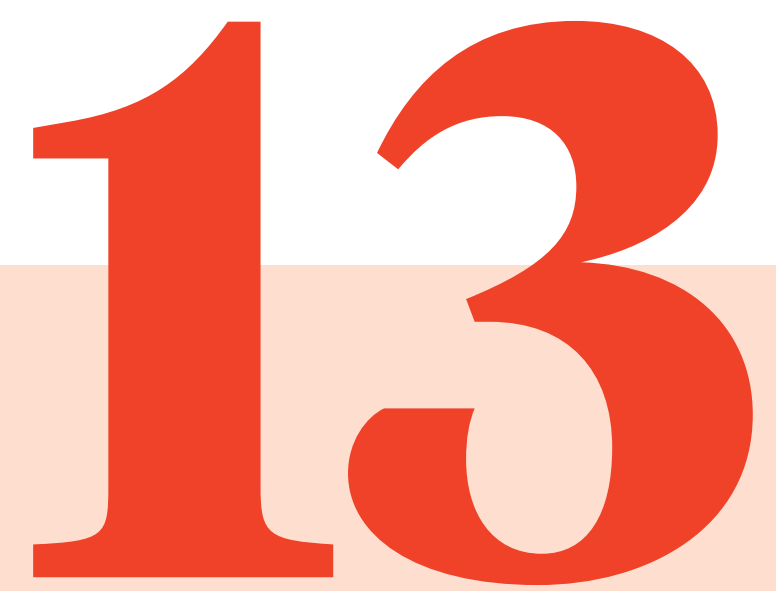

\section{The Design of a}

\section{Phone Attachment \\ that Evokes an}

\section{Initiation of Use}




\subsection{Introduction}

This chapter addresses the research question:

1.3.1 How can we design an everyday object that restrains movement of the less affected arm to facilitate and initiate the use of the affected arm?

Two design concepts based on the outcomes of the design workshop were chosen to be further refined and evaluated in a pluralistic walkthrough with four chronic stroke survivors. One design concept is based on a coercive influence, while the second design is based on a persuasive design influence. The physical prototypes developed as part of this study were created to be used on a smartphone to initiate the use of the affected arm and hand.

\subsection{Background}

To overcome learned nonuse and evoke an initiation of use in the long term the user must undergo a behaviour change. The behaviour contract is used as part of the transfer package as part of CIMT. It is a formal written document signed by the therapist and the stroke survivor and outlines that the survivor will use the affected arm for specific daily activities as much as is feasible and as long as it is safe (Morris et al., 2006, pp. 262-263; Taub, 2013, pp. 129-130). The design strategies developed use the behaviour contract in the form of packaging or elements of the design prototype. It is outside the scope of this study to confirm whether they evoked a behaviour change in the user; however, inclusion of such an element appears to be essential for invoking an initiation of use, see Chapter 4.

\subsection{Methods}

A RTD methodology was used for this study. Serial design experiments were evaluated with the criteria-based thesis model. Two refined design prototypes were evaluated using a pluralistic walkthrough with chronic stroke survivors. The 
feedback provided was analysed using thematic analysis. See Figure 110 for an overview of the different steps.

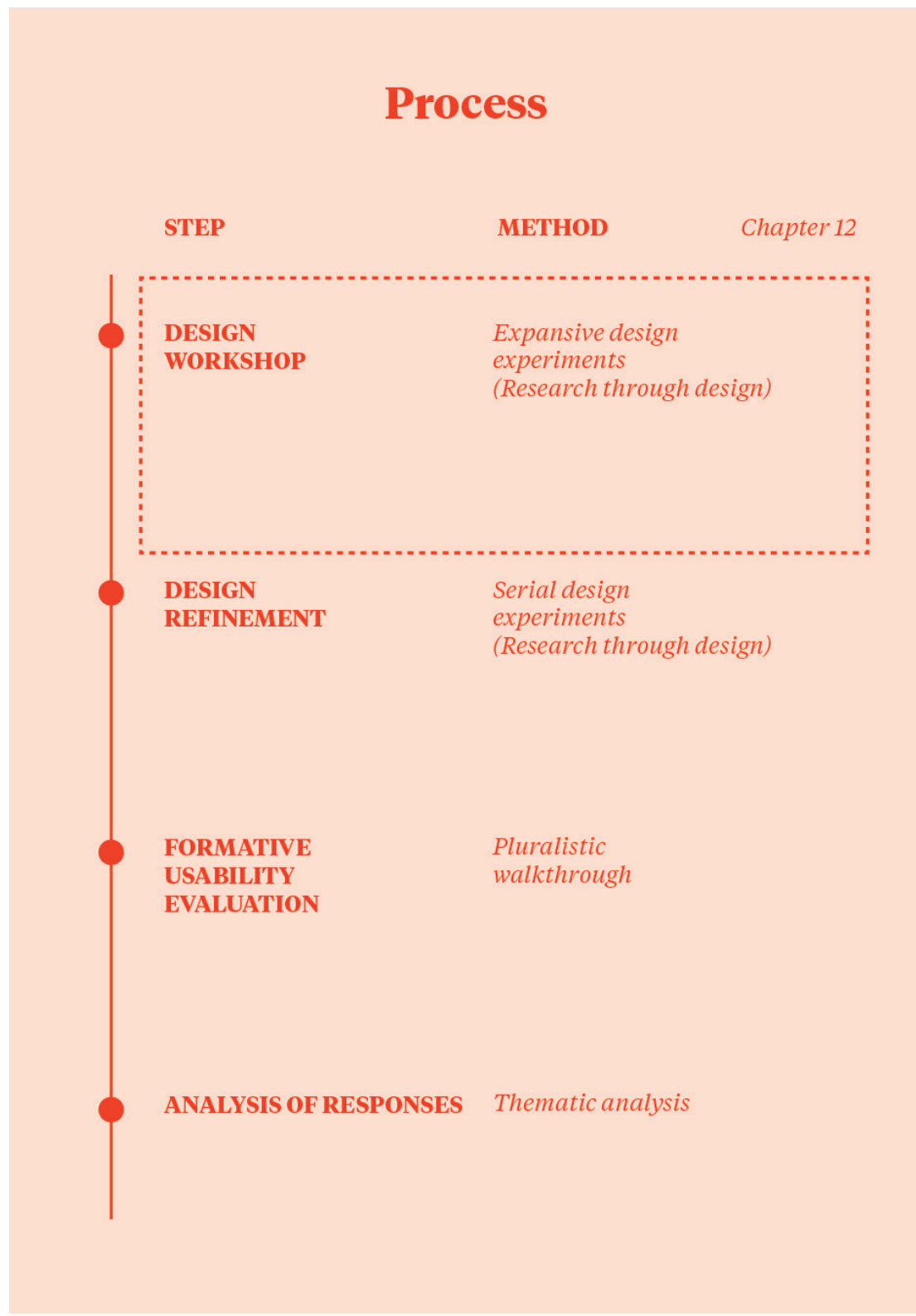

Figure 110:

Steps and employed methods.

\subsubsection{Participants}

Participants who took part in the previous interviews, see chapter 11, were invited to take part in the usability evaluation process of this study. Each participant was informed about the study and provided written consent. Please 
refer to Table 15 for a description of the study sample. Four participants took part in the study, three were male $(n=3)$, and one was female $(n=1)$.

Table 15: Study sample.

\begin{tabular}{|c|c|c|c|c|c|c|}
\hline & Gender & Age & Effect of the stroke & $\begin{array}{l}\text { Time since } \\
\text { stroke }\end{array}$ & $\begin{array}{l}\text { Dominant } \\
\text { side }\end{array}$ & Devices used \\
\hline 01 & male & 74 & $\begin{array}{l}\text { Mild impairments of the } \\
\text { upper and lower limb on } \\
\text { the left side }\end{array}$ & 2 years & $\begin{array}{l}\text { Right } \\
\text { handed }\end{array}$ & $\begin{array}{l}\text { Laptop, } \\
\text { Android } \\
\text { smartphone }\end{array}$ \\
\hline 02 & male & 70 & $\begin{array}{l}\text { Moderate impairments of } \\
\text { the upper and lower limb } \\
\text { on the left side }\end{array}$ & 12 years & $\begin{array}{l}\text { Right } \\
\text { handed }\end{array}$ & $\begin{array}{l}\text { Feature } \\
\text { phone, } \\
\text { landline }\end{array}$ \\
\hline 03 & male & 77 & $\begin{array}{l}\text { Moderate impairments of } \\
\text { the upper and lower limb } \\
\text { on the right side }\end{array}$ & 10 years & $\begin{array}{l}\text { Right } \\
\text { handed }\end{array}$ & $\begin{array}{l}\text { Laptop, } \\
\text { feature } \\
\text { phone }\end{array}$ \\
\hline 04 & female & 72 & $\begin{array}{l}\text { Mild impairments of the } \\
\text { upper and lower limb on } \\
\text { the right side, reduced } \\
\text { sensation on the right side }\end{array}$ & 8 months & $\begin{array}{l}\text { Left } \\
\text { handed }\end{array}$ & $\begin{array}{l}\text { Laptop, } \\
\text { iPad, Apple } \\
\text { smartphone }\end{array}$ \\
\hline
\end{tabular}

\subsubsection{Research through design}

The two design prototypes were refined based on the initial design concepts because of their focus on the physical form of the phone: a persuasive design influence in the form of "physical and visual prompts/cues" and a coercive design influence in the form of "punishment". The prototypes were refined in a serial design process (Krogh et al., 2015 p. 46). The initial set of design criteria (Rodríguez Ramírez, 2017, p. 14) was refined and referred to in the design process, see Figure 111. 


\begin{tabular}{|c|c|}
\hline 01: Restrain & $\begin{array}{l}\text { The interaction with the object needs to restrain movement } \\
\text { in order to remind the user to use the affected arm and hand }\end{array}$ \\
\hline 02: Purpose & The object needs to be used within an activity of daily living \\
\hline 03: Repetition & $\begin{array}{l}\text { The interaction with the object needs to be repetitive in form } \\
\text { of random practice }\end{array}$ \\
\hline 04: Challenge & $\begin{array}{l}\text { The interaction with the object needs to become progressive- } \\
\text { ly challenging and offer different levels of complexity }\end{array}$ \\
\hline $\begin{array}{l}\text { 05: Functional } \\
\text { feedforward }\end{array}$ & The design of the object should be based on a familiar object \\
\hline $\begin{array}{l}\text { 06: Augmented } \\
\text { feedforward }\end{array}$ & $\begin{array}{l}\text { Augmented feedforward should contribute to reminding the } \\
\text { user to use the affected side of the body }\end{array}$ \\
\hline $\begin{array}{l}\text { 07: Inherent feed- } \\
\text { forward }\end{array}$ & $\begin{array}{l}\text { Inherent feedforward should indicate how the user can } \\
\text { interact with the object and that the movement needs to be } \\
\text { carried out with one particular side of the body }\end{array}$ \\
\hline $\begin{array}{l}\text { 08: Functional } \\
\text { feedback }\end{array}$ & $\begin{array}{l}\text { Functional feedback should indicate the successful } \\
\text { completion of a task }\end{array}$ \\
\hline $\begin{array}{l}\text { 09: Augmented } \\
\text { feedback }\end{array}$ & $\begin{array}{l}\text { Augmented feedback should provide quantitative feedback } \\
\text { about the movement }\end{array}$ \\
\hline $\begin{array}{l}\text { 10: Inherent } \\
\text { feedback }\end{array}$ & $\begin{array}{l}\text { Inherent feedback should contribute to a moderate level of } \\
\text { effort }\end{array}$ \\
\hline $\begin{array}{l}\text { 11: Adherence in- } \\
\text { creasing techniques }\end{array}$ & The design should evoke a behaviour change in the user \\
\hline 12: Movement & $\begin{array}{l}\text { The design should allow to hold the device with the affected } \\
\text { hand to initiate the use of it }\end{array}$ \\
\hline 13: Compensation & The design should minimise compensatory movement \\
\hline
\end{tabular}

Figure 111: Set of design criteria. 


\subsubsection{Pluralistic walkthroughs}

Two design prototypes were evaluated using a pluralistic walkthrough with chronic stroke survivors. Prototypes were developed for participants with an affected left hand and for participants with an affected right hand. The task that was focused on was picking up the prototype, see the interview guide below. After the testing, the participants were asked to point out which of the design prototypes they liked the least. The packaging with the behaviour contract was shown at the end of the testing to avoid biasing the results.

The following interview guide was used:

Duration: 30 minutes

Task: Picking up the phone, holding it and tapping on an icon at the front.

The participant is asked to pick up the smartphone without any attachment, followed by prototype one and two.

1. Could you please pick up the object?

2. Could you please describe to me how the use of the object makes you feel?

3. Do you find it easier to hold the object with your affected hand or with the less affected hand?

4. Would you use such an object?

The packaging and behaviour contract are shown to the participant.

5. Could you please describe what your thoughts are regarding the packaging?

6. The packaging contains this behaviour contract that you would be asked to sign. Would you sign something like that?

7. Are there any elements that you would like to change?

\subsubsection{Analysis}

The feedback and videos of the pluralistic walkthrough were analysed using thematic analysis in a deductive form (Braun \& Clarke, 2006, p. 83) according to 
the themes of initiation of use of the affected arm and hand, general usability issues, and semantic interpretation.

\subsection{Results}

\subsubsection{RTD results}

Two design prototypes were refined and tested with participants. Each prototype was produced for both left-handed use right-handed use. It could be simply attached to the back of a smartphone. Please refer to Appendix D, design Workbook 03, for a detailed description of the developed design sketches and prototypes. For testing the designs, non-functional smartphones were created to determine anticipated initiation of use during the 30-minute testing session.

\subsubsection{Smartphone}

A smartphone based on a Huawei smartphone was developed for the testing. It was $14.5 \mathrm{~cm}$ x $7.5 \mathrm{~cm}$, had a smooth surface and was non-functional but had a screen with some icons on the front, see Figures 112 and 113. The smartphone was chosen because the interviews revealed that often both hands were required to hold it and interact with the screen. The focus on a bilateral task is one of the design strategies used to restrain movement with an object. Therefore, it was chosen to be used in combination with the concepts developed during the design workshop to evoke an initiation of use.

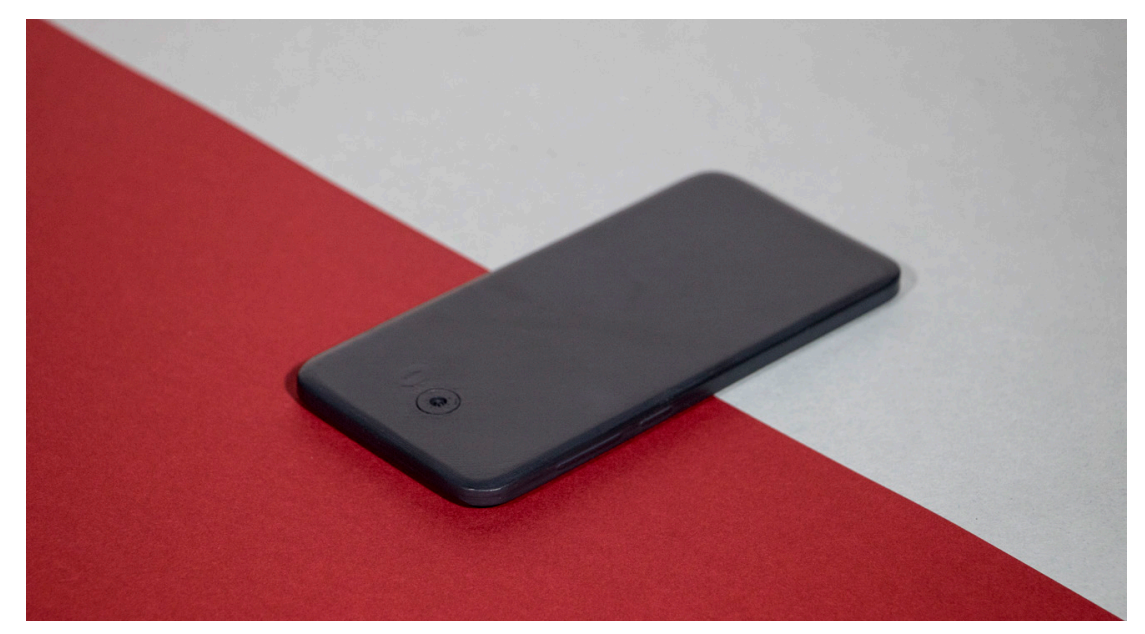

Figure 112: Standard smartphone used for testing, P0O. 


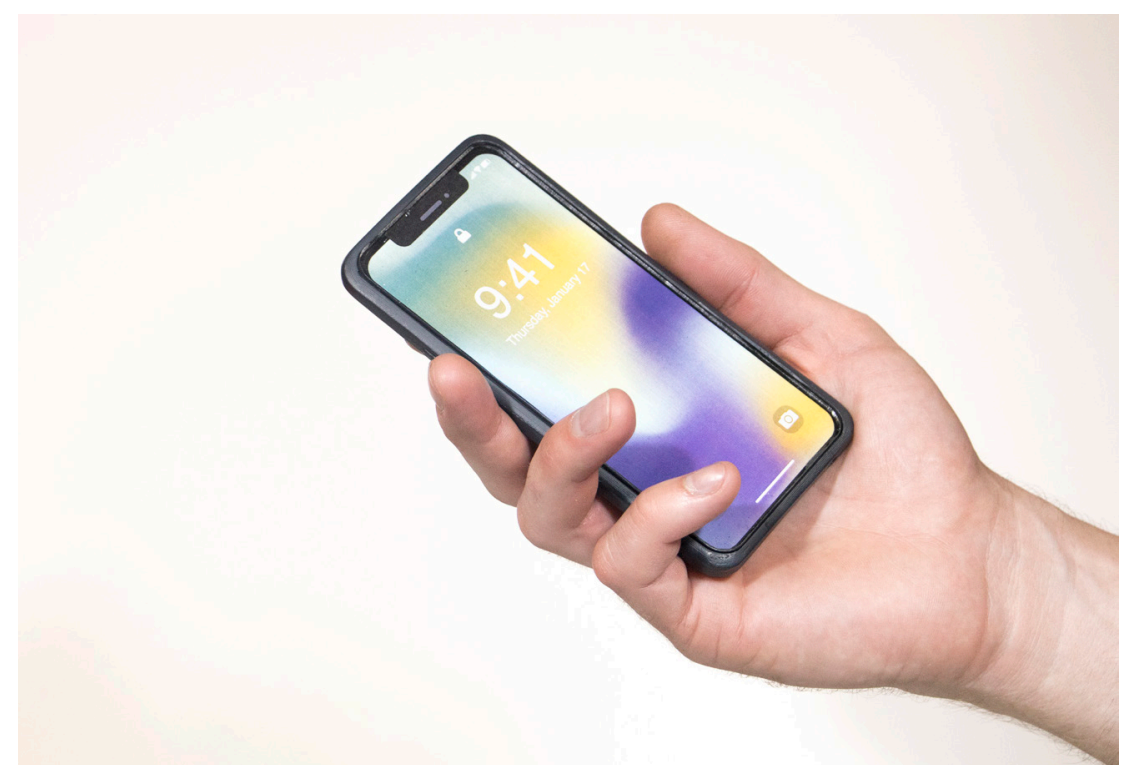

Figure 113:

Non-functional

interface.

\subsubsection{Persuasive design concept}

The persuasive design concept relies on visual and physical prompts in the form of ergonomic restrictions to evoke an initiation of use. Sketches led to the development of early form prototypes using a tabletop 3D printer and further refined versions of the design concepts. Refer to design Workbook 03 for a detailed description of the sketches and design iterations. The final design prototype, P01, consisted of three grip elements attached with double-sided tape to the back of the smartphone prototype, see Figures 114 to 117. The design creates an uneven surface at the back, which limits the ability to use it on a flat surface to compensate for the affected arm, see Figure 117. 


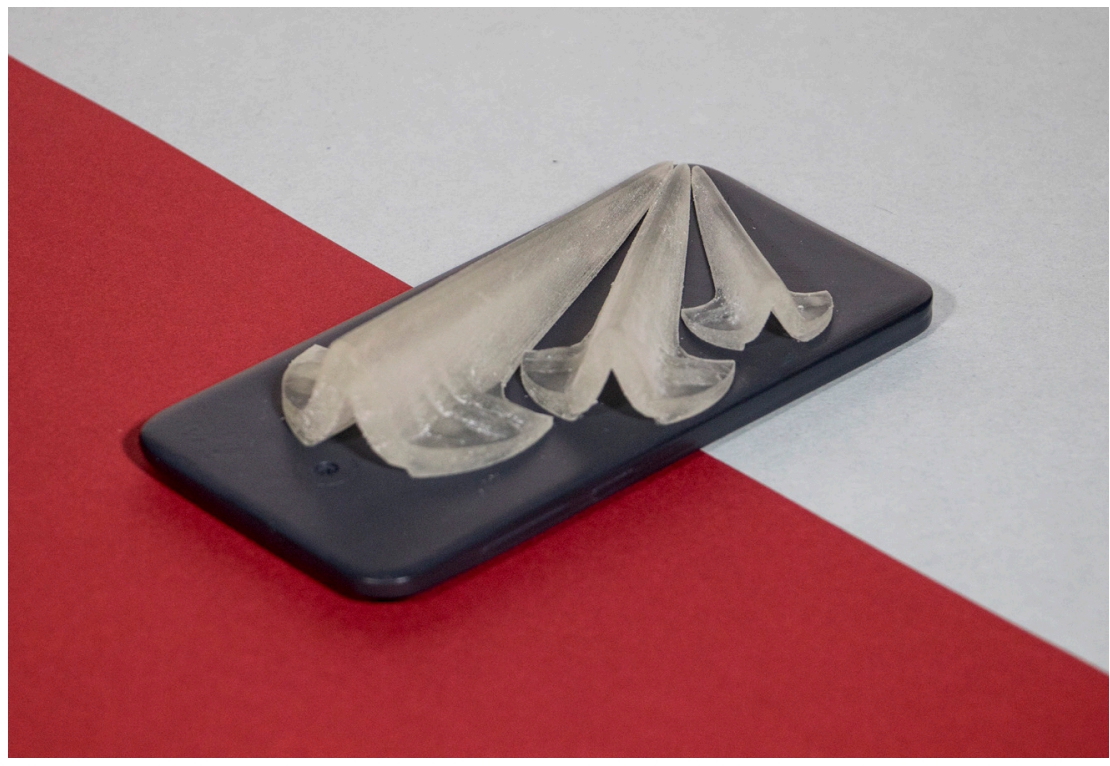

Figure 114:

Three grip elements of

“Manta”, prototype P01.

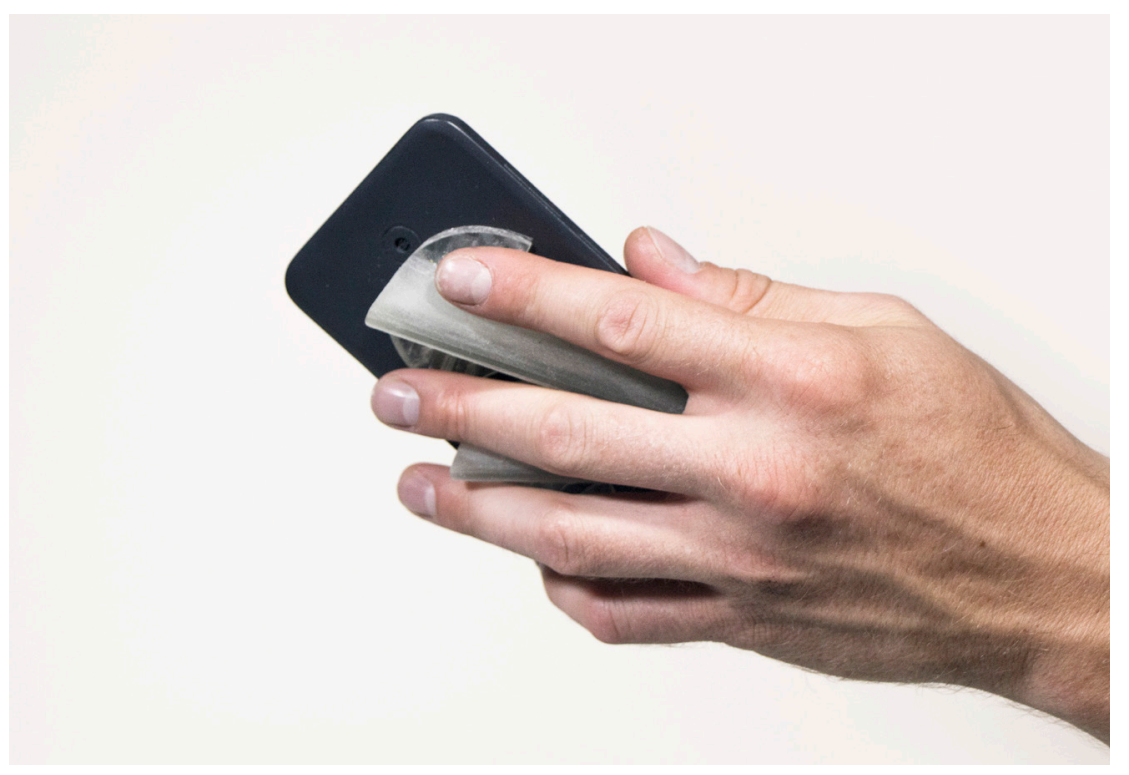

Figure 115:

"Manta" for left-handed use. 


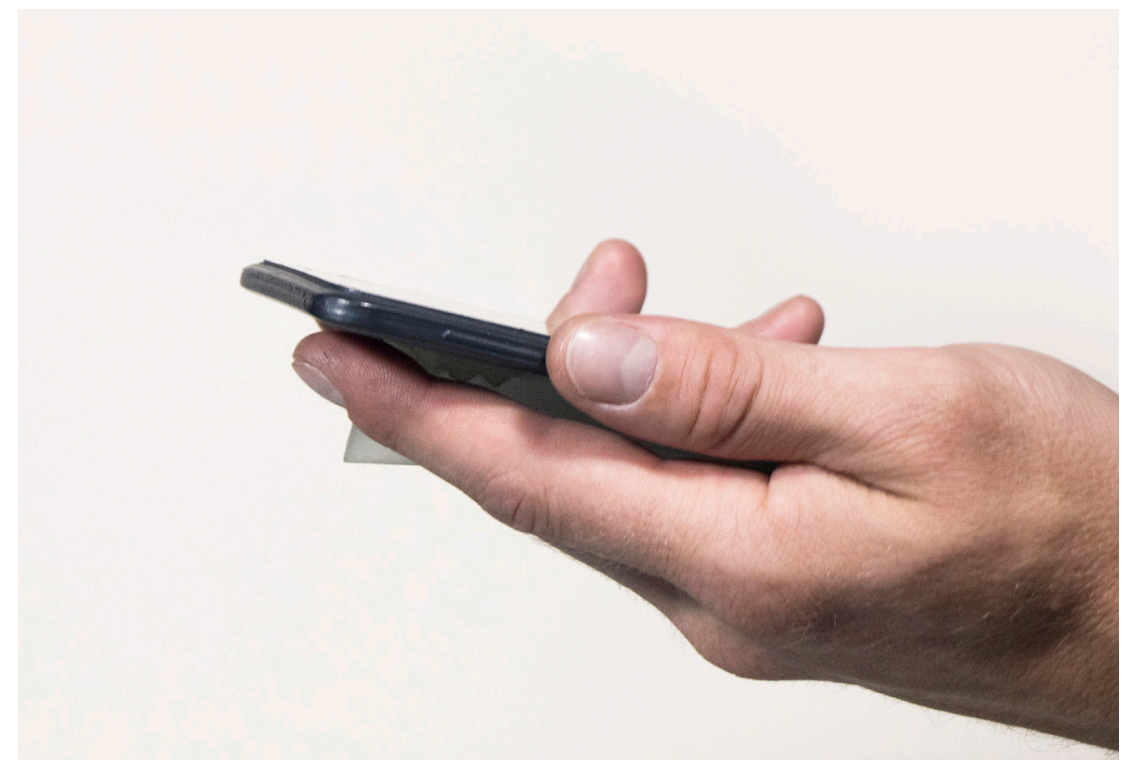

Figure 116:

The design elements are decreasing in size.

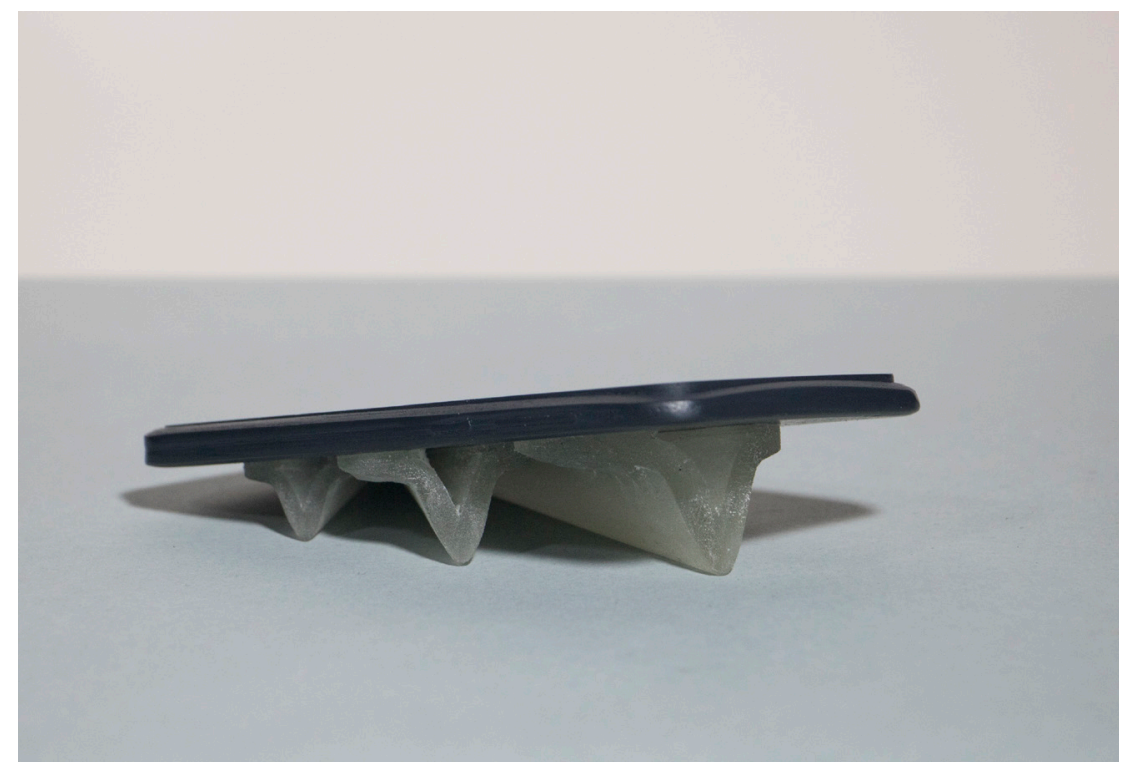

Figure 117:

The design incorporates an uneven back to limit compensatory movement patterns.

The packaging for the design prototype was developed for the usability evaluation to include a behaviour change contract. The packaging also aimed to create a context that addresses product-related stigma. de Barros, Duarte, and Cruz, (2011, p. 109) reported that the context in which the products are shown and associated labelling influences perception of the object. Therefore, the packaging did not include any reference to stroke or elderly people. Packaging for the lefthanded and for the right-handed design was produced, see Figure 118. Each lot 
of packaging contained a behaviour contract, see Figure 119, reminding the user to use the affected arm and hand as much as possible while using the design prototype.

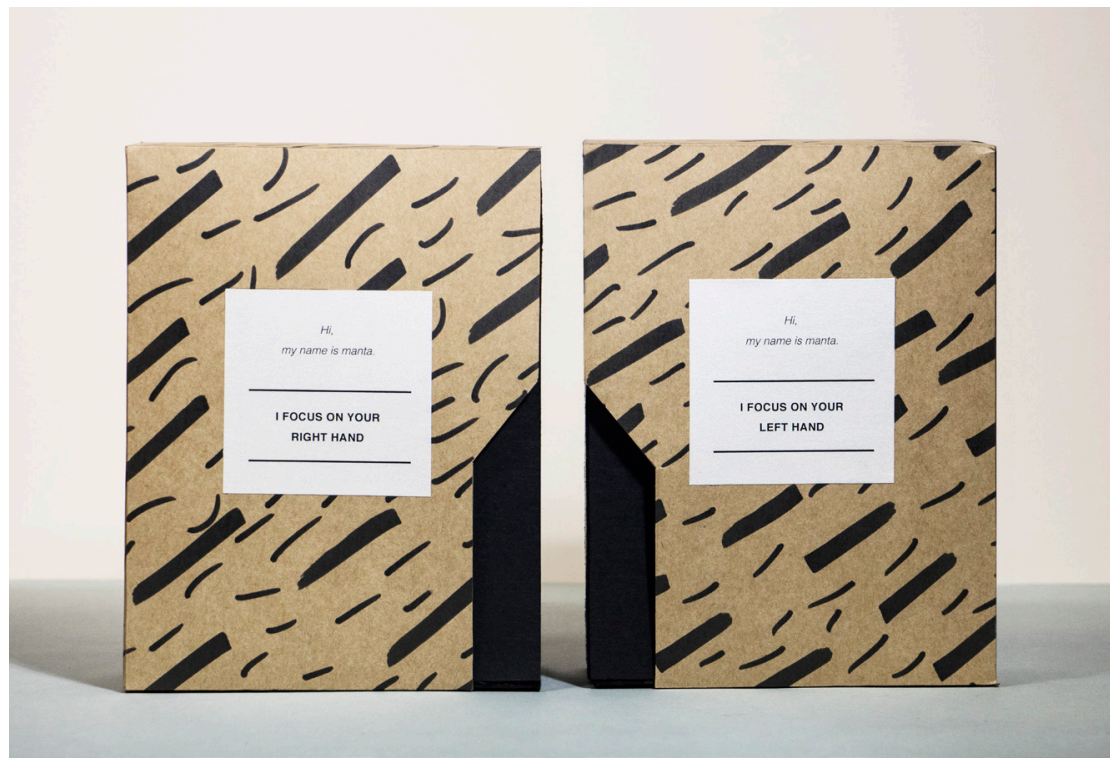

Figure 118:

The two sets of

packaging for the

persuasive design prototype.

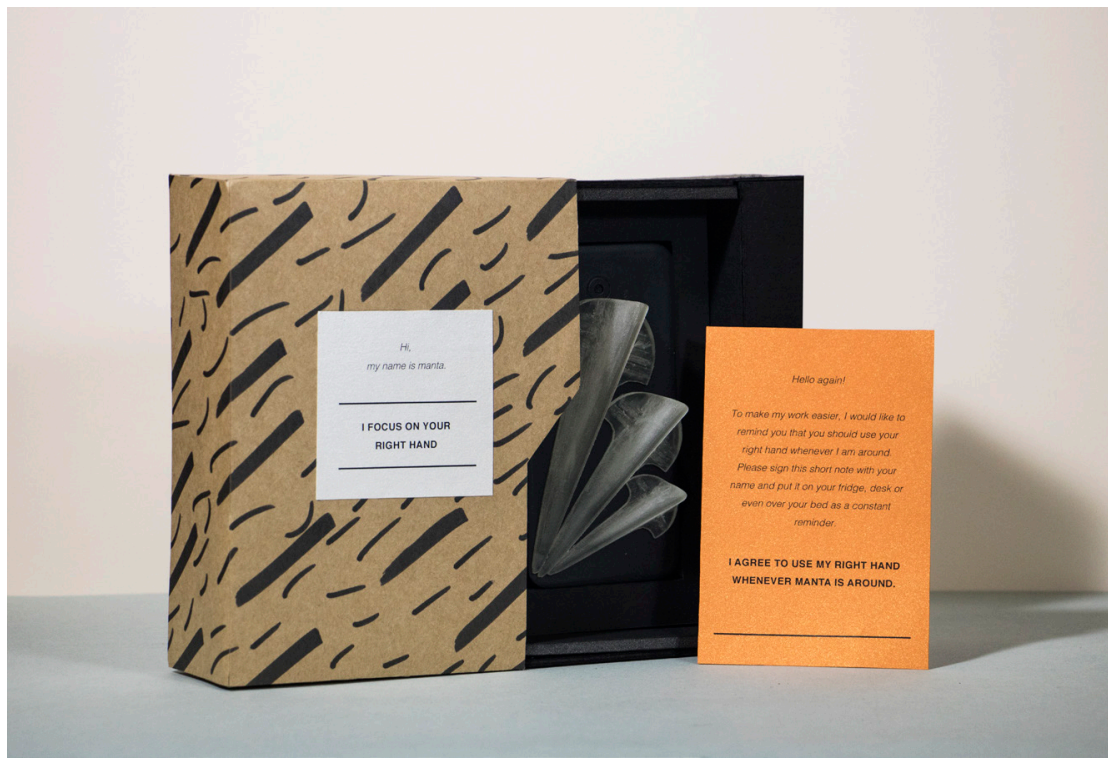

Figure 119:

Packaging and

behaviour note for P01. 


\subsubsection{Coercive design concept}

The coercive design concept relies on "punishment" in the form of uncomfortable use. The initial design concept produced during the workshop described an electric shock each time the less affected hand was used to compensate for the affected arm and hand. The sketches focused more on an uncomfortable feeling to make testing the design with participants feasible. Sketches led to the development of early form prototypes using a tabletop 3D printer and further refined versions of the design concepts. The final design prototype, P02, consisted of two golden cacti attached with double-sided tape to the back of the smartphone prototype, see Figure 120. The design created an uneven surface at the back of the phone to decrease the possibility of putting the phone on an even surface to use it. A second set of packaging was created for the coercive design prototype, see Figure 121.

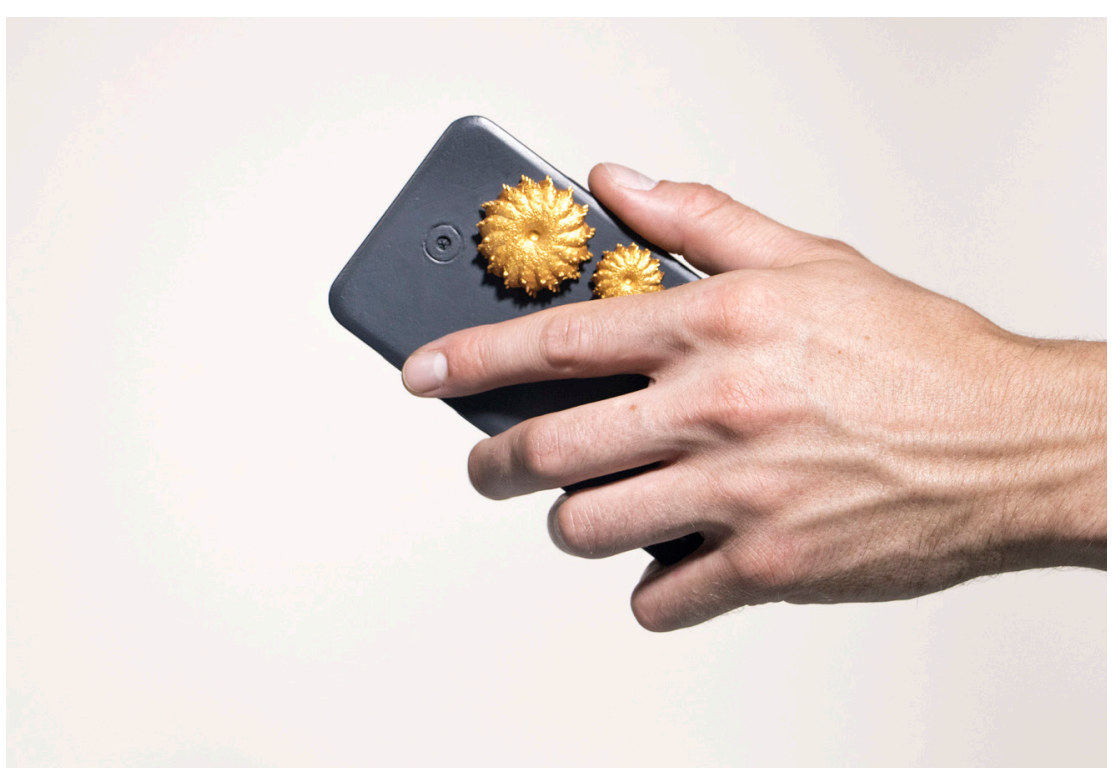

Figure 120:

“Cactacea”, prototype P02. 


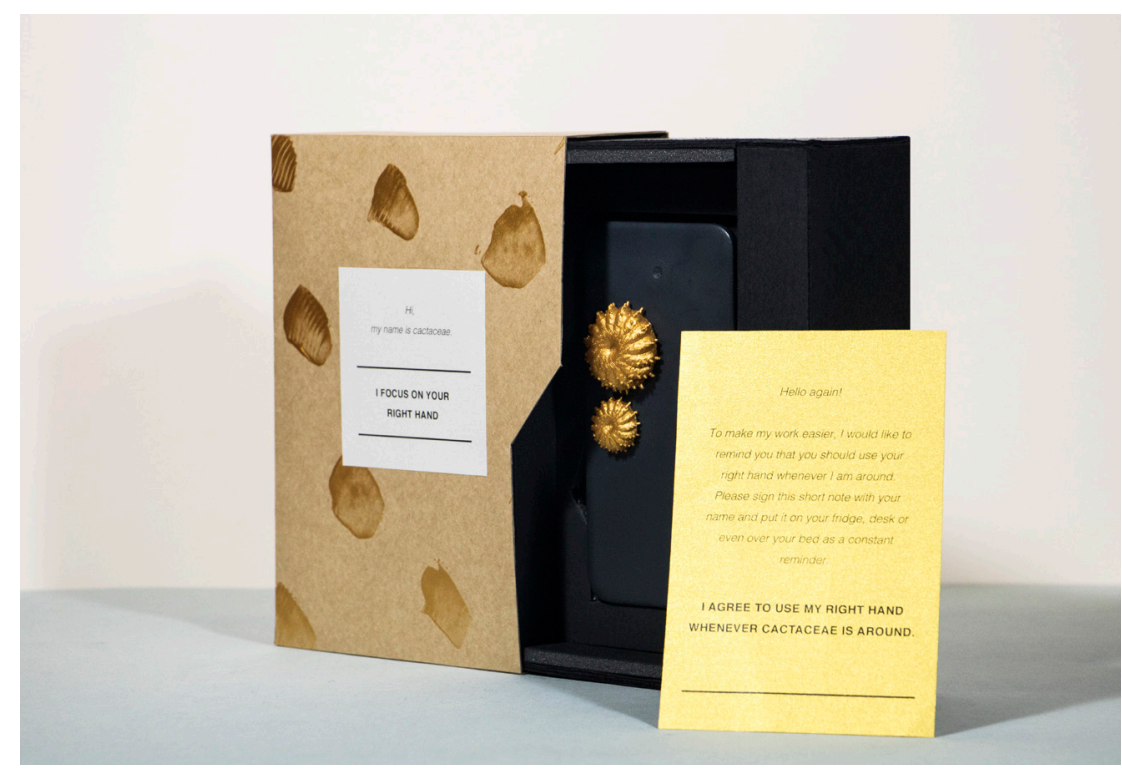

Figure 121:

Packaging and

behaviour note for PO2.

\subsubsection{Design criteria evaluation}

The two design prototypes were evaluated against the design criteria, see Figure 122. Criteria 06, 07, 10, 11, and 12 required a formative evaluation with stroke survivors and will be focused on in the following section. Criteria 08 and 09 were not included in the design but could contribute to long-term engagement in the process. 


\begin{tabular}{|c|c|c|c|}
\hline & & P 01 & P02 \\
\hline 01: Restrain & $\begin{array}{l}\text { The interaction with the object needs to restrain } \\
\text { movement in order to remind the user to use the } \\
\text { affected arm and hand }\end{array}$ & \multicolumn{2}{|c|}{$\begin{array}{l}\text { The use of the } \\
\text { smartphone is already } \\
\text { repetitive }\end{array}$} \\
\hline 02: Purpose & $\begin{array}{l}\text { The object needs to be used within an activity of } \\
\text { daily living }\end{array}$ & \multicolumn{2}{|c|}{$\begin{array}{l}\text { Objects are used with a } \\
\text { standard phone }\end{array}$} \\
\hline 03: Repetition & $\begin{array}{l}\text { The interaction with the object needs to be } \\
\text { repetitive in form of random practice }\end{array}$ & \multicolumn{2}{|c|}{$\begin{array}{l}\text { The use of the } \\
\text { smartphone is already } \\
\text { repetitive }\end{array}$} \\
\hline 04: Challenge & $\begin{array}{l}\text { The interaction with the object needs to become } \\
\text { progressively challenging and offer different } \\
\text { levels of complexity }\end{array}$ & \multicolumn{2}{|c|}{$\begin{array}{l}\text { Increased challenge } \\
\text { can be evoked by } \\
\text { reducing the elements } \\
\text { e.g. the grips }\end{array}$} \\
\hline $\begin{array}{l}\text { 05: Functional } \\
\text { feedforward }\end{array}$ & $\begin{array}{l}\text { The design of the object should be based on a } \\
\text { familiar object }\end{array}$ & \multicolumn{2}{|c|}{$\begin{array}{l}\text { Design can simply be } \\
\text { combined with a } \\
\text { standard phone }\end{array}$} \\
\hline $\begin{array}{l}\text { 06: Augmented } \\
\text { feedforward }\end{array}$ & $\begin{array}{l}\text { Augmented feedforward should contribute to } \\
\text { reminding the user to use the affected side of the } \\
\text { body }\end{array}$ & \multicolumn{2}{|c|}{$\begin{array}{l}\text { Evaluated with stroke } \\
\text { survivors }\end{array}$} \\
\hline $\begin{array}{l}\text { 07: Inherent } \\
\text { feedforward }\end{array}$ & $\begin{array}{l}\text { Inherent feedforward should indicate how the } \\
\text { user can interact with the object and that the } \\
\text { movement needs to be carried out with one } \\
\text { particular side of the body }\end{array}$ & \multicolumn{2}{|c|}{$\begin{array}{l}\text { Evaluated with stroke } \\
\text { survivors }\end{array}$} \\
\hline $\begin{array}{l}\text { 08: Functional } \\
\text { feedback }\end{array}$ & $\begin{array}{l}\text { Functional feedback should indicate the } \\
\text { successful completion of a task }\end{array}$ & $\begin{array}{l}\text { Not } \\
\text { included }\end{array}$ & $\begin{array}{l}\text { Not } \\
\text { included }\end{array}$ \\
\hline $\begin{array}{l}\text { 09: Augmented } \\
\text { feedback }\end{array}$ & $\begin{array}{l}\text { Augmented feedback should provide quantitative } \\
\text { feedback about the movement }\end{array}$ & $\begin{array}{l}\text { Not } \\
\text { included }\end{array}$ & $\begin{array}{l}\text { Not } \\
\text { included }\end{array}$ \\
\hline $\begin{array}{l}\text { 10: Inherent } \\
\text { feedback }\end{array}$ & $\begin{array}{l}\text { Inherent feedback should contribute to a } \\
\text { moderate level of effort }\end{array}$ & \multicolumn{2}{|c|}{$\begin{array}{l}\text { Evaluated with stroke } \\
\text { survivors }\end{array}$} \\
\hline $\begin{array}{l}\text { 11: Adherence } \\
\text { increasing } \\
\text { techniques }\end{array}$ & $\begin{array}{l}\text { The design should evoke a behaviour change in } \\
\text { the user }\end{array}$ & \multicolumn{2}{|c|}{$\begin{array}{l}\text { Evaluated with stroke } \\
\text { survivors }\end{array}$} \\
\hline 12: Movement & $\begin{array}{l}\text { The design should allow to hold the device with } \\
\text { the affected hand to initiate the use of it }\end{array}$ & \multicolumn{2}{|c|}{$\begin{array}{l}\text { Evaluated with stroke } \\
\text { survivors }\end{array}$} \\
\hline $\begin{array}{l}\text { 13: Compensa- } \\
\text { tion }\end{array}$ & $\begin{array}{l}\text { The design should minimise compensatory } \\
\text { movement }\end{array}$ & \multicolumn{2}{|c|}{$\begin{array}{l}\text { Evaluated with stroke } \\
\text { survivors }\end{array}$} \\
\hline
\end{tabular}

Figure 122: Evaluation of the design against the design criteria set. 


\subsubsection{Pluralistic walkthrough}

\subsubsection{Usability evaluation}

Each participant was informed about the purpose of the study and provided informed consent. The participants were asked to sit for the individual evaluation and had the three prototypes placed in front of them. The prototypes were chosen based on the side that was affected by the stroke. Each testing started with picking up the standard phone P00, followed by P01 and P02, see Figure 123. The participants were told that they could pick up the phone in any way that felt comfortable to them. After the testing, they were asked to point out which one they liked the most and which one they liked the least.

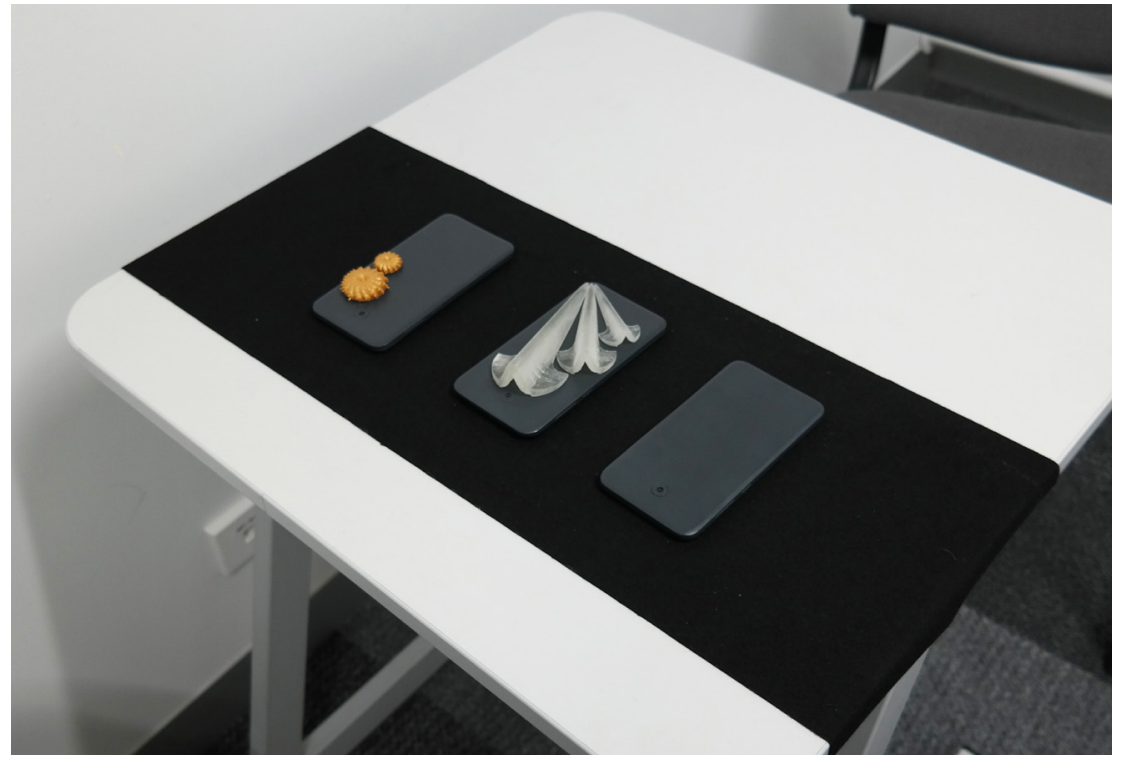

Figure 123:

Prototype P00, P01, and $\mathrm{PO} 2$.

\subsubsection{Analysis}

The feedback was analysed using deductive thematic analysis according to the themes of restraining effect, initiation of use, usability issues that impact on the use of the phone and could potentially impact on the initiation of use and the packaging and behaviour contract. Table 16 shows an overview of the results of the usability evaluation. Figure 124 shows the rating of the prototypes according to the participant's preference. 
Table 16: Formative usability evaluation.

\begin{tabular}{|c|c|c|c|c|c|c|c|}
\hline & Gender & Affected side & $\begin{array}{l}\text { Dominant } \\
\text { side }\end{array}$ & Prototype & $\begin{array}{l}\text { Restraining } \\
\text { effect }\end{array}$ & $\begin{array}{l}\text { Initiation of } \\
\text { use }\end{array}$ & $\begin{array}{l}\text { Behaviour } \\
\text { contract }\end{array}$ \\
\hline \multirow{3}{*}{01} & male & Left side & $\begin{array}{l}\text { Right } \\
\text { handed }\end{array}$ & 00 & None & $\begin{array}{l}\text { Picks it up: } \\
\text { No } \\
\text { Holds it: Yes }\end{array}$ & \multirow{3}{*}{$\begin{array}{l}\text { Does not } \\
\text { need remind- } \\
\text { ing. Agrees } \\
\text { with the idea } \\
\text { but would not } \\
\text { put it up on } \\
\text { the fridge }\end{array}$} \\
\hline & & & & 01 & $\begin{array}{l}\text { None: } \\
\text { Use with the } \\
\text { less affect- } \\
\text { ed side is } \\
\text { described as } \\
\text { more com- } \\
\text { fortable }\end{array}$ & $\begin{array}{l}\text { Picks it up: } \\
\text { No } \\
\text { Holds it: Yes }\end{array}$ & \\
\hline & & & & 02 & $\begin{array}{l}\text { None: Texture } \\
\text { feels comfort- } \\
\text { able }\end{array}$ & $\begin{array}{l}\text { Picks it up: } \\
\text { No } \\
\text { Holds it: Yes }\end{array}$ & \\
\hline \multirow{3}{*}{02} & male & Left side & $\begin{array}{l}\text { Right } \\
\text { handed }\end{array}$ & 00 & None & $\begin{array}{l}\text { Picks it up: } \\
\text { No } \\
\text { Holds it: No }\end{array}$ & \multirow{3}{*}{$\begin{array}{l}\text { Does not } \\
\text { need remind- } \\
\text { ing. Does } \\
\text { not think } \\
\text { it would be } \\
\text { necessary. }\end{array}$} \\
\hline & & & & 01 & None & $\begin{array}{l}\text { Picks it up: } \\
\text { No } \\
\text { Holds it: } \\
\text { Yes, but } \\
\text { holds it up- } \\
\text { side down. } \\
\text { Easier to } \\
\text { hold than } \\
\text { POO }\end{array}$ & \\
\hline & & & & 02 & $\begin{array}{l}\text { None: Tries } \\
\text { to hold onto } \\
\text { it during the } \\
\text { testing }\end{array}$ & $\begin{array}{l}\text { Picks it up: } \\
\text { No } \\
\text { Holds it: } \\
\text { Yes. Holds } \\
\text { onto the } \\
\text { design ele- } \\
\text { ments }\end{array}$ & \\
\hline \multirow{3}{*}{03} & male & Right side & $\begin{array}{l}\text { Right } \\
\text { handed }\end{array}$ & 00 & None & $\begin{array}{l}\text { Picks it up: } \\
\text { No } \\
\text { Holds it: No }\end{array}$ & \multirow{3}{*}{$\begin{array}{l}\text { Does not } \\
\text { need remind- } \\
\text { ing. Would } \\
\text { not sign the } \\
\text { behaviour } \\
\text { contract. }\end{array}$} \\
\hline & & & & 01 & $\begin{array}{l}\text { None: Doesn't } \\
\text { use the affect- } \\
\text { ed hand at all }\end{array}$ & $\begin{array}{l}\text { Picks it up: } \\
\text { No } \\
\text { Holds it: No }\end{array}$ & \\
\hline & & & & 02 & $\begin{array}{l}\text { None: Doesn't } \\
\text { use the affect- } \\
\text { ed hand at all }\end{array}$ & $\begin{array}{l}\text { Picks it up: } \\
\text { No } \\
\text { Holds it: No }\end{array}$ & \\
\hline 04 & female & Right side & $\begin{array}{l}\text { Left hand- } \\
\text { ed }\end{array}$ & 00 & None & $\begin{array}{l}\text { Picks is up: } \\
\text { No } \\
\text { Holds it: Yes }\end{array}$ & $\begin{array}{l}\text { Does not } \\
\text { need remind- } \\
\text { ing. Would } \\
\text { not sign the } \\
\text { behaviour } \\
\text { contract. }\end{array}$ \\
\hline
\end{tabular}




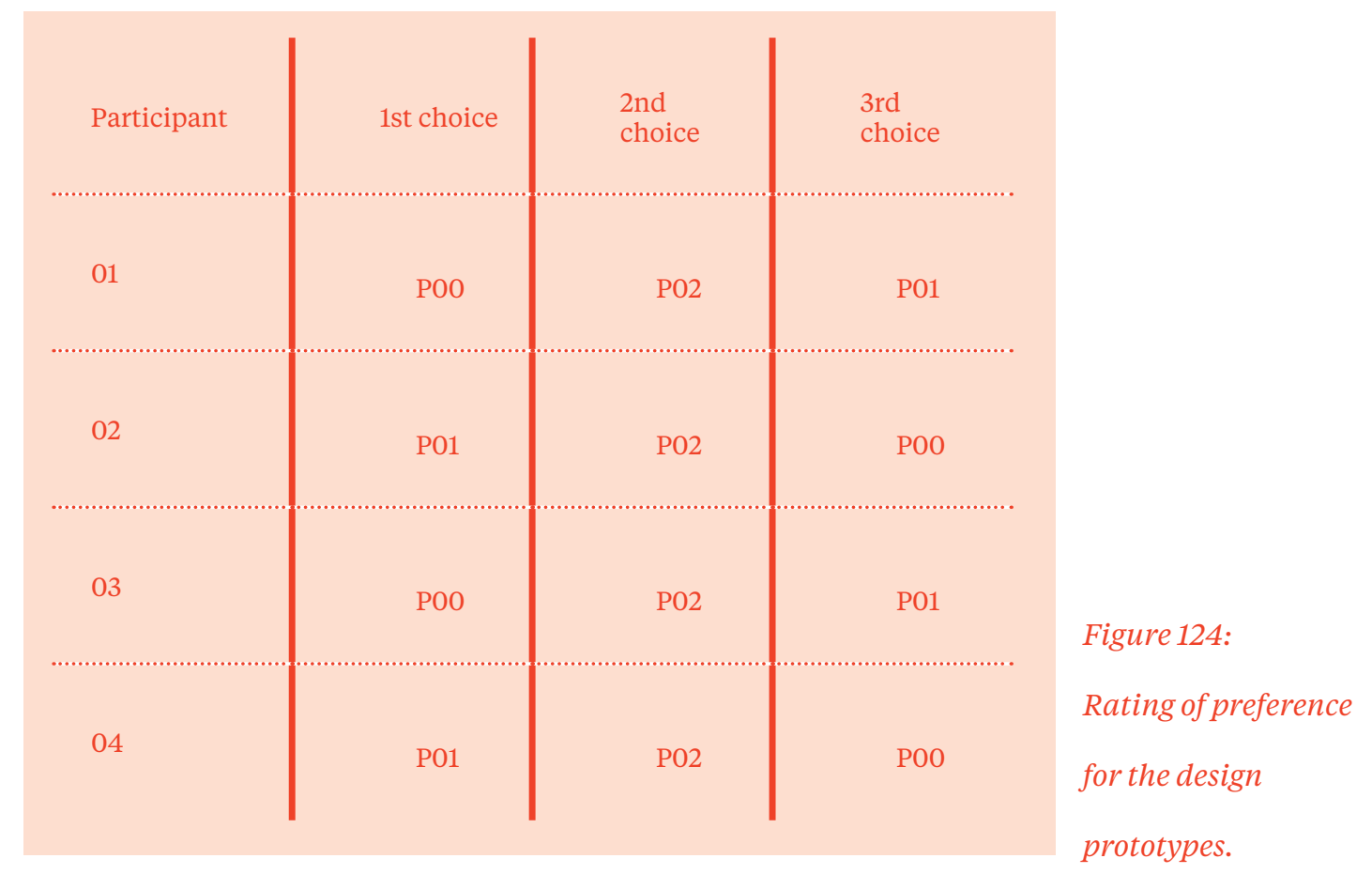

13.4.3.2.1 Restraining effect

Prototype P00, the standard smartphone, did not contain any kind of restraint and was used to see how the participants would pick up a standard phone. It had, therefore, no restraining effect on any of the participants. However, two of the participants initiated use of the affected hand to hold the phone.

Persuasive design P01

None of the participants used P01 in the intended form, but instead gripped the phone at the sides to give it a stable position inside the hand. The intended hand position was with slightly extended fingers at the back of the phone taking account of a possible decreased strength of the hand and fingers, see Figure 125. 


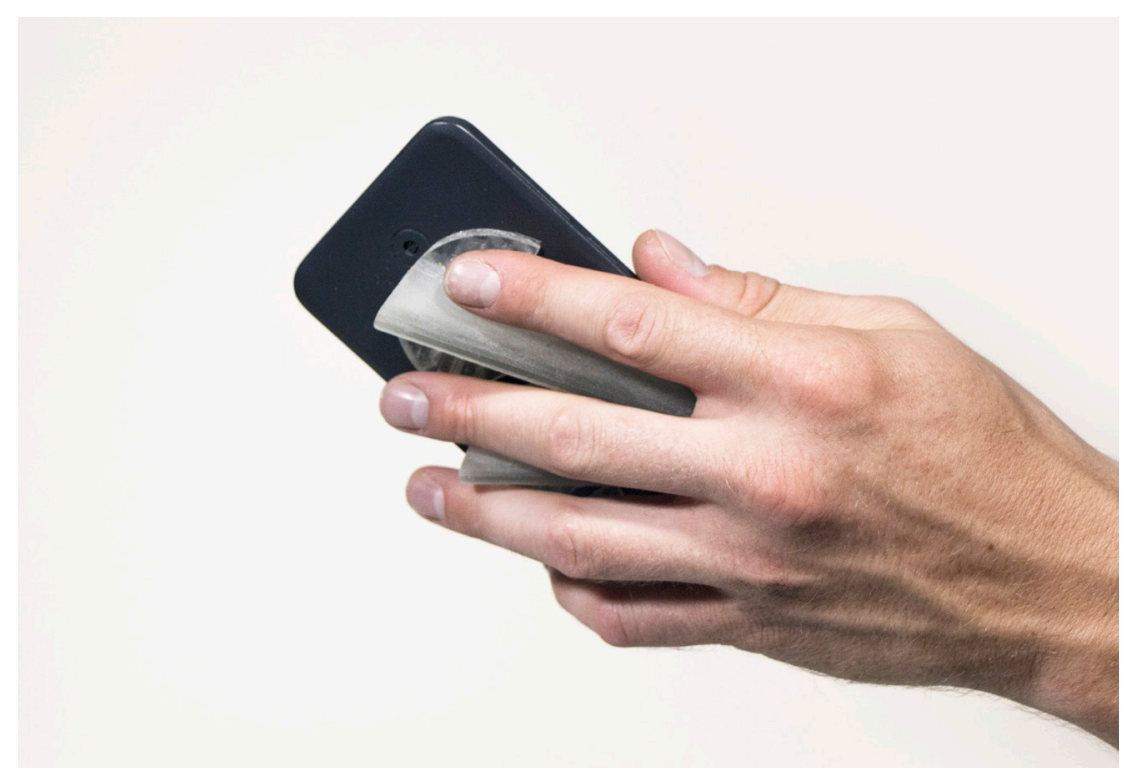

Figure 125:

Intended use of P01.

Two of the participants picked up the phone with the affected hand and transferred it to the less affected side to hold it. One of the participants picked it up with the affected side but had significant issues extending the fingers in a way that would allow a comfortable position. Instead, he held onto the design prototypes at the back and rotated the phone into a landscape position. He described holding a phone as quite difficult in general because his shoulder would tense up immediately. The fourth participant did not use his affected arm and hand while using the prototype.

\section{Coercive design $\mathrm{PO} 2$}

None of the participants described touching P02 as uncomfortable. Rather than trying to position the fingers away from the rough texture of the object the participants had the fingers close to or even on top of the object to use it as a kind of grip while holding the phone.

\subsection{Initiation of use}

Each of the participants picked up the phone prototypes with the less affected hand and transferred it to the affected side to hold it. Two of the four participants used the affected hand to hold the standard phone POO and pretended to click on one of the icons on the screen. The influence of P01 and P02 will be 
outlined below.

\section{Persuasive design}

P01 evoked an initiation of use in three of the four participants. The fourth participant used only his less affected hand and even put P01 on his left thigh to compensate for the uneven back of the phone. One of the participants used the affected hand to hold P01 but gripped onto the design element which meant the phone was rotated upside down.

One of the participants described P01 as offering more grip support compared to P00 and P02. The participant pointed out that P01 was the only design that could be picked up with the affected hand. The participant further described P01 as offering an easier way to hold onto the phone because of the roughness of the prototype surface. According to her, part of the problem with holding a smartphone is often that it is slim line and there is nothing to hold onto.

Coercive design

P02 evoked an initiation of use in three of the four participants. None of the participants experienced the design as uncomfortable. One of the participants used the design elements as a kind of handle and had the phone rotated upside down. The fourth participant did not initiate use of the affected hand but just used his less affected side to hold the prototype.

\subsection{Usability issues}

There were some general usability issues pointed out regarding semantic interpretation, orientation of the design elements, size of the prototypes, and device specific elements. A general issue was willingness to use the device. One participant who had issues opening up his hand explained that even if he were able to use the phone he would not chose to do so because he was used to his feature phone. 


\section{Semantic interpretation}

Semantic interpretation in the form of inherent, functional, and augmented feedforward allows the user to decipher the action possibilities of the object. None of the participants understood that P01 encourages opening the hand to place the fingers between the design elements, and that P02 is a sharp-looking object. One of the participants initially thought P01 was some kind of sea shell that someone had placed on the phone. Another participant preferred the visual appearance of P01 over P02 and described P01 as looking like a kind of shark or lilies.

I thought that is a piece of art that are shelves. But why would you put shelves on it?

Participant 01 pointing at P01

\section{Orientation}

The phone prototypes were gripped by two of the participants at the side rather than at the back. P01 was described as not being comfortable and that a convex form would fit better into the hand. P02 was described as offering too little support.

It doesn't affect my grip because my grip is mainly on the sides. And it is not making it more secure.

Participant 01 holding PO2

Size

P01 was criticised by two participants for its size, which they said would make storing it in the pocket quite tricky. However, one of the participants did slip it into the front pocket of his shirt and was surprised that it fit in there. 
I wouldn't want to carry it around.

Participant 01 looking at P01

\section{Device specific elements}

One of the participants had to open the fingers of his affected hand with his less affected hand to slide the phone into his hand. He pointed out that the thumb of the affected hand would always touch the smartphone screen and interfere with its functionality. Another participant mentioned that his hand grip is often quite tight and that meant he would tend to accidentally change the phone's volume. He suggested that the off switch for the phone should ideally be at the top rather than at the side.

\subsection{Packaging and behaviour contract}

All the participants mentioned that they would try to use the affected arm and hand as often as possible in everyday situations even before the behaviour contract was presented to them. Consequently, they did not see any need to sign a document that would ask them to use the affected hand while interacting with the design prototypes. One participant said he believed the behaviour contract would be beneficial. It needs to be considered, though, that there might have been a potential recruitment bias. All the participants were recruited through a research facility and were quite engaged in their therapies.

I think the psychology of having someone sign it, is good. That's very good because you have the commitment there [...] The mantra and the signing are very, very good. But I am not a fridge person.

Participant 01 about the behaviour contract

One of the participants pointed out that he would probably not read it at all when opening the package. 
[I think it is] useless. It wouldn’t work for me. It's like getting instructions when you turn the radio on.

Participant 03 about the behaviour contract

All the participants described the packaging as visually appealing. One of the participants preferred to have no packaging at all. Another participant suggested an option of returning the packaging to the shop for reuse.

\subsection{Discussion}

The persuasive, as well as the coercive design prototypes, evoked an initiation of use in the participants but require further refinements to increase the usability of the designs. The results of P01 will be discussed first, followed by the discussion of P02 and the influence of the behaviour change contract.

\subsubsection{Persuasive influence}

The evaluation of the design prototype against the design criteria shows that the design does not incorporate any form of augmented and functional feedback. The functional feedback is missing because the design is used in combination with a smartphone the user already owns. The functional feedback is provided by the smartphone rather than the design element. Additional augmented feedback could increase the usability of the design and clarify how the object needs to be used.

\subsubsection{Initiation of use and restraint}

The persuasive design P01, which uses physical and visual prompts in the form of ergonomic restrictions, did not restrain movement in the intended way. However, the restraining effect in the form of a bilateral task requiring the use of both limbs to hold the phone did evoke an initiation of use in three of the four participants. Participant four did not use his affected hand but started to show compensatory movement by putting the phone on his thighs to be able to tap the screen of the prototype. 
P01 was chosen as the preferred design solution by two of the participants. One participant had significant issues holding the phone comfortably in his affected hand. Further refinements of the design to provide support for participants with limited fine motor skills could increase the usability of the design. The second participant with a high level of fine motor skills who preferred P01 pointed out that it was the only design she would be able to pick up from a table with her affected hand. The prototype seemed to offer the potential to evoke an initiation of use that involves more complex fine motor skills.

\subsubsection{General usability issues}

The feedback from the usability evaluation indicated P01 was perceived as aesthetically pleasing, but it was not clear how the object needed to be used. The design lacked clear feedforward elements. One of the participants even asked at the beginning of the evaluation process if the object was something he could eat. None of the participants used the grip elements in the intended way but instead gripped the phone on the sides, where little support was provided.

A further point of criticism concerned the perceived comfort of holding and carrying the prototype. Two participants described P01 as their least liked design because of its form, which would make carrying it in a pocket difficult.

A further design iteration should incorporate feedforward elements indicating that the fingers need to be placed between the different objects, allow for a comfortable holding position, and decrease the overall size of the prototype.

\subsubsection{Coercive influence}

\subsubsection{Initiation of use and restraint}

The coercive design P02, which was based on "punishment" in the form of the object's uncomfortable texture did not restrain movement in the intended way. The restraining effect in the form of a bilateral task, on the other hand, evoked an initiation of use in three of the four participants. Participant four, again, did not use his affected hand at all to use the prototype. At that stage, the design was not perceived as coercive, and it remains therefore unclear whether such an influence 
would be tolerated by users. Future studies need to investigate if the coercive influence of the design would reinforce initiation of use.

\subsubsection{General usability issues}

It needs to be emphasised that the design aimed to evoke an uncomfortable feeling when touched, but none of the participants described it as being so. Rather, they experienced the surface as "textured". Some of the participants even put their hand over the object or gripped it to hold the phone. Further design iterations of P02 that aim to reinforce the coercive nature of the design should investigate alternative surfaces and material qualities perceived as uncomfortable to touch, for example sandpaper.

P02 was not criticised for its size; however, some of the participants held onto the design element to hold the phone. To allow such a use of the device the size of the prototypes should be increased. Such a change of design would need to depart from the coercive design influence since the element would be used more like a handle than something that should not be touched.

\subsubsection{Behaviour change contract}

The designs aimed to evoke a behaviour change in the user to overcome learned nonuse of the affected arm and hand in the long term. Participants' feedback indicates that they agreed with the purpose of the element, but they would not need it. One of the participants pointed out that it looked like an instruction for use that he would not pay any attention to.

All the participants mentioned that they would not need a reminder because they were all aware of their limitations and tried to use and train the affected arm as much as possible in daily activities. Even though the behaviour contract might not have had any effect on the participants of this study, it has the potential to reinforce initiation of use among users who sometimes forget to use the affected arm and hand.

Determining effective behaviour change is outside the scope of this study. However, the behaviour change contract is used as part of CIMT to evoke an 
initiation of use outside the clinical setting (Morris et al., 2006, pp. 262-263; Taub, 2013, pp. 129-130) and could potentially increase the effect of the design prototypes. Further design iterations should aim to incorporate the behaviour change contracts into the design to offer a constant reminder and include participants who have low motivation to use the affected arm and hand in daily activities.

\subsection{Further Design Iterations}

The two participants who preferred P01 showed different signs of impairment. One participant had quite severe problems in opening the affected hand, while the other one showed a high degree of fine motor skills. Two different design iterations will be outlined in the following section to address observed usability.

\subsubsection{Design iteration C01}

This design concept uses the ergonomic restrictions developed as part of P01 but in a different orientation, see Figure 126.

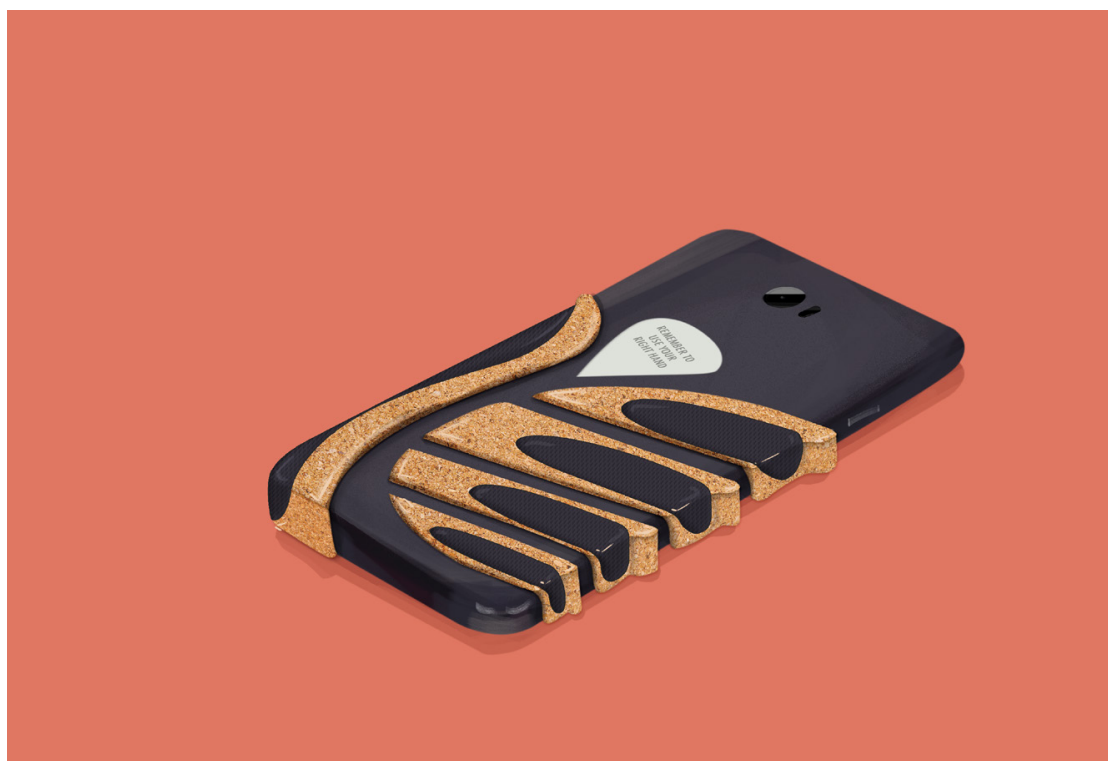

Figure 126:

Design iteration of

C01 that uses the

bilateral restraint

in combination with

ergonomic restrictions.

The behaviour contract

is included as a sticker. 
Figure 127:

Design iteration of

C01 that uses the

bilateral restraint

in combination with

ergonomic restrictions.

The behaviour contract

is included in as a

sticker.

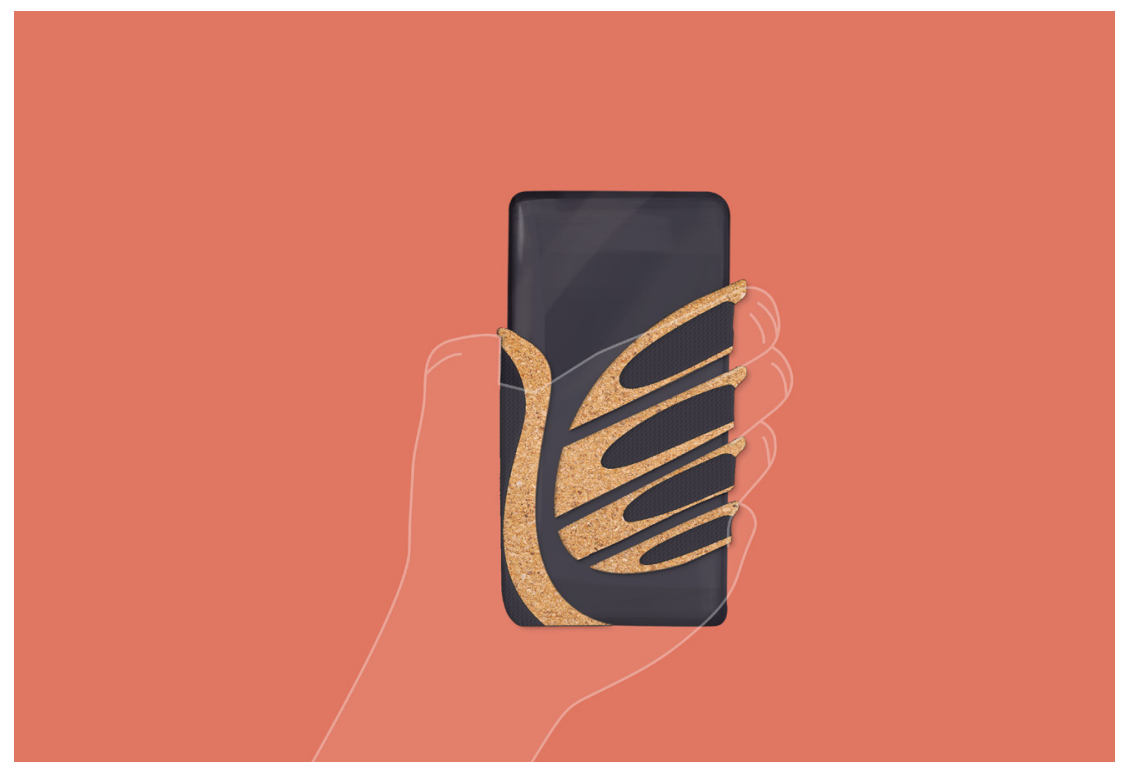

The new design supports gripping the side of the phone as two of the four participants did during the evaluation process. The handle elements are separate, allowing them to be placed on the phone to match the finger width of the participants; see Figure 127. The different elements are attached with doublesided tape onto the device, allowing them to be placed on different kinds of smartphones. The tape is attached in a way that it allows the user to easily peel it off and stick the design elements onto the smartphone. The behaviour contract was included in the design as a sticker, which can be removed if not needed. C01 uses cork as the primary material, combined with textured inlays to decrease the overall weight of the design prototype and limit the likelihood of dropping the phone. Textured inlays were added to C01 to avoid a slippery surface, which was previously mentioned as a significant issue.

\subsubsection{Design iteration $\mathrm{CO2}$}

The second design iteration C02 based on P01 aimed to address limited finger movement. The design had a handle at the back that can be used as a grip when picking up the phone and later to support the affected hand to decrease the risk of dropping the phone, see Figures 128 to 129. A focus of this design concept was to enable use of the affected arm and hand to evoke an initiation of use. 
The restraining effect is elicited through the bilateral task that requires using the affected hand to hold the phone. The handle aims to limit compensatory movement patterns since it is not possible to put the device flat on a surface. The ergonomic restriction influenced the position of the handle, slightly to the left, but was not expected to be the primary restraining factor. The behaviour contract was included as a sticker.

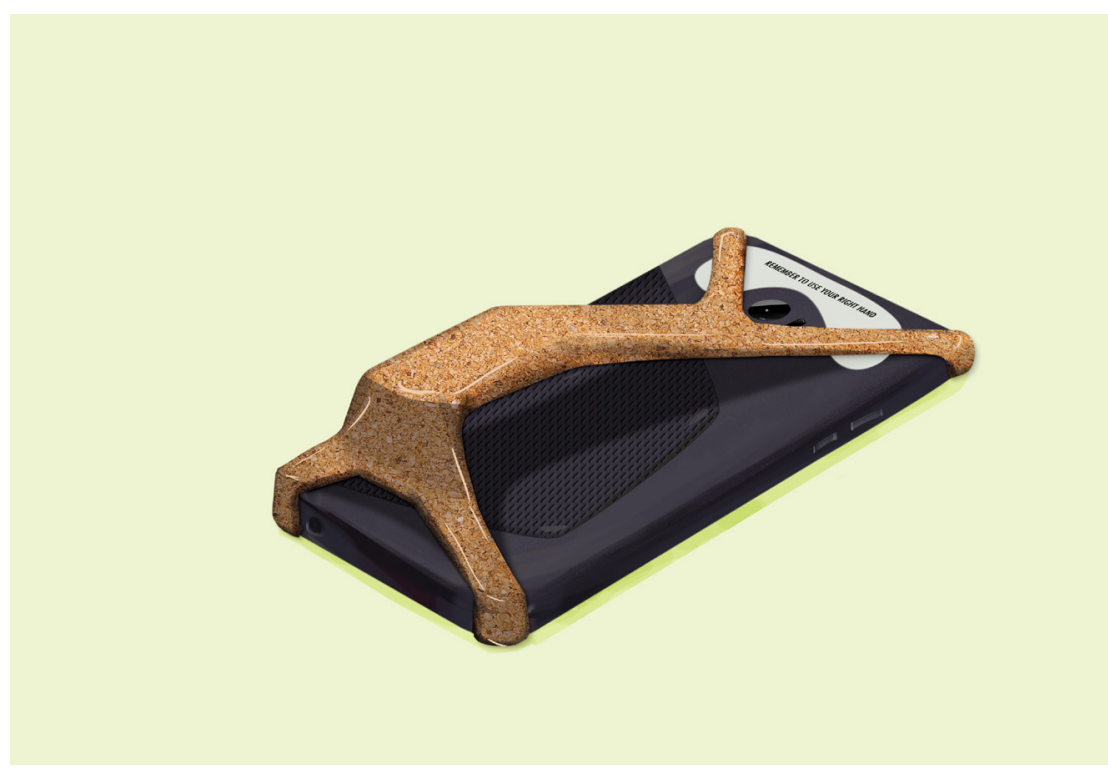

Figure 128:

Design prototype CO2, which uses a handle to initiate use of the affected hand.
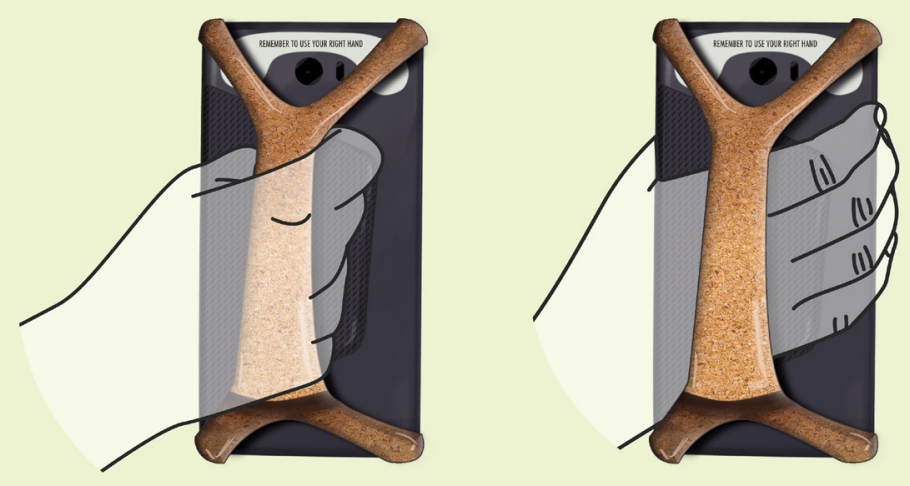

Figure 129:

Design prototype CO2 can be used as a handle or support for the affected side. 


\subsection{Conclusion}

A RTD methodology led to some design concepts that aimed to restrain movement while interacting with a smartphone to evoke an initiation of use. Two design prototypes, one with a coercive influence and one with a persuasive influence, were developed. Both prototypes required the use of both limbs and were tested by four chronic stroke survivors. Three of the four participants initiated use of the affected hand to hold the two design prototypes. The initiation of use appeared to be caused by focusing on a bilateral task rather than the anticipated coercive and persuasive design influences. The behaviour contract was perceived as beneficial, but all the participants stated they already focused on the use of the affected side in daily activities. Further research involving participants who have not been involved in the current research needs to investigate whether the actual design concepts would increase initiation of use and if the proposed design changes would increase the usability of the prototypes. Long-term studies would enable the results of this study to be validated and could investigate the use of a behaviour contract in daily activities.

\subsection{Limitations}

Participants for the study were recruited through physiotherapists in Auckland and were involved in this study prior to the usability testing. It needs to be considered that all the participants were highly motivated to use the affected arm and hand in daily activities. This motivation might have influenced the evaluation process. Further limitations are the limited amount of time during which participants could use the design and the small number of participants. A further limitation might be using a smartphone for the evaluation process. One of the participants pointed out that he would never use a smartphone in daily activities because he was used to his feature phone. The low motivation to use a smartphone might have influenced his behaviour in the testing session during which he only used the affected arm and hand. 


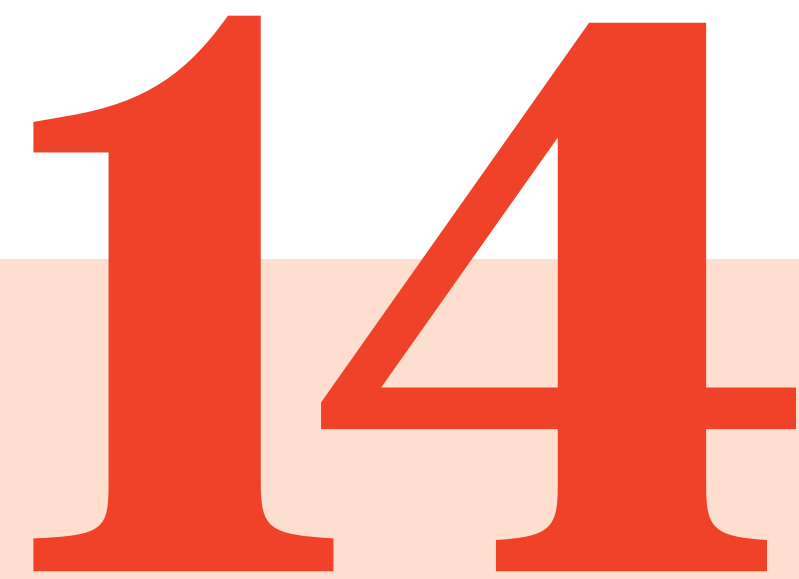

General Discussion 
I will reflect in this chapter on the research questions, contributions to, and limitations of this research. I highlighted at the start of the thesis that the process of making is an essential part of addressing the research questions and investigating possible design solutions that initiate the use of the affected arm and introduce restraint. Under the pragmatist perspective, the designerly inquiry offers the opportunity to develop a deeper understanding for the intended user, create, and validate solutions that address the observed problem (Biesenthal, 2014, p. 648; Dalsgaard, 2014, p. 150). This is particularly important where there do not exist objects that can be used to test the hypothesis. For this project, there were no existing everyday objects that intended to facilitate initiation of use of the affected limb in stroke survivors with learned nonuse. Designing the objects is an intrinsic and necessary activity to address the research question. The process of making was accompanied by reflections on the results and contributions to the body of knowledge, which requires a rigorous, precise, and structured approach (Biesenthal, 2014, p. 650) and addresses criticism on the pragmatist epistemology. The main research question of this study was addressed with three further research questions.

Main research question:

1. How can an everyday object that chronic stroke survivors use in activities of daily living restrain movement of the less affected upper limb to initiate use of the affected arm and hand?

Further research sub-questions:

1.1.1 How is movement of the upper limb restrained during rehabilitation to initiate use of the affected arm and hand in stroke survivors?

1.2.1 Which everyday objects are used by stroke survivors during activities of daily living that they like to take part in, and therefore may assist in facilitating use of the affected arm? 
1.3.1 How can we design an everyday object that restrains movement of the less affected arm to facilitate and initiate the use of the affected arm?

\subsection{Learned Nonuse and Initiation of Use}

This section will discuss the results relating to the following research question, which was addressed with thirteen semi-structured interviews with health professionals.

1.1.1 How is movement of the upper limb restrained during rehabilitation to initiate use of the affected arm and hand in stroke patients?

\subsubsection{Development of learned nonuse}

This research focused on initiation of use of the affected arm in chronic stroke survivors to overcome learned nonuse through a tailored rehabilitation approach. The results of this study indicate that learned nonuse of the affected arm and hand takes place over time and is an acquired behaviour of the stroke survivor reinforced through physiological factors, the structure of current rehabilitation, individual behaviour, and social factors.

The interviewed therapists often referred to the initial description of learned nonuse, which is based on studies with primates, to outline its influence on humans. The initial studies highlighted the fact that learned nonuse depends on multiple factors such as punishment, failure, and pain (Taub \& Morris, 2001, pp. 279-280. The factors contribute to and reinforce the self-taught suppression of using the affected arm and hand. Further studies focusing on the experience of chronic stroke survivors suggest that the factors of planning, initiating, and completing a movement; fatigue; a lack of confidence and control over the situation; fear of negative consequences; as well as fear of becoming dependent on others contribute to the phenomenon (Taule \& Råheim, 2014, pp. 2113-2114; Wallenbert \& Jonsson, 2005, pp. 220-223). The social pressure to appear normal 
can further reinforce the self-taught behaviour by trying to hide the condition (Lynch et al. 2008, pp. 519-520; Salter et al., 2008, p. 600; Taule \& Råheim, 2014, p. 2115). Another potential factor is the attempt by others to ease the stroke survivor's life and start to compensate for the lost functionality by taking over tasks for them (Lynch et al., 2008, p. 520), and from objects that intend to help, but end up facilitating compensation.

I have used the schematic model by Taub et al. (1994, p. 284) to outline the different factors that contribute to an initiation of use. The contributions of this study are highlighted in red, see Figure 130. Green highlights refer to previous studies that outline factors contributing to learned nonuse.

The results of the interviews with health professionals indicated that in clinical practice the concept of learned nonuse is strongly associated with the use of CIMT and its theoretical foundation. The term learned nonuse was just named in the context of this specific intervention. The results of this study suggest that other disciplines such as social sciences refer to the same phenomenon under different terms; for example, the lack of habit formation (Wallenbert \& Jonsson, 2005, p. 222). Further research could potentially extend the list of factors that contribute to learned nonuse after a stroke. A consensus on the same terminology would allow combining insights from different disciplines to develop a comprehensive model of factors that contribute to learned nonuse post-stroke. 


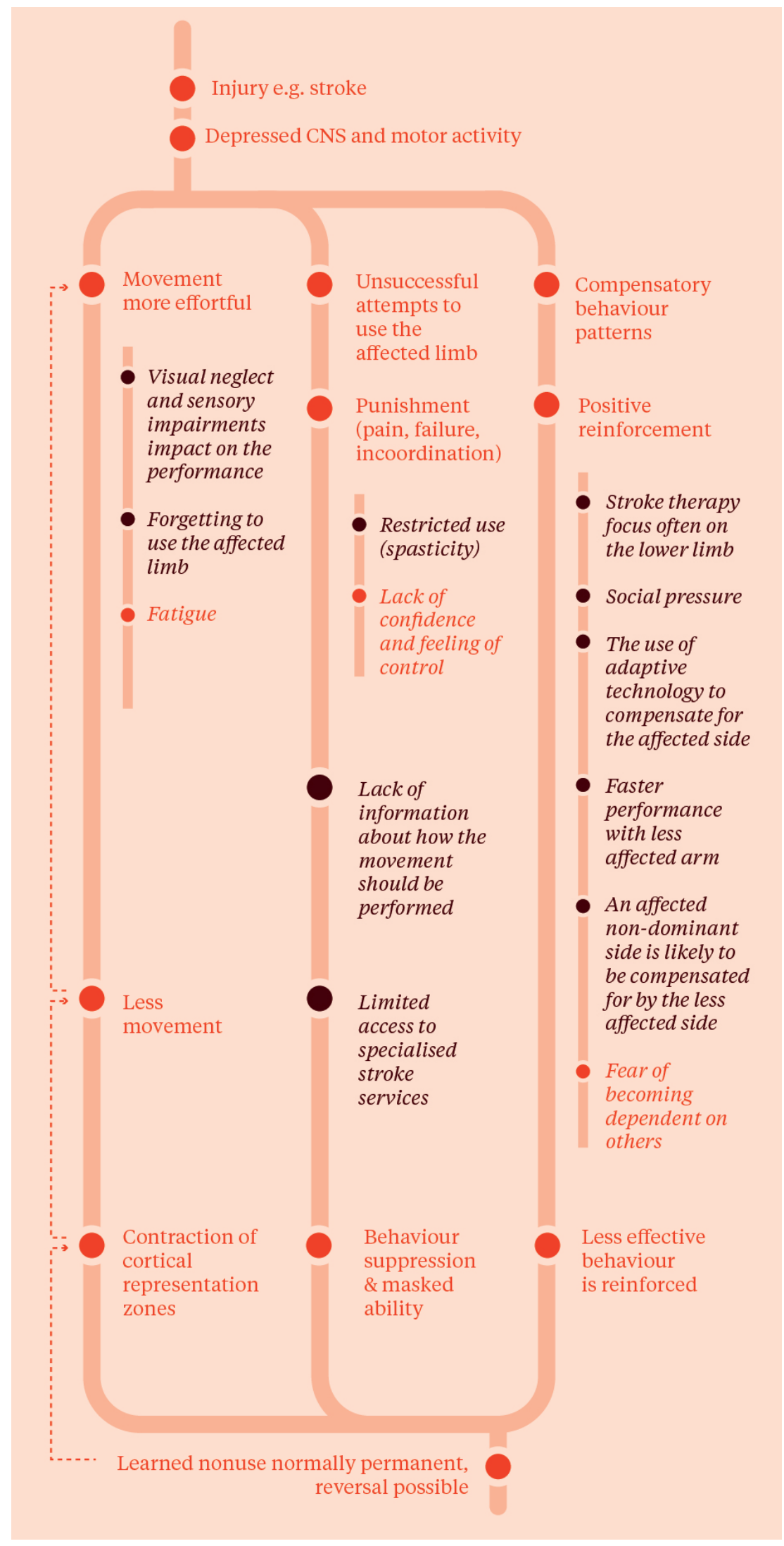

Figure 130:

Factors influencing

the development

of learned nonuse

adapted from Taub et

al. (1994, p. 284). The

contributions of this

study are highlighted

in black italic. Red \&

red italic highlights

refer to previous studies

that outline factors

contributing to learned

nonuse. 


\subsubsection{Initiation of use and the use of restraint}

To overcome learned nonuse and initiate use of the affected arm and hand a restraint can be used. In clinical practice this seems to be done mainly as part of CIMT. The restraint is applied on the less affected arm and hand to motivate the stroke survivor to use the affected side. The thematic analysis of the interviews with stroke therapists and researchers indicated that to overcome learned nonuse three main steps need to be followed.

1. Assessing the amount of learned nonuse;

2. Choosing appropriate strategies to evoke the initiation of use based on assessed motor capabilities and motivational input; and

3. Evoking a behaviour change to transfer gains made in the process into real-world situations.

The description of CIMT correlates with those three steps (Taub et al., 2006, p. 259; Taub, 2012, p. 161; Taub, 2013, pp. 129-131). The schematic model by Taub et al. (1994, p. 285) is often referred to. I have outlined additional factors that contribute to overcoming learned nonuse based on this schema, see Figure 131. The additional factors are based on the results of the interviews with therapists and researchers. The use of restraint is part of "B: Initiation of use/Increased motivation”. Its use in clinical practice will be outlined in the following section.

\subsubsection{Use of restraint}

The results of this study indicate that physical restraint is mainly used as part of CIMT in the form of a soft mitt that is applied on the less affected arm and hand to overcome the learned nonuse. The restraint reminds the patient to use the affected arm and hand rather than physically restraining movement. Its role is the one of an extrinsic motivator (Dancause et al., 2015, p. 339) that reinforces the initiation of use, see Figure 146. It is a behavioural restraint that reminds the stroke survivor to use the affected arm and hand rather than physically restraining the movement. In clinical practice, the mitt is the preferred form of restraint as it 


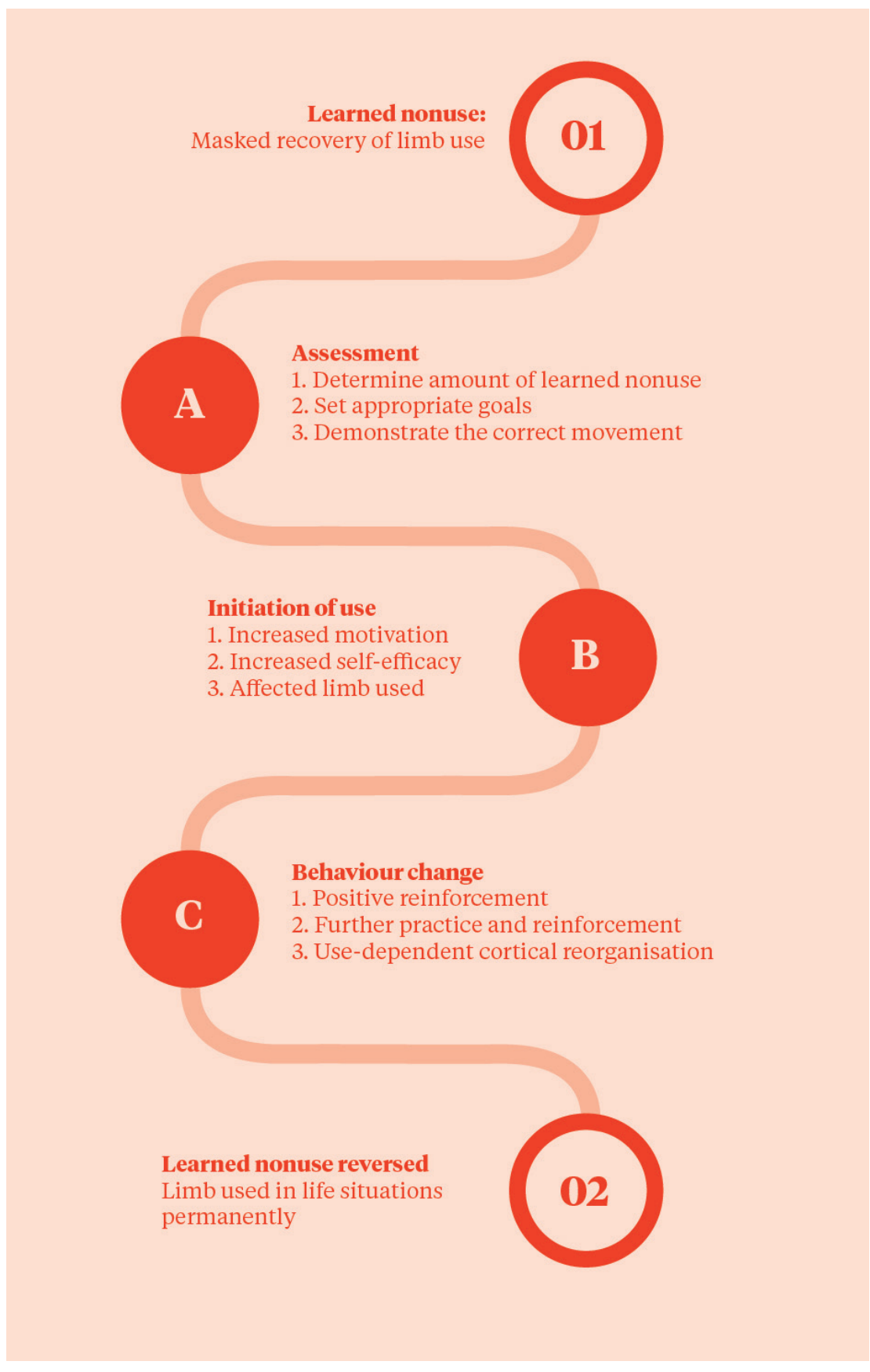

Figure 131:

Overcoming learned nonuse adapted from Taubet al. (1994, $p$. 285). Initiation of use is outlined as part of ' $B$ '.

maintains the ability to extend the arm in the case of balance issues (Taub et al., 1998, p. 159; Uswatte et al., 2006, p. 149).

To overcome the learned nonuse, the stroke survivor must undergo a behaviour change to transfer gains made in the rehabilitation setting into the real world (Morris et al., 2006, pp. 265-266; Taub, 2012, pp. 163-164). CIMT, therefore, 
uses additional elements like the transfer package to evoke a behaviour change (Morris et al., 2006, pp. 261-264).

\subsubsection{Self-efficacy}

A unique element to evoke an initiation of use among chronic stroke survivors that was pointed out by the therapists is the focus on feasible goals and the belief of the stroke survivors that performance of the movement is possible. This belief is called self-efficacy and relates to a subjective cognition of being able to perform a particular behaviour (Clark \& Zimmerman, 2014, p. 485; Dancause et al., 2015, p. 341). To initiate use of the affected arm and hand the stroke survivor needs to believe that it is just the learned nonuse that is impacting on using the affected arm and hand. The components that aim to evoke an initiation of use mentioned during the interviews can be mapped to the following elements of selfefficacy (Bandura, 1997):

- Mastery experiences;

- Vicarious experiences; and

- Verbal persuasion.

Self-efficacy beliefs rely on acquirable skills, increasing the belief of the individual that they can gain these skills, and refining activities in a way that makes them achievable (Bandura 1997, p. 105). However, little is known about effective strategies to create self-efficacy in stroke survivors in regard to the upper limb (Dancause et al., 2015, pp. 341-342). The validation of self-efficacy components included in the design prototypes was outside the scope of this study.

\subsubsection{Initial design criteria}

The results of the analysis of the semi-structured interviews led to a set of seven design criteria (Rodríguez Ramírez, 2017) that was referred to in the design process of investigating possible ways to restrain movement to evoke an initiation of use; see Figure 132. The design criteria were applied during the development of the design to guide the process and evaluate concepts. 


\begin{tabular}{|c|c|}
\hline $\begin{array}{l}\text { 01: } \\
\text { Restrain }\end{array}$ & $\begin{array}{l}\text { The interaction with the object needs to restrain } \\
\text { movement to evoke an initiation of use }\end{array}$ \\
\hline $\begin{array}{l}\text { 02: } \\
\text { Purpose }\end{array}$ & $\begin{array}{l}\text { The object needs to be used within an activity of daily } \\
\text { living }\end{array}$ \\
\hline $\begin{array}{l}\text { 03: } \\
\text { Repetition }\end{array}$ & The interaction with the object needs to be repetitive \\
\hline $\begin{array}{l}\text { 04: } \\
\text { Feedback }\end{array}$ & Feedback needs to be provided as part of the interaction \\
\hline $\begin{array}{l}\text { 05: } \\
\text { Challenge }\end{array}$ & $\begin{array}{l}\text { The interaction with the object needs to become } \\
\text { progressively challenging }\end{array}$ \\
\hline $\begin{array}{l}\text { 06: } \\
\text { Self-efficacy }\end{array}$ & $\begin{array}{l}\text { The interaction with the object needs to contribute to } \\
\text { developing self-perceived self-efficacy of the user }\end{array}$ \\
\hline $\begin{array}{l}\text { 07: } \\
\text { Usability }\end{array}$ & $\begin{array}{l}\text { The interaction with the object needs to be achievable for } \\
\text { a stroke survivor with motor impairments }\end{array}$ \\
\hline
\end{tabular}

Figure 132:

Initial design criteria.

Some of the criteria could not be evaluated during the design process and required the involvement of users; for example, criteria 06 and 07. It further transpired that criterion 06 was too complex due to the lack of knowledge concerning effective ways to evoke self-efficacy in the context of upper limb stroke rehabilitation.

Design criteria that focused on specific details were easier to address in the design process. Criteria 04 was, for example, quite broad in the first criteria set and was further refined in augmented, inherent, and functional feedback (Wensveen et al., 2004, pp. 179-180). Feedforward was not mentioned by the stroke therapists, 
but was added as a design criterion since it had the potential to contribute to an initiation of use. Feedforward is also referred to as part of the conceptual model that one has of an object. It clarifies real and perceived affordance that the object offers through the use of signifiers (Norman, 2013, p. 14) and allows a connection between action and function of an object for the user (Locher et al., 2010, p. 72).

\subsection{Daily Activities Post Stroke}

This section will discuss the results relating to the following research question:

1.2.1 Which everyday objects are used by stroke survivors during activities of daily living that they like to take part in, and therefore may assist in facilitating use of the affected arm?

Interviews with health professionals, an online survey, and six semistructured interviews with stroke survivors were conducted to address this research question.

\subsubsection{ADL}

The findings of this study indicated a discrepancy between activities that stroke survivors prioritise immediately after the stroke and those that they would like to take part in once they are living at home again. The stroke therapists described several tasks that contribute to dignity, for example being able to wash oneself, while the stroke survivors in the online survey identified more complex daily activities, for example driving and leisure tasks such as sport.

This study suggests that meaningful activities change over time, because the survivor has gained an understanding of what kinds of activities are possible to perform once they return home. A recent review confirms that stroke survivors change their behaviour patterns after the stroke and increase the amount of physical activities during the chronic stage (Fini et al., 2017, p. 711). An alternative 
explanation is the setting in which stroke rehabilitation is provided. Goals for the rehabilitation process immediately after the stroke have to be or ideally should be SMART: specific, measurable, activity-related, realistic, and time-limited (Wilson, 2008, p. 145) to be achievable in the restricted time frame available to therapists. Stroke survivors, on the other hand, define the recovery process as a way to return to their previous lifestyle (Doolittle, 1992, p. 124; Plant et al., 2016, pp. 923-924).

The importance of social and leisure activities needs to be highlighted at this stage. The role in the recovery process has been reported in previous studies (Carlsson et al., 2009, p. 780; Luke et al., 2015, p. 1705; Mauss \& Robinson et al. 2009, p. 1559), as well as the demand to include related activities in the rehabilitation process (Narayan Arya et al., 2012, pp. 205-206; Robison, 2009, pp. 1564-1565). There needs to be consideration though that such activities often require a high level of complexity, which might not be possible to achieve after a stroke. It has been reported that stroke survivors often do not resume previous activities that were meaningful before the stroke because they feel uncomfortable in the public environment or refuse to take part in an activity in a restricted way (Robison et al., 2009, p. 1564). It further needs to be considered that multiple factors influence the performance of leisure activities such as the type of leisure task, social and support system, personal, environmental and economic barriers, or transportation (Gillen, 2016, p. 301). The inclusion of more complex activities can potentially increase engagement in the rehabilitation process; however, given the current financial resources that stroke survivors can access, it seems such an approach may not be feasible to implement in clinical practice in New Zealand.

\subsubsection{Everyday objects used as part of ADL}

The everyday objects that were named in the online survey as being essential in daily activities are objects used for telecommunication tasks, for example, a smartphone. Six semi-structured interviews with chronic stroke survivors were conducted to gain a deeper understanding of the motivation as well as barriers influencing its use. The results suggest that the object can evoke 
a feeling of safety, facilitate reintegration into the social and work environment, are part of leisure activities, help to connect with others and can even be used as active contributions in the rehabilitation process. Studies have reported similar motivational drivers to use ICT devices (Gustavsson et al., 2018, pp. 564-566;

Kamwesiga et al., 2017, pp. 441-446), but there is little focus on the barriers that impact on the use of such devices. The results of the current study are shown in Figure 133 and Figure 134 as a schematic overview.

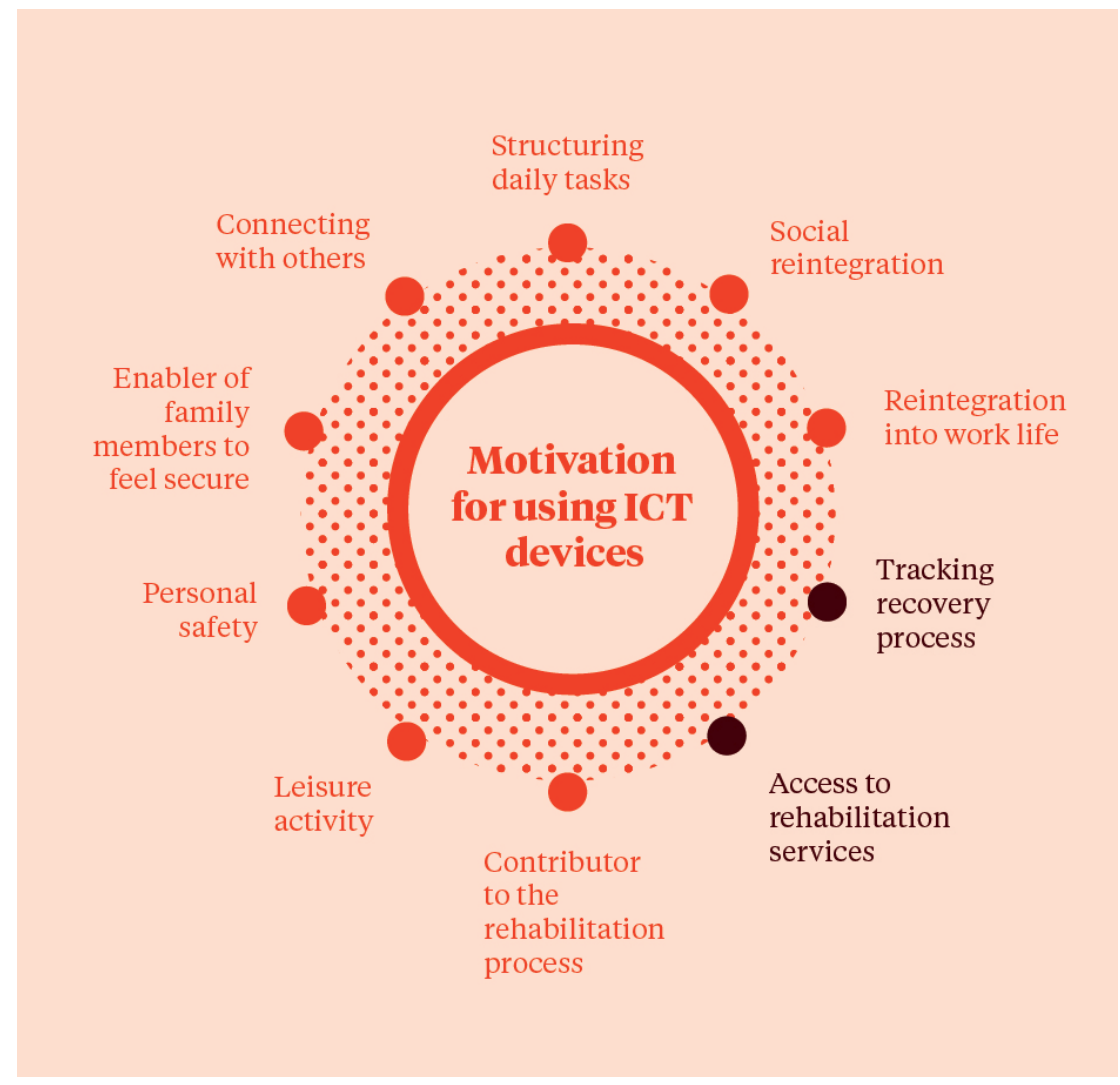

Figure 133:

Motivation for

using information

communication

devices (ICT) such as

a smartphone after

the stroke. The factors

"Tracking recovery" and

"Access to rehabilitation

services" were named in

previous studies. 


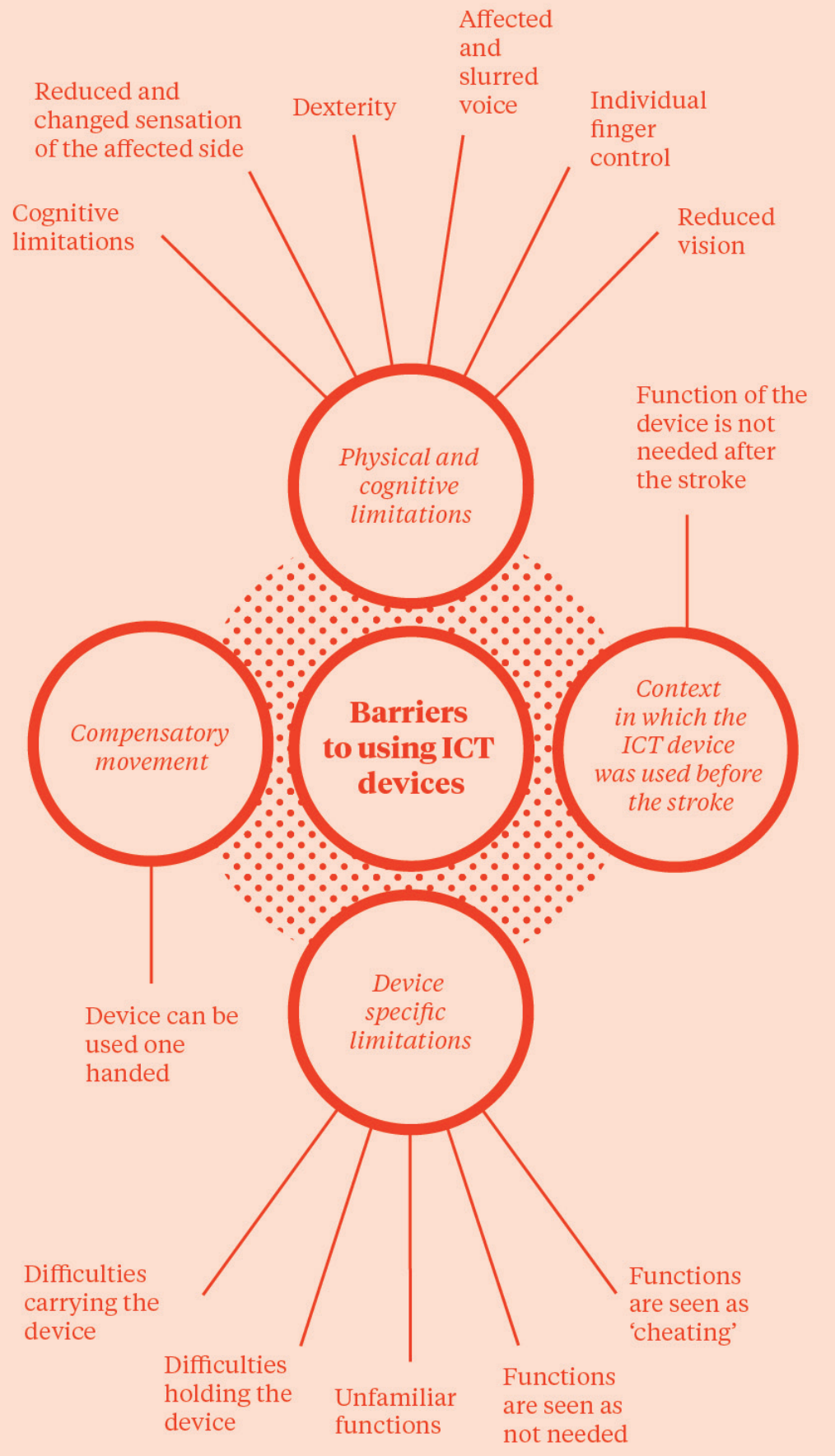

Figure 134:

Barriers that impact on the use of information communication devices (ICT) after a stroke. 
In the context of this research, ICT devices offer potential to evoke an initiation of use because of their smaller size, allowing easy integration into everyday situations (Kamwesiga et al. 2017, p. 447). They are also a familiar object in modern society and have no product-related stigma attached to them, in contrast to other assistive technologies, for example, the wheelchair (Kielhofner, 2002, p. 106). Stroke survivors are often highly motivated to continue using the devices after the stroke (Gustavsson et al.2018, p. 566), which can further contribute to the initiation process.

The focus of this current study was on the physical form of the device and barriers that currently impacted on the use of the devices that needed to be considered in the design process, see Figure 134. Barriers caused by the software of the device were outside the scope of this study and therefore not addressed. One barrier identified in regard to the use of an iPhone 6 was that when the affected hand started to shake, letters were typed multiple times. Further research could contribute to the development of software to address this and similar stroke specific limitations.

When examining the results, there was just a small number of stroke survivors involved in the semi-structured interviews. The findings of this study offer a starting point for further research projects which could extend and validate barriers to using ICT devices.

\subsection{Design Prototypes and Theorising of Results}

This section will discuss the following research question:

1.3.1 How can we design an everyday object that restrains movement of the less affected arm to facilitate an initiation of use of the affected arm? 
This research focused on a RTD process and can be divided into the two main steps of:

1. Development of prototypes to externalise possible solutions and use of the prototypes as a form of inquiry

2. Theorising the results in the form of bridging concepts

The following sections will outline the generated knowledge and contributions to the field of design.

\subsubsection{Limited use only}

The results of the RTD process in the form of serial and expansive design experiments (Krogh et al., 2015, pp. 46-47) were theorised in the form of bridging concepts (Dalsgaard \& Dindler, 2014, p. 1637) that designers can refer to as a source of inspiration. This form of intermediate knowledge that needs to prove its value in practice is based on the pragmatism perspective used in this current study (Dalsgaard \& Dindler, 2014, pp. 1638-1639). The designs were tested with clinicians and the strategies were tested with designers as it was important to make those strategies useful for designers.

It needs to be emphasised at this stage that a restraining effect alone is not sufficient to evoke the intended initiation of use in the long-term. Learned nonuse is an acquired behaviour in stroke survivors that needs to be addressed with behaviour change components. The interaction with the design should, therefore, take account of additional elements to evoke a long-term effect, such as a repetitive movement and the incorporation of a behaviour change contract - see Figure 135 for a schematic overview of the design process. The following sections will focus on the different steps that need to be considered. Validation of the intended behaviour change is outside the scope of this study and requires further long-term studies. 


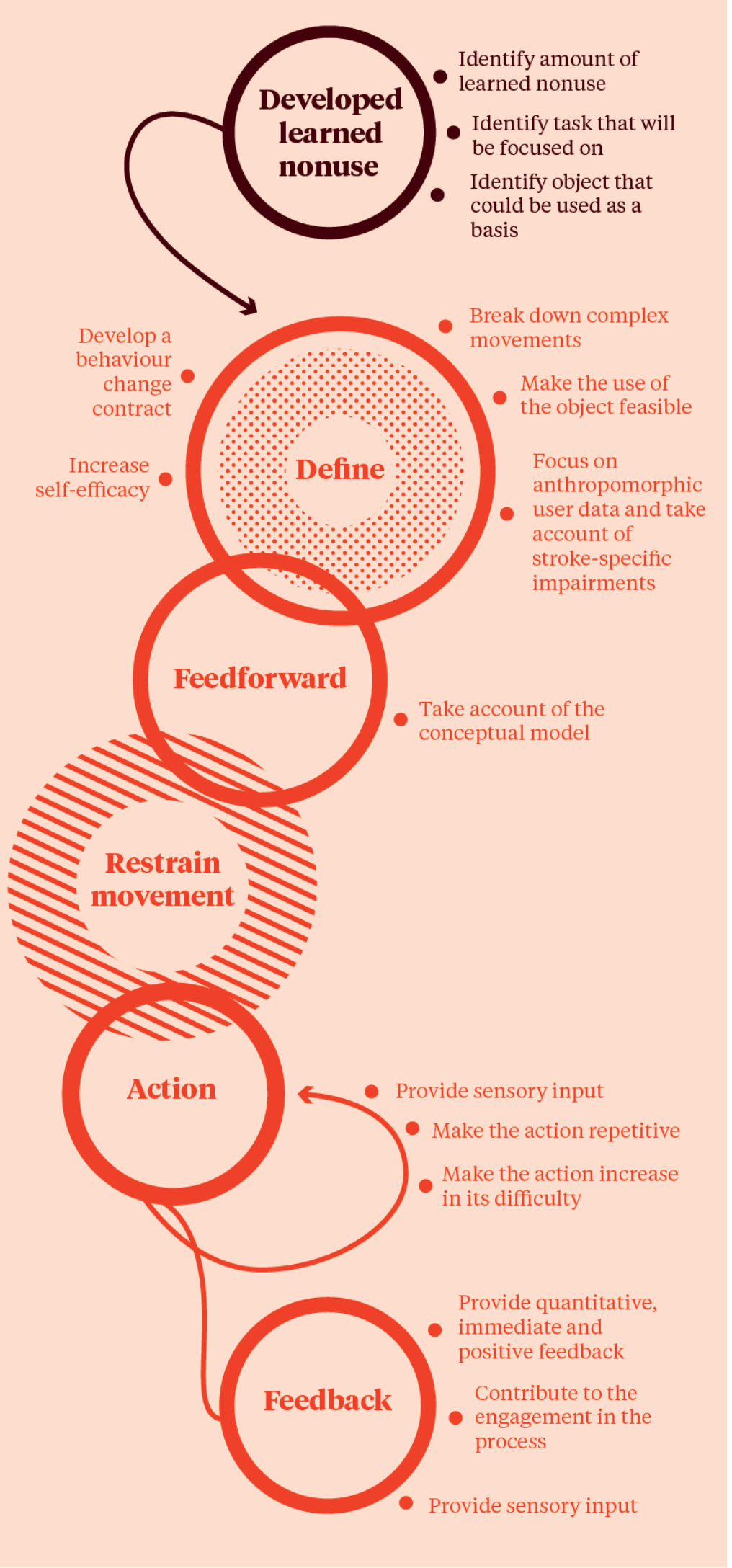

Figure 135:

Overview of design elements. 


\subsubsection{Identify the developed learned nonuse}

The first step in the process is to define which movement the user wants to focus on and establish that the movement is impacted by the learned nonuse. This step is performed as part of CIMT using the motor activity log (MAL) which is a semi-structured interview containing an amount scale and how well scale (Morris et al., 2006, p. 262). The assessment of the amount of learned nonuse was not a focus of this study but use of the MAL has been shown to provide reliable and valid measurements (Uswatte, Taub, Morris, Light, \& Thompson, 2006). Based on the outcomes of the assessment, it needed to be determined which object involved in daily tasks could be used as a basis for the design process.

\subsubsection{Define}

The design process starts with making use of the object achievable to contribute to the self-efficacy of the user and evoke an initiation of use. Increasing the belief of the individual that they can perform the behaviour often is dependent on refining the activity in a way that makes goals achievable (Bandura, 1997, p. 105). The design strategies focused on observations and storyboards to help to break down complex movement patterns involved in performing a particular task. Further elements that needed to be considered in this process were anthropomorphic data and stroke-specific impairments, for example, decreased wrist extension.

Breaking down the movement and making it feasible:

1. Carry out the movement

2. Observe it

3. Break it down into its components

4. Focus on a simple movement or just one element

To overcome learned nonuse in the long-term, the user must undergo a behaviour change to transfer gains made in the rehabilitation setting into the real world (Morris et al., 2006, pp. 265-266; Taub, 2012, pp. 163-164). The behaviour contract used as part of CIMT is a formal agreement signed by the therapist and 
the stroke patient on the first day of the treatment. I have included the behaviour contract because it determines the amount and context of use of the affected arm. The behaviour contract should outline when the affected arm and hand should be used and can be implemented in the following ways.

The use of the behaviour contract:

- The behaviour contract is part of the actual design

- The behaviour contract is part of the packaging of the design

- The behaviour contract is provided as an additional element

\subsubsection{Feedforward}

The evaluation of the radio prototype with stroke survivors highlighted the fact that the design prototype needs to take account of the already established conceptual model that one has of the object (Norman, 1999, pp. 25-31). The impact of the stroke might require modifications and adjustments that allow the use of the objects, but the conceptual model needs to remain the same in order to secure an intuitive use and initiation of use. The element of feedforward was not explicitly defined as part of the digital design method cards but has been used as part of the design criteria of the second radio and the phone prototypes. Based on the results of Chapter 6, the different feedforward elements should be addressed in the following way:

\section{Feedforward elements:}

- Functional feedforward should communicate the general purpose of the object and how it should be used in a specific ADL.

- Augmented feedforward should convince and remind the user that the use of the affected arm is possible.

- Inherent feedforward should communicate the kind of actions that are possible to perform with the object and remind the user to use the affected side

Functional and inherent feedforward of the design can refer to stereotypes, 
similar products, metaphors, characters, conventions, and clichés (Crilly et al., 2004, pp. 565-567) that allow the user to refer to an already established conceptual model of the object. Cultural and physical constraints (Norman, 1990, pp. 12-13) can be used to exemplify the restrained interaction. Augmented feedfoward in clinical practice is provided in the form of coaching and modelling how the movement should look (Morris et al., 2006, p. 260). Educational material that is provided alongside the design prototype could be beneficial in explaining how the movement needs to be performed, but requires further research.

\subsubsection{Restrain the movement}

The following strategies are based on the design workshop with industrial design students, see Chapter 7, and were tested by designers using digital design method cards, see Chapter 8 . The second design workshop, see Chapter 12 , that focused on the development of restraining phone designs resulted in the conceptual development of further design elements. The developed ideas were not tested under the pragmatist perspective due to time constraints and require the development of physical design prototypes to validate their restraining effect. Figure 136 shows an overview of the expected influence that the strategies have on the user based on the "Classification of Influence Based on Intended User Experience” (Tromp et al., 2011, p. 12).

Restraining the movement:

- Ergonomic restrictions that encourage the use of a specific side of the body

- Bilateral tasks that involve the use of both arms and hands: by keeping the unaffected arm occupied, the affected limb needs to be used to perform the task

- Intuitive use that relies on the already established conceptual models that the user has of the object 


\section{Restraining design elements}

to evoke an initiation of use

of the affected arm and hand

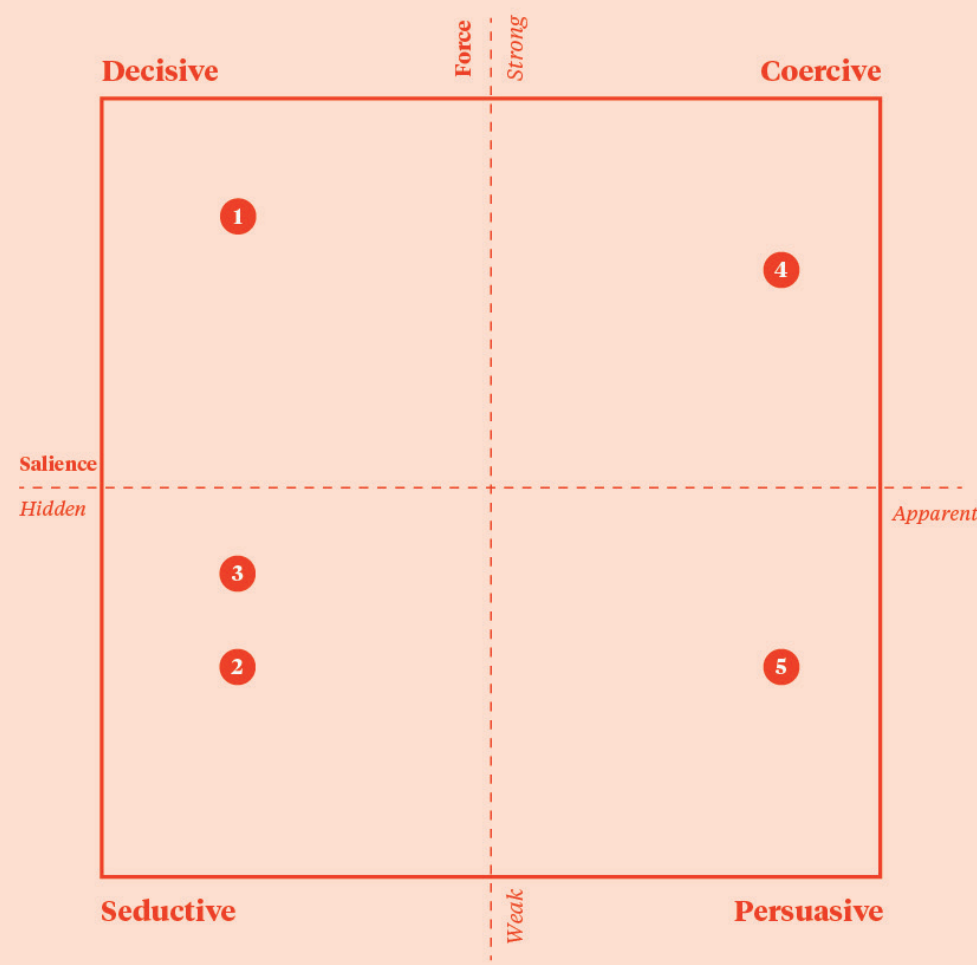

(1) Physical prompts/ cues in the form of ergonomic restrictions

(2) Bilateral tasks that involve the use of both arms

3 Intuitive use

(4) Limiting the functionality

5 Physical \& visual prompts/ cues to remind the user

Figure 136:

Overview of restraining design elements. 
- Limiting functionality to make the use of the affected arm and hand necessary

- Visually and verbally reminding the user to use the affected arm and hand

\section{Influence of the restraint}

The usability evaluation of the radio with stroke survivors indicated that the restraining influence during the interaction was tolerated by the participants in the individual testing; however, one participant during the group testing expressed concerns towards the restraining effect indicating a possible coercive influence. The line between a persuasive and a coercive influence on a user can be difficult to draw (Fogg, 2003, p. 21) and depends on the voluntariness of the user (Smids, 2012, p. 131). Users can choose to accept or disregard the designer's proposed way of doing things (Redström, 2006, p. 115), which means the anticipated persuasive influence can be experienced as coercive when it conflicts with one's individual freedom (Tromp et al., 2011, p.17). To overcome its potentially coercive nature, the object needs to offer freedom of choice to the user (Smids, 2012, p. 126).

At this stage, it remains unclear whether a persuasive influence is the most efficient way to evoke an initiation of use. The two smartphone prototypes aimed to evoke a persuasive and a coercive influence to validate their varying effects. The coercive design was not experienced as such and further research should aim to develop appropriate design solutions. Future research could potentially focus on Tromp et al. (2011, p. 14) framework that outlines two forms of coercive influence as a starting point for new design iterations:

- Creating a perceivable barrier for the undesired behaviour in the form of pain; and

- Making the unacceptable user behaviour overt by evoking a feeling of shame. 
This research has focused on creating an uncomfortable feeling, which aligns with the first kind of influence. However, the social environment of the user affords potential to create a coercive influence that contributes to an initiation of use. Further research could use the outlined restraining techniques as a starting point for investigating their contribution to an initiation of use.

\subsubsection{Action}

The use of the object should encourage increased repetition and challenge to contribute to neuroplasticity in the brain. It is not enough to simply perform a movement once, and repetition is a key component in the context of rehabilitation (Kleim, \& Jones, 2008, p. S229). The second factor of challenge is based on increased intensity, which affects the induction of neural plasticity. Overuse of the affected arm and hand should be avoided and could potentially worsen the condition (Kleim, \& Jones, 2008, p. S230). The design strategies of repetition and challenge can be implemented in the following way:

\section{Repetition:}

- Focus on an already repetitive task such as cutting

- Limit the functionality to increase the repetition, such as letting just a little bit of water be poured out of a bottle so the movement needs to be repeated.

\section{Progress:}

- The user decides him/herself when they want to enter a more challenging level

- The designed object determines when the user has to enter a more challenging level There are two other main forms of including progress in a design:

- The progress is included in just one design element

- The progress is provided by using multiple elements 
Another factor that should be considered is the use of texture as part of the object. Decreased strength and impaired fine motor skills often impact on the performance of daily tasks after a stroke. Therapists mentioned during the interviews that they would add Velcro or sticky tape to smooth surfaces to make the task feasible to perform. Texture can be used as part of the design in two distinct ways.

\section{Texture:}

- To increase friction and ease the use of an object

- To address tactile discrimination deficits

One tactile discrimination training that could be used as a source of inspiration is by Carey et al. (2011) named SENSe. The training focuses on texture discrimination limb position sense training and tactile object recognition (Carey et al., 2011, p. 306) and could offer further guidance in the design process.

\subsubsection{Feedback}

The provision of augmented feedback is an important part of the rehabilitation process and is provided in the form of knowledge of result after the movement, for example the amount of repetitions that were performed (Morris et al., 2006, p. 260). The design object can incorporate feedback in three different forms:

\section{Feedback}

- Inherent feedback that is caused by the action itself such as the clicking noise when you push a button

- Functional feedback that refers to the actual function of the object such as that the TV is showing you moving images

- Augmented feedback that is not part of the actual function but is provided to give an indication of the internal state such as that the device enters a "standby" phase 
Augmented feedback was incorporated as part of the second radio prototype as an app that visualised the weekly and monthly progress of the participant. One participant mentioned that she would use such an app while the other three participants pointed out that using the device would be too complicated for them. Further research is needed to investigate how augmented feedback could be incorporated to provide an effective way of tracking recovery progress as described by Gustavsson et al. (2018, p. 565). 


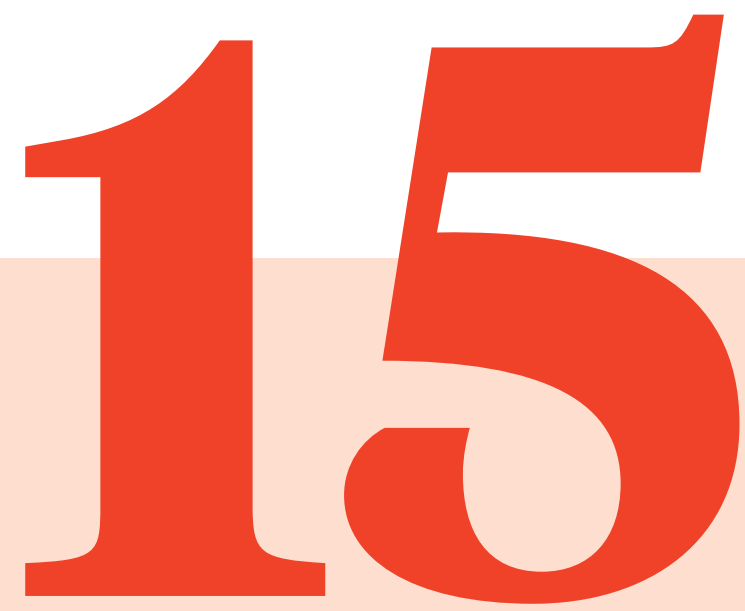

\section{Limitations}


In this section I would like to reflect on some of the methods that were used as part of this research.

\subsection{Focus on Qualitative Methods}

The use of formative usability evaluation methods has addressed my subjective role in the process. The employed qualitative methods offered a rich description of the situation at hand and the results of this study can be a starting point for further explorations employing quantitative methods. A summative evaluation of further design iterations could contribute to quantifying the initiation of use evoked be the design prototypes but was outside the scope of this study.

It needs to be emphasised that the research question required the use of designerly inquiry to develop suitable solutions. The process of theorising the results and outlining the different insights gathered through the multimethod approach have further addressed criticism of pragmatism regarding its scientific rigor. The outlined results of daily activities post stroke and design strategies are, however, by no means extensive. Further research could validate and extend the proposed contributions to knowledge.

\subsection{Design Prototypes}

The development of design prototypes is a crucial element and used as a form of inquiry from the pragmatist perspective (Dalsgaard, 2017, p. 24). The prototypes are manifestations of possible solutions that address the observed problem. The development of these solutions was accompanied by a number of challenges that will be outlined in the following section.

\subsubsection{The object as a form of inquiry}

The primary purpose of the design prototypes was to address the research question (Hengeveld, Frens, \& Deckers, 2016, p. 333) rather than having them ready for production. This lack of refinement has been reported in studies 
that employed a RTD methodology (Hengeveld et al., 2016, p. 333; Hengeveld, Overbeeke, Moor, \& Hummels, 2011, p. 203; Mullaney \& Stolterman, 2014, p. 1045). This "good enough" approach allows more experimental freedom without the restrictions of the real world and aligns with the pragmatist perspective under which the design prototypes were used as a form of inquiry. However, a refinement of the proposed design prototypes would allow transfer of knowledge into a practical application that contributes to the rehabilitation process for chronic stroke survivors outside of the clinical environment.

\subsubsection{Objects with a rehabilitative purpose}

The interviews with stroke therapists indicated that everyday objects specifically designed for stroke survivors most often focuses on assistive devices that compensate for the loss of motor functionality. The prototypes described in this research are the first of their kind and were essential in creating possible ways of restraining movement to initiate use of the affected hand. It was unclear at the beginning of the research how such an object should look. Assistive technology can be associated with product-related stigma (de Barros et al., 2011, p. 109; Pettersson et al., 2005, p. 166; Renda et al., 2015, p. 666) and to avoid such stigma, the first design prototypes did not closely resemble a stereotypical radio. However, to evoke an initiation of use the object needs to incorporate the established conceptual model that the user has of the object.

The separation between objects used to rehabilitate the affected arm and hand and objects used to compensate for lost motor ability appears from a theoretical perspective quite simple. In the design process, however, this separation can be challenging. Throughout the process, I tried to identify everyday objects which aim to contribute to the rehabilitation process rather than being assistive design solutions. However, the usability testing sessions indicated that the design prototypes need to incorporate some assistive design features in order to make the use of the object feasible. A broader focus on assistive design solutions and universal design approaches could have potentially helped to identify relevant 
design precedents in the context of this research.

During the evaluation processes of the radio and the smartphone device, some of the participants demonstrated compensatory movement patterns. One participant started to rotate the hip to use the radio while another one used his thighs when putting down the smartphone. Further studies should investigate how the restraining effect needs to be delivered to minimise those adverse movements and determine which assistive design components contribute to the development of learned nonuse to limit possible compensatory movement patterns.

\subsubsection{Use of design criteria}

The criteria based thesis model (Rodríguez Ramírez, 2017, p. 14) offered a systematic approach to the design process. Reflecting on the design process, design criteria with a defined focus were easier to assess. For example, the first set of design criteria focused on the criterion of "providing feedback" while the revised criteria outlined three different forms of feedback. Further, a number of criteria could not be assessed by me and required the involvement of users, for example the evaluation of feedforward elements and especially the movement that was needed to interact with the object. The presented design criteria offer the potential to influence the design process and be used as a point of reference for further design iterations.

\subsubsection{Evaluation of design solutions}

The evaluation of the design prototypes was restricted to thirty minutes during which the participants were informed about the purpose and structure of the study, provided informed consent, and evaluated the design prototypes. During the evaluation of the radio prototype, participants felt slightly hesitant to criticise the design prototypes and elements of the intended interaction. A possible solution would have been to involve the participants throughout the whole process or show many prototypes and existing products for comparison without telling which ones were mine. However, three of the stroke survivors who 
tested the radio prototype lived in Australia, which made it impossible to test the designs regularly with them.

For the evaluation process of the smartphone prototypes, two design prototypes and a standard smartphone were developed to offer more choices to the participants. This approach provided a richer description of criticism and feedback on the developed design prototypes compared to the formative evaluation of the radio prototype. Further long-term studies that allow the participants to take the prototypes home with them could offer crucial insights into the usability of the prototypes.

\subsubsection{Design for stroke survivors}

A recent HCI study focused on the design of everyday objects for upper limb stroke rehabilitation (Magnusson et al., 2017) and employed a co-design approach. The authors emphasise the importance of involving multiple stakeholders such as family members, stroke survivors, and health professionals (Magnusson et al. 2017, pp. 599-600) in the process. This is particularly true if the design is supposed to be used in the home environment. Due to the complicated ethical and slow recruitment processes, such an approach was outside the scope of this study. Further research should include primary caregivers and family members in the design process and evaluation of design concepts. A closer collaboration with local stroke organisations could allow creation of a co-working space in which designers, caregivers and stroke survivors share ideas and insights.

\subsection{Stroke Rehabilitation}

\subsubsection{Literature vs clinical practice}

Resource limitations often obstruct the implementation of research insights into clinical practice. New Zealand's stroke management guidelines recommend CIMT as an intervention. The results of this study indicate that it is not a commonly used intervention in New Zealand. It was therefore not possible to gather feedback from stroke survivors who have experienced the restraint as 
part of their rehabilitation process. Further studies are needed to determine their perspective regarding the use of the restraint. However, the implementation of CIMT as a standard intervention in New Zealand might be difficult due to limited resources available to stroke survivors.

\subsubsection{Study sample of health professionals}

There seems to be a traditional separation between the role of occupational therapists and physiotherapists in the context of a stroke. It was pointed out that physiotherapists traditionally focused on the rehabilitation side while occupational therapists focus on the compensation side. Occupational therapists would often be involved at a late stage of rehabilitation with a stronger focus on everyday objects and the home environment. During the recruitment process for the interviews with health professionals, it was not apparent which group of health professionals would use the restraint as part of clinical practice. The restraint contributes to the rehabilitation process ergo is the domain of physiotherapists. However, learned nonuse needs time to develop and would, therefore, be more likely to be visible at the later stage of the stroke rehabilitation; ergo when occupational therapists have a stronger involvement. Both professions were interviewed as part of this study to develop an understanding of challenges and barriers throughout the process. Both professions described focusing on functional improvements for the stroke survivor, and both professions had used the physical restraint as part of their practice. It is difficult to give a recommendation as to which group of professionals might benefit the most from using CIMT because of the small sample size of the current study.

The interviews with health professionals were a crucial element in understanding clinical practice and barriers in the rehabilitation process. The interview results need to be considered in light of the study sample. Two participants worked in Australia where the health system, and stroke guidelines might be slightly different. Furthermore, most of the participants were located close to Wellington, the capital of New Zealand, and in Auckland, New Zealand's 
largest city. Access to stroke services might, therefore, be different in those cities compared to provincial and rural areas of the country. Since none of the stroke therapists were located in a rural area, there might be a potential bias included in the results.

Another factor that needs to be considered is that the intended use of the design prototypes is in the home setting of the stroke survivor. Home-based interventions were mentioned during the interviews with health therapists as being a crucial element in rehabilitation. Nonetheless, there seems to be no consensus on one specific therapy approach. In New Zealand, access to stroke services for chronic stroke survivors with mild impairments seems to be quite limited. Most of the health professionals who took part in this study had, therefore, more experience in working with acute and subacute stroke survivors. One of the therapists mentioned that only highly motivated survivors with sufficient funding would try to improve motor impairments in the chronic stage, which indicates a lack of support for this stroke population.

\subsubsection{Study sample of stroke survivors}

The inclusion and exclusion criteria used for this study outlined that participants needed to be able to stand independently to take part in the study. Some participants in the online survey stated they had limitations of the upper and lower limb, which offers a possible explanation for the fact that walkingrelated activities were prioritised in the online survey. The inclusion and exclusion criteria of this study ensured the safe use of the design prototypes. There was no formal assessment of participant's abilities involved in the study and participants could take part based on their therapist's recommendation.

\subsection{User Generated Ideas}

The developed design strategies could potentially be used by stroke survivors to develop products that address their specific needs. In the context of assistive technology, it was mentioned during the interviews with stroke therapists 
that survivors often face financial limitations and that, especially in New Zealand, there is an established do-it-yourself (DIY) culture. This confirms previous studies that indicate that stroke survivors start creating their own solutions (de Barros \& Duarte, 2009, p. 80; de Barros, Duarte, \& Cruz, 2010, p. 4). One example that showcases DIY in a similar context is the Spinalistips which is a Swedish website where people with spinal cord injuries share ideas on how to make assistive devices themselves (The Spinalist Foundation, 2016).

Studies indicate that user-generated product ideas can contain a higher component of novelty compared to designer-generated products, and generate more sales (Nishikawa et al. 2013, p. 160). The design strategies developed in this study that evoke an initiation of use could potentially be refined and included as parameters in automated and customisable design approaches; for example, to produce an individualised design with the use of 3D printers. A possible scenario is the development of websites similar to the Spinalistips with the difference that it offers customisable designs. The designs would be able to incorporate elements that contribute to the initiation of use of the affected arm and hand while taking account of factors that contribute to an individual's selfhood (Orth \& van den Hoven, 2016, pp. 321-322) and express one's identity rather than being a reminder of the impairment (de Barros et al., 2010, p. 8).

Symbolism, which is an important factor in the design of an object, has not been addressed as part of the current study but could benefit from a customisable manufacturing approach. The attached symbolism is hard to predict but can contribute to a high level of attachment (Odom, Pierce, Stolterman, \& Blevis, 2009, pp. 1060-1061) and consequently use of the object. Digital manufacturing techniques provide an opportunity to tailor the function and material quality of designs to strengthen the attachment that the user has to the object. However, the design strategies would need further refinement and simplification to be used in the proposed context.

In summary, more design prototypes need to be developed based on design 
strategies, which consequently need to be tested to confirm that they evoke initiation of use of the affected arm while avoiding compensatory movement patterns. The evaluation of these prototypes should aim to validate a longterm effect and include testing the design prototypes in the participants' home environment. The involvement of end-users in the design development process could increase the novelty and usability of the designs. 


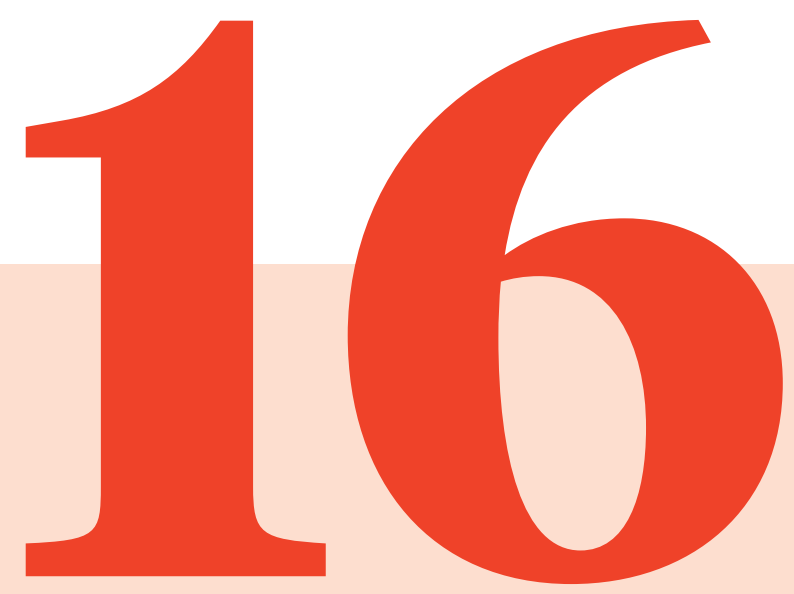

\section{Conclusion}


The development of learned nonuse post-stroke is influenced by multiple factors, for example social and physiological, and is a learned behaviour of the stroke survivor. Findings of this study confirm that initiation of use of the affected arm and hand can be evoked by restraining the less affected arm and hand. However, physical restraint in clinical practice seems to be primarily used as part of CIMT and aims to remind the user to use the affected arm and hand rather than physically restraining its use. To initiate use of the affected side, the stroke survivor needs to have increased self-efficacy and believe that the use of the affected arm and hand is possible. CIMT further uses behaviour change techniques to overcome the learned nonuse and secure long-term goals developed in the rehabilitation process. However, CIMT is not administered widely and everyday objects may offer a potential alternative to facilitate initiation of use. This study employed a multimethod approach from the pragmatist perspective, which indicated that digital devices used for communication purposes, such as the smartphone, have the potential to contribute to the initiation of use because of their significant role in daily activities. The devices are part of activities that contribute to communication, a sense of belonging, social integration, self-directed rehabilitation, and safety after a stroke. A RTD methodology was used to address the research question and led to five different design strategies that restrained movement to initiate use of the affected arm and hand. The designs were evaluated by health professionals and chronic stroke survivors to evaluate the intended initiation of use. Their feedback indicated that the objects contributed to initiation of use of the affected arm and hand but require further refinement to increase the usability of the designs. The evaluation process further indicated that it is essential to take account of the conceptual model that the user has of the object. The use of the object needs to be feasible for the stroke survivor, provide sufficient feedforward to initiate the use of the affected arm and hand, increase self-efficacy, provide a repetitive and increasingly challenging movement, provide sensory input, and provide feedback to secure engagement in 
the process. The behaviour change used as part of CIMT was incorporated in the form of a behaviour contract within the design components. Further studies are needed to validate the intended behaviour change that the contract aims to evoke. Such an evaluation has been outside the scope of this study. The theorising of the design process in the form of bridging concepts led to a series of further design strategies that were tested by designers according to the pragmatist perspective. The evaluators indicated that the design strategies could be used in a self-directed way but needed to be provided in smaller components to enable empathy of the designer for the user and lead to design prototypes that can be tested by chronic stroke survivors.

This research has contributed to the field of design by outlining strategies that evoke an initiation of use in chronic stroke survivors. The development of everyday objects based on those strategies could contribute to self-directed rehabilitation for chronic stroke survivors and address the issue of learned nonuse of the upper limb impacting on the performance of daily activities. 


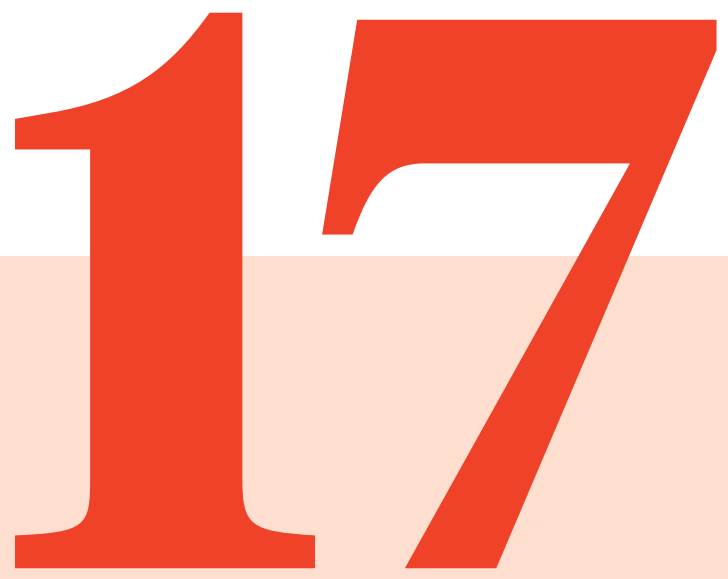

References 
ableX healthcare limited. (2017). Plug-in \& play rehabilitation. Retrieved 20 July 2017, from https://ablex.healthcare/the-system/index.html

Ada, L., Canning, C. G., Carr, J. H., Kilbreath, S. L., \& Shepherd, R. B. (1994). Chapter 12: Task-specific training of reaching and manipulation. In Advances in Psychology. 105, 239-265. Elsevier. https://doi.org/10.1016/ S0166-4115(08)61281-9

Adamit, T., Maeir, A., Ben Assayag, E., Bornstein, N. M., Korczyn, A. D., \& Katz, N. (2015). Impact of first-ever mild stroke on participation at 3 and 6 months post-event: The TABASCO study. Disability and Rehabilitation, 37(8), 667673. https://doi.org/10.3109/09638288.2014.923523

Adkins, D. L., Voorhies, A. C., \& Jones, T. A. (2004). Behavioral and neuroplastic effects of focal endothelin-1 induced sensorimotor cortex lesions. Neuroscience, 128(3), 473-486. https://doi.org/10.1016/j. neuroscience.2004.07.019

Akrich, M., \& Latour, B. (1992). A summary of a convenient vocabulary for the semiotics of human and nonhuman assemblies. In W. E. Bijker \& J. Law, Shaping Technology/Building Society: Studies in Sociotechnical Change. 259-265. Cambridge Massachussetts: Cambridge University Press.

Algurén, B., Lundgren-Nilsson, Å., \& Sunnerhagen, K. S. (2009). Facilitators and barriers of stroke survivors in the early post-stroke phase. Disability and Rehabilitation, 31(19), 1584-1591. https://doi. org/10.1080/09638280802639004

Allred, R. P., Maldonado, M. A., Hsu and, J. E., \& Jones, T. A. (2005). Training the 'less-affected' forelimb after unilateral cortical infarcts interferes with functional recovery of the impaired forelimb in rats. Restorative Neurology and Neuroscience, 23(5-6), 297-302.

Alves, V., \& Roque, L. (2011). A deck for sound design in games: Enhancements based on a design exercise, 1. ACM Press. https://doi. org/10.1145/2071423.2071465 
Andrew, N., Kilkenny, M., Naylor, R., Purvis, T., \& Cadilhac, D. (2015). The relationship between caregiver impacts and the unmet needs of survivors of stroke. Patient Preference and Adherence, 1065. https://doi.org/10.2147/PPA. S85147

Andrews, K., \& Steward, J. (1979). Stroke recovery: He can but does he? Rheumatology, 18(1), 43-48. https://doi.org/10.1093/rheumatology/18.1.43

Arya, K. N., Verma, R., Garg, R. K., Sharma, V. P., Agarwal, M., \& Aggarwal, G. G. (2012). Meaningful task-specific training (MTST) for stroke rehabilitation: a randomized controlled trial. Topics in Stroke Rehabilitation, 19(3), 193-211. https://doi.org/10.1310/tsr1903-193

Asheim, J., Divitini, M., Kjøllesdal, A., \& Mora, S. (2016). Tiles cards: A card-based design game for smart objects ecosystems. In SERVE@AVI. (pp.19-24). Bari, Italy.

Atkinson, R., \& Flint, J. (2011). Snowball sampling. In M. Lewis-Beck, A. Bryman, \& T. Futing Liao (Eds.), The SAGE Encyclopedia of Social Science Research Methods. Thousand Oaks: Sage Publications, Inc. https://doi. org/10.4135/9781412950589.n931

Atler, K. (2016). The experiences of everyday activities post-stroke. Disability and Rehabilitation, 38(8), 781-788. https://doi.org/10.3109/09638288.2015.106160 3

Balaam, M., Rennick Egglestone, S., Fitzpatrick, G., Rodden, T., Hughes, A., Wilkinson, A., ... Burridge, J. (2011). Motivating mobility: Designing for lived motivation in stroke rehabilitation. Proceedings of the SIGCHI Conference on Human Factors in Computing Systems, 3073-3082.

Baldwin, C., \& Brusco, N. K. (2011). The effect of vocational rehabilitation on return-to-work rates post stroke: A systematic review. Topics in Stroke Rehabilitation, 18(5), 562-572. https://doi.org/10.1310/tsr1805-562

Bandura, A. (1997). Self-efficacy: the exercise of control. New York: W.H. Freeman. Bang, A. L., Krogh, P. G., Ludvigsen, M., \& Markussen, T. (2012). The role of 
hypothesis in constructive design research. In Proceedings of The Art of Research IV,1-11.

Baxter, M. (1995). Product design: practical methods for the systematic development of new products. London: Stanley Thornes: CRC Press.

Berenguer, A., Goncalves, J., Hosio, S., Ferreira, D., Anagnostopoulos, T., \& Kostakos, V. (2017). Are smartphones ubiquitous?: An in-depth survey of smartphone adoption by seniors. IEEE Consumer Electronics Magazine, 6(1), 104-110. https://doi.org/10.1109/MCE.2016.2614524

Bernhardt, J. (2004). Inactive and alone: Physical activity within the first 14 days of acute stroke unit care. Stroke, 35(4), 1005-1009. https://doi.org/10.1161/01. STR.0000120727.40792.40

Bernhardt, J., Chan, J., Nicola, I., \& Collier, J. M. (2007). Little therapy, little physical activity: Rehabilitation within the first 14 days of organized stroke unit care. Journal of Rehabilitation Medicine, 39(1), 43-48. https://doi. org/10.2340/16501977-0013

Best, C., van Wijck, F., Dennis, J., Smith, M., Donaghy, M., Fraser, H., ... Mead, G. (2012). A survey of community exercise programmes for stroke survivors in Scotland: Survey of community Exercise after Stroke. Health \& Social Care in the Community, 20(4), 400-411. https://doi.org/10.1111/j.13652524.2011.01043.x

Bettger, J. P., Zhao, X., Bushnell, C., Zimmer, L., Pan, W., Williams, L. S., \& Peterson, E. D. (2014). The association between socioeconomic status and disability after stroke: Findings from the adherence eValuation after ischemic stroke longitudinal (AVAIL) registry. BMC Public Health, 14(1). https://doi.org/10.1186/1471-2458-14-281

Biesenthal, C. (2014). Pragmatism. In D. Coghlan \& M. Brydon-Miller, The SAGE Encyclopedia of Action Research. 648-650. 2455 Teller Road, Thousand Oaks, California 91320: SAGE Publications Ltd. https://doi. org/10.4135/9781446294406.n287 
Bionik. (2017). Upper extremity rehabilitation. Retrieved 21 January 2017, from http://bionikusa.com/healthcarereform/upper-extremity-rehabilitiation

Biskjaer, M. M., \& Dalsgaard, P. (2012). Toward a constraint-oriented pragmatism understanding of design creativity. In DS 73-1 Proceedings of the 2nd International Conference on Design Creativity, 1, 65-74. Glasgow, UK: The Design Society.

Blakey, G. (2014). CIMT Shaping video. Retrieved October 31, 2016, from https:// www.youtube.com/watch?v=YBFf1x29NvA

Bogousslavsky, J. (2003). William Feinberg Lecture 2002: Emotions, mood, and behavior after stroke. Stroke, 34(4), 1046-1050. https://doi.org/10.1161/01. STR.0000061887.33505.B9

Borchers, J. O. (2001). A pattern approach to interaction design. AI \& Society, 15(4), 359-376. https://doi.org/10.1007/BF01206115

Brannigan, C., Galvin, R., Walsh, M. E., Loughnane, C., Morrissey, E.-J., Macey, C., ... Horgan, N. F. (2017). Barriers and facilitators associated with return to work after stroke: A qualitative meta-synthesis. Disability and Rehabilitation, 39(3), 211-222. https://doi.org/10.3109/09638288.2016.1141242

Braun, V., \& Clarke, V. (2006). Using thematic analysis in psychology.

Qualitative Research in Psychology, 3(2), 77-101. https://doi. org/10.1191/1478088706qp063oa

Brown, T. (2017). The power of the powers of ten. Retrieved 12 December 2017, from https://designthinking.ideo.com/?p=1034

Brown, M., Levack, W., McPherson, K. M., Dean, S. G., Reed, K., Weatherall, M., \& Taylor, W. J. (2014). Survival, momentum, and things that make me 'me': Patients' perceptions of goal setting after stroke. Disability and Rehabilitation, 36(12), 1020-1026. https://doi.org/10.3109/09638288.2013.82 5653

Buchanan, R. (1992). Wicked problems in design thinking. Design Issues, 8(2), 5. https://doi.org/10.2307/1511637 
Bugge, C., Alexander, H., \& Hagen, S. (1999). Stroke patients' informal caregivers: Patient, caregiver, and service factors that affect caregiver strain. Stroke, 30(8), 1517-1523. https://doi.org/10.1161/01.STR.30.8.1517

Buma, F., Kwakkel, G., \& Ramsey, N. (2013). Understanding upper limb recovery after stroke. Restorative Neurology and Neuroscience, 31(6), 707-722. https:// doi.org/10.3233/RNN-130332

Bütefisch, C., Hummelsheim, H., Denzler, P., \& Mauritz, K.-H. (1995). Repetitive training of isolated movements improves the outcome of motor rehabilitation of the centrally paretic hand. Journal of the Neurological Sciences, 130(1), 59-68. http://doi.org/10.1016/0022-510X(95)00003-K

Canadian Partnership for Stroke Recovery. (2016). Living with stroke. Retrieved October 31, 2016, from https://www.strokengine.ca/living-with-stroke/

Carey, L., Macdonell, R., \& Matyas, T. A. (2011). SENSe: Study of the effectiveness of neurorehabilitation on sensation: A randomized controlled trial. Neurorehabilitation and Neural Repair, 25(4), 304-313. https://doi. org/10.1177/1545968310397705

Carey, J. R., Bhatt, E., \& Nagpal, A. (2005). Neuroplasticity promoted by task complexity. Exercise and Sport Sciences Reviews, 33(1), 24-31.

Cardoso, C., \& Badke-Schaub, P. (2011). The influence of different pictorial representations during idea deneration. The Journal of Creative Behavior, 45(2), 130-146. https://doi.org/10.1002/j.2162-6057.2011.tb01092.x

Carlsson, G. E., Möller, A., \& Blomstrand, C. (2009). Managing an everyday life of uncertainty - A qualitative study of coping in persons with mild stroke. Disability and Rehabilitation, 31(10), 773-782. https://doi. org/10.1080/09638280802638857

Carod-Artal, F. J., González-Gutiérrez, J. L., Herrero, J. A. E., Horan, T., \& Seijas, E. V. D. (2002). Functional recovery and instrumental activities of daily living: Follow-up 1-year after treatment in a stroke unit. Brain Injury, 16(3), 207216. https://doi.org/10.1080/02699050110103337 
Carter, K., Anderson, C., Hacket, M., Feigin, V., Barber, P. A., Broad, J. B., ... on behalf of the Auckland Regional Community Stroke (ARCOS) Study Group. (2006). Trends in ethnic disparities in stroke incidence in Auckland, New Zealand, during 1981 to 2003. Stroke, 37(1), 56-62. https://doi.org/10.1161/01. STR.0000195131.23077.85

Carter, N., Bryant-Lukosius, D., DiCenso, A., Blythe, J., \& Neville, A. J. (2014). The use of triangulation in qualitative research. Oncology Nursing Forum, 41(5), 545-547. https://doi.org/10.1188/14.ONF.545-547

Casey, D., \& Murphy, K. (2009). Issues in using methodological triangulation in research: Dympna Casey and Kathy Murphy explore the advantages and disadvantages of using triangulation. Nurse Researcher, 16(4), 40-55. https:// doi.org/10.7748/nr2009.07.16.4.40.c7160

Chan, J., Dow, S. P., \& Schunn, C. D. (2015). Do the best design ideas (really) come from conceptually distant sources of inspiration? Design Studies, 36, 31-58. https://doi.org/10.1016/j.destud.2014.08.001

Chatterjee, S., Byun, J., Pottathil, A., Moore, M. N., Dutta, K., \& Xie, H. (2012). Persuasive sensing: A novel in-home monitoring technology to assist elderly adult diabetic patients. In M. Bang \& E. L. Ragnemalm (Eds.), Persuasive Technology. Design for Health and Safety, 7284, 31-42. Berlin, Heidelberg: Springer Berlin Heidelberg. Doi: 10.1007/978-3-642-31037-9_3

Chatterjee, S., \& Price, A. (2009). Healthy living with persuasive technologies: Framework, issues, and challenges. Journal of the American Medical Informatics Association, 16(2), 171-178. Doi: 0.1197/jamia.M2859

Cheng, P., Mugge, R., \& Schoormans, J. P. L. (2014). A new strategy to reduce design fixation: Presenting partial photographs to designers. Design Studies, 35(4), 374-391. https://doi.org/10.1016/j.destud.2014.02.004

Chi, P.-Y. (Peggy), Chen, J.-H., Chu, H.-H., \& Lo, J.-L. (2008). Enabling calorieaware cooking in a smart kitchen. In H. Oinas-Kukkonen, P. Hasle, M. Harjumaa, K. Segerståhl, \& P. Øhrstrøm (Eds.), Persuasive Technology, 5033, 
116-127. Berlin, Heidelberg: Springer Berlin Heidelberg. Doi.:10.1007/978-3540-68504-3_11

Choi-Kwon, S., \& Kim, J. S. (2011). Poststroke fatigue: An emerging, critical issue in stroke medicine: Reviews. International Journal of Stroke, 6(4), 328-336. https://doi.org/10.1111/j.1747-4949.2011.00624.x

Chung, D., \& Liang, R.-H. (2015). Understanding the usefulness of ideation tools with the grounding lenses, 13-22. ACM Press. https://doi. $\operatorname{org} / 10.1145 / 2739999.2740002$

Cirstea, M. C., \& Levin, M. F. (2007). Improvement of arm movement patterns and endpoint control depends on type of feedback during practice in stroke survivors. Neurorehabilitation and Neural Repair, 21(5), 398-411. https://doi. org/10.1177/1545968306298414

Clark, N. M., \& Zimmerman, B. J. (2014). A social cognitive view of self-regulated learning about health. Health Education \& Behavior, 41(5), 485-491. https:// doi.org/10.1177/1090198114547512

Clarke, P. J., Lawrence, J. M., \& Black, S. E. (2000). Changes in quality of life over the first year after stroke: Findings from the Sunnybrook stroke study. Journal of Stroke and Cerebrovascular Diseases, 9(3), 121-127. https://doi. org $/ 10.1053 /$ jscd.2000.5868

Cobley, C. S., Fisher, R. J., Chouliara, N., Kerr, M., \& Walker, M. F. (2013). A qualitative study exploring patients' and carers' experiences of early supported discharge services after stroke. Clinical Rehabilitation, 27(8), 750-757. https://doi.org/10.1177/0269215512474030

Coleman, R., Clarkson, J., \& Cassim, J. (2007). Design for inclusivity: A practical guide to accessible, innovative and user-centred design. Aldershot: Burlington, VT: Gower; Ashgate Pub.

Coleman, R., Lebbon, C., \& Myerson, J. (2003). Design and empathy. In J. Clarkson, S. Keates, R. Coleman, \& C. Lebbon (Eds.), Inclusive Design, 478-499. London: Springer London. https://doi.org/10.1007/978-1-4471-0001-0_29 
Combs, S. A., Finley, M. A., Henss, M., Himmler, S., Lapota, K., \& Stillwell, D. (2012). Effects of a repetitive gaming intervention on upper extremity impairments and function in persons with chronic stroke: A preliminary study. Disability and Rehabilitation, 34(15), 1291-1298. https://doi.org/10.310 9/09638288.2011.641660

Cramer, S. C. (2008). Repairing the human brain after stroke: I. mechanisms of spontaneous recovery. Annals of Neurology, 63(3), 272-287. https://doi. org/10.1002/ana.21393

Coupar, F., Pollock, A., Legg, L. A., Sackley, C., \& van Vliet, P. (2012). Home-based therapy programmes for upper limb functional recovery following stroke. The Cochrane Database of Systematic Reviews, 5, CD006755. https://doi. org/10.1002/14651858.CD006755.pub2

Creswell, J. W. (2014). Research design: Qualitative, quantitative, and mixed methods approaches (4th ed). Thousand Oaks: SAGE Publications.

Creswell, J. W. (2015). A concise introduction to mixed methods research. Los Angeles: SAGE.

Cross, N. (1982). Designerly ways of knowing. Design Studies, 3(4), 221-227. https:// doi.org/10.1016/0142-694X(82)90040-0

Crotty, M. (1998). The foundations of social research: meaning and perspective in the research process. London; Thousand Oaks, Calif.: Sage Publications.

Crilly, N., Moultrie, J., \& Clarkson, P. J. (2004). Seeing things: Consumer response to the visual domain in product design. Design Studies, 25(6), 547-577. https://doi.org/10.1016/j.destud.2004.03.001

Crosbie, J. H., Lennon, S., Basford, J. R., \& McDonough, S. M. (2007). Virtual reality in stroke rehabilitation: Still more virtual than real. Disability and Rehabilitation, 29(14), 1139-1146. https://doi. org/10.1080/09638280600960909

Csikszentmihalyi, M., \& Halton, E. (1981). The meaning of things: Domestic symbols and the self. Cambridge [Eng.]; New York: Cambridge University Press. 
Dalsgaard, P. (2014). Pragmatism and design thinking. International Journal of Design, 11(1), 143-155.

Dalsgaard, P. (2017). Instruments of inquiry: Understanding the nature and role of tools in design. International Journal of Design, 11(1), 21-33.

Dalsgaard, P., \& Dindler, C. (2014). Between theory and practice: Bridging concepts in HCI research, 1635-1644. ACM Press. https://doi. org/10.1145/2556288.2557342

Dancause, N., Nadeau, S., \& Rossignol, S. (Eds.). (2015). Sensorimotor rehabilitation: at the crossroads of basic and clinical sciences (1. ed). Amsterdam: Elsevier.

Daniel, L., Howard, W., Braun, D., \& Page, S. J. (2012). Opinions of constraintinduced movement therapy among therapists in southwestern Ohio. Topics in Stroke Rehabilitation, 19(3), 268-275. https://doi.org/10.1310/tsr1903-268

Daniel, K., Wolfe, C. D. A., Busch, M. A., \& McKevitt, C. (2009). What are the social consequences of stroke for working-aged adults?: A systematic review. Stroke, 40(6), e431-e440. https://doi.org/10.1161/STROKEAHA.108.534487

de Barros, A. C., \& Duarte, C. (2009). Assistive devices: Stroke patients’ design. ACM Press. https://doi.org/10.1145/1640233.1640248

de Barros, A. C., Duarte, C., \& Cruz, J. B. (2009). Designers and stroke research. Australasian Medical Journal, 10(1), 104-111. https://doi.org/10.4066/ AMJ.2009.151

de Barros, A. C., Duarte, C., \& Cruz, J. (2011). The influence of context on product judgement - presenting assistive products as consumer goods. International Journal of Design, 5(3), 99-112.

de Barros, A. C., Duarte, C., \& Cruz, J. B. (2010). 'I made it myself' - DIY in the design of assistive devices for stroke patients. 7th International Conference on Design \& Emotion, Chicago, IL, USA, 4-7 October 2010. 1-13. Chicago: IIT Institute of Design. 
Deloitte Touche Tohmatsu Limited. (2017). Technology, Media and Telecommunications Predictions 2018. China: Deloitte Touche Tohmatsu Limited. Retrieved from https://www2.deloitte.com/cn/en/pages/ technology-media-and-telecommunications/articles/tmt-predictions-2018. html

Demaerschalk, B. M., Hwang, H.-M., \& Leung, G. (2010). US cost burden of ischemic stroke: A systematic literature review. The American Journal of Managed Care, 16(7), 525-533.

DePiero, T. J. (2011a). Hemiparesis. In J. S. Kreutzer, J. DeLuca, \& B. Caplan (Eds.), Encyclopedia of Clinical Neuropsychology, 1228-1228. New York, NY: Springer New York. Retrieved from http://link.springer.com/10.1007/978-0387-79948-3_456

DePiero, T. J. (2011b). Hemiplegia. In J. S. Kreutzer, J. DeLuca, \& B. Caplan (Eds.), Encyclopedia of Clinical Neuropsychology, 1228-1228. New York, NY: Springer New York. Retrieved from http://link.springer.com/10.1007/978-0387-79948-3_457

Dewald, J. P., Pope, P. S., Given, J. D., Buchanan, T. S., \& Rymer, W. Z. (1995). Abnormal muscle coactivation patterns during isometric torque generation at the elbow and shoulder in hemiparetic subjects. Brain: A Journal of Neurology, 118 (2), 495-510.

Doolittle, N. D. (1991). Clinical ethnography of lacunar stroke: Implications for acute care. The Journal of Neuroscience Nursing: Journal of the American Association of Neuroscience Nurses, 23(4), 235-240.

Doolittle, N. D. (1992). The experience of recovery following lacunar stroke. Rehabilitation Nursing: The Official Journal of the Association of Rehabilitation Nurses, 17(3), 122-125.

Doyle, S., Bennett, S., Fasoli, S. E., \& McKenna, K. T. (2010). Interventions for sensory impairment in the upper limb after stroke. In The Cochrane Collaboration (Ed.), Cochrane Database of Systematic Reviews. 
Chichester, UK: John Wiley \& Sons, Ltd. Retrieved from http://doi.wiley. com/10.1002/14651858.CD006331.pub2

Dromerick, A. W., Lang, C. E., Birkenmeier, R. L., Wagner, J. M., Miller, J. P., Videen, T. O., ... Edwards, D. F. (2009). Very early constraint-induced movement during stroke rehabilitation (VECTORS): A single-center RCT. Neurology, 73(3), 195-201. https://doi.org/10.1212/WNL.0b013e3181ab2b27

Drummond, A. E., Parker, C. J., Gladman, J. R., \& Logan, P. A. (2001). Development and validation of the Nottingham Leisure Questionnaire (NLQ). Clinical Rehabilitation, 15(6), 647-656. https://doi.org/10.1191/0269215501cr438oa Duncan, P. W., Zorowitz, R., Bates, B., Choi, J. Y., Glasberg, J. J., Graham, G. D., ... Reker, D. (2005). Management of adult stroke rehabilitation care: A clinical practice guideline. Stroke, 36(9), e100-e143. https://doi.org/10.1161/01. STR.0000180861.54180.FF

Eckert, C., \& Stacey, M. (2000). Sources of inspiration: A language of design. Design Studies, 21(5), 523-538. https://doi.org/10.1016/S0142-694X(00)00022-3

Eco Innovators. (2013). Design play cards. Retrieved 2 October 2017, from http:// com.core77designawards.c77ad2012.s3.amazonaws.com/uploads/6432/ originals/45745142cc4d7d6d5.pdf

Edwards, D. F., Hahn, M., Baum, C., \& Dromerick, A. W. (2006). The impact of mild stroke on meaningful activity and life satisfaction. Journal of Stroke and Cerebrovascular Diseases, 15(4), 151-157. https://doi.org/10.1016/j. jstrokecerebrovasdis.2006.04.001

Ehn, P., \& Ullmark, P. (2017). Educating the reflective design researcher. In L. Vaughan (Ed.), Practice based design research, 77-86. New York: Bloomsbury Academic.

Ericsson, K. A., \& Simon, H. A. (1998). How to study thinking in everyday life: Contrasting think-aloud protocols with descriptions and explanations of thinking. Mind, Culture, and Activity, 5(3), 178-186. https://doi.org/10.1207/ s15327884mca0503_3 
Eriksson, L., Lindstrom, B., \& Ekenberg, L. (2010). Patients' experiences of telerehabilitation at home after shoulder joint replacement. Journal of Telemedicine and Telecare, 17(1), 25-30. https://doi.org/10.1258/ jtt.2010.100317

Erzberger, C., \& Prein, G. (1997). Triangulation: Validity and empirically-based hypothesis construction. Quality and Quantity, 31(2), 141-154. https://doi. org/https://doi.org/10.1023/A:1004249313062

Fager, S. K., \& Burnfield, J. M. (2014). Patients' experiences with technology during inpatient rehabilitation: Opportunities to support independence and therapeutic engagement. Disability and Rehabilitation: Assistive Technology, 9(2), 121-127. https://doi.org/10.3109/17483107.2013.787124

Fan, J., He, J., \& Tillery, S. I. H. (2006). Control of hand orientation and arm movement during reach and grasp. Experimental Brain Research, 171(3), 283-296. https://doi.org/10.1007/s00221-005-0277-6

Fang, Y., Daly, J. J., Sun, J., Hvorat, K., Fredrickson, E., Pundik, S., ... Yue, G. H. (2009). Functional corticomuscular connection during reaching is weakened following stroke. Clinical Neurophysiology, 120(5), 994-1002. https://doi.org/10.1016/j.clinph.2009.02.173

Farmer, T., Robinson, K., Elliott, S. J., \& Eyles, J. (2006). Developing and implementing a triangulation protocol for qualitative health research. Qualitative Health Research, 16(3), 377-394. https://doi. org/10.1177/1049732305285708

Feigin, V. L., Forouzanfar, M. H., Krishnamurthi, R., Mensah, G. A., Connor, M., Bennett, D. A., ... Murray, C. (2014). Global and regional burden of stroke during 1990-2010: Findings from the Global Burden of Disease Study 2010. The Lancet, 383(9913), 245-255. https://doi.org/10.1016/S0140-6736(13)61953-4

Feigin, V. L., McPherson, K., Barker-Collo, S., \& Krishnamurthi, R. (2014). Measuring and reducing the stroke burden in New Zealand: Editorial. 
International Journal of Stroke, 9(1), 5-5. https://doi.org/10.1111/ijs.12122

Fini, N. A., Holland, A. E., Keating, J., Simek, J., \& Bernhardt, J. (2017). How physically active are people following stroke? Systematic review and quantitative synthesis. Physical Therapy, 97(7), 707-717. https://doi. org/10.1093/ptj/pzx038

Fink, J. (2006). Ethnic trends in stroke in New Zealand: Closing the gaps or widening? The New Zealand Medical Journal, 119(1245), 1-3.

Fitzmaurice, G. W., Ishii, H., \& Buxton, W. A. S. (1995). Bricks: Laying the foundations for graspable user interfaces, 442-449. ACM Press. Doi: $10.1145 / 223904.223964$

Fleet, A., Che, M., MacKay-Lyons, M., MacKenzie, D., Page, S., Eskes, G., ... Boe, S. (2014). Examining the use of constraint-induced movement therapy in Canadian neurological occupational and physical therapy. Physiotherapy Canada, 66(1), 60-71. https://doi.org/10.3138/ptc.2012-61

Fogg, B. J. (2003). Persuasive technology: Using computers to change what we think and do. Amsterdam; Boston: Morgan Kaufmann Publishers.

Fogg, B. J. (2009a). A behavior model for persuasive design. ACM Press. Doi: 10.1145/1541948.1541999

Fogg, B. J. (2009b). Creating persuasive technologies: An eight-step design process. ACM Press. Doi.: 10.1145/1541948.1542005

Fogg, B. J. (2018). The behaviour model. Retrieved September 28, 2018, from https://www.behaviormodel.org/.

Fogg, B. J., \& Hreha, J. (2010). Behavior wizard: A method for matching target behaviors with solutions. In T. Ploug, P. Hasle, \& H. Oinas-Kukkonen (Eds.), Persuasive Technology (Vol. 6137, pp. 117-131). Berlin, Heidelberg: Springer Berlin Heidelberg. Doi: 10.1007/978-3-642-13226-1_13

Forty, A. (1986). Objects of desire, 1750-1980. London: Thames and Hudson.

Forty, A. (1992). Objects of desire: Design and society since 1750. New York, N.Y: Thames and Hudson. 
Frayling, C., \& Royal College of Art. (1993). Research in art and design. London: Royal College of Art.

Gaver, B., \& Bowers, J. (2012). Annotated portfolios. Interactions, 19(4), 40. https:// doi.org/10.1145/2212877.2212889

Gaver, W. (2011). Making spaces: How design workbooks work, 1551. ACM Press. https://doi.org/10.1145/1978942.1979169

Gaver, W. (2012). What should we expect from research through design? In Proceedings of the SIGCHI conference on human factors in computing systems, 937-946. Austin, Texas, USA: ACM Press. https://doi. org/10.1145/2207676.2208538

Geyh, S., Cieza, A., Schouten, J., Dickson, H., Frommelt, P., Omar, Z., ... Stucki, G. (2004). ICF Core Sets for stroke. Journal of Rehabilitation Medicine, 36(0), 135-141. https://doi.org/10.1080/16501960410016776

Gibson, J. J. (1977). The theory of affordance. In R. E. Shaw \& J. Bransford (Eds.), Perceiving, acting and knowing: towards an ecological psychology, 62-82. New York: Lawrence Erlbaum Associates.

Gibson, J. J. (2011). The ecological approach to visual perception (17th pr). New York: Psychology Press.

Gillen, G. (Ed.). (2016). Stroke rehabilitation: a function-based approach (Fourth edition). St. Louis, Missouri: Elsevier, Inc.

Given, L. M. (2008). The SAGE encyclopedia of qualitative research methods (Vols. 1-0). Thousand Oaks, CA: SAGE Publications Ltd doi: 10.4135/9781412963909

Givon, N., Zeilig, G., Weingarden, H., \& Rand, D. (2016). Video-games used in a group setting is feasible and effective to improve indicators of physical activity in individuals with chronic stroke: a randomized controlled trial. Clinical Rehabilitation, 30(4), 383-392. https://doi. org/10.1177/0269215515584382

Golembewski, M., \& Selby, M. (2010). Ideation decks: A card-based design ideation tool, 89. ACM Press. https://doi.org/10.1145/1858171.1858189 
Gommans, J., McNaughton, H., Hanger, C., Bennett, P., Spriggs, D., \& Baskett, J. J. (2003). Stroke rehabilitation services in New Zealand. The New Zealand Medical Journal (Online), 119(1245). Retrieved from http://www.nzma.org. nz/journal/119-1245/2305/

Gonçalves, M., Badke-Schaub, P., \& Cardoso, C. (2013). Through the looking glass of inspiration: Case studies on inspirational search processes of novice designers. Presented at the IASDR 2013, Tokyo, Japan.

Gonçalves, M., Cardoso, C., \& Badke-Schaub, P. (2014). What inspires designers? Preferences on inspirational approaches during idea generation. Design Studies, 35(1), 29-53. https://doi.org/10.1016/j.destud.2013.09.001

Gonçalves, M., Cardoso, C., \& Badke-Schaub, P. (2016). Inspiration choices that matter: the selection of external stimuli during ideation. Design Science, 2. https://doi.org/10.1017/dsj.2016.10

Gosman-Hedström, G., Claesson, L., \& Blomstrand, C. (2002). Assistive devices in elderly people after stroke: A longitudinal, randomized study - The Göteborg 70+ stroke study. Scandinavian Journal of Occupational Therapy, 9(3), 109-118. https://doi.org/10.1080/11038120260246941

Green, W. S. (1999). Essential conditions for the acceptance of user trialing as a design tool. In W. S. Green \& B. Jordan (Eds.), Human Factors in Product Design: Current Practice and Future Trends, 8-14. London: Taylor \& Francis.

Grube, M. M., Koennecke, H.-C., Walter, G., Thummler, J., Meisel, A., Wellwood, I., ... on behalf of the Berlin Stroke Register. (2012). Association between socioeconomic status and functional impairment 3 months after ischemic stroke: The Berlin Stroke Register. Stroke, 43(12), 3325-3330. https://doi. org/10.1161/STROKEAHA.112.669580

Gresham, G. E. (1986). Stroke outcome research. Stroke; a Journal of Cerebral Circulation, 17(3), 358-360.

Gresham, G. E., Alexander, D., Bishop, D. S., Giuliani, C., Goldberg, G., Holland, A., ... Trombly, C. A. (1997). Rehabilitation. Stroke, 28(7), 1522-1526. https://doi. 
org/10.1161/01.STR.28.7.1522

Gustafsson, A., \& Gyllenswärd, M. (2005). The power-aware cord: Energy awareness through ambient information display, 1423. ACM Press. Doi: $10.1145 / 1056808.1056932$

Gustavsson, M., Ytterberg, C., Nabsen Marwaa, M., Tham, K., \& Guidetti, S. (2018). Experiences of using information and communication technology within the first year after stroke - a grounded theory study. Disability and Rehabilitation, 40(5), 561-568. https://doi.org/10.1080/09638288.2016.12640 12

Hackett, M. L., Yapa, C., Parag, V., \& Anderson, C. S. (2005). Frequency of depression after stroke: A systematic review of observational studies. Stroke, 36(6), 1330-1340. https://doi.org/10.1161/01.STR.0000165928.19135.35

Han, C. E., Kim, S., Chen, S., Lai, Y.-H., Lee, J.-Y., Osu, R., ... Schweighofer, N. (2013). Quantifying arm nonuse in individuals poststroke. Neurorehabilitation and Neural Repair, 27(5), 439-447. https://doi. org/10.1177/1545968312471904

Hardie, K., Hankey, G. J., Jamrozik, K., Broadhurst, R. J., \& Anderson, C. (2003). Ten-year survival after first-ever stroke in the Perth community stroke study. Stroke, 34(8), 1842-1846. https://doi.org/10.1161/01. STR.0000082382.42061.EE

Hartke, R. J., \& Trierweiler, R. (2015). Survey of survivors' perspective on return to work after stroke. Topics in Stroke Rehabilitation, 22(5), 326-334. https://doi. org/10.1179/1074935714Z.0000000044

Hartman-Maeir, A., Soroker, N., Ring, H., Avni, N., \& Katz, N. (2007). Activities, participation and satisfaction one-year post stroke. Disability and Rehabilitation, 29(7), 559-566. https://doi.org/10.1080/09638280600924996

Hartson, H. R., Andre, T. S., \& Williges, R. C. (2001). Criteria for evaluating usability evaluation methods. International Journal of Human-Computer Interaction, 13(4), 373-410. https://doi.org/10.1207/S15327590IJHC1304_03 
Haynes, S. R., \& Carroll, J. M. (2007). Theoretical design science in humancomputer interaction: A practical concern? Artifact, 1(3), 159-171. https:// doi.org/10.1080/17493460701872016

Hengeveld, B., Frens, J., \& Deckers, E. (2016). Artefact matters. The Design Journal, 19(2), 323-337. https://doi.org/10.1080/14606925.2016.1129175

Hengeveld, B., Overbeeke, C., Moor, de J.M.H, \& Hummels, C. C. (2011). Designing LinguaBytes: a tangible language learning system for non-or hardly speaking toddlers. Technische Universiteit Eindhoven, Eindhoven.

Herring, S. R., Chang, C.-C., Krantzler, J., \& Bailey, B. P. (2009). Getting inspired!: Understanding how and why examples are used in creative design practice, 87-96. ACM Press. https://doi.org/10.1145/1518701.1518717

Ho, P. (2016). Flow. Interactions, 24(1), 80-80. Doi: 10.1145/3021384

Hocoma. (2017). Armeo ${ }^{\circledR}$ therapy concept. Retrieved 20 January 2017, from https:// www.hocoma.com/usa/us/products/armeo/

Hodson, T., Gustafsson, L., Cornwell, P., \& Love, A. (2017). Post-acute hospital healthcare services for people with mild stroke: A scoping review. Topics in Stroke Rehabilitation, 24(4), 288-298. https://doi.org/10.1080/10749357.2016. 1267831

Holden, M. K. (2005). Virtual environments for motor rehabilitation: Review. CyberPsychology \& Behavior, 8(3), 187-211. https://doi.org/10.1089/ cpb.2005.8.187

Hoyle, M., Gustafsson, L., Meredith, P., \& Ownsworth, T. (2012). Participation after stroke: Do we understand all the components and relationships as categorised in the ICF? Brain Impairment, 13(1), 4-15. https://doi. org/10.1017/BrImp.2012.9

Höök, K., \& Löwgren, J. (2012). Strong concepts: Intermediate-level knowledge in interaction design research. ACM Transactions on Computer-Human Interaction, 19(3), 1-18. https://doi.org/10.1145/2362364.2362371

Hubbard, I. J., Parsons, M. W., Neilson, C., \& Carey, L. M. (2009). Task-specific 
training: Evidence for and translation to clinical practice. Occupational Therapy International, 16(3-4), 175-189. https://doi.org/10.1002/oti.275

Hudson, W. (2005). Playing your cards right: Getting the most from card sorting for navigation design. Interactions, 12(5), 56. https://doi. org/10.1145/1082369.1082410

Huijben-Schoenmakers, M., Gamel, C., \& Hafsteinsdóttir, T. B. (2009). Filling up the hours: How do stroke patients on a rehabilitation nursing home spend the day? Clinical Rehabilitation, 23(12), 1145-1150. https://doi. org/10.1177/0269215509341526

Hussein, A. (2009). The use of triangulation in social sciences research: Can qualitative and quantitative methods be combined. Journal of Comparative Social Work, (1), 1-12.

ICF Research Branch. (2013). Comprehensive ICF core set for stroke. Retrieved 13 July 2017, from https://www.icf-research-branch.org/download/send/12-card iovascularandrespiratoryconditions/199-comprehensive-icf-core-set-stroke Ideo. (2003, November). Method cards. Retrieved 2 October 2017, from https:// www.ideo.com/post/method-cards

IJsselsteijn, W., de Kort, Y., Midden, C., Eggen, B., \& van den Hoven, E. (2006). Persuasive technology for human well-being: Setting the scene. In W. A. IJsselsteijn, Y. A. W. de Kort, C. Midden, B. Eggen, \& E. van den Hoven (Eds.), Persuasive Technology, 3962, 1-5. Berlin, Heidelberg: Springer Berlin Heidelberg. Doi: 10.1007/11755494_1

Intercollegiate Working Party for Stroke. (2012). National clinical guideline for stroke (4th ed.). London: Royal College of Physicians of London. International Organization for Standardization. (1998). ISO 9241-11. Ergonomic requirements for office work with visual display terminals (VDTs)-Part II guidance on usability.

Ishii, H., \& Ullmer, B. (1997). Tangible bits: Towards seamless interfaces between people, bits and atoms, 234-241. ACM Press. Doi.: 10.1145/258549.258715 
ISO. (1998). ISO 9241-11: Guidance on Usability. Geneva: ISO.

Jansson, D. G., \& Smith, S. M. (1991). Design fixation. Design Studies, 12(1), 3-11. https://doi.org/10.1016/0142-694X(91)90003-F

Jones, F., \& Riazi, A. (2011). Self-efficacy and self-management after stroke: A systematic review. Disability and Rehabilitation, 33(10), 797-810. https://doi. org/10.3109/09638288.2010.511415

Jones, T. A. (2017). Motor compensation and its effects on neural reorganization after stroke. Nature Reviews Neuroscience, 18(5), 267-280. https://doi. org/10.1038/nrn.2017.26

Jordan, P. W. (1998). Human factors for pleasure in product use. Applied Ergonomics, 29(1), 25-33. https://doi.org/10.1016/S0003-6870(97)00022-7

Kamwesiga, J. T., Tham, K., \& Guidetti, S. (2017). Experiences of using mobile phones in everyday life among persons with stroke and their families in Uganda - a qualitative study. Disability and Rehabilitation, 39(5), 438-449. https://doi.org/10.3109/09638288.2016.1146354

Kannengiesser, U., \& Gero, J. S. (2012). A process framework of affordances in design. Design Issues, 28(1), 50-62. http://doi.org/10.1162/DESI_a_00123

Kiata, L., \& Kerse, N. (2004). Intercultural residential care in New Zealand. Qualitative Health Research, 14(3), 313-327. https://doi. org/10.1177/1049732303261820

Kielhofner, G. (2002). A model of human occupation: theory and application (3rd ed). Baltimore, MD: Lippincott Williams \& Wilkins.

Kies, J. K., Williges, R. C., \& Rosson, M. B. (1998). Coordinating computersupported cooperative work: A review of research issues and strategies. Journal of the American Society for Information Science, 49(9), 776791. https://doi.org/10.1002/(SICI)1097-4571(199807)49:9<776::AIDASI3>3.0.CO;2-D

Kim, H., Heo, J., Shim, J., Kim, M., Park, S., \& Park, S. (2007). Contextual research on elderly users' needs for developing universal design mobile phone. In 


\section{Stephanidis (Ed.), Universal Access in Human Computer Interaction.} Coping with Diversity, 4554, 950-959. Berlin, Heidelberg: Springer Berlin Heidelberg. https://doi.org/10.1007/978-3-540-73279-2_106

Kim, H., Miller, L. M., Fedulow, I., Simkins, M., Abrams, G. M., Byl, N., \& Rosen, J. (2013). Kinematic data analysis for post-stroke patients following bilateral versus unilateral rehabilitation with an upper limb wearable robotic system. IEEE Transactions on Neural Systems and Rehabilitation Engineering, 21(2), 153-164. https://doi.org/10.1109/TNSRE.2012.2207462

Kimchi, J., Polivka, B., \& Stevenson, J. S. (1991). Triangulation: Operational definitions. Nursing Research, 40(6), 364-366.

Kisiel-Sajewicz, K., Fang, Y., Hrovat, K., Yue, G. H., Siemionow, V., Sun, C.-K., ... Daly, J. J. (2011). Weakening of synergist muscle coupling during reaching movement in stroke patients. Neurorehabilitation and Neural Repair, 25(4), 359-368. Doi: $10.1177 / 1545968310388665$

Kleim, J. A., \& Jones, T. A. (2008). Principles of experience-dependent neural plasticity: Implications for rehabilitation after brain damage. Journal of Speech Language and Hearing Research, 51(1), S225. https://doi. org/10.1044/1092-4388(2008/018)

Knapp, H. D., Taub, E., \& Berman, A. J. (1963). Movements in monkeys with deafferented forelimbs. Experimental Neurology, 7(4), 305-315. https://doi. org/10.1016/0014-4886(63)90077-3

Krakauer, J. W. (2006). Motor learning: Its relevance to stroke recovery and neurorehabilitation. Current Opinion in Neurology, 19(1), 84-90. https://doi. org/10.1097/01.wco.0000200544.29915.cc

Krakauer, J. W., Carmichael, S. T., Corbett, D., \& Wittenberg, G. F. (2012). Getting neurorehabilitation right: What can be learned from animal models? Neurorehabilitation and Neural Repair, 26(8), 923-931. https://doi. org/10.1177/1545968312440745

Krakauer, J. W. (2015). The applicability of motor learning to neurorehabilitation. 
In V. Dietz \& N. Ward (Eds.), Oxford Textbook of Neurorehabilitation (pp. 55-64). Oxford University Press. https://doi.org/10.1093/ med/9780199673711.003.0007

Krebs, H. I., Ferraro, M., Buerger, S. P., Newbery, M. J., Makiyama, A., Sandmann, M., ... Hogan, N. (2004). Rehabilitation robotics: Pilot trial of a spatial extension for MIT-Manus. Journal of NeuroEngineering and Rehabilitation, 1(5), 1-15.

Krogh, P. G., Markussen, T., \& Bang, A. L. (2015). Ways of drifting-five methods of experimentation in research through design. In A. Chakrabarti (Ed.), ICoRD'15 - Research into Design Across Boundaries, 1 (39-50). New Delhi: Springer India. https://doi.org/10.1007/978-81-322-2232-3_4

Kurniawan, S. (2008). Older people and mobile phones: A multi-method investigation. International Journal of Human-Computer Studies, 66(12), 889-901. https://doi.org/10.1016/j.ijhcs.2008.03.002

Kwakkel, G., Kollen, B., \& Lindeman, E. (2004). Understanding the pattern of functional recovery after stroke: facts and theories. Restorative Neurology and Neuroscience, 22(3-5), 281-299.

Lafrenière, D., Dayton, T., \& Muller, M. (1999). Variations of a theme: Card-based techniques for participatory analysis and design, 151. ACM Press. https://doi. $\operatorname{org} / 10.1145 / 632716.632807$

Lang, C. E., MacDonald, J. R., Reisman, D. S., Boyd, L., Jacobson Kimberley, T., Schindler-Ivens, S. M., ... Scheets, P. L. (2009). Observation of amounts of movement practice provided during stroke rehabilitation. Archives of Physical Medicine and Rehabilitation, 90(10), 1692-1698. https://doi. org/10.1016/j.apmr.2009.04.005

Langhorne, P., Bernhardt, J., \& Kwakkel, G. (2011). Stroke rehabilitation. The Lancet, 377(9778), 1693-1702. https://doi.org/10.1016/S0140-6736(11)60325-5

Langhorne, P., Coupar, F., \& Pollock, A. (2009). Motor recovery after stroke: A systematic review. The Lancet Neurology, 8(8), 741-754. https://doi. 
org/10.1016/S1474-4422(09)70150-4

Larsson Lund, M., Lövgren-Engström, A., \& Lexell, J. (2011). Using everyday technology to compensate for difficulties in task performance in daily life: Experiences in persons with acquired brain injury and their significant others. Disability and Rehabilitation: Assistive Technology, 6(5), 402-411. https://doi.org/10.3109/17483107.2011.574309

Laschke, M., Hassenzahl, M., Diefenbach, S., \& Tippkämper, M. (2011). With a little help from a friend: A shower calendar to save water, 633. ACM Press. Doi: $10.1145 / 1979742.1979659$

Laver, K. E., Schoene, D., Crotty, M., George, S., Lannin, N. A., \& Sherrington, C. (2013). Telerehabilitation services for stroke. In The Cochrane Collaboration (Ed.), Cochrane Database of Systematic Reviews. Chichester, UK: John Wiley \& Sons, Ltd. Retrieved from http://doi.wiley.com/10.1002/14651858. CD010255.pub2

Lavrakas, P. (2008). Internet Surveys. In Encyclopedia of Survey Research Methods. 2455 Teller Road, Thousand Oaks California 91320 United States of America: Sage Publications, Inc. https://doi.org/10.4135/9781412963947.n234

Law, M., Baptiste, S., McColl, M., Opzoomer, A., Polatajko, H., \& Pollock, N. (1990). The Canadian Occupational Performance Measure: An outcome measure for occupational therapy. Canadian Journal of Occupational Therapy, 57(2), 82-87. https://doi.org/10.1177/000841749005700207

Lazy-hands grips. (2018). Retrieved 22 February 2018, from https://www.lazyhands.com/product-category/cell-phone-hand-grips/2-loop-phone-grips/

Lebbon, C., \& Coleman, R. (2003). A designer-centred approach. In J. Clarkson, S. Keates, R. Coleman, \& C. Lebbon (Eds.), Inclusive Design, 500-518. London: Springer London. https://doi.org/10.1007/978-1-4471-0001-0_30

Lebeer, J. (1998). How much brain does a mind need? Scientific, clinical, and educational implications of ecological plasticity. Developmental Medicine and Child Neurology, 40(5), 352-357. 
Lee, M., Son, J., Kim, J., Pyun, S.-B., Eun, S.-D., \& Yoon, B. (2016). Comparison of individualized virtual reality- and group-based rehabilitation in older adults with chronic stroke in community settings: A pilot randomized controlled trial. European Journal of Integrative Medicine, 8(5), 738-746. https://doi.org/10.1016/j.eujim.2016.08.166

Lenz, E., Diefenbach, S., \& Hassenzahl, M. (2013). Exploring relationships between interaction attributes and experience, 126. ACM Press. https://doi. org/10.1145/2513506.2513520

Lemke, M., Rodríguez Ramírez, E., \& Robinson, B. (2017a). Limited use only. How can the design of an everyday object help chronic stroke patients to overcome the learned nonuse of the upper limb? The Design Journal, 20, S2397-S2417. https://doi.org/10.1080/14606925.2017.1352754

Lemke, M., Ramírez, E.-R., \& Robinson, B. (2017b). How can constraint-induced movement therapy for stroke patients be incorporated into the design of a tangible interface? The case study of the 'Biggest Hit.' The Design Journal, 20, S2315-S2335. https://doi.org/10.1080/14606925.2017.1352747

Levack, W. M. M., Dean, S. G., Siegert, R. J., \& McPherson, K. M. (2011). Navigating patient-centered goal setting in inpatient stroke rehabilitation: How clinicians control the process to meet perceived professional responsibilities. Patient Education and Counseling, 85(2), 206-213. https:// doi.org/10.1016/j.pec.2011.01.011

Levin, M. F., Kleim, J. A., \& Wolf, S. L. (2009). What do motor 'recovery' and 'compensation' mean in patients following stroke? Neurorehabilitation and Neural Repair, 23(4), 313-319. https://doi.org/10.1177/1545968308328727

Levin, M. F., Michaelsen, S. M., Cirstea, C. M., \& Roby-Brami, A. (2002). Use of the trunk for reaching targets placed within and beyond the reach in adult hemiparesis. Experimental Brain Research, 143(2), 171-180. https://doi. org/10.1007/s00221-001-0976-6

Lewis-Beck, M., Bryman, A., \& Futing Liao, T. (2004). Convenience sample. In 
The SAGE Encyclopedia of Social Science Research Methods. 2455 Teller Road, Thousand Oaks California 91320 United States of America: Sage Publications, Inc. https://doi.org/10.4135/9781412950589.n176

Linsey, J. S., Tseng, I., Fu, K., Cagan, J., Wood, K. L., \& Schunn, C. (2010). A study of design fixation, its mitigation and perception in engineering design faculty. Journal of Mechanical Design, 132(4), 41003-1-41003-12. https://doi. $\operatorname{org} / 10.1115 / 1.4001110$

Lister, R. (1999). Loss of ability to drive following a stroke: The early experiences of three elderly people on discharge from hospital. The British Journal of Occupational Therapy, 62(11), 514-520. https://doi. org/10.1177/030802269906201107

Locher, P., Overbeeke, K., \& Wensveen, S. (2010). Aesthetic interaction: A framework. Design Issues, 26(2), 70-79.

Lockton, D., Harrison, D., \& Stanton, N. A. (2010). Design with Intent: 101 patterns for influencing behaviour through design. Berkshire, UK: Equifine.

Logan, P. A., Gladman, J. R., Drummond, A. E., \& Radford, K. A. (2003). A study of interventions and related outcomes in a randomized controlled trial of occupational therapy and leisure therapy for community stroke patients. Clinical Rehabilitation, 17(3), 249-255. https://doi. org/10.1191/0269215503cr593oa

Lucero, A., \& Arrasvuori, J. (2010). PLEX Cards: A source of inspiration when designing for playfulness, 28-37. ACM Press. https://doi. org/10.1145/1823818.1823821

Luker, J., Lynch, E., Bernhardsson, S., Bennett, L., \& Bernhardt, J. (2015). Stroke survivors' experiences of physical rehabilitation: A systematic review of qualitative studies. Archives of Physical Medicine and Rehabilitation, 96(9), 1698-1708.e10. https://doi.org/10.1016/j.apmr.2015.03.017

Lum, P. S., Burgar, C. G., Loos, M. V. der, Shor, P. C., Majmundar, M., \& Yap, R. (2006). MIME robotic device for upper-limb neurorehabilitation in subacute 
stroke subjects: A follow-up study. The Journal of Rehabilitation Research and Development, 43(5), 631. https://doi.org/10.1682/JRRD.2005.02.0044 Lynch, E., Butt, Z., Heinemann, A., Victorson, D., Nowinski, C., Perez, L., \& Cella, D. (2008). A qualitative study of quality of life after stroke: The importance of social relationships. Journal of Rehabilitation Medicine, 40(7), 518-523. https://doi.org/10.2340/16501977-0203

MacKenzie, H. M., \& Viana, R. (2016). Constraint-induced therapies. In B. Ovbiagele (Ed.), Ischemic Stroke Therapeutics, 217-223. Cham: Springer International Publishing. Retrieved from http://link.springer. com/10.1007/978-3-319-17750-2_21

Magill, R. A., \& Hall, K. G. (1990). A review of the contextual interference effect in motor skill acquisition. Human Movement Science, 9(3-5), 241-289. Doi: $10.1016 / 0167-9457(90) 90005-X$

Magnusson, C., Caltenco, H. A., McGookin, D., Kytö, M., Hjaltadóttir, I., Hafsteinsdóttir, T. B., ... Bjartmarz, I. (2017). Tangible interaction for stroke survivors: Design recommendations, 597-602. ACM Press. https://doi. $\operatorname{org} / 10.1145 / 3024969.3025073$

Mallory, B. S. (2006). Stroke. In G. Cooper (Ed.), Essential Physical Medicine and Rehabilitation. 33-57. Totowa, NJ: Humana Press. Retrieved from http:// link.springer.com/10.1007/978-1-59745-100-0_2

Månsson, M., Fredriksson, B., \& Bränholm, I.-B. (1995). On life satisfaction and activity preferences in long-term survivors after stroke. Scandinavian Journal of Occupational Therapy, 2(2), 51-55. https://doi. org/10.3109/11038129509106668

Markussen. (2017). Building theory through design. In L. Vaughan (Ed.), Practice based design research, 87-98. New York: Bloomsbury Academic.

Mayo, N. E., Wood-Dauphinee, S., Côté, R., Durcan, L., \& Carlton, J. (2002). Activity, participation, and quality of life 6 months poststroke. Archives of Physical Medicine and Rehabilitation, 83(8), 1035-1042. 
Mauss, I. B., \& Robinson, M. D. (2009). Measures of emotion: A review. Cognition \& Emotion, 23(2), 209-237. https://doi.org/10.1080/02699930802204677

McGinley, C., \& Dong, H. (2011). Designing with information and empathy: Delivering human information to designers. The Design Journal, 14(2), 187-206. https://doi.org/10.2752/175630611X12984592780005

McKay, J., \& Mensah, G. A. (2004). The atlas of heart disease and stroke. Geneva: World Health Organization.

McKevitt, C., Redfern, J., Mold, F., \& Wolfe, C. (2004). Qualitative studies of stroke: A systematic review. Stroke, 35(6), 1499-1505. https://doi.org/10.1161/01. STR.0000127532.64840.36

McNaughton, H., Feigin, V., Kerse, N., Barber, P. A., Weatherall, M., Bennett, D., ... on behalf of the Auckland Regional Community Stroke Study Group. (2011). Ethnicity and functional outcome after stroke. Stroke, 42(4), 960-964. https://doi.org/10.1161/STROKEAHA.110.605139

McNaughton, H., McRae, A., Green, G., Abernethy, G., \& Gommans, J. (2014). Stroke rehabilitation services in New Zealand: A survey of service configuration, capacity and guideline adherence. The New Zealand Medical Journal, 127(1402), 10-19.

MedTech CoRE, \& CMDT. (2018). Flagship 5: Assistive and rehabilitation technologies. Retrieved January 1, 2018, from https://www.cmdt.org.nz/ flagship5

Mercierand, C., \& Bourbonnais, D. (2004). Relative shoulder flexor and handgrip strength is related to upper limb function after stroke. Clinical Rehabilitation, 18(2), 215-221. Doi: 10.1191/0269215504cr724oa

Merians, A. S., Poizner, H., Boian, R., Burdea, G., \& Adamovich, S. (2006). Sensorimotor training in a virtual reality environment: Does it improve functional recovery poststroke? Neurorehabilitation and Neural Repair, 20(2), 252-267. https://doi.org/10.1177/1545968306286914

Meyer, M., Foley, N., Pereira, S., Salter, K., \& Teasell, R. (2012). Organized stroke 
rehabilitation in Canada: Redefining our objectives. Topics in Stroke Rehabilitation, 19(2), 149-157. https://doi.org/10.1310/tsr1902-149

Michaelsen, S., Jacobs, S., Roby-Brami, A., \& Levin, M. (2004). Compensation for distal impairments of grasping in adults with hemiparesis. Experimental Brain Research, 157(2). https://doi.org/10.1007/s00221-004-1829-x

Michie, S., van Stralen, M. M., \& West, R. (2011). The behaviour change wheel: A new method for characterising and designing behaviour change interventions. Implementation Science, 6(1), 42. https://doi.org/10.1186/1748$5908-6-42$

Ministry of Health. (2009). Mortality and demographic data 2006. Wellington: Ministry of Health.

Ministry of Health. (2013). New Zealand Health Survey: annual update of key findings 2012/13. Wellington, N.Z.: Ministry of Health. Retrieved from http:// www.health.govt.nz/publication/new-zealand-health-survey-annualupdate-key-findings-2012-13

Ministry of Health - Manatū Hauora. (2017). About the Committees. Retrieved 20 June 2017, from http://ethics.health.govt.nz/about-committees

Molin, L. (2004). Wizard-of-Oz prototyping for co-operative interaction design of graphical user interfaces. 425-428. ACM Press. https://doi. org/10.1145/1028014.1028086

Morgan, W. G. (1974). The shaping game: A teaching technique. Behavior Therapy, 5(2), 271-272. https://doi.org/10.1016/S0005-7894(74)80144-9

Morris, D. M., Taub, E., \& Mark, V. W. (2006). Constraint-induced movement therapy: Characterizing the intervention protocol. Europa Medicophysica, 42(3), 257-268.

Morse, J. M. (2009). Mixing qualitative methods. Qualitative Health Research, 19(11), 1523-1524. https://doi.org/10.1177/1049732309349360

Mott, F., \& Sherrington. (1894). Experiments upon the influence of sensory nerves upon movement and nutrition of the limbs. Preliminary communication. 
In Proceedings of the Royal Society of London (1854-1905), (57), 481-488. London.

Mougenot, C., Bouchard, C., Aoussat, A., \& Westerman, S. (2008). Inspiration, images and design: An investigation of designers' information gathering strategies. Journal of Design Research, 7(4), 331-351.

Mukherjee, D., Levin, R. L., \& Heller, W. (2006). The cognitive, emotional, and social sequelae of stroke: Psychological and ethical concerns in poststroke adaptation. Topics in Stroke Rehabilitation, 13(4), 26-35. https://doi. org/10.1310/tsr1304-26

Mullaney, T., \& Stolterman, E. (2014). Why "design research practice” is not design as we know it. In Y. K. Lim, L. Redström, E. Stolterman, \& A. Valtonen (Eds.), Proceedings of DRS 2014: Design's Big Debates, 1039-1048. Umeå: Umeå University. Retrieved from http://urn.kb.se/ resolve?urn=urn:nbn:se:umu:diva-113501

Murphy, T. H., \& Corbett, D. (2009). Plasticity during stroke recovery: From synapse to behaviour. Nature Reviews Neuroscience, 10(12), 861-872. https:// doi.org/10.1038/nrn2735

Murray, J., Ashworth, R., Forster, A., \& Young, J. (2003). Developing a primary care-based stroke service: A review of the qualitative literature. The British Journal of General Practice: The Journal of the Royal College of General Practitioners, 53(487), 137-142.

Narayan Arya, K., Verma, R., Garg, R. K., Sharma, V. P., Agarwal, M., \& Aggarwal, G. G. (2012). Meaningful task-specific training (MTST) for stroke rehabilitation: A randomized controlled trial. Topics in Stroke Rehabilitation, 19(3), 193211. https://doi.org/10.1310/tsr1903-193

Nef, T., Guidali, M., Klamroth-Marganska, V., \& Riener, R. (2009). ARMin Exoskeleton robot for stroke rehabilitation. In O. Dössel \& W. C. Schlegel (Eds.), World Congress on Medical Physics and Biomedical Engineering, September 7 - 12, 2009, Munich, Germany (Vol. 25/9, pp. 127-130). Berlin, 
Heidelberg: Springer Berlin Heidelberg. Retrieved from http://link.springer. com/10.1007/978-3-642-03889-1_35

Nickpour, F., \& Dong, H. (2009). Anthropometrics without numbers! An investigation of designers' use and preference of people data. In Include2009. London: Royal College of Art.

Nielsen, J., \& Molich, R. (1990). Heuristic evaluation of user interfaces, 249-256. ACM Press. https://doi.org/10.1145/97243.97281

Norman, D. A. (1990). The design of everyday things (1st Doubleday/Currency ed). New York: Doubleday.

Norman, D. A. (1999). Affordance, conventions, and design. Interactions, 6(3), 38-43. https://doi.org/10.1145/301153.301168

Norman, D. A. (2005). Emotional design: Why we love (or hate) everyday things. New York: Basic Books.

Obermair, C., Reitberger, W., Meschtscherjakov, A., Lankes, M., \& Tscheligi, M. (2008). perFrames: Persuasive picture frames for proper posture. In $\mathrm{H}$. Oinas-Kukkonen, P. Hasle, M. Harjumaa, K. Segerståhl, \& P. Øhrstrøm (Eds.), Persuasive Technology, 5033, 128-139. Berlin, Heidelberg: Springer Berlin Heidelberg. Doi: 10.1007/978-3-540-68504-3_12

Oinas-Kukkonen, H., \& Harjumaa, M. (2009). Persuasive systems design: Key issues, process model, and system features. Communications of the Association for Information Systems, 24, Article 28(1), 485-500.

Osiurak, F., Rossetti, Y., \& Badets, A. (2017). What is an affordance? 40 years later. Neuroscience \& Biobehavioral Reviews, 77, 403-417. https://doi.org/10.1016/j. neubiorev.2017.04.014

Ostendorf, C. G., \& Wolf, S. L. (1981). Effect of forced use of the upper extremity of a hemiplegic patient on changes in function. A single-case design. Physical Therapy, 61(7), 1022-1028.

OwnFone. (2018). OwnFone. Retrieved 1 January 2018, from https://stroke. myownfone.com 
Ozkan, O., \& Dogan, F. (2013). Cognitive strategies of analogical reasoning in design: Differences between expert and novice designers. Design Studies, 34(2), 161-192. https://doi.org/10.1016/j.destud.2012.11.006

Page, S. J., Levine, P., Sisto, S., Bond, Q., \& Johnston, M. V. (2002). Stroke patients' and therapists' opinions of constraint-induced movement therapy. Clinical Rehabilitation, 16(1), 55-60. https://doi.org/10.1191/0269215502cr473oa

Panyan, M. C. (1980). How to use shaping. Lawrence, KS: H \& H Enterprises.

Pape, T. L.-B., Kim, J., \& Weiner, B. (2002). The shaping of individual meanings assigned to assistive technology: A review of personal factors. Disability and Rehabilitation, 24(1-3), 5-20. https://doi.org/10.1080/09638280110066235

Parker, C. J., Gladman, J. R., \& Drummond, A. E. (1997). The role of leisure in stroke rehabilitation. Disability and Rehabilitation, 19(1), 1-5.

Patton, M. Q. (1999). Enhancing the quality and credibility of qualitative analysis. Health Services Research, 34(5 Pt 2), 1189-1209.

Pedlow, K., Lennon, S., \& Wilson, C. (2014). Application of constraint-induced movement therapy in clinical practice: An online survey. Archives of Physical Medicine and Rehabilitation, 95(2), 276-282. https://doi. org/10.1016/j.apmr.2013.08.24

Pereira, E., Kolb, S., Schäfers, K., Schreiber, S., Weckbach, V., Goodwin, W., ... Kohler, K. (2016). STARS: enlightenment in the office space for behavioral change, 1640-1645. ACM Press. Doi: 10.1145/2968219.2968527

Pernecky, T. (2016). Epistemology and metaphysics for qualitative research. 55 City Road: SAGE Publications.

Petrovčič, A., Taipale, S., Rogelj, A., \& Dolničar, V. (2017). Design of mobile phones for older adults: An empirical analysis of design guidelines and checklists for feature phones and smartphones. International Journal of HumanComputer Interaction, 1-14. https://doi.org/10.1080/10447318.2017.1345142

Pettersson, I., Berndtsson, I., Appelros, P., \& Ahlström, G. (2005). Lifeworld perspectives on assistive devices: Lived experiences of spouses of persons 
with stroke. Scandinavian Journal of Occupational Therapy, 12(4), 159-169. https://doi.org/10.1080/11038120510031789

Plant, S. E., Tyson, S. F., Kirk, S., \& Parsons, J. (2016). What are the barriers and facilitators to goal-setting during rehabilitation for stroke and other acquired brain injuries? A systematic review and meta-synthesis. Clinical Rehabilitation, 30(9), 921-930. https://doi.org/10.1177/0269215516655856

Pollock, A., Baer, G., Campbell, P., Choo, P. L., Forster, A., Morris, J., ... Langhorne, P. (2014). Physical rehabilitation approaches for the recovery of function and mobility following stroke. In The Cochrane Collaboration (Ed.), Cochrane Database of Systematic Reviews. Chichester, UK: John Wiley \& Sons, Ltd. Retrieved from http://doi.wiley.com/10.1002/14651858.CD001920. pub3

Pollock, A., Baer, G., Pomeroy, V., \& Langhorne, P. (2007). Physiotherapy treatment approaches for the recovery of postural control and lower limb function following stroke. The Cochrane Database of Systematic Reviews, (1), CD001920. https://doi.org/10.1002/14651858.CD001920.pub2

Pollock, A., Farmer, S. E., Brady, M. C., Langhorne, P., Mead, G. E., Mehrholz, J., \& van Wijck, F. (2014). Interventions for improving upper limb function after stroke. In The Cochrane Collaboration (Ed.), Cochrane Database of Systematic Reviews. Chichester, UK: John Wiley \& Sons, Ltd. Retrieved from http://doi.wiley.com/10.1002/14651858.CD010820.pub2

Popay, J., Rogers, A., \& Williams, G. (1998). Rationale and standards for the systematic review of qualitative literature in health services research. Qualitative Health Research, 8(3), 341-351. https://doi. org/10.1177/104973239800800305

Prabhakaran, S., Zarahn, E., Riley, C., Speizer, A., Chong, J. Y., Lazar, R. M., ... Krakauer, J. W. (2007). Inter-individual variability in the capacity for motor recovery after ischemic stroke. Neurorehabilitation and Neural Repair, 22(1), 64-71. https://doi.org/10.1177/1545968307305302 
Prakash, V., Ganesan, M., Vasanthan, R., \& Hariohm, K. (2017). Do commonly used functional outcome measures capture activities that are relevant for people with stroke in India? Topics in Stroke Rehabilitation, 24(3), 200-205. https:// doi.org/10.1080/10749357.2016.1234190

Quinn, T., Harrison, \& McArthur. (2013). Assessment scales in stroke: Clinimetric and clinical considerations. Clinical Interventions in Aging, 201. https://doi. org/10.2147/CIA.S32405

Rand, D., Schejter-Margalit, T., Dudkiewicz, I., Kizony, R., Zeilig, G., \& Kizony, R. (2013). The use of the iPad for poststroke hand rehabilitation; A pilot study. 109-113. IEEE. https://doi.org/10.1109/ICVR.2013.6662068

Regmi, K. (2014). Triangulation in Healthcare Research: What Does It Achieve? 1 Oliver's Yard, 55 City Road, London EC1Y 1SP United Kingdom: SAGE Publications, Ltd. https://doi.org/10.4135/978144627305014534931

Renda, G., Jackson, S., Kuys, B., \& Whitfield, T. W. A. (2015). The cutlery effect: Do designed products for people with disabilities stigmatise them? Disability and Rehabilitation: Assistive Technology, 1-7. https://doi.org/10.3109/1748310 7.2015.1042077

Rensink, M., Schuurmans, M., Lindeman, E., \& Hafsteinsdottir, T. (2009). Taskoriented training in rehabilitation after stroke: Systematic review. Journal of Advanced Nursing, 65(4), 737-754.

Research New Zealand. (2015). A Report on a Survey of New Zealanders' Use of Smartphones and other Mobile Communication Devices 2015 (Survey No. 3). Wellington: Research New Zealand. Retrieved from http://www.researchnz. com/pdf/special\%20reports/research\%20new\%20zealand\%20special\%20 report\%20-\%20use\%20of\%20smartphones.pdf

Resnik, D. B. (2015). Some reflections on evaluating institutional review board effectiveness. Contemporary Clinical Trials, 45, 261-264. https://doi. $\operatorname{org} / 10.1016 /$ j.cct.2015.09.018

Reynolds, P. S., Crenshaw, C. T., Lefkowitz, D. S., Shelton, B. J., Preisser, J. S., \& 
Tegeler, C. H. (2001). A practical stroke severity scale predicts hospital outcomes. Journal of Stroke and Cerebrovascular Diseases, 10(5), 231-235. https://doi.org/10.1053/jscd.2001.29824

Rivera, H. (2018). Traffic lights. Retrieved January 1, 2018, from https://unsplash. com/photos/CiUiXZZYQGI

Robbiy, \& Photocase. (2016). Susan. Retrieved 13 April 2016, from https://www. photocase.com/photos/271993-human-being-woman-blue-old-adultsfeminine-senior-citizen-photocase-stock-photo

Robison, J., Wiles, R., Ellis-Hill, C., McPherson, K., Hyndman, D., \& Ashburn, A. (2009). Resuming previously valued activities post-stroke: Who or what helps? Disability and Rehabilitation, 31(19), 1555-1566. https://doi. org/10.1080/09638280802639327

Robson, B., Harris, R., \& Te Rōpū Rangahau Hauora a Eru Pōmare. (2007). Hauora, Māori Standards of Health. A Study of the Years, 2000-2005 IV. Wellington, N.Z.: Te Rōpū Rangahau Hauora a Eru Pōmare.

Rochette, A., Desrosiers, J., Bravo, G., St-Cyr-Tribble, D., \& Bourget, A. (2007). Changes in participation after a mild stroke: Quantitative and qualitative perspectives. Topics in Stroke Rehabilitation, 14(3), 59-68. https://doi. org/10.1310/tsr1403-59

Rochette, A., Desrosiers, J., \& Noreau, L. (2001). Association between personal and environmental factors and the occurrence of handicap situations following a stroke. Disability and Rehabilitation, 23(13), 559-569.

Rodríguez Ramírez, E. (2011). Elements of surprise: Industrial designers' strategies for eliciting surprise through interaction (Doctor of Philosophy). Victoria University of Wellington, Wellington, N.Z.

Rodríguez Ramírez, E. (2014). Industrial design strategies for eliciting surprise. Design Studies, 35(3), 273-297. https://doi.org/10.1016/j.destud.2013.12.001 Rodríguez Ramírez, E. (2017). A postgraduate thesis model for research through design based on design criteria. The International Journal of Designed 
Objects, 11(4), 11-27. https://doi.org/10.18848/2325-1379/CGP/v11i04/11-27

Rose, A., Rosewilliam, S., \& Soundy, A. (2017). Shared decision making within goal setting in rehabilitation settings: A systematic review. Patient Education and Counseling, 100(1), 65-75. https://doi.org/10.1016/j.pec.2016.07.030

Rosen, M. J. (1999). Telerehabilitation. NeuroRehabilitation, 12(1), 11-26.

Sacco, R. L., Kasner, S. E., Broderick, J. P., Caplan, L. R., Connors, J. J., Culebras, A., ... on behalf of the American Heart Association Stroke Council, Council on Cardiovascular Surgery and Anesthesia, Council on Cardiovascular Radiology and Intervention, Council on Cardiovascular and Stroke Nursing, Council on Epidemiology and Prevention (2013). An updated definition of stroke for the 21st Century: A statement for healthcare professionals from the American Heart Association/American Stroke Association. Stroke, 44(7), 2064-2089. https://doi.org/10.1161/STR.0b013e318296aeca

Säde, S. (2001). Cardboard mock-ups and conversations: studies on user-centered product design. University of Art and Design Helsinki UIAH, Helsinki.

Salkind, N. (2010). Descriptive statistics. In Encyclopedia of Research Design. 2455 Teller Road, Thousand Oaks California 91320 United States: SAGE Publications, Inc. https://doi.org/10.4135/9781412961288.n111

Salter, K. L., Foley, N. C., Jutai, J. W., \& Teasell, R. W. (2007). Assessment of participation outcomes in randomized controlled trials of stroke rehabilitation interventions. International Journal of Rehabilitation Research, 30(4), 339-342. https://doi.org/10.1097/MRR.0b013e3282f144b7

Salter, K., Hellings, C., Foley, N., \& Teasell, R. (2008). The experience of living with stroke: A qualitative meta-synthesis. Journal of Rehabilitation Medicine, 40(8), 595-602. https://doi.org/10.2340/16501977-0238

Samsa, G. P., \& Matchar, D. B. (2004). How strong is the relationship between functional status and quality of life among persons with stroke? Journal of Rehabilitation Research and Development, 41(3A), 279-282.

Sanchez, R. J., Jiayin Liu, Rao, S., Shah, P., Smith, R., Rahman, T., ... 
Reinkensmeyer, D. J. (2006). Automating arm movement training following severe stroke: Functional exercises with quantitative feedback in a gravity-reduced environment. IEEE Transactions on Neural Systems and Rehabilitation Engineering, 14(3), 378-389. https://doi.org/10.1109/ TNSRE.2006.881553

Scherer, Marcia J. (2005). Assessing the benefits of using assistive technologies and other supports for thinking, remembering and learning. Disability and Rehabilitation, 27(13), 731-739.

Seshadri, S., Beiser, A., Kelly-Hayes, M., Kase, C. S., Au, R., Kannel, W. B., \& Wolf, P. A. (2006). The lifetime risk of stroke: Estimates from the Framingham study. Stroke, 37(2), 345-350. https://doi.org/10.1161/01.STR.0000199613.38911.b2

Sevaldson, B. (2010). Discussions and movements in design research: A systems approach to practice research in design. 2010@FORMakademisk, 3(1), 8-35. Shin, J.-H., Kim, M.-Y., Lee, J.-Y., Jeon, Y.-J., Kim, S., Lee, S., ... Choi, Y. (2016). Effects of virtual reality-based rehabilitation on distal upper extremity function and health-related quality of life: A single-blinded, randomized controlled trial. Journal of NeuroEngineering and Rehabilitation, 13(1). https://doi.org/10.1186/s12984-016-0125-X

Siangliulue, P., Chan, J., Gajos, K. Z., \& Dow, S. P. (2015). Providing timely examples improves the quantity and quality of generated ideas, 83-92. ACM Press. https://doi.org/10.1145/2757226.2757230

Skinner, B. F. (1974). About behaviourism. New York: Knopf.

Smids, J. (2012). The voluntariness of persuasive technology. In M. Bang \& E. L. Ragnemalm (Eds.), Persuasive Technology. Design for Health and Safety, 7284, 123-132. Berlin, Heidelberg: Springer Berlin Heidelberg. Doi: 10.1007/978-3-642-31037-9_11

Stehle, C., \& Albrecht-Buehler, C. (2008). Developing more desirable products for stroke survivors. Topics in Stroke Rehabilitation, 15(2), 109-117. https://doi. org/10.1310/tsr1502-109 
Sterr, A., Freivogel, S., \& Schmalohr, D. (2002). Neurobehavioral aspects of recovery: Assessment of the learned nonuse phenomenon in hemiparetic adolescents. Archives of Physical Medicine and Rehabilitation, 83(12), 17261731. https://doi.org/10.1053/apmr.2002.35660

Stewart, J. C., \& Cramer, S. C. (2013). Patient-reported measures provide unique insights into motor function after stroke. Stroke, 44(4), 1111-1116. https://doi. org/10.1161/STROKEAHA.111.674671

Stienen, A. H. A., Hekman, E. E. G., Van der Helm, F. C. T., Prange, G. B., Jannink, M. J. A., Aalsma, A. M. M., \& Van der Kooij, H. (2007). Dampace: Dynamic force-coordination trainer for the upper extremities, 820-826. IEEE. https:// doi.org/10.1109/ICORR.2007.4428519

Stolterman, E., \& Wiberg, M. (2010). Concept-driven interaction design research. Human-Computer Interaction, 25(2), 95-118. https://doi. org/10.1080/07370020903586696

Stroke Foundation of New Zealand, \& New Zealand Guidelines Group. (2011). New Zealand Clinical Guidelines for Stroke Management 2010. Wellington, N.Z.: Stroke Foundation of New Zealand. Retrieved from http://www.stroke.org. nz/resources/NZClinicalGuidelinesStrokeManagement2010ActiveContents. pdf

Sue, V., \& Ritter, L. (2012). Conducting online surveys. 2455 Teller Road, Thousand Oaks California 91320 United States: SAGE Publications, Inc. https://doi. $\operatorname{org} / 10.4135 / 9781506335186$

Sumathipala, K., Radcliffe, E., Sadler, E., Wolfe, C. D., \& McKevitt, C. (2012). Identifying the long-term needs of stroke survivors using the International Classification of Functioning, Disability and Health. Chronic Illness, 8(1), 31-44. https://doi.org/10.1177/1742395311423848

Sunderland, A., \& Tuke, A. (2005). Neuroplasticity, learning and recovery after stroke: A critical evaluation of constraint-induced therapy. Neuropsychological Rehabilitation, 15(2), 81-96. https://doi. 
org/10.1080/09602010443000047

Sveen, U., Bautz-Holter, E., Margrethe Sodring, K., Bruun Wyller, T., \& Laake, K. (1999). Association between impairments, self-care ability and social activities 1 year after stroke. Disability and Rehabilitation, 21(8), 372-377. https://doi.org/10.1080/096382899297477

Taub, E. (2012). The behavior-analytic origins of constraint-induced movement therapy: An example of behavioral neurorehabilitation. The Behavior Analyst / MABA, 35(2), 155-178.

Taub, E. (2013). Promoting acceptance of efficacious behavior analysis interventions by clinical communities: The example of CI therapy. European Journal of Behaviour Analysis, 14(1), 127-193.

Taub, E., Crago, J. E., Burgio, L. D., Groomes, T. E., Cook, E. W., DeLuca, S. C., \& Miller, N. E. (1994). An operant approach to rehabilitation medicine: Overcoming learned nonuse by shaping. Journal of the Experimental Analysis of Behavior, 61(2), 281-293. https://doi.org/10.1901/jeab.1994.61-281

Taub, E., Crago, J. E., \& Uswatte, G. (1998). Constraint-induced movement therapy: A new approach to treatment in physical rehabilitation. Rehabilitation Psychology, 43(2), 152-170. https://doi.org/10.1037/0090-5550.43.2.152

Taub, E., Lum, P. S., Hardin, P., Mark, V. W., \& Uswatte, G. (2005). AutoCITE: Automated delivery of CI Therapy with reduced effort by therapists. Stroke, 36(6), 1301-1304. https://doi.org/10.1161/01.STR.0000166043.27545.e8

Taub, E., \& Morris, D. M. (2001). Constraint-induced movement therapy to enhance recovery after stroke. Current Atherosclerosis Reports, 3(4), 279-286. https:// doi.org/10.1007/s11883-001-0020-0

Taub, E., Uswatte, G., \& Elbert, T. (2002). New treatments in neurorehabilitation founded on basic research. Nature Reviews Neuroscience, 3(3), 228-236. https://doi.org/10.1038/nrn754

Taub, E., Uswatte, G., King, D. K., Morris, D., Crago, J. E., \& Chatterjee, A. (2006). A placebo-controlled trial of constraint-induced movement therapy for upper 
extremity after stroke. Stroke, 37(4), 1045-1049. https://doi.org/10.1161/01. STR.0000206463.66461.97

Taub, E., Uswatte, G., Mark, V. W., \& Morris, D. M. M. (2006). The learned nonuse phenomenon: Implications for rehabilitation. Europa Medicophysica, 42(3), 241-256.

Taule, T., \& Råheim, M. (2014). Life changed existentially: A qualitative study of experiences at 6-8 months after mild stroke. Disability and Rehabilitation, 36(25), 2107-2119. https://doi.org/10.3109/09638288.2014.904448

Teasell, R., Mehta, S., Pereira, S., McIntyre, A., Janzen, S., Allen, L., ... Viana, R. (2012). Time to rethink long-term rehabilitation management of stroke patients. Topics in Stroke Rehabilitation, 19(6), 457-462. https://doi. org/10.1310/tsr1906-457

Tellier, M., \& Rochette, A. (2009). Falling through the cracks: A literature review to understand the reality of mild stroke survivors. Topics in Stroke Rehabilitation, 16(6), 454-462. https://doi.org/10.1310/tsr1606-454

Tetteroo, D. A., Markopoulos, P., Timmermans, A., \& Seelen, H. (2014). TagTrainer: Supporting exercise variability and tailoring in technology supported upper limb training. Journal of NeuroEngineering and Rehabilitation, 11(1).

The Spinalist Foundation. (2016). Spinalistips. Retrieved 1 January 2017, from http://www.spinalistips.se

Torning, K. (2013). A review of four persuasive design models. International Journal of Conceptual Structures and Smart Applications, 1(2), 17-27. Doi.: 10.4018/ijcssa.2013070103

Torning, K., \& Oinas-Kukkonen, H. (2009). Persuasive system design: State of the art and future directions,1. ACM Press. Doi: 10.1145/1541948.1541989

Treger, I., Shames, J., Giaquinto, S., \& Ring, H. (2007). Return to work in stroke patients. Disability and Rehabilitation, 29(17), 1397-1403. https://doi. org/10.1080/09638280701314923

Trombly, C. A., \& Ma, H. (2002). A synthesis of the effects of occupational therapy 
for persons with stroke, Part I: Restoration of roles, tasks, and activities. The American Journal of Occupational Therapy: Official Publication of the American Occupational Therapy Association, 56(3), 250-259.

Tromp, N., Hekkert, P., \& Verbeek, P.-P. (2011). Design for socially responsible behavior: A classification of influence based on intended user experience. Design Issues, 27(3), 3-19. https://doi.org/10.1162/DESI_a_00087

Tyson, S. F., Hanley, M., Chillala, J., Selley, A., \& Tallis, R. C. (2006). Balance disability after stroke. Physical Therapy, 86(1), 30-38.

Tyson, S., \& Turner, G. (2000). Discharge and follow-up for people with stroke: What happens and why. Clinical Rehabilitation, 14(4), 381-392. https://doi. org/10.1191/0269215500cr331oa

Ullmer, B., \& Ishii, H. (2000). Emerging frameworks for tangible user interfaces. IBM Systems Journal, 39(3.4), 915-931. Doi: 10.1147/sj.393.0915

University of Alabama at Birmingham. (2014). CI therapy at UAB: Pre- and posttreatment. Retrieved October 31, 2016, from https://www.youtube.com/ watch?v=zjQSZRX2oLE

Uswatte, G., Taub, E., Morris, D., Barman, J., \& Crago, J. (2006). Contribution of the shaping and restraint components of constraint-induced movement therapy to treatment outcome. NeuroRehabilitation, 21(2), 147-156.

Uswatte, G., Taub, E., Morris, D., Light, K., \& Thompson, P. A. (2006). The Motor Activity Log-28: Assessing daily use of the hemiparetic arm after stroke. Neurology, 67(7), 1189-1194. https://doi.org/10.1212/01. wnl.0000238164.90657.c2

van den Bos, G. A. M. (2002). Socioeconomic variations in the course of stroke: Unequal health outcomes, equal care? Journal of Epidemiology \& Community Health, 56(12), 943-948. https://doi.org/10.1136/jech.56.12.943 Verbeek, P.-P. (2015). Beyond interaction: A short introduction to mediation theory. Interactions, 22(3), 26-31. http://doi.org/10.1145/2751314

Viana, R., \& Teasell, R. (2012). Barriers to the implementation of constraint- 
induced movement therapy into practice. Topics in Stroke Rehabilitation, 19(2), 104-114. https://doi.org/10.1310/tsr1902-104

Wallenbert, I., \& Jonsson, H. (2005). Waiting to get better: A dilemma regarding habits in daily occupations after stroke. The American Journal of Occupational Therapy: Official Publication of the American Occupational Therapy Association, 59(2), 218-224.

Warner, G., Packer, T., Villeneuve, M., Audulv, A., \& Versnel, J. (2015). A systematic review of the effectiveness of stroke self-management programs for improving function and participation outcomes: Self-management programs for stroke survivors. Disability and Rehabilitation, 37(23), 21412163. https://doi.org/10.3109/09638288.2014.996674

Wensveen, S. A. G., Djajadiningrat, J. P., \& Overbeeke, C. J. (2004). Interaction frogger: A design framework to couple action and function through feedback and feedforward. ACM Press. https://doi. org/10.1145/1013115.1013140

Wensveen, S., \& Matthews, B. (2015). Prototypes and prototyping in design research. In P. A. Rodgers \& J. Yee (Eds.), The Routledge companion to design research, 262-276. New York: Routledge, Taylor \& Francis Group.

White, J. H., Miller, B., Magin, P., Attia, J., Sturm, J., \& Pollack, M. (2012). Access and participation in the community: A prospective qualitative study of driving post-stroke. Disability and Rehabilitation, 34(10), 831-838. https:// doi.org/10.3109/09638288.2011.623754

Whiting, L. S. (2008). Semi-structured interviews: guidance for novice researchers. Nursing Standard, 22(23).

WHO | Disabilities. (n.d.). Retrieved 21 August 2016, from http://www.who.int/ topics/disabilities/en/

Wilson, B. A. (2008). Neuropsychological rehabilitation. Annual Review of Clinical Psychology, 4(1), 141-162. https://doi.org/10.1146/annurev. clinpsy.4.022007.141212 
Wilson, C. (2014). User interface inspection methods: a user-centered design method. Amsterdam; Boston: Elsevier/Morgan Kaufmann.

Wolf, T. J., Baum, C., \& Conner, L. T. (2009). Changing face of stroke: Implications for occupational therapy practice. The American Journal of Occupational Therapy: Official Publication of the American Occupational Therapy Association, 63(5), 621-625.

Wolf, S. L., Winstein, C. J., Miller, J. P., Taub, E., Uswatte, G., Morris, D., ... EXCITE Investigators (2006). Effect of constraint-induced movement therapy on upper extremity function 3 to 9 months after stroke: The EXCITE randomized clinical trial. JAMA, 296(17), 2095. https://doi.org/10.1001/ jama.296.17.2095

Wolfe, C. D. (2000). The impact of stroke. British Medical Bulletin, 56(2), 275-286.

Wölfel, C., \& Merritt, T. (2013). Method card design dimensions: A survey of card-based design tools. In P. Kotzé, G. Marsden, G. Lindgaard, J. Wesson, \& M. Winckler (Eds.), Human-Computer Interaction - INTERACT 2013, 8117, 479-486. Berlin, Heidelberg: Springer Berlin Heidelberg. https://doi. org/10.1007/978-3-642-40483-2_34

Wongx, B. D. (2006). Stehaufmann. Retrieved January 1, 2018, from https:// commons.wikimedia.org/wiki/File:Stehaufmann

Wood, J. P., Connelly, D. M., \& Maly, M. R. (2010). 'Getting back to real living': a qualitative study of the process of community reintegration after stroke. Clinical Rehabilitation, 24(11), 1045-1056. https://doi. org/10.1177/0269215510375901

World Health Organization. (2002). Towards a common language for functioning, disability and health/ICF. Retrieved from http:/www.who.int/ classifications/icf/icfbeginnersguide.pdf

World Health Organization. (2017). ICF Browser. Retrieved 6 August 2017, from http://apps.who.int/classifications/icfbrowser/

Wright, K. B. (2006). Researching internet-based populations: Advantages and 
disadvantages of online survey research, online questionnaire authoring software packages, and web survey services. Journal of Computer-Mediated Communication, 10(3). https://doi.org/10.1111/j.1083-6101.2005.tb00259.x

Wyller, T. B., Sveen, U., Sødring, K. M., Pettersen, A. M., \& Bautz-Holter, E. (1997). Subjective well-being one year after stroke. Clinical Rehabilitation, 11(2), 139-145. https://doi.org/10.1177/026921559701100207

Xie, S. (2016). Advanced robotics for medical rehabilitation: Current state of the art and recent advances. Cham: Springer.

Yoon, J., Desmet, P. M. A., \& Pohlmeyer, A. E. (2016). Developing usage guidelines for a card-based design tool. Archives of Design Research, 29(4), 5. https://doi. org/10.15187/adr.2016.11.29.4.5s

Zackowski, K. M. (2004). How do strength, sensation, spasticity and joint individuation relate to the reaching deficits of people with chronic hemiparesis? Brain, 127(5), 1035-1046. Doi: 10.1093/brain/awh116

Zarahn, E., Alon, L., Ryan, S. L., Lazar, R. M., Vry, M.-S., Weiller, C., ... Krakauer, J. W. (2011). Prediction of motor recovery using initial impairment and fMRI 48 h poststroke. Cerebral Cortex, 21(12), 2712-2721. https://doi.org/10.1093/ cercor/bhr047

Zimmerman, J., \& Forlizzi, J. (2008). The role of design artifacts in design theory construction. Artifact, 2(1), 41-45. https://doi. $\operatorname{org} / 10.1080 / 17493460802276893$

Zimmerman, J., Stolterman, E., \& Forlizzi, J. (2010). An analysis and critique of research through design: Towards a formalization of a research approach. ACM Press. https://doi.org/10.1145/1858171.1858228 
LIMITED USE ONLY 


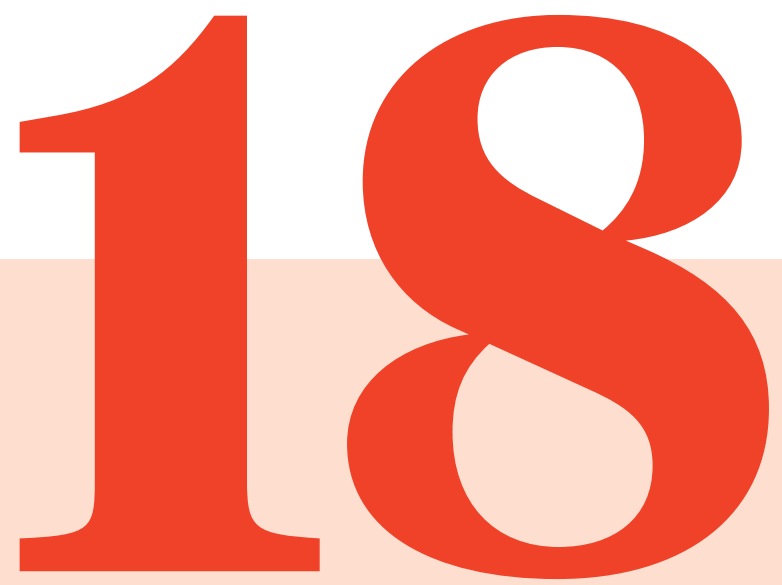

\section{Appendices}




\section{Appendix A Ethics application}




\section{Design of Study and Protocol}

Tangible interaction with everyday objects for home-based upper-limb stroke rehabilitation

\section{Study Summary}

This project is a study to develop and assess interactive design prototypes that can be used by stroke survivors in a self-directed way outside the clinical environment (e.g. their home). The interactive design prototypes are based on everyday objects in combination with digital technology to enhance the use of the affected body part. The outcome of this research is to determine what kinds of activities of daily living stroke survivors enjoy participating in while living at home. Based on these outcomes digital, interactive design prototypes will be developed that can be used by stroke survivors in activities of daily living. These interactive design prototypes encourage the use of the affected hand and arm. A further outcome of this study is the usability assessment of these design prototypes to validate the clinical usability and alignment with therapeutical goals. The research will be carried out by Mailin Lemke, PhD student at the School, Victoria University of Wellington. Clinical guidance will be provided by academic clinical physiotherapists with specialist research experience in the field of stroke rehabilitation. The research participants are survivors of stroke who will provide information about activities of daily living that they enjoy taking part in and feedback on their experience (i.e. the usability) of the design prototypes. This will enable iterative changes to be made and re-evaluated within this research.

\section{Study question}

Stroke causes significant morbidity and mortality in New Zealand (Feigin et al., 2014). Survivors of stroke can have limited access to rehabilitation therapies following their discharge from hospital and there is limited availability of stroke rehabilitation in the community (Gommans et al., 2008; McNaughton et al., 2014a). One strategy is to introduce self-directed rehabilitation. This has been defined as "a stroke person's goal-directed journey of recovery following stroke, totally or largely independent of professional rehabilitation clinician input" (McNaughton et al, 2014b). One of the risks of self-directed rehabilitation is that it can be conducted incorrectly at standards well below the therapists' expectations. To avoid this, sensor technology and software developments have allowed computerized systems to establish goal setting, feedback and performance measurement specifically for lower-limb rehabilitation post stroke (Mawson et al., 2014; Parker et al., 2014).

Stroke causes significant morbidity and mortality in New Zealand (Ministry of Health, 2013). Stroke rehabilitation of the upper limb primarily focuses on the first six months post stroke and stroke rehabilitation in the community to improve the affected body side are rare. One strategy is to introduce self-directed rehabilitation. This has been defined as "a stroke person's goal-directed journey of recovery following stroke, totally or largely independent of professional rehabilitation clinician input" (McNaughton et al, 2014b). Doing a repetitive movement without getting the impression that it is repetitive is a core element in stroke rehabilitation. Among the different rehabilitation approaches is the constraint-induced therapy proven to be beneficial in the rehabilitation process but it is labour intensive and expensive. Using this therapy approach in combination with everyday objects and digital technology in the rehabilitation process would benefit from the patient's motivation to engage with the object. The focus would be on the interaction with the everyday object rather than on the pure delivery of rehabilitation. Using digital technology with those objects provides the opportunity to enhance the use of the affected arm by restricting the functionality of the object to a specific part of the body (Reiss, Wolf, Hammel, McLeod, \& Williams, 2012; Taub et al., 2000: Wolf, Lecraw, Barton, and Jann, 1989).

The overall research aim is:

To investigate the potential of tangible interfaces to facilitate self-delivered home-based CIT (constraint-induced therapy) for upper-limb stroke rehabilitation.

The research questions are 
1. How can everyday objects that the stroke patient use during activities of daily living be used for the rehabilitation process?

2. How can constraint-induced therapy be incorporated in a tangible interface that involves activities of daily living?

3. Does the design facilitate CIT and how can it be improved?

\section{Study design}

The study is to develop interactive design prototypes based on everyday objects that the stroke survivors use during activities of daily living and incorporate them in the rehabilitation process. These design prototypes offer a self-directed rehabilitation to stroke survivors who are living at home. The intended setting for these objects is the home where the objects can contribute to the recovery of the impaired arm and hand.

The study includes interviewing stroke survivors about activities of daily living that they enjoy participating in. The inclusion criteria for this study are: the experience of a stroke at least 6 months prior to the interview, living at home, an active wrist and finger, the ability to walk independently, an age of 40-75 years, and good general health. Exclusion criteria are signs of severely impaired verbal communication, inability to give consent, severe neurocognitive deficits, excessive pain in any joint of the paretic arm, terminal illness, or life-threatening comorbidities (Wolf et al., 2006; Barzel et al.,2015). The exclusion criteria ensure that the stroke survivors are able to take part in the interviews and testing of the design prototypes. There is no formal screening process involved in this study. The anticipation is that stroke survivors who are impaired and fit the inclusion criteria are the ones who will make contact with the researchers. The inclusion criteria will be outlined on the recruitment flyer.

The study includes four different methods to answer the research questions: interviews with stroke survivors prior and after the development of the interactive design prototypes, an online questionnaire to collect quantitative data concerning activities of daily living and everyday objects, design ethnography in the form of observation of therapy sessions, and cultural probes which will be provided to the stroke survivors.

Interviews:

The stroke survivors will be interviewed twice during this study.

The first semi-structured interviews investigate how stroke survivors engage in rehabilitation at home, and what kinds of objects they like to use during everyday tasks and if they receive a homebased rehabilitation.

The second interview is taking place after the design prototypes are completed.

This method includes the stroke survivors as research participants providing their feedback and suggestions to the designers (Mawson and Mountain, 2011; Williamson et al., 2015)

The stroke survivors will be asked to test the devices and invited to give feedback and recommendations. Clinicians will assess the testing undertaken by the stroke survivors to secure the therapeutic validity and usability of the prototypes.

Testing of design prototypes will not involve participants taking part in rehabilitation activities, such as assisted walking, that require direct expert clinical supervision. The therapeutic activities will focus on the control of one affected limb and will be performed to ensure the safety of the participants. It is intended that the use of the design prototypes will be tested while participants are seated to avoid falls or other injuries. Participants have the right to withdraw their consent and discontinue their involvement at any stage.

The interactive design prototypes will be evaluated by the stroke survivors to ensure that limb movements and tasks align to therapeutic goals and translate into controllable movements for the return of useful function to an affected limb. These are activities of daily living such as the ability to turn a tap on and off or to turn a door handle and open a door. The usability testing ensures that 
users can achieve their goals with effectiveness, efficiency and satisfaction in a specified context of use (Wasserkrug et al., 2009).

\section{Online questionnaire}

Using an online questionnaire offers the possibility to collect data concerning activities of daily living and everyday objects that are meaningful to stroke patients. The questionnaire covers questions concerning the impact that the stroke had on the individual and possible stroke therapy that they receive. A strong focus is on activities of daily living that people used to engage in prior to the stroke and post the event to get a better understanding about the kind of activities that are meaningful and important to them. The questionnaire further contains details based on the 'UAB CI Therapy Research Group Grade Table' which is an essential part of constraint-induced movement therapy and helps to determine the severity of the stroke. The questionnaire comprises 19 stroke and demographic related open as well as closed questions and takes 10-15 minutes to complete. Benefits of an online questionnaire are that it preserves the participant's confidentiality, can be completed at the respondent's convenience and can be administered in a standard manner (Leung, 2001). It further offers the possibility to be sent out to stroke clubs nationally and internationally to ease the recruitment process.

\section{Ethnography}

In order to determine what kinds of objects stroke survivors use in activities of daily living the method of ethnography in the form of observations will be used. Observations offer the possibility to gain a deeper understanding of what kind of objects are used by therapists in the rehabilitation process and how the stroke survivors use everyday objects during the rehabilitation. It is pointed out in the literature that task specific training is beneficial in the rehabilitation process, but there is no detailed description of which kinds of objects are used and which specific tasks are trained (Hubbard, Parsons, Neilson, \& Carey, 2009; Pollock et al., 2014). The observations will take place in the environment of the participant's home. During the observations the stroke survivor conducts activities of daily living. The aim of this method is to get a better understanding what kinds of objects play an essential role in activities of daily living.

\section{Cultural probes}

The stroke survivors will further be provided with a cultural probes kit. Cultural probes offer the opportunity to collect data that has a documentary and life-realistic character. Participants are provided with a self-documentary probe kit, consisting of, for example, a disposable camera with instructions on what to photograph, diaries or postcards to write down feelings and thoughts that are valuable to the research. Using probes requires the participants to be creative, to think about what they take for granted (Graspemo et al., 2004; Hassling et al., 2005; Hemmings et al., 2002).

Participants will be recruited through local stroke clubs and can be considered representative of the stroke survivors in the community who would use self-directed rehabilitation. The interviews and ethnography study will take place at the participant's home. The researchers will work in teams of at least two persons when visiting the stroke survivor at their home. The therapeutic goal of the each of the design prototypes will be described to potential participants. The participants will, in part, selfselect based on individual therapeutic goals aligning to each of the design prototypes.

Participant data collection will include non-identifying demographic information and relevant clinical information relating to the stroke, physical disabilities and rehabilitation goals. The experience of the participants using the design prototypes will be collected using audio recorded semi-structured interviews. The data collected with the online questionnaire will contain non-identifiable details and be stored on a platform owned by the Victoria University of Wellington. Just the primary investigator will have access to this data. Participants' use of the design prototypes will be recorded with using video and photography to ensure use is clinically appropriate. This recording will just focus on the hands of the participant and the design prototypes. The comments and the way the participant 
interacts with the object is beneficial to improve the functionality of the design prototypes and helps to make sure that the functionality suits the intended purpose. The video recording will purely be used to analyse the interaction with the devices. The recording will not be used in presentations or teaching lessons.

\section{Scientific Justification}

Worldwide, stroke affects 15 million people and is the third most likely cause of death (McKay and Mensah, 2004). While the worldwide rate of stroke is declining, this rate is four times slower in New Zealand where it is a leading factor for long-term disability and a topic of particular concern (Feigin et al., 2014). In addition the different ethnic groups in New Zealand are affected in different ways by stroke; with some groups, for example Pacific Islander and Māori, being more affected or having longterm dependence on care (McNaughton et al., 2011).

Stroke survivors are most likely to suffer from physical and cognitive limitations and impairments caused by their stroke. Around $20 \%$ of all new stroke survivors are limited in their mobility and require institutional care while $30 \%$ of stroke patients can return to their homes (McNaughton et al., 2014). Approximately $70 \%$ of patients experience altered arm function after a stroke. $40 \%$ of stroke patients are left with a persistent lack of function in the affected arm (Intercollegiate Working Party for Stroke and Royal College of Physicians of London, 2012).

The 'Stroke Foundation of New Zealand' and the 'New Zealand Guidelines Group' (2011) recommends that all stroke patients should be offered a rehabilitation service after hospital discharge. The rehabilitation continues after discharge and community rehabilitation services, as well as home-based rehabilitation, are recommended whenever those services are needed and available to overcome motor impairment and increase the rehabilitation process (Stroke Foundation of New Zealand and New Zealand Guidelines Group, 2011).

The tasks that get trained during the rehabilitation vary. Task specific training rather than conventional stroke rehabilitation is associated with an increased improvement of motor functionality. The emphasis is on training a specific task rather than remediating impairment such as muscle weakness. The training induces neuroplastic changes at several levels in the brain using spared regions, which have not been affected by the stroke (Arya et al. 2012; Hubbard et al. 2009). Task specific training often uses 'real world' tasks or everyday tasks as the therapeutic medium in functional recovery (Hubbard et al. 2009). Essential elements of this form of rehabilitation are that the tasks are specific to the patient, randomly assigned, positively reinforced and lead to the relearning of the whole task (Hubbard et al. 2009).

Among the different therapy approaches is the constraint-induced therapy (CIT) form mentioned as being the one with the most potential to improve motor functionality (Langhorne et al., 2011). This therapy method does not yet use external devices or digital technology and is provided in a clinical setting. It is recommended by the New Zealand clinical guidelines for stroke management that patients with difficulties using their upper limb should receive constraint-induced therapy. The therapy can be provided as a single therapy or in combination with others. Patients are encouraged by their caregivers and therapists to use the affected arm and hand as much as possible (Stroke Foundation of New Zealand and New Zealand Guidelines Group, 2011)

Task practice is an important component of the CIT (Barzel et al., 2015), however some of the tasks are relatively less meaningful and they are performed just with the affected arm. 'Task specific' is defined as a training or therapy where patients 'practice context-specific motor tasks and receive some form of feedback' (Teasell et al., 2008). Neuroplastic changes were found to be greater when the task was important to the individual, that is, meaningful in nature (Daly and Ruff, 2007). The 'task specific' training can restore motor function by using spared regions of the brain, which are usually next to the lesion and recruiting accompanying parts of the brain (Nudo et al., 2000).

An example of a 'task specific' training is the lifting and bringing a water glass towards the mouth with the impaired arm while using the non-affected arm to stabilize the water bottle (Arya et al. 2012). 
Other examples are household tasks such as 'eating, opening and closing jars and spring-loaded clothespins' (Hamzei et al., 2006).

This emphasis of task specific training in constraint-induced therapy does not focus on the object that plays an important role in it. Using those objects in combination with digital technology to deliver constraint-induced therapy is a promising foundation to facilitate self-directed rehabilitation (Giaccardi, 2015; Pollock et al., 2014; Van Peppen et al., 2004).

The approach of this research is to ensure that the devices and software systems will be designed to align clinical therapeutic goals to the goals of the participants and achieving goals is measurable and can be disclosed to the participants and can be assessed by clinicians. Observations of use will be made to ensure that achieving goals is not influenced by the use of any unaffected limb.

The engagement of Māori and Pacific island participants in this research will be undertaken by the recruitment invitation process. Invitations for participant involvement will be made through the stroke clubs from Wellington South through to Upper Hutt and the Kapiti Coast.

For Pacific Islanders and Māori is the risk to experience a stroke compared to European New Zealand inhabitants 1.4 higher (McNaughton et al., 2011). The importance to this study of including Māori participants is that there is not just an increased risk of experiencing a stroke for but also disparities in the outcomes of rehabilitation for Māori affected by stroke (Dyall et al., 2008) and self-directed rehabilitation can improve medium-term stroke outcomes in both Māori and Pacific people (Harwood et al., 2012).

\section{Protocol}

Study Aim:

To investigate the potential of tangible interfaces to facilitate self-delivered home-based for upperlimb stroke rehabilitation based on the constraint induced therapy approach.

\section{Data Required:}

Demographic data.

Rationale. The data will be collected to describe the research participant groups.

Data will be collected by self-reporting at interview, cultural probes and ethnography. Clinical notes will not be accessed.

Data collected. Age, gender, ethnicity, time since stroke, side of brain affected, physical disability (i.e. limb affected and how it is affected), any other affects (e.g. speech, memory), use and access to computers before and after stroke. 


\section{Performance data.}

Rationale. The data will be collected to include factors such as time to set-up devices for use, speed and accuracy of movements, percentage of tasks or movements meeting clinical therapy expectations and duration of involvement with devices and games.

Data collected. Quantifiable data will be collected by software imbedded within the computer systems. Data relating to usability, ergonomics and clinical expectation will be recorded using video and still camera capture

\section{Experience of user data}

Rationale. User experience informs the usefulness of the devices and software from a user-behaviour perspective. This allows changes to be made and reassessed.

Data collected. Participants will be invited to describe their experience using the design prototypes and will be asked questions regarding ease or difficulty of use, comfort or discomfort relating to use, and also invited to make suggestions for improvement. Data will be collected by video or audio recording and still images showing just the hands and design prototypes may also be used.

Data Storage and confidentiality.

All data will be securely stored as digital files within the Victoria University data system for 5 years after collection.

All data stored will have any identifiable information removed. Given the research methods used in ergonomics, images are likely to be included in either thesis, publications or presentations. This will be with the agreement of participants and images will be altered so that participants are not identifiable. Images and videos will be recorded using cameras provided by the School of Design, Victoria University of Wellington. Any images and videos will be deleted from the cameras afterwards.

\section{Participant numbers}

Qualitative analysis and usability research requires smaller numbers of participants, typically between 6 to 10, compared to quantitative research, where 30 to 40 participants are required.

As a result, this study will require around 8 people to participate in the qualitative data collection using the methods of interviews and usability testing, design ethnography, and cultural probes.

For the online questionnaires a quantitative approach will be chosen requiring a higher response rate. A minimum of 80 responses is anticipated.

\section{References}

Arya, K.N., Verma, R., Garg, R.K., Sharma, V.P., Agarwal, M., Aggarwal, G.G., 2012. Meaningful taskspecific training (MTST) for stroke rehabilitation: a randomized controlled trial. Top. Stroke Rehabil. 19, 193-211. doi:10.1310/tsr1903-193

Barzel, A., Ketels, G., Stark, A., Tetzlaff, B., Daubmann, A., Wegscheider, K., van den Bussche, H., Scherer, M., 2015. Home-based constraint-induced movement therapy for patients with upper limb dysfunction after stroke (HOMECIMT): a cluster-randomised, controlled trial. Lancet Neurol. 14, 893-902. doi:10.1016/S1474-4422(15)00147-7

Daly, J.J., Ruff, R.L., 2007. Construction of Efficacious Gait and Upper Limb Functional Interventions Based on Brain Plasticity Evidence and Model-Based Measures For Stroke Patients. Sci. World J. 7, 2031-2045. doi:10.1100/tsw.2007.299

Dyall, L., Feigin, V., Brown, P., Roberts, M., 2008. Stroke: A picture of health disparities in New Zealand. Soc. Policy J. N. Z. 178-191.

Feigin, V.L., McPherson, K., Barker-Collo, S., Krishnamurthi, R., 2014. Measuring and reducing the stroke burden in New Zealand: Editorial. Int. J. Stroke 9, 5-5. doi:10.1111/ijs.12122

Giaccardi, E., 2015. Designing the connected everyday. interactions 22, 26-31. doi:10.1145/2692982

Gommans, J., Barber, P.A., Hanger, H.C., Bennett, P., 2008. Rehabilitation after stroke: changes between 2002 and 2007 in services provided by district health boards in New Zealand. N. Z. Med. J. 121, 26-33.

Graspemo, G., Hassling, L., Nordfeldt, S., Eriksson, H., Timpka, T., 2004. Design of interactive health drama built on social realism. Stud. Health Technol. Inform. 107, 879-883. 
Hamzei, F., Liepert, J., Dettmers, C., Weiller, C., Rijntjes, M., 2006. Two different reorganization patterns after rehabilitative therapy: An exploratory study with fMRI and TMS. Neurolmage 31, 710-720. doi:10.1016/j.neuroimage.2005.12.035

Harwood, M., Weatherall, M., Talemaitoga, A., Barber, P.A., Gommans, J., Taylor, W., McPherson, K., McNaughton, H., 2012. Taking charge after stroke: promoting self-directed rehabilitation to improve quality of life - a randomized controlled trial. Clin. Rehabil. 26, 493-501. doi:10.1177/0269215511426017

Hassling, L., Nordfeldt, S., Eriksson, H., Timpka, T., 2005. Use of cultural probes for representation of chronic disease experience: exploration of an innovative method for design of supportive technologies. Technol. Health Care Off. J. Eur. Soc. Eng. Med. 13, 87-95.

Hemmings, T., Clarke, K., Rouncefield, M., Rodden, T., 2002. Probing the Probes. Presented at the Proceedings of the Participation Design Conference, CPSR Press, Malmö, Sweden, pp. 42-50.

Hubbard, I.J., Parsons, M.W., Neilson, C., Carey, L.M., 2009. Task-specific training: evidence for and translation to clinical practice. Occup. Ther. Int. 16, 175-189. doi:10.1002/oti.275

Intercollegiate Working Party for Stroke, Royal College of Physicians of London, 2012. National clinical guideline for stroke.

Langhorne, P., Bernhardt, J., Kwakkel, G., 2011. Stroke rehabilitation. The Lancet 377, 1693-1702. doi:10.1016/S0140-6736(11)60325-5

Leung, Wai-Ching. "How to design a questionnaire." student BMJ 9.11 (2001): 187-189.

Mawson, S., Mountain, G., 2011. The SMART rehabilitation system for stroke self-management : issues and challenges for evidence-based health technology research. J. Phys. Ther. Educ. 25, 48-53.

Mawson, S., Nasr, N., Parker, J., Zheng, H., Davies, R., Mountain, G., 2014. Developing a personalised self-management system for post stroke rehabilitation; utilising a user-centred design methodology. Disabil. Rehabil. Assist. Technol. 9, 521-528. doi:10.3109/17483107.2013.840863

McKay, J., Mensah, G.A., 2004. The atlas of heart disease and stroke. World Health Organization, Geneva.

McNaughton, H., Feigin, V., Kerse, N., Barber, P.A., Weatherall, M., Bennett, D., Carter, K., Hackett, M., Anderson, C., on behalf of the Auckland Regional Community Stroke Study Group, 2011. Ethnicity and Functional Outcome After Stroke. Stroke 42, 960-964. doi:10.1161/STROKEAHA.110.605139

McNaughton, H., McRae, A., Green, G., Abernethy, G., Gommans, J., 2014. Stroke rehabilitation services in New Zealand: a survey of service configuration, capacity and guideline adherence. N. Z. Med. J. 127, 10-19.

Ministry of Health, 2013. New Zealand Health Survey: Annual update of key findings 2012/13. Ministry of Health, Wellington, N.Z.

Nudo, R.J., Friel, K.M., Delia, S.W., 2000. Role of sensory deficits in motor impairments after injury to primary motor cortex. Neuropharmacology 39, 733-742. doi:10.1016/S0028-3908(99)002543

Parker, J., Mawson, S., Mountain, G., Nasr, N., Davies, R., Zheng, H., 2014. The provision of feedback through computer-based technology to promote self-managed post-stroke rehabilitation in the home. Disabil. Rehabil. Assist. Technol. 9, 529-538. doi:10.3109/17483107.2013.845611

Pollock, A., Baer, G., Campbell, P., Choo, P.L., Forster, A., Morris, J., Pomeroy, V.M., Langhorne, P., 2014. Physical rehabilitation approaches for the recovery of function and mobility following stroke, in: The Cochrane Collaboration (Ed.), Cochrane Database of Systematic Reviews. John Wiley \& Sons, Ltd, Chichester, UK.

Reiss, A.P., Wolf, S.L., Hammel, E.A., McLeod, E.L., Williams, E.A., 2012. Constraint-Induced Movement Therapy (CIMT): Current Perspectives and Future Directions. Stroke Res. Treat. 2012, 1-8. doi:10.1155/2012/159391

Stroke Foundation of New Zealand, New Zealand Guidelines Group, 2011. New Zealand clinical guidelines for stroke management 2010. Stroke Foundation of New Zealand, Wellington, N.Z.

Taub, E., Uswatte, G., van der Lee, J.H., Lankhorst, G.J., Bouter, L.M., Wagenaar, R.C., 2000. Constraint-Induced Movement Therapy and Massed Practice Response. Stroke 31, 983-991. doi:10.1161/01.STR.31.4.983-c 
Teasell, R.W., Foley, N.C., Salter, K.L., Jutai, J.W., 2008. A Blueprint for Transforming Stroke Rehabilitation Care in Canada: The Case for Change. Arch. Phys. Med. Rehabil. 89, 575-578. doi:10.1016/j.apmr.2007.08.164

Van Peppen, R.P.S., Kwakkel, G., Wood-Dauphinee, S., Hendriks, H.J.M., Van der Wees, P.J., Dekker, J., 2004. The impact of physical therapy on functional outcomes after stroke: what's the evidence? Clin. Rehabil. 18, 833-862.

Wasserkrug, S., Dalvi, N., Munson, E.V., Gogolla, M., Sirangelo, C., Fischer-Hübner, S., Ives, Z., Velegrakis, Y., Bevan, N., Jensen, C.S., Snodgrass, R.T., 2009. Usability, in: Liu, L., öZsu, M.T. (Eds.), Encyclopedia of Database Systems. Springer US, Boston, MA, pp. 3247-3251.

Williamson, T., Kenney, L., Barker, A.T., Cooper, G., Good, T., Healey, J., Heller, B., Howard, D., Matthews, M., Prenton, S., Ryan, J., Smith, C., 2015. Enhancing public involvement in assistive technology design research. Disabil. Rehabil. Assist. Technol. 10, 258-265. doi:10.3109/17483107.2014.908247

Wolf, S.L., Lecraw, D.E., Barton, L.A., Jann, B.B., 1989. Forced use of hemiplegic upper extremities to reverse the effect of learned nonuse among chronic stroke and head-injured patients. Exp. Neurol. 104, 125-132.

Wolf, S.L., Winstein, C.J., Miller, J.P., Taub, E., Uswatte, G., Morris, D., Giuliani, C., Light, K.E., NicholsLarsen, D., EXCITE Investigators, for the, 2006. Effect of Constraint-Induced Movement Therapy on Upper Extremity Function 3 to 9 Months After Stroke: The EXCITE Randomized Clinical Trial. JAMA 296, 2095. doi:10.1001/jama.296.17.2095 
02 June 2016

Dear Miss Lemke

$\begin{array}{lll}\text { Re: } & \text { Ethics ref: } & 16 / C E N / 37 \\ & \text { Study title: } & \text { Tangible interaction with everyday objects for home-based upper-limb } \\ & & \text { stroke rehabilitation }\end{array}$

I am pleased to advise that this application has been approved by the Central Health and Disability Ethics Committee. This decision was made through the HDEC-Full Review pathway.

\section{Conditions of HDEC approval}

HDEC approval for this study is subject to the following conditions being met prior to the commencement of the study in New Zealand. It is your responsibility, and that of the study's sponsor, to ensure that these conditions are met. No further review by the Central Health and Disability Ethics Committee is required.

Standard conditions:

1. Before the study commences at any locality in New Zealand, all relevant regulatory approvals must be obtained.

2. Before the study commences at any locality in New Zealand, it must be registered in a clinical trials registry. This should be a WHO-approved (such as the Australia New Zealand Clinical Trials Registry, www. anzctr.org.au). However https://clinicaltrials.gov/ is acceptable provided registration occurs prior to the study commencing at any locality in New Zealand.

3. Before the study commences at a given locality in New Zealand, it must be authorised by that locality in Online Forms. Locality authorisation confirms that the locality is suitable for the safe and effective conduct of the study, and that local research governance issues have been addressed. 


\section{After HDEC review}

Please refer to the Standard Operating Procedures for Health and Disability Ethics Committees (available on www.ethics. health.govt.nz) for HDEC requirements relating to amendments and other post-approval processes.

Your next progress report is due by 01 June 2017.

\section{Participant access to ACC}

The Central Health and Disability Ethics Committee is satisfied that your study is not a clinical trial that is to be conducted principally for the benefit of the manufacturer or distributor of the medicine or item being trialled. Participants injured as a result of treatment received as part of your study may therefore be eligible for publicly-funded compensation through the Accident Compensation Corporation (ACC).

Please don't hesitate to contact the HDEC secretariat for further information. We wish you all the best for your study.

Yours sincerely.

Mrs Helen Walker

Chairperson

Central Health and Disability Ethics Committee

Encl: appendix A: documents submitted appendix B: statement of compliance and list of members 
Appendix A

Documents submitted

\begin{tabular}{|c|c|c|}
\hline Document & Version & Date \\
\hline CV for $\mathrm{Cl}$ : CV of Co-ordinator Investigator & $\mathrm{CV}$ & 12 February 2016 \\
\hline Other (No Description Entered) & $\begin{array}{l}\text { Invitation } \\
\text { Flyer }\end{array}$ & 11 February 2016 \\
\hline Participant Information Sheet / Interviews & 01 & 08 March 2016 \\
\hline Participant Information Sheet / Cultural probes & 01 & 08 March 2016 \\
\hline Participant Information Sheet / Design ethnography & 01 & 08 March 2016 \\
\hline Covering Letter & 01 & 08 March 2016 \\
\hline Protocol & 01 & 08 March 2016 \\
\hline Flyer Interview & 01 & 08 March 2016 \\
\hline Flyer Design Ethnography & 01 & 08 March 2016 \\
\hline Flyer cultural probes & 01 & 08 March 2016 \\
\hline $\begin{array}{l}\text { Evidence of scientific review: Review of the research committee at } \\
\text { the School of Design including recommended changes }\end{array}$ & 01 & 08 March 2016 \\
\hline Semi structured interview questions & 01 & 10 March 2016 \\
\hline \multicolumn{3}{|l|}{ Application } \\
\hline Covering Letter: Covering letter: Responses to requested changes & 2 & 16 May 2016 \\
\hline Updated Information sheet and consent form & 02 & 16 May 2016 \\
\hline Response to Request for Further Information & & 18 May 2016 \\
\hline
\end{tabular}




\section{Appendix B}

\section{Statement of compliance and list of members}

\section{Statement of compliance}

The Central Health and Disability Ethics Committee:

- is constituted in accordance with its Terms of Reference

- operates in accordance with the Standard Operating Procedures for Health and Disability Ethics Committees, and with the principles of international good clinical practice (GCP)

- is approved by the Health Research Council of New Zealand's Ethics Committee for the purposes of section 25(1)(c) of the Health Research Council Act 1990

- is registered (number 00008712) with the US Department of Health and Human Services' Office for Human Research Protection (OHRP).

\section{List of members}

\begin{tabular}{|l|l|l|l|}
\hline Name & Category & Appointed & Term Expires \\
\hline Mrs Helen Walker & $\begin{array}{l}\text { Lay (consumer/community } \\
\text { perspectives) }\end{array}$ & $01 / 07 / 2015$ & $01 / 07 / 2018$ \\
\hline Dr Angela Ballantyne & Lay (ethical/moral reasoning) & $30 / 07 / 2015$ & $30 / 07 / 2018$ \\
\hline Dr Melissa Cragg & Non-lay (observational studies) & $30 / 07 / 2015$ & $30 / 07 / 2018$ \\
\hline Dr Peter Gallagher & $\begin{array}{l}\text { Non-lay (health/disability service } \\
\text { provision) }\end{array}$ & $30 / 07 / 2015$ & $30 / 07 / 2018$ \\
\hline Mrs Sandy Gill & $\begin{array}{l}\text { Lay (consumer/community } \\
\text { perspectives) }\end{array}$ & $30 / 07 / 2015$ & $30 / 07 / 2018$ \\
\hline Dr Patries Herst & Non-lay (intervention studies) & $27 / 10 / 2015$ & $27 / 10 / 2018$ \\
\hline Dr Dean Quinn & Non-lay (intervention studies) & $27 / 10 / 2015$ & $27 / 10 / 2018$ \\
\hline Dr Cordelia Thomas & Lay (ethical/moral reasoning) & $19 / 05 / 2014$ & $19 / 05 / 2017$ \\
\hline
\end{tabular}

Unless members resign, vacate or are removed from their office, every member of HDEC shall continue in office until their successor comes into office (HDEC Terms of Reference) 


\title{
Your ACTRN (registration number): ACTRN12616001014448
}

\author{
info@actr.org.au \\ Tue 8/2/2016 12:57 PM \\ To:Mailin Lemke <lemkemail@myvuw.ac.nz>;
}

Dear Mailin Lemke,

Re: Tangible interaction with everyday objects for homebased upper limb stroke rehabilitation

Thank you for submitting the above trial for inclusion in the Australian New Zealand Clinical Trials Registry (ANZCTR).

Your trial has now been successfully registered and allocated the ACTRN: ACTRN12616001014448

Web address of your trial: http://www.ANZCTR.org.au/ACTRN12616001014448.aspx

Date submitted: 27/07/2016 11:44:50 AM

Date registered: 2/08/2016 10:57:35 AM

Registered by: Mailin Lemke

Principal Investigator: Mailin Lemke

If you have already obtained Ethics approval for your trial, please send a copy of at least one Ethics Committee approval letter to info@actr.org.au or by fax to (+61 2) 9565 1863, attention to ANZCTR.

Note that updates should be made to the registration record as soon as any trial information changes or new information becomes available. Updates can be made at any time and the quality and accuracy of the information provided is the responsibility of the trial's primary sponsor or their representative (the registrant). For instructions on how to update please see http://www.anzctr.org.au/Support/HowToUpdate.aspx.

Please also note that the original data lodged at the time of trial registration and the tracked history of any changes made as updates will remain publicly available on the ANZCTR website.

The ANZCTR is recognised as an ICMJE acceptable registry (http://www.icmje.org/faq.pdf) and a Primary Registry in the WHO registry network (http://www.who.int/ictrp/network/primary/en/index.html).

If you have any enquiries please send a message to info@actr.org.au or telephone +6129562 5333.

Kind regards,

ANZCTR Staff

$\mathrm{T}:+61295625333$

$\mathrm{F}:+61295651863$

E: info@actr.org.au

W: www.ANZCTR.org.au

Scanned by MailMarshal - M86 Security's comprehensive email content security solution.

IMPORTANT NOTICE: This e-mail and any attachment to it are intended only to be read or used by the named addressee. It is confidential and may contain legally privileged information. No confidentiality or privilege is waived or lost by any mistaken transmission to you. The CTC is not responsible for any unauthorised alterations to this e-mail or attachment to it. Views expressed in this message are those of the individual sender, and are not necessarily the views of the CTC. If you receive this e-mail in error, please immediately delete it and notify the sender. You must not disclose, copy or use any part of this e- 
Health

and

Disability

Ethics

Committees
Health and Disability Ethics Committees

Ministry of Health 133 Molesworth Street

PO Box 5013

Wellington 6011

08004 ETHICS hdecs@moh.govt.nz

24 January 2017

Dear Miss Lemke

Re: Ethics ref:

Study title:
16/CEN/37/AM01

Tangible interaction with everyday objects for home-based upper-limb stroke rehabilitation

I am pleased to advise that this amendment has been approved by the Central Health and Disability Ethics Committee. This decision was made through the HDEC Expedited Review pathway.

Please don't hesitate to contact the HDEC secretariat for further information. We wish you all the best for your study.

Yours sincerely,

Mrs Helen Walker

Chairperson

Central Health and Disability Ethics Committee

Encl: appendix $A$ : documents submitted

appendix $B$ : statement of compliance and list of members 
Appendix A

Documents submitted and approved

\begin{tabular}{|l|l|l|}
\hline Document & Version & Date \\
\hline $\begin{array}{l}\text { PIS/CF: Updated participant information sheet for online } \\
\text { questionnaires }\end{array}$ & 2 & 09 January 2017 \\
\hline Survey/questionnaire: Online questionnaire & 1 & 08 December 2016 \\
\hline Post Approval Form & 01 & 01 \\
\hline $\begin{array}{l}\text { Protocol: Updated research protocol including the new method of } \\
\text { data collection }\end{array}$ & 2 & 09 January 2017 \\
\hline Covering letter: Covering letter & 2 & 09 January 2017 \\
\hline Response to Request for Further Information & & \\
\hline
\end{tabular}




\section{Participant Information Sheet Interviews}

Study title:

Locality:

Lead investigator:
Tangible interaction with everyday objects for home-based upper-limb stroke rehabilitation

Wellington

Ethics committee ref.:

16/CEN/37

Contact phone number:

You are invited to take part in a study on 'Tangible interaction with everyday objects for home-based upper-limb stroke rehabilitation'.

Whether or not you take part is your choice. If you don't want to take part, you don't have to give a reason, and it wori't affect the care you receive. 'f you do wart to take part now, but change your mind later, you can pull out of the study at any time.

This Participant Information Sheet will help you decide if you'd like to take part. It sets out why we are doing the study, what your participation would involve, what the benefits and risks to you might be, and what would happen after the study ends. We will go through this information with you and answer any questions you may have.

You do not have to decide today whether or not you will participate in this study. Before you decide you may want to talk about the study with other people, such as family, whānau, friends, or healthcare providers. Feel free to do this.

If you agree to take part in this study, you will be asked to sign the Consent Form on the last page of this document. You will be given a copy of both the Participant Information Sheet and the Consent Form to keep.

This document is 7 pages long, including the Consent Form.

Please make sure you have read and understood all the pages.

\section{WHAT IS THE PURPOSE OF THE STUDY?}

This study is to develop interactive design prototypes based on everyday objects in combination with digital technology. These design prototypes offer a self-directed rehabilitation to stroke survivors who are living at home. The intended setting for these objects is the home where the objects can contribute to the recovery of the impaired arm and hand.

We want to know how you use everyday objects in activities of daily living. What kind of activities you like to do when you are at home, and what kinds of everyday objects you use in those activities. 
These design prototypes are developed as part of a Ph.D. thesis. This project is supported by the New Zealand International Doctoral Research Scholarship. Additional funding comes from the VUW Faculty of Science Strategic Research Fund. The research is also funded through the Center of Research Excellence in Medical Technology.

Any other questions you have can be answered by Dr. Brian Robinson (04 4636155 ). This research has been approved by the Health and Disability Ethics Committee.

\section{WHAT WILL MY PARTICIPATION IN THE STUDY INVOLVE?}

We invite you to take part in this study because you have had a stroke in the past and may have limited use of one of your arm and hand. Your stroke occurred at least 6 months prior to the first interview, you are living at home, have an active wrist and finger, are able to walk independently and have a good general health. If you agree to participate in this study there will be two researchers who will come to your home.

The study involves three different steps. You can decide if you would like to take part in all of the steps or just individual ones.

Step 01: Interviews

If you agree to take part in the interviews we will interview you twice at a place of your convenience, for example your home. During the first interview, we will ask some questions about such as how old you are, your ethnic background and how the stroke affects you. We will ask some further questions about the kind of rehabilitation that you take part in to improve your affected arm and hand and what kind of objects you use in activities of daily living. We will record what you say.

A couple of months later you will be interviewed a second time. We will ask you to give your opinion and feedback on some design prototypes. These design prototypes are based on household objects that you use during activities of daily living e.g. gardening or watching TV. The design prototypes encourage you to use your affected arm and hand more often during activities of daily living. Your feedback and suggestions for changes are important to enhance the functionality and usability of the prototypes. We will record what you say and we will take a video and photographs of you using the design prototypes. This is to make sure that using the interactive design prototypes will be useful for stroke recovery and not cause any harm. These recordings will just focus on your hands and the design prototypes. Your comments and the way you interact with the object is beneficial to improve the functionality of the design prototypes. It helps to make sure that the functionality suits the intended purpose. The video recording will purely be used to analyse the interaction with the devices. The recording will not be used in presentations or teaching lessons. Stroke rehabilitation therapists will review these recordings.

We will ask for your thoughts on using the design prototypes. We will audio record what you say. This should take no longer than 30 minutes. You may be invited to take part again if you would like to help us test changes. You can use the prototypes up to 30 minutes. You can stop using the prototypes at any point. The prototypes will be returned to the researcher after testing. 
If you agree to take part in this step we will provide you with a set of objects. The set will contain a disposable camera, diaries or postcards to write down feelings and thoughts that are valuable to the research. We are interested to see what kind of objects you often use in activities of daily living e.g. a teakettle or a remote control. We will give you some instructions on how to use the camera and when to use it. You can use the diary and postcards to add notes and explain why you like to do certain activities and what kind of objects are important or meaningful to you. You can use the set for up to two weeks. You can stop using the set at any point. After two weeks we will collect the set and analyse the notes and photos that you collected.

Step 03: Observations of daily living

If you agree to take part in this step two researchers will visit you in your home. We are interested to see how you structure your day and what kind of activities you do. We will observe how you do daily activities such as making a cup of coffee, gardening or preparing a meal. The observations will take place on just one day for up to four hours. You can ask us to stop at any time.

We will take photographs of you during those activities and the objects you use for example a fork or a gardening tool. These photos will just focus on your hands and the objects you use. The activities and the objects that play an important role in them are beneficial to improve the functionality of the design prototypes, which are getting developed as part of this study. It helps to make sure that the functionality suits the intended purpose. The recording will not be used in presentations or teaching lessons. Stroke rehabilitation therapists will review these photos.

Your involvement in the study will only be known by the researchers. All audio and videos recordings and photographs will be taken using cameras and audio recorders belonging to the School of Design. The recordings and images will be taken off these cameras and recorders immediately after the sessions and then kept secure in the University computer system. What you say to us will also be written into documents (transcribed) and this will also be kept secure.

\section{WHAT ARE THE POSSIBLE BENEFITS AND RISKS OF THIS STUDY?}

We know that people who have had a stroke cannot always access stroke rehabilitation therapy on a regular basis. They have to travel to clinics or hospitals. We also know that stroke rehabilitation is most effective when it is carried out for several hours throughout the day, every day.

This study is to support people who have had a stroke and to provide stroke rehabilitation therapy in their home. The interactive design prototypes do not replace any other form of therapy that you may receive. The aim of this study is not to use the interactive design prototypes as therapeutic devices but to find out whether they are usable as such. 


\section{WHO PAYS FOR THE STUDY?}

This project is supported by the New Zealand International Doctoral Research Scholarship. Additional funding comes from the VUW Faculty of Science Strategic Research Fund. The research is also funded through the Center of Research Excellence in Medical Technology. You will not incur any costs by taking part and we will travel to you.

\section{WHAT IF SOMETHING GOES WRONG?}

If you were injured in this study, which is unlikely, you would be eligible for compensation from ACC just as you would be if you were injured in an accident at work or at home. You will have to lodge a claim with ACC, which may take some time to assess. If your claim is accepted, you will receive funding to assist in your recovery.

\section{WHAT ARE MY RIGHTS?}

You are volunteering to take part. You do not have to take part in this study and you can withdraw at any time.

We can show you the video recording and photographs of you that we have collected. We can also give you a copy of what we have recorded you saying to us about using the interactive rehabilitation devices.

It is unlikely that participating will affect your health but if it does, we will contact you immediately.

We will not identify you in any presentations of the work.

\section{WHAT HAPPENS AFTER THE STUDY OR IF I CHANGE MY MIND?}

After you have taken part and change your mind about being involved, please contact the lead investigator, Mailin Lemke, and any data, information and images associated with your participation will be destroyed.

We will securely store the information and any data you have provided for ten (10) years and it will then be destroyed. We can present the findings of this study at stroke clubs within a year of conducting the study. We can also send you a summary of the student's thesis describing the outcome of the study. We may also present this study with other similar studies we are conducting at conferences or in books or journals.

\section{WHO DO I CONTACT FOR MORE INFORMATION OR IF I HAVE CONCERNS?}

If you have any questions, concerns or complaints about the study at any stage, you can contact:

Mailin Lemke, Ph.D. student, School of Design, Victoria University of Wellington 0226470388

lemkemail@myvuw.ac.nz

If you want to talk to someone who isn't involved with the study, you can contact an independent health and disability advocate on: 
Phone: $\quad 0800555050$

Fax: $\quad 08002$ SUPPORT (0800 2787 7678)

Email: $\quad$ advocacy@hdc.org.nz

For Maori health support please contact:

Katherine Reweti-Russell

Katherine.Reweti-Russell@ccdhb.org.nz

You can also contact the health and disability ethics committee (HDEC) that approved this study on:

Phone: $\quad 08004$ ETHICS

Email: $\quad$ hdecs@moh.govt.nz 


\section{Consent Form}

If you need an INTERPRETER, please tell us.

\section{Please tick to indicate you consent to the following)}

I have read, or have had read to me in my first language, and I understand the Participant Information Sheet.

Yes $\square$

I have been given sufficient time to consider whether or not to participate in this study.

Yes $\square$

I have had the opportunity to use a legal representative, whanau/ family support or a friend to help me ask questions and understand the study.

I am satisfied with the answers I have been given regarding the study and I have a copy of this consent form and information sheet.

Yes

I understand that taking part in this study is voluntary (my choice) and that I may withdraw from the study at any time without this affecting my medical care.

Yes

I consent to the research staff collecting and processing my information, including information about my health.

Yes

I understand that my participation in this study is confidential and that no material, which could identify me personally, will be used in Yes any reports on this study.

I understand the compensation provisions in case of injury during the study.

Yes

I know who to contact if I have any questions about the study in general.

Yes

I understand my responsibilities as a study participant.

Yes $\square$

I understand that the information I have provided will be destroyed 10 years after the research is finished.

Yes $\square$

I agree to take part in step 01: an audio-recorded interview. 
I agree that during step 01: the testing of the design devices will be video recorded. This recording will just show the design prototypes and my hands while I use the devices.

I agree to take part in step 02: Documentation of daily living

Yes $\square \quad$ No $\square$

I agree to take part in step 03: Observations of daily living

Yes $\square \quad$ No $\square$

I would like to receive a transcript of my interview

Yes $\square$

No $\square$

I would like to receive a copy of the final report

Yes $\square \quad$ No $\square$

and have added my email address below.

Declaration by participant:

I hereby consent to take part in this study.

Participant's name:

Signature:

Date:

\section{Declaration by member of research team:}

I have given a verbal explanation of the research project to the participant, and have answered the participant's questions about it.

I believe that the participant understands the study and has given informed consent to participate.

Researcher's name: 


\section{Participant Information Sheet Online questionnaire}

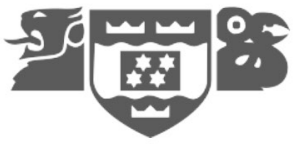

Study title:

Tangible interaction with everyday objects for home-based upper-limb stroke rehabilitation

Locality:

Wellington

Ethics committee ref.:

16/CEN/37/AM01

Lead investigator: Mailin Lemke

Contact phone number:

0226470388

You are invited to take part in a study on 'Tangible interaction with everyday objects for home-based upper-limb stroke rehabilitation'.

Whether or not you take part is your choice. If you don't want to take part, you don't have to give a reason, and it won't affect the care you receive. If you do want to take part now, but change your mind later, you can pull out of the study at any time.

This Participant Information Sheet will help you decide if you'd like to take part. It sets out why we are doing the study, what your participation would involve, what the benefits and risks to you might be, and what would happen after the study ends.

You do not have to decide today whether or not you will participate in this study. Before you decide you may want to talk about the study with other people, such as family, whānau, friends, or healthcare providers. Feel free to do this.

If you agree to take part in this study, you will be asked to sign the Consent Form by clicking on 'Yes I consent and have read and understood the Consent Form' as part of the online questionnaire.

This document is 5 pages long, including the Consent Form. Please make sure you have read and understood all the pages.

\section{WHAT IS THE PURPOSE OF THE STUDY?}

This study is to investigate what kind of activities of daily living you like to do at home after experiencing a stroke. We want to know how you use everyday objects in activities of daily living as well as what kind of activities you like to do when you are at home.

This project is supported by the New Zealand International Doctoral Research Scholarship. Additional funding comes from the VUW Faculty of Science Strategic Research Fund. The research is also funded through the Center of Research Excellence in Medical Technology. 
We invite you to take part in this study because you have had a stroke in the past and may have limited use of one of your arm and hand. You are living at home, have an active wrist and finger, are able to walk independently and have a good general health. If you agree to take part in this online questionnaire you are asked to respond and fill out some questions, which might take $10-15$ minutes.

We will ask some questions such as how old you are, your ethnic background and how the stroke affects you. We will ask some further questions about the kind of rehabilitation that you take part in to improve your affected arm and hand and what kind of objects you use in activities of daily living.

\section{WHAT ARE THE POSSIBLE BENEFITS AND RISKS OF THIS STUDY?}

We know that people who have had a stroke cannot always access stroke rehabilitation therapy on a regular basis. They have to travel to clinics or hospitals. We also know that stroke rehabilitation is most effective when it is carried out for several hours throughout the day, every day. The rehabilitation should as well take account of tasks that are meaningful to you.

This study is to support people who have had a stroke and to provide stroke rehabilitation therapy in their home. We want to find out what kind of activities are important and meaningful to you. Based on your feedback and suggestions design prototypes can be developed that can be used during the rehabilitation process and offer a meaningful form of training.

\section{WHO PAYS FOR THE STUDY?}

This project is supported by the New Zealand International Doctoral Research Scholarship. Additional funding comes from the VUW Faculty of Science Strategic Research Fund. The research is also funded through the Center of Research Excellence in Medical Technology. You will not incur any costs by taking part and we will travel to you.

\section{WHAT IF SOMETHING GOES WRONG?}

There are no risks associated with this study. Your information will be non-identifiable and stored on a secure platform that just the primary investigator has access to.

\section{WHAT ARE MY RIGHTS?}

You are volunteering to take part. You do not have to take part in this study and you can stop filling out the online questionnaire anytime.

We will not identify you in any presentations of the work. 


\section{WHAT HAPPENS AFTER THE STUDY OR IF I CHANGE MY MIND?}

After you have taken part and change your mind about being involved, please contact the lead investigator, Mailin Lemke lemkemail@myvuw.ac.nz, and any data associated with your participation will be destroyed.

We will securely store the information and any data you have provided for five (5) years and it will then be destroyed. We can present the findings of this study at stroke clubs within a year of conducting the study. We may also present this study with other similar studies we are conducting at conferences or in books or journals.

\section{WHO DO I CONTACT FOR MORE INFORMATION OR IF I HAVE CONCERNS?}

If you have any questions, concerns or complaints about the study at any stage, you can contact:

Mailin Lemke, Ph.D. student, School of Design, Victoria University of Wellington 0226470388

lemkemail@myvuw.ac.nz

If you want to talk to someone who isn't involved with the study, you can contact an independent health and disability advocate on:

\begin{tabular}{|c|c|}
\hline Phone: & 0800555050 \\
\hline Fax: & 08002 SUPPORT (0800 27877678 ) \\
\hline
\end{tabular}

For Maori health support please contact your health provider and they will refer you to the representative Maori health support group.

You can also contact the health and disability ethics committee (HDEC) that approved this study on:

$\begin{array}{ll}\text { Phone: } & 08004 \text { ETHICS } \\ \text { Email: } & \text { hdecs@moh.govt.nz }\end{array}$




\section{Consent Form}

If you need an INTERPRETER, please tell us.

If you are unable to provide interpreters for the study, please clearly state this in the Participant Information Sheet

\section{Please tick to indicate you consent to the following (Add or delete as appropriate)}

I have read, or have had read to me in my first language, and I understand the Participant Information Sheet.

Yes $\square \quad$ No $\square$

I have been given sufficient time to consider whether or not to participate in this study.

Yes $\square \quad$ No $\square$

I have had the opportunity to use a legal representative, whanau/ family support or a friend to help me ask questions and understand the study.

Yes $\square \quad$ No $\square$

I am satisfied with the answers I have been given regarding the study and I have a copy of this consent form and information sheet.

Yes $\square \quad$ No $\square$

I understand that taking part in this study is voluntary (my choice) and that I may withdraw from the study at any time without this Yes $\square \quad$ No $\square$ affecting my medical care.

I consent to the research staff collecting and processing my information, including information about my health.

Yes $\square \quad$ No $\square$

If I decide to withdraw from the study, I agree that the information collected about me up to the point when I withdraw may continue to be processed.

I understand that my participation in this study is confidential and that no material, which could identify me personally, will be used in $\quad$ Yes $\square \quad$ No $\square$ any reports on this study.

I know who to contact if I have any questions about the study in general.

Yes $\square \quad$ No $\square$

I understand my responsibilities as a study participant.

Yes $\square \quad$ No $\square$

I understand that the information I have provided will be destroyed 5 years after the research is finished.

Yes

No $\square$ 
Declaration by participant:

I hereby consent to take part in this study.

Participant's name:

Signature:

Date:

Declaration by member of research team:

I have given a verbal explanation of the research project to the participant, and have answered the participant's questions about it.

I believe that the participant understands the study and has given informed consent to participate.

Researcher's name:

Signature:

Date: 


\section{Tangible interaction with everyday objects for home-based upper limb stroke rehabilitation}

\section{INFORMATION SHEET FOR PARTICIPANTS}

Thank you for your interest in this project. Please read this information before deciding whether or not to take part. If you decide to participate, thank you. If you decide not to take part, thank you for considering my request.

\section{Who am I?}

My name is Mailin Lemke and I am a Doctoral student in Industrial Design at the Victoria University of Wellington. This research project is work towards my dissertation.

\section{What is the aim of the project?}

This thesis aims to investigate how meaningful objects could be used in a self-directed approach by chronic stroke patients who live at home. This project aims to determine how meaningful objects could be used in the rehabilitation process to increase the engagement in the rehabilitation process.

This research (0000022410) has been approved by the Victoria University of Wellington Human Ethics Committee.

\section{How can you help?}

If you agree to take part I will interview you twice at a place of your convenience.

\section{Part:}

I will ask you questions about stroke rehabilitation approaches and meaningful tasks and objects that play a significant role in the rehabilitation process. The interview will take 45 minutes. I will audio record the interview and write it up later.

The different interviews that I will conduct for this thesis will influence the design prototypes that I am developing as part of my thesis.

\section{Part:}

I will present to you different objects that can be used by stroke patients in a home-based rehabilitation approach. I will ask you to give feedback on the design prototypes. You are then asked to use the devices and suggest changes and improvements that could enhance the 
usability. The interview and testing will take 45 minutes. I will audio record the interview and write it up later. I will further video record how you use the designs. This recording will just focus on your hands and the design prototypes. Your comments and the way you interact with the object is beneficial to improve the functionality of the design prototypes and helps to make sure that the functionality suits the intended purpose. The video recording will purely be used to analyse the interaction with the devices. The recording will not be used in presentations or teaching lessons.

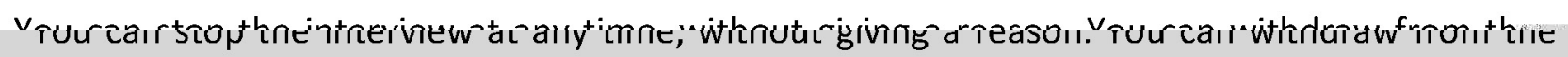
study after the interview by the 31.12.2016. If you withdraw, the information you provided will be destroyed or returned to you.

\section{What will happen to the information you give?}

This research is confidential. I will not name you or your organisation in any reports, and I will not include any information that would identify you. Only my supervisors and I will read the notes or transcript of the interview. The interview transcripts, summaries and any recordings will be kept securely and destroyed 2 years after the research ends.

\section{What will the project produce?}

The information from my research will be used in my Ph.D. dissertation. You will not be identified in my report. I may also use the results of my research for conference presentations, and academic reports. I will take care not to identify you in any presentation or report.

\section{If you accept this invitation, what are your rights as a research participant?}

You do not have to accept this invitation if you don't want to. If you do decide to participate, you have the right to:

- $\quad$ choose not to answer any question;

- $\quad$ ask for the recorder to be turned off at any time during the interview;

- $\quad$ withdraw from the study up until the 31.12.2016

- $\quad$ ask any questions about the study at any time;

- $\quad$ receive a copy of your interview recording (if it is recorded);

- $\quad$ read over and comment on a written summary of your interview;

- $\quad$ agree on another name for me to use rather than your real name;

- be able to read any reports of this research by emailing the researcher to request a copy. 
If you have any questions or problems, who can you contact?

If you have any questions, either now or in the future, please feel free to contact either:

\section{Student:}

Mailin Lemke

lemkemail@myvuw.ac.nz

\section{Supervisor:}

Dr Edgar Rodriguez

Programme Director, Industrial Design

School of Design

(04) 4636245

edgar.rodriguez@vuw.ac.nz

\section{Human Ethics Committee information}

If you have any concerns about the ethical conduct of the research you may contact the Victoria University HEC Convener: Associate Professor Susan Corbett. Email susan.corbett@vuw.ac.nz or telephone+64-4-463 5480. 


\section{Tangible interaction with everyday objects for home-based upper limb stroke rehabilitation}

\section{INFORMATION SHEET FOR PARTICIPANTS}

Thank you for your interest in this project. Please read this information before deciding whether or not to take part. If you decide to participate, thank you. If you decide not to take part, thank you for considering my request.

\section{Who am I?}

My name is Mailin Lemke and I am a Doctoral student in Industrial Design at the Victoria University of Wellington. This research project is work towards my dissertation.

\section{What is the aim of the project?}

This thesis aims to investigate how everyday objects could be used in a self-directed way by chronic stroke patients who live at home. I try to determine how these objects could be used in the rehabilitation process to increase the engagement in the rehabilitation process. As part of the research I am working on some design strategies that other designers can refer to in order to create objects that incorporate a rehabilitative purpose.

This research has been approved by the Victoria University of Wellington Human Ethics Committee.

\section{How can you help?}

If you agree to take part, I will ask you to test the design strategies which are provided in form of an online website. I would like to find out if they are suitable to be used in a self-directed way by someone who has never designed for stroke patients before. After you have tested the strategies I would like you to give some feedback and recommend changes to improve the usability of the strategies. To gather your feedback, I will interview you in a public place, such as a café. The interview will take up to 30 minutes. I will audio record the interview and write it up later. You can stop the interview at any time, without giving a reason. You can withdraw from the study after the interview by the 31.12.2017. If you withdraw, the information you provided will be destroyed or returned to you.

\section{What will happen to the information you give?}

This research is confidential. I will not name you in any reports, and I will not include any information that would identify you. Only my supervisors and I will read the notes or transcript 
of the interview. The interview transcripts, summaries and any recordings will be kept securely and destroyed 2 years after the research ends.

I will not name you in the final report but your organisation might be named (and you have the authority to agree to this on behalf of the organisation).

\section{What will the project produce?}

The information from my research will be used in my Ph.D. dissertation. You will not be identified in my report. I may also use the results of my research for conference presentations, and academic reports. I will take care not to identify you in any presentation or report.

\section{If you accept this invitation, what are your rights as a research participant?}

You do not have to accept this invitation if you don't want to. If you do decide to participate, you have the right to:

- $\quad$ choose not to answer any question;

- $\quad$ ask for the recorder to be turned off at any time during the interview;

- $\quad$ withdraw from the study up until the 31.12.2017

- $\quad$ ask any questions about the study at any time;

- $\quad$ receive a copy of your interview recording (if it is recorded);

- $\quad$ read over and comment on a written summary of your interview;

- $\quad$ agree on another name for me to use rather than your real name;

- be able to read any reports of this research by emailing the researcher to request a copy.

\section{If you have any questions or problems, who can you contact?}

If you have any questions, either now or in the future, please feel free to contact either:

\section{Student:}

Mailin Lemke

lemkemail@myvuw.ac.nz

\section{Supervisor:}

Dr Edgar Rodriguez

Programme Director, Industrial Design

School of Design

(04) 4636245

edgar.rodriguez@vuw.ac.nz

\section{Human Ethics Committee information}

If you have any concerns about the ethical conduct of the research you may contact the Victoria University HEC Convener: Associate Professor Susan Corbett. Email susan.corbett@vuw.ac.nz or telephone +64-4-463 5480. 


\section{Tangible interaction with everyday objects for home-based upper limb stroke rehabilitation CONSENT TO INTERVIEW}

This consent form will be held for 5 years.

Researcher: Mailin Lemke, School of Design, Victoria University of Wellington

- I have read the Information Sheet and the project has been explained to me. My questions have been answered to my satisfaction. I understand that I can ask further questions at any time.

- I agree to take part in an audio recorded interview.

I understand that:

- I may withdraw from this study after the interview by the 31.12.2017, and any information that I have provided will be returned to me or destroyed.

- The information I have provided will be destroyed 2 years after the research is finished.

- $\quad$ Any information I provide will be kept confidential to the researcher and the supervisor. I understand that the results will be used for a Ph.D. report and a summary of the results may be used in academic reports and/or presented at conferences.

- My name will not be used in reports, nor will any information that would identify me.

- I would like a copy of the transcript of my interview:

Yes No

- I would like a summary of my interview:

Yes No

- I would like to receive a copy of the final report Yes No

and have added my email address below.

Signature of participant:

Name of participant:

Date:

Contact details: 
Default Question Block

Dear Participant,

You are invited to take part in a study on 'Tangible interaction with everyday objects for home-based upperlimb stroke rehabilitation'. Thank you in advance for taking the time to complete this survey to help us bette understand your life and living situation. The survey will take approx. 10-15 minutes to complete.

This study is to develop everyday devices that people use, such as a radio or a lamp, that can be used by people who have experienced a stroke that will also help with their rehabilitation. These are devices that are used at home and using them in a particular way may contribute to the recovery of the affected arm and hand. This questionnaire helps us to understand what kind of activities you like to take part in when you are at home. Based on your responses different design prototypes will be developed that we can test with people who are recovering from a stroke.

If you need more information the Participant Information Sheet can be found through this attached link

\section{https://goo.gV/4tsOFk}

This research is part of a Ph.D. thesis. This project is supported by the New Zealand International Doctoral Research Scholarship. Additional funding comes from the VUW Faculty of Science Strategic Research Fund. The research is also funded through the Center of Research Excellence in Medical Technology.

Any other questions you have can be answered by Dr. Brian Robinson (04 4636155$)$. This research has been approved by the Health and Disability Ethics Committee.

\section{Consent}

By submitting this form you agree to the following terms outlined in this consent form: https://goo.gl/4tsOFk

Yes I consent, and have read and understood the consent form

\section{Age}

How old are you?

\section{Gender}

\section{Male}

Female

\section{Residence}

In which country do you live?

\section{Living situation}

Where do you currently live?
Athome
In an inpatient rehabilitation facility (IRF)
In a long-term acute care hospital (LTACH)
In a skilled nursing facility (SNF)
In a home-health agency (HHA)
Hospice
Other 


\section{Preference}

Which side of the body do you prefer to use?

\footnotetext{
I am right-handed

I am left-handed
}

\section{Stroke}

How long since you had a stroke?
0-3 months
3-6 months
6-9 months
9-12 months
$12+$ months

\section{Stroke effects}

How does the stroke affect moving your arms and legs?

$\square$ Affects my right arm

$\square$ Affects my left arm

$\square$ Affects my right leg

$\square$ Affects my left leg

Does not affect my arms and legs

Flexibility of the affected arm and hand

Which descriptions describes the flexibility of your affected arm and hand?

\footnotetext{
I can easily pick up coins from a flat surface

I can easily pick up and drop a tennis ball

I can easily pick up a washing cloth with at least two fingers but not a tennis ball

I can easily pick up a washing cloth but just with two fingers

I can move the fingers and extend the wrist but I can't pick things up

None of the above
}

\section{Rehabilitation}

Did you receive any rehabilitation after the stroke?

Yes
No

\section{Form of rehabilitation}

What kind of rehabilitation did you receive?

\section{Rehabilitation intensity}

How frequently did you receive rehabilitation after the stroke?

\section{Everyday activities of daily living}


We want to know what kind of activities you like to do when you are at home, and what kinds of everyday objects you use in those activities. Those activities can be for example cooking, dressing or brushing your teeth.

Everyday activities before the stroke

What kind of everyday activities did you enjoy taking part in before the stroke?

Everyday activities after the stroke

What kind of everyday activities do you enjoy taking part in now after the stroke?

Objects in everyday activities

What kinds of object (for example this may be a telephone, pictures, photograph albums etc.) play a part in those activities?

Everyday activities after the stroke that are difficult

What kind of everyday activities have become quite difficult after the stroke?

(2)

Everyday activities that you miss

What kind of everyday activities would you like to take part in again?

\section{Everyday activities that you don't miss}

What kind of activities would you rather not take part in anymore?

\section{Hobbies}

We would like to know what kind of hobbies you like to participate in your free time. Hobbies could be for example knitting, golf, chess, playing an instrument, computer games or walking.

Hobbies before the stroke

What kind of hobbies did you enjoy taking part in before the stroke? 
Hobbies after the stroke

What kind of hobbies do you enjoy taking part in now after stroke?

\section{Results}

If you would like to receive a summary of the results of this research please write down your email address here.

Thanks for your time 


\section{Appendix B}

\section{Workbook 1}


Introduction

Stroke affects 15 million people world wide there are no devices a vailable to stroke curvivers and is the third not likely cause of death. that could be used in a sey-directed way in Stroke surwiors are expected to platean the home onvironment. The use of digital in thenr recovery within the fist six months technology to delier thir form of stroho rehabiliktiva ofter the stroke. As a result primary re- If ens the possibility to deliver a neaningul form habilitation is only provided durigthis of rehabilitation. This workbook presents the itarative period. However, the response differs bepending process founsing on an interactive radio which on the lengh, intensity and form of the re. restrains the marement by just working if the habilitation and are likely to express affected arm and hand is used in the interaction. compensatory movement of the affected aim \&

hand resulting in a 'larerned noruse'. During constrant-induced movement theropy the less affected arm and hand is restrained. 


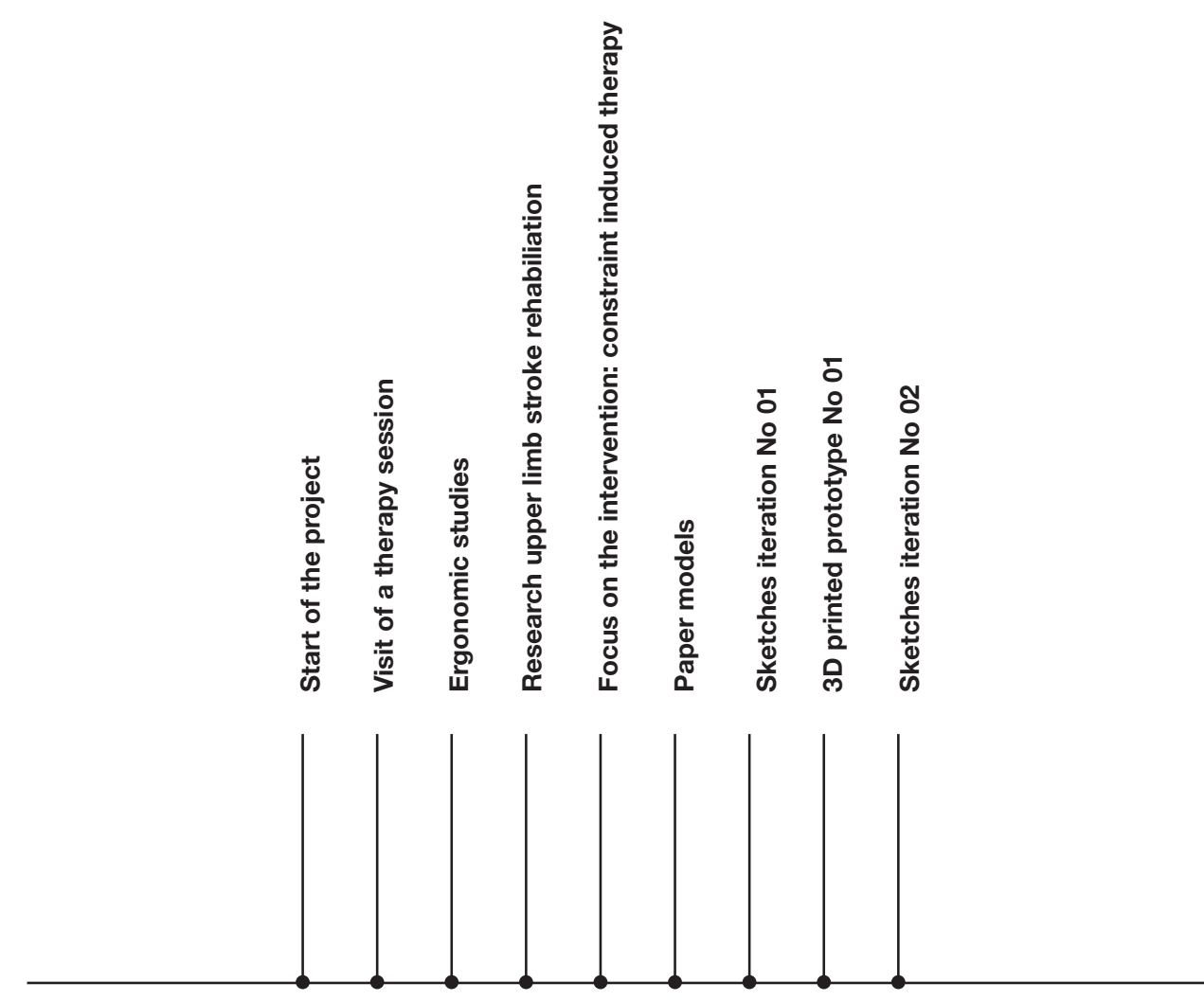

Project development

Timelne 


$$
\text { |ulwil }
$$




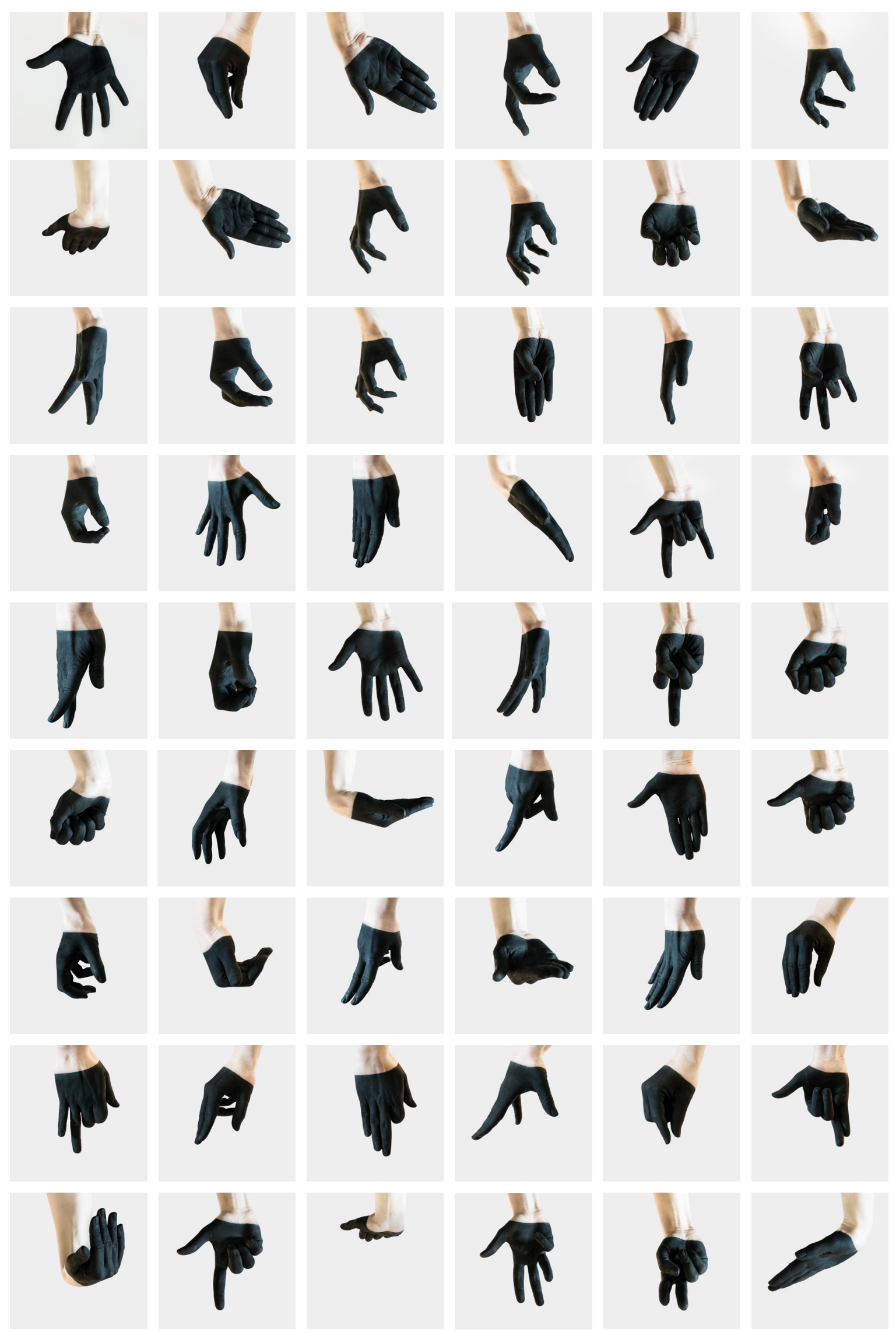


Egonomic studies

The pioget started with same exploratory reseosch

inwolung the visit of a therapt sesson involung

chrovic stoke surviers with impariments of

the upper linb. I chose tofocus on a reach

to grasp movement whuh was trained

in the the ropy session.

istarted with a rouple of movement studies

to investigate the components that are

rectessory to carry out the novement. 


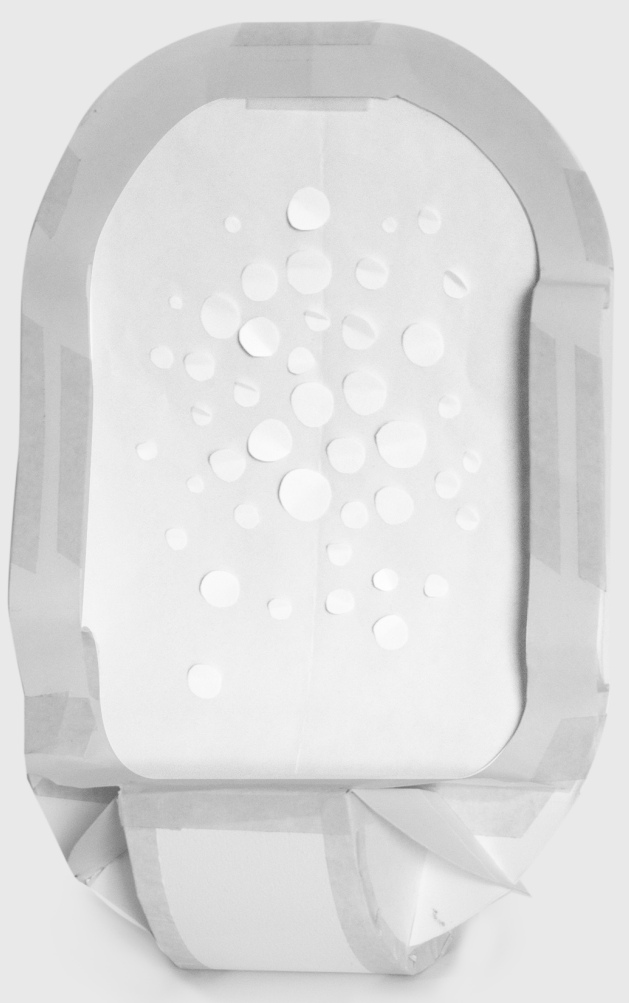

Paper prototype No or

Paper prototype

Repetition is key during the rehabilitation to elicit remoplastic changes in the brain. I mach two different paper prototypes that are based on my initial ergonomic studies. The form of the prototypes is based on the idea to evoke a repetitive movement of the object similar to the on of the children's toys that move back wards \& fol woods once hut. 
Paper protelype No 02 


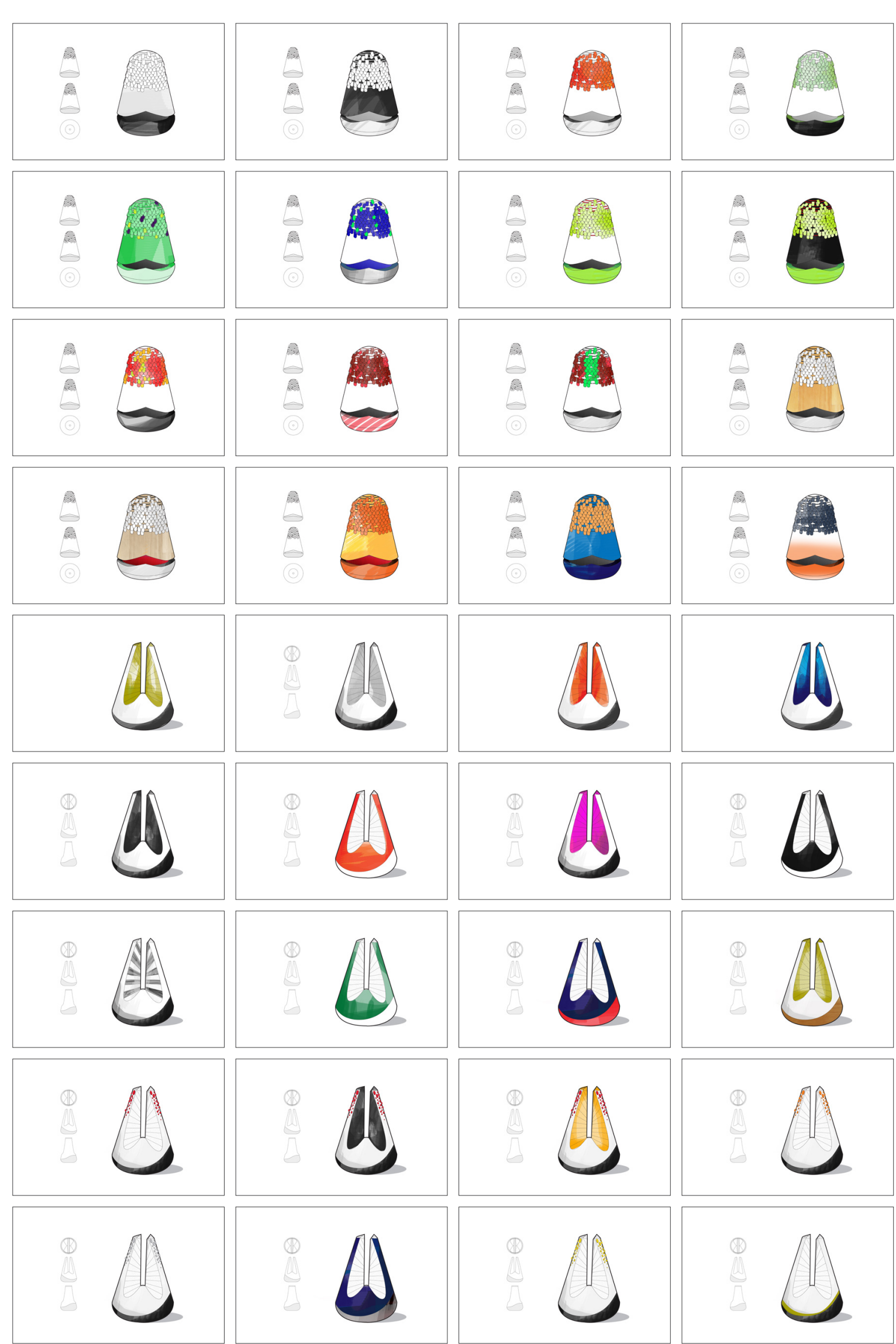


Prototpe No 01 Stetches

The functionality of the object is the one of a radio. The radio is gften an ossentid part in people's everyday life but in case some of the user strugfle to use it, it is not hye threatening. The object reeds to be used in a repatitive way througout the day. The coacept of the radis is that it jest turns on when the affected arm is used to interact with it. If the less affected orm is a sed it remains quite. The user is en couraged to use the affected orm to get the radio waking 


$$
\Delta
$$




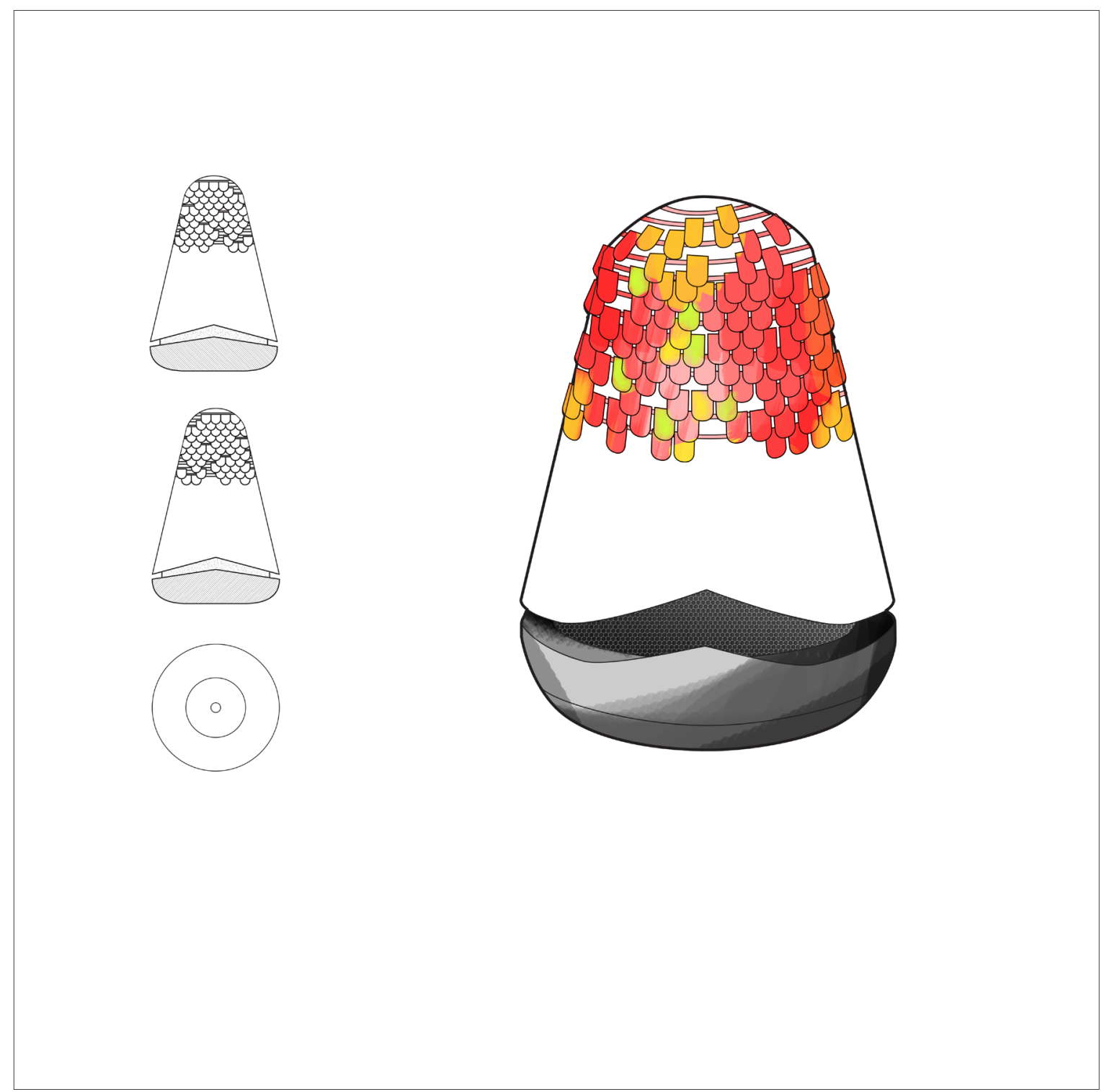

Prototipe No ol stectch 02 


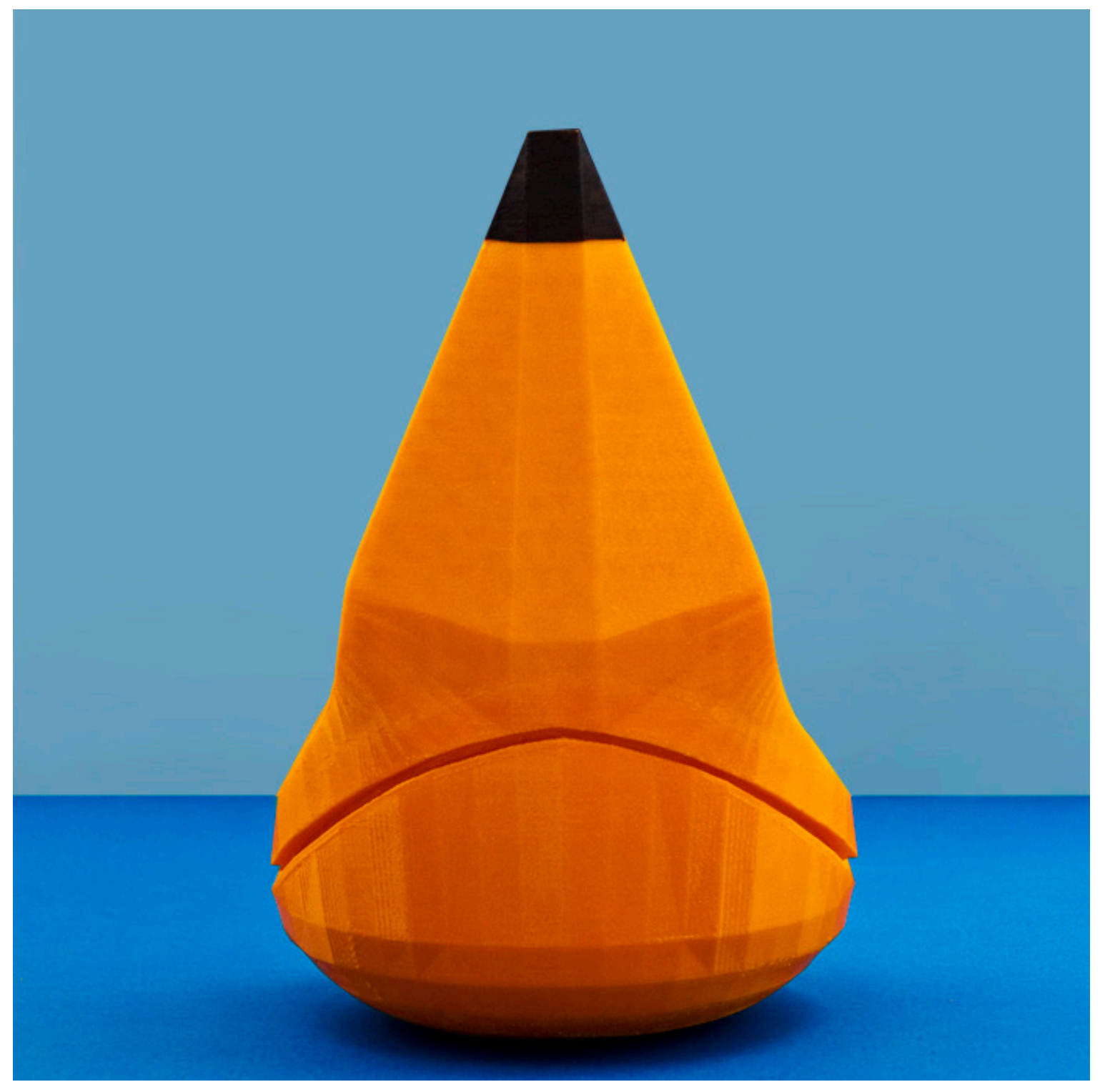

Prototye Do 01 3D print

The firt radus prototype got pisted with ABS filament in a tablety $3 D$ ponter in two separate parts. He ovedil fom of the object is the one of an allupse. It thraned the movement that the radio performed looked rather shaky once being hit. 


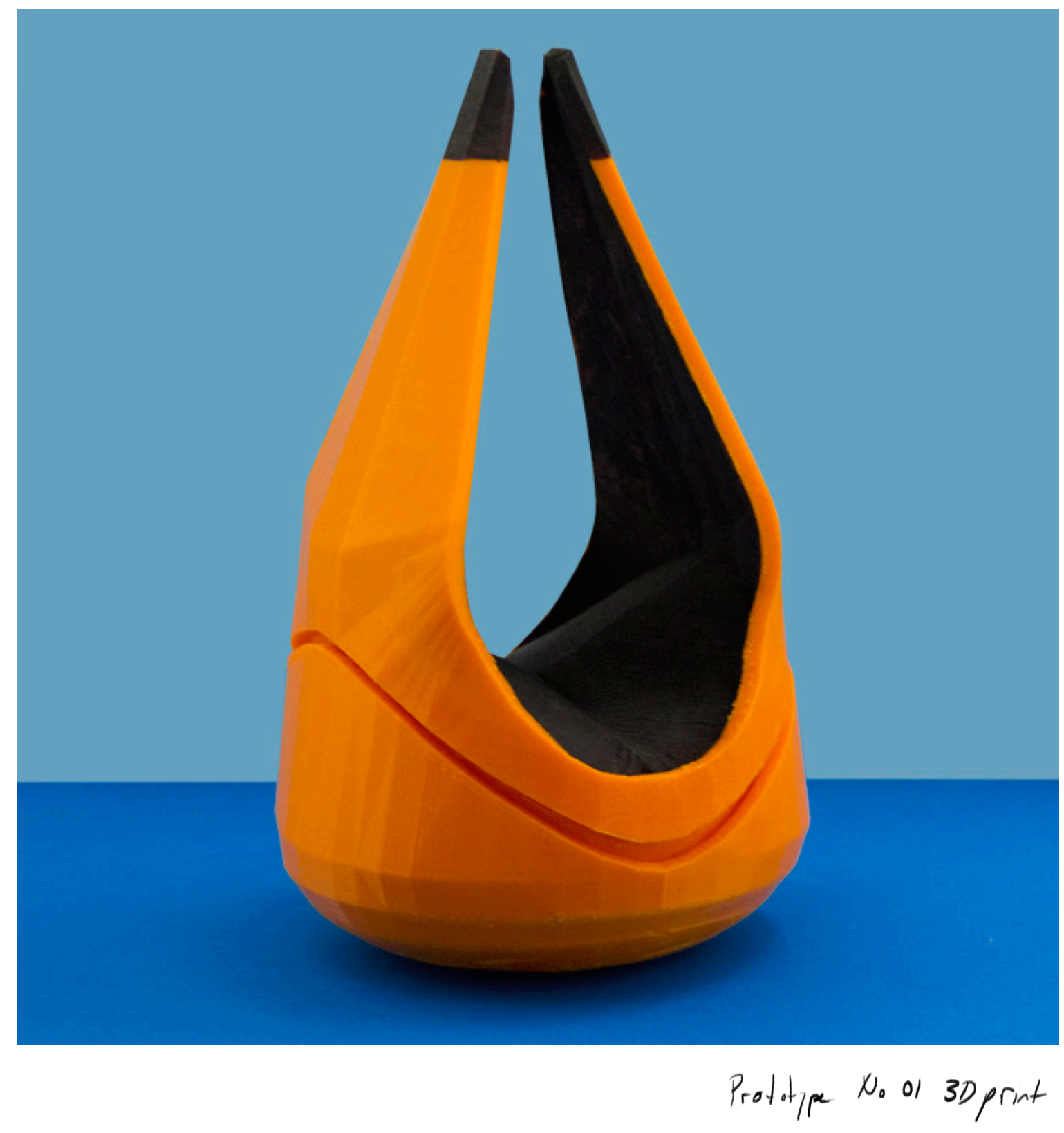




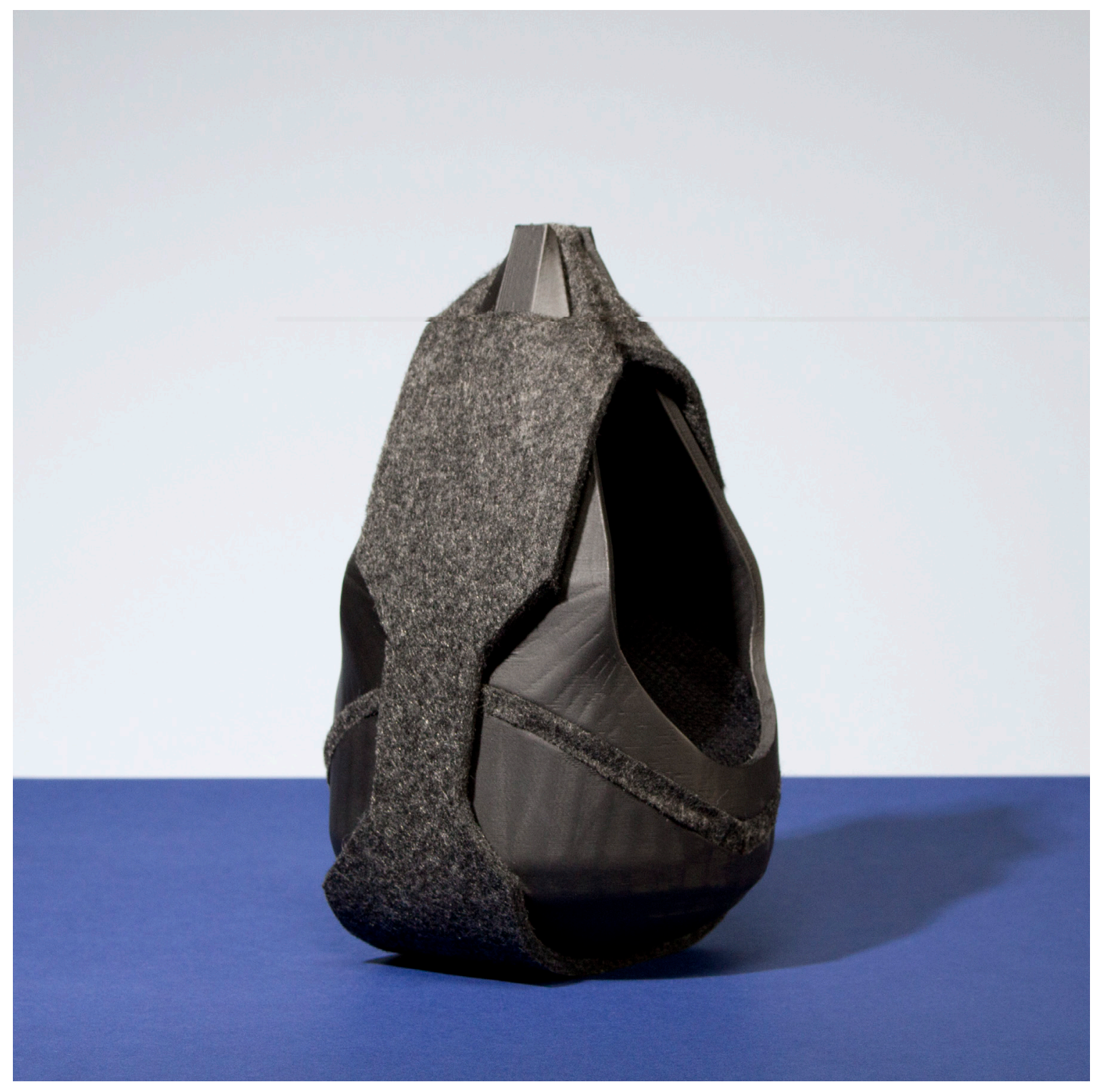

Prototye No ol 3Dprint

I mate a slevere in a second teration of the radio. the morement became smoother \& queter through the matenial but was still not as smooth asluanted it to be I also considered using different kinds of sleevers to oddress sonesthetic defiuts that stroce- suinivas quite often experence in ther hands. 


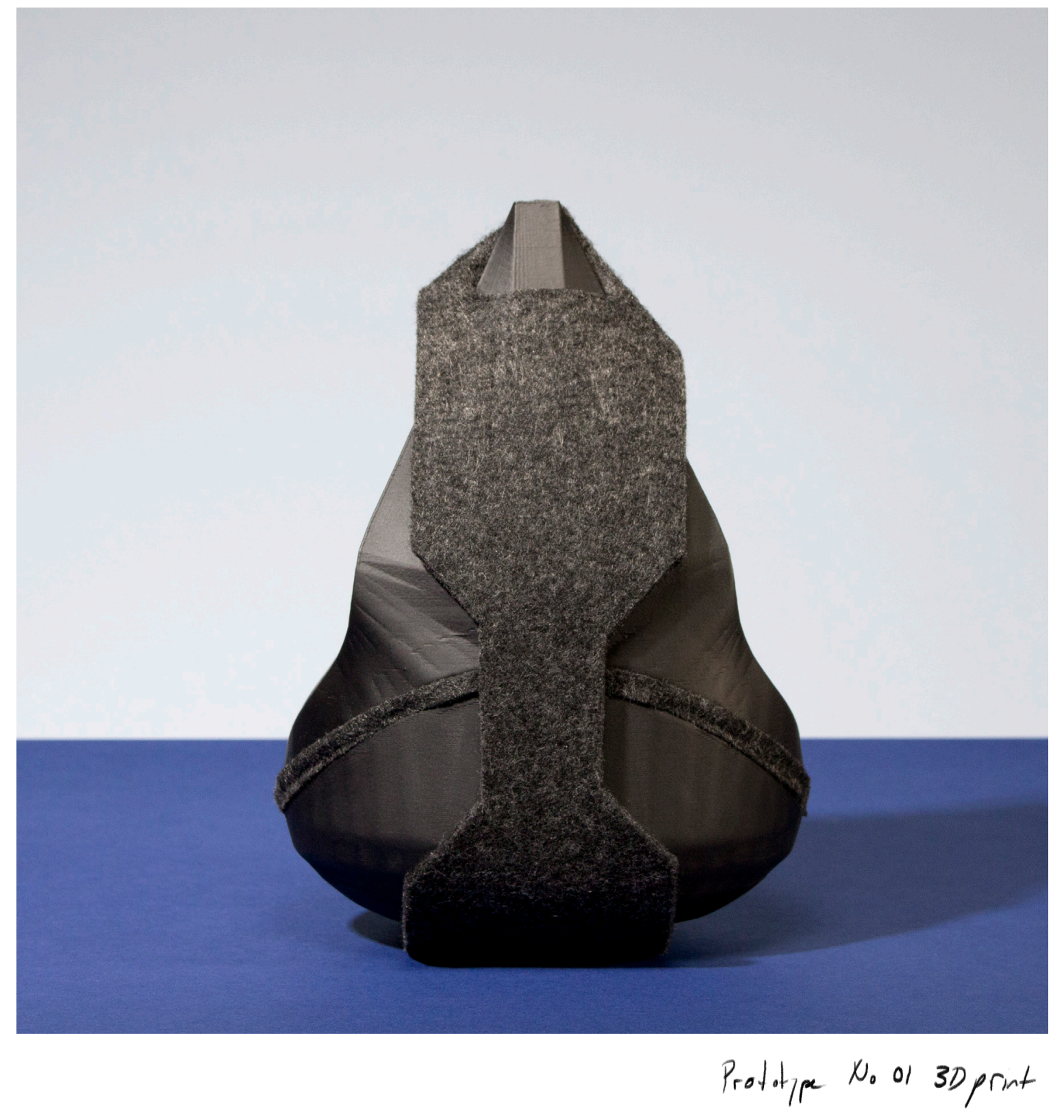




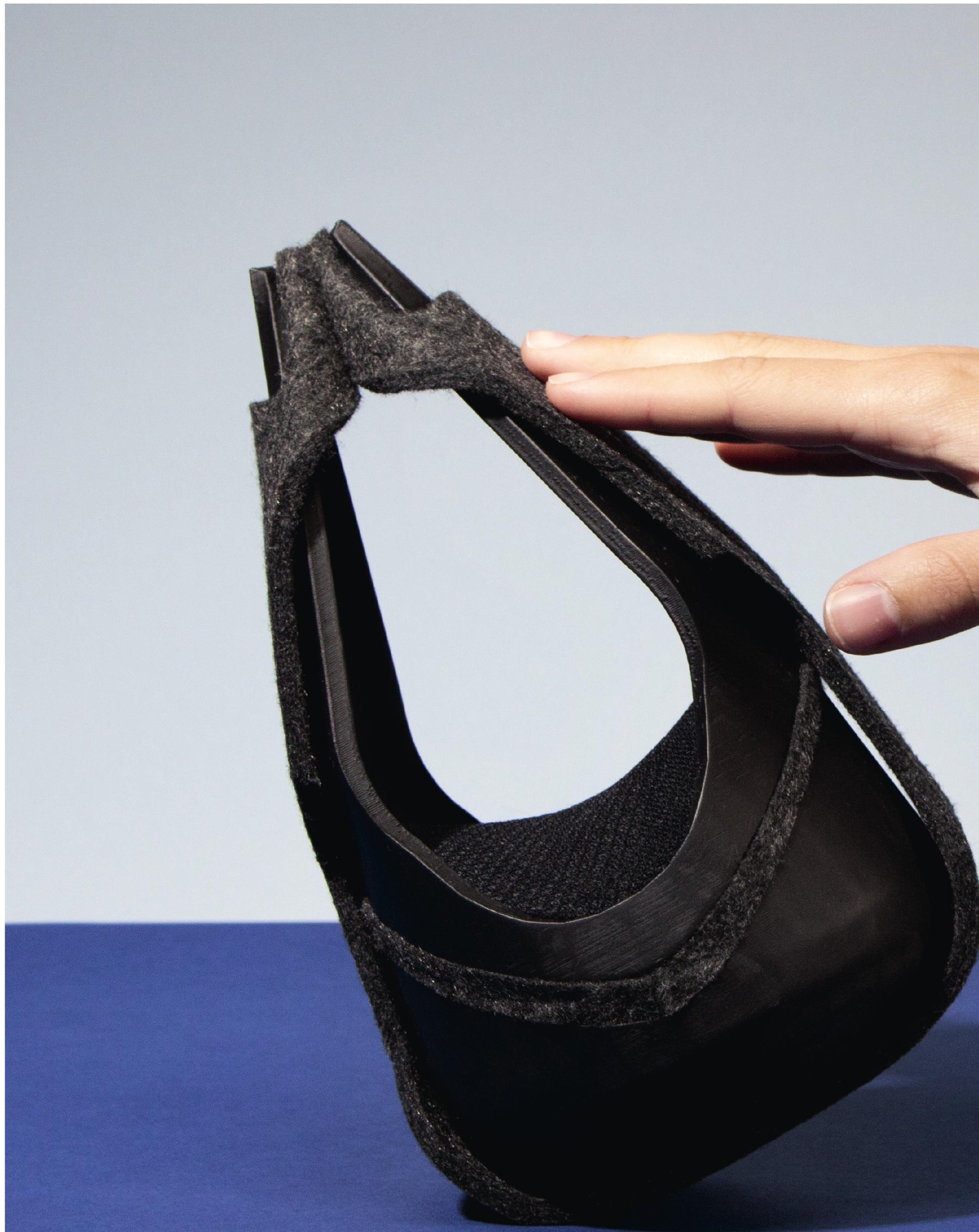


$\uparrow$ 


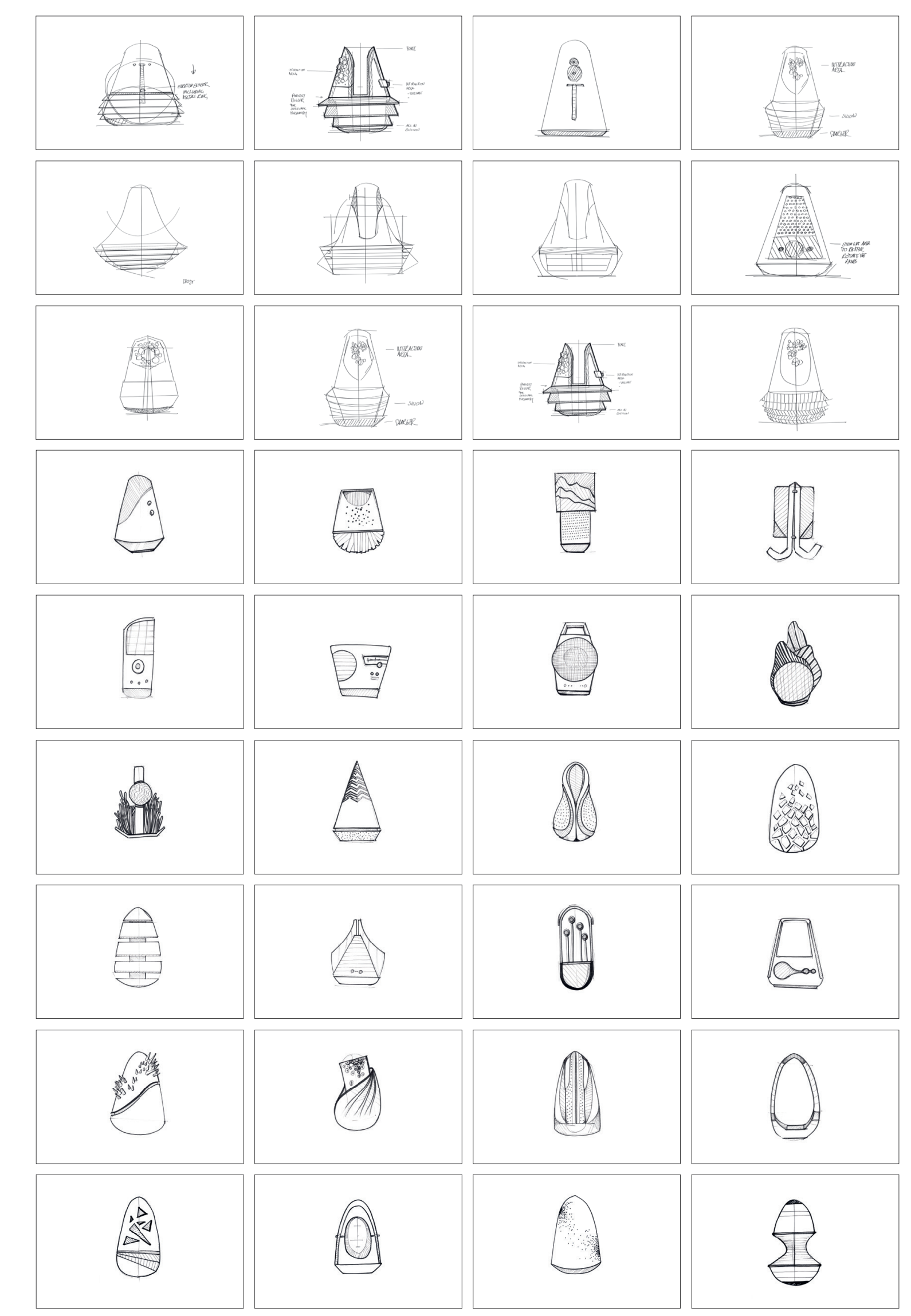


Piotolper No 02 Sketches

1 changed the top part of the racks to

increase the area where the user con touch

and interact with the object. The bigger surface

offers more room for the needed electronic components. 


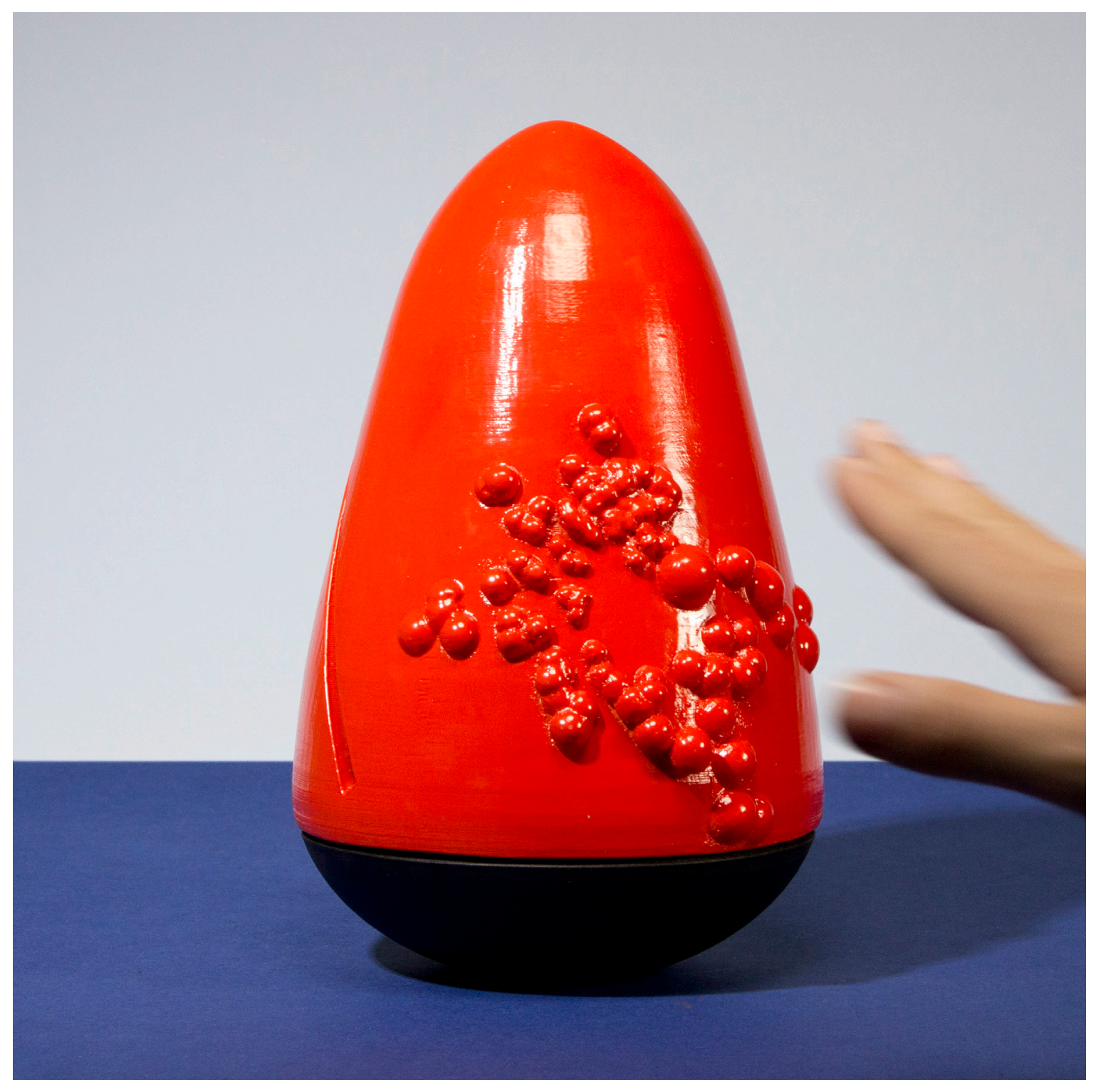

Prototype 02 30point 


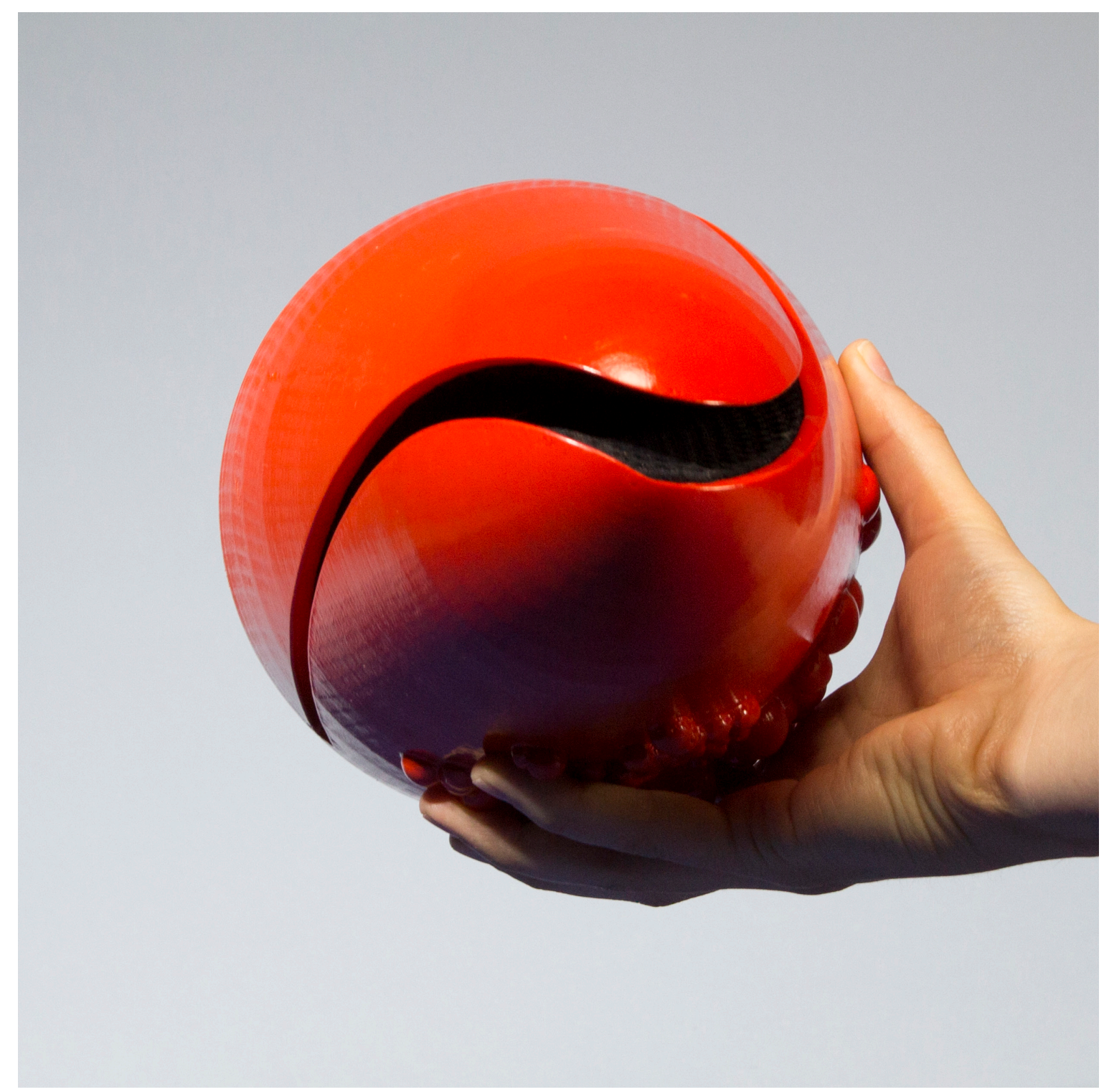

Prototype 02 30point 


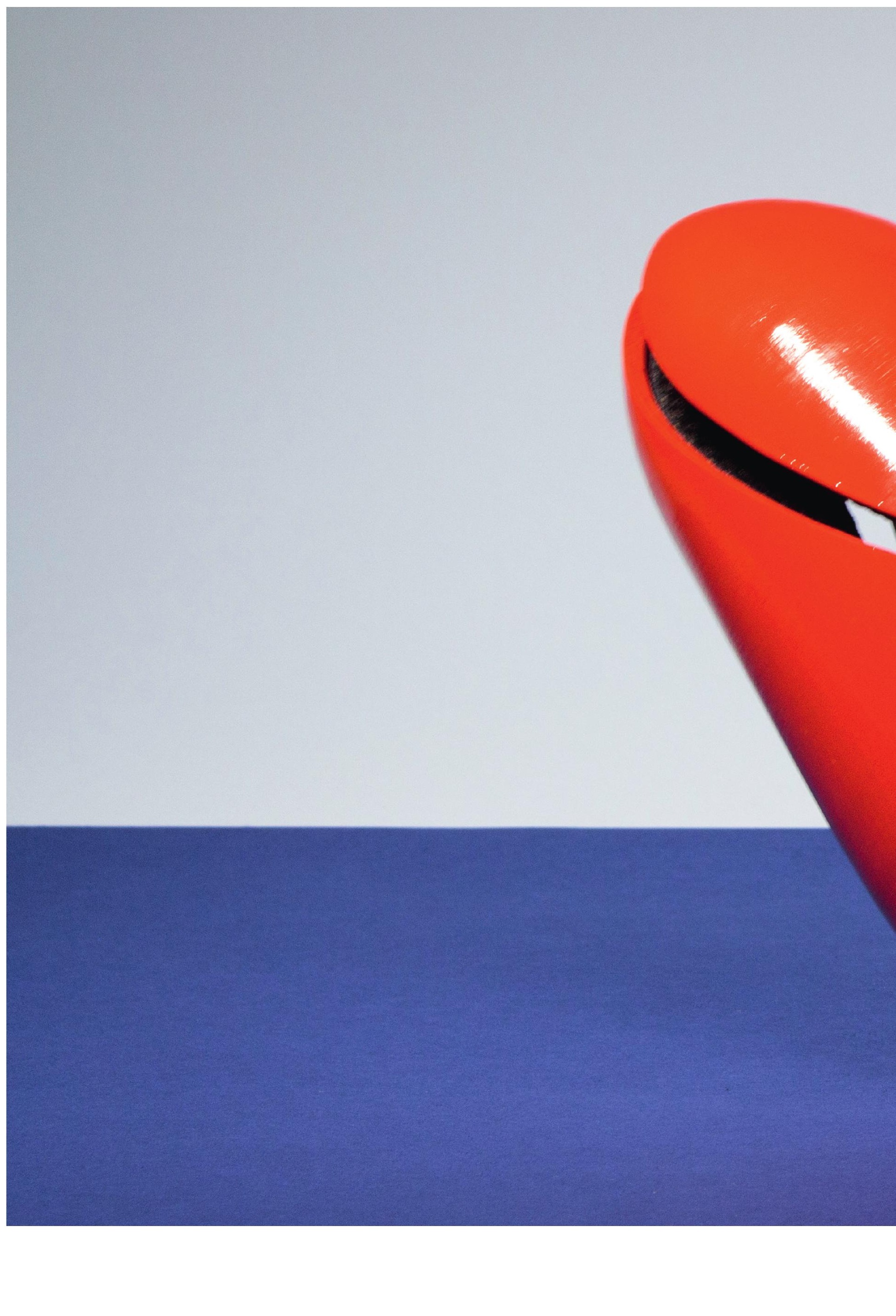




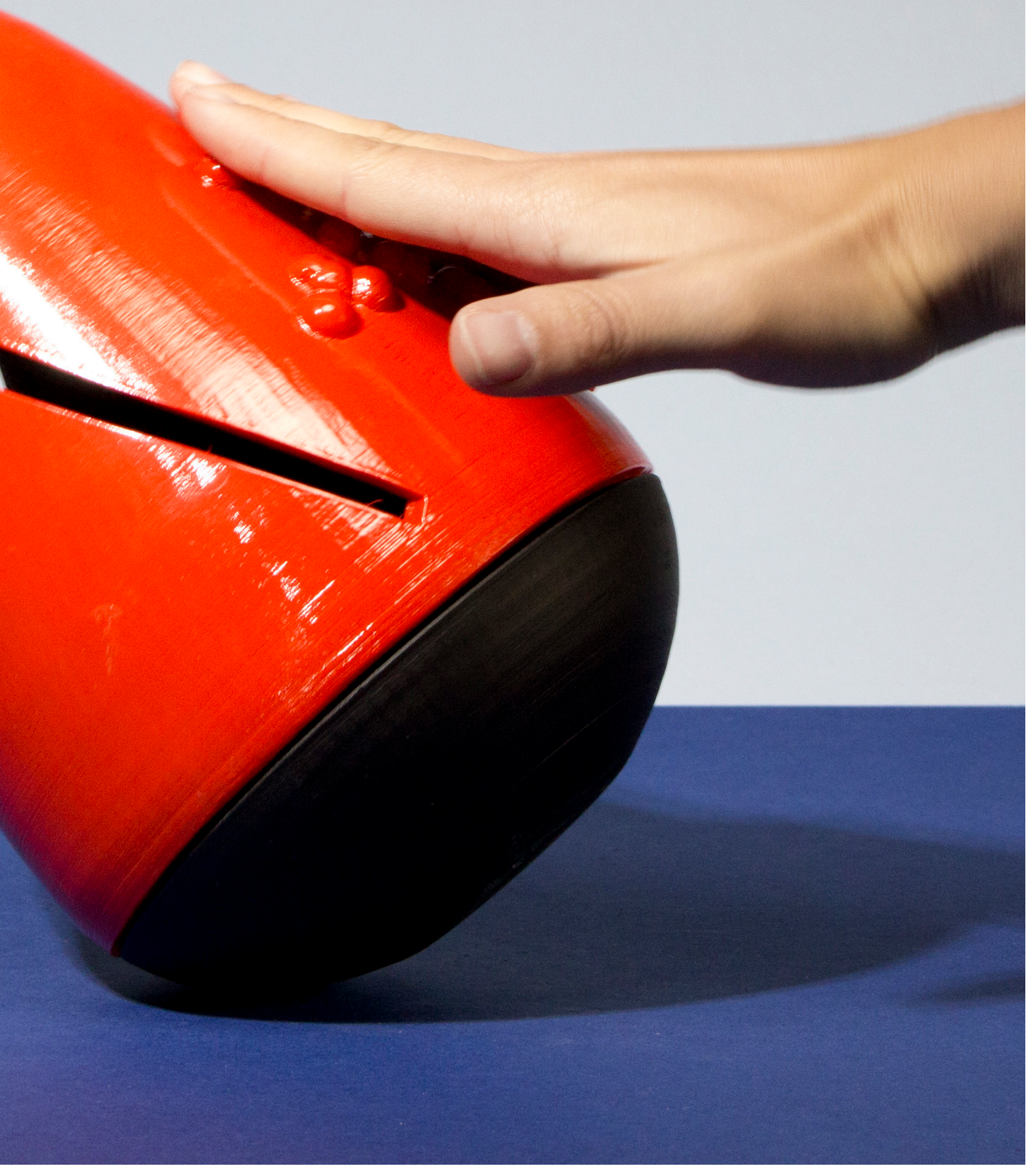




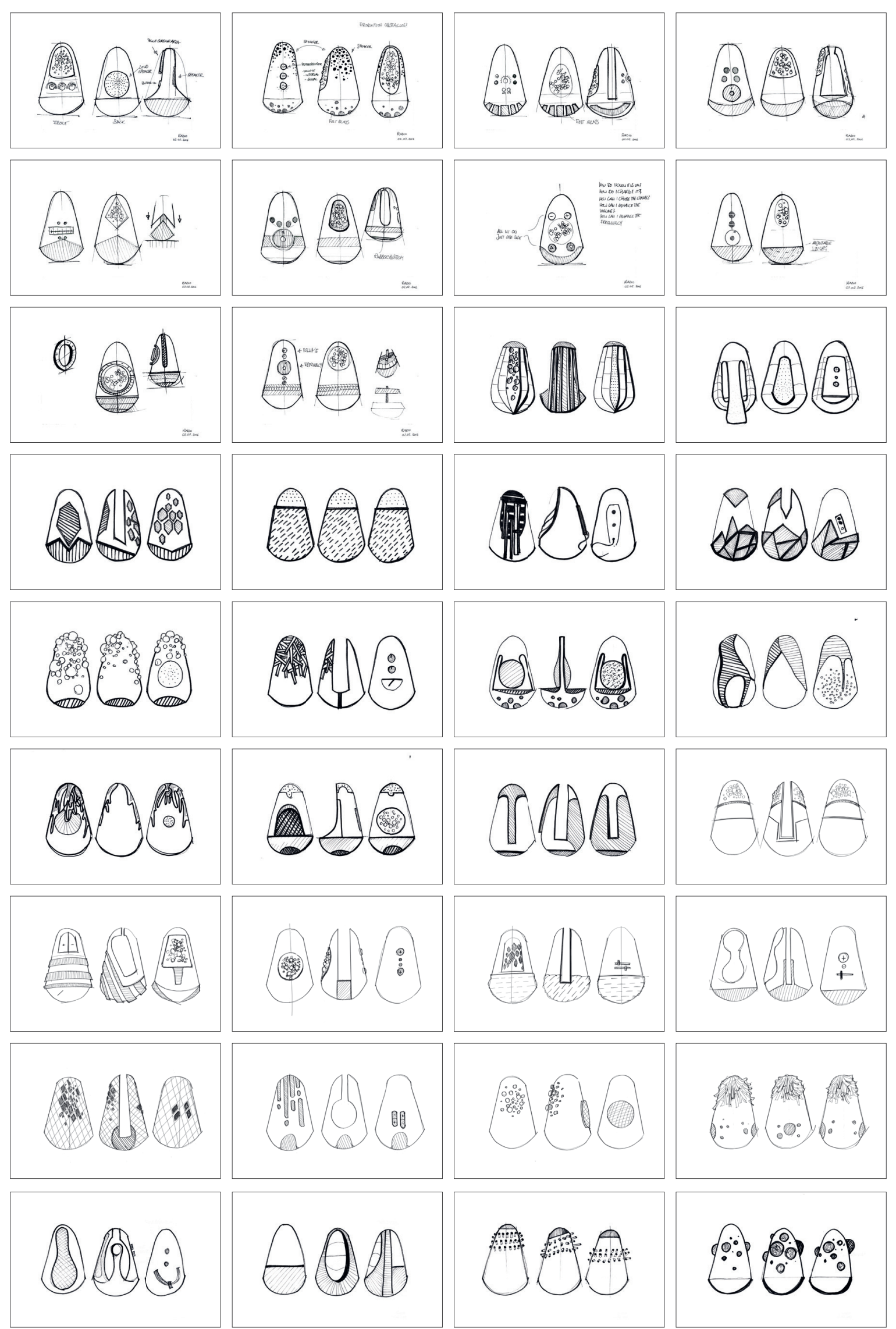


Prototype NoOS stectes

I hid more sketches tryig to come up with

now approa hes by adling tetures and sujpares

to the top part. Adding wore teture g/furs

a better gip duning the riterachin and can

be used to oddress somatosenbry cleficits. 


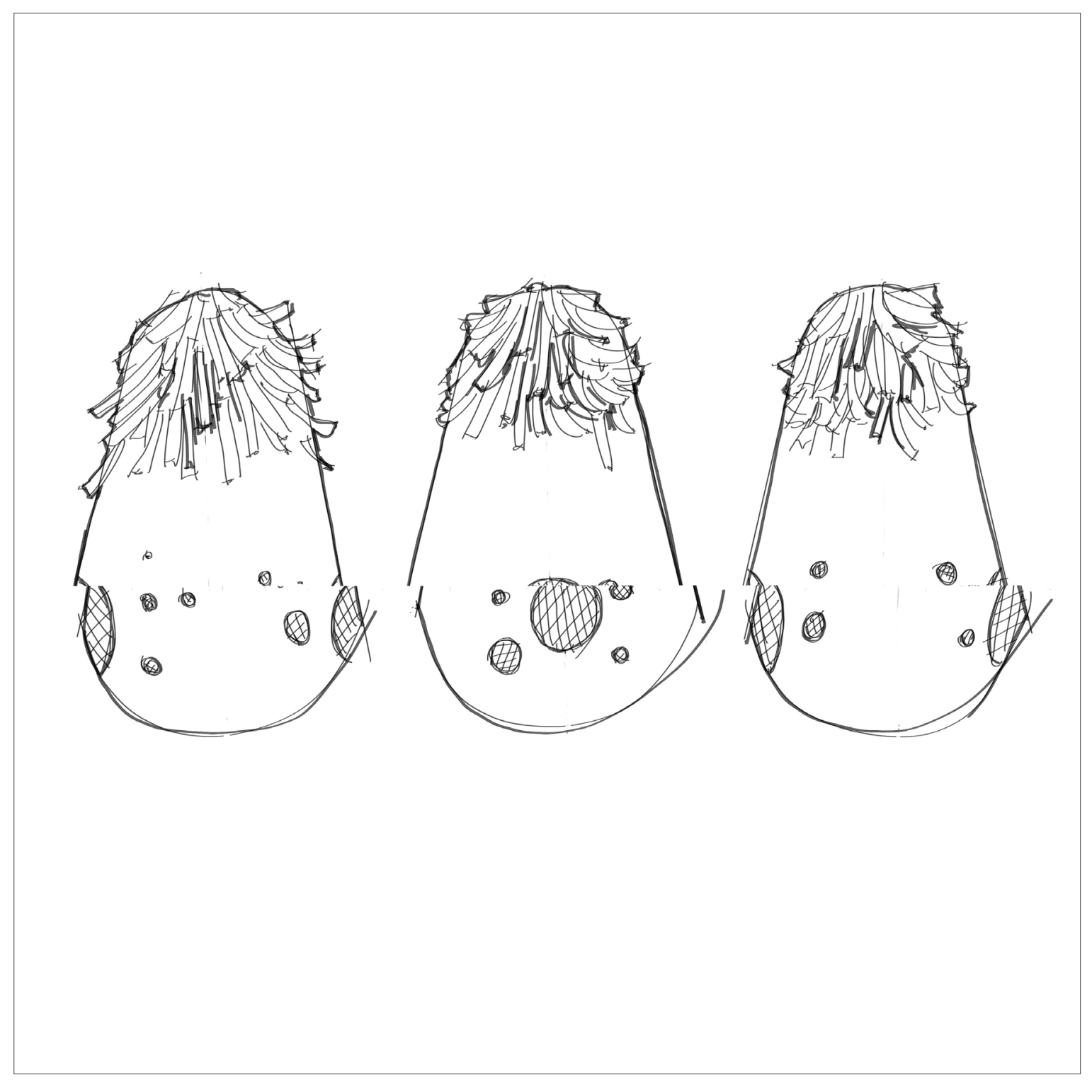

Protolype 03 sketcles 

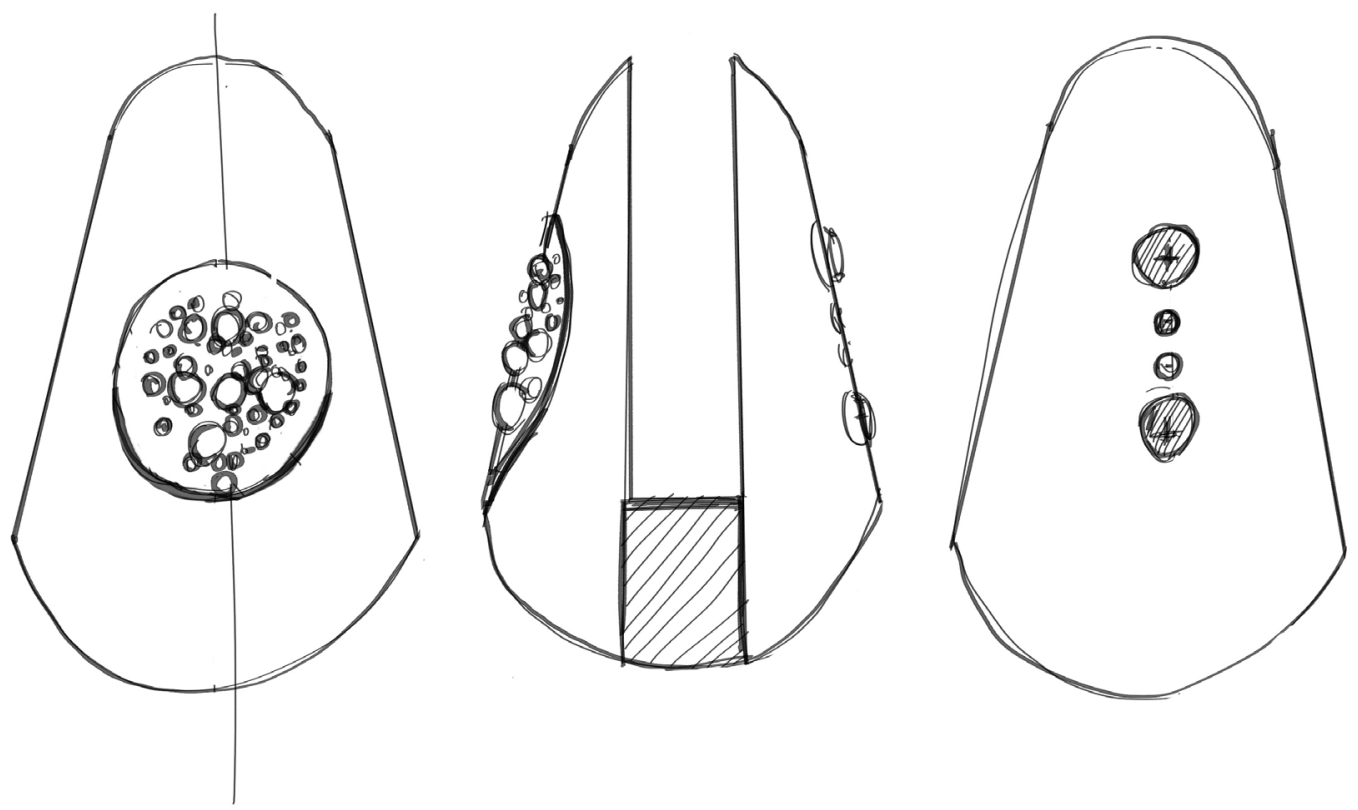

Protolype 03 sketcles 


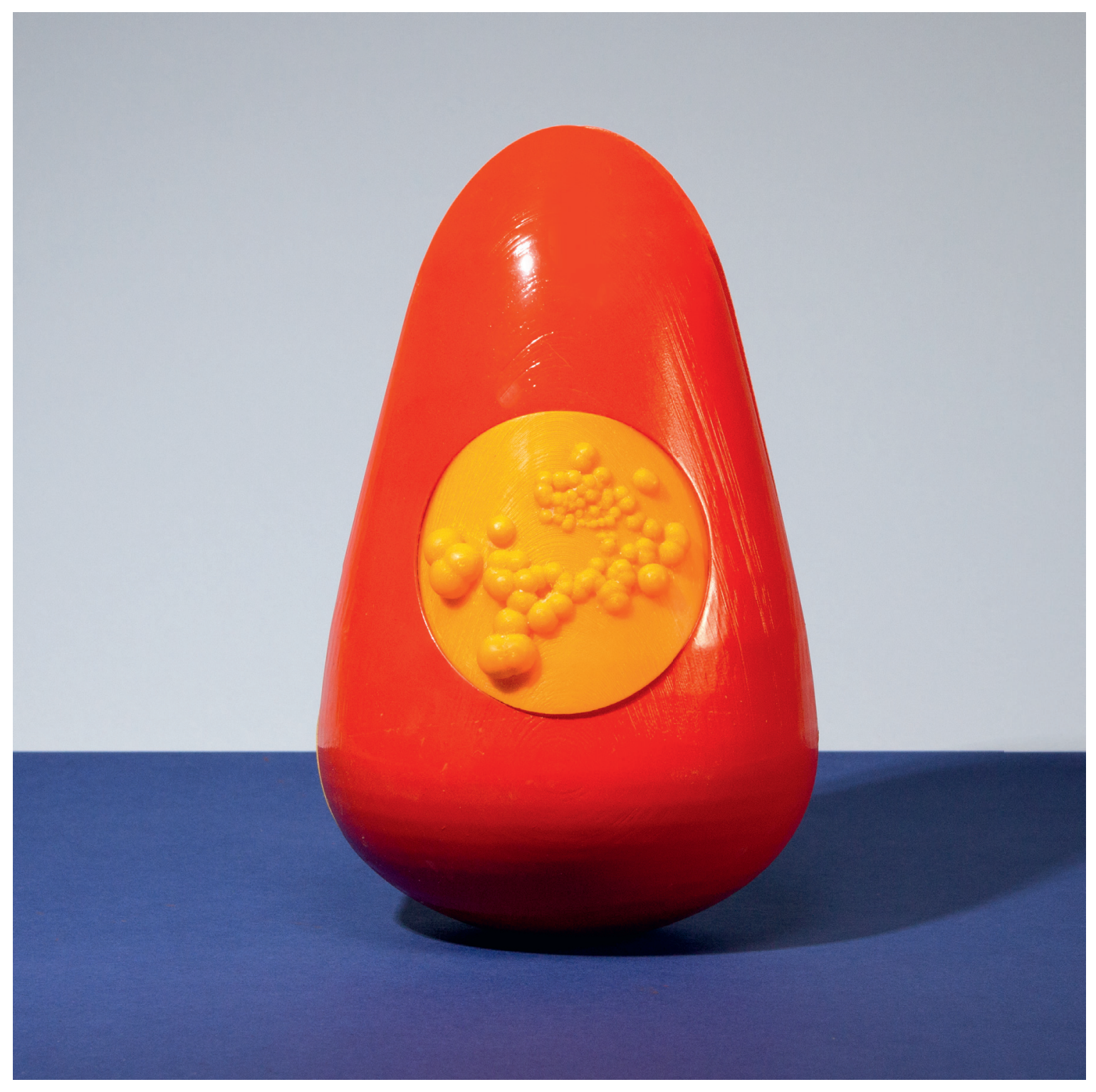

Prolotype os tront view
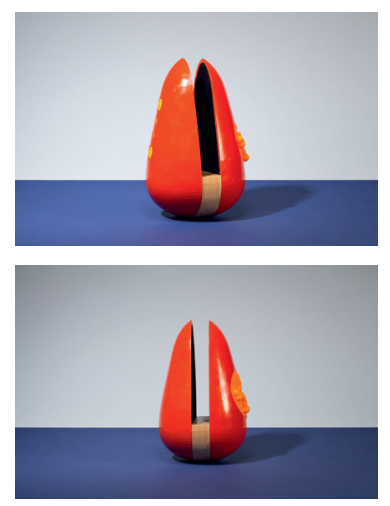


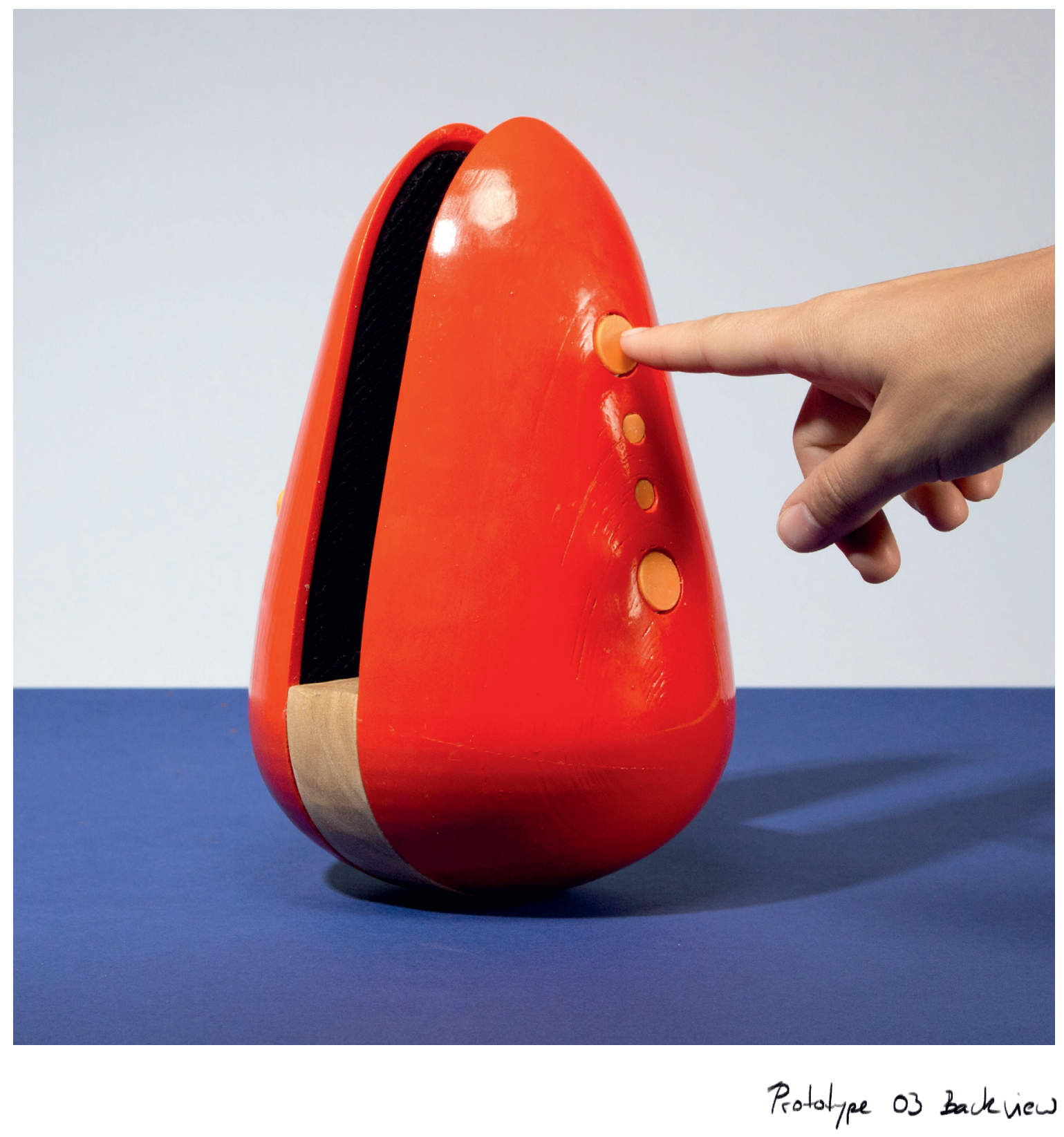




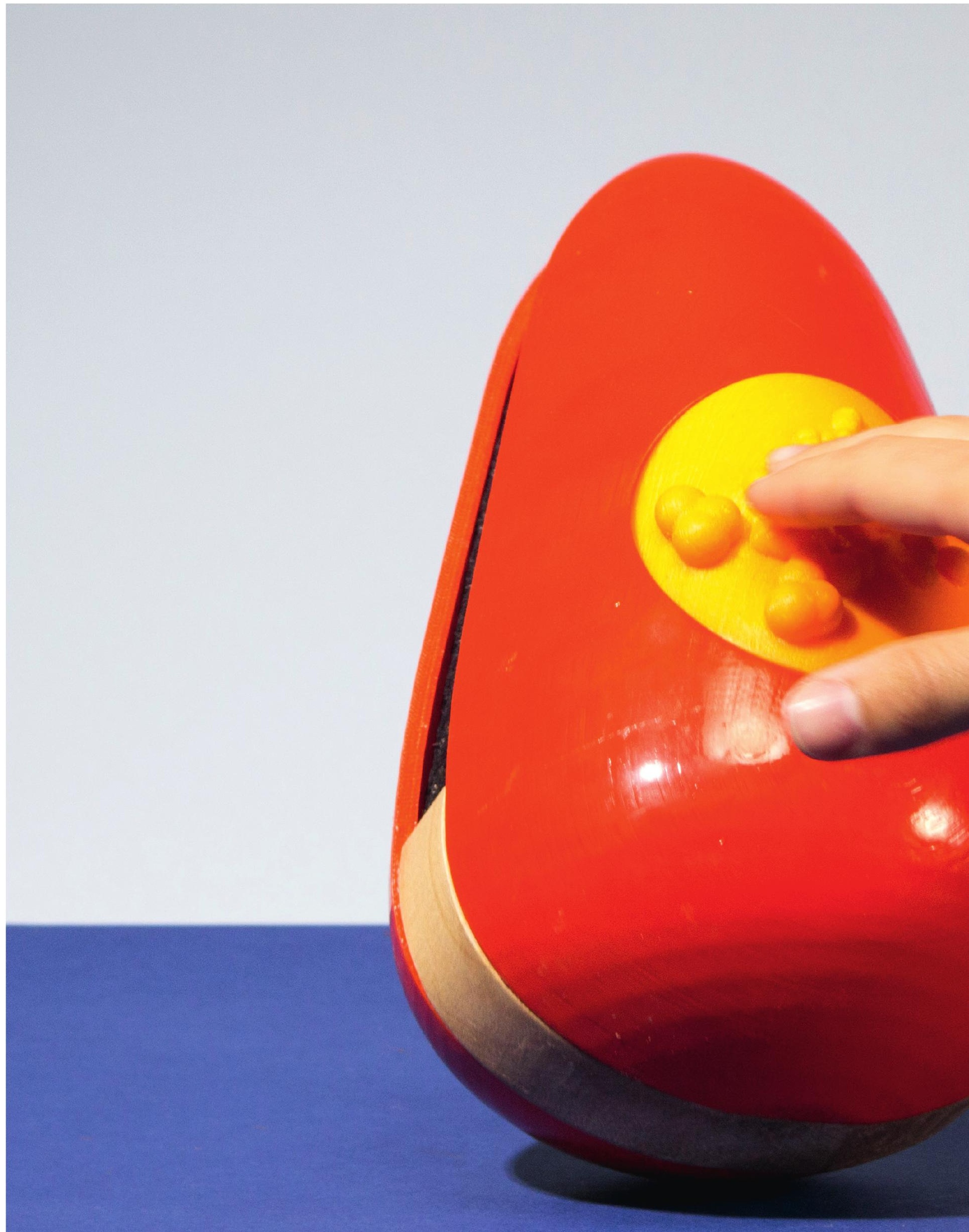




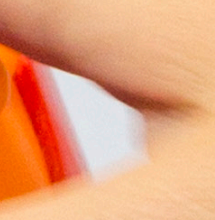




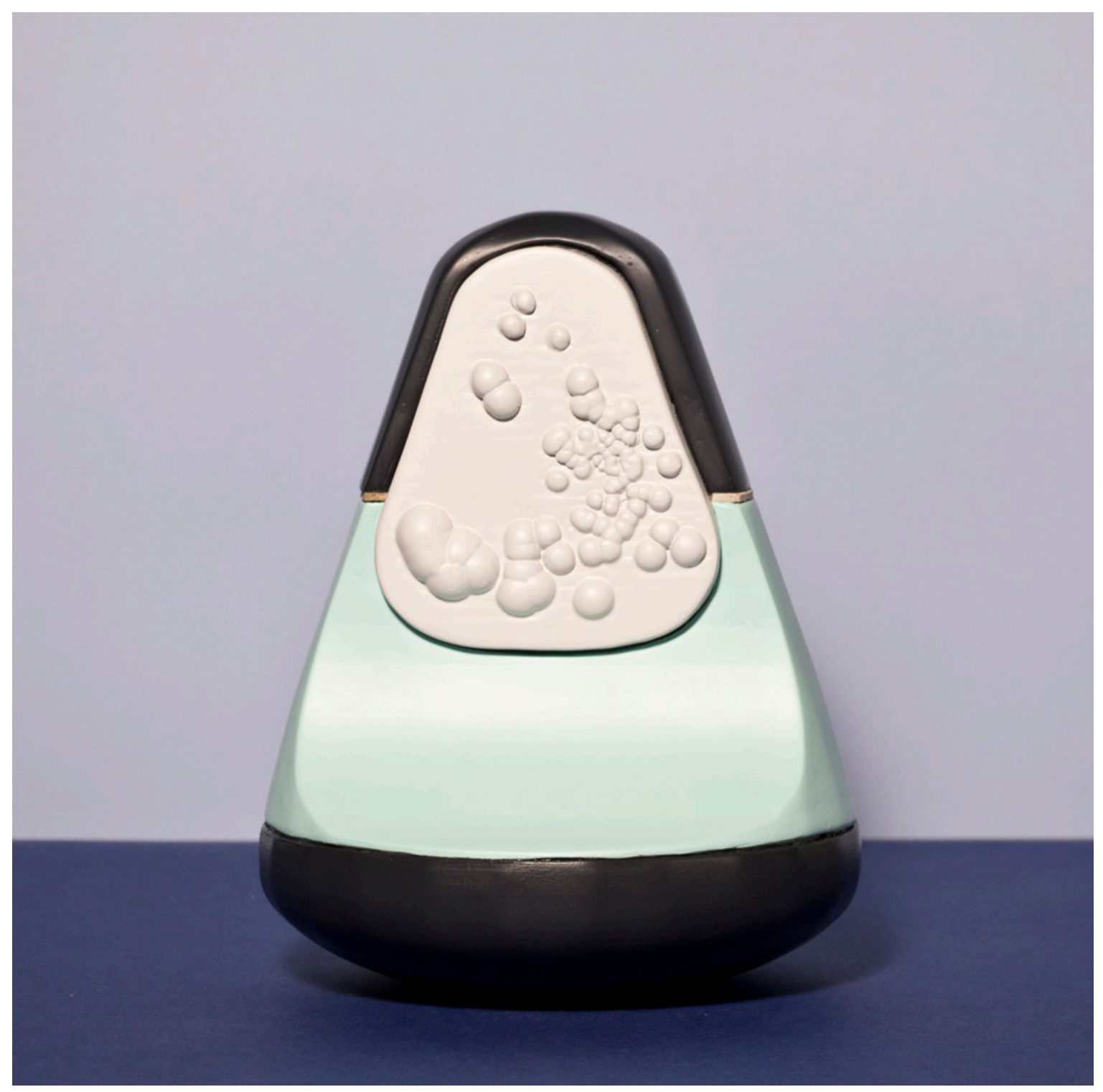

Protolype o4 Frantview

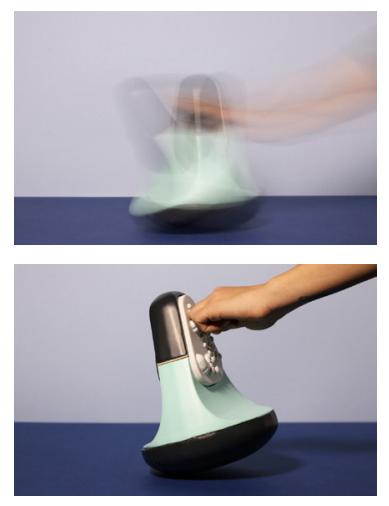

For the rextiteration, I chose to wark on e shmmer silhouette and increased the weight of the rado by adding band into the base before glueing both parts together the movement turred out to ke smosthor but the top part did not offer enough space for all the reded hardware components. 


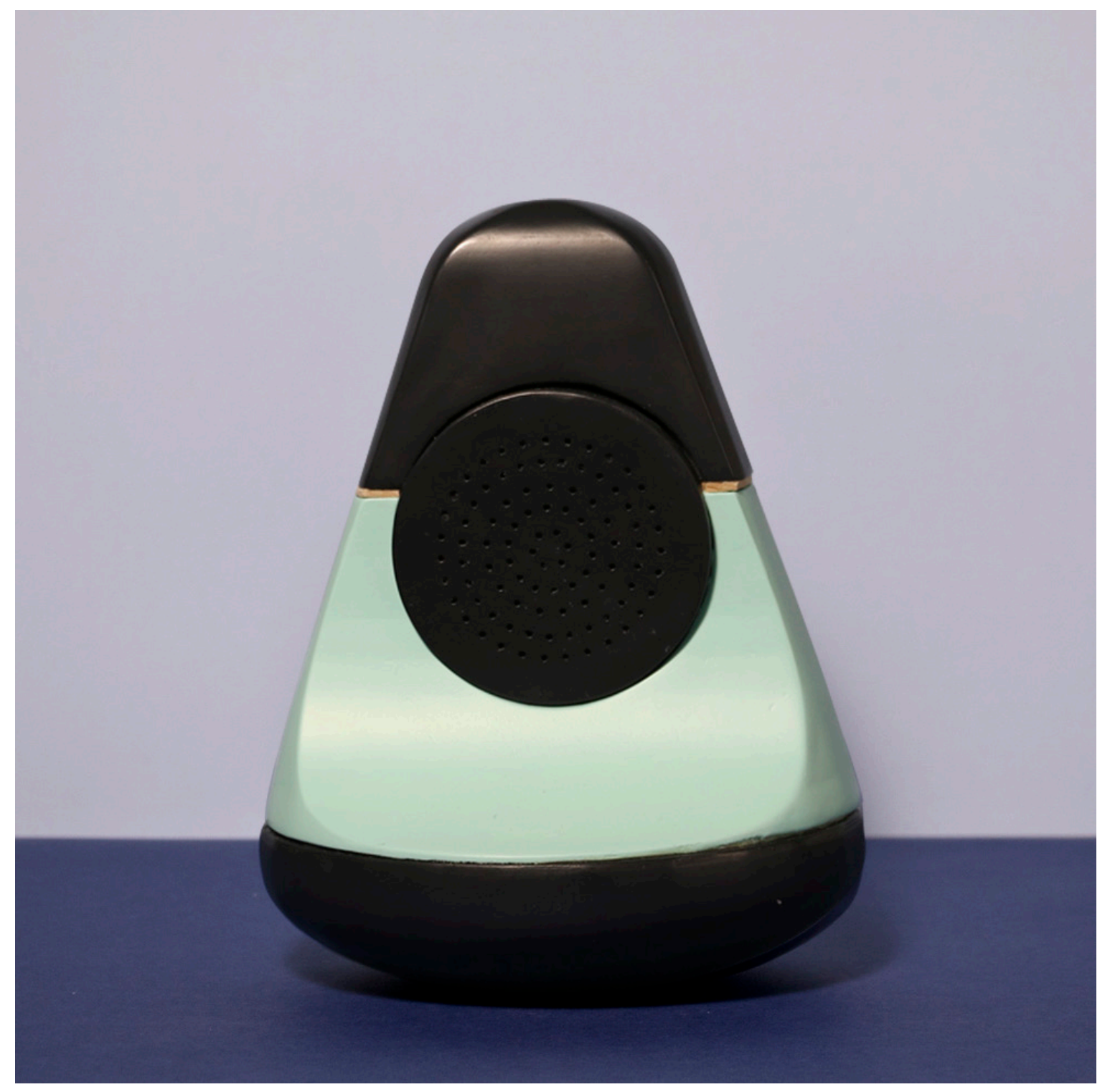

Protolyee on Backiew 


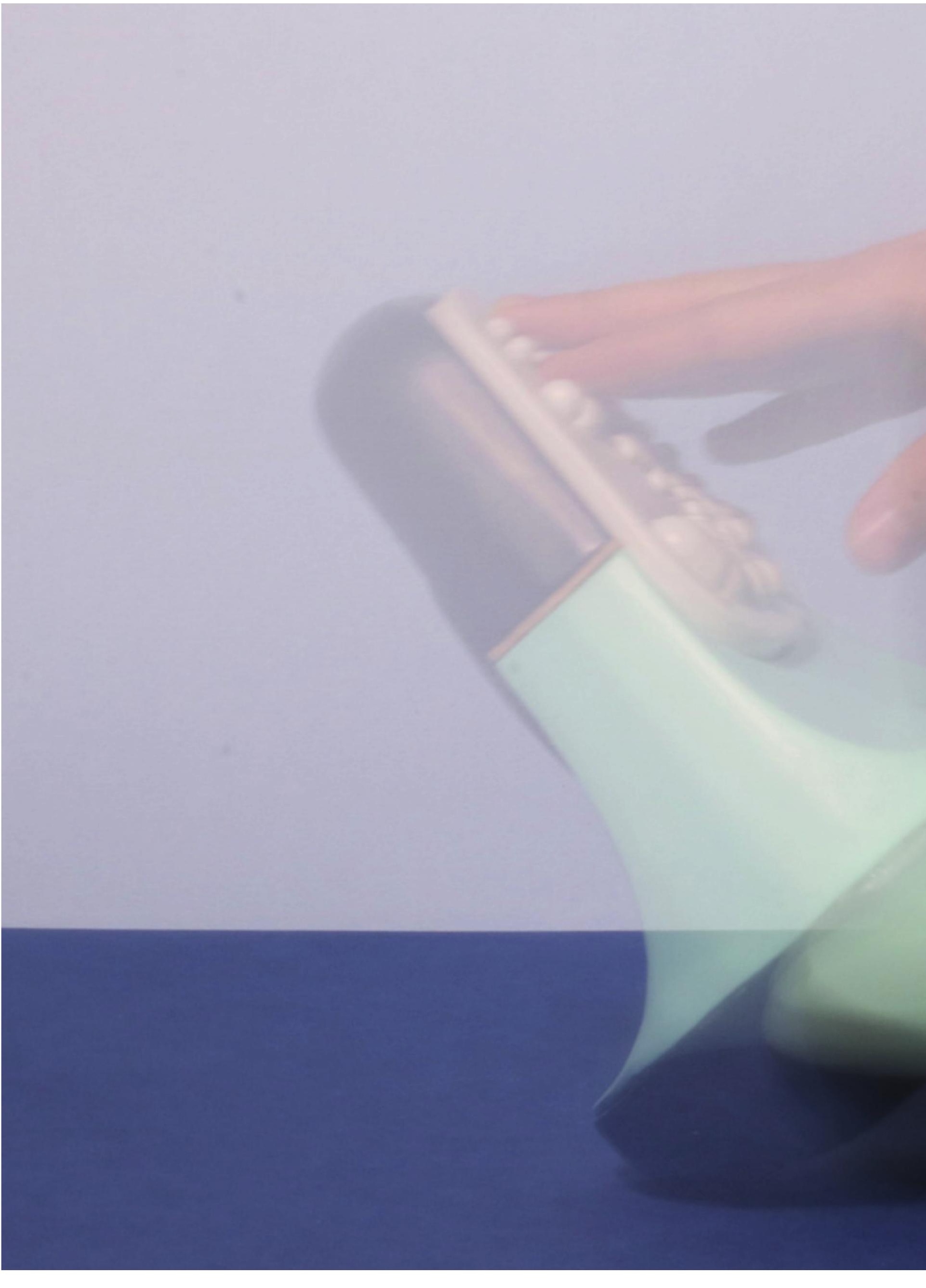





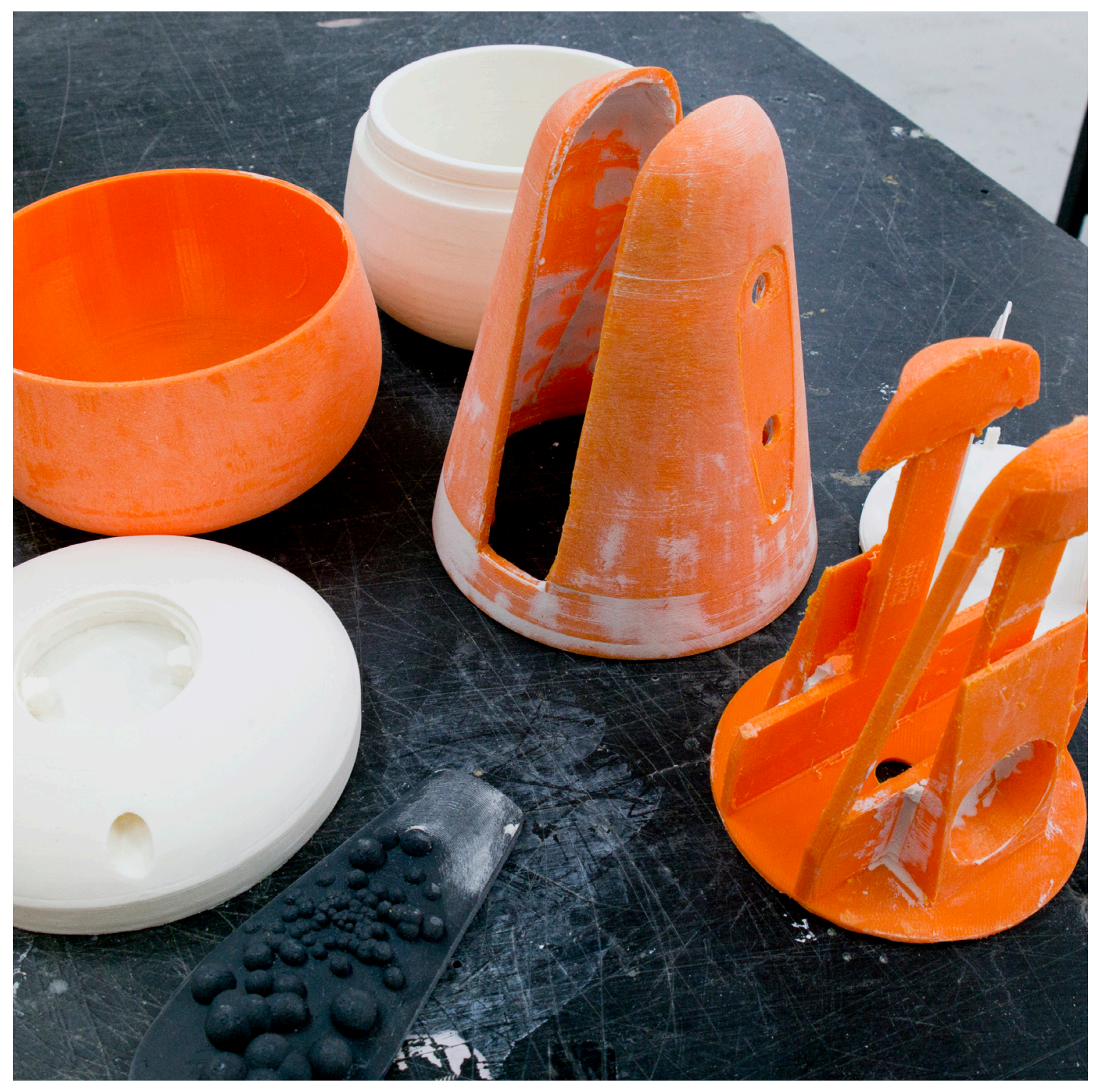

Prototype 05 Model making 


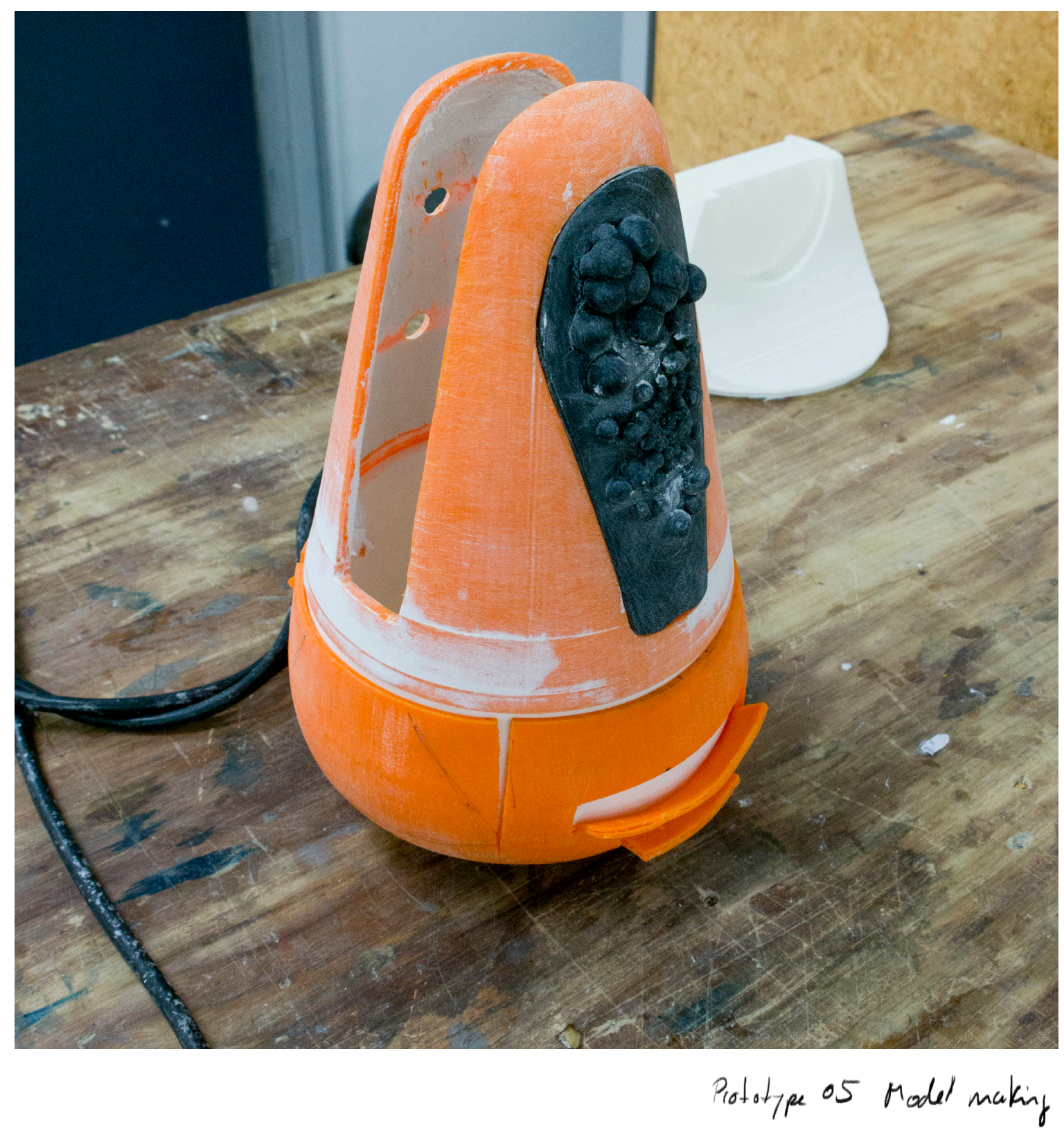




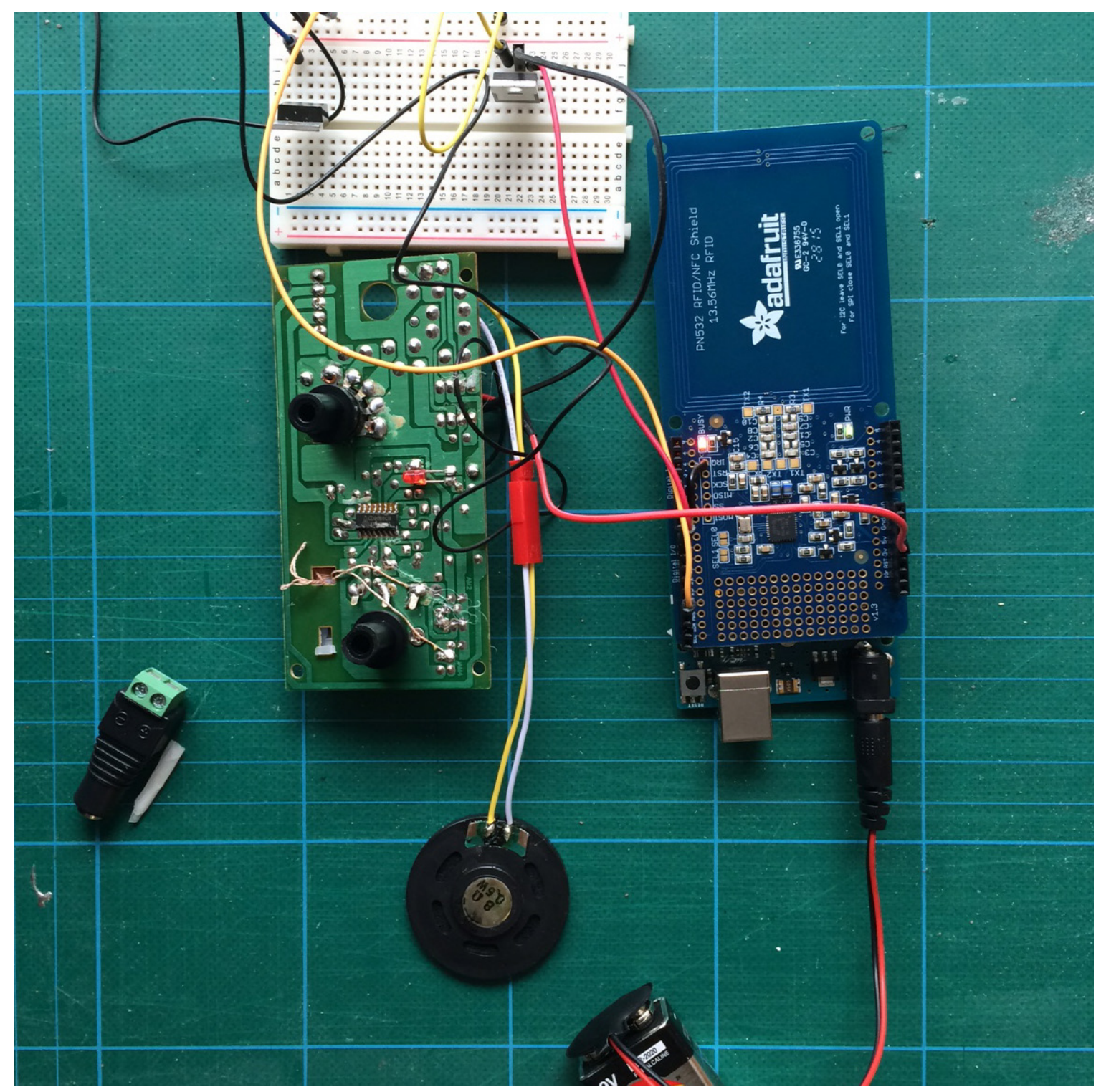

Electromis used for the restraning affect

I used NFC technology to restrain the movenent The user is wearing an WTEC bracelet. If the cuser uses the wrong. less affected-side the racho will just not turn on. The electronics involve an Arduino lna boord, NFC shield and a radios boord. 


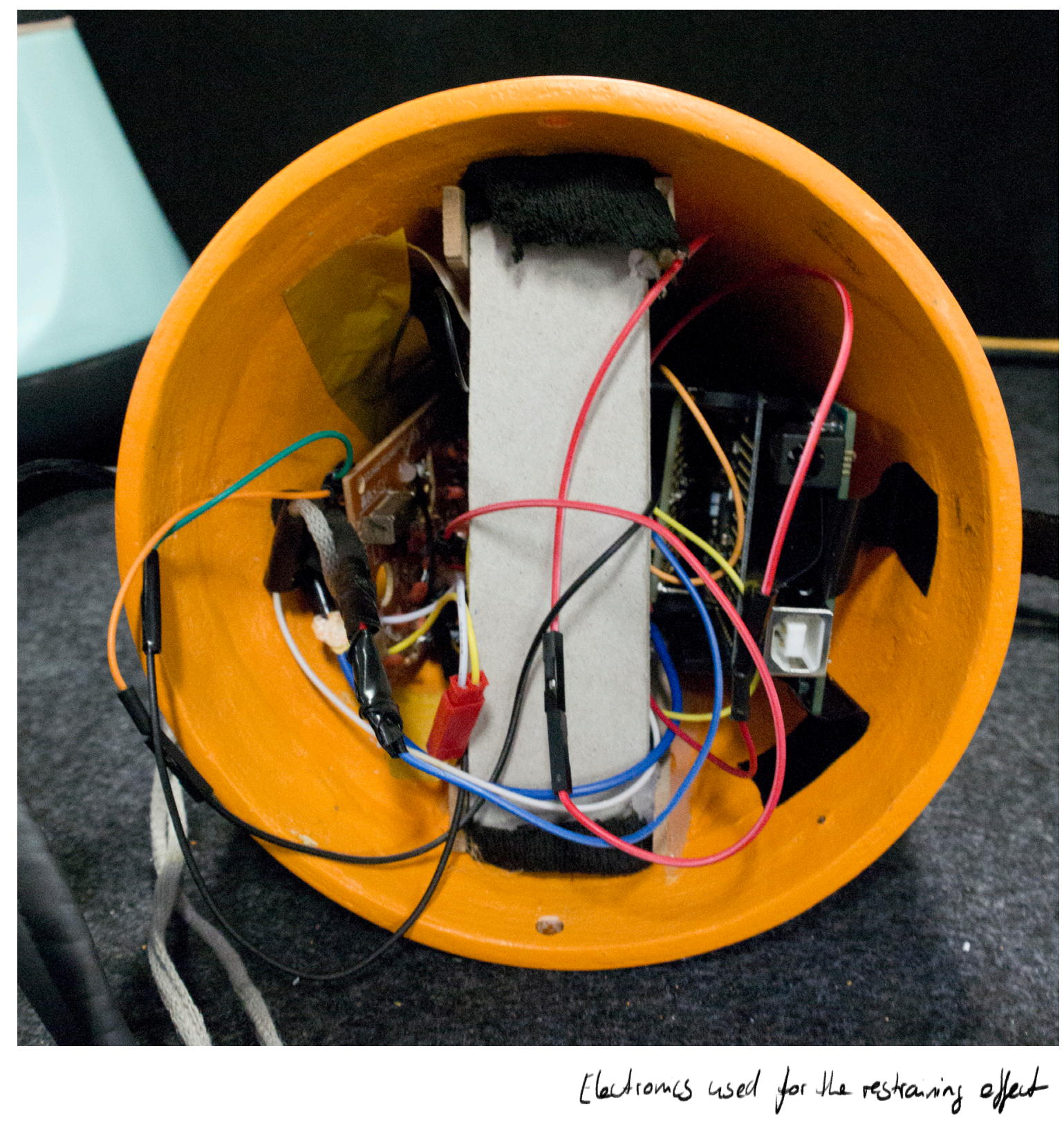

The inside of the radis offens enough room for

all the components. The rachio board is

sinated on the left while the Ardenino and

NFL shield on top of it are situated on the

Might side 


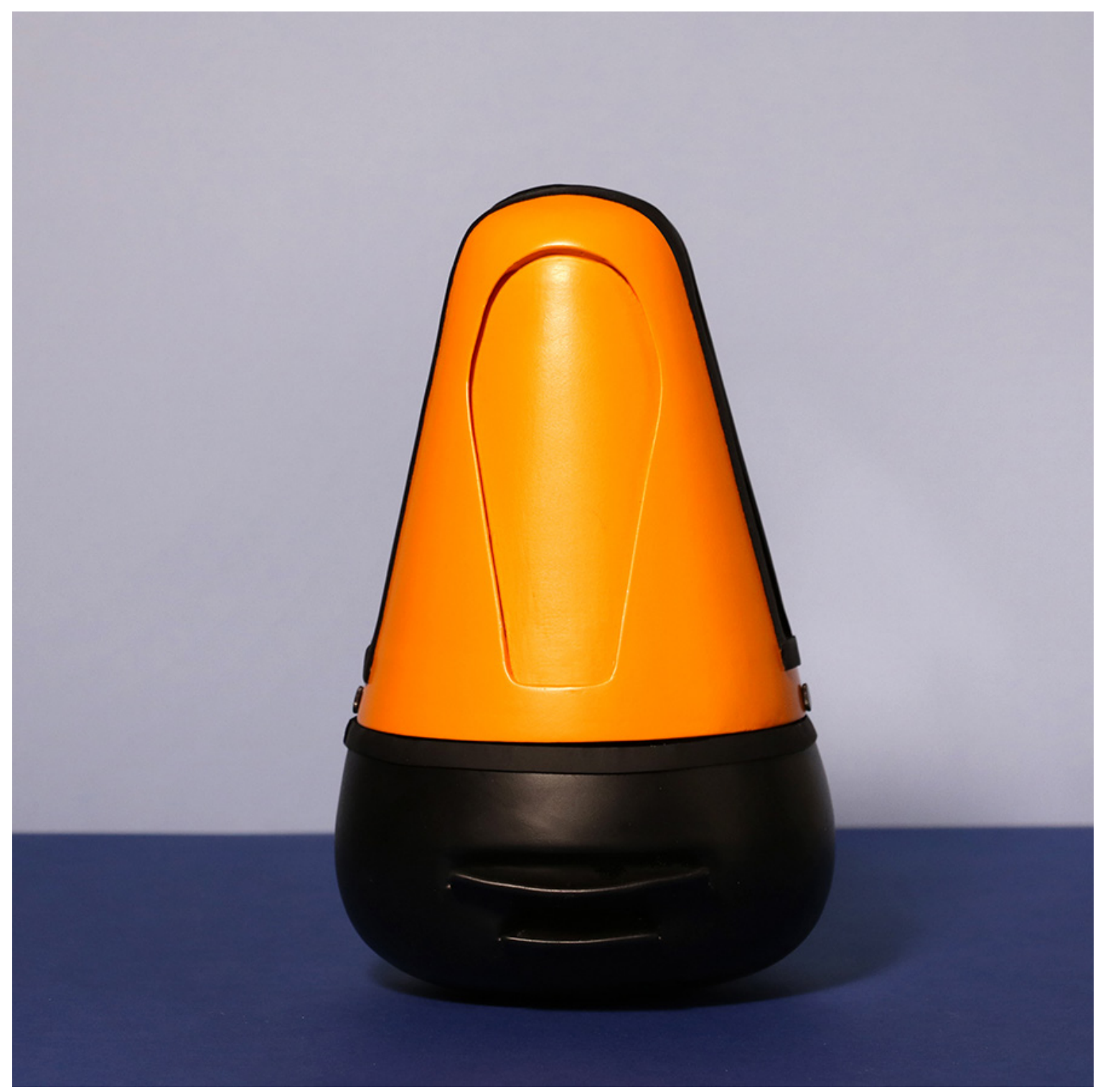

Protalype 05 Frontivew

The radio consiots of two main parts: a top and bubbles are based on the parametnc desyn a botton. The bottom part contains weights that is based on a sonnd fi6. The user can to increase the training difficulty. The user decich which tind of iiteraction area 5 can stait with a lot of weights and once attached to the racho.

propress starts to set in the waght can be increased. On the top part, the main interaction made out of silicon can be detaled. The 


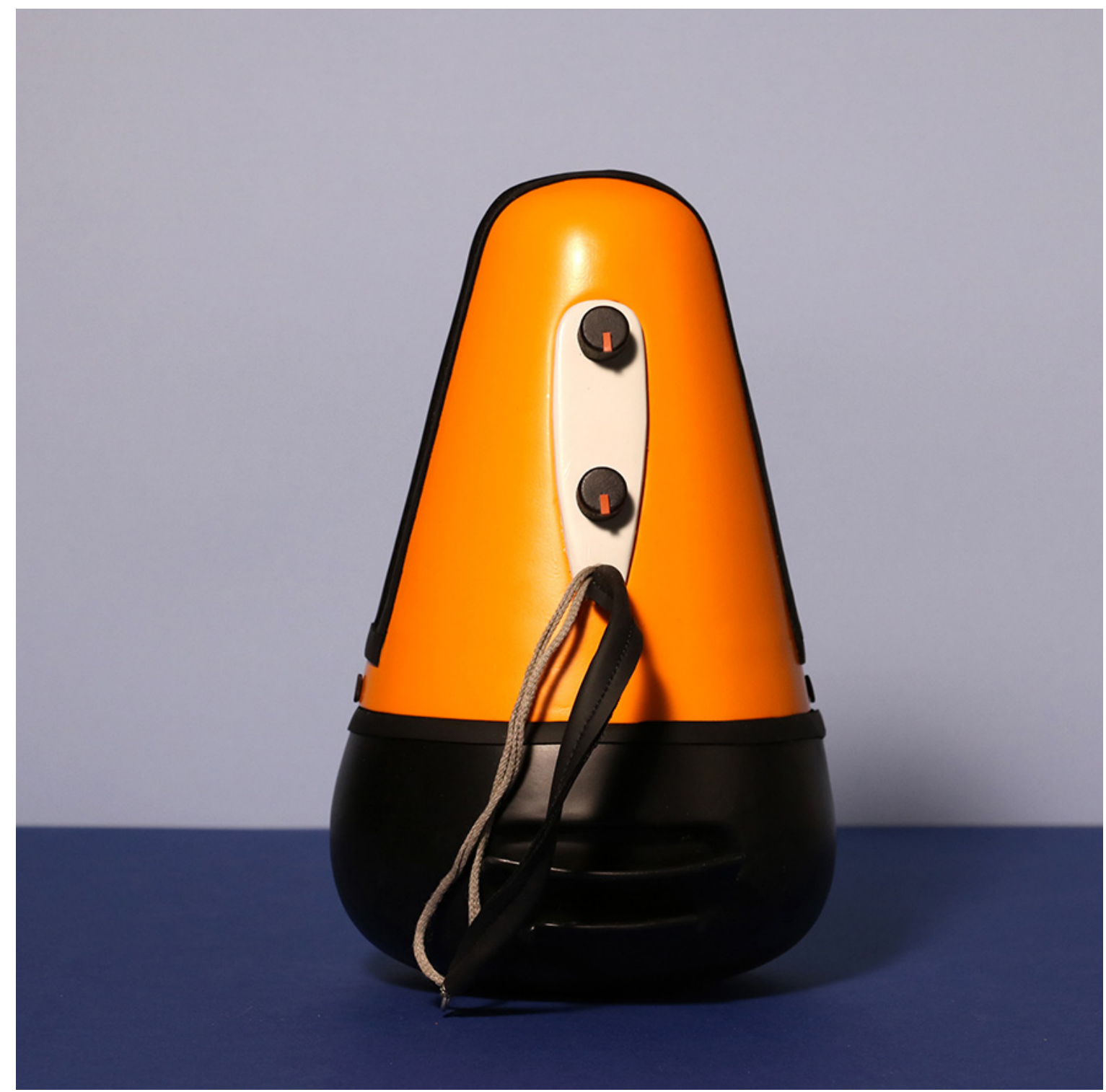

Problatype o5 Backinew

The backside of the radio offers the opporturity movement in orde, to elicit neuvoplastic tochange the rachostation, adjust the volume changes in the buan thet lead to recorery. and change the training intercal. The oadio the more ofter a movement is carned recoginses if the corm is used and turns on out the ketter the chances that the for a couple of mimutes before shuthy down user recovers from some of the struke sympomer. ogain. The user is osked to do a repetiture 


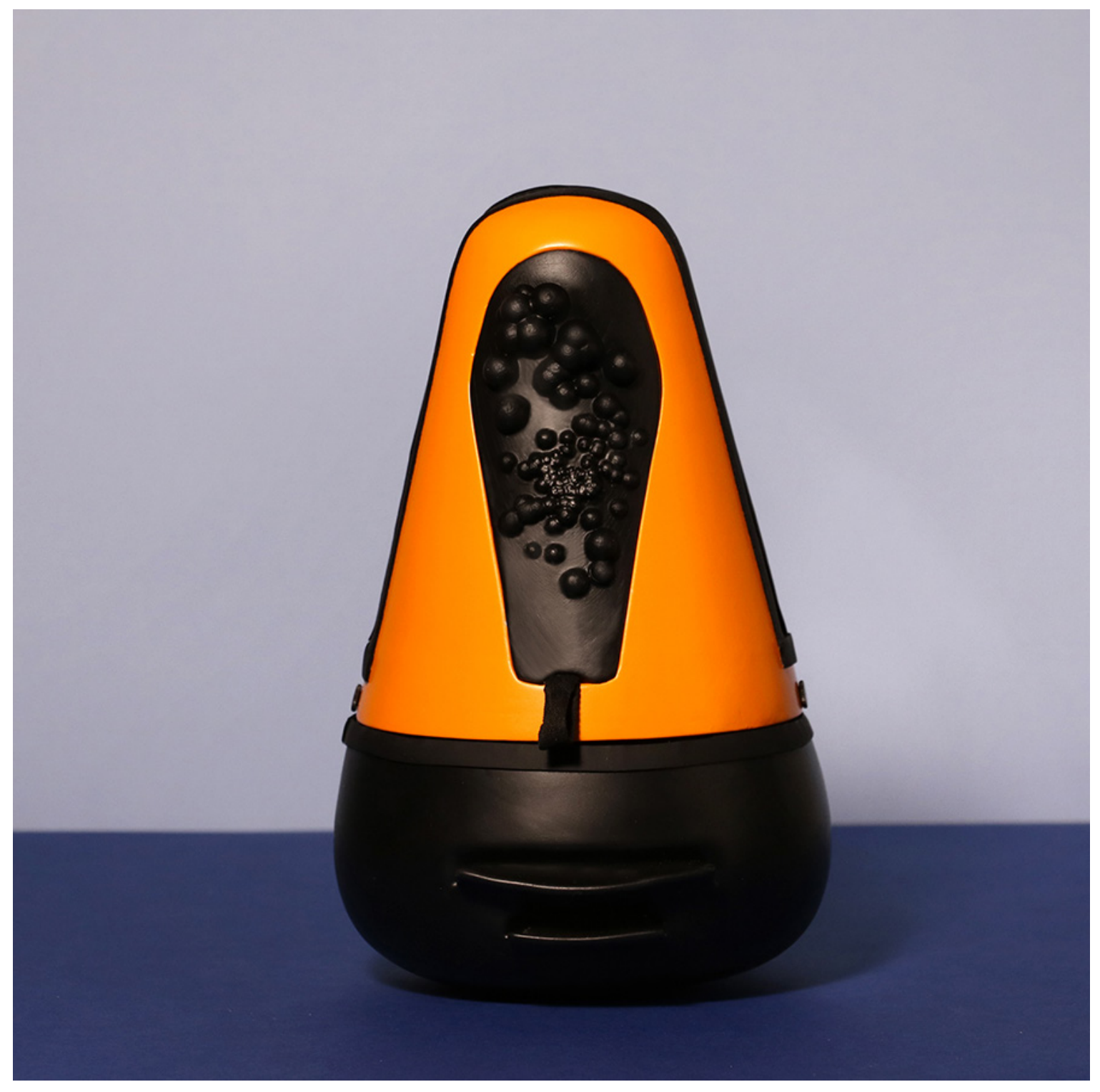

Protolype 05 troutriew 


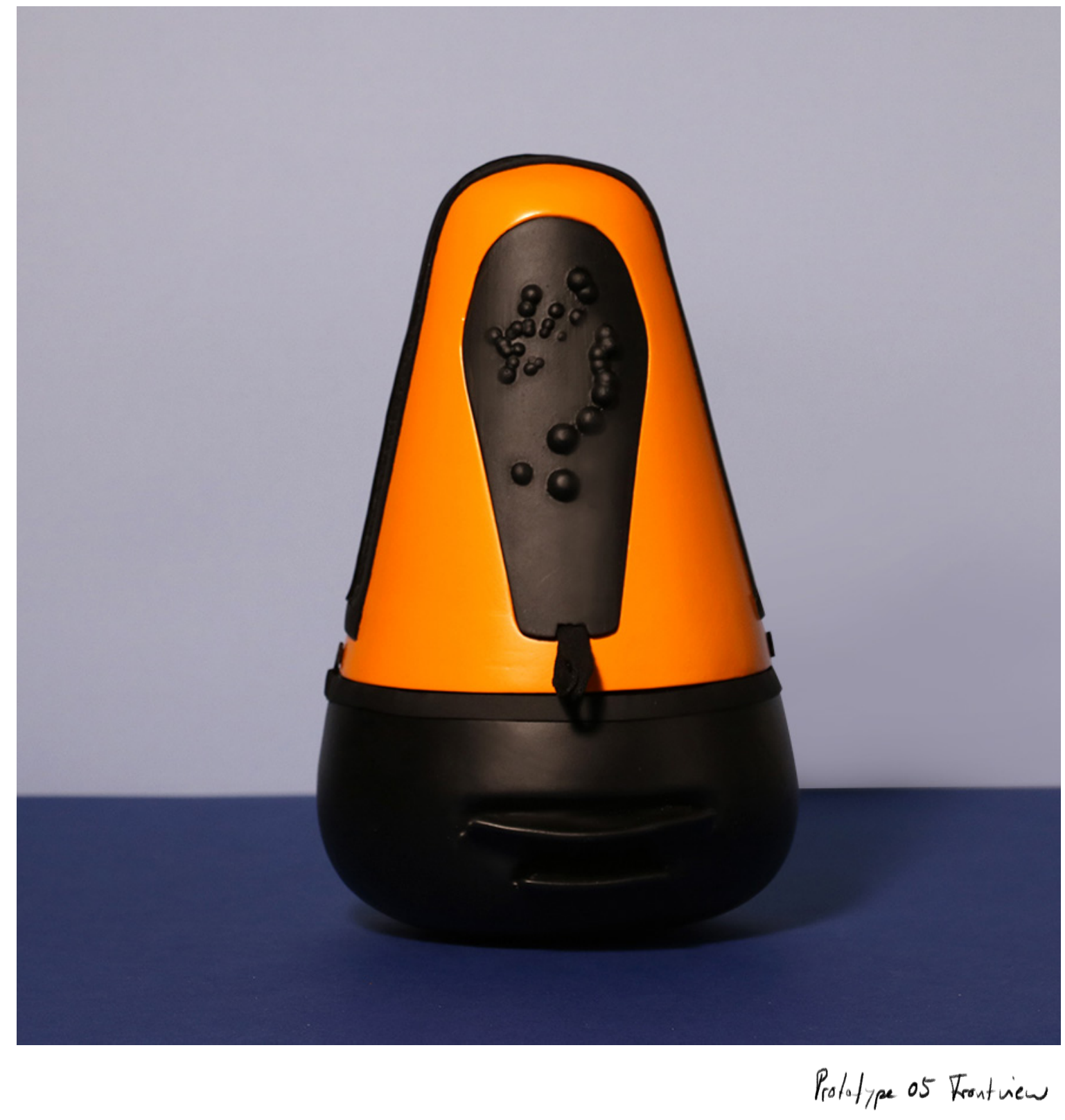




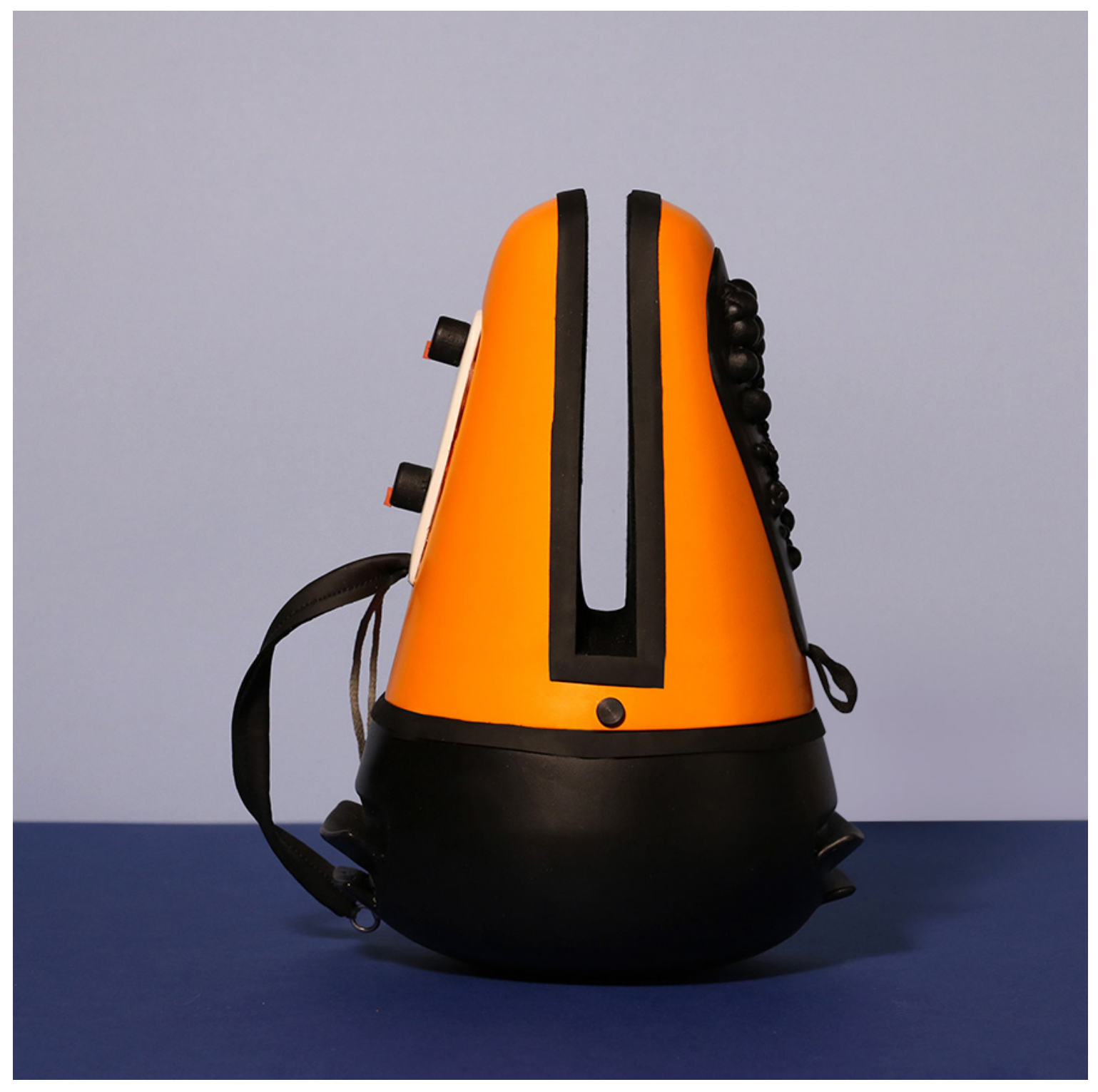

Protolype o5 Sileniew

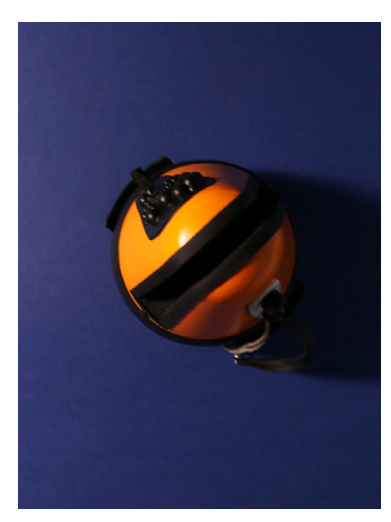

Protolype 05 Topuiew 


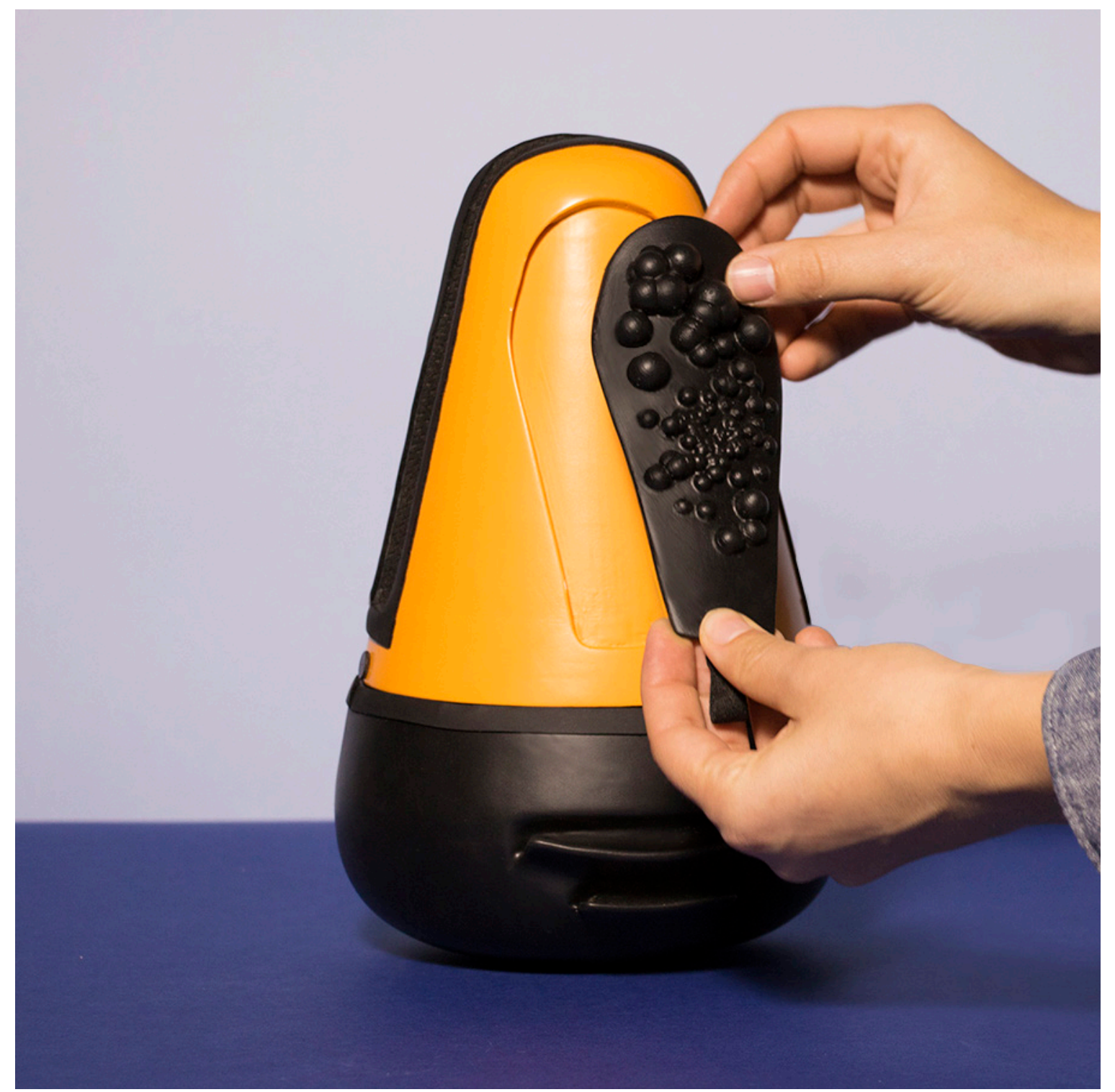

Prodolype 05 Interaction agen

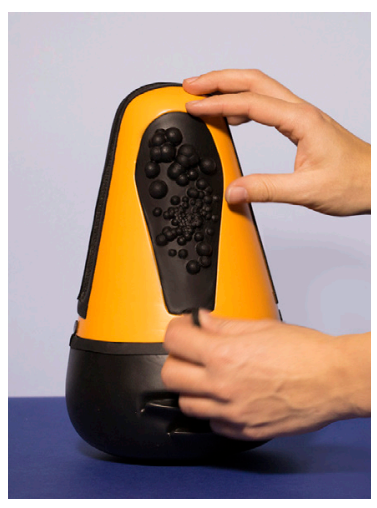




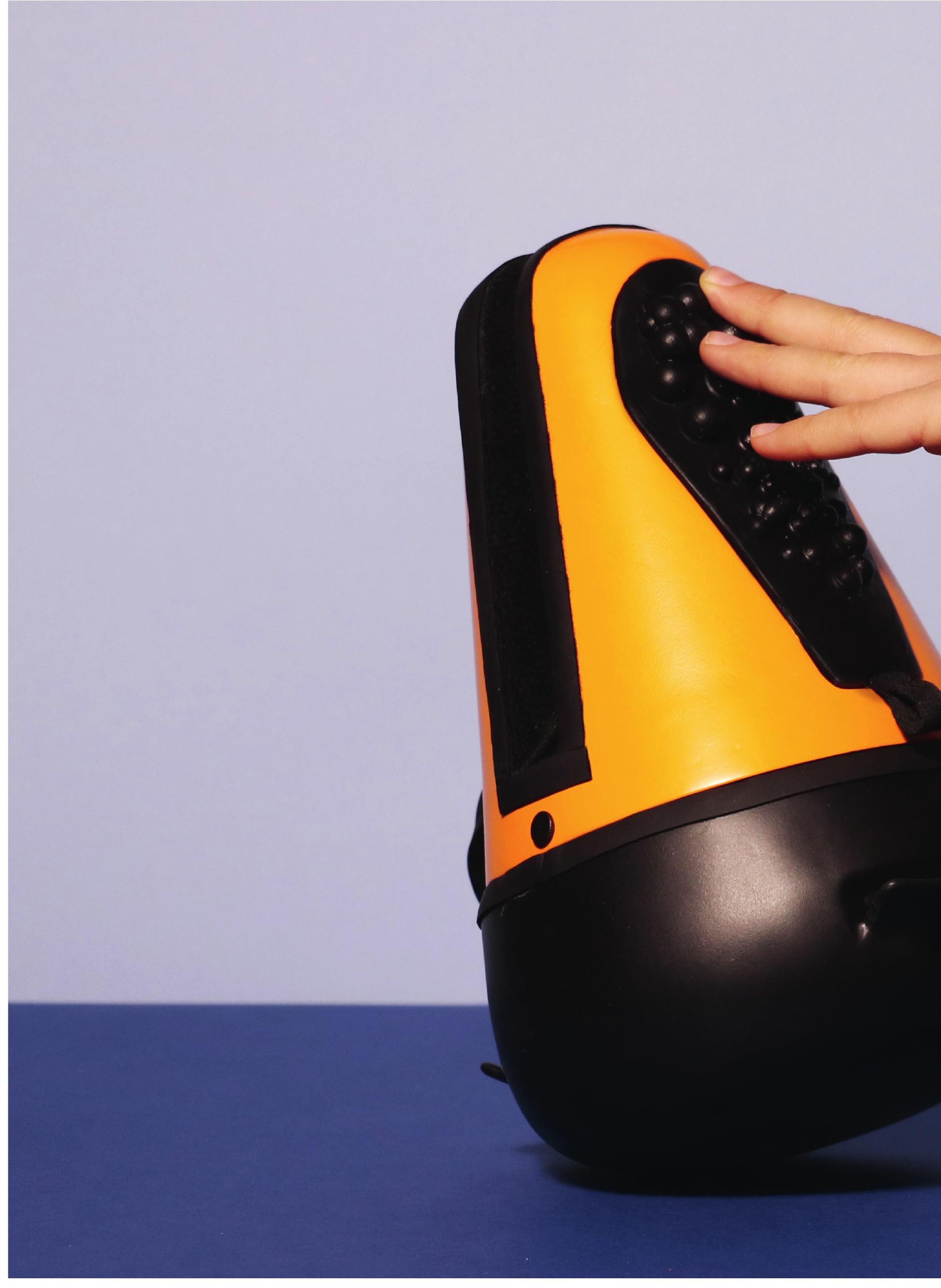




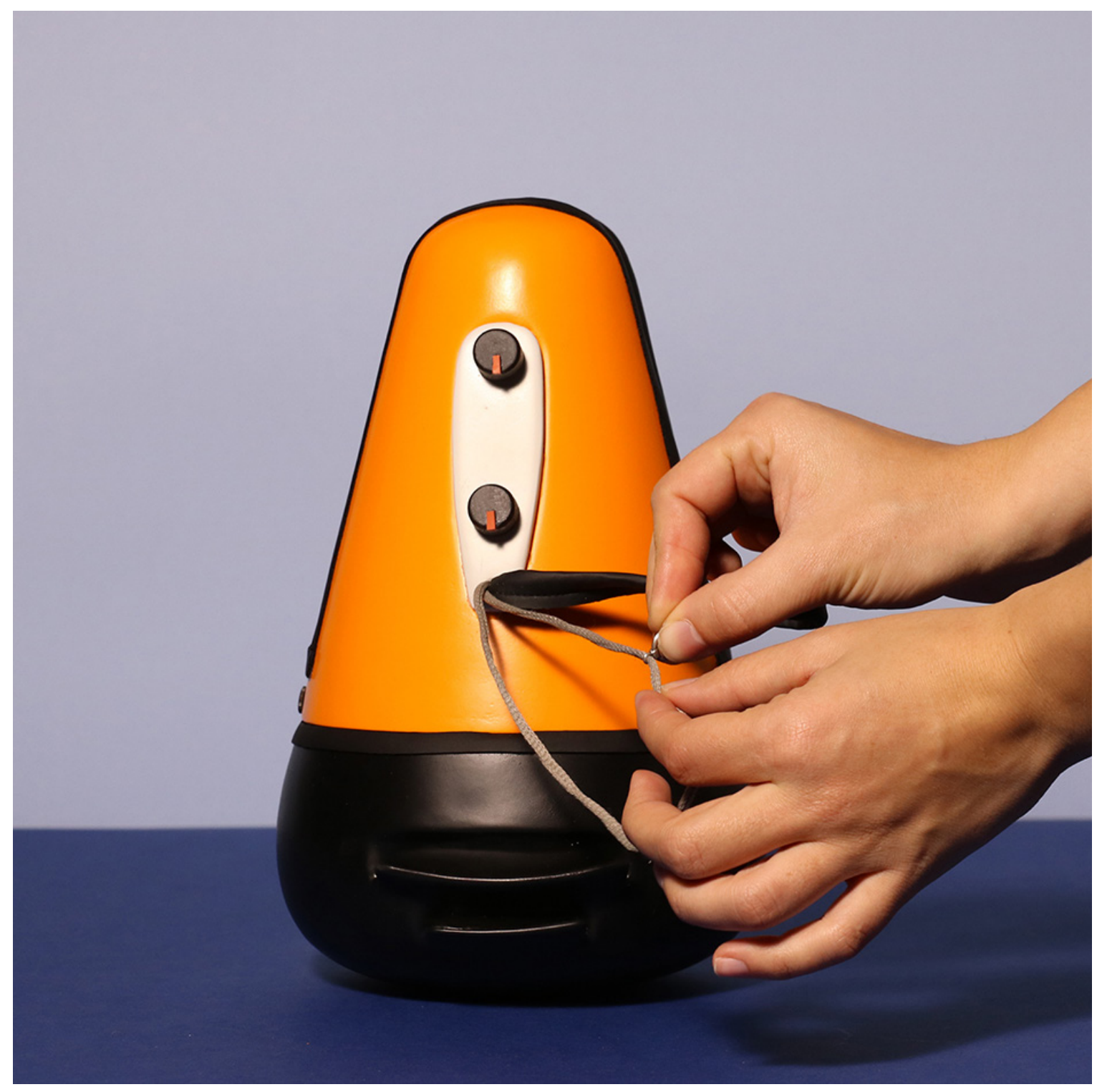

Prototype 05 Soff potentio metar to change the troming

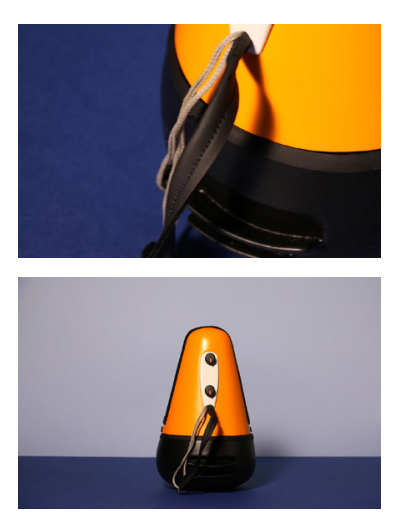

The user can decide the traing intensity by pulling on a soft potentionster at the back of the radio. 


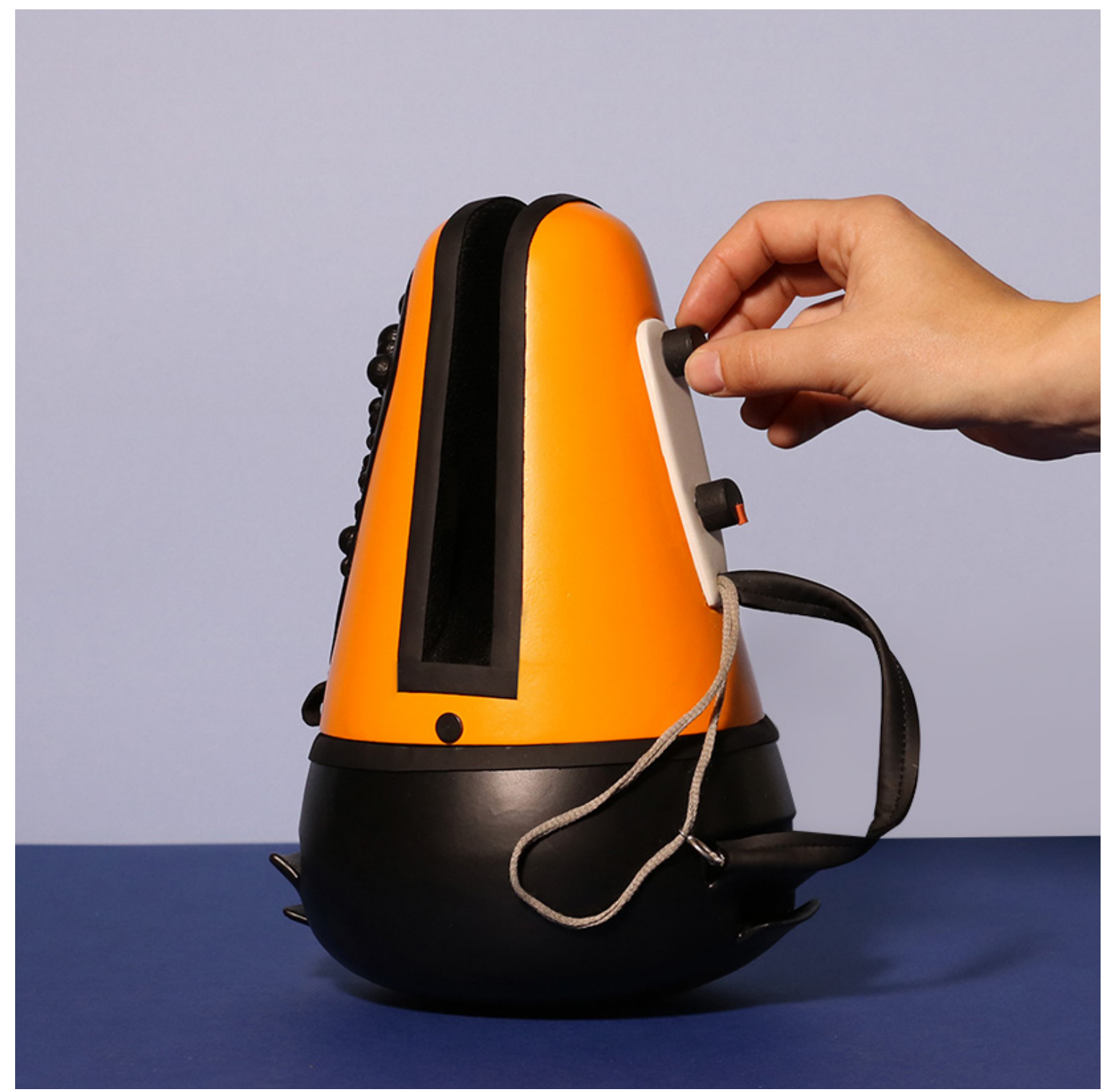

Protolype o5 Side via

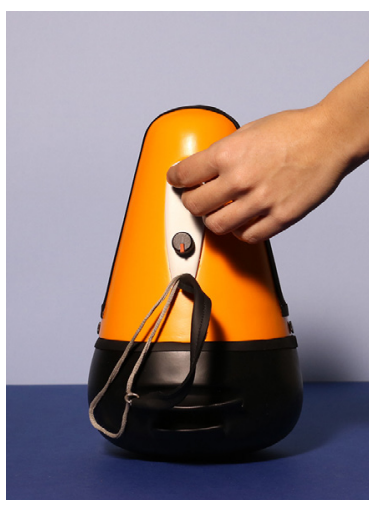




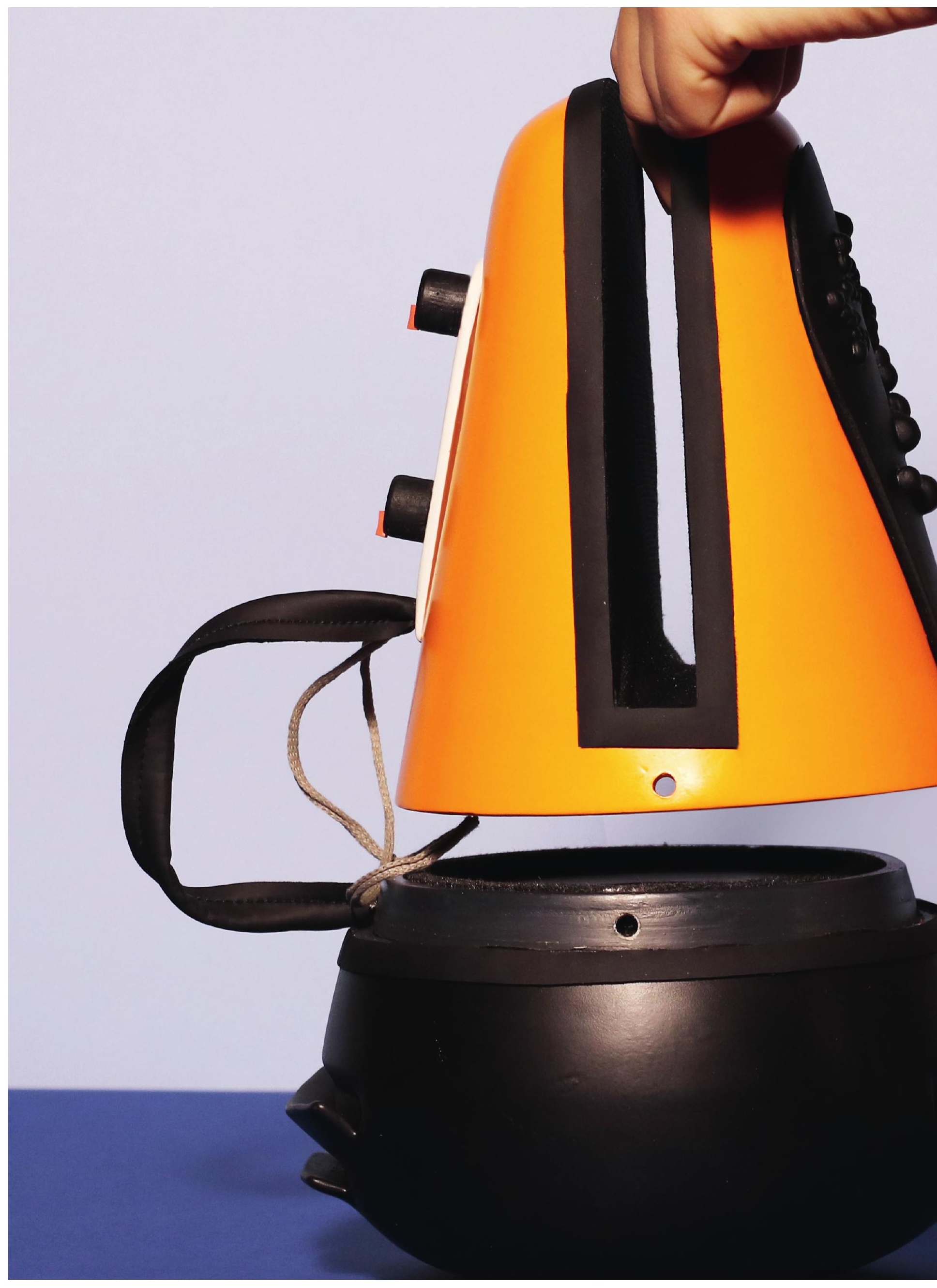




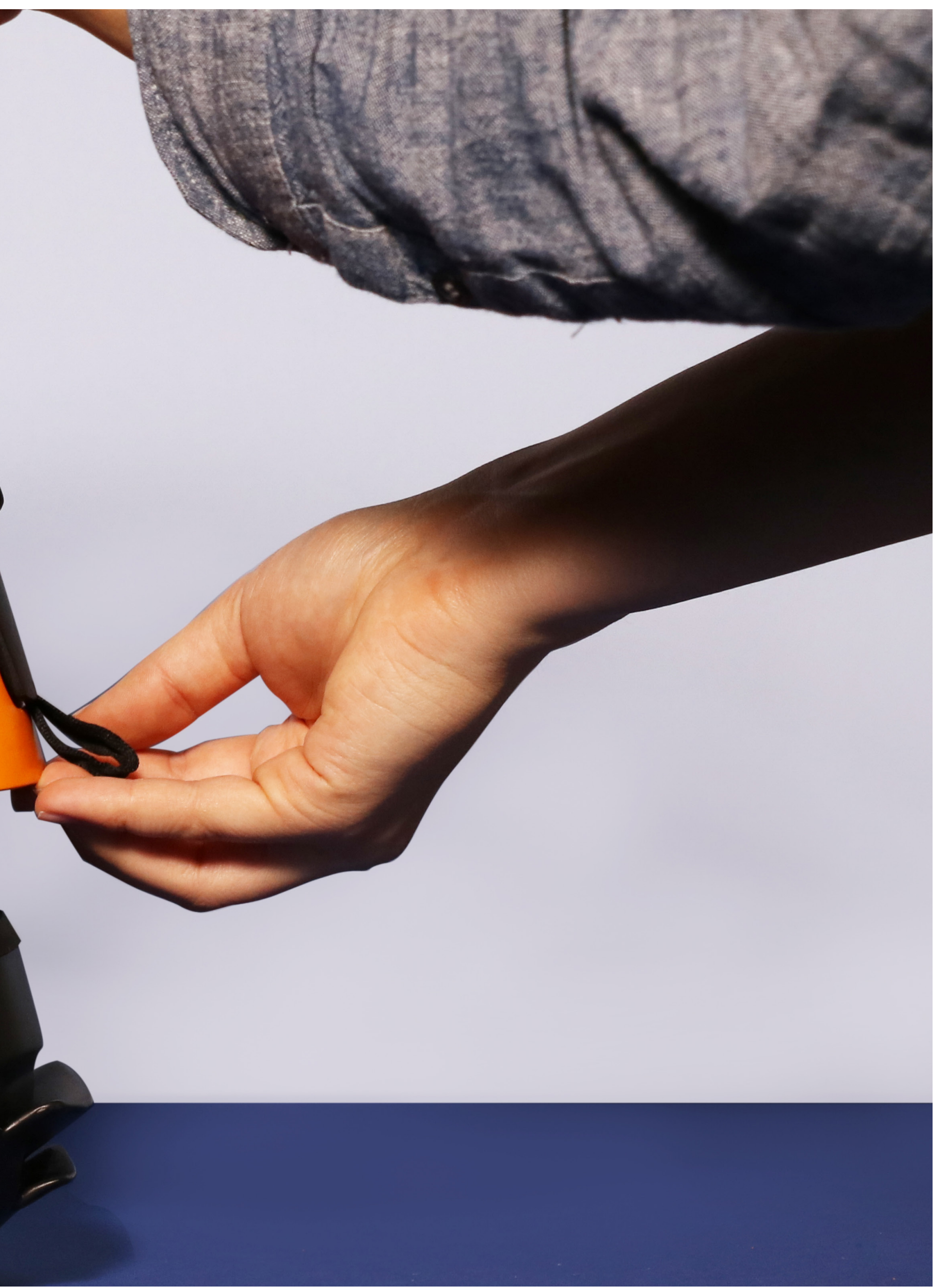




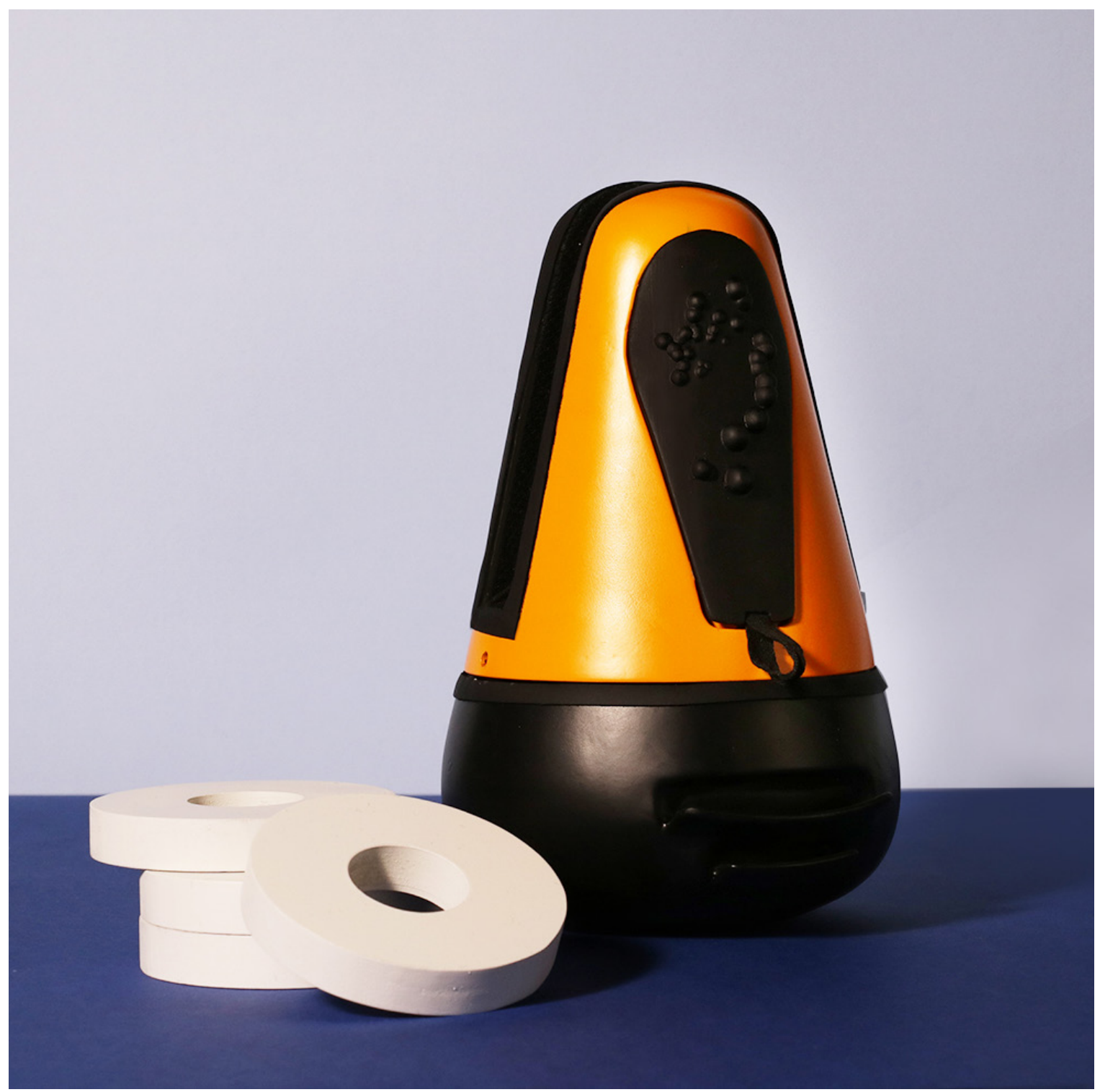

Prototype 05 weights

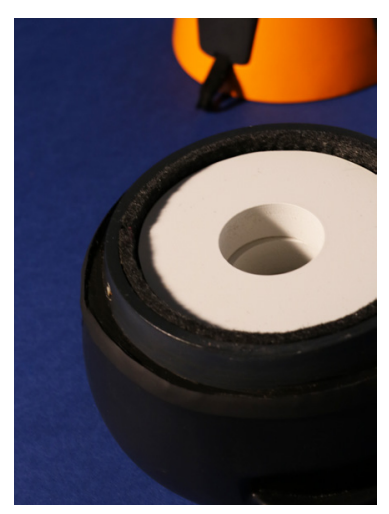

The difficulty of the traning reeds to increase over time so the user is able to pigress in the rhabilatation process the radio incorporates piogress by using different weigths in the base that cun $b$ adeled or taken out depending on the progress. 


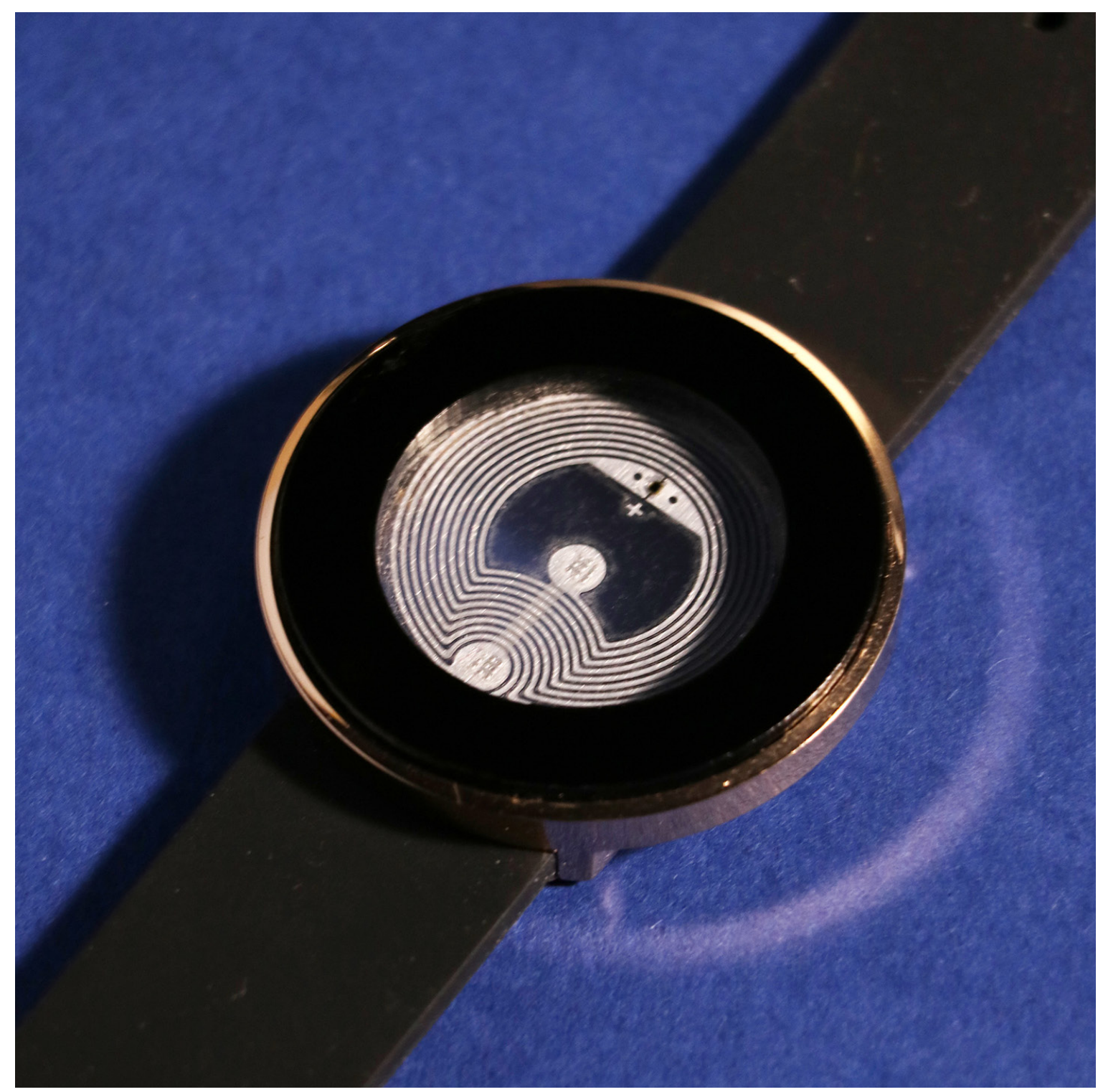

NFC bracelet

I used NFC technology to restrain the movenent

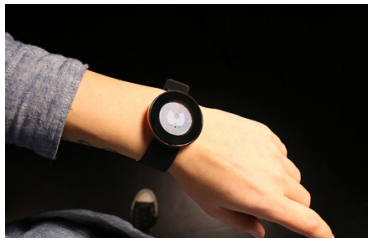

The user is wearing on DTFC bracelet. If the

user uses the wrong. less affected-side

the racho will just not turn on. The

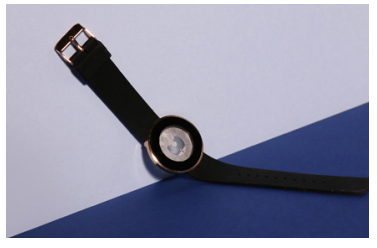

electroncs involve on Arduino lno bavd,

NFC shield and a radis boord. 


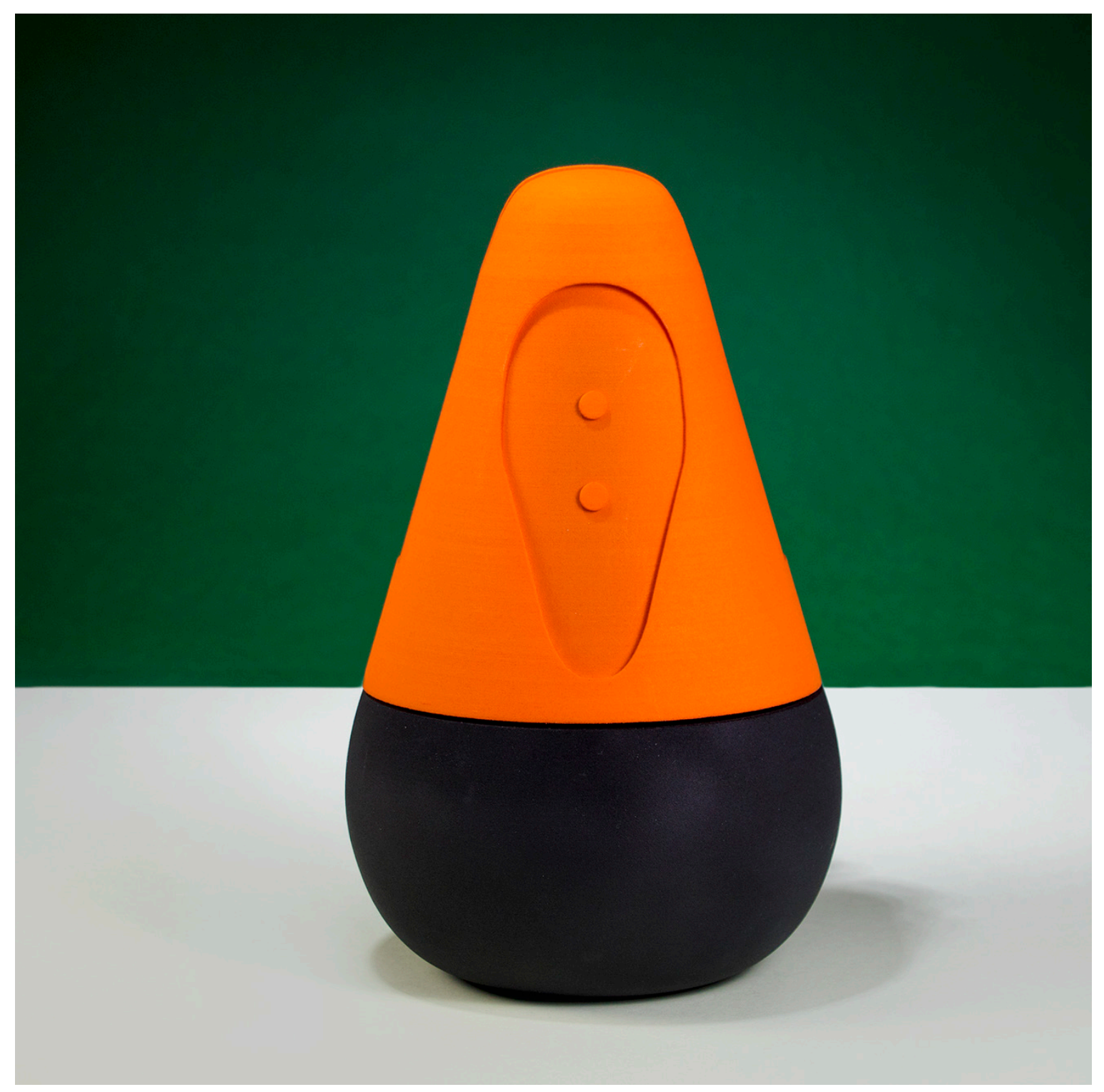

Final design prototype

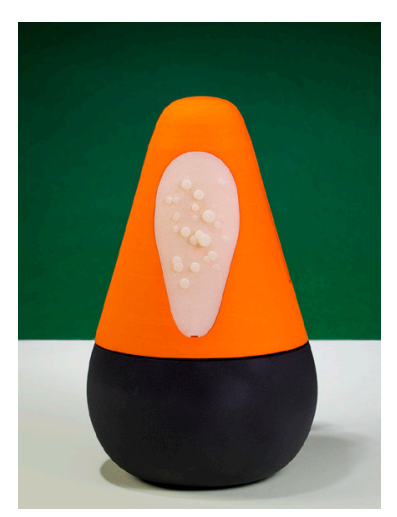




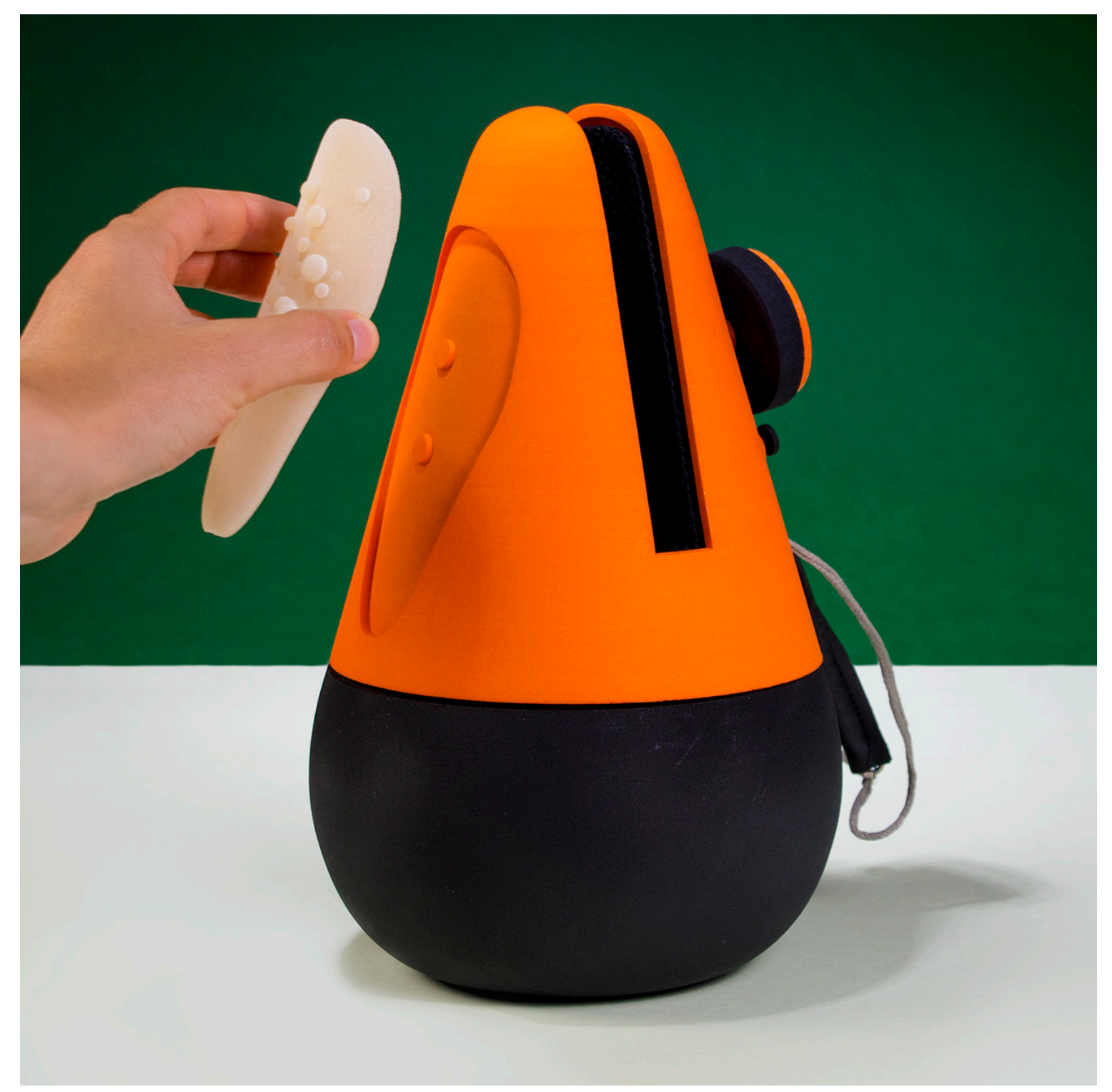

Final design prototype

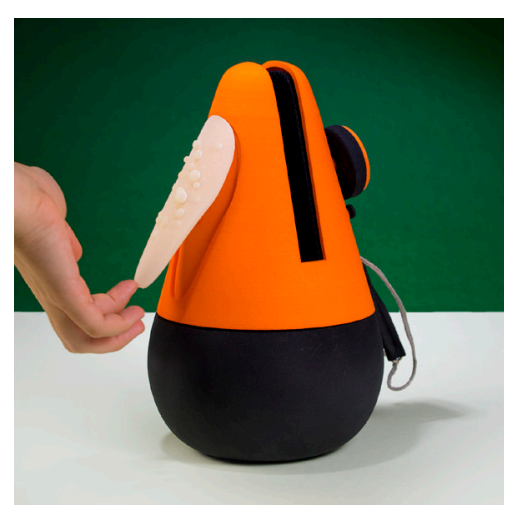




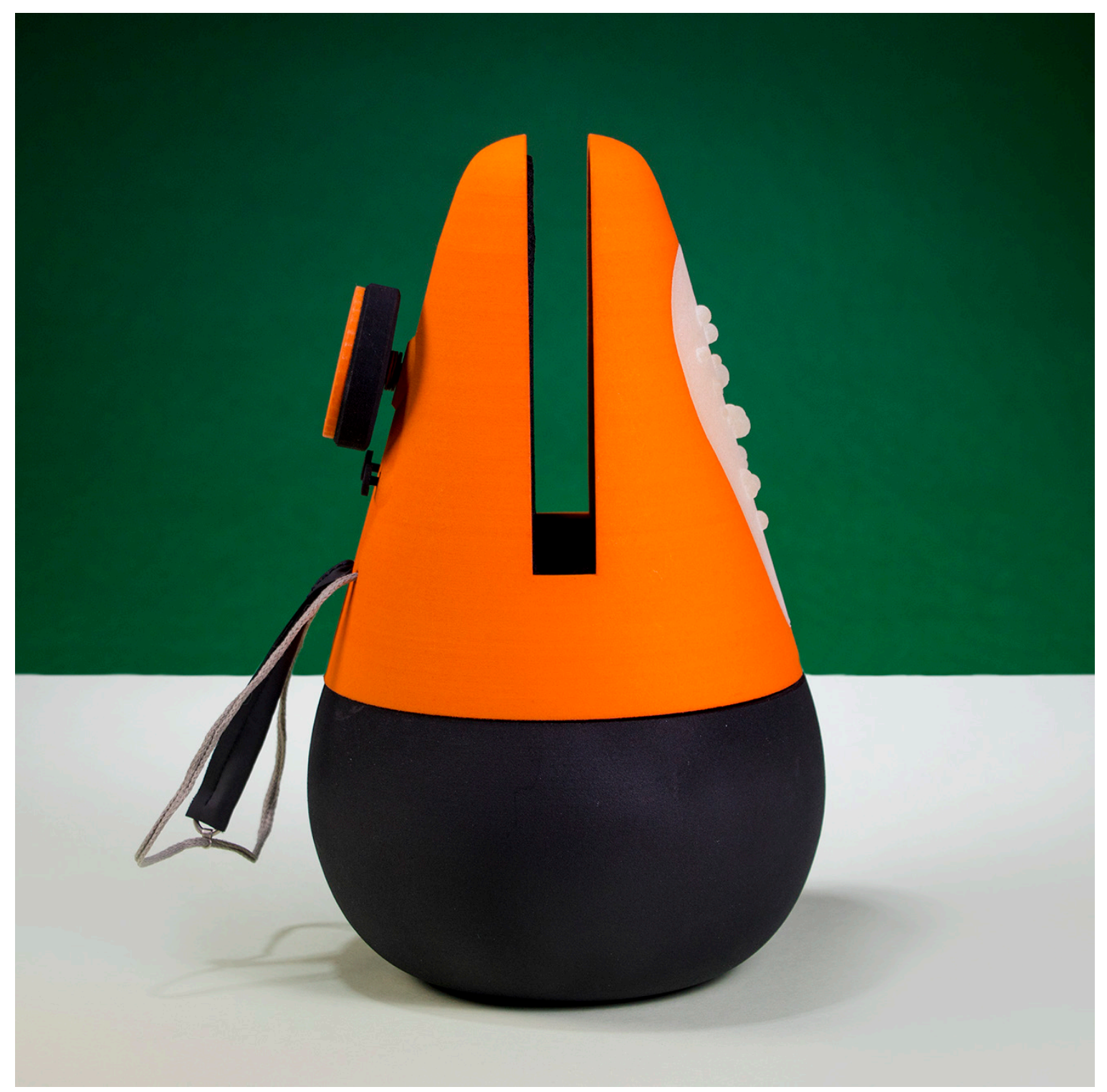

Final design proldzpe Sideview 


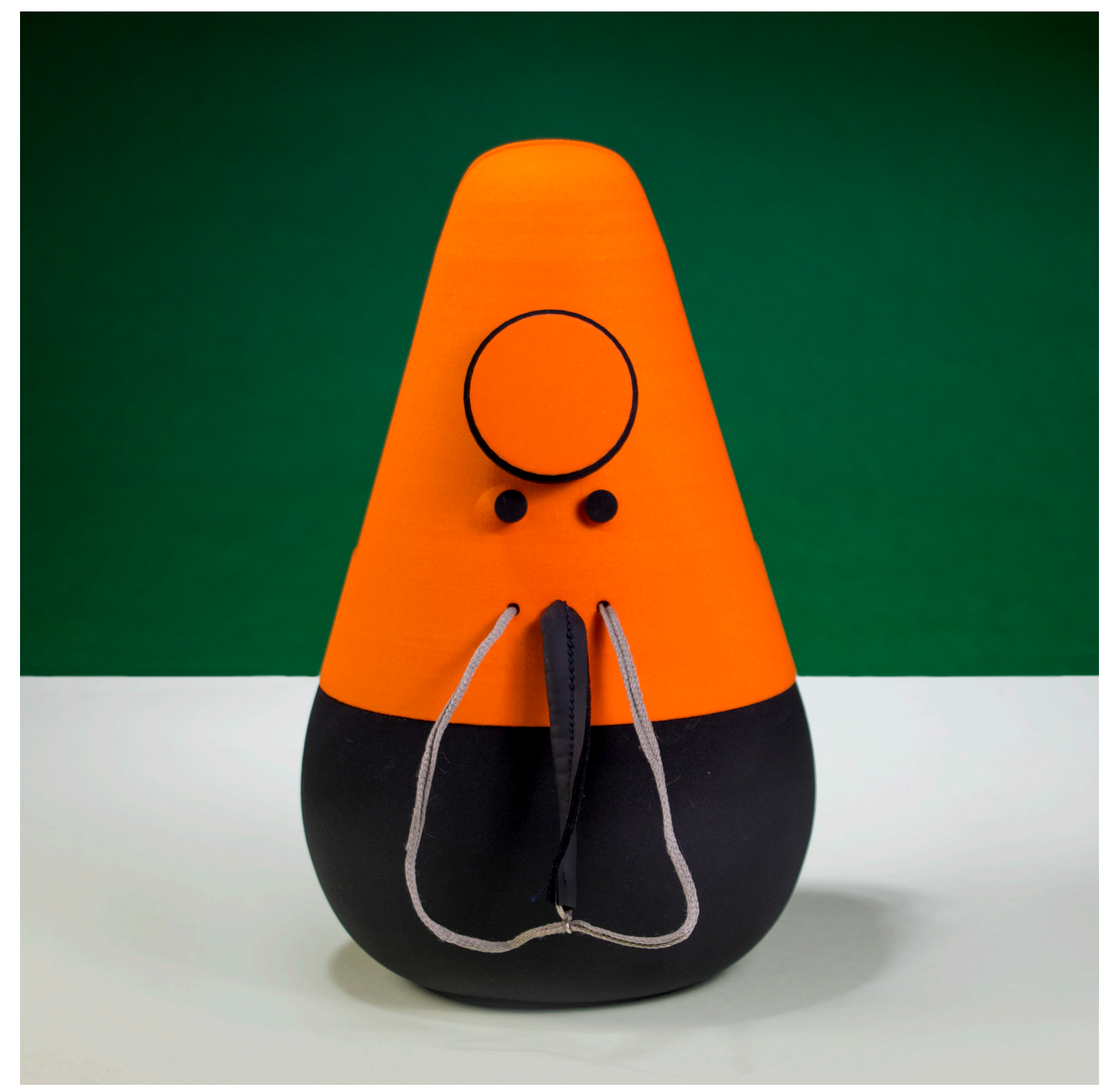

Finol design protdjpe Backeview 


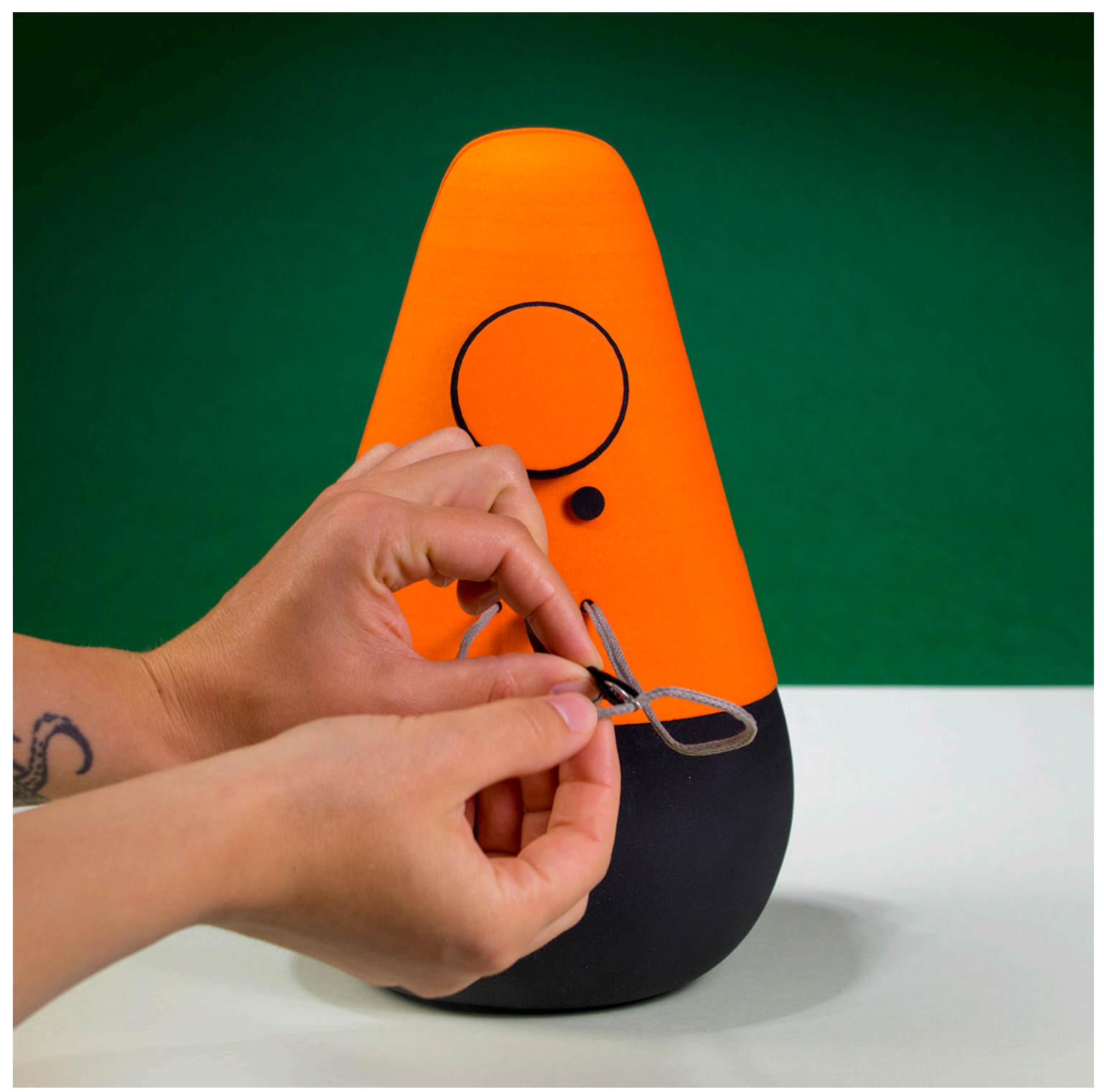

Find design proldepp: Chanjing the traning stling

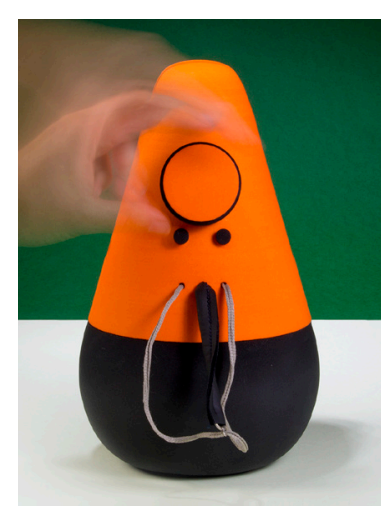

Finol design proldype: Changing the volume 


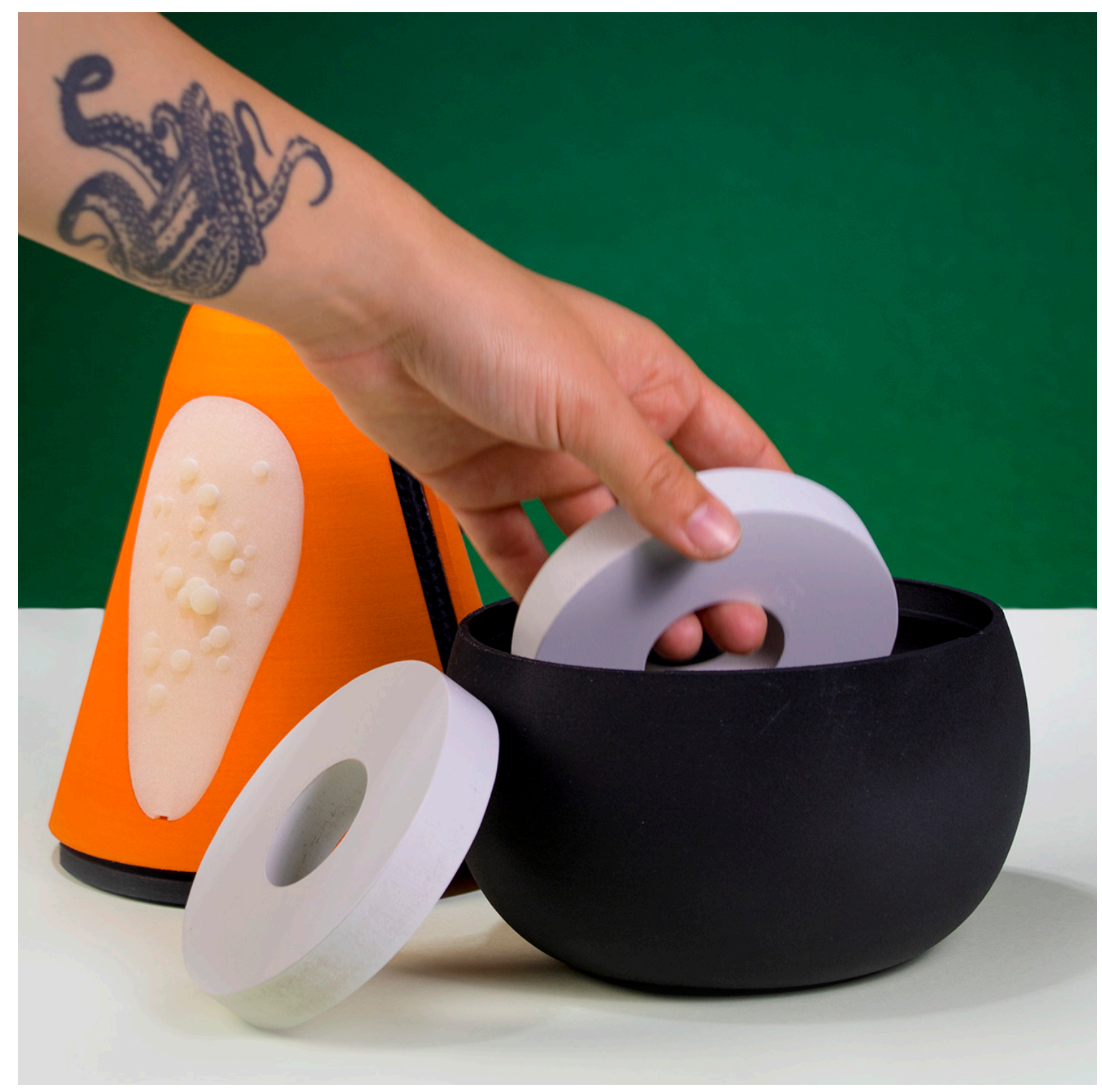

Final design prototype

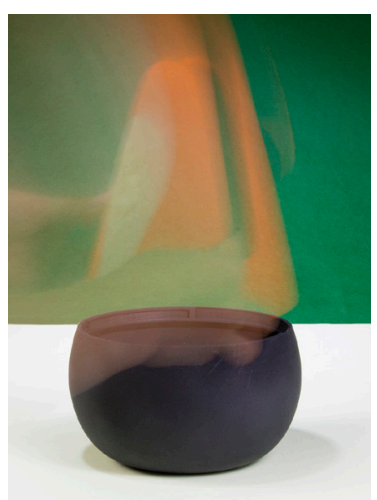





\section{Appendix C Workbook 2}



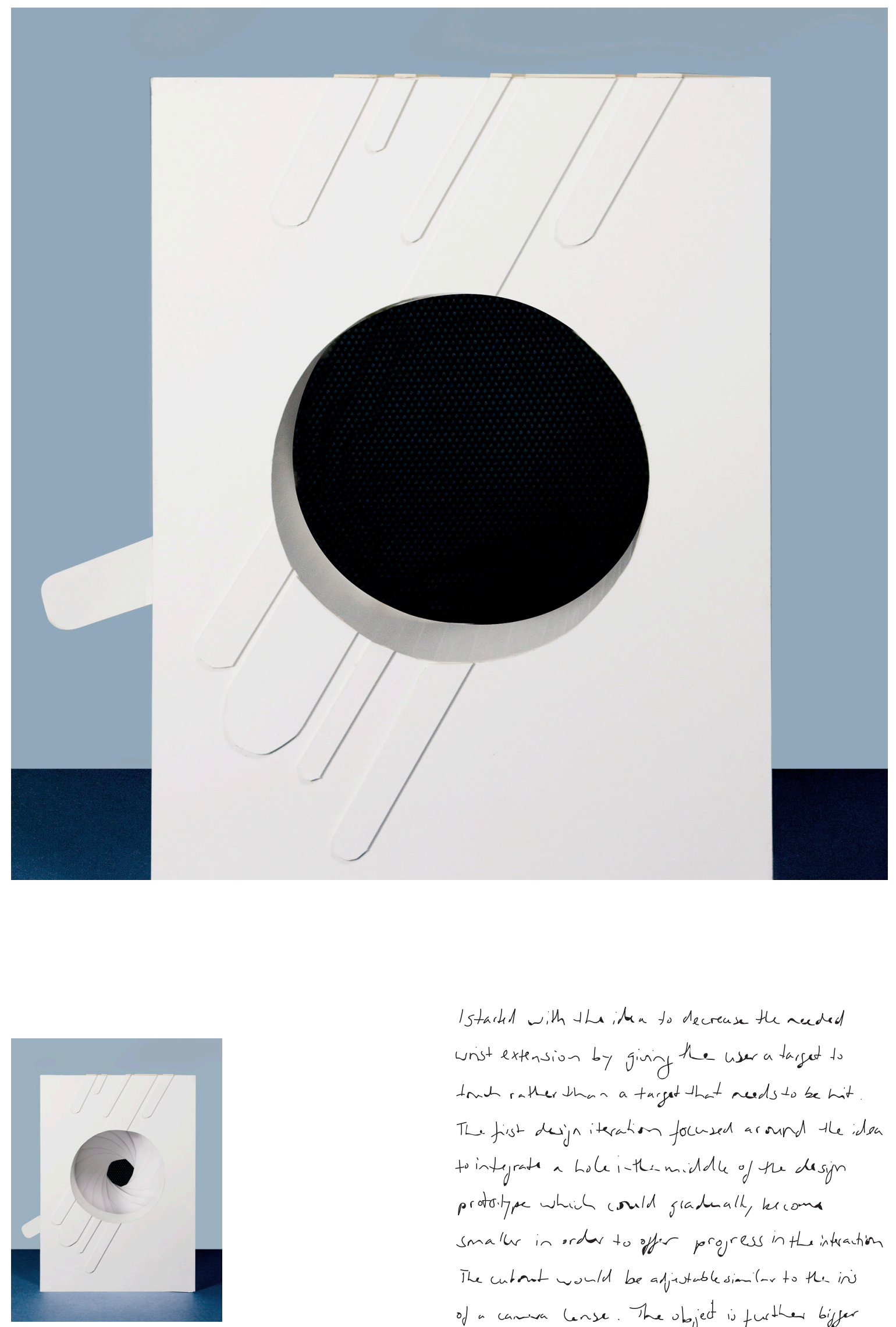

Istarkd with the itan to decrease the needed wrist extension by giving he user a target to truch rather than a target that needs to be hit The fist derijn iteration focused around the idea to integrate a hole inthemiddle of the design protolype which could giadealh, ke coma smath in order to offer progress in the interaction The cutant would be afjustablesionilar to the ins of a camera lanse. The object is further bigger than 'The bijgest Hit+I' to increase the stability of the object and decrease the risk of diapping it the the floor. 

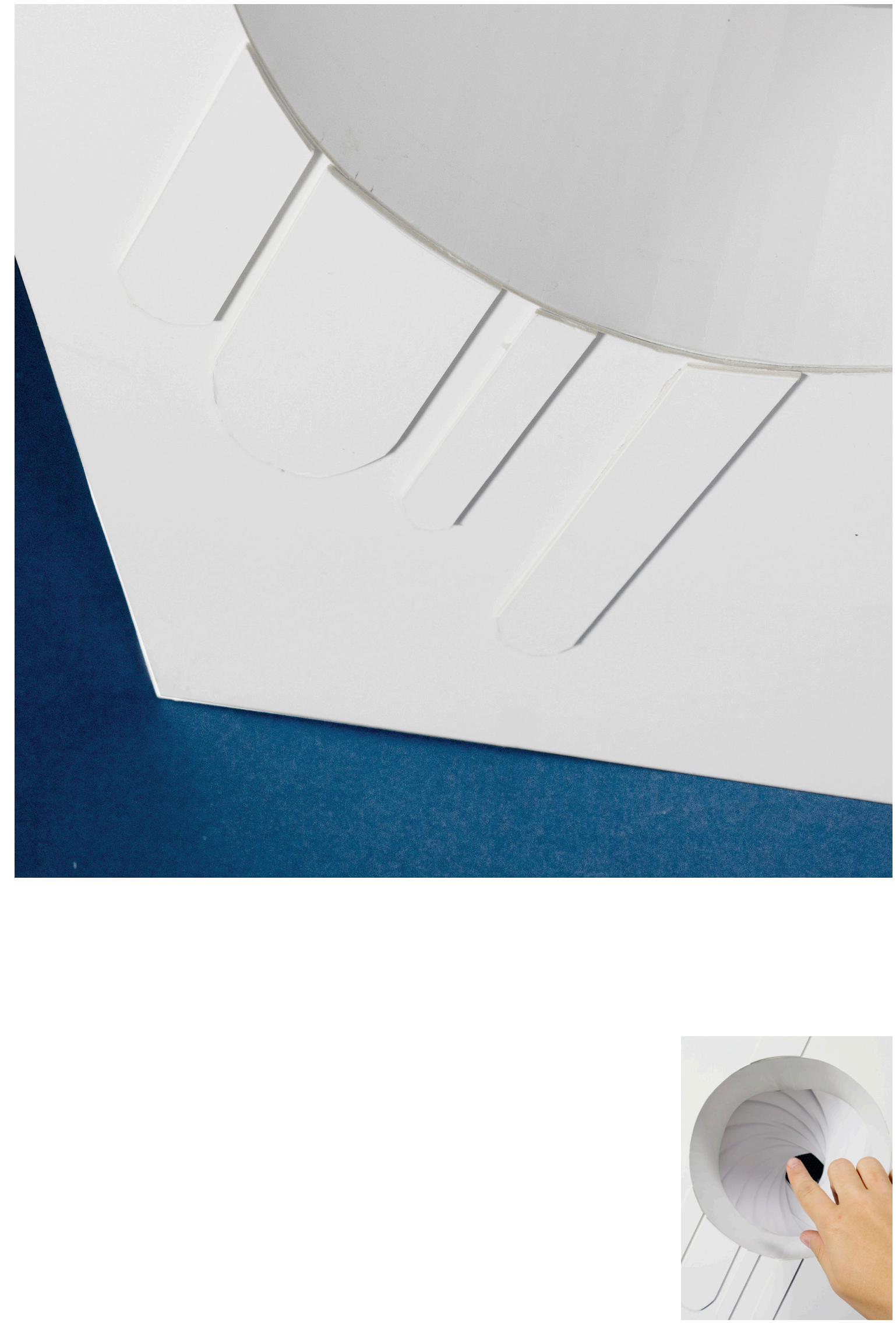


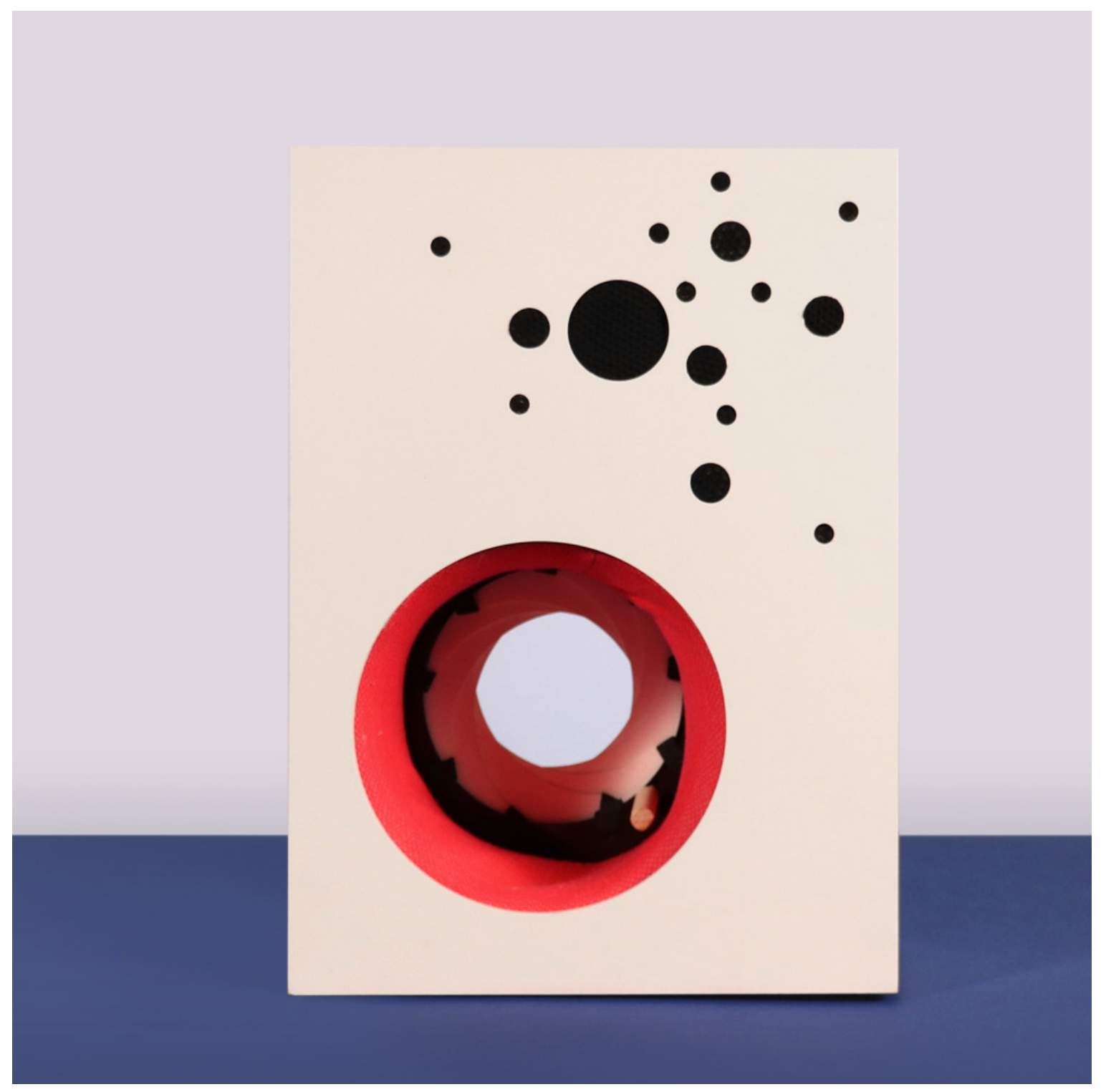

Pidolype ol

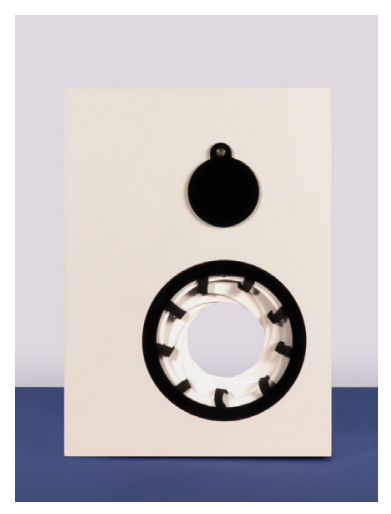




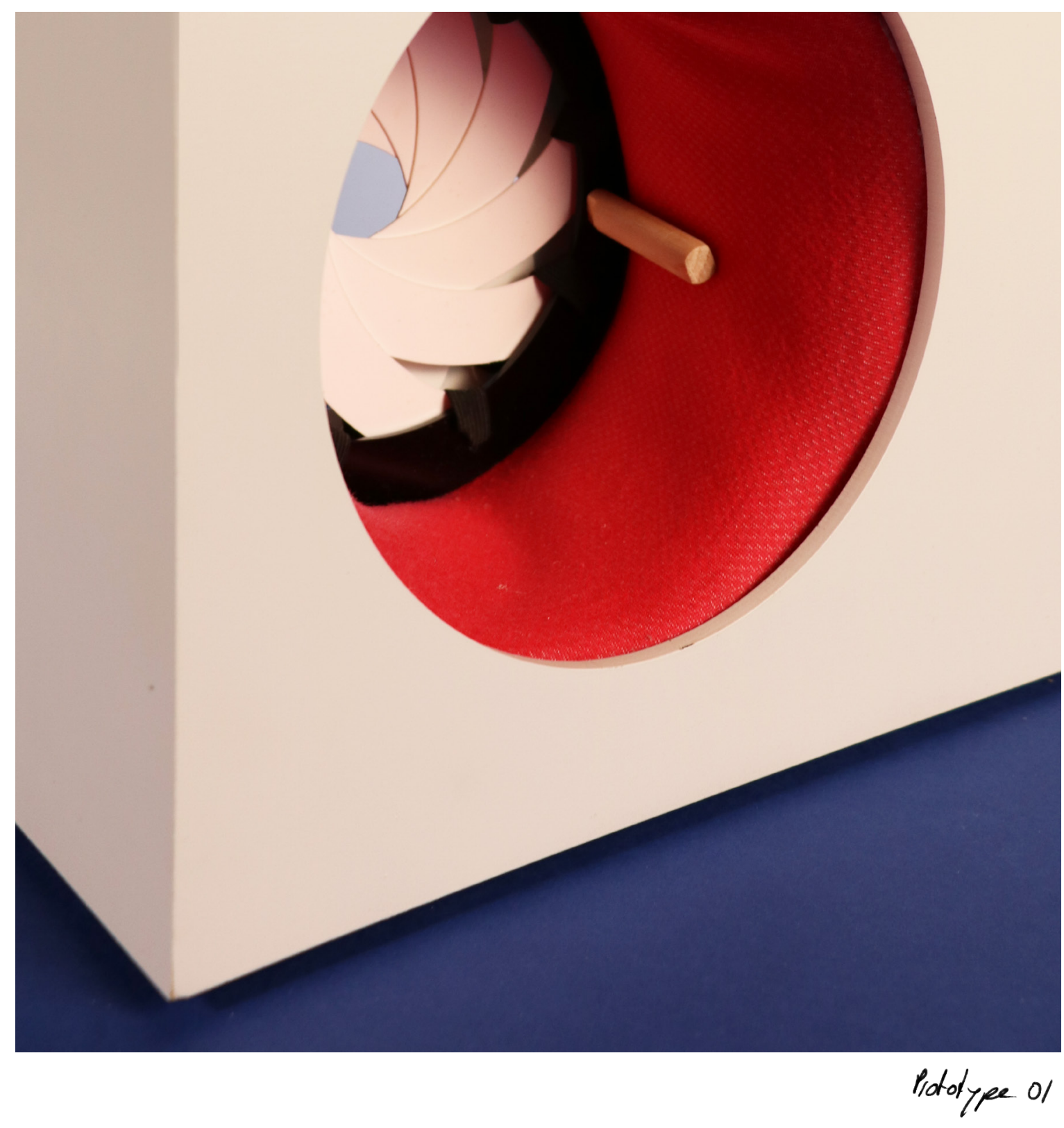

The isteraction with the object can piogressively be made more diffecult by clecreasing the sire of the hole that the user has to reach throught touch in order to turn on the radio.

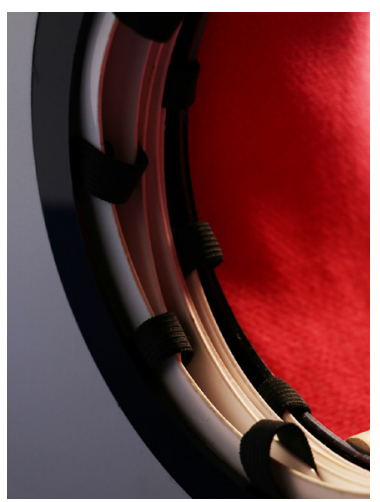



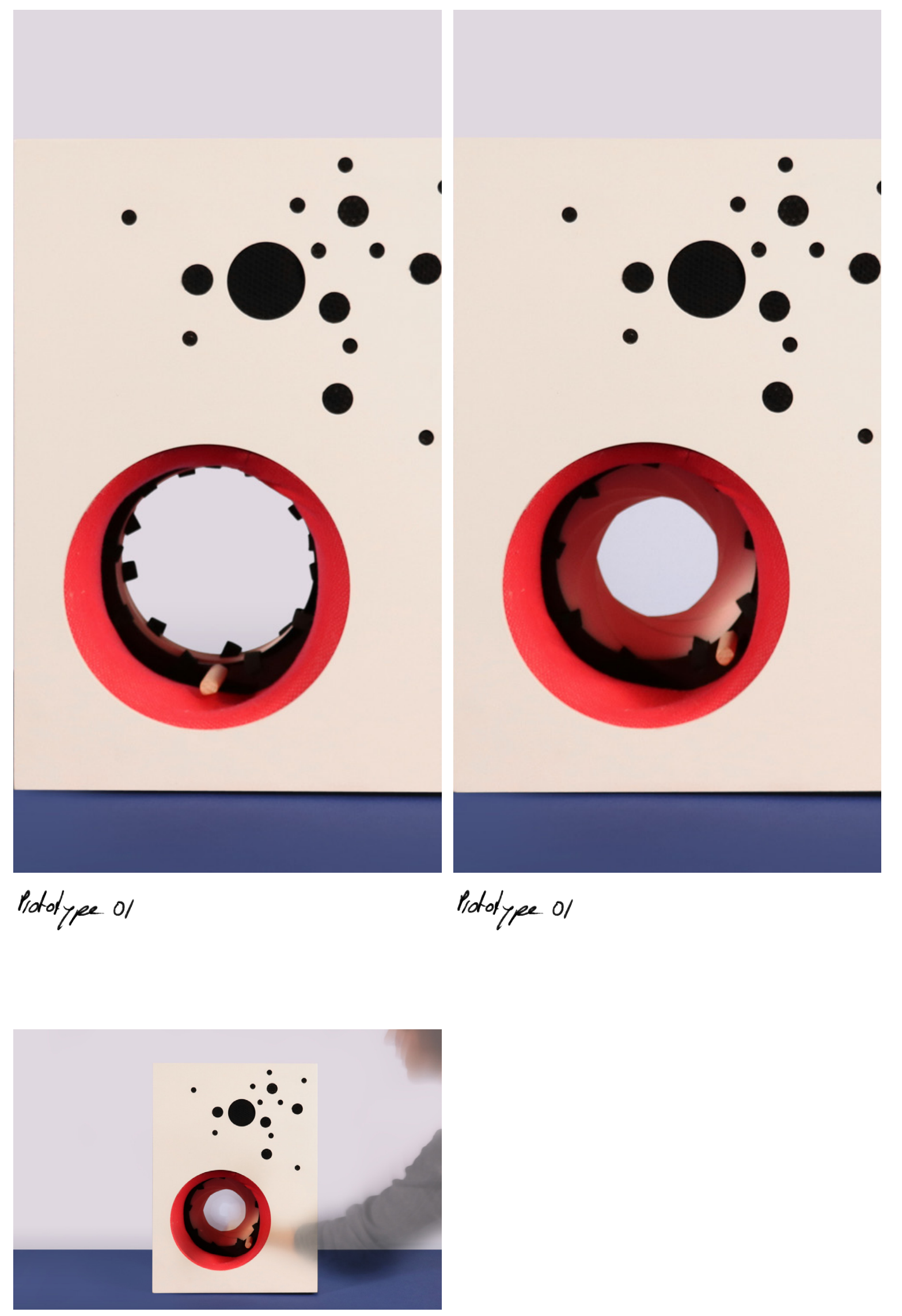


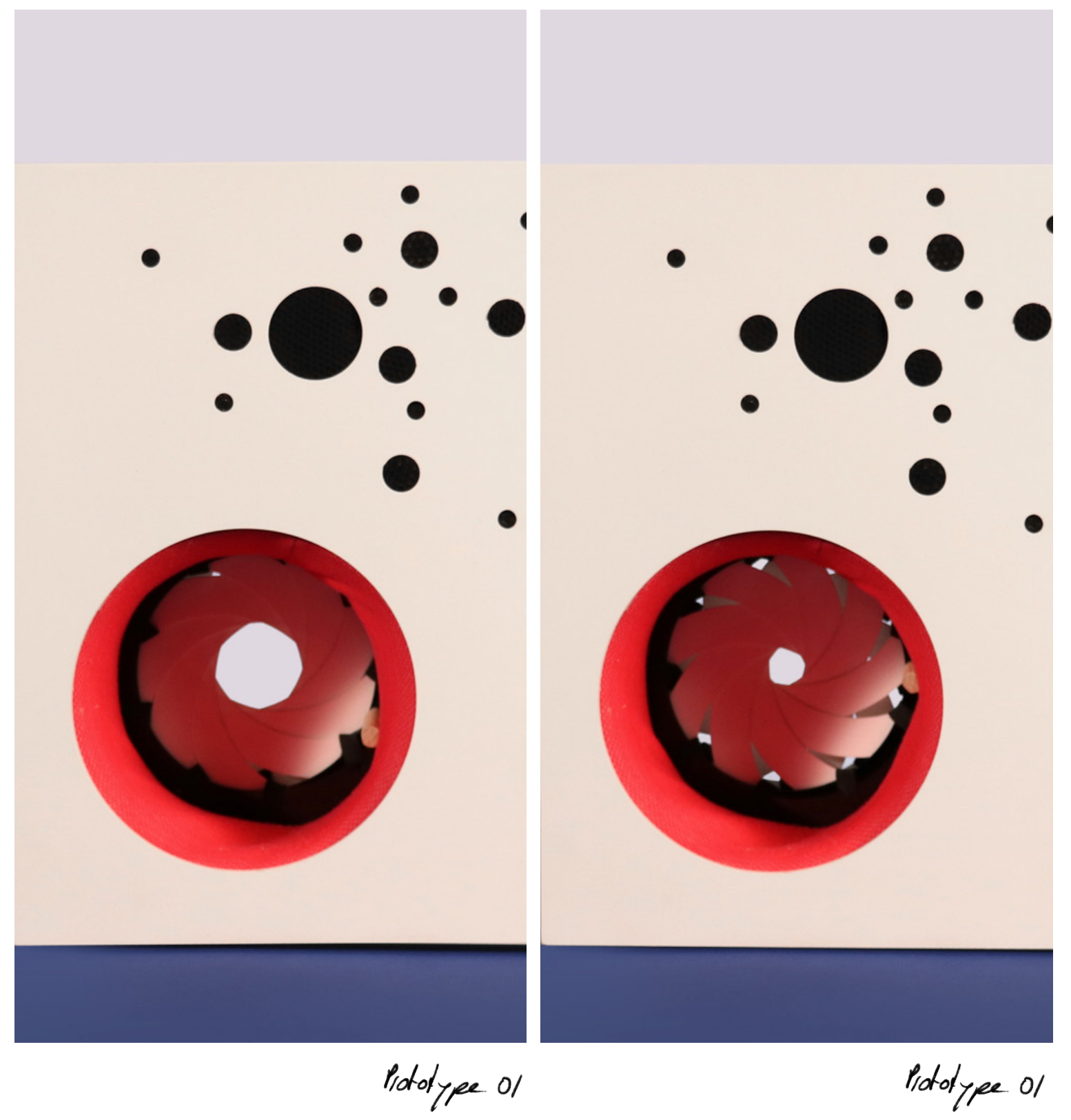




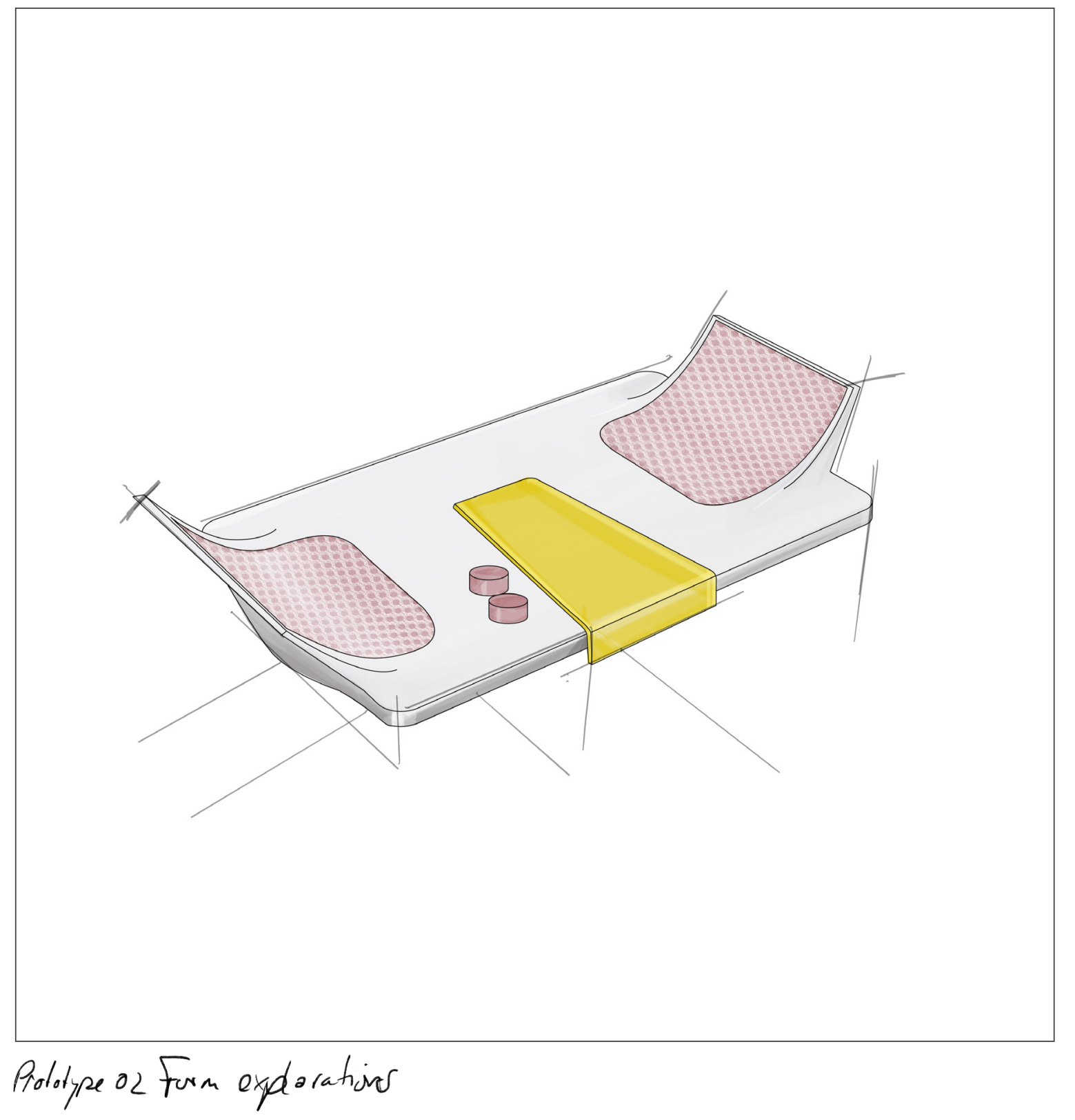

Dunng the testing of the first prototppe Ifound that the reference of the camern lense did not work as smoothly as anticipoted and that the objact due to the hole and the cut-out resembled a minitare washny machne. Itherefre decibd tochange the overall form in a way so the user has to move the arm downwards ratter than fowwords 


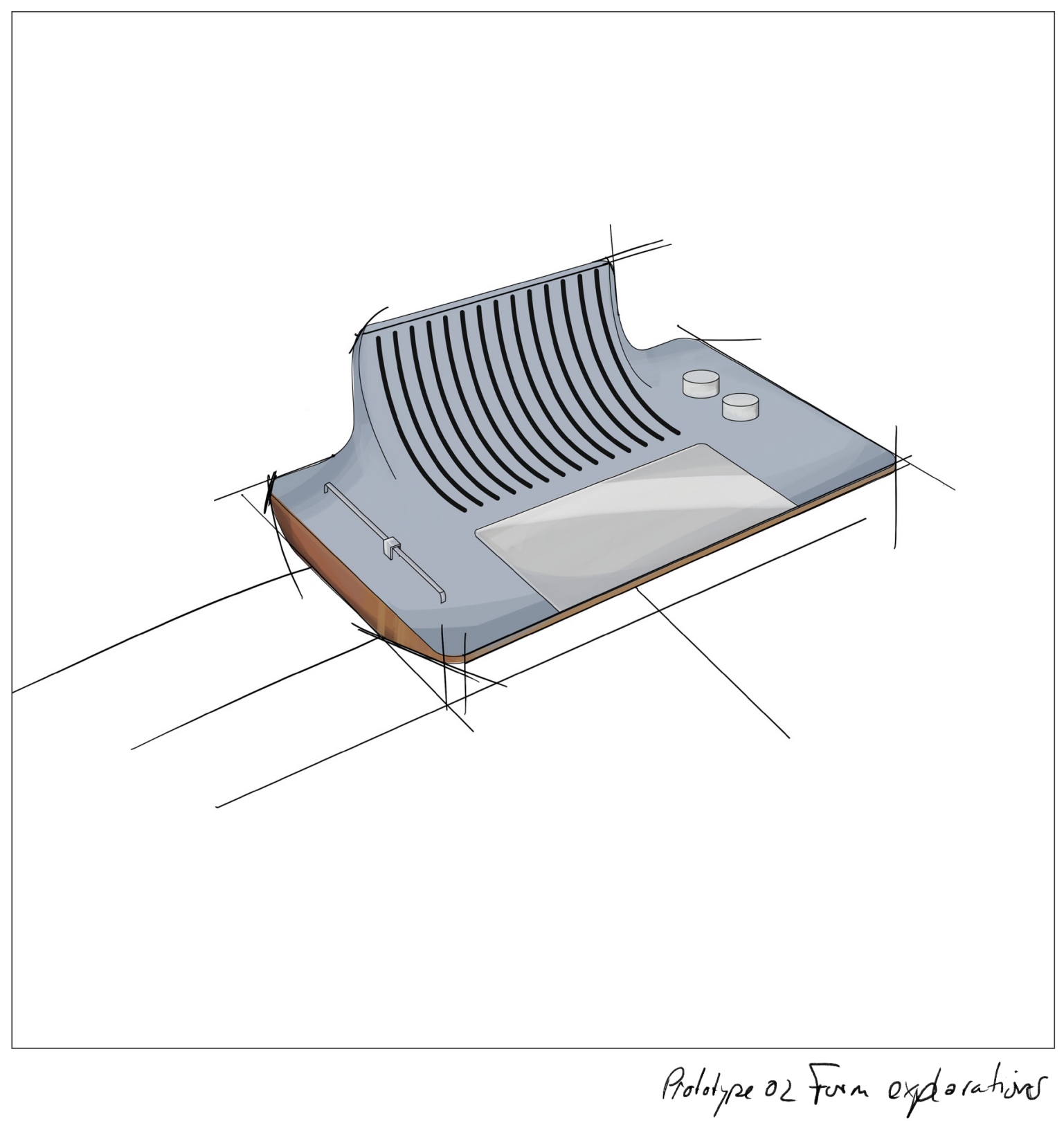



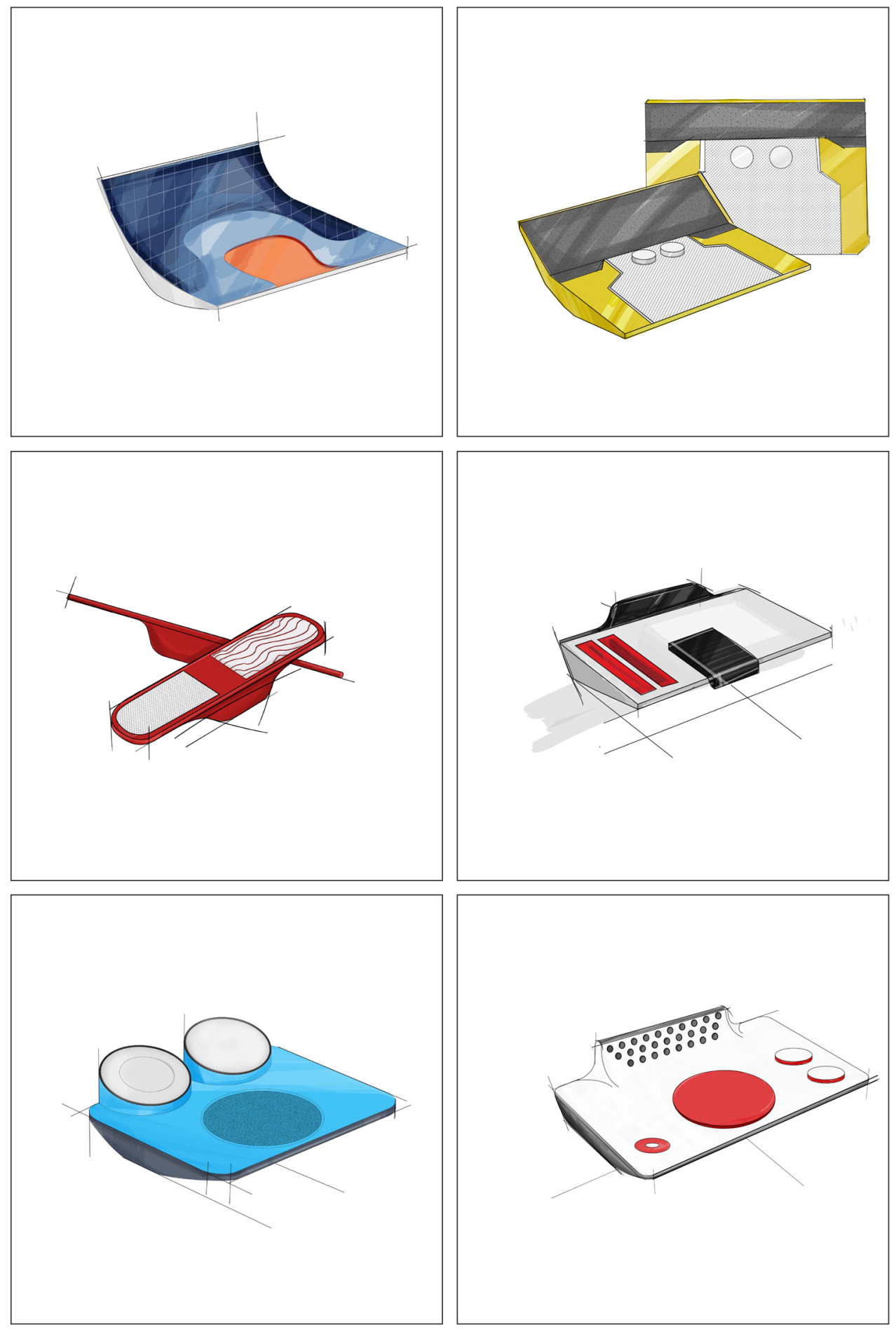

Prololyse 02 form expla rations 

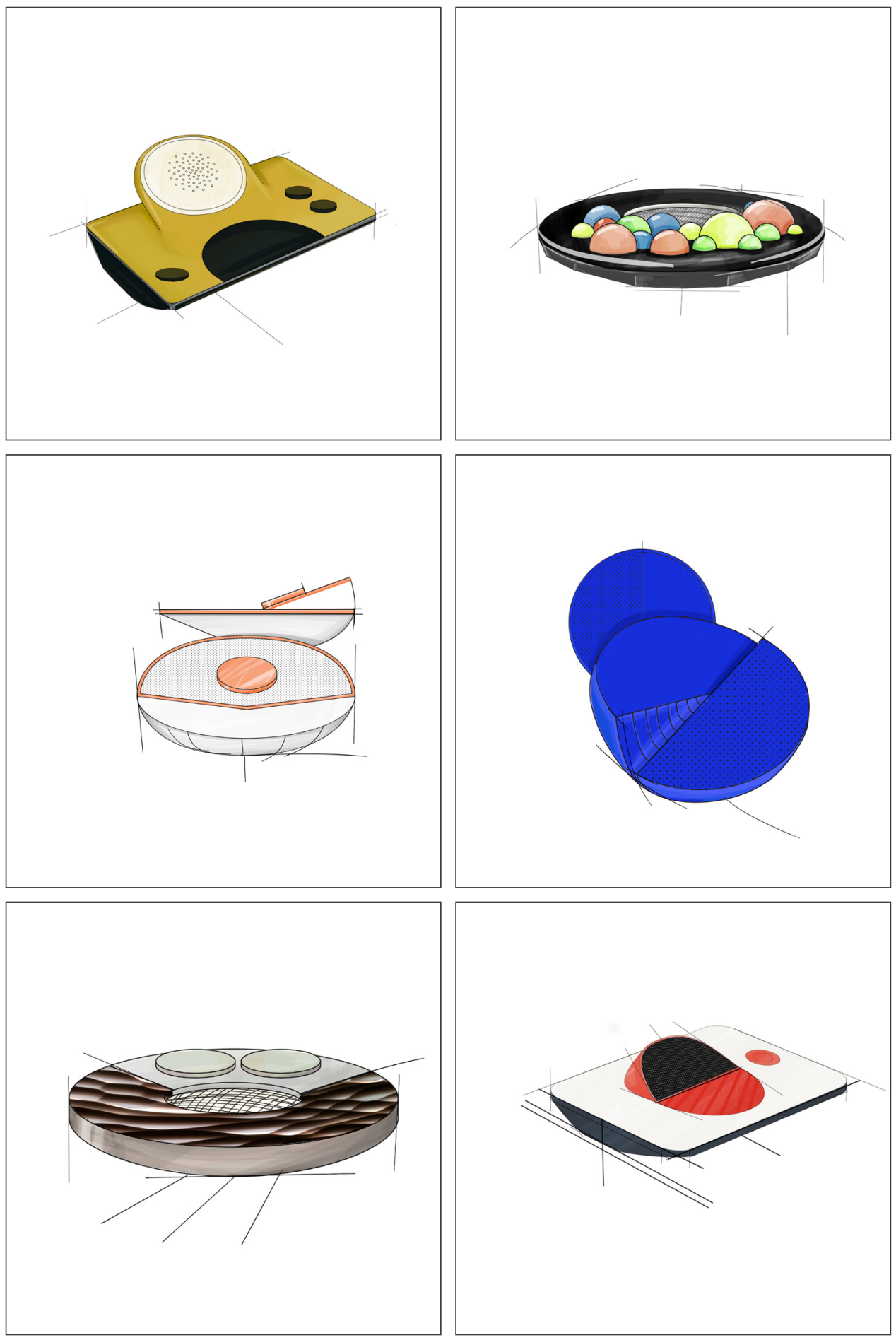

Prolotype ol Form expla a ations 


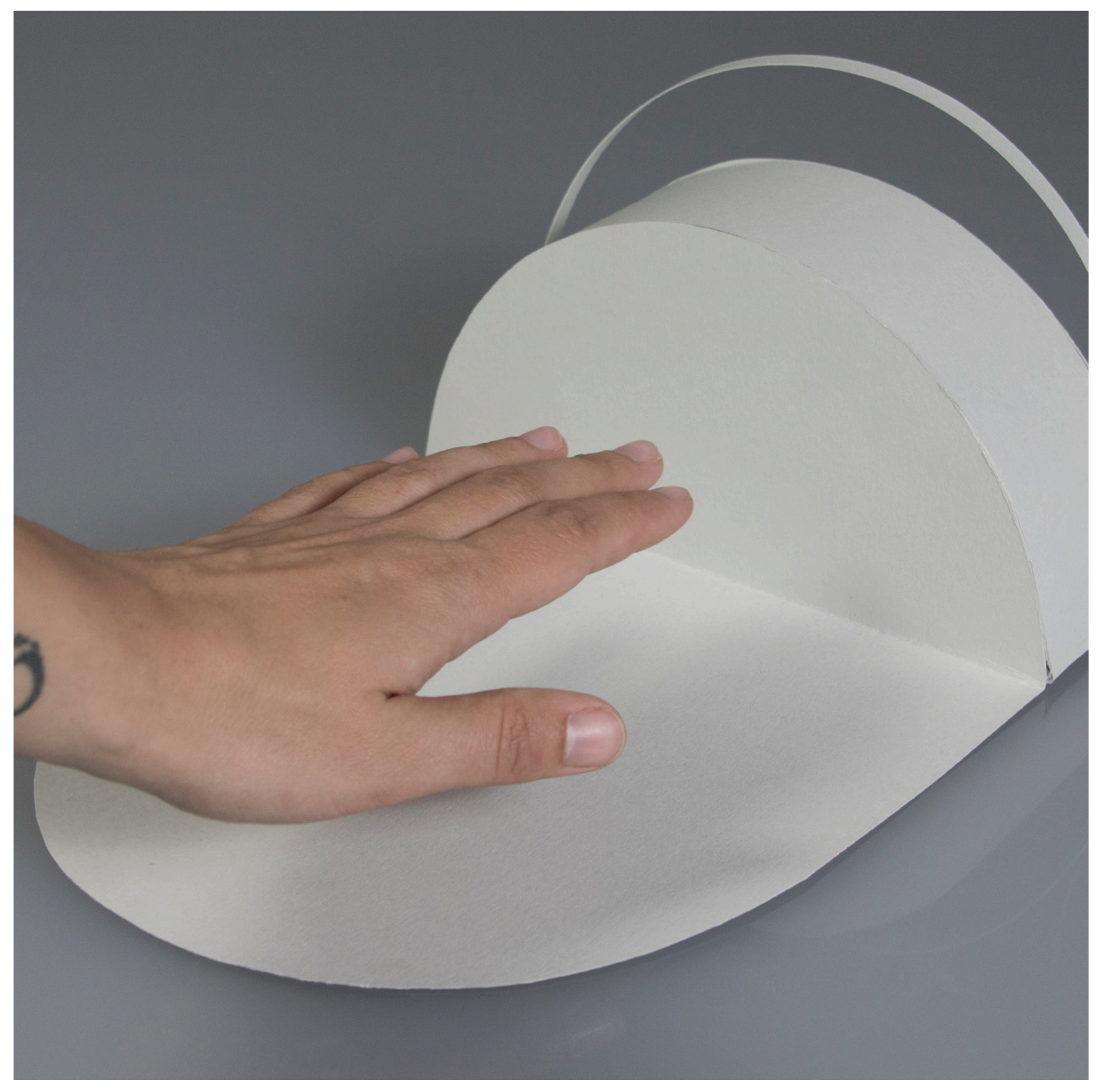




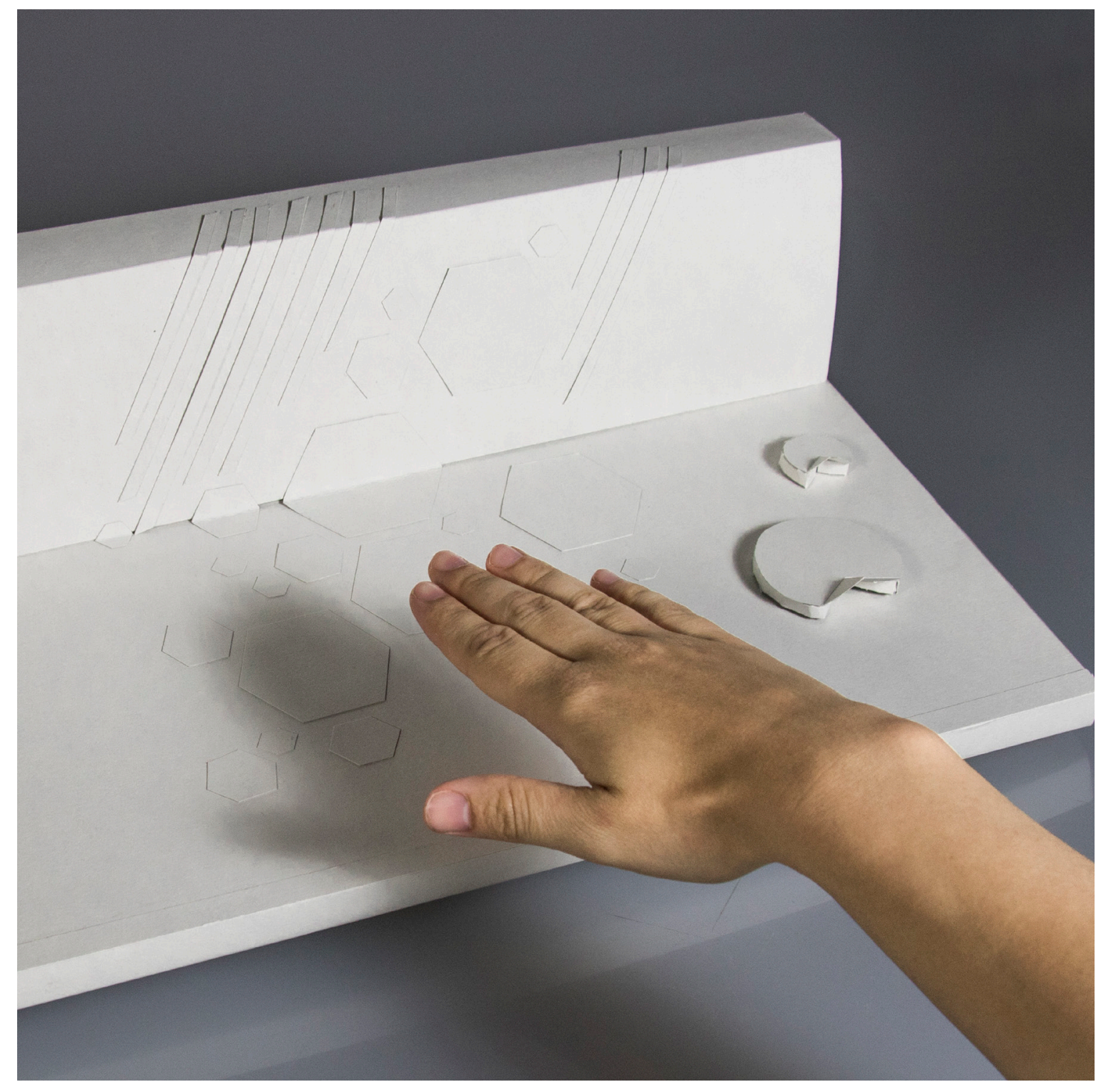




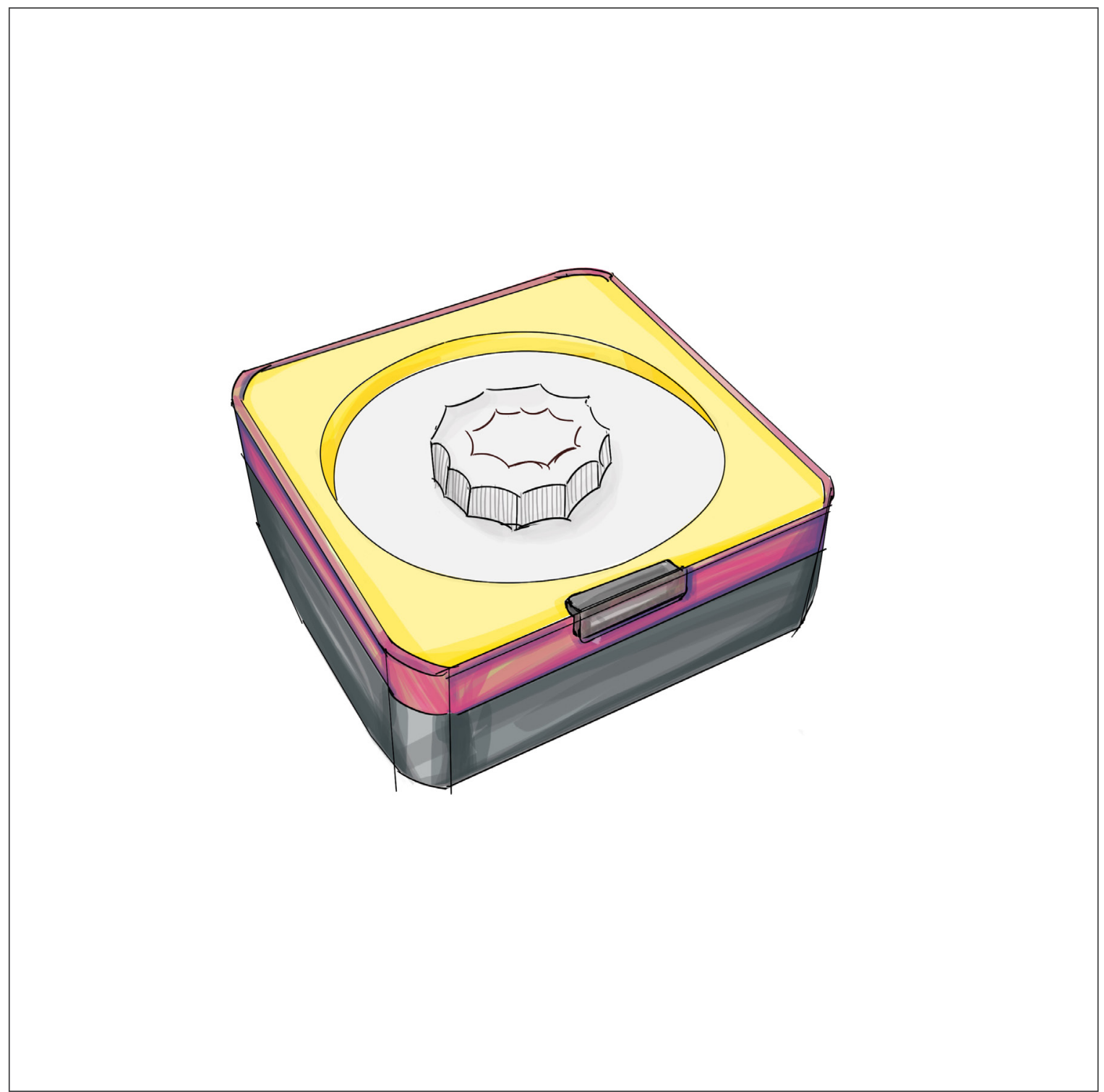

Pidatype os Shettes

I decided to reduce the overall form of the radis

to derease the space that the object would

need in the home environment. In order to

increase the functional feedformard I decided

to increase the man button on the front surface

that allous to repulate the volume of the ridis. 


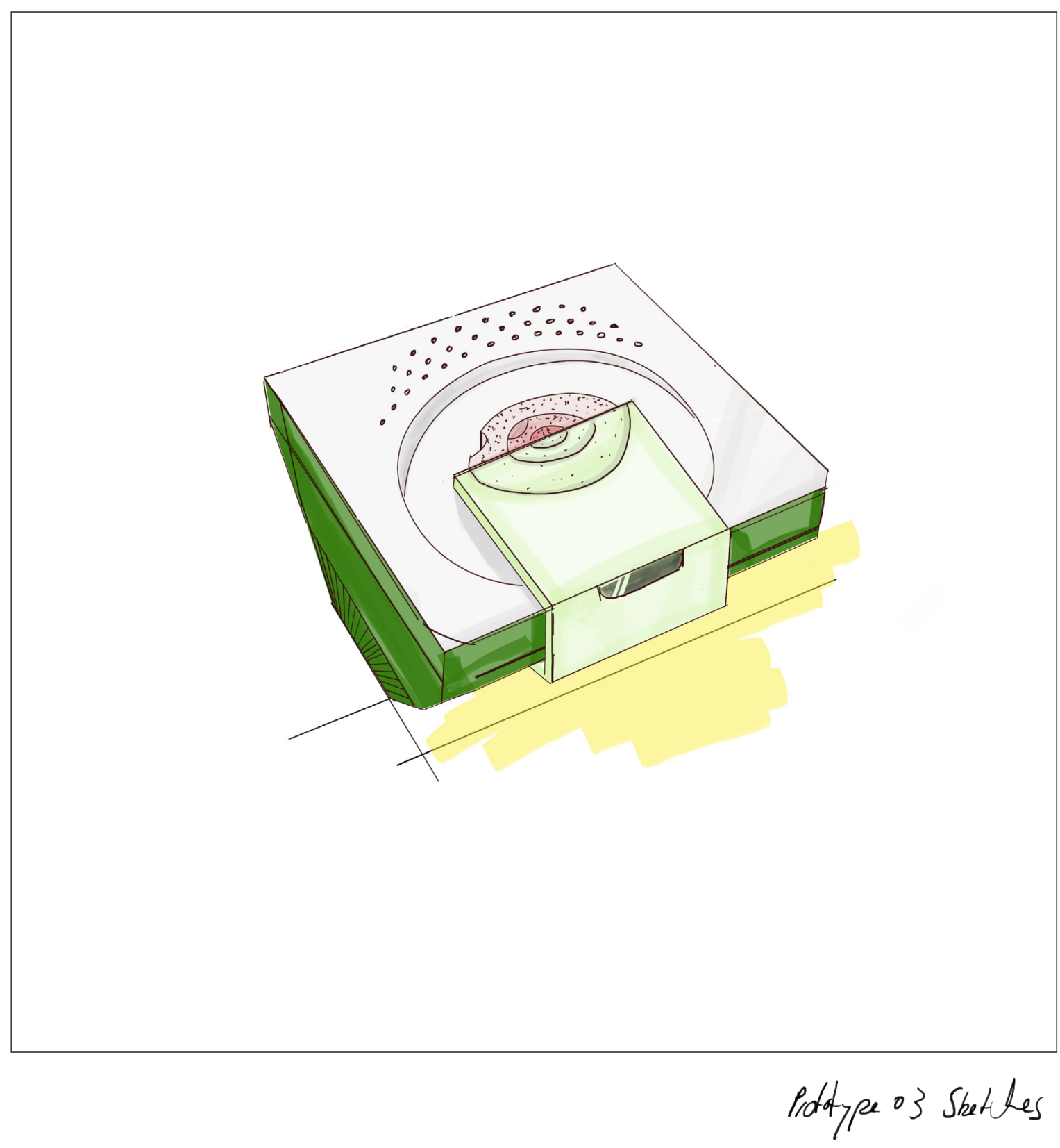




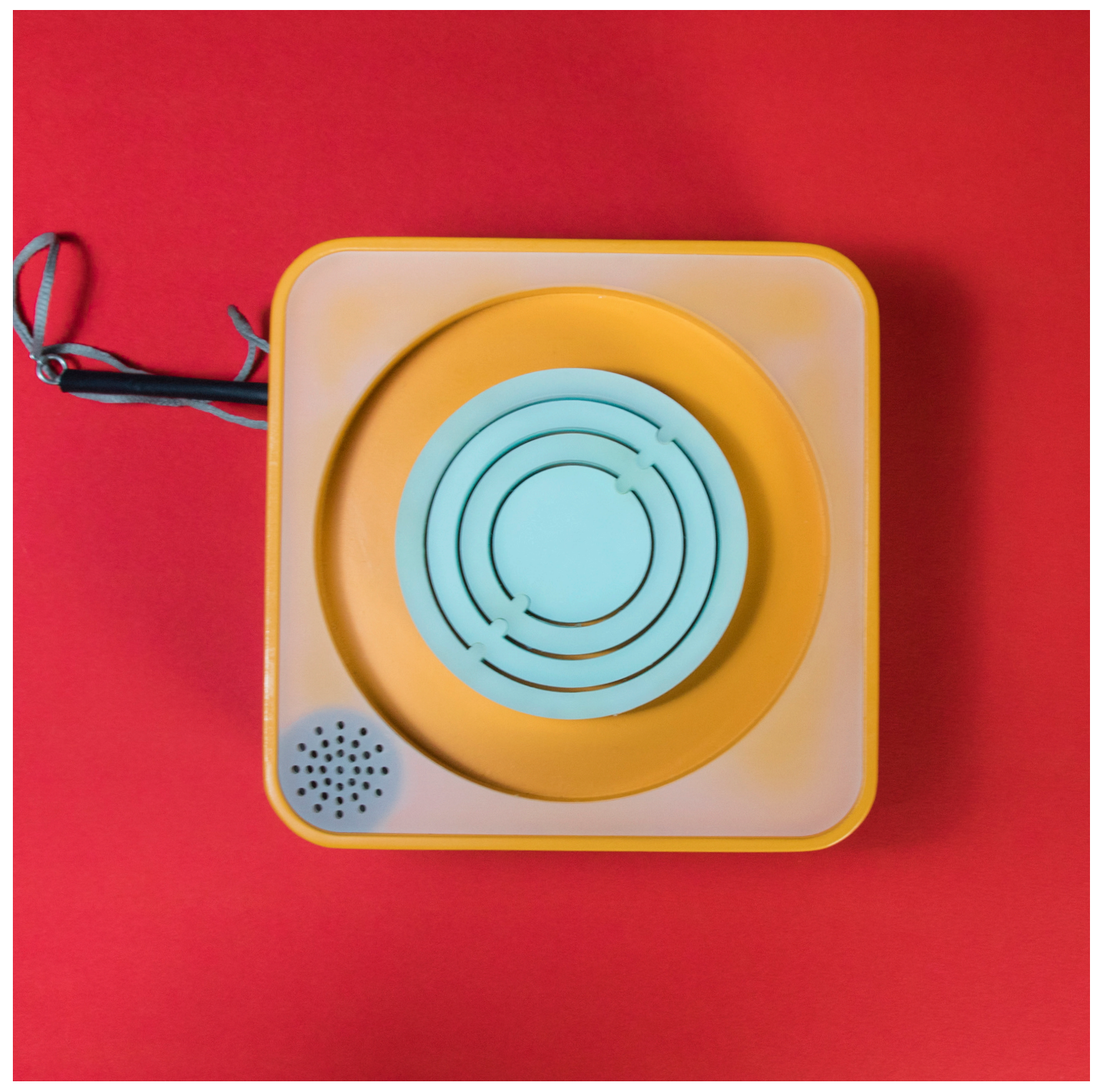

Prototype o3 Vopriew

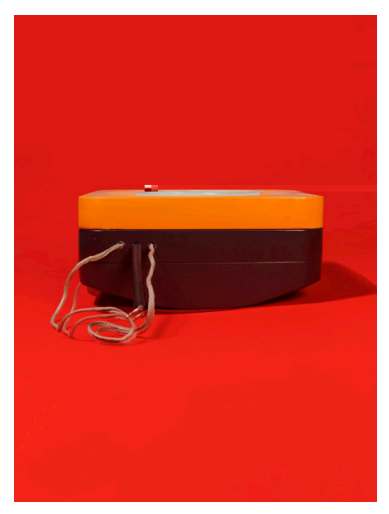

Prototype os Sideview

The user can decrease the size of the main

colume buttion is order to inrease the

difficulty of the inferactions. 


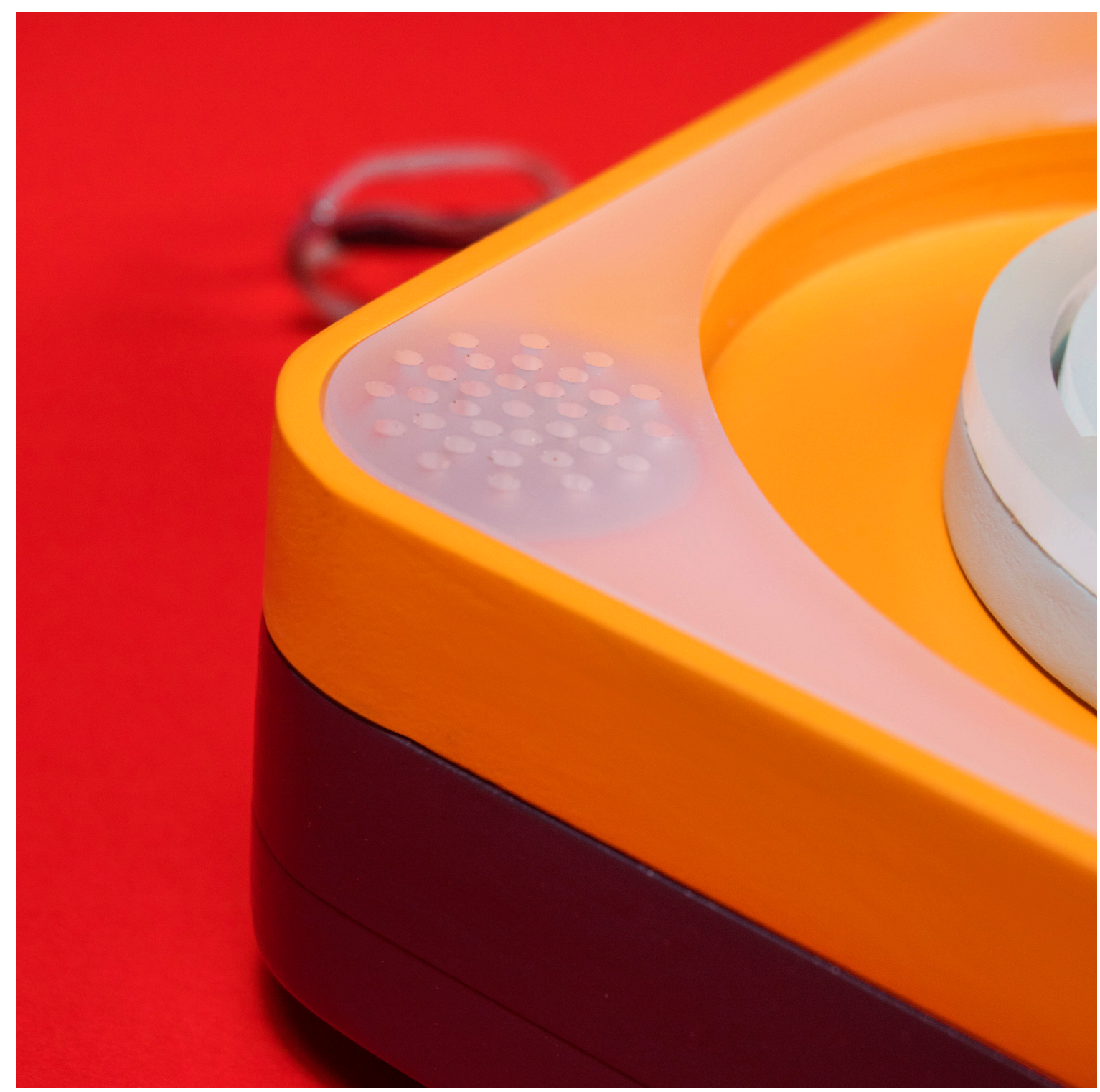

Prototype os Detail of the functional feedformerd

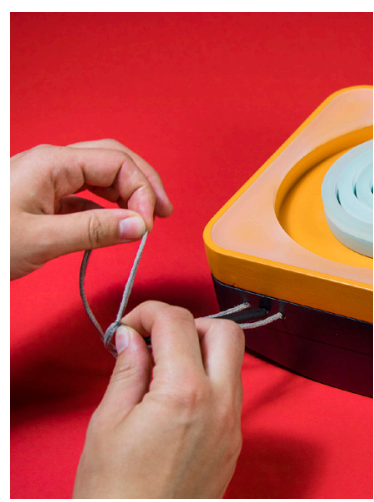

Prototyee os Detail of Changing the training interval 

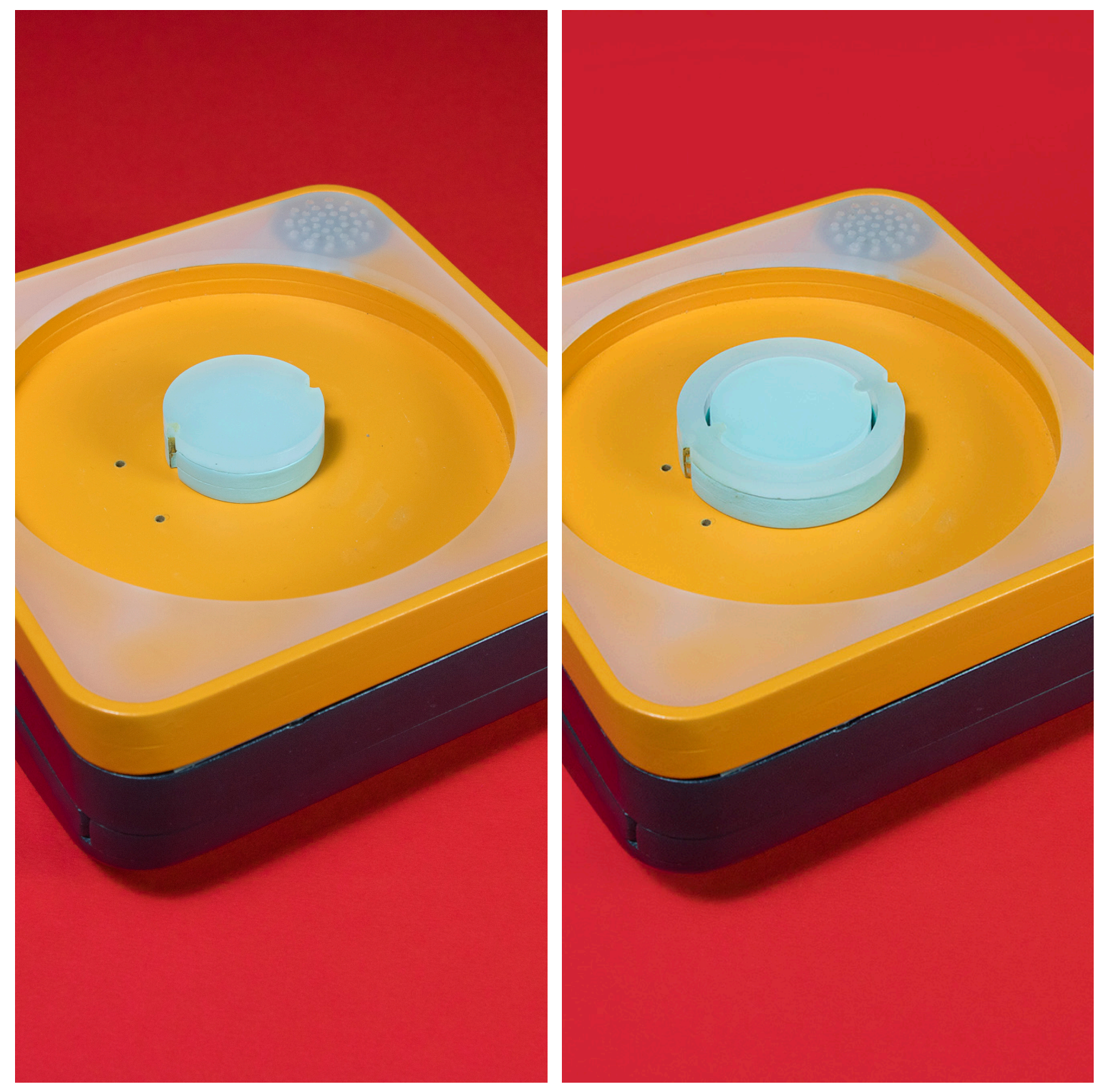

Prototype 03 Proves I

Prototype o3 Progress II

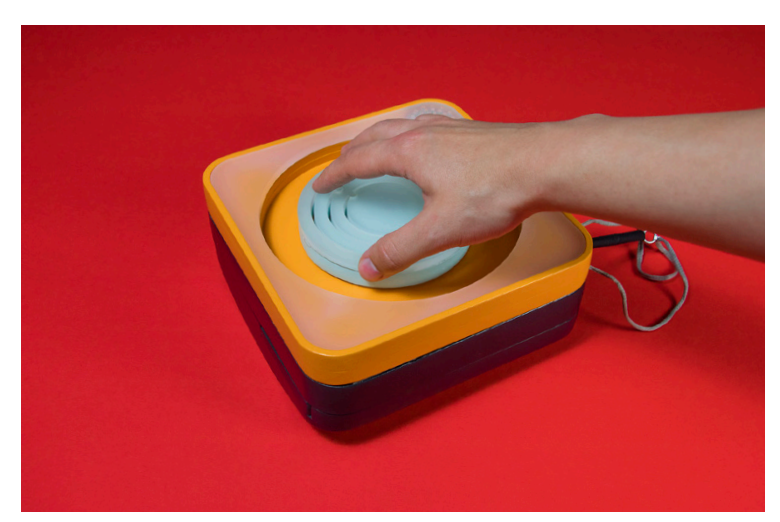




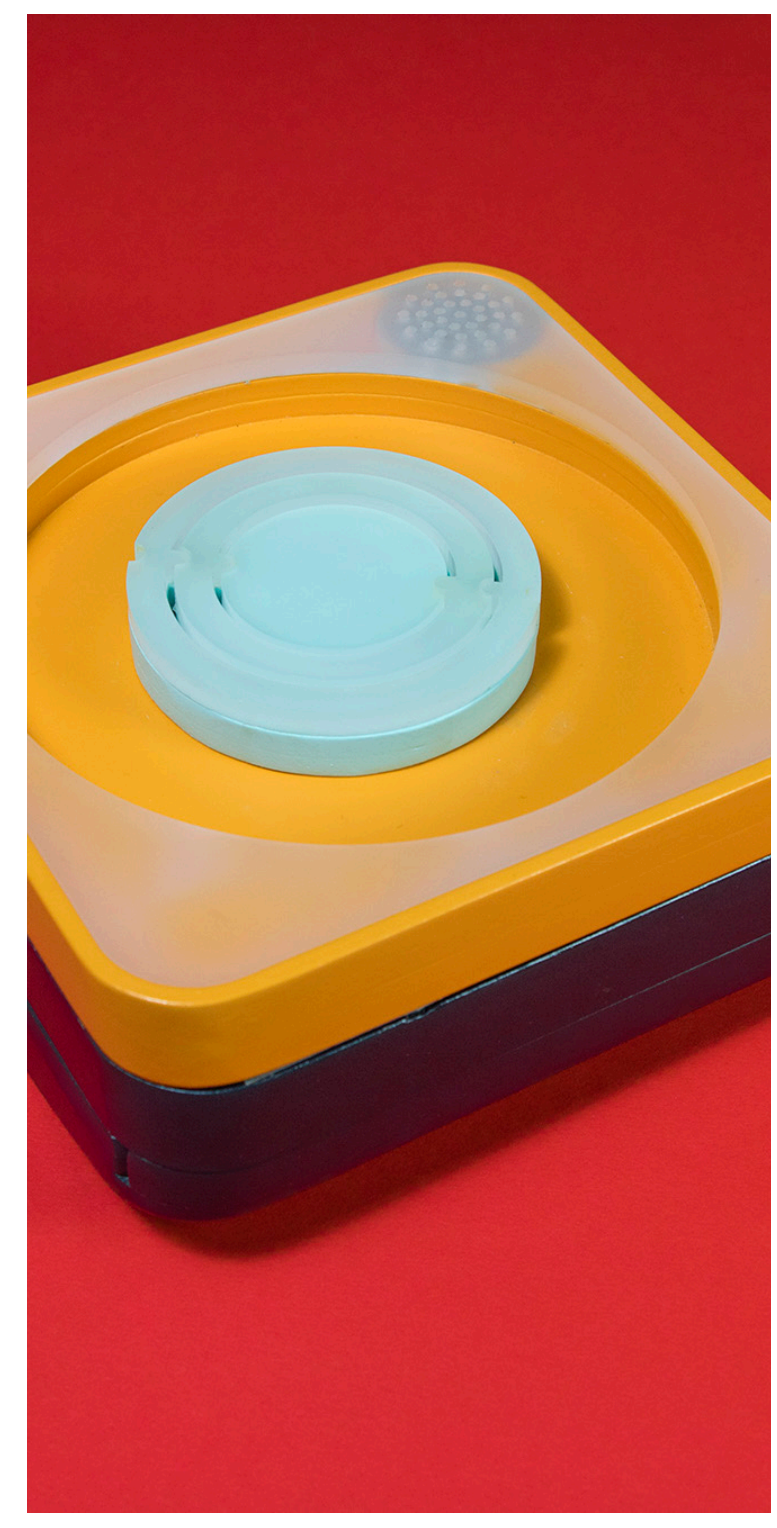

Prototype o3 Proress If

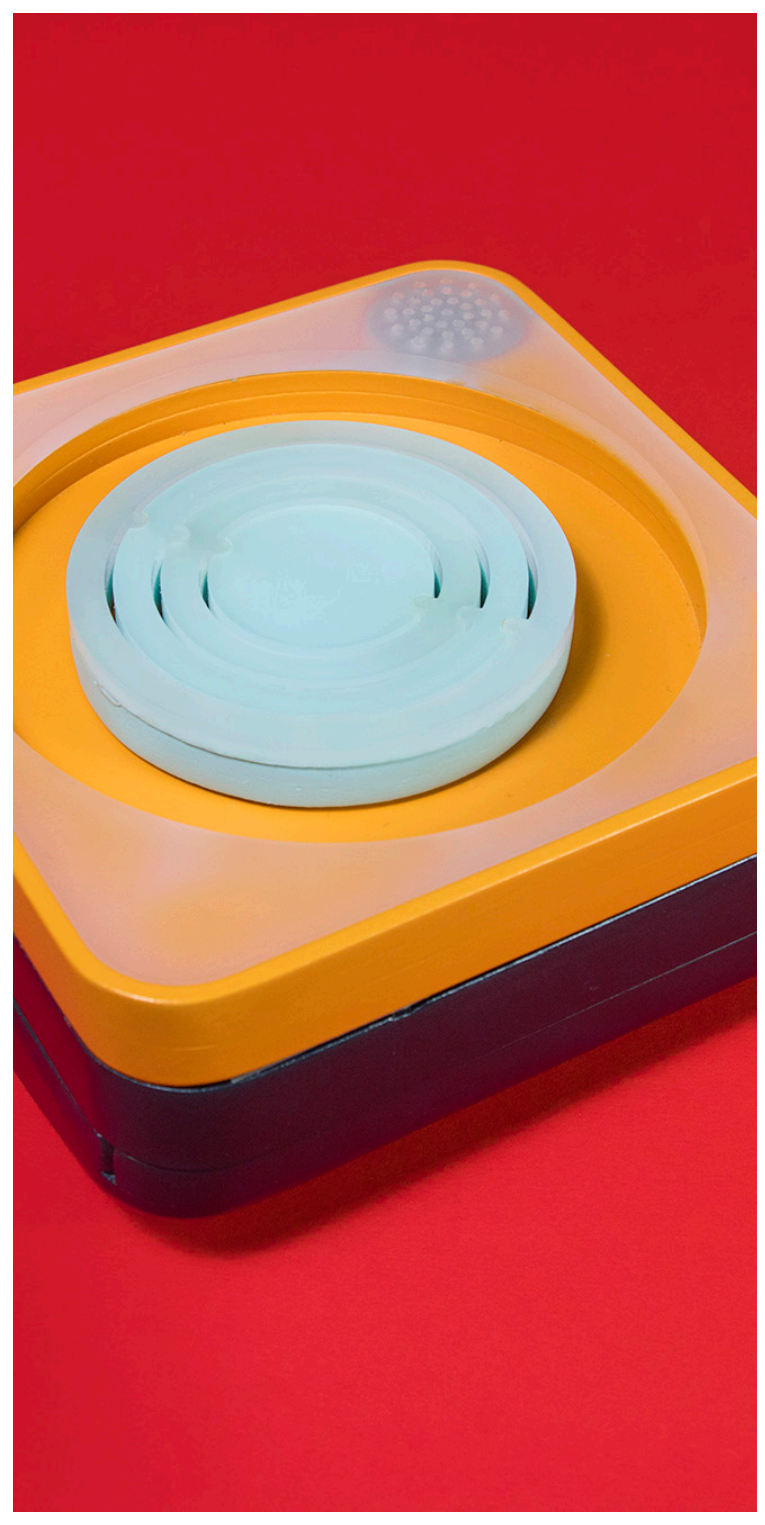

Prototype 03 Progress At 


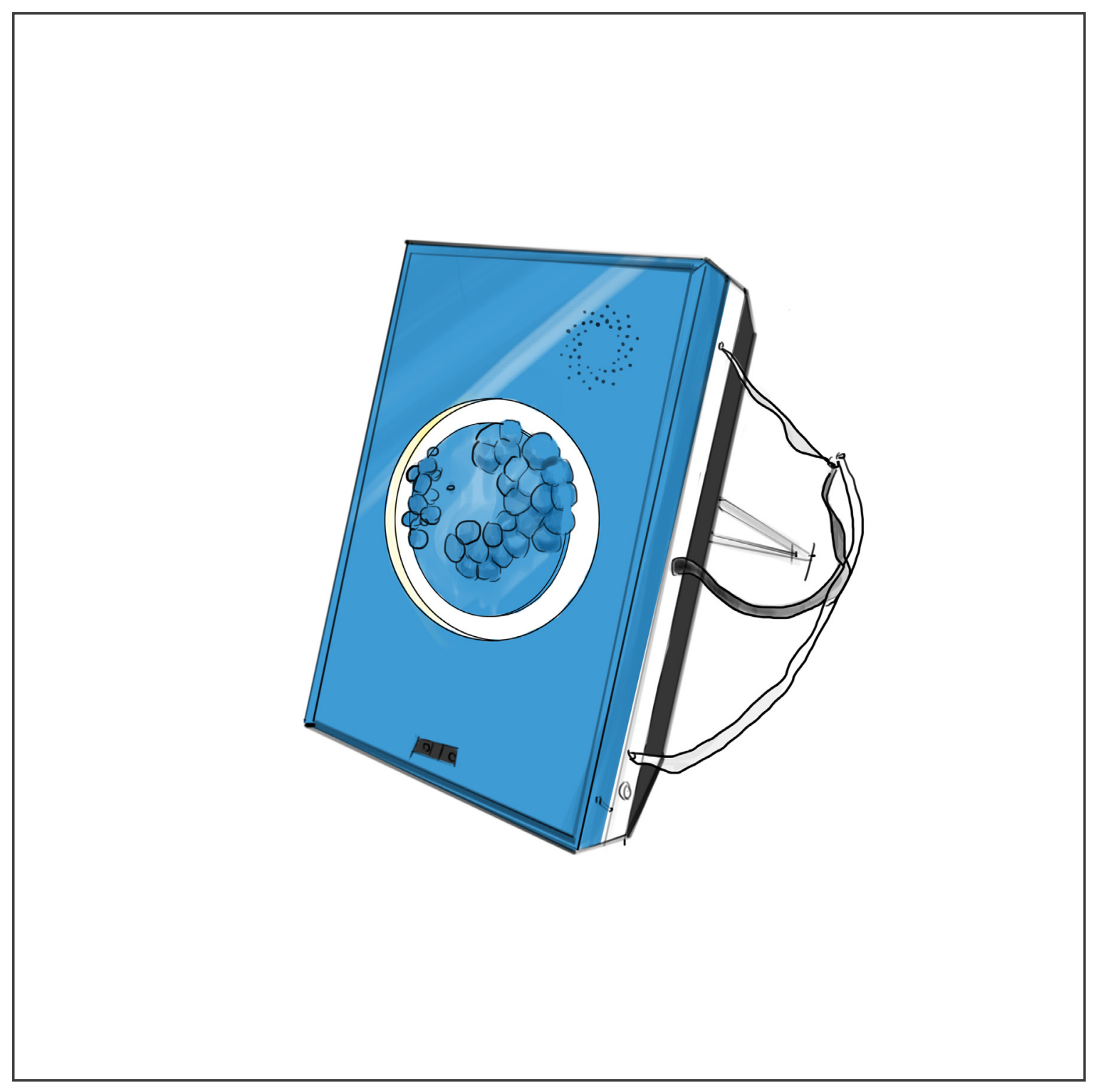

Protoxpe ol sketcles 


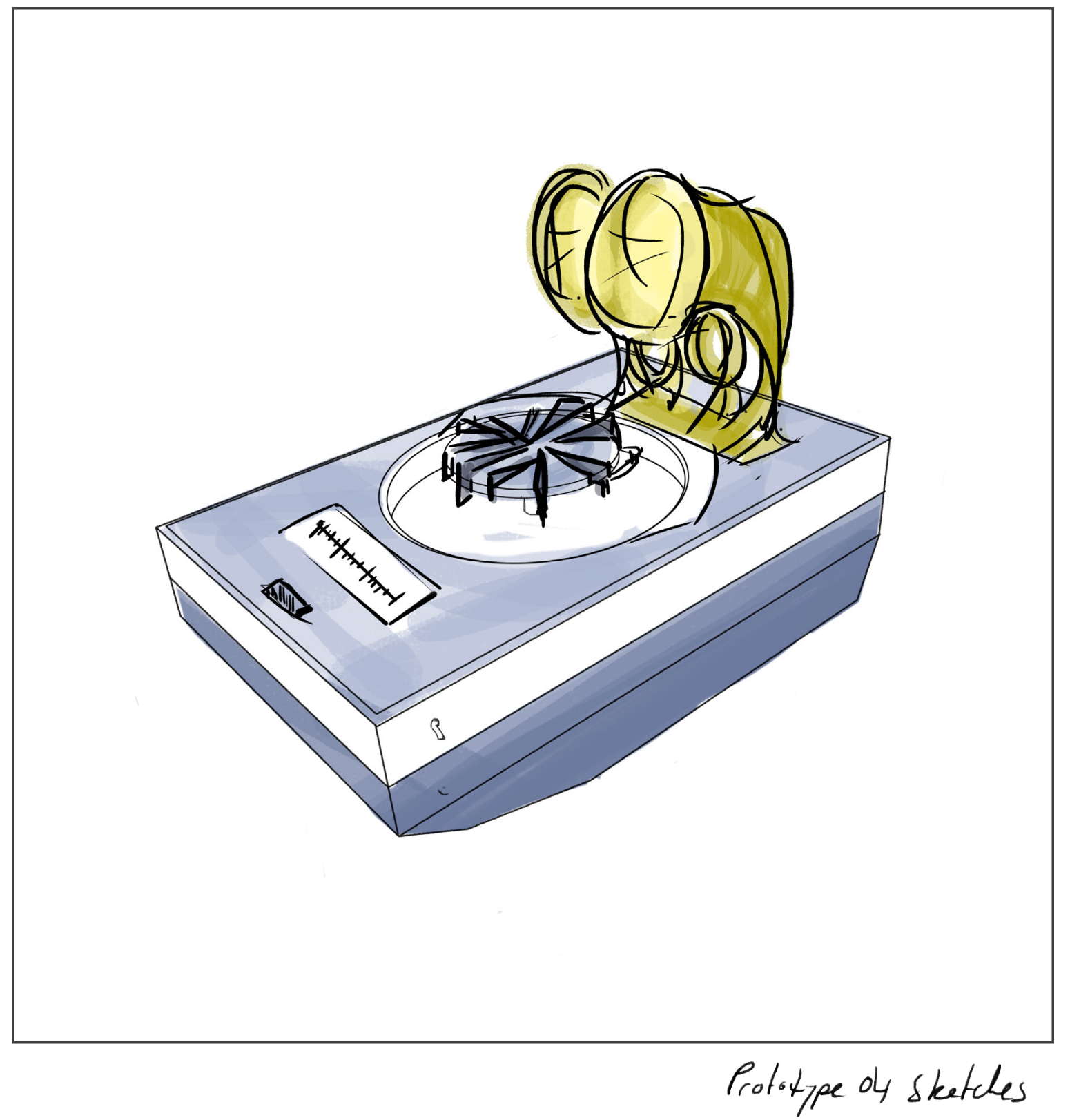




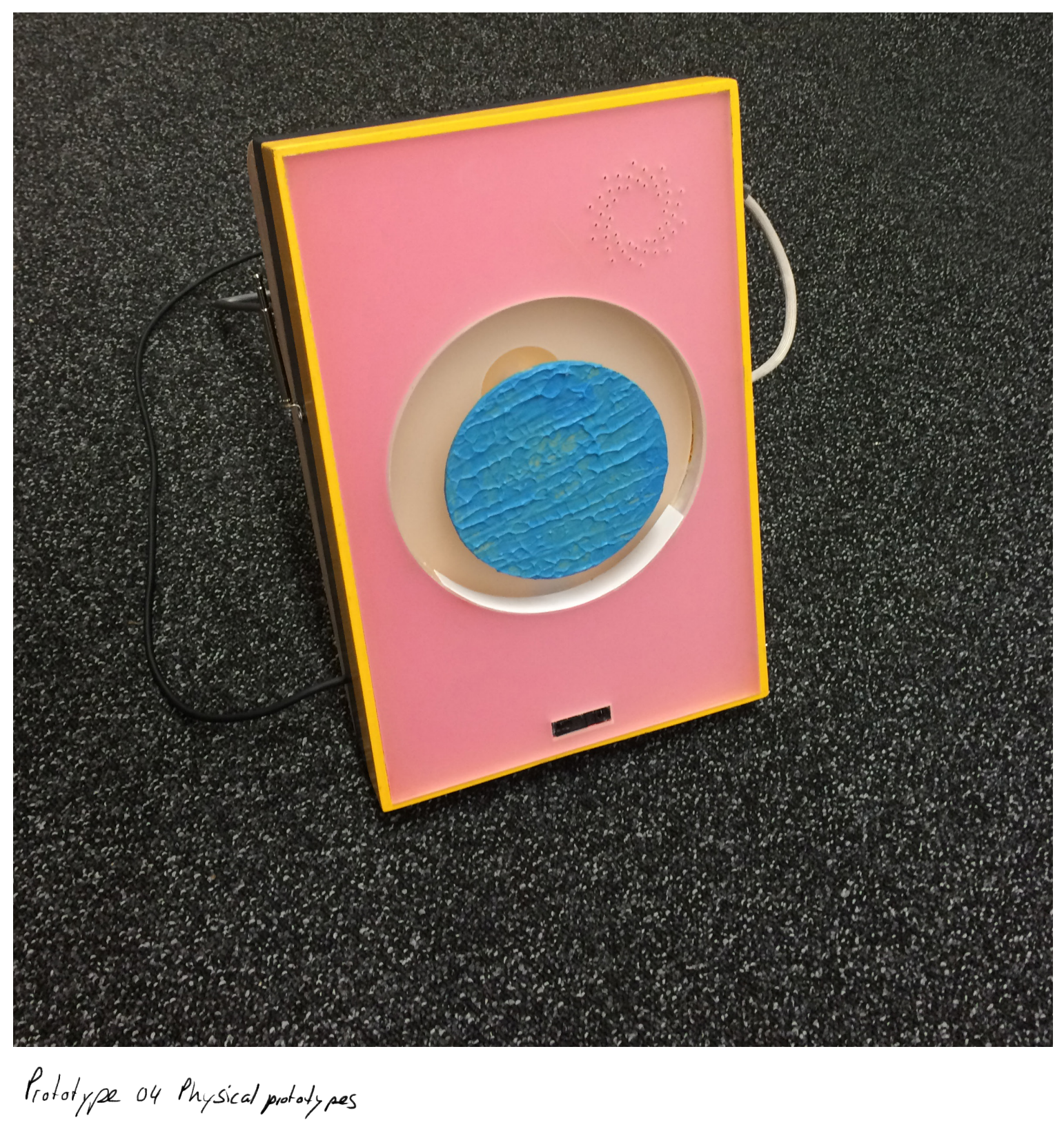




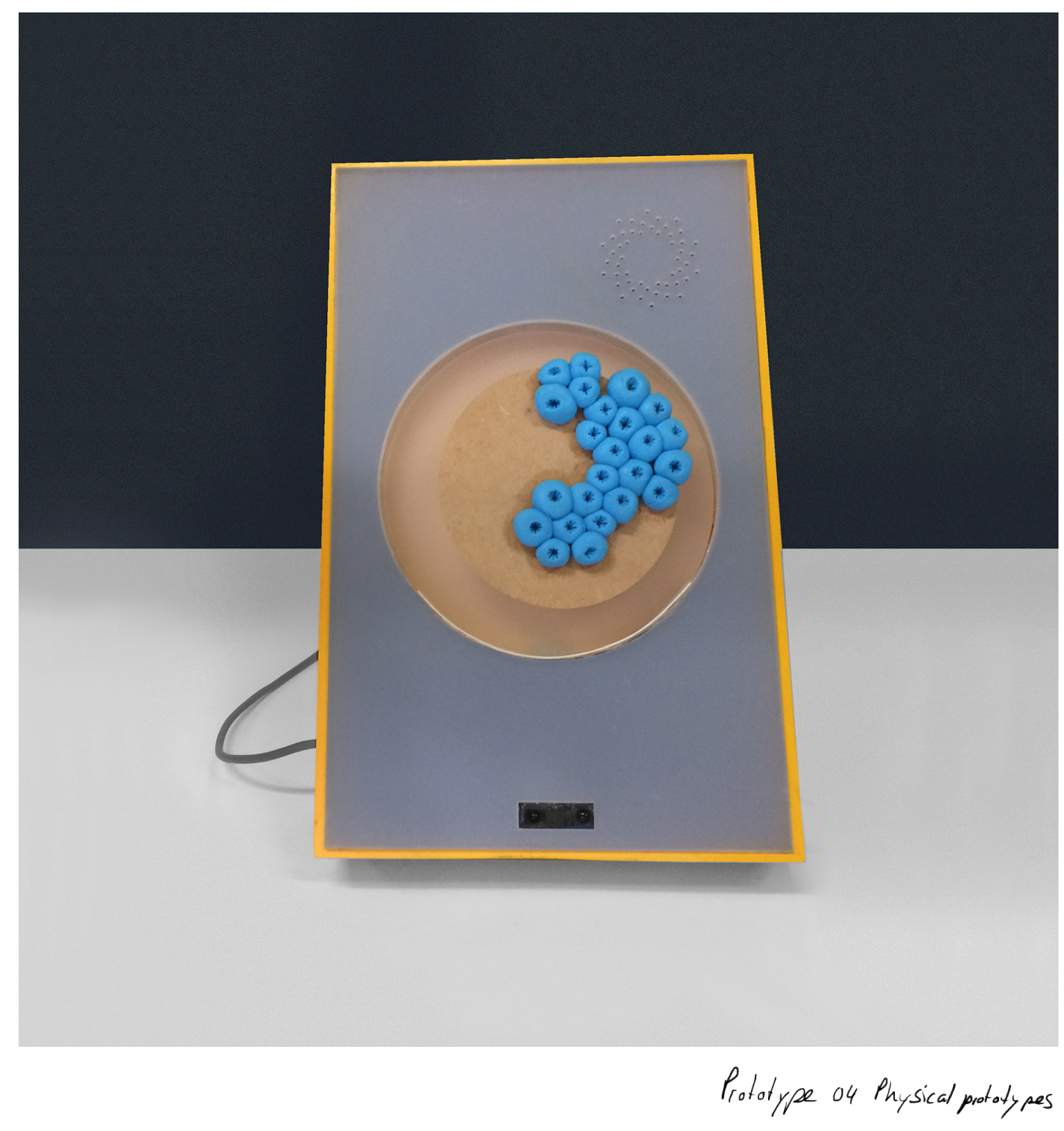




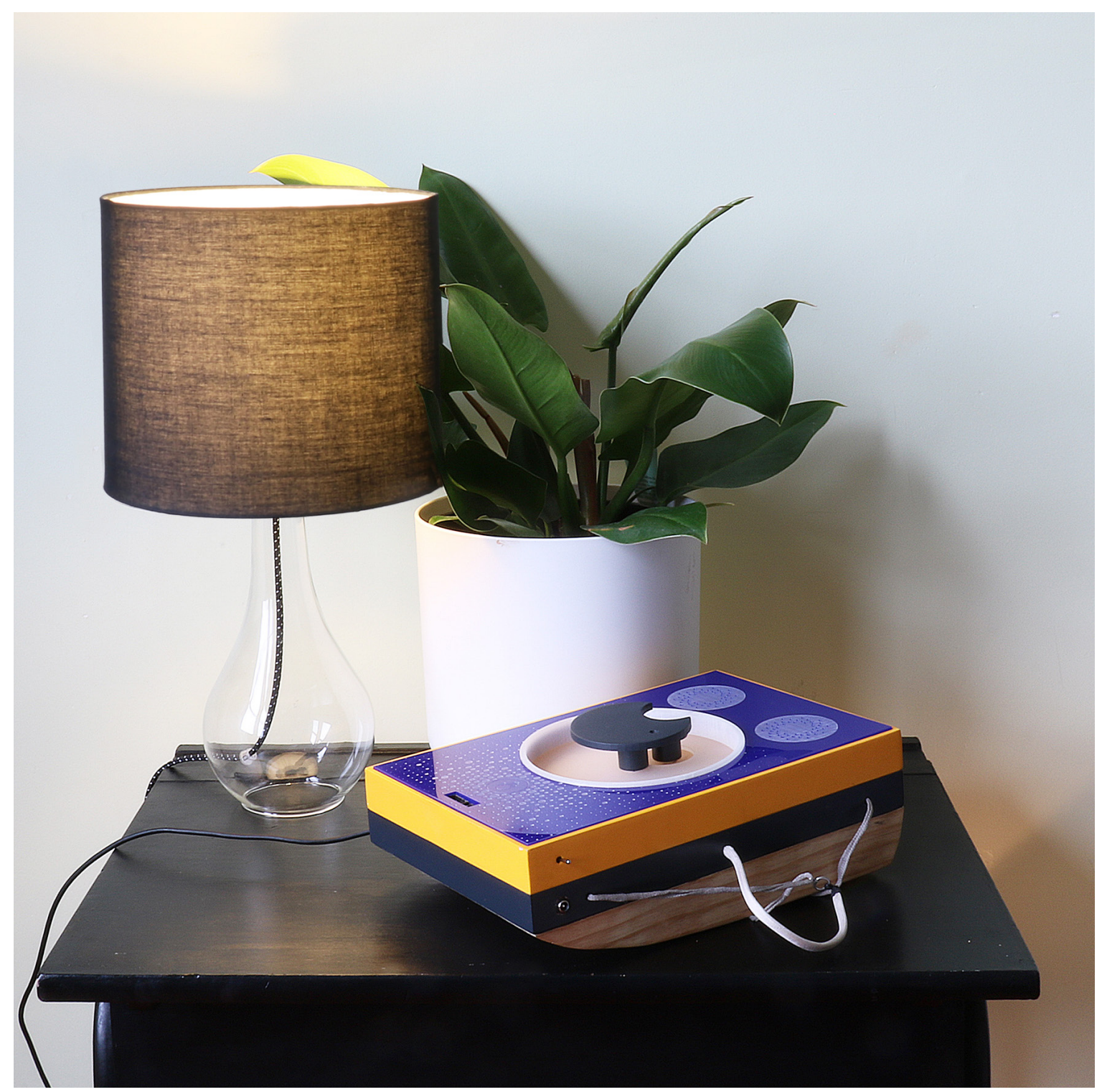

Final design protatype without the stand to decrease wrist extension

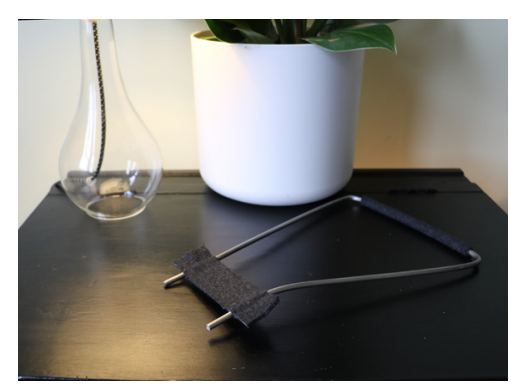




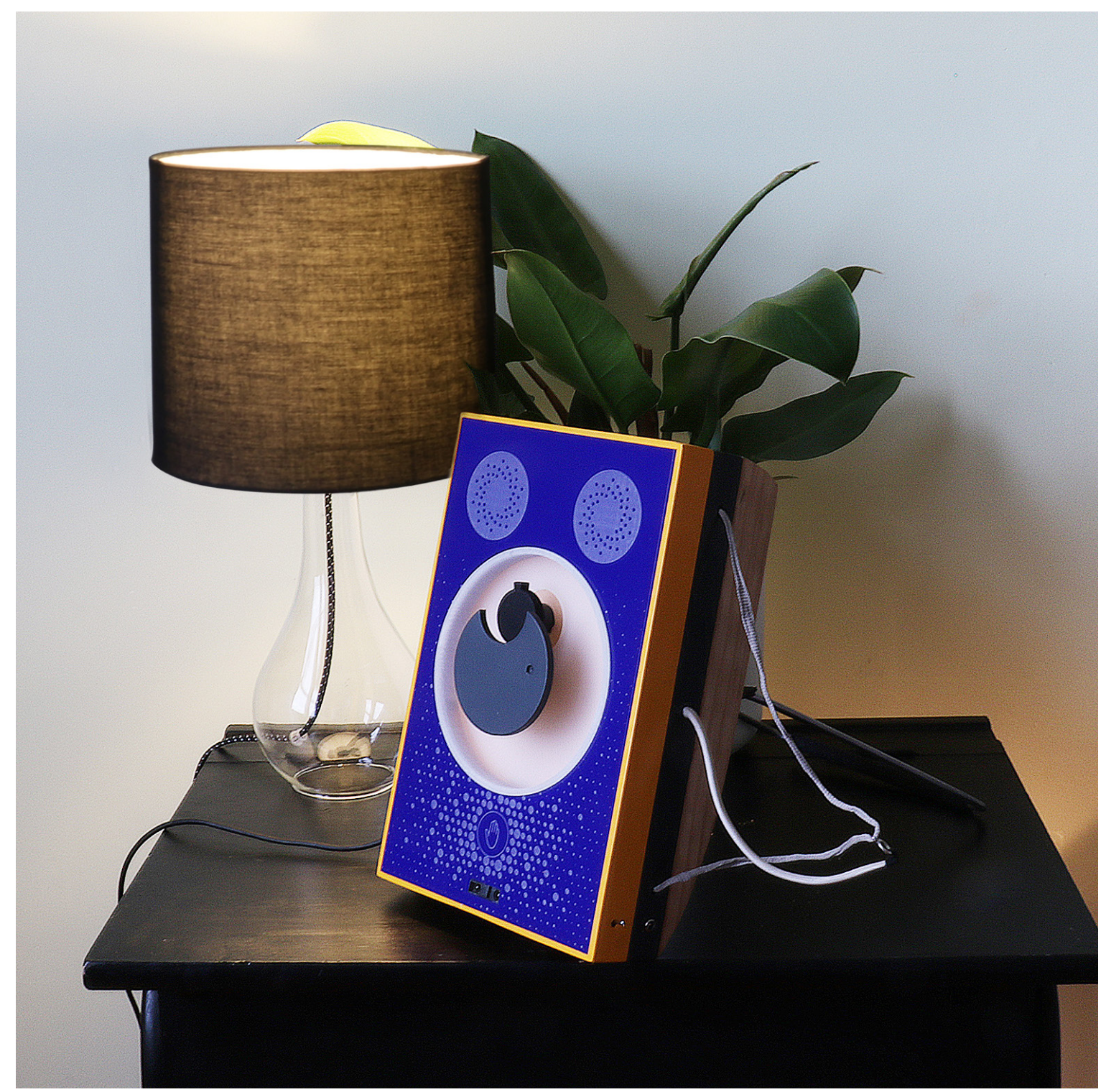

Final dosign probtype with the added stand 


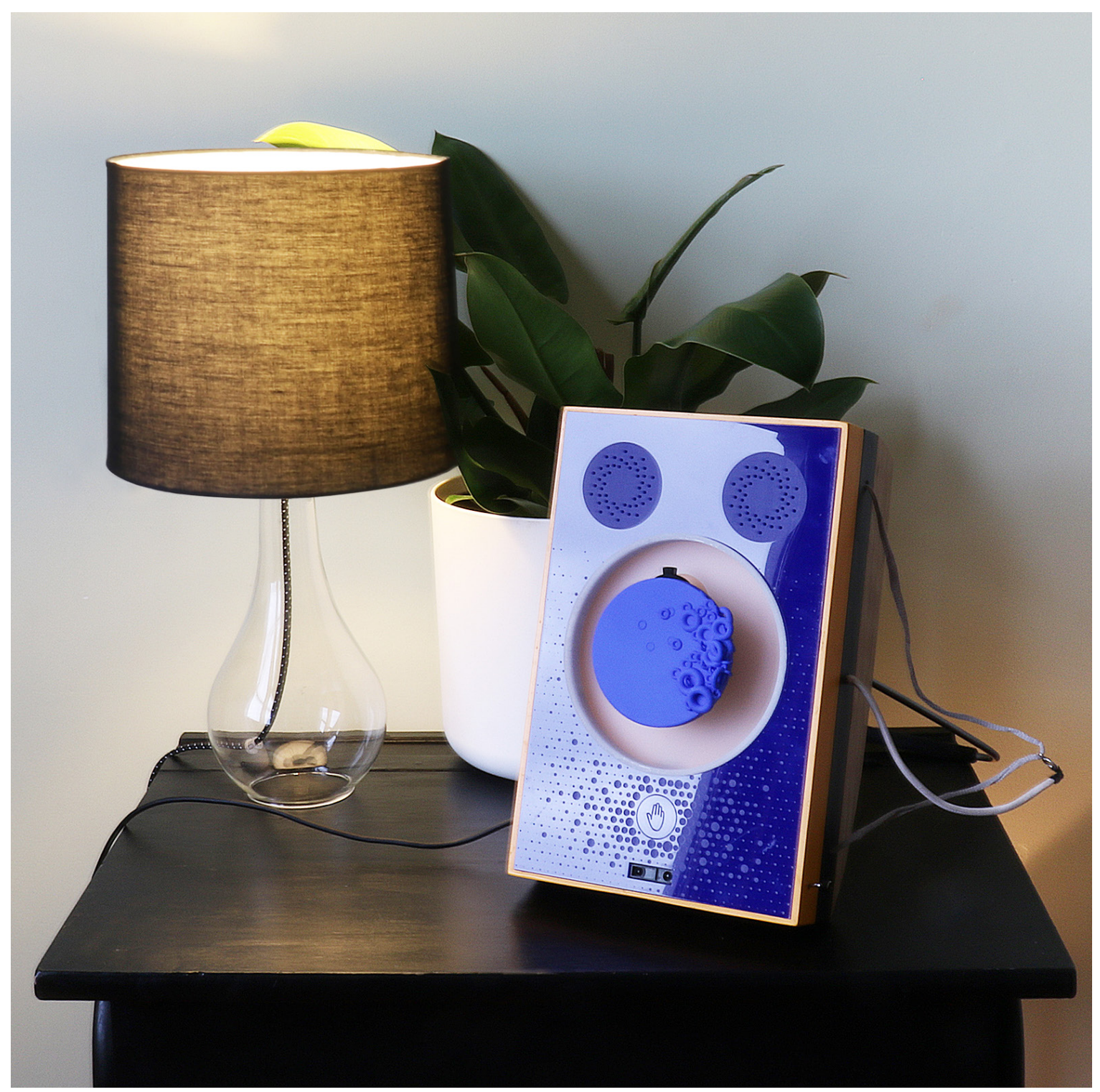

Final dosign prototipe with the added stand 


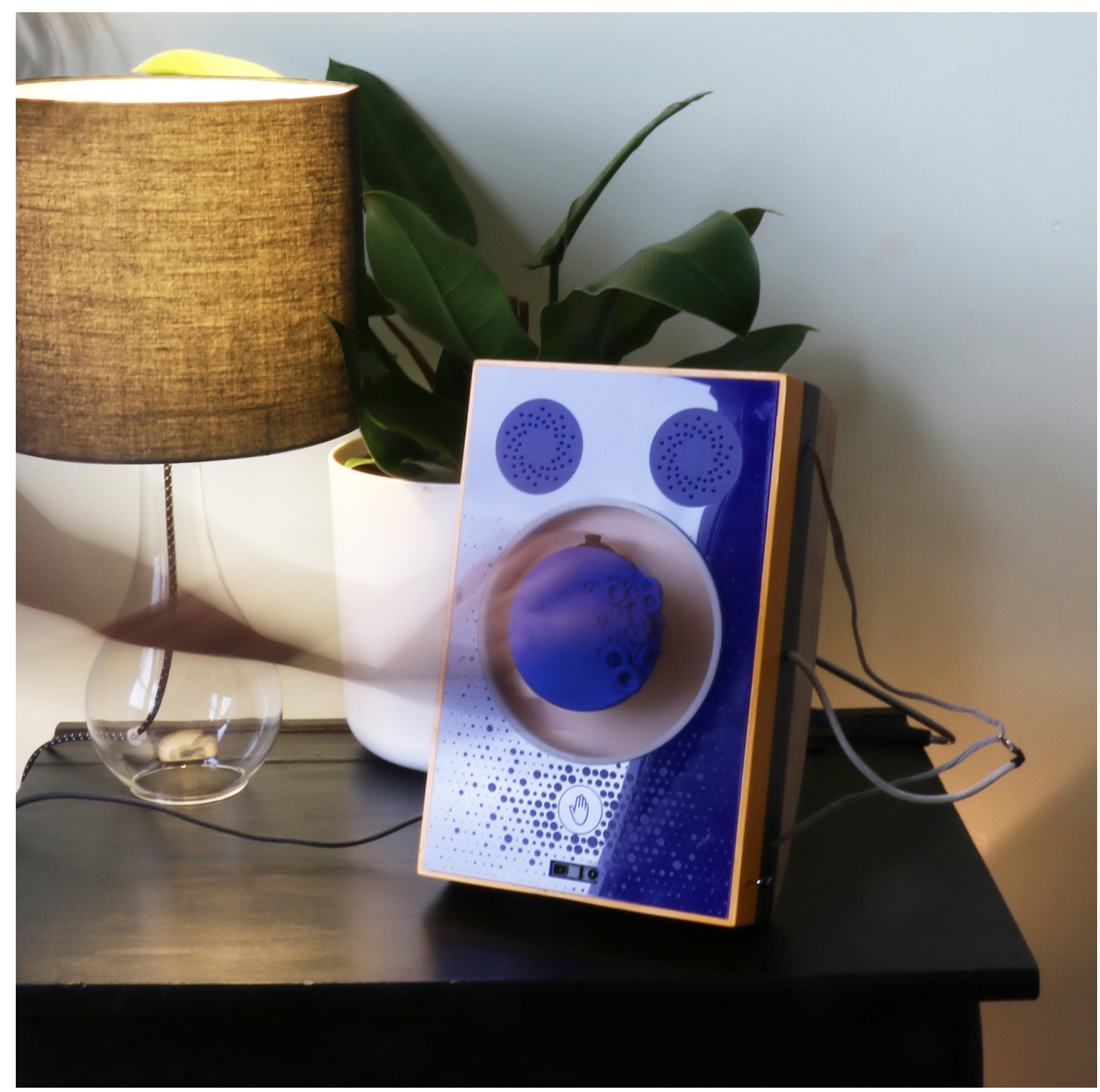

The user can rotate the man butlon to adjuest the volume of the radio 


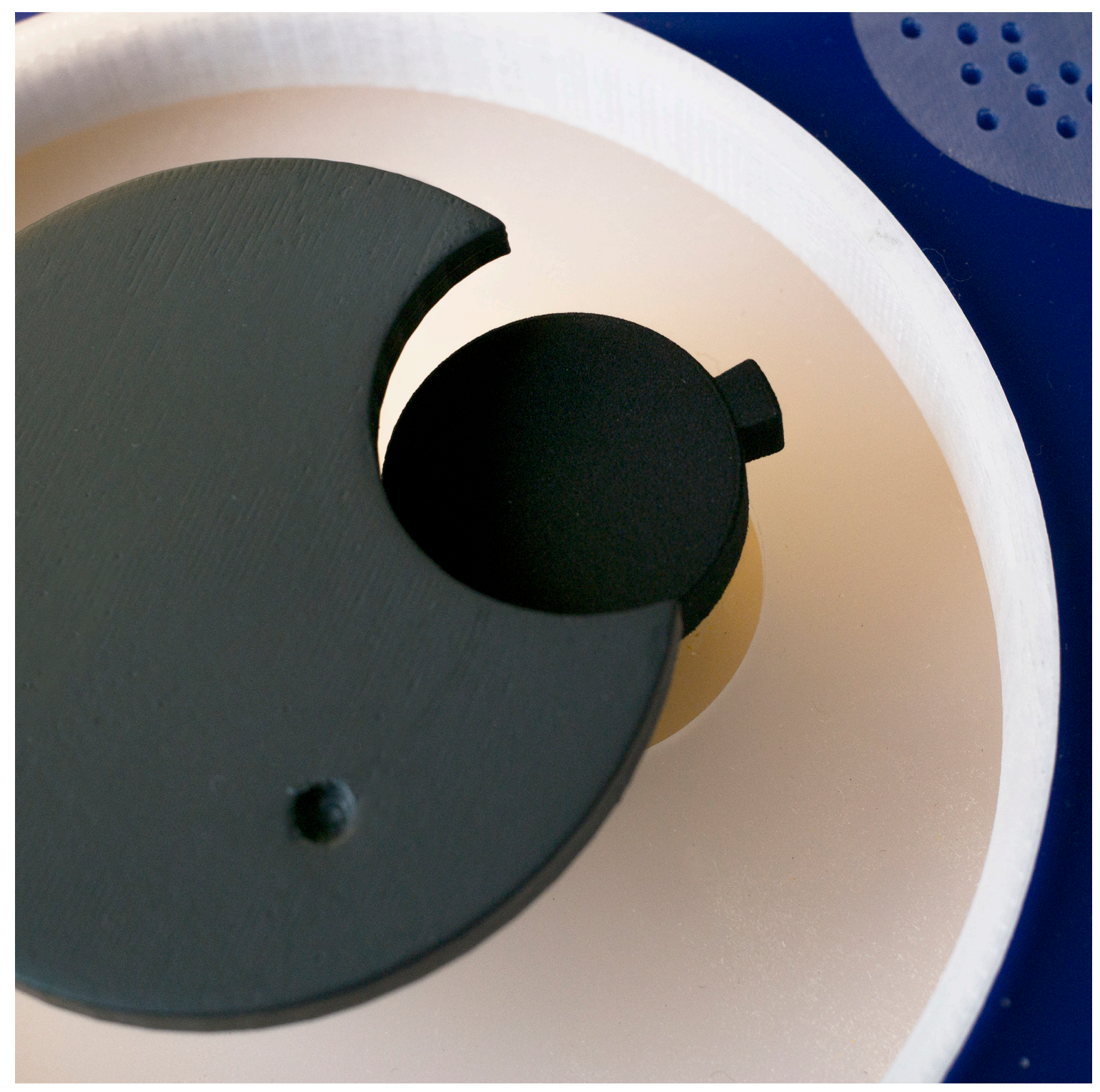

Debuils of the buttens to chang to volume \& dunned

The button that needs to be rotated to change the vodume

of the radio cun be changed according to the usi's

peference and lacte of sensation in the hands. 1 did

a series of design experiments to test possible pethns

and orentation on the buttons. 


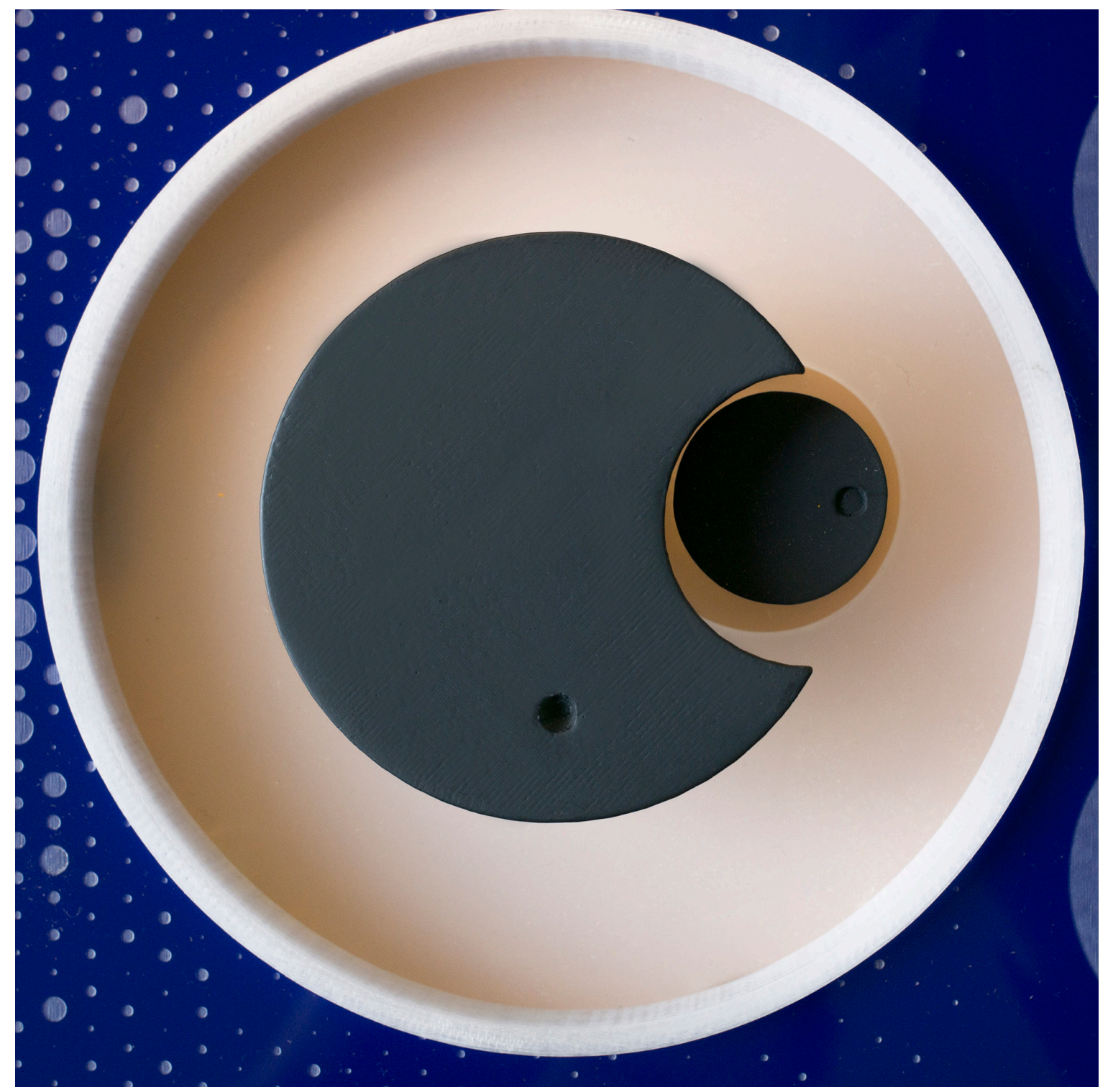

Debails of the buttons to chang to volume \& dunned 


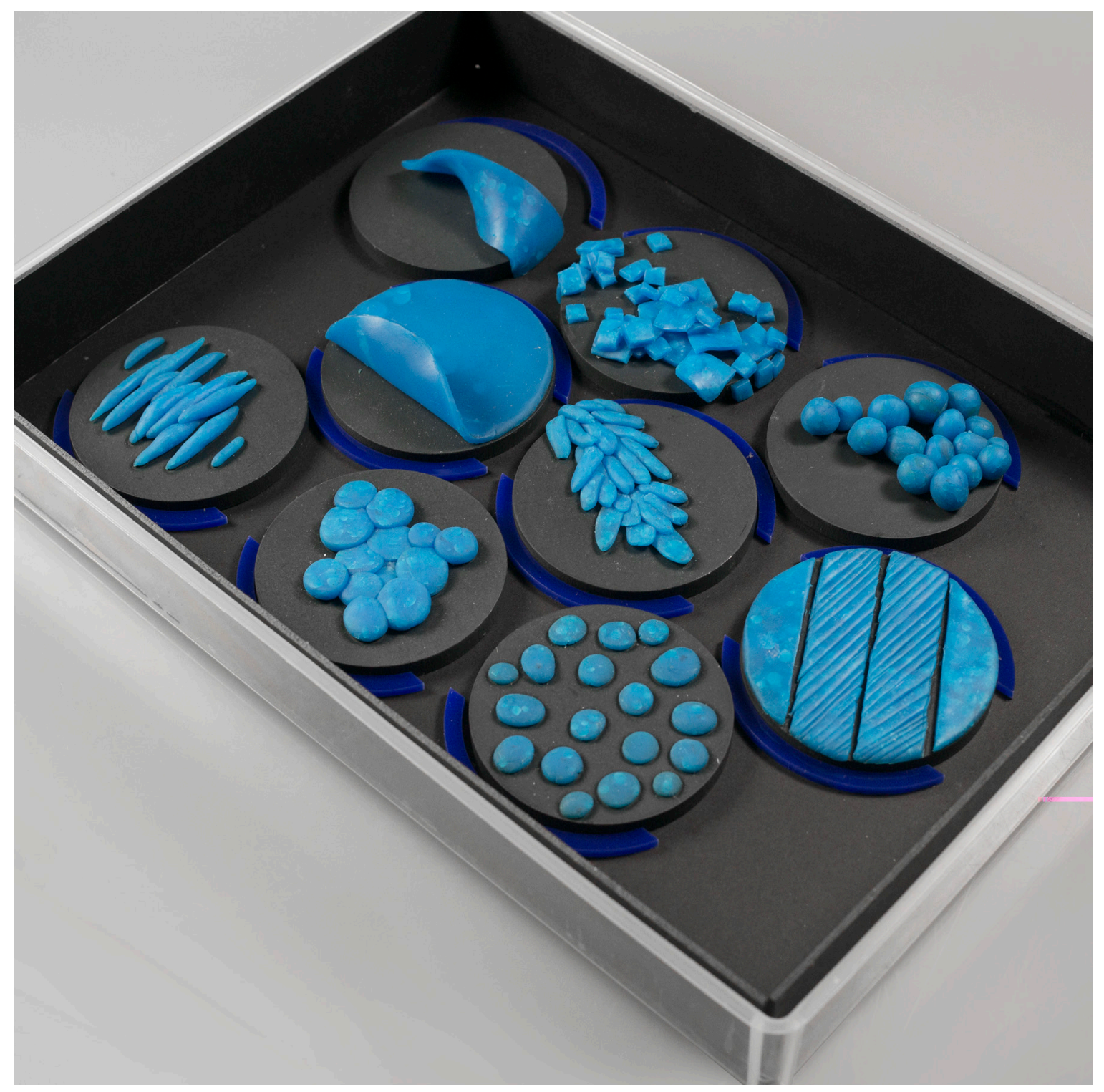

Desin experiments: Small buttons

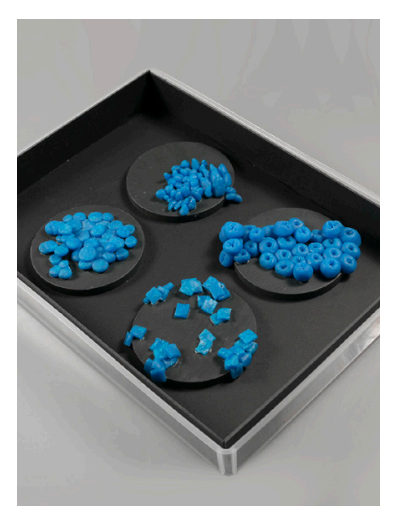

Desin experiments: Large buttons
The button that reedsto be rotated to change the vodure of the radio cun be changed according to the ust's prefernce and lack of sensation in the hands. I did a series of design experiments to test possible pathns and orentation on the buttons. 


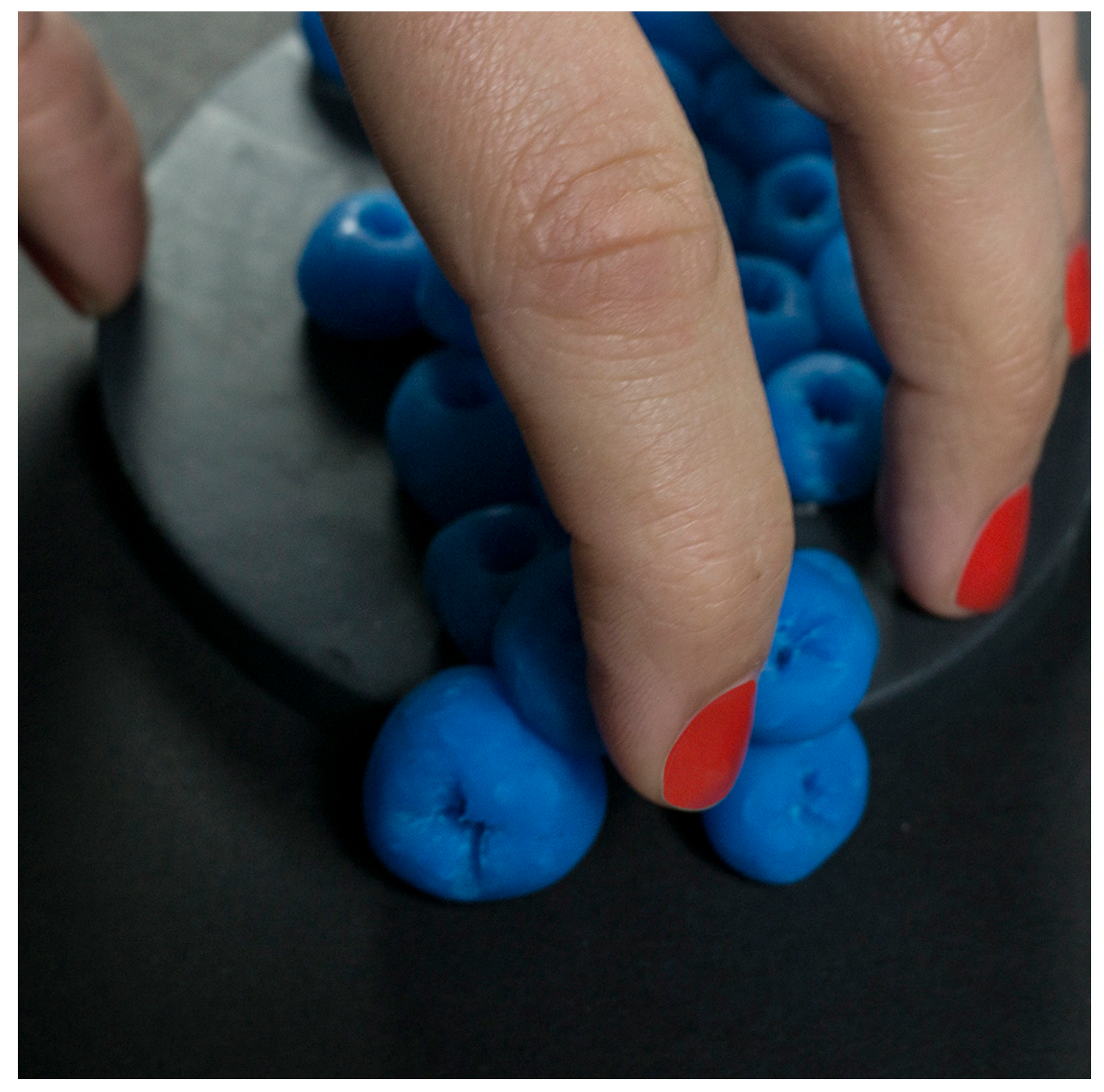

Details of the contact area

Throght the experineats ifound out that the

texture should ideally be situated around the

bution rather than purely ou the top.

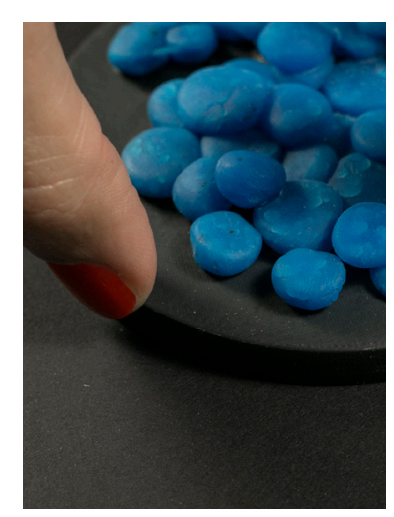




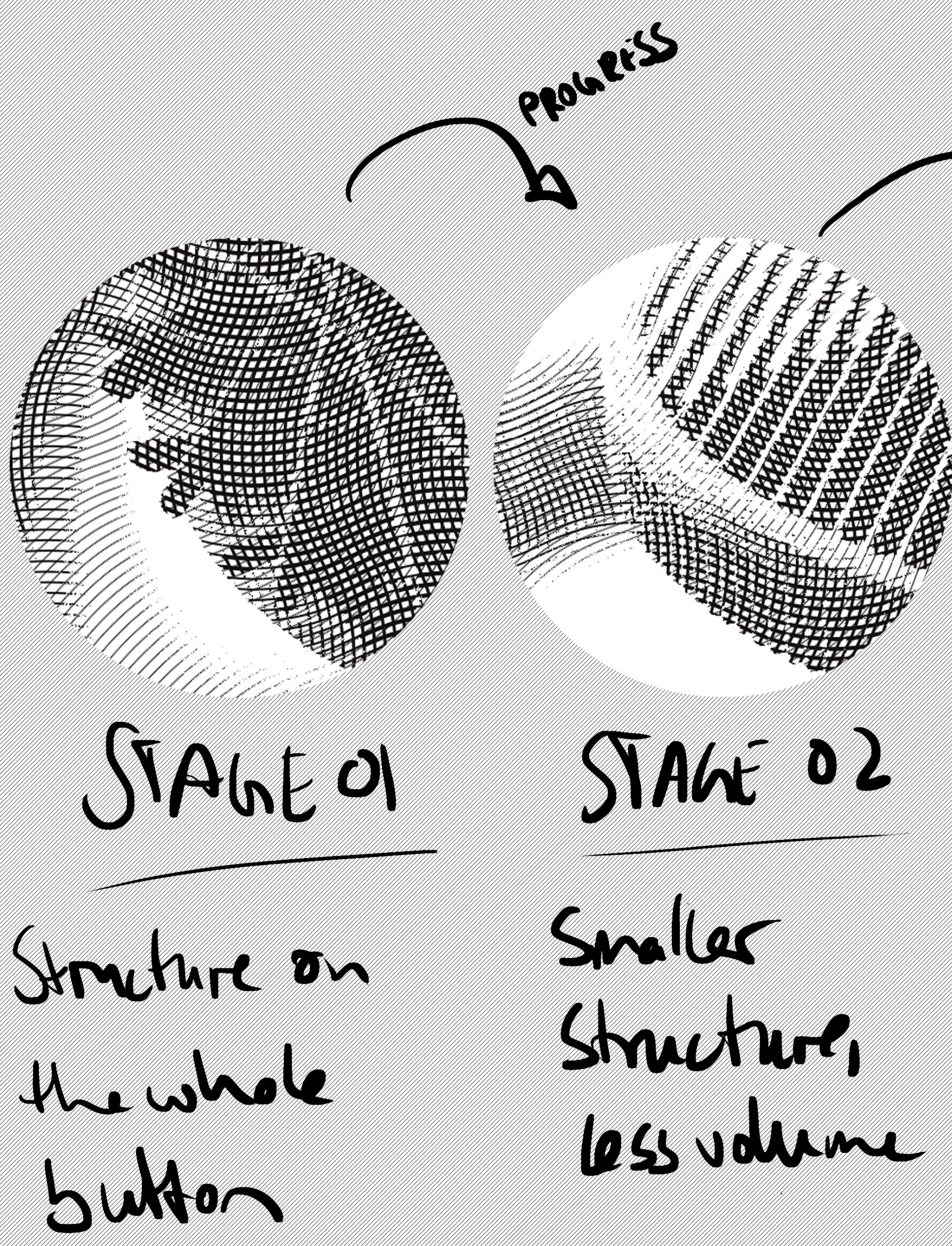




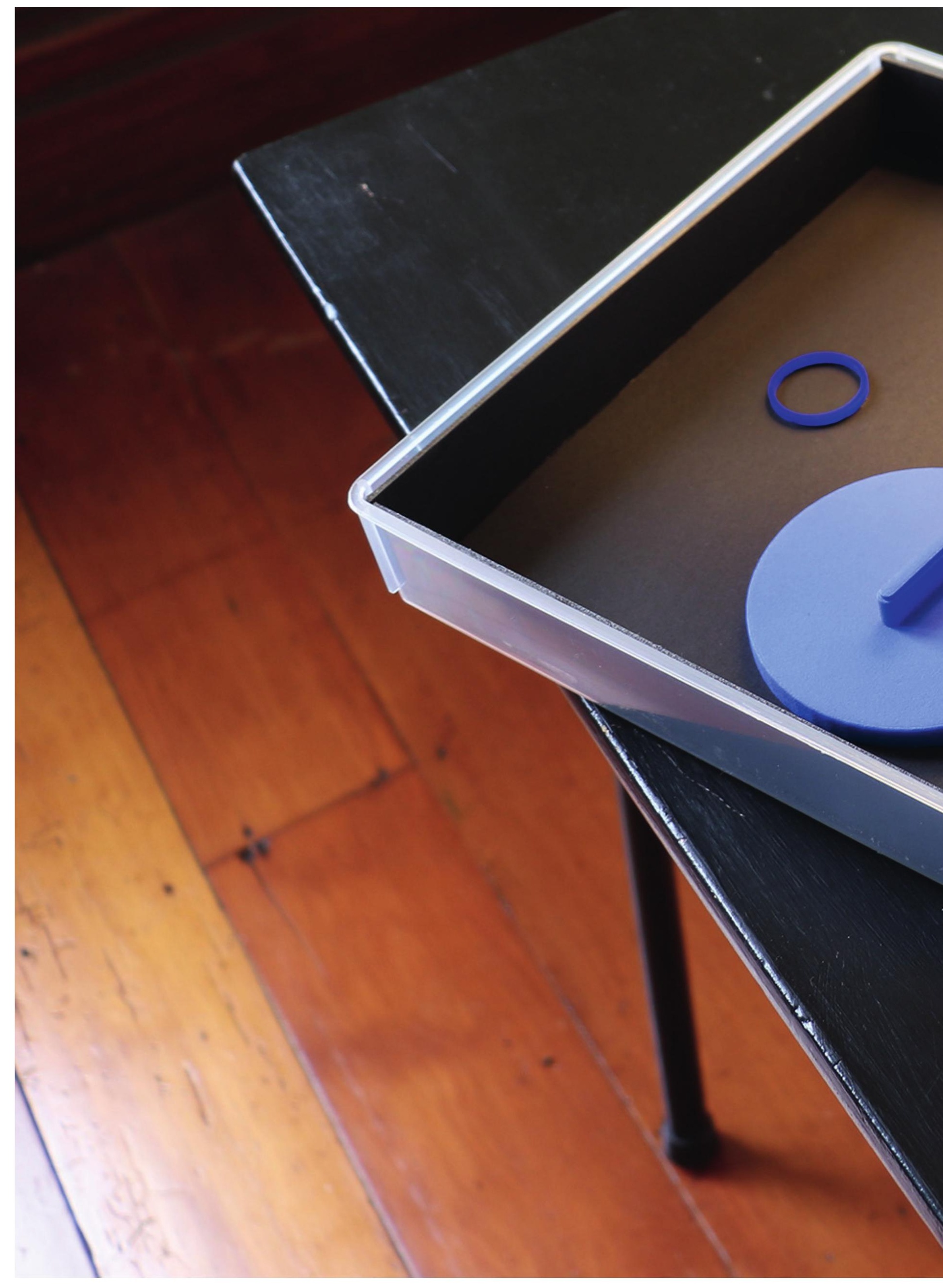




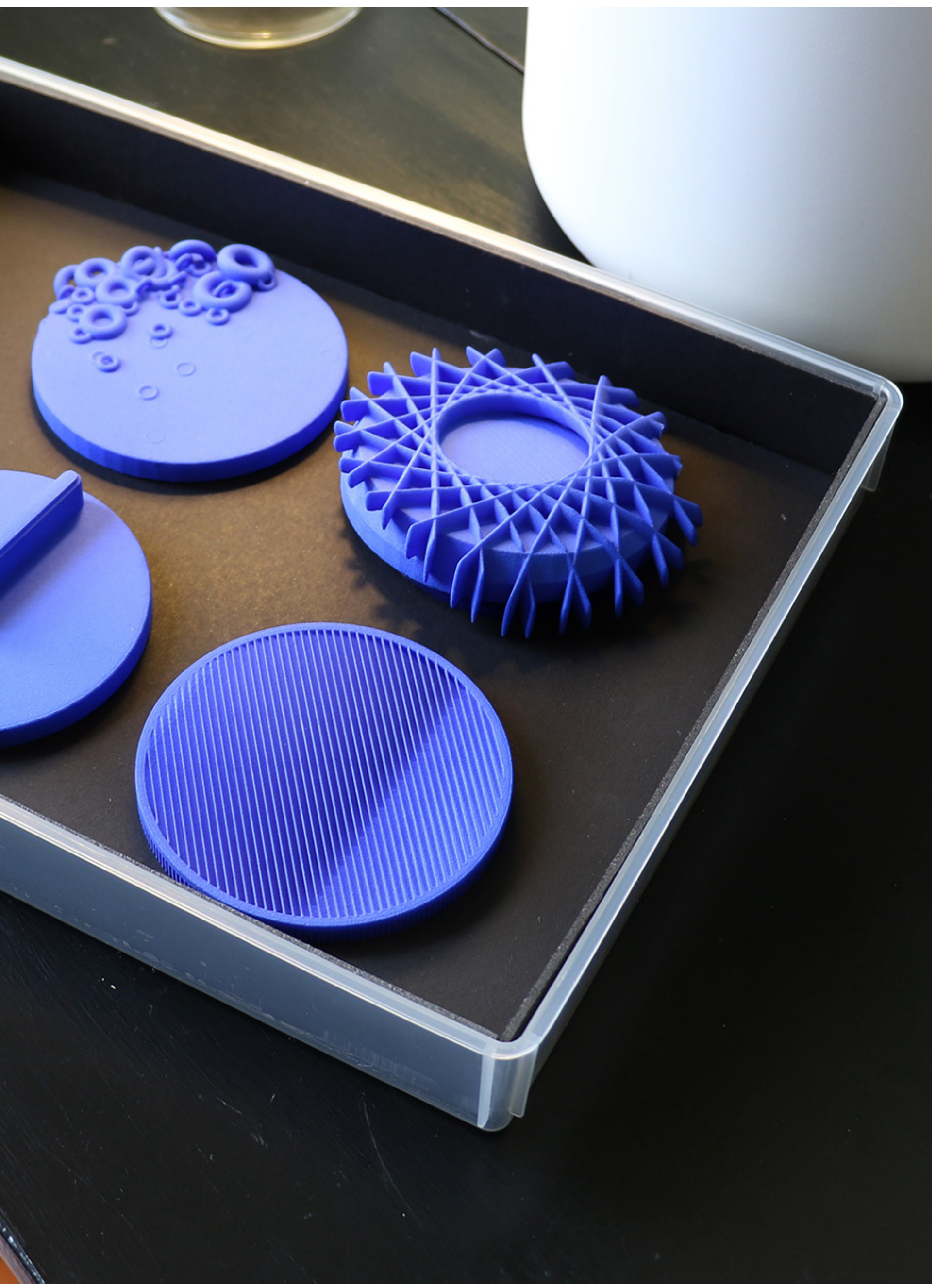




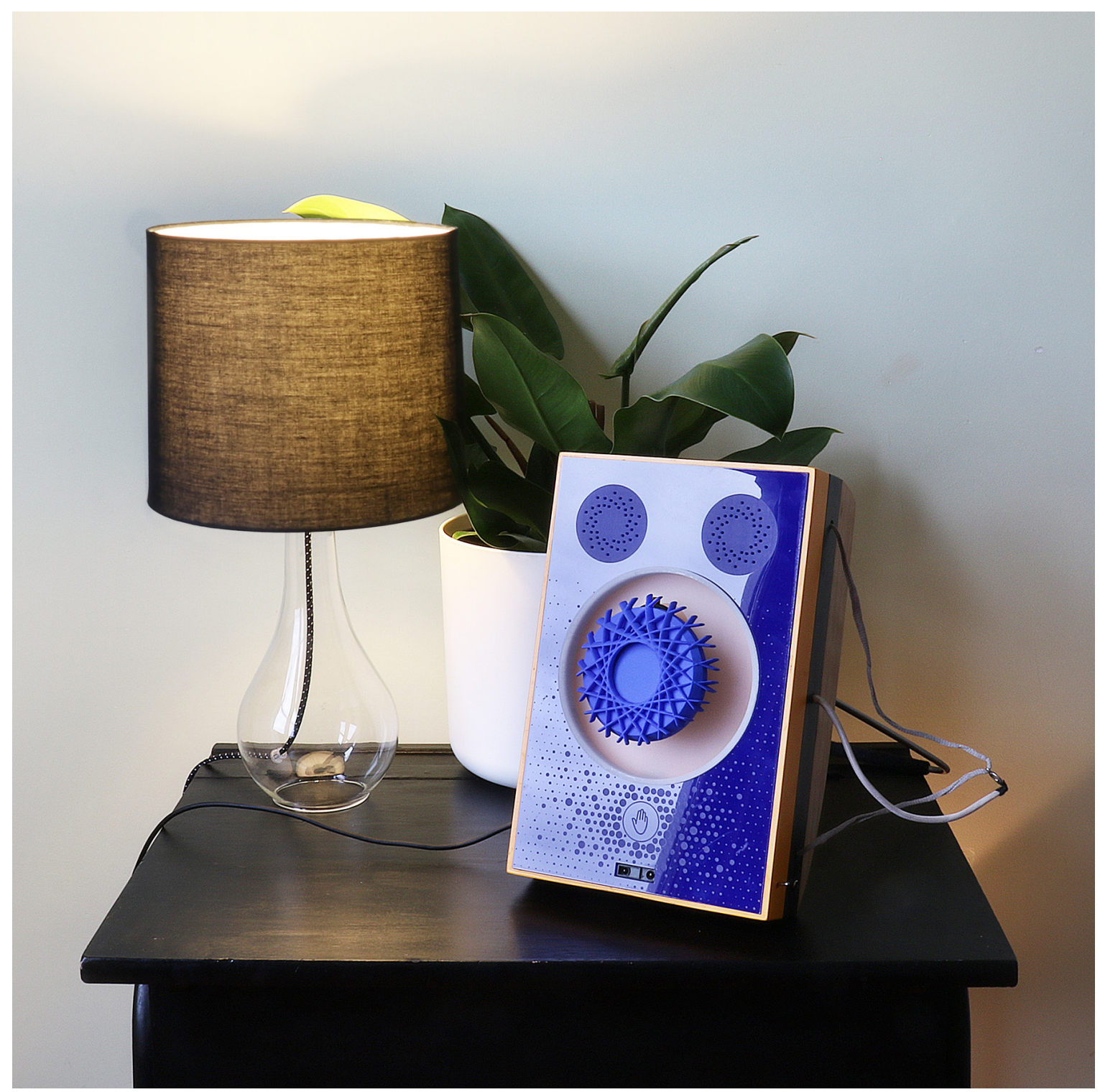

Volume button Stage I

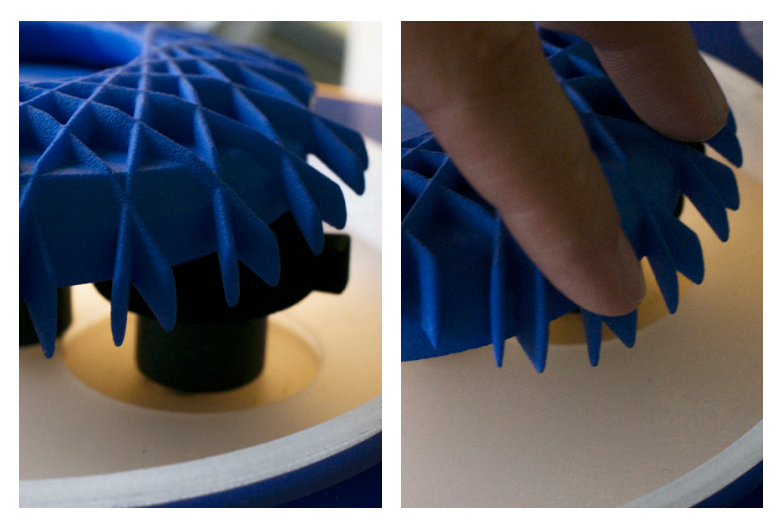




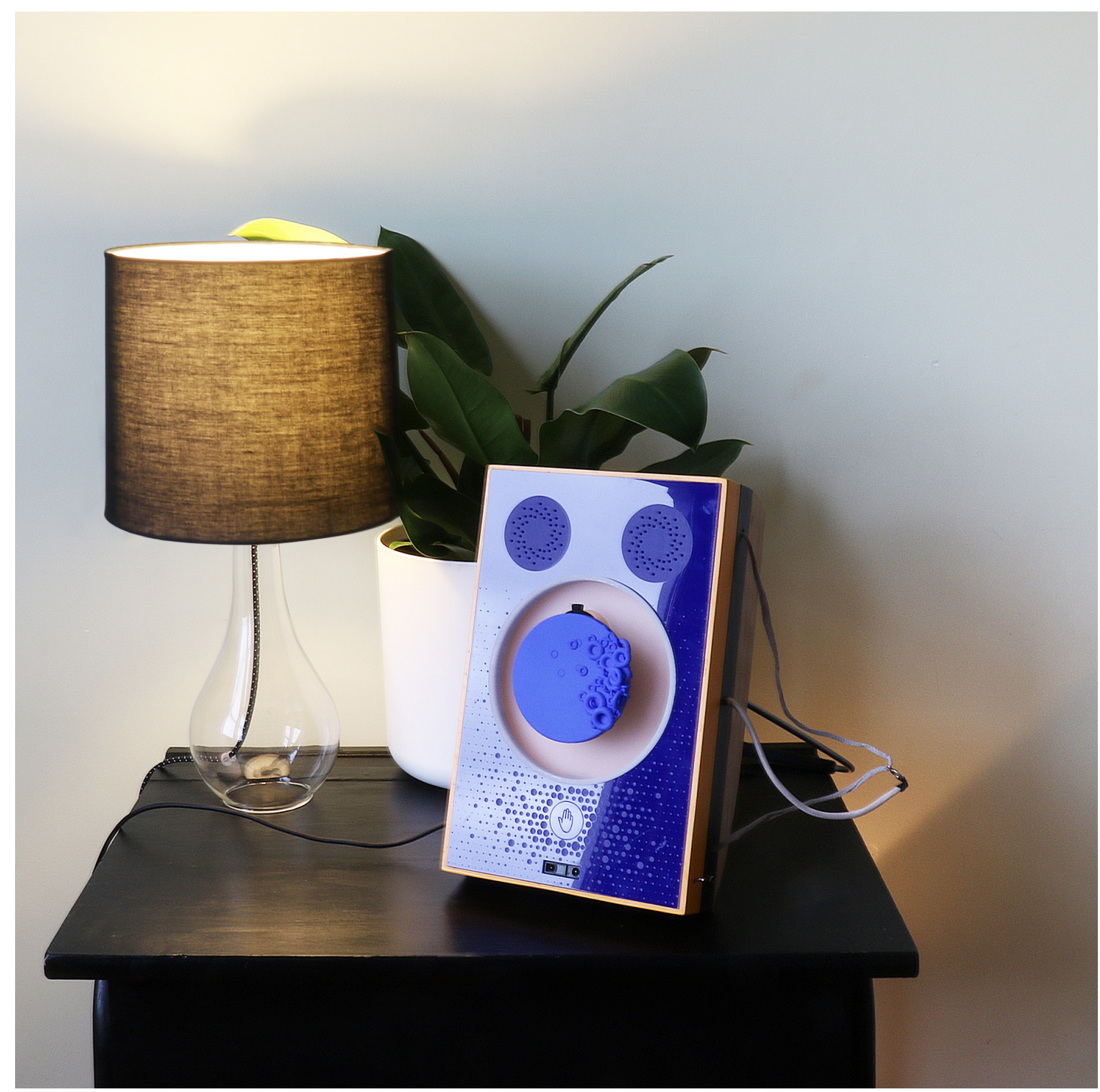

Volume button Stage II
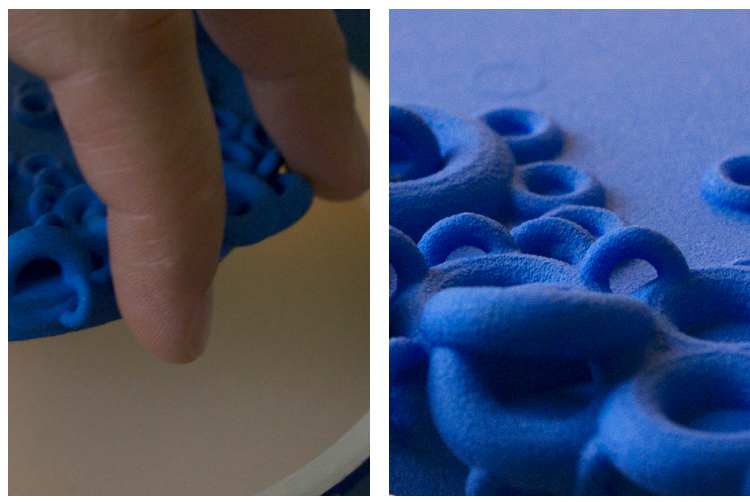


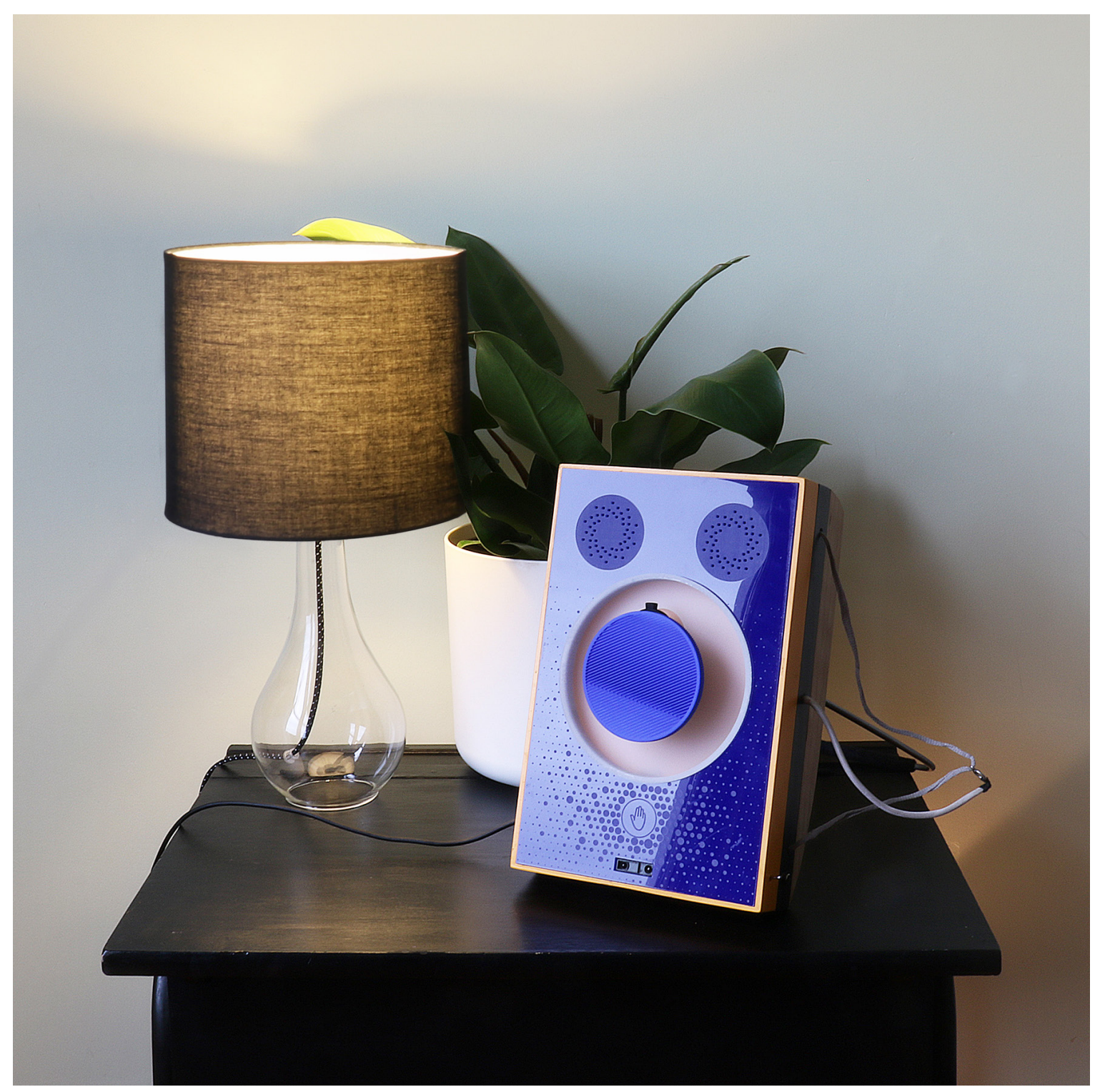

Volume button Stage III

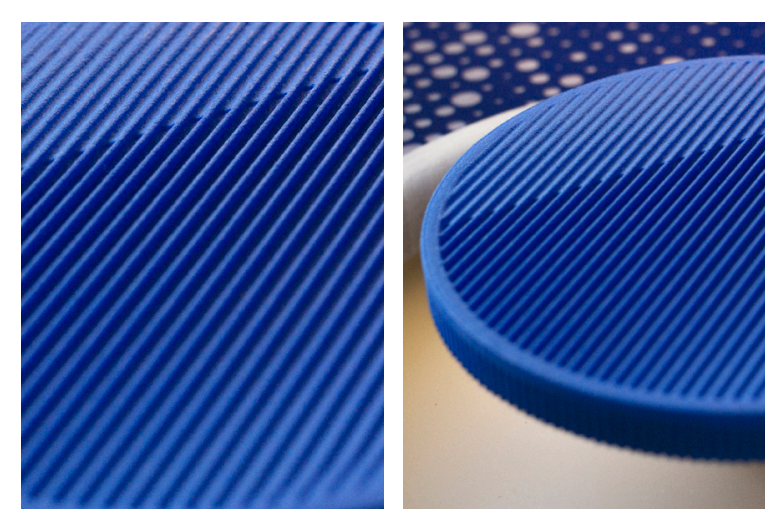




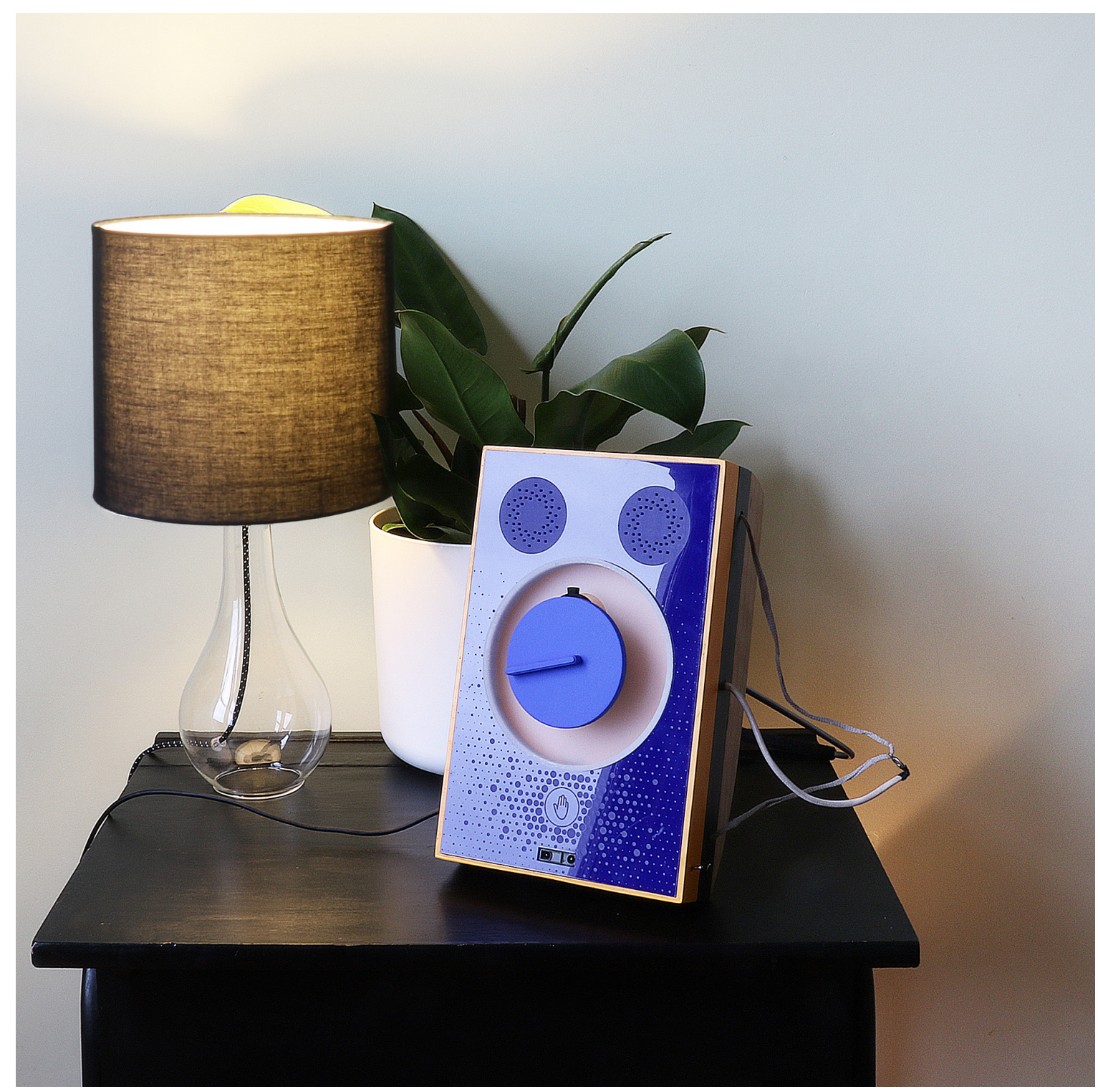

Volume button Stage II
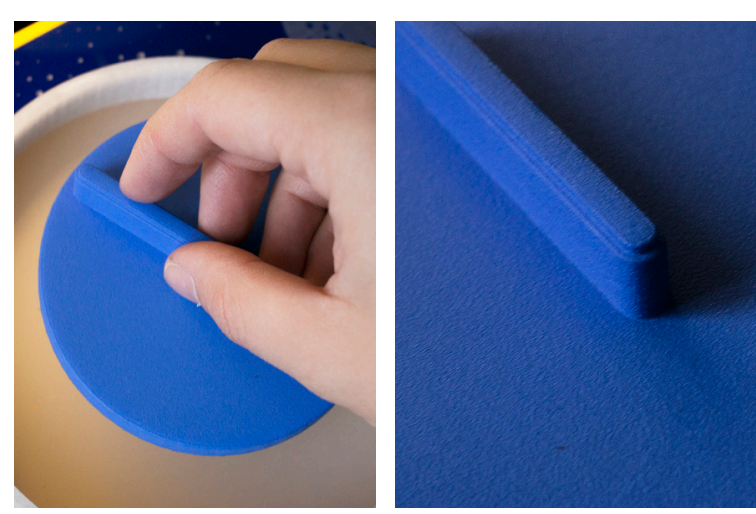


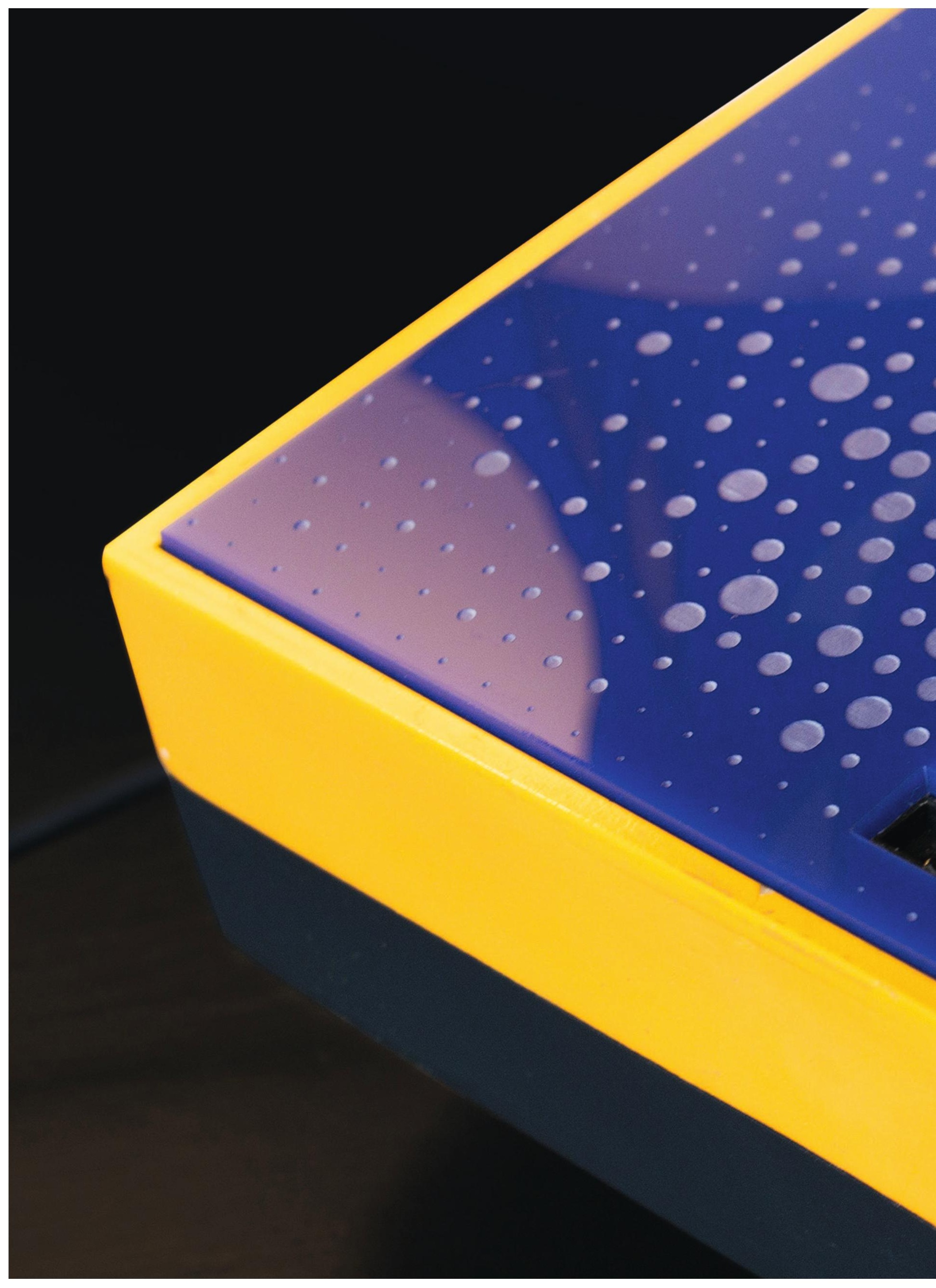




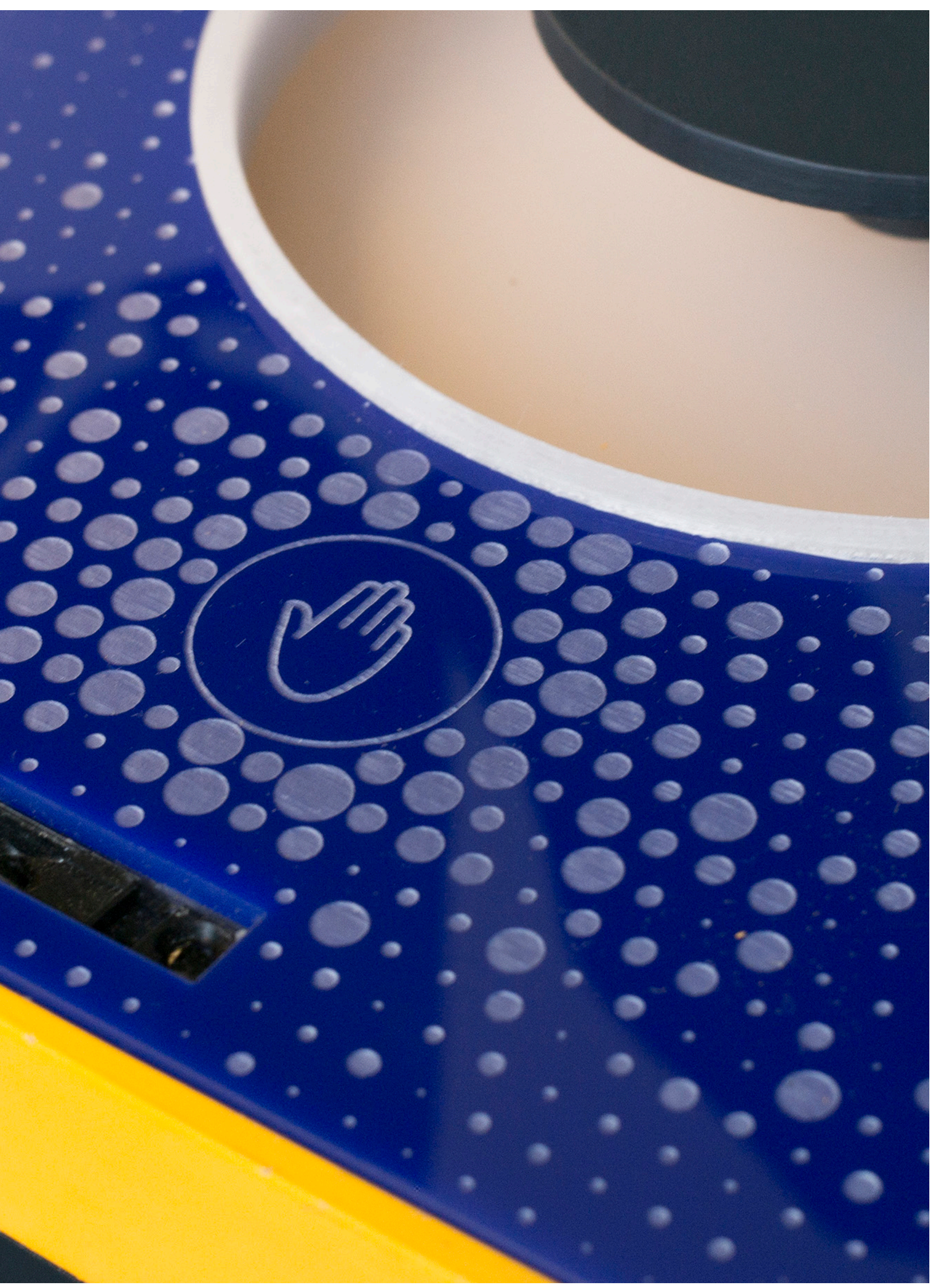





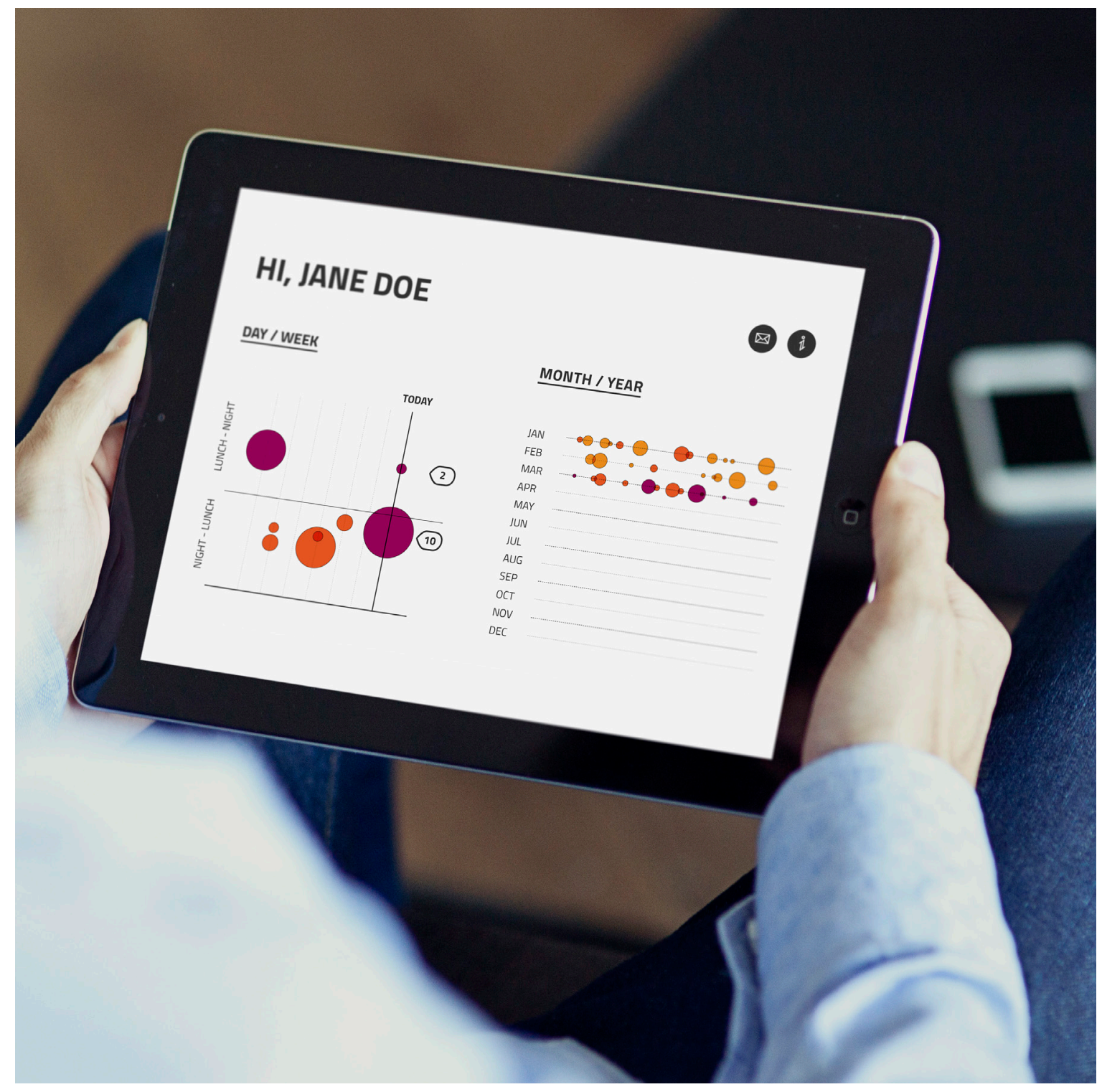




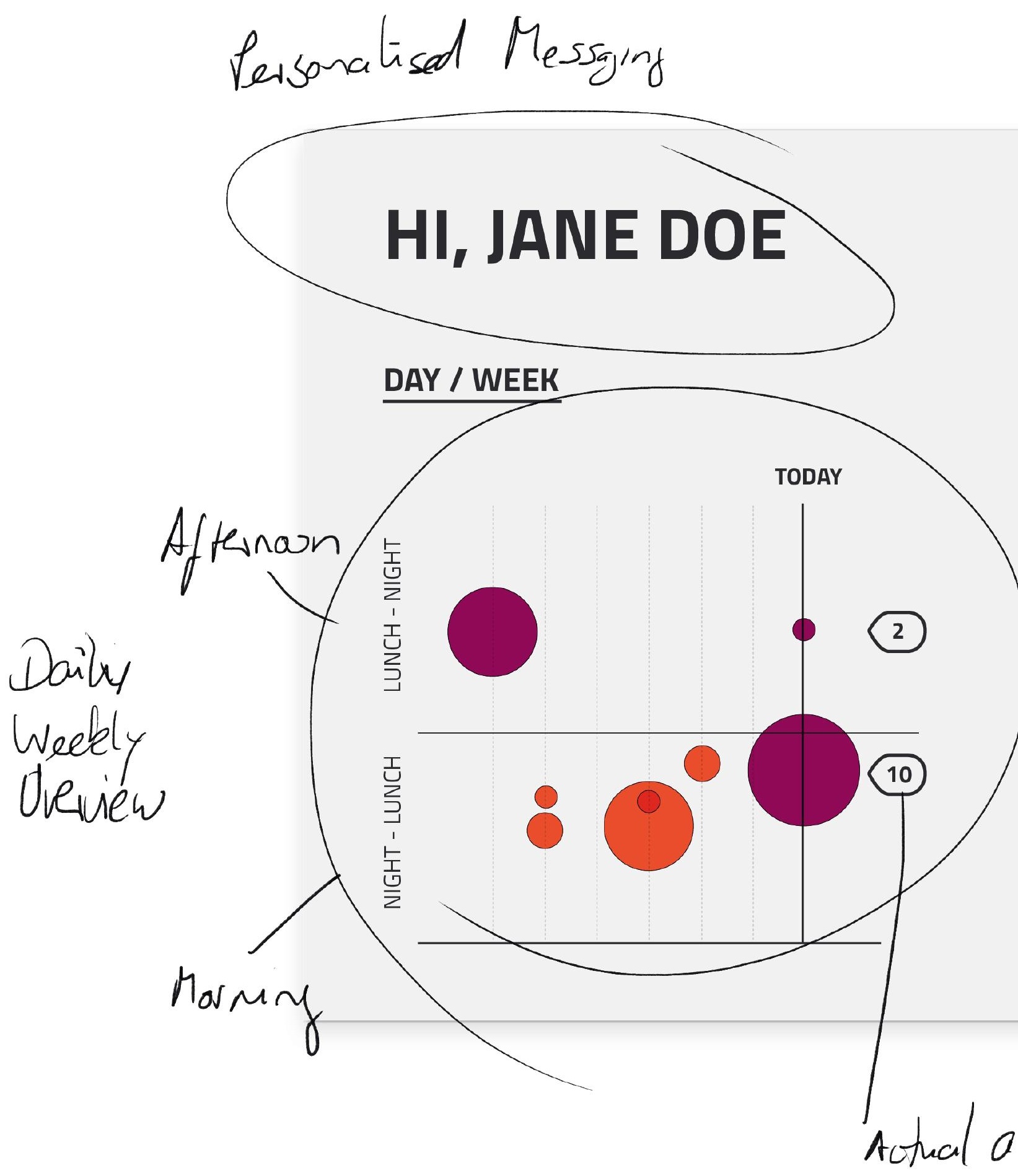




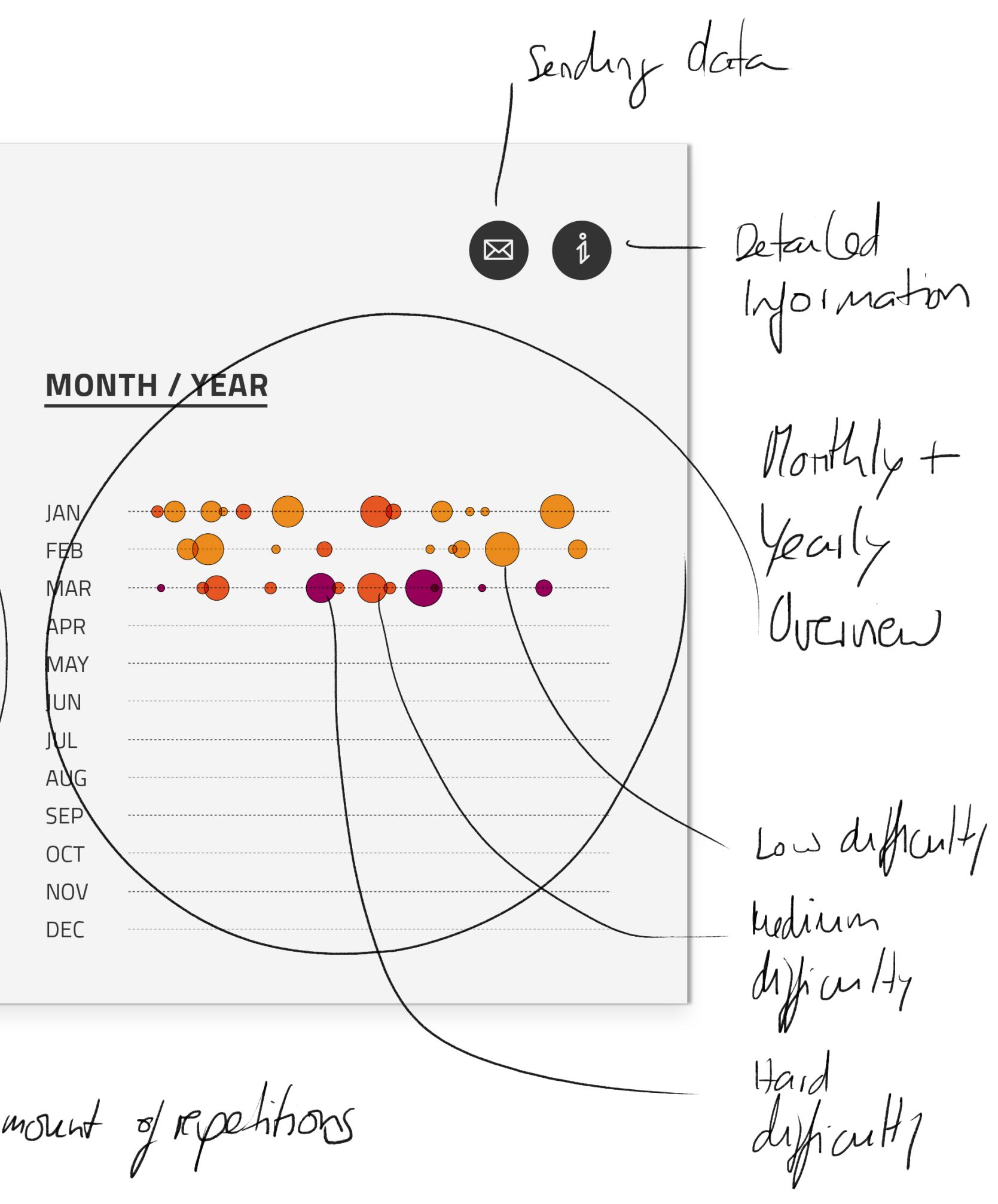




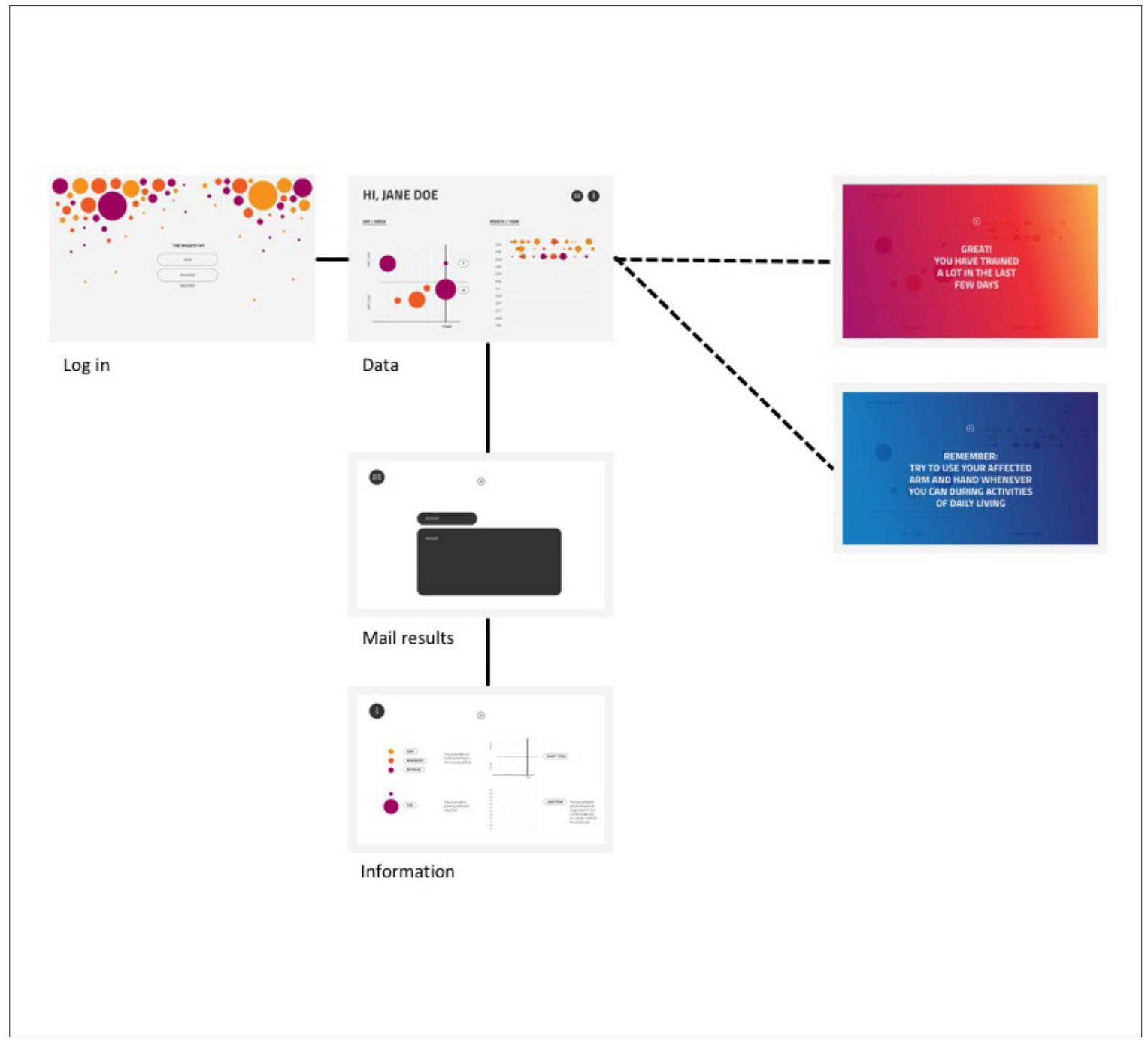

Struletogram 


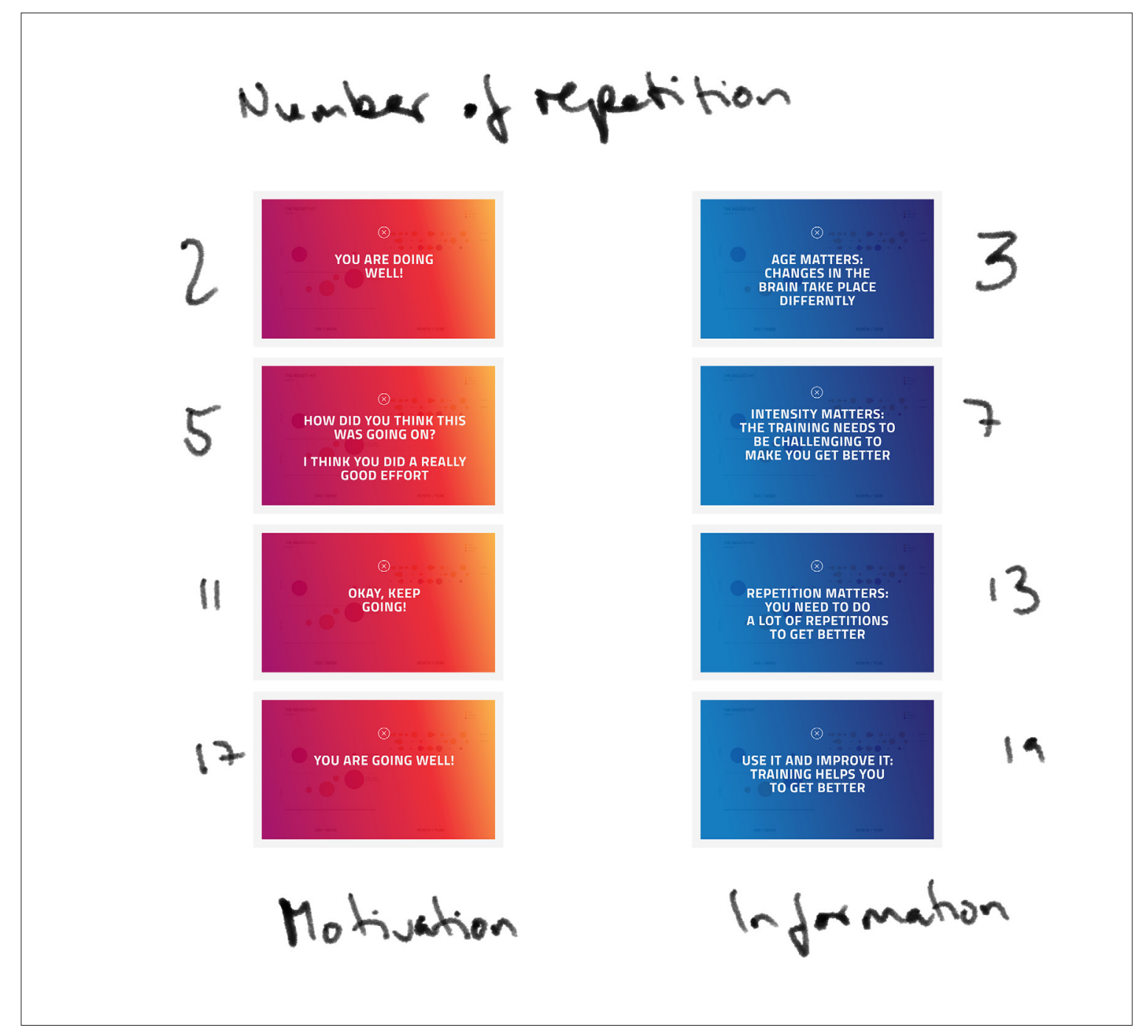

Motivation + Information Input 



\section{Appendix D Workbook 3}




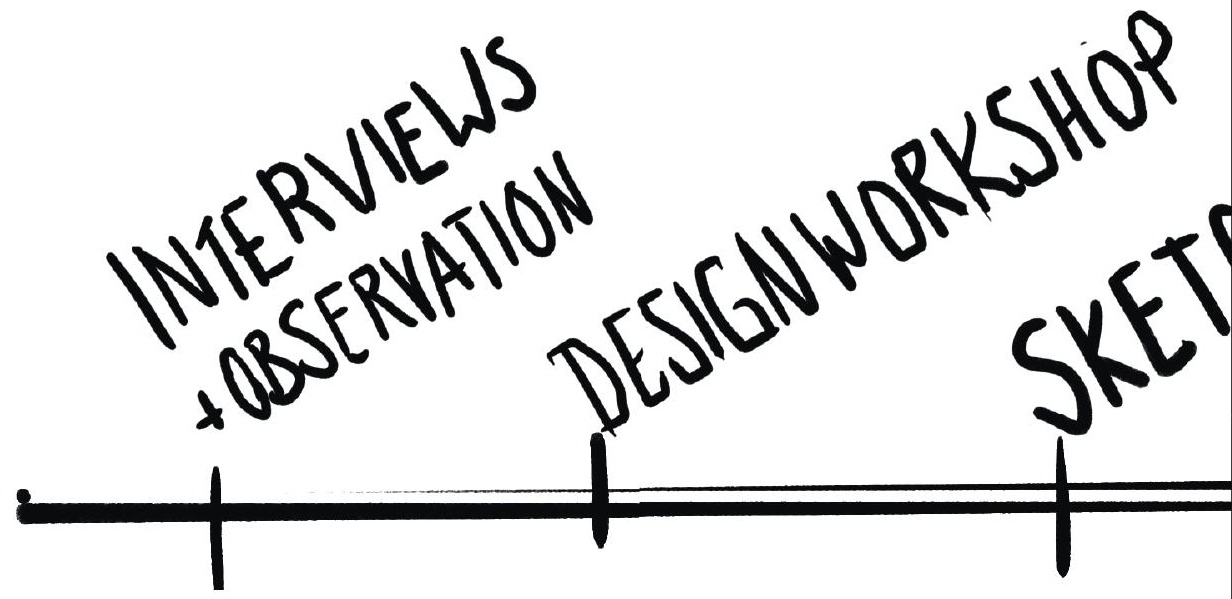

Timelne 


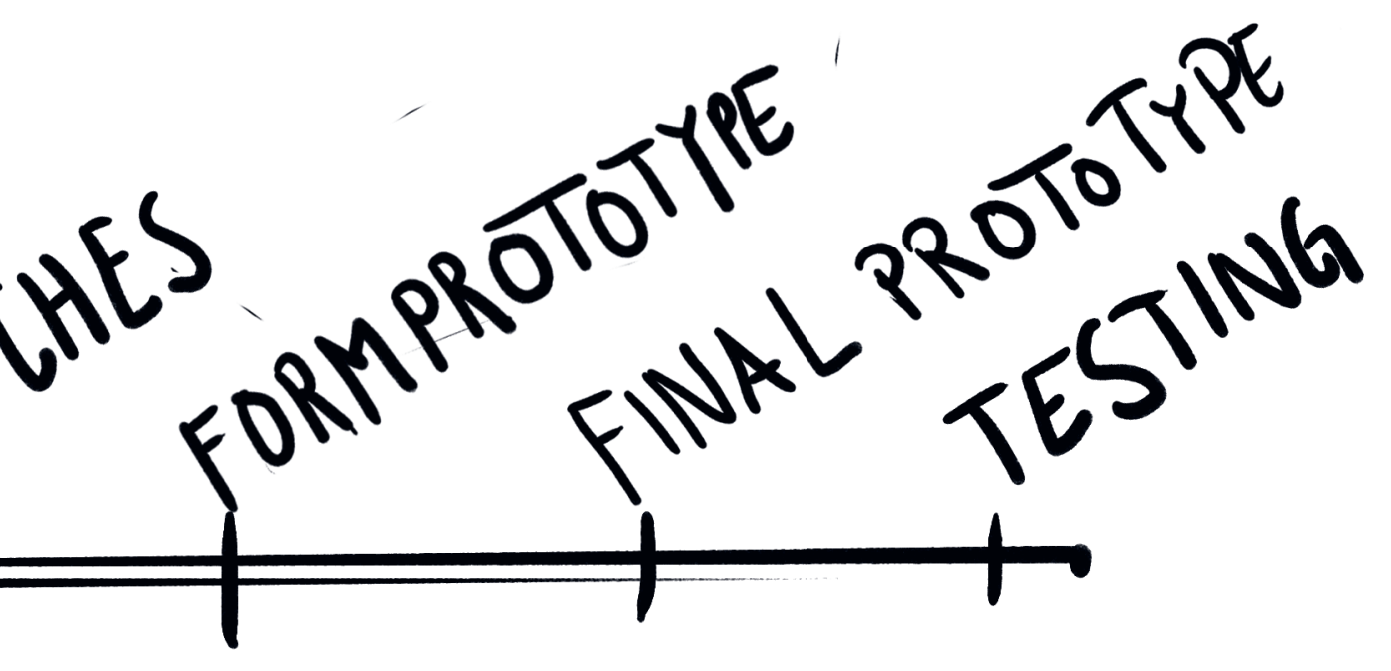


$-g !$

How can the use of a phone be reskained to evoke on initiation of use?

is

Man focus of the design workshop 


\author{
INTRODUCTION \\ I \\ BRANNSTORM ING \\ ACVIVITY \\ I \\ SKETCHINL \\ - CRAZT EIGHTO \\ I \\ SKETCHIUG \\ REFINING CONCEPTS \\ WRAPPING UP
}




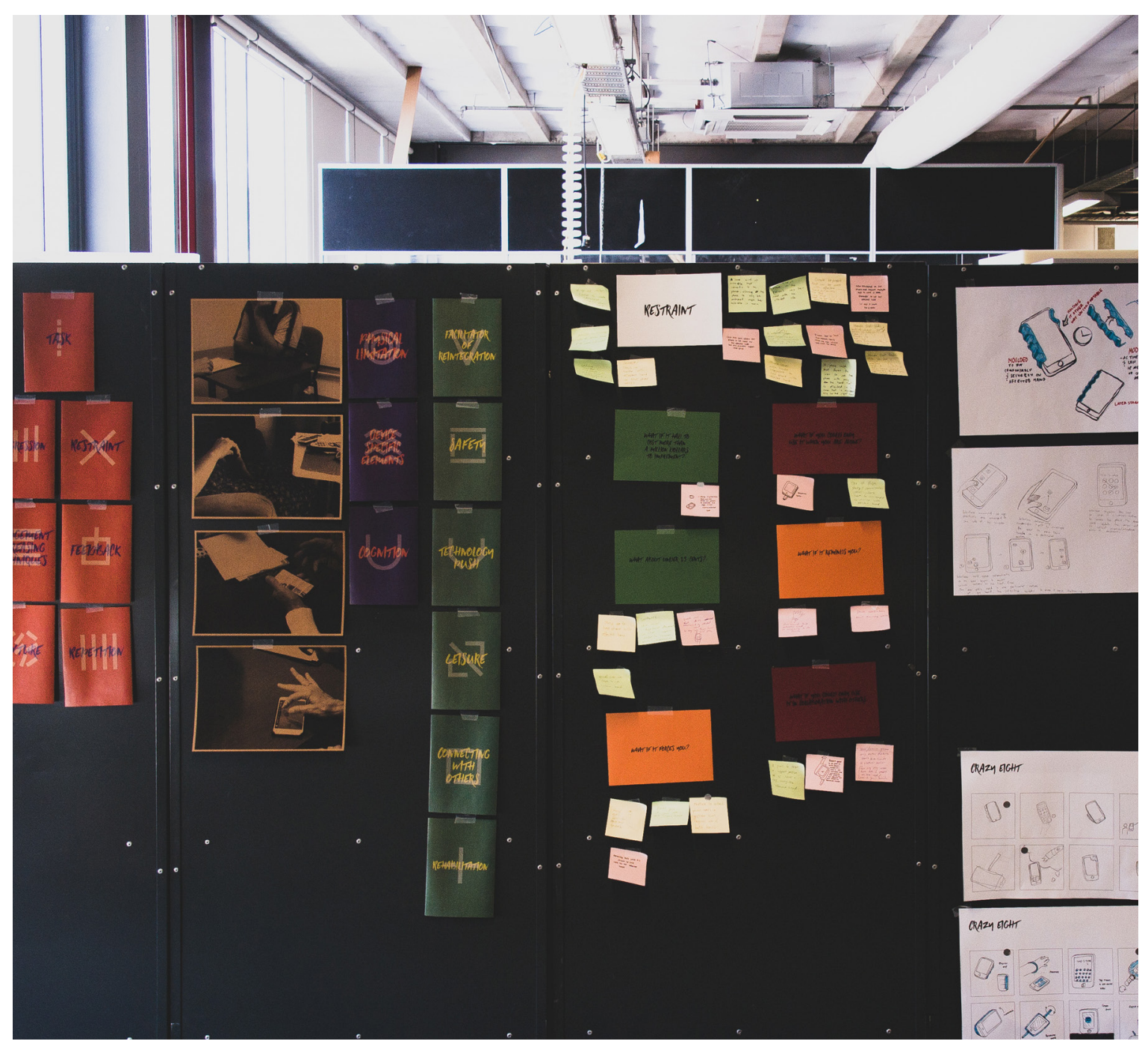

Brain storming actinty \& power of ten
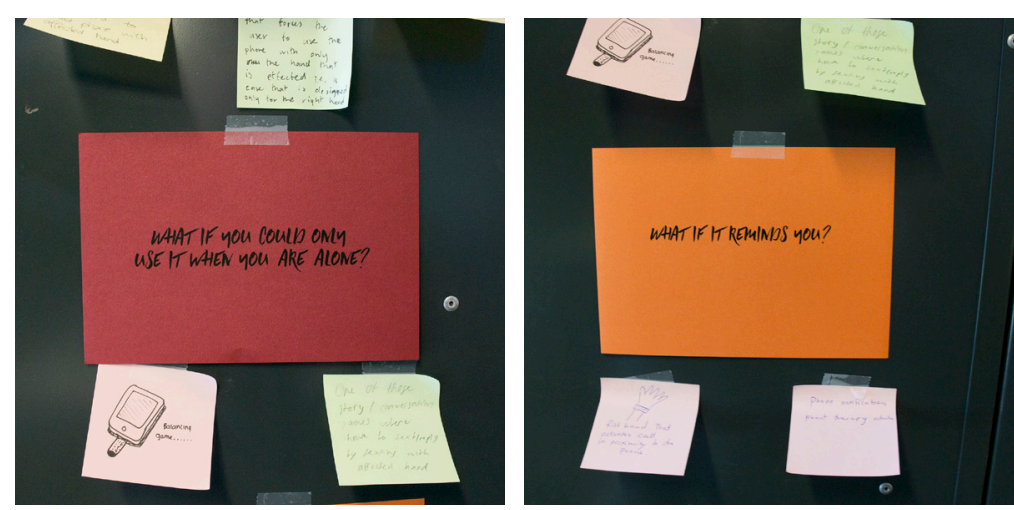


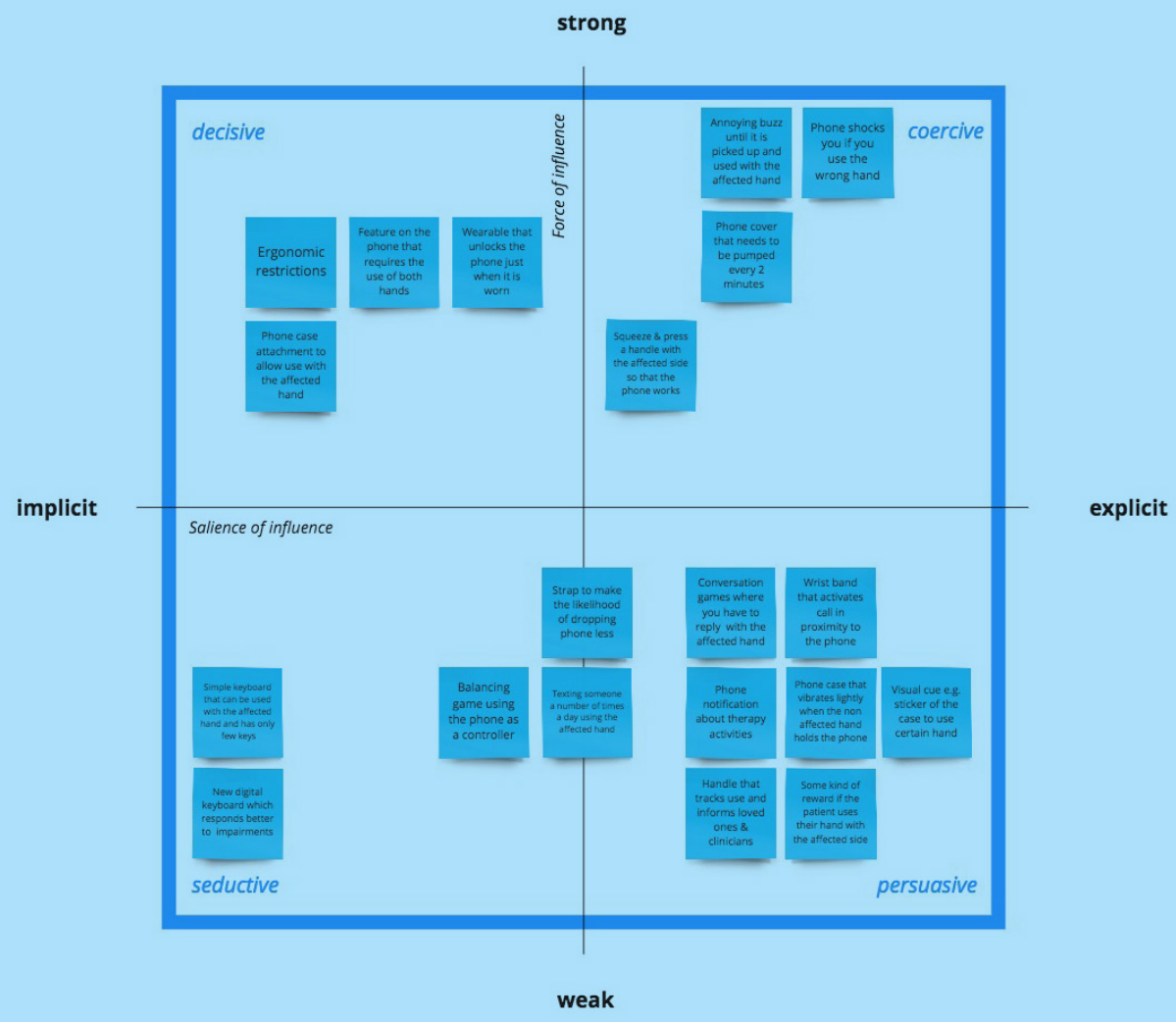

Design concepts and thair expected nfunence on theuer 


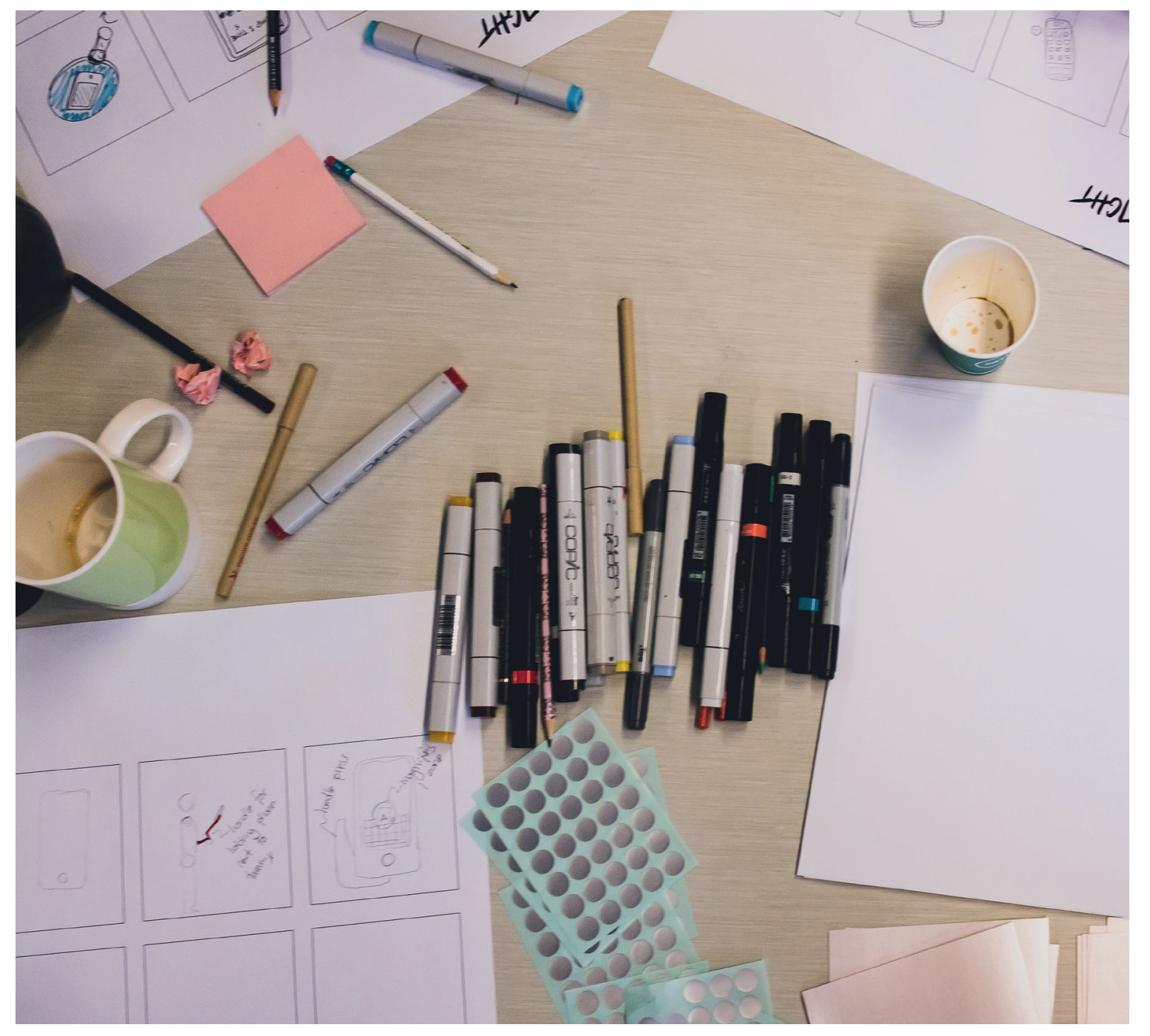

The steatehny actinty the power of aight led to a series of desgn concopts In a seond step the deyign cancopts were refined, see the right sicle 


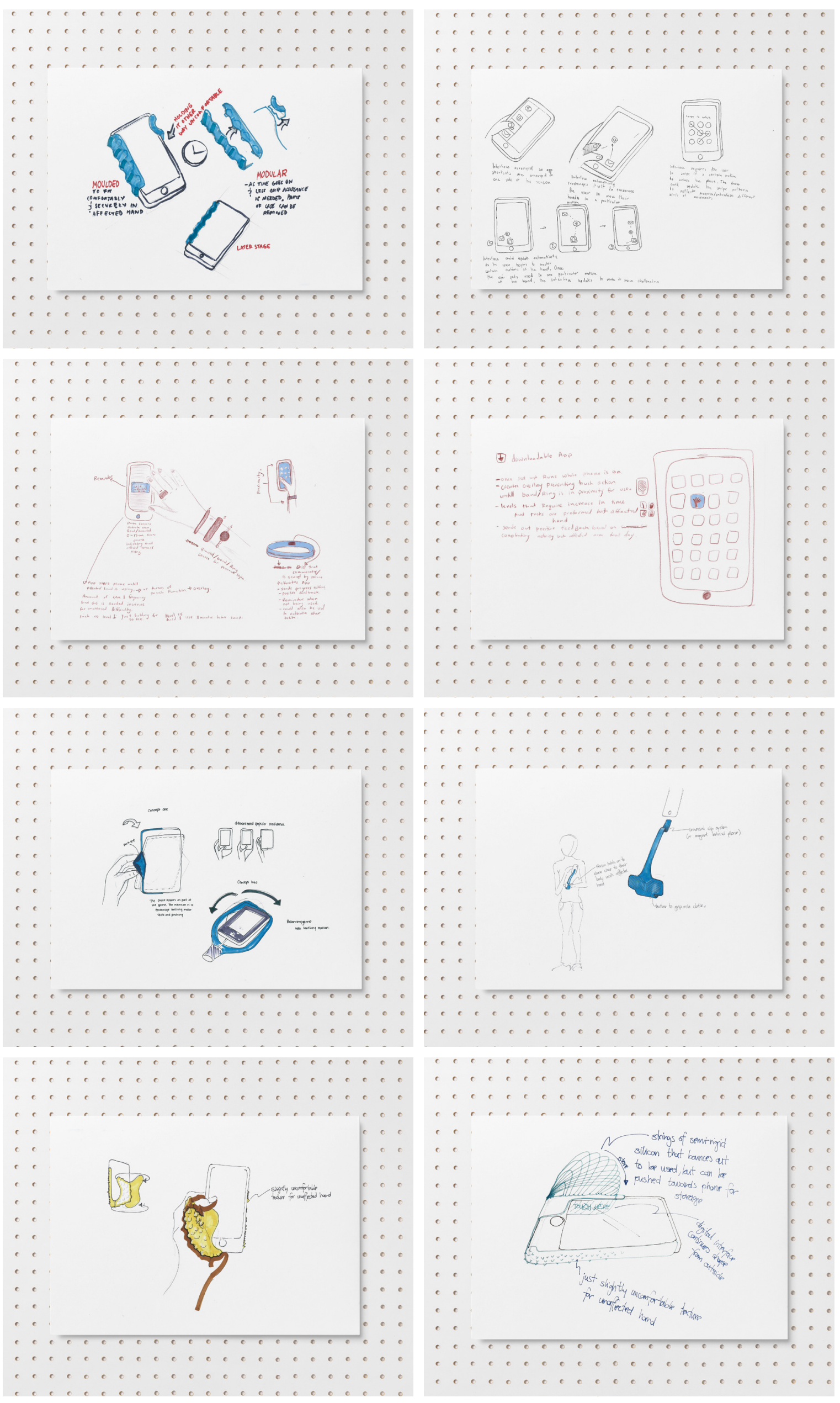




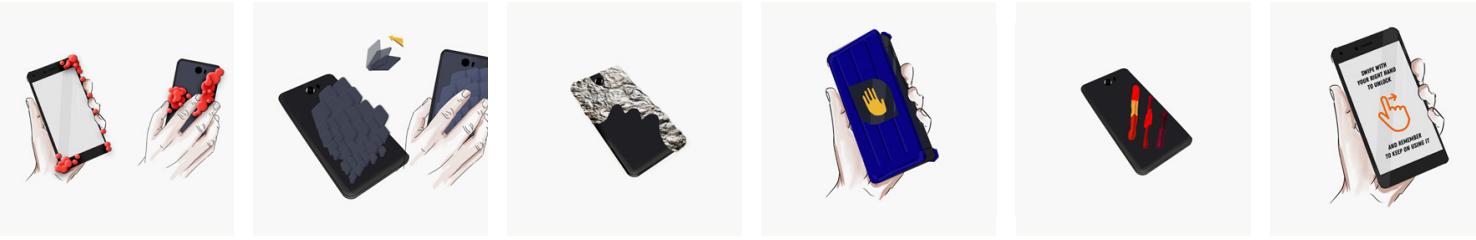

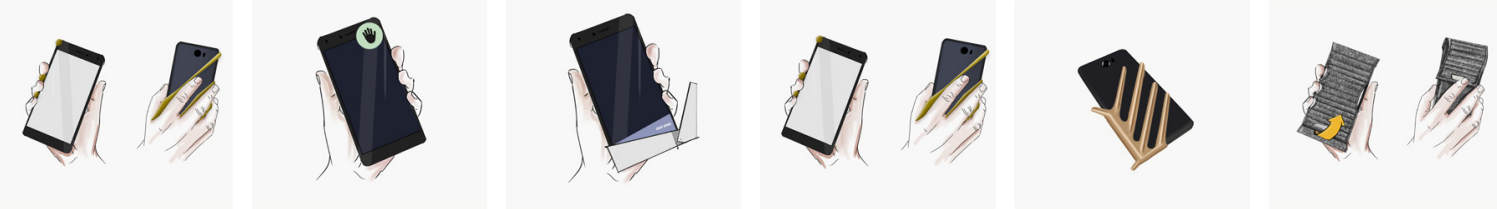

01000

1) a 1200

Q

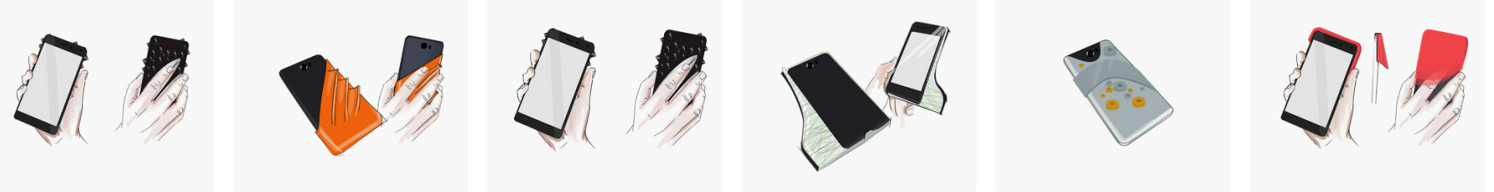


Based on the canalysis of the developed

design concepts two different concepls were

chosen for further steetchos. One desyn arms

to evoke a persuosice in/fuence white the

second on is coerave. 


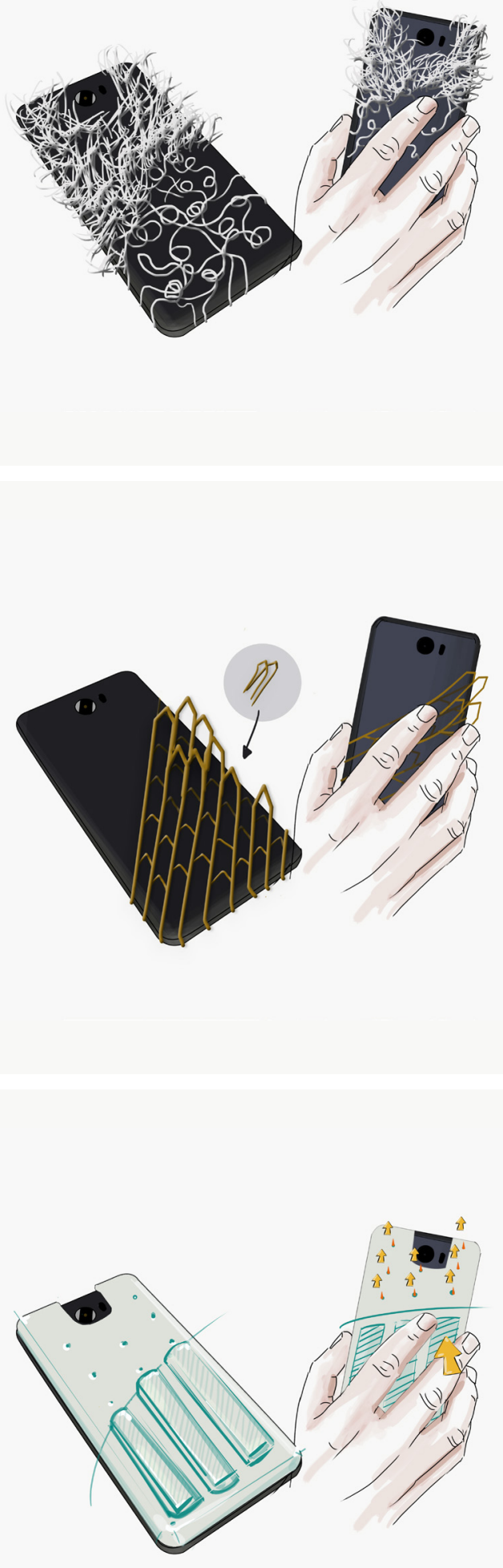

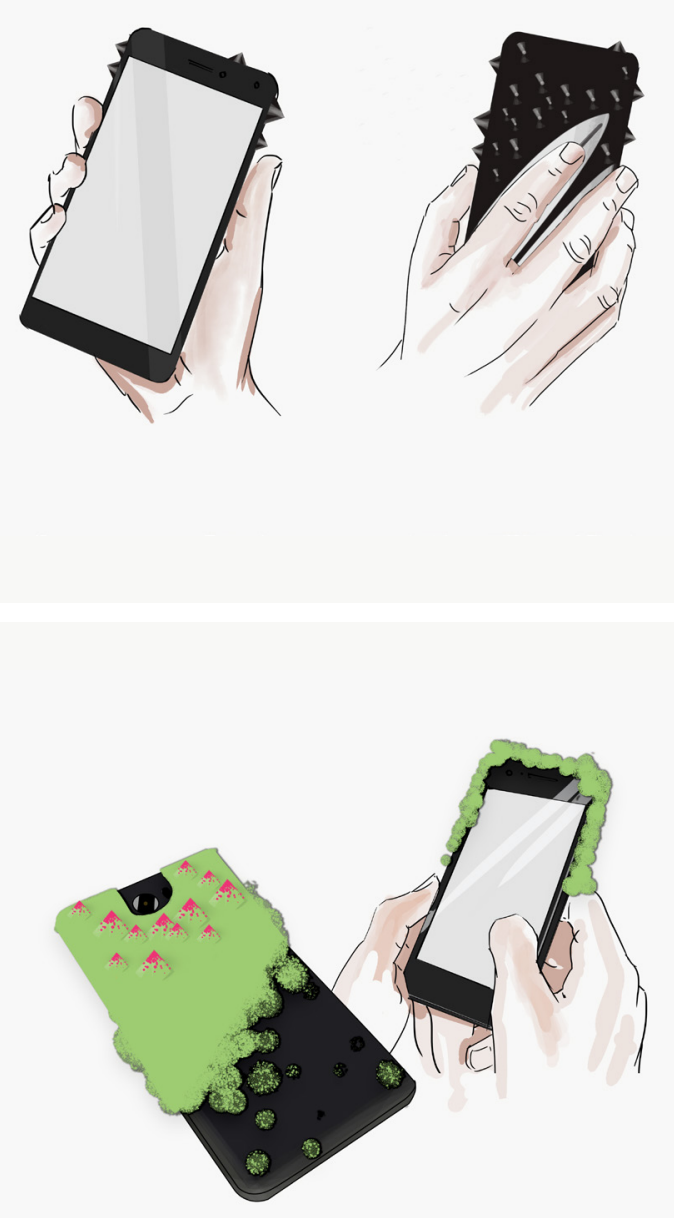


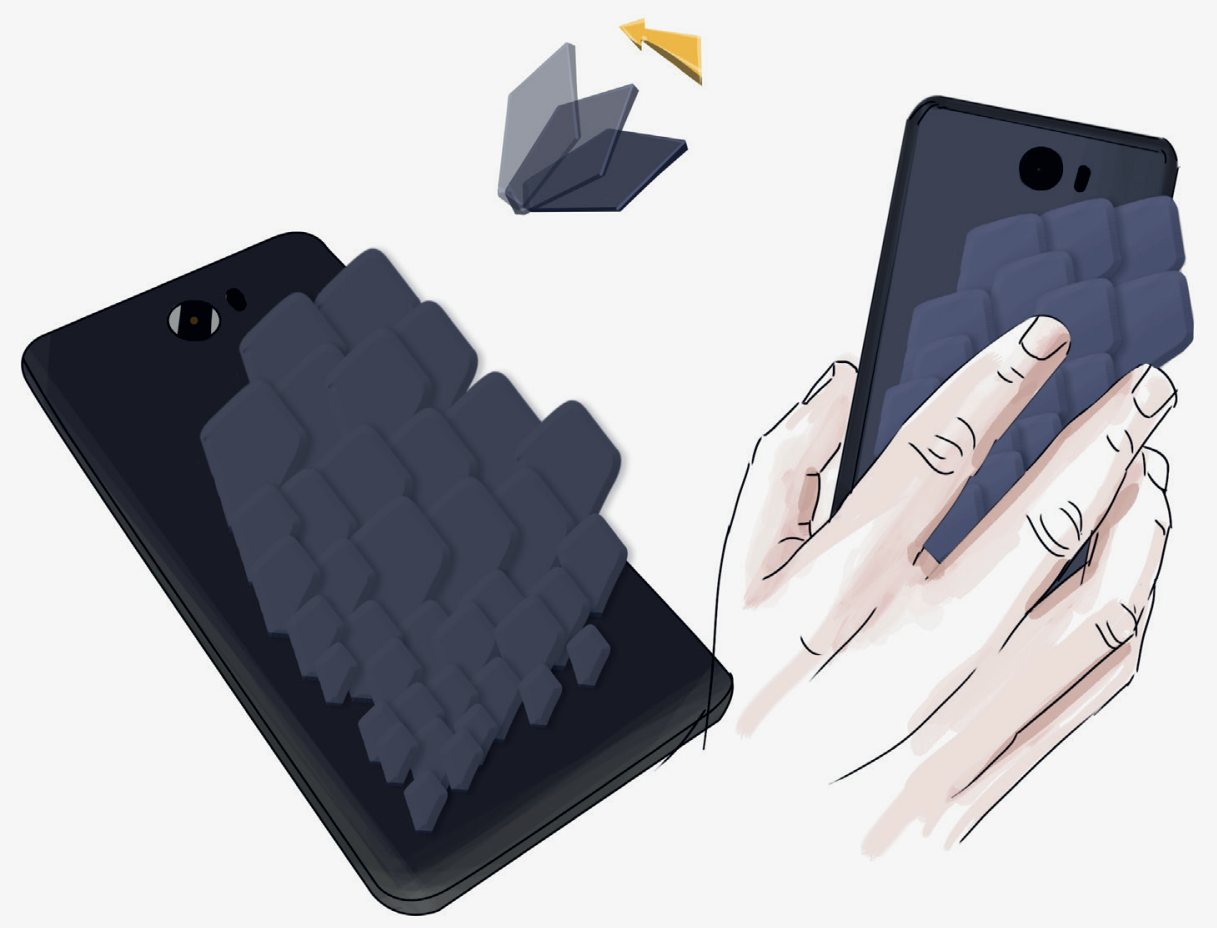

Concept os (topleff).

The design has a spoky tetture situated at the top.

(oncept or (botto m left):

A spiky texture at the top becones softer towards the botfom of the phone. (oncept ot (right)

The lexture on the phove just impacts on the use of the phore if the less affected hand is used. It the affected hand holds t the texture is simply pressed down. 

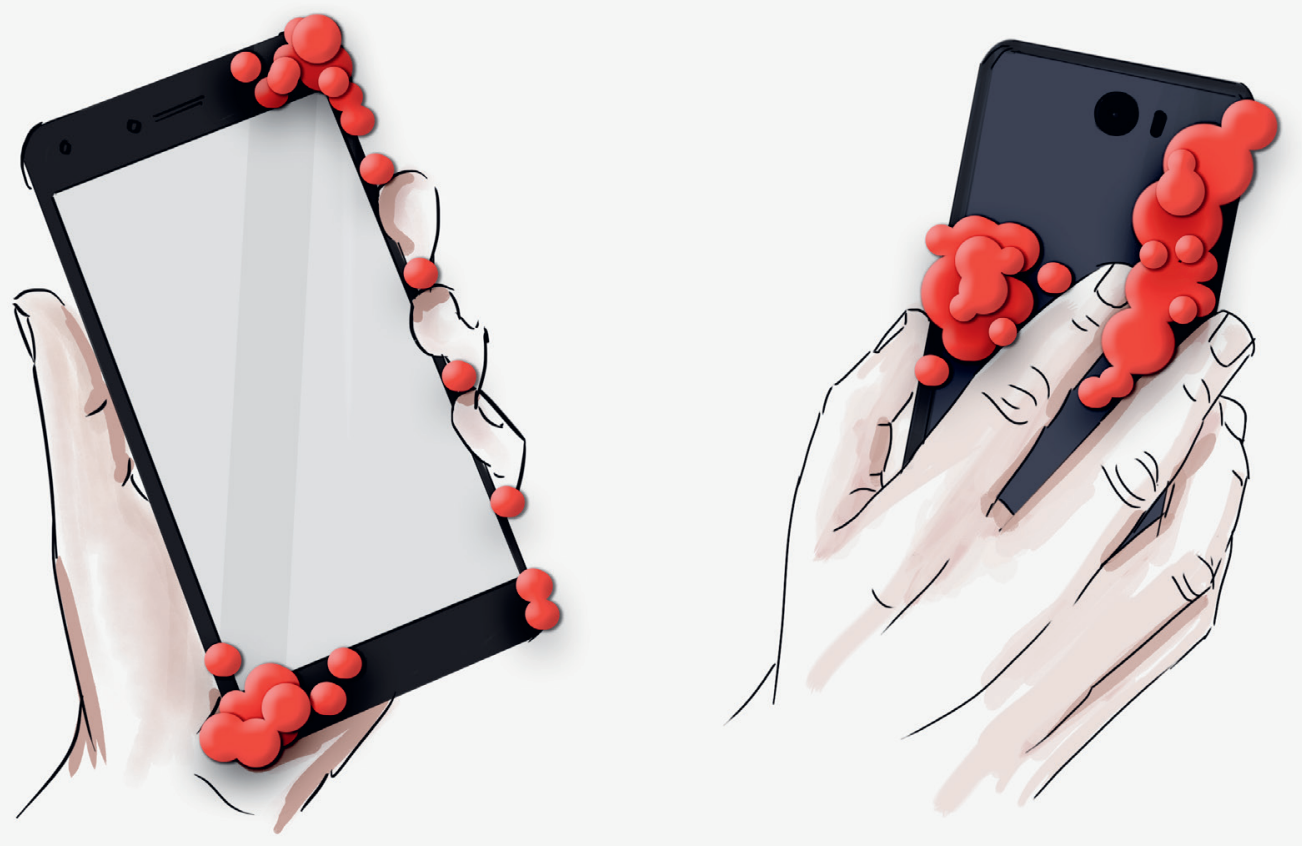

Conch os ( left)

A red, soft lethe is used to provide ongasome estuctions

Con capt or (tonight)

Elgorome restrictions at the bottom of the phone restrain the movement and enable

Concept 10 (middle right)

The handle on one side limits the ability to hold the phone on that side.

a comparable holding position with the gelled loncoptll (bother night) hand.

A strap allows to hold the phone surely. 

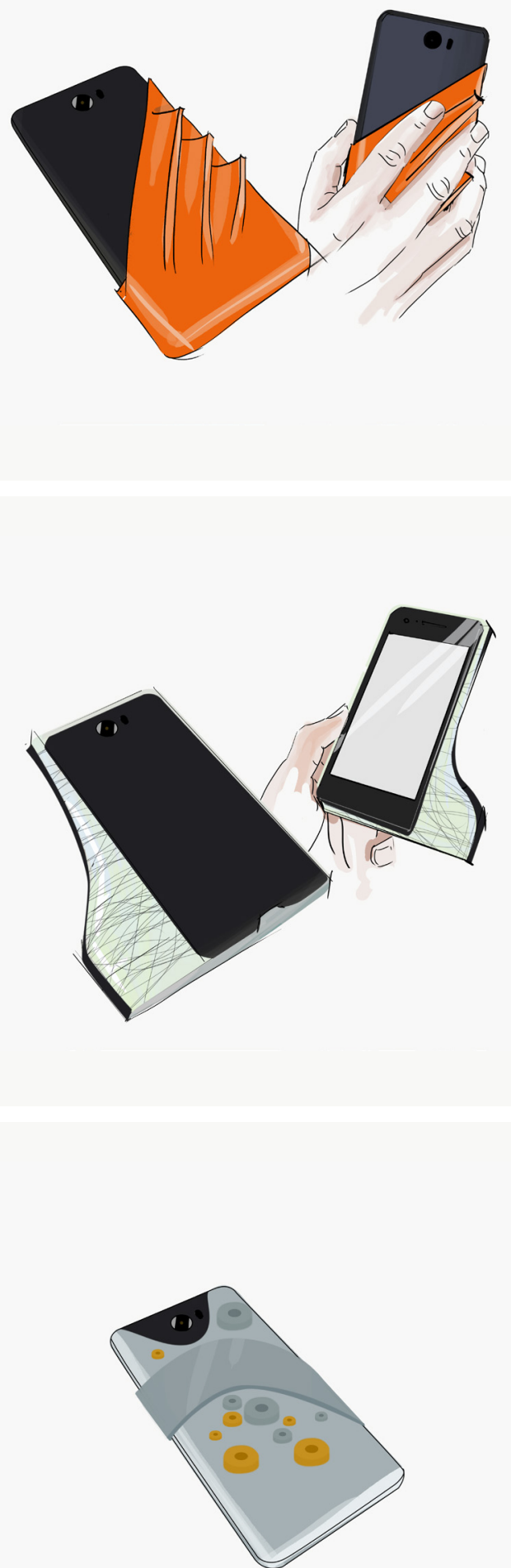


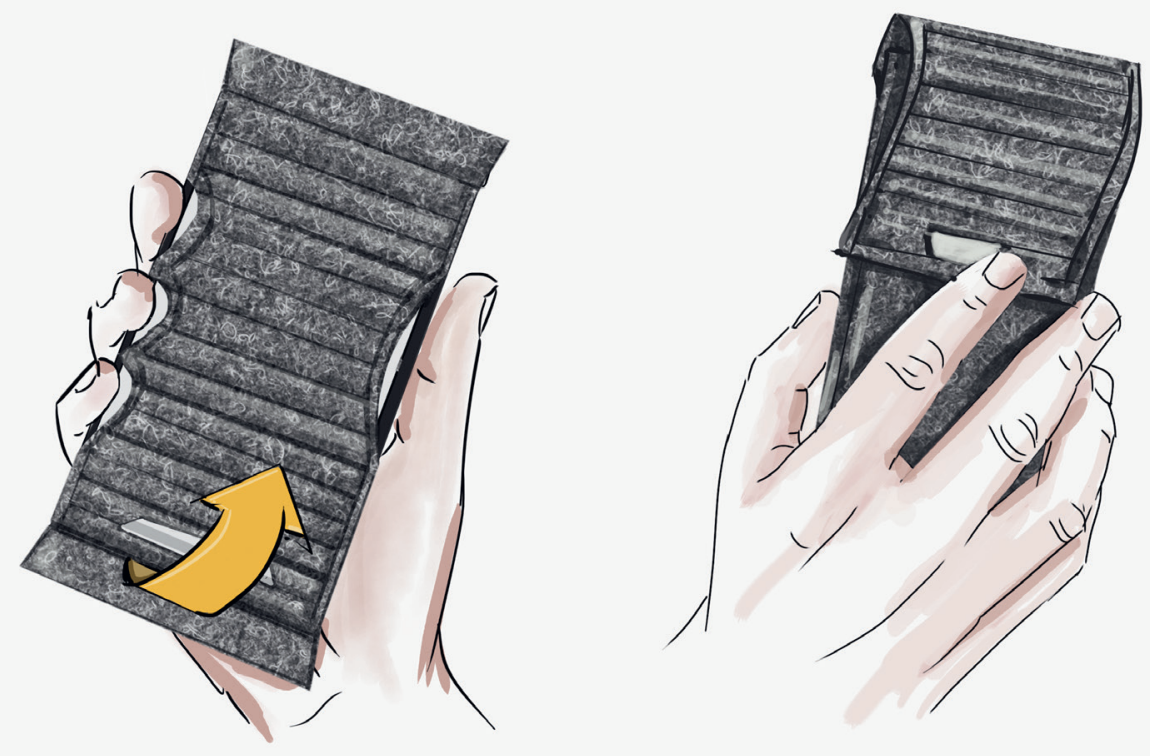

Concept 12- M (top to bottom left): Concept 15 ( night)

The designs he visual remanders in the form The protection case of the phove can easily of stikers or screensavers to use the transjormed into a support handle at the affected hand. back of the phoce 

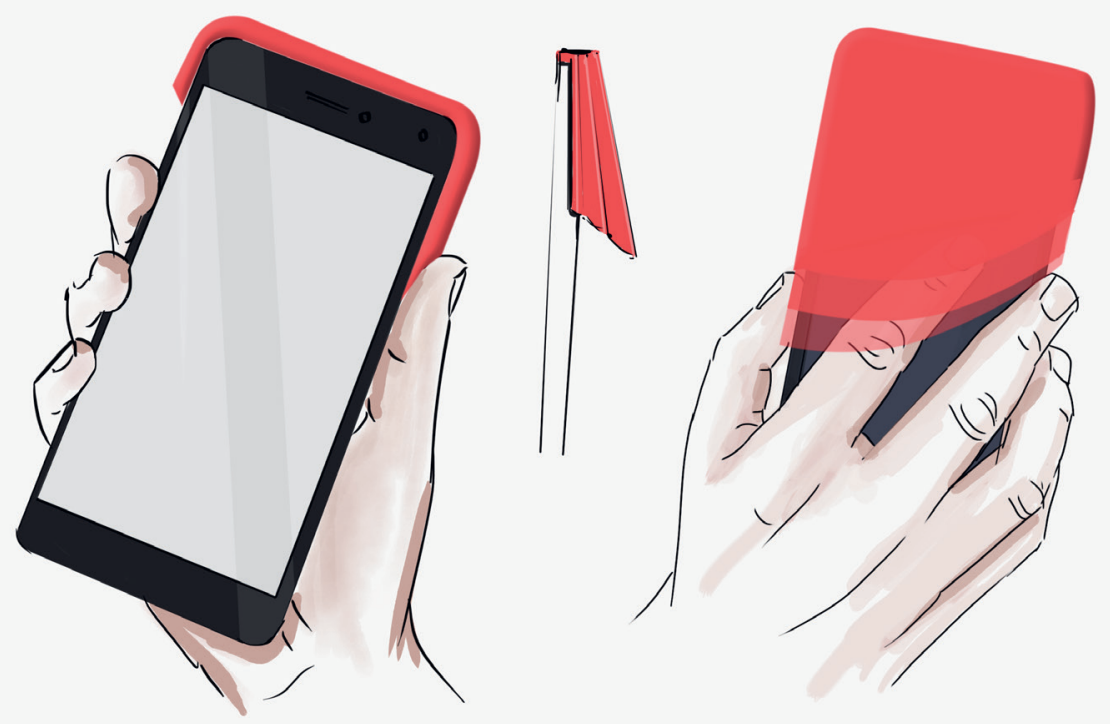

Comet $16(6 / t)$

The red clement offers support while holding the (concept 18 (middlenght)

phone. The design haas a sight angle to allow The support at the back can just be used with a better use with the affected hand. the affected index finger.

(once/ 17 (toping)

Concept 19 ( bottom nits)

The shops at the front need to bo moved to the Smatisteps at the back allow a more comparlast back to support the effected hand. hand position with the affected hand. 

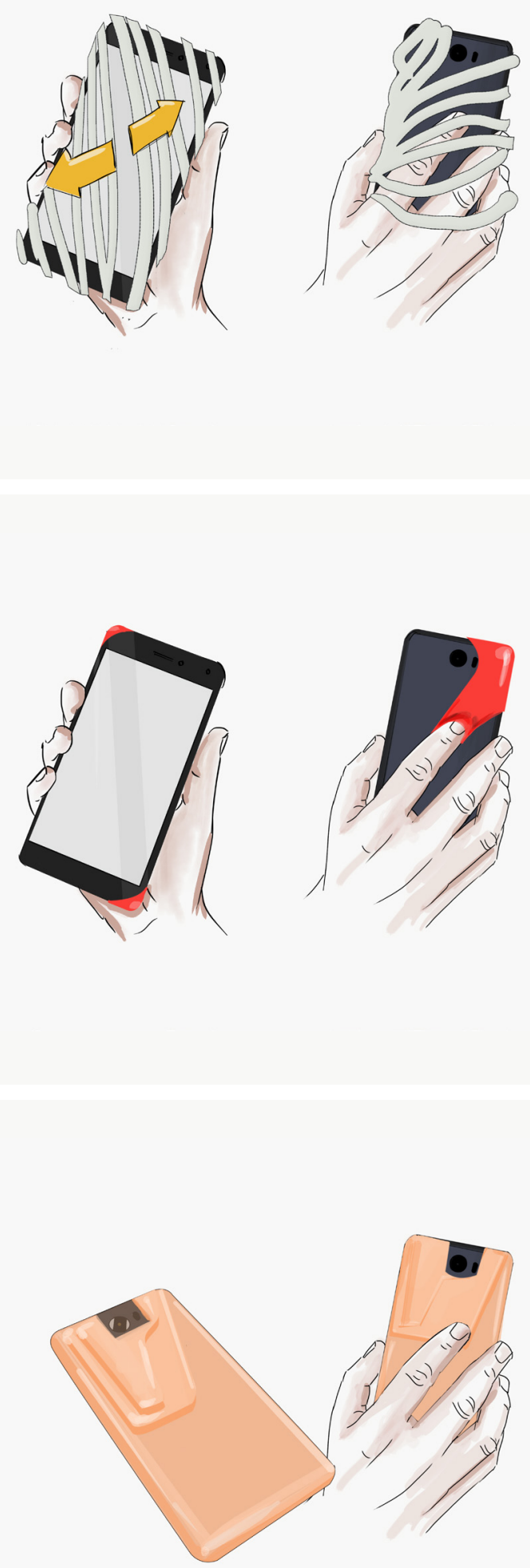

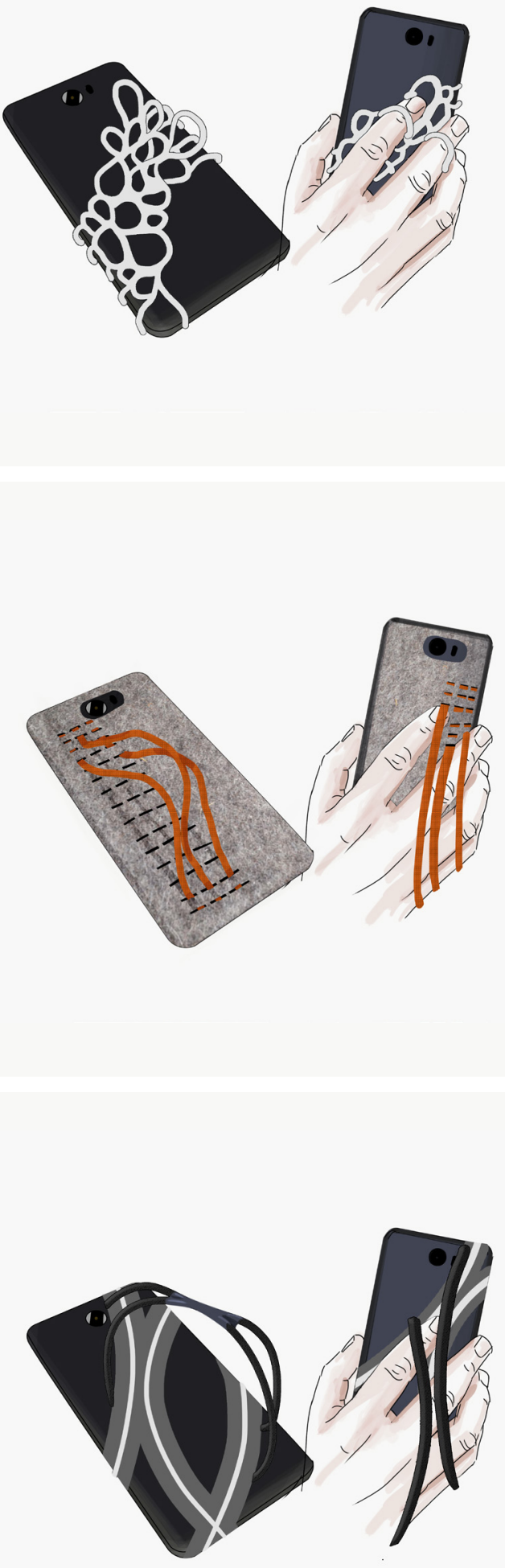

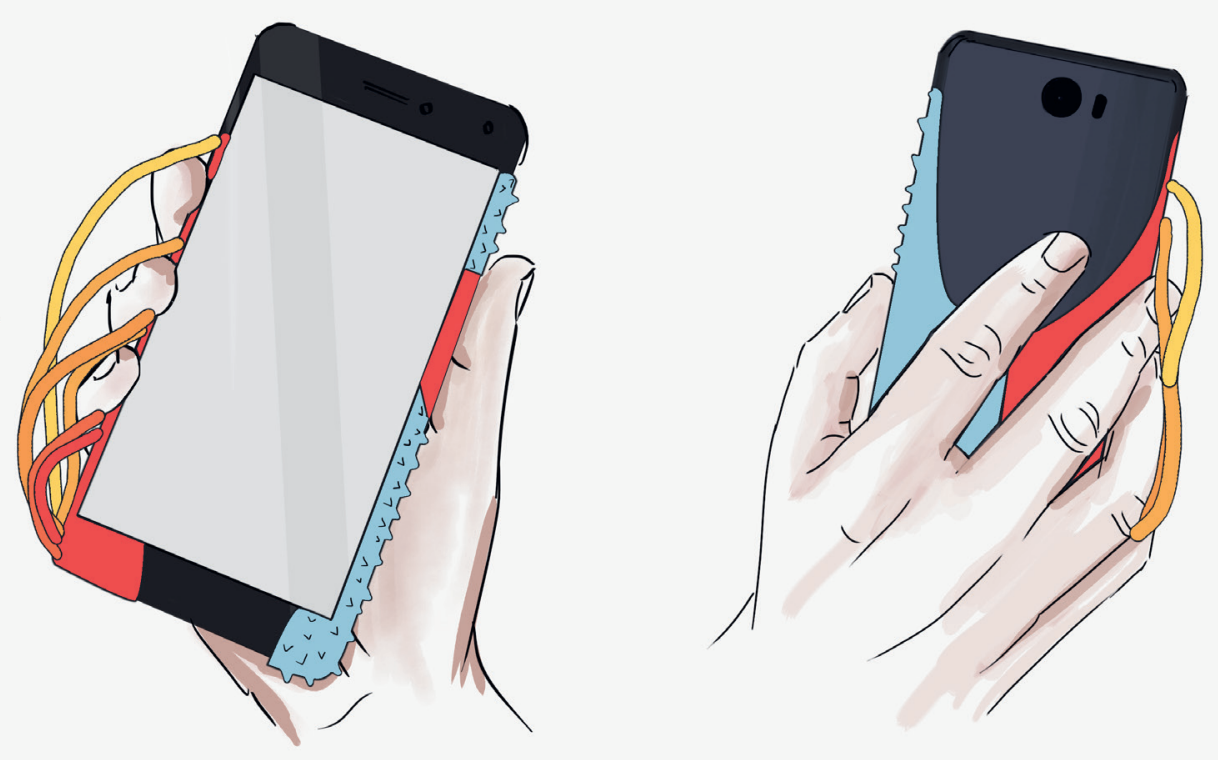

Concept $20-22$ (topto botton left).

(oncepi Ls (rijht)

The three different design concopt use stijos that The desin has strips to safly hold the phove. A spiky allow to hold the phore safely. The way the strijs texture evokes an uncomplortable freding in case the are situaled ains to restrain novemest. user tries to hold the phove with the less affected hand. 


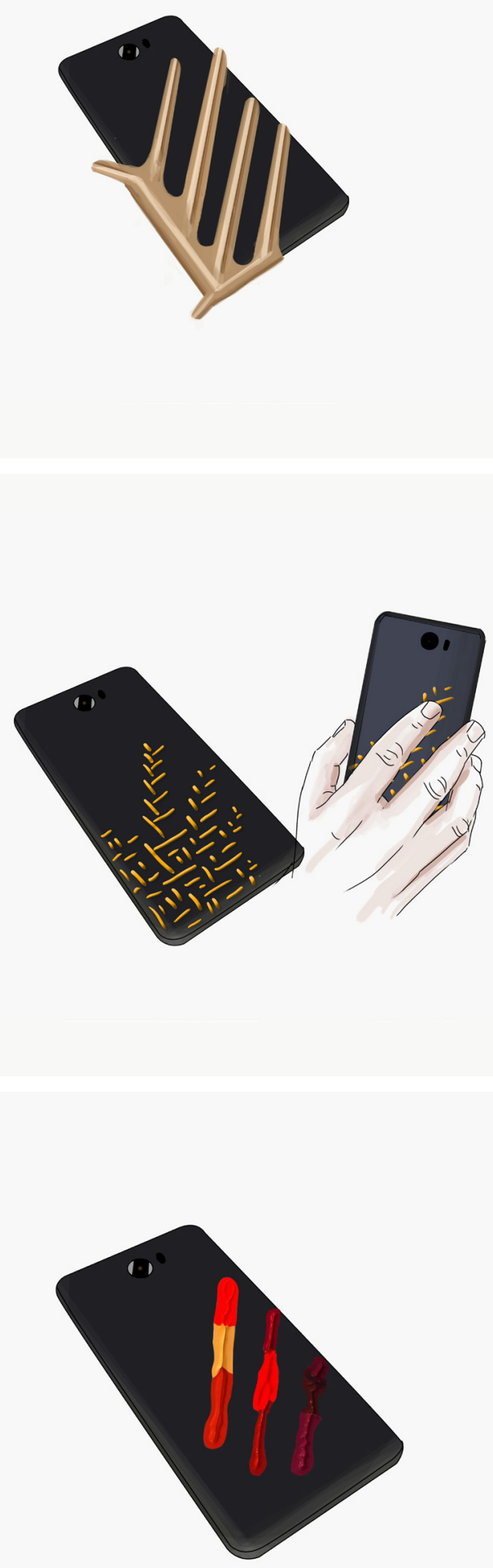


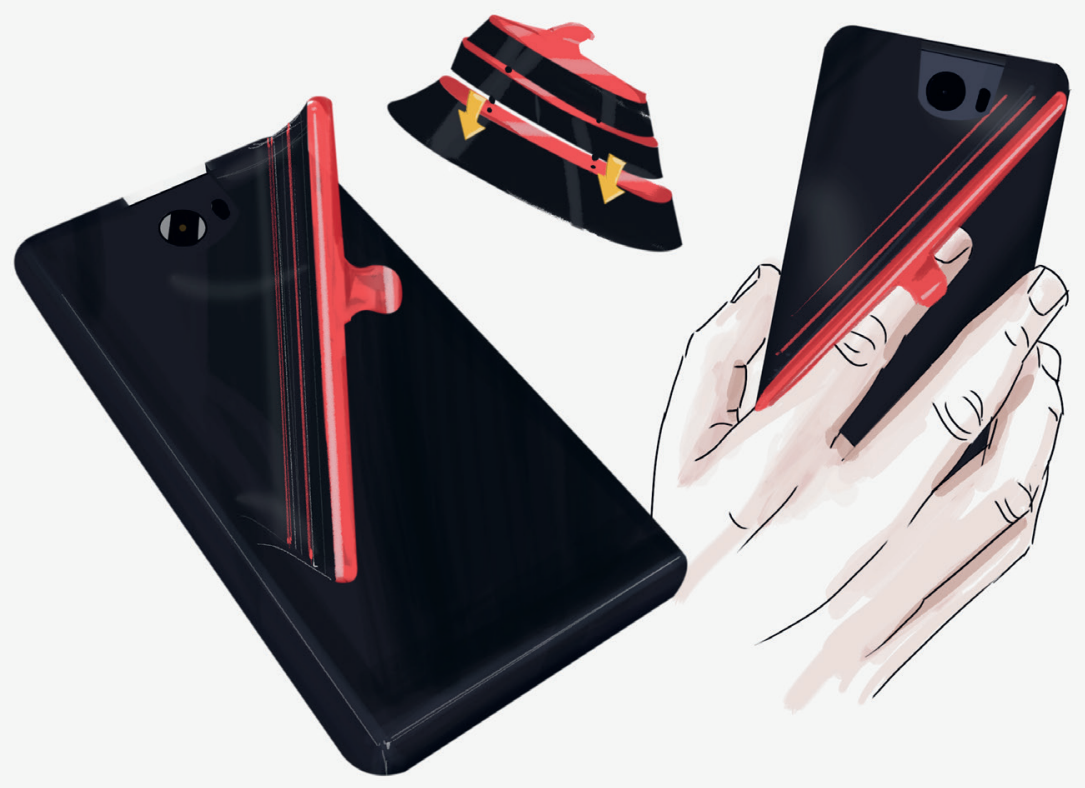

(oncep $28(6) 1)$

The plore has an ergonomic reshnchors at the baik

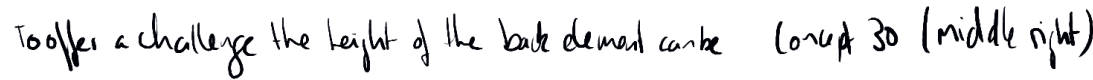

adjested. Etronomic retrictions in the form of staps.

Concepzaftop nght) (oncept 31 (bottom night)

An ergonomic resticition that offers support for the inder concept for on adderssbole wlece every thing is shated finge of the affected had. $\quad$ on the right (the a fected) side of the screen. 

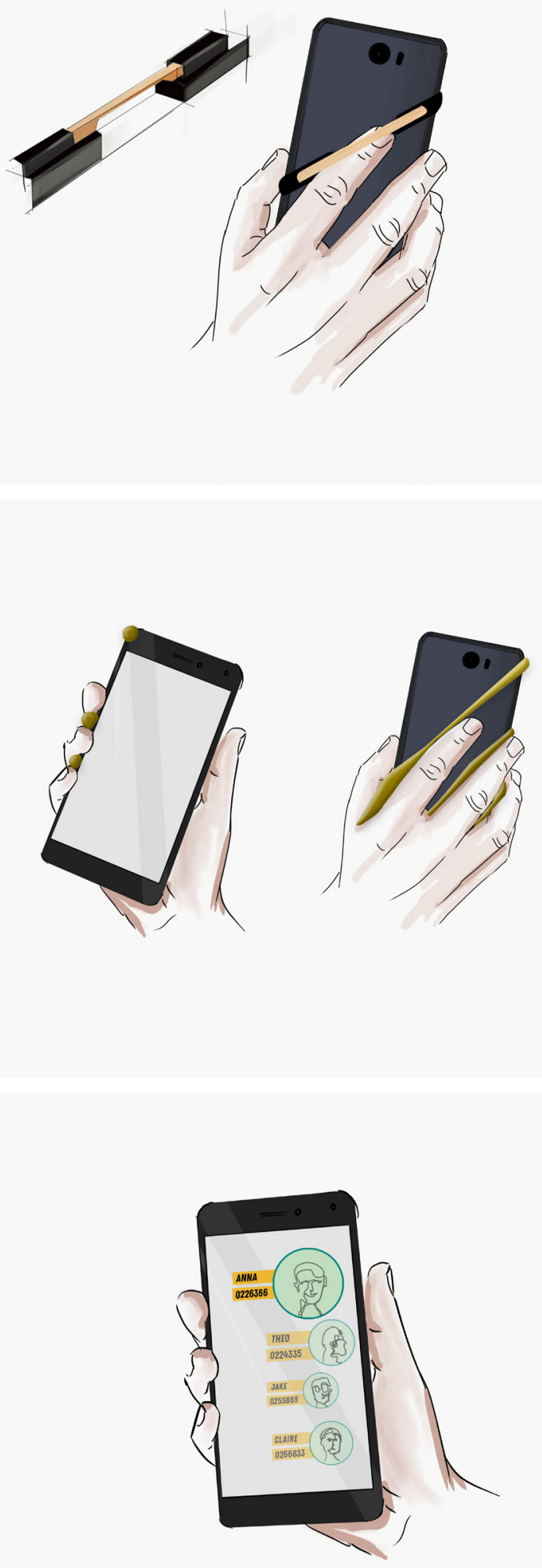

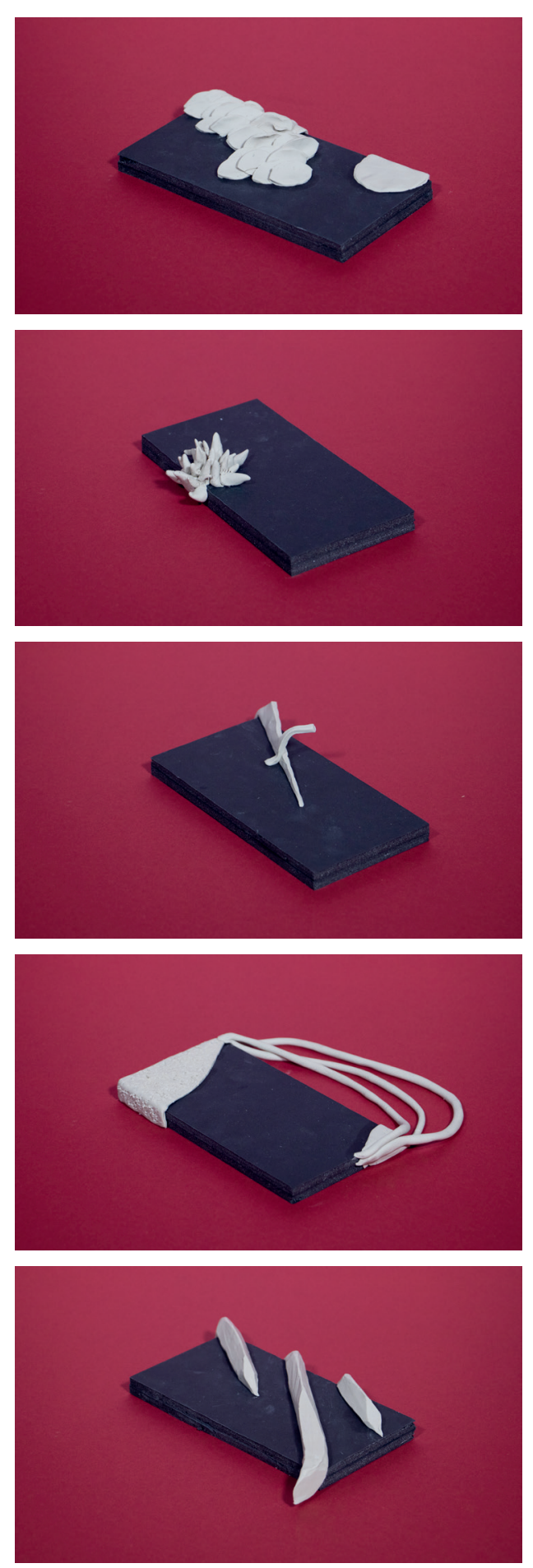

I dereloped a number of clay models to test the restraining effect of the dosign 

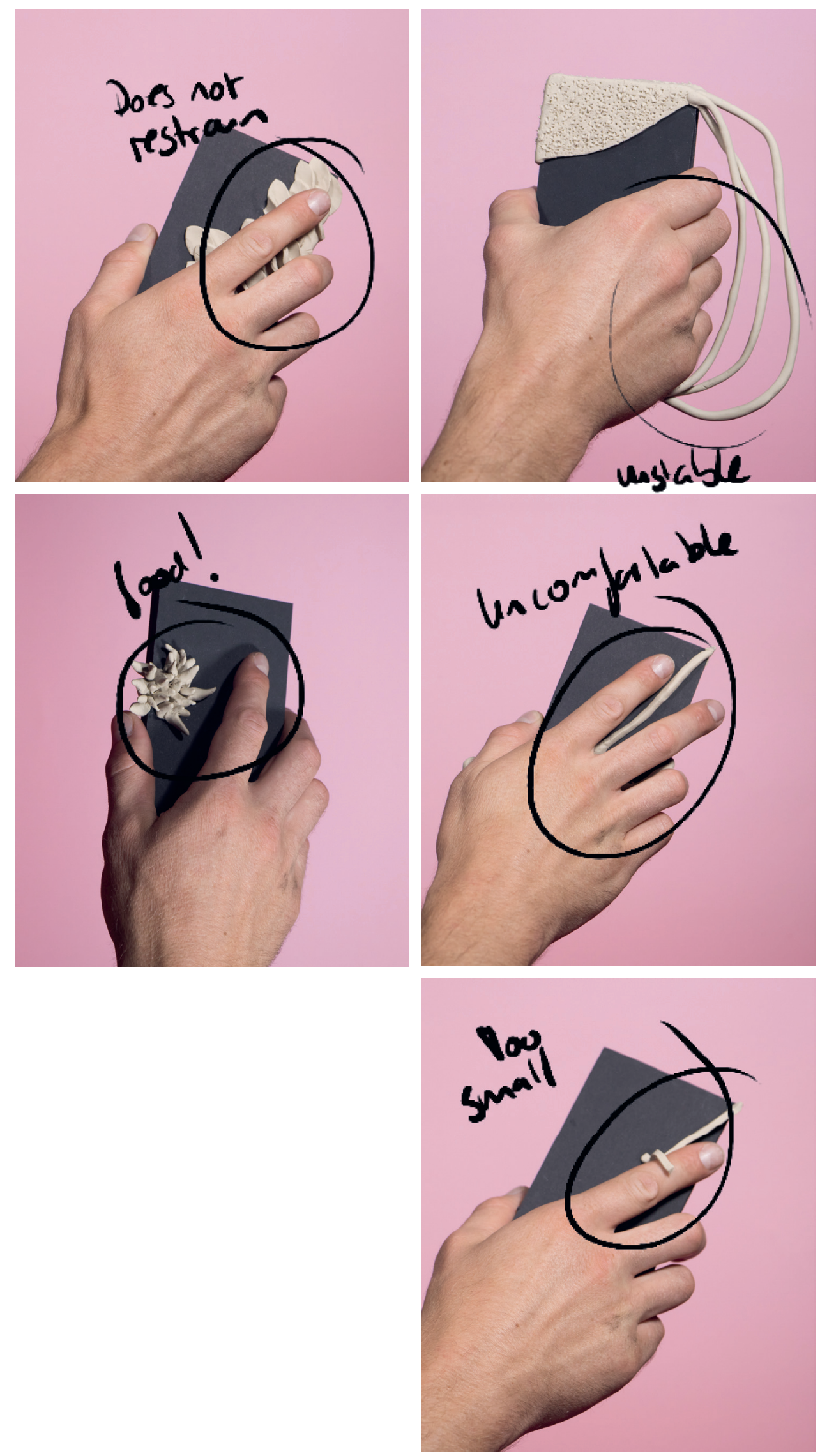

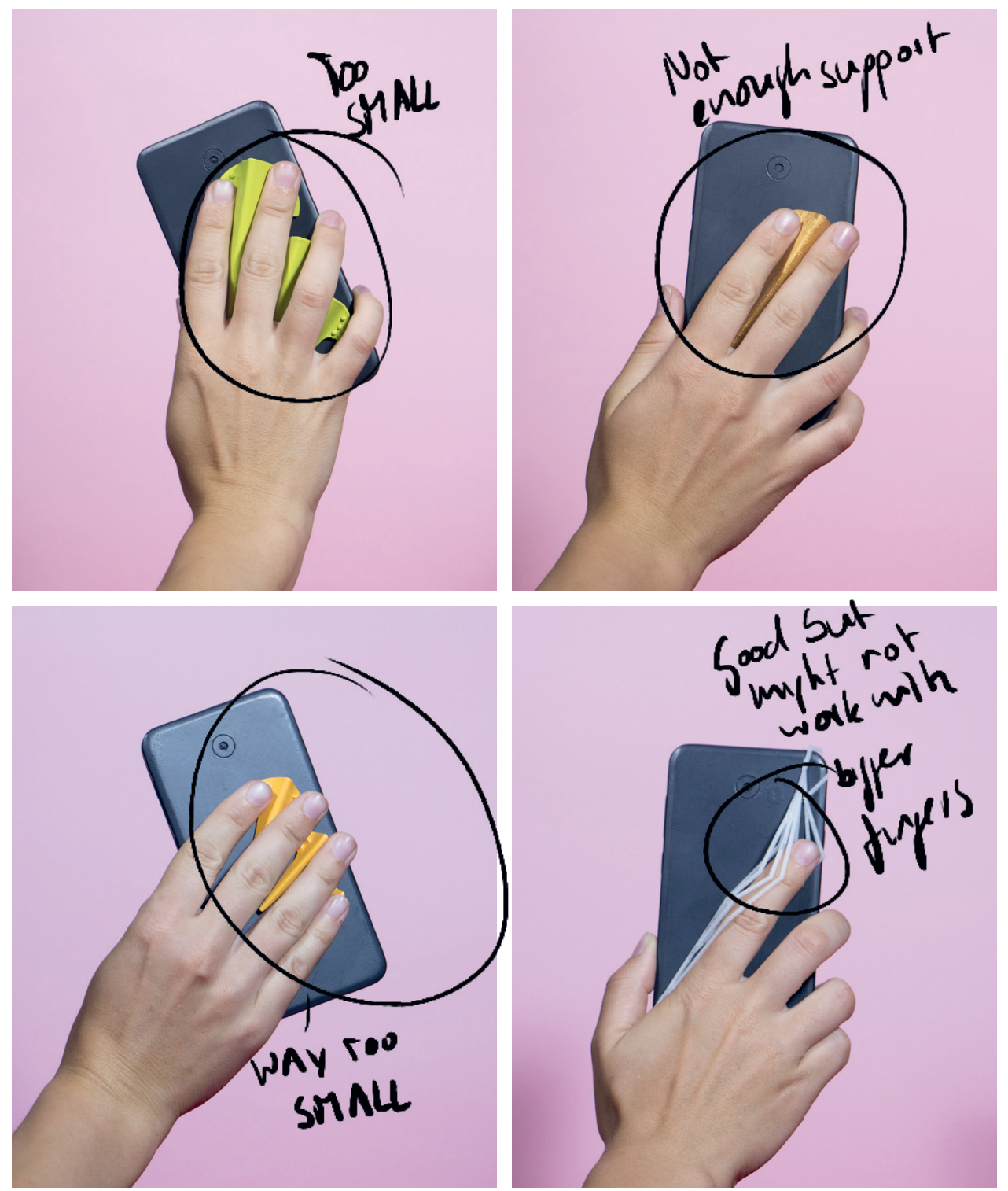

quitler design repirements were produced with the ve of a tabletop 3D printer 


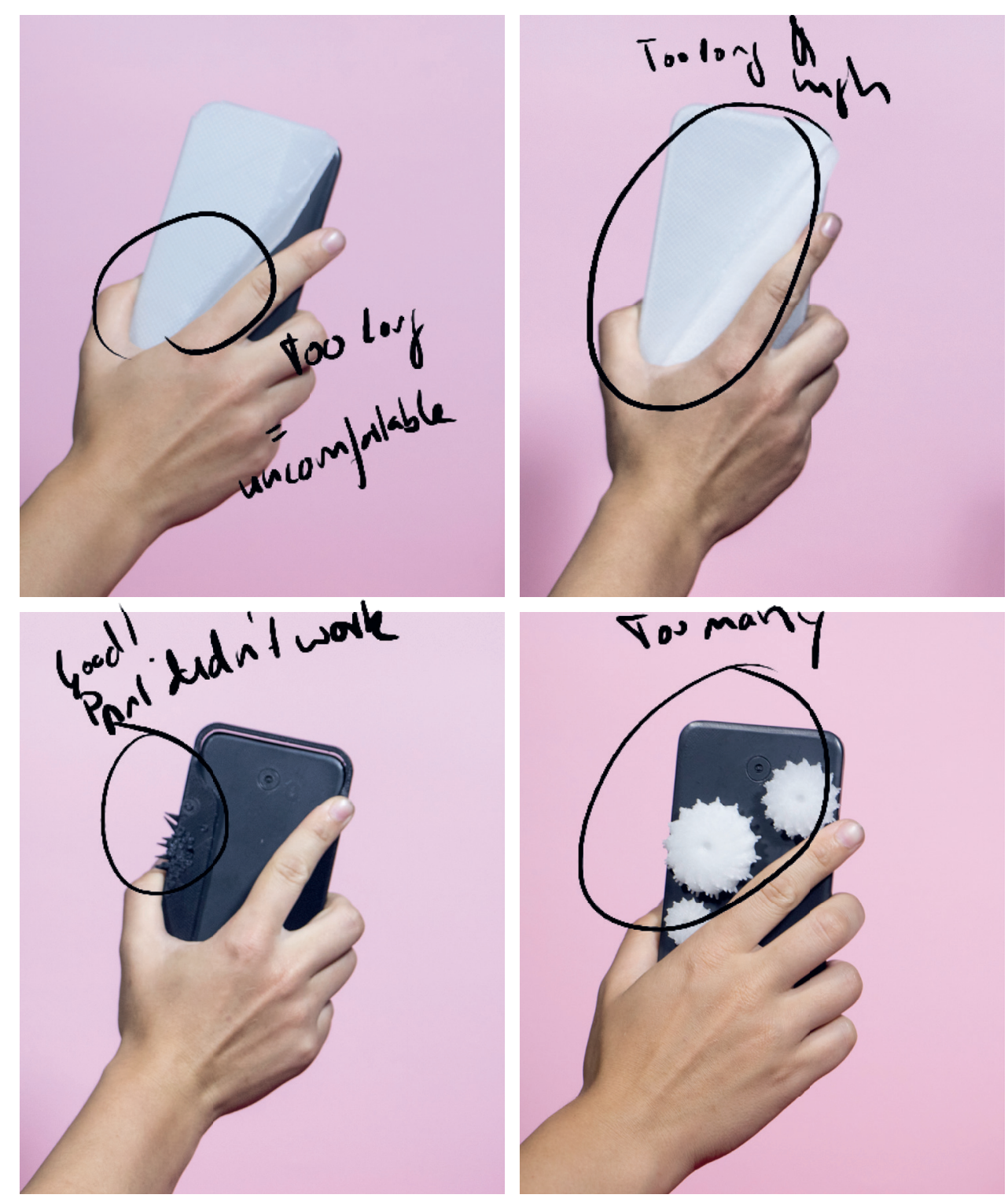




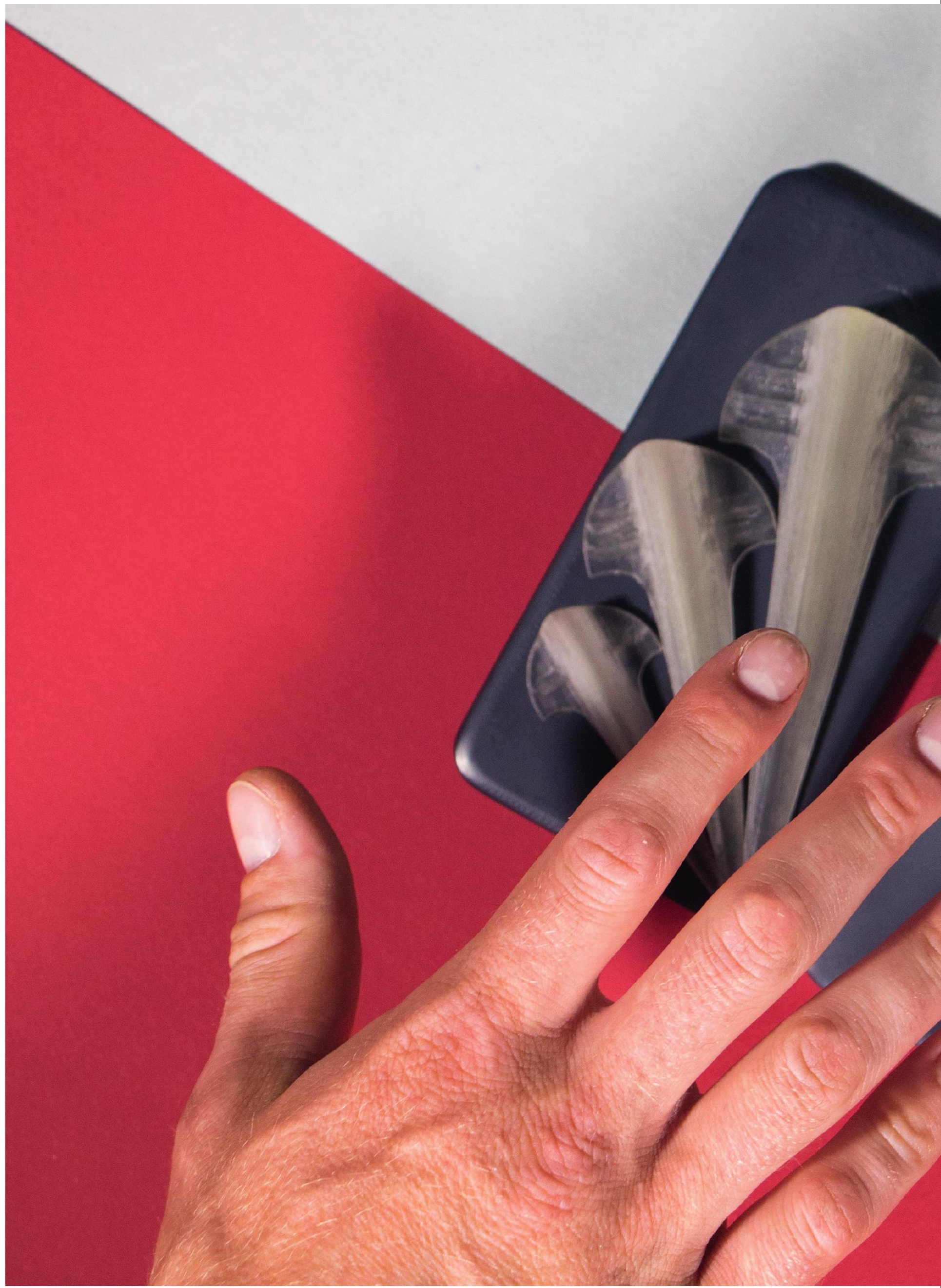


The attempt to hold if from this side would be uncomfortable or even painful 
strength of the 3D proved tho ms Form is inspired by the thorns of cacti

objects are positioned in a way that allows a comfortable portion with the affected hand 


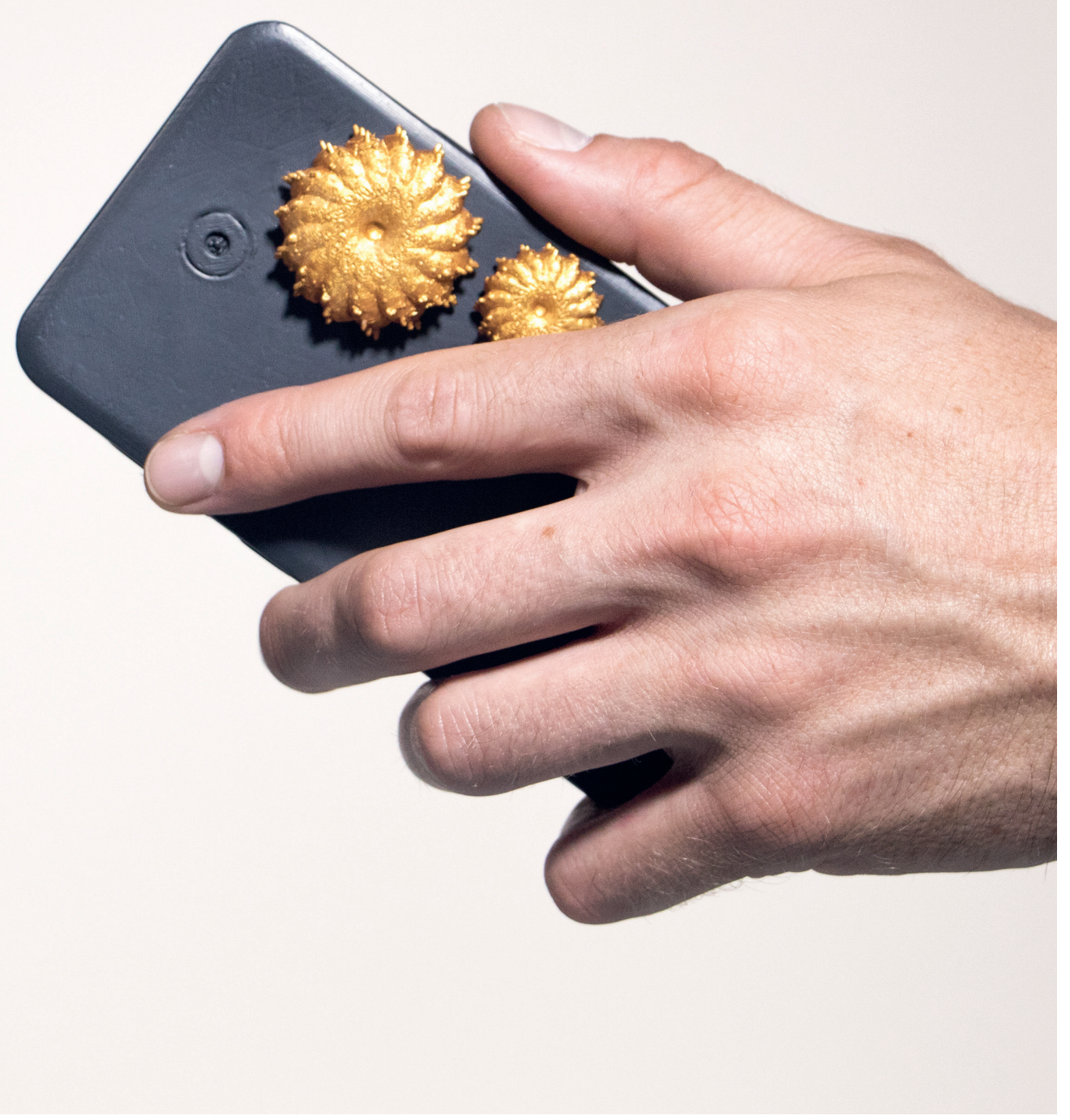

The form of the coesure desing prolotype is inspired by the texture \& suipare of a cactus. 

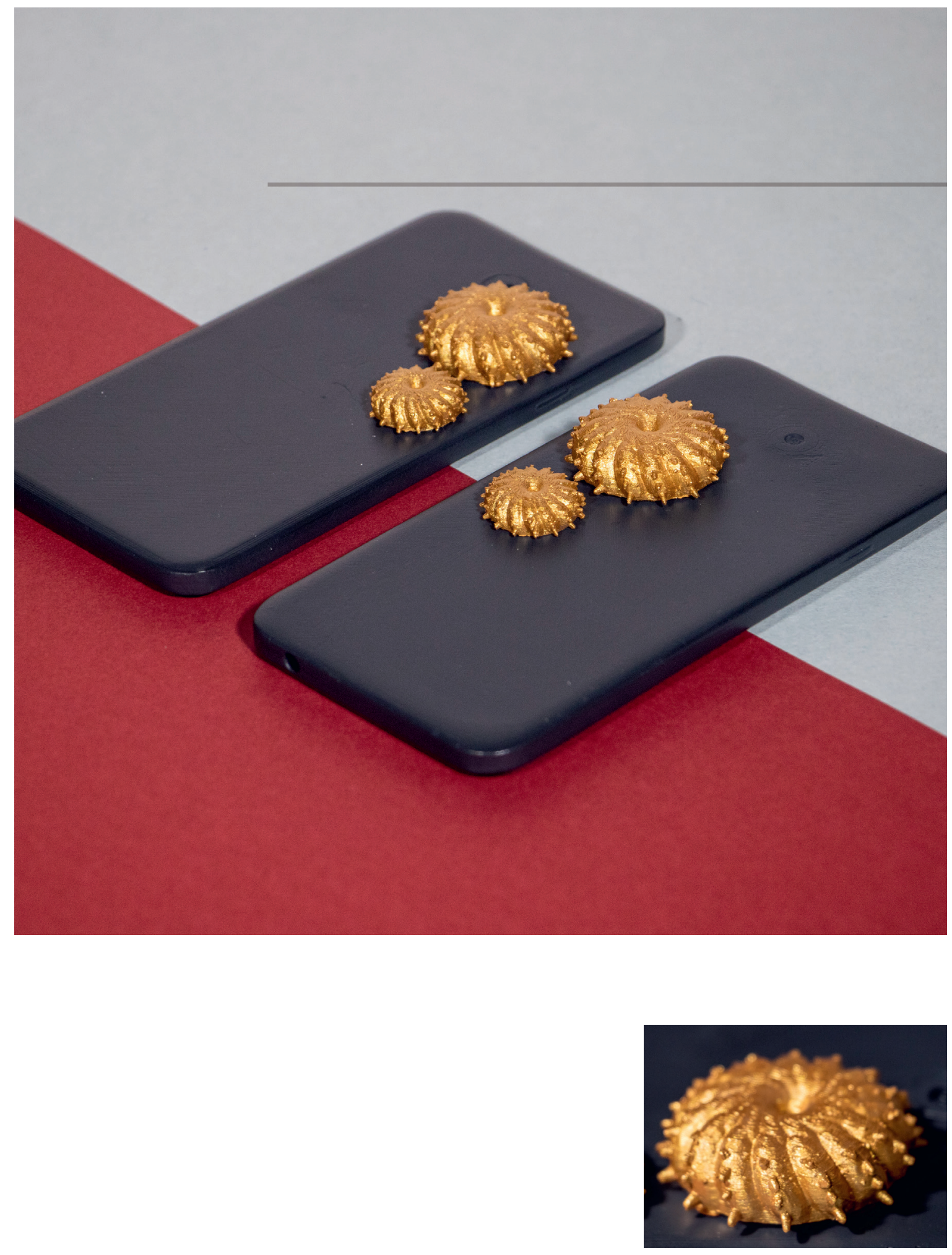

Left-handed Q night handed orientation on the smastphore. 


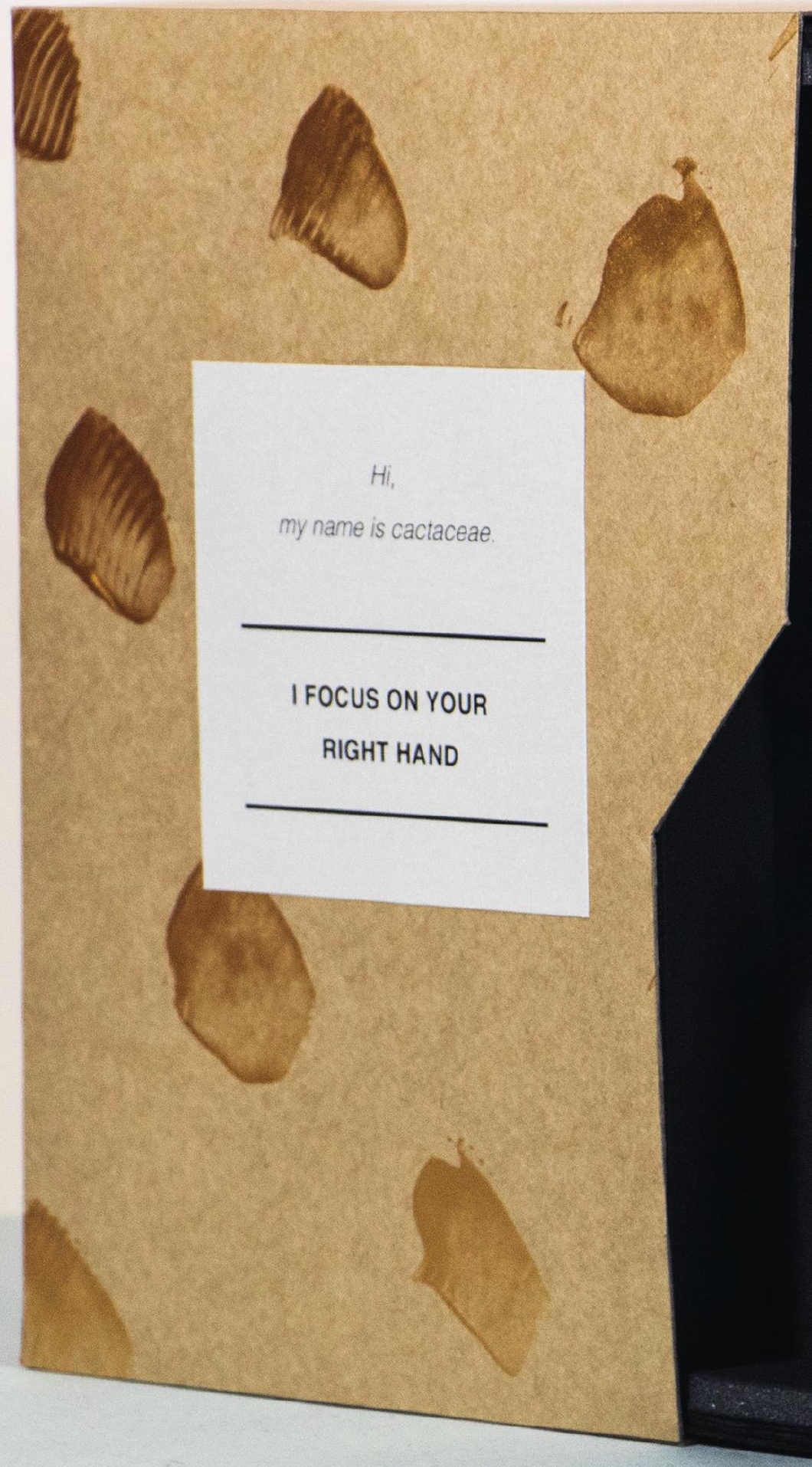




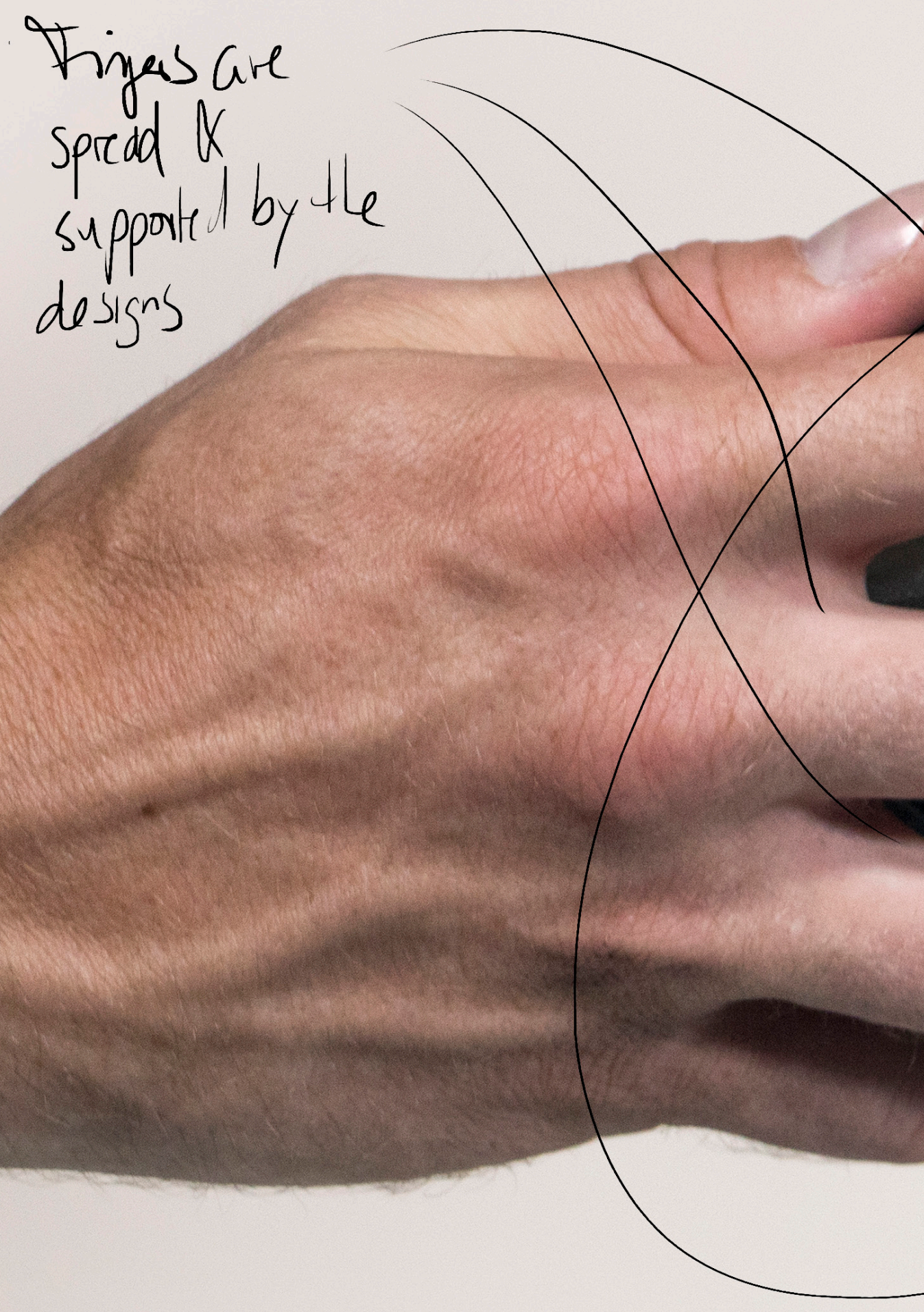


Prnted with a lonnex 3Dpmter

Plve Mifkent sized handle's can be dued on any land of smaitphore/ feature phor Texture decreases nise of doppingtle 


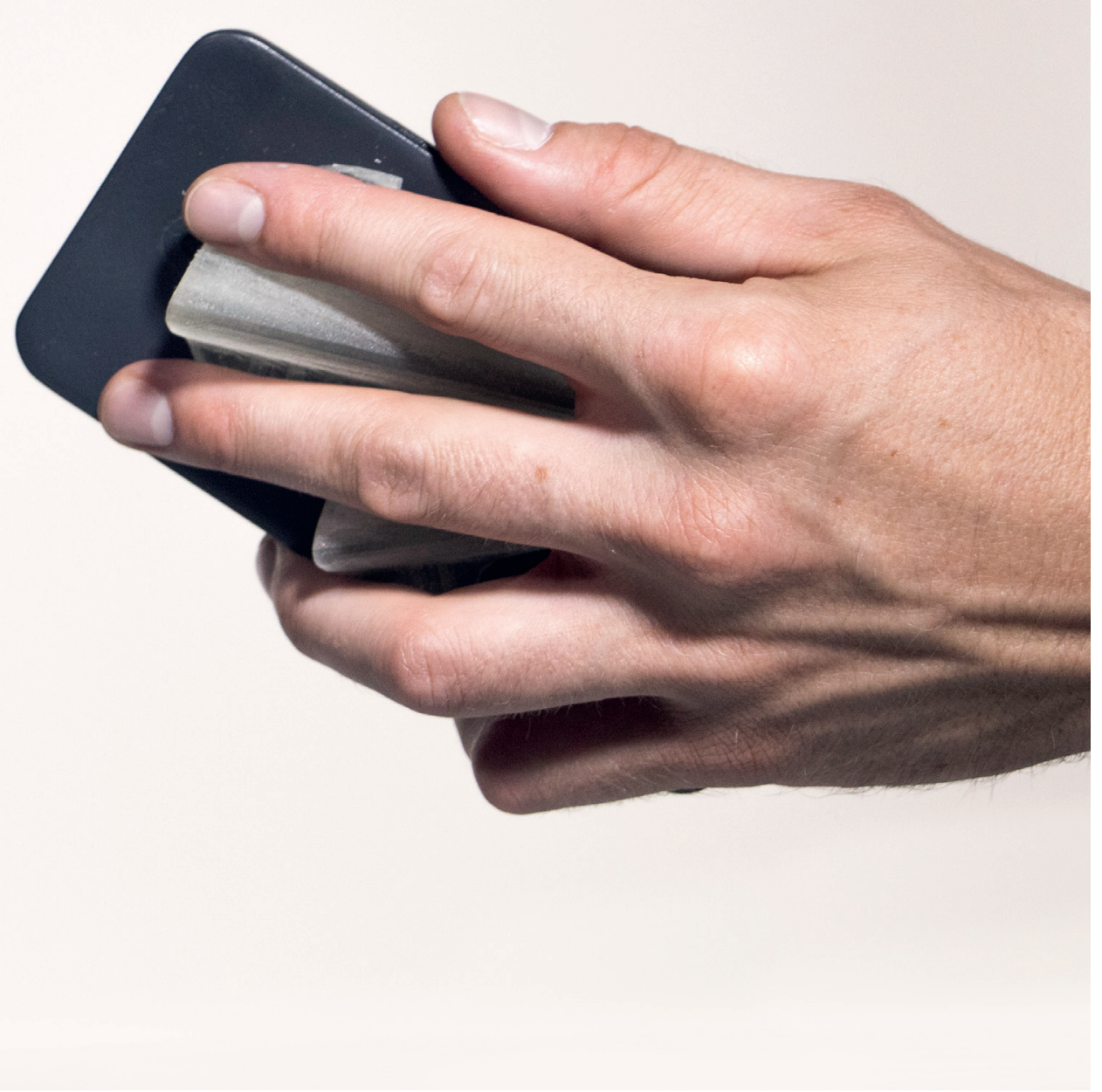

The persuasive design prolatype is infuenced by the form of a sting ray. 

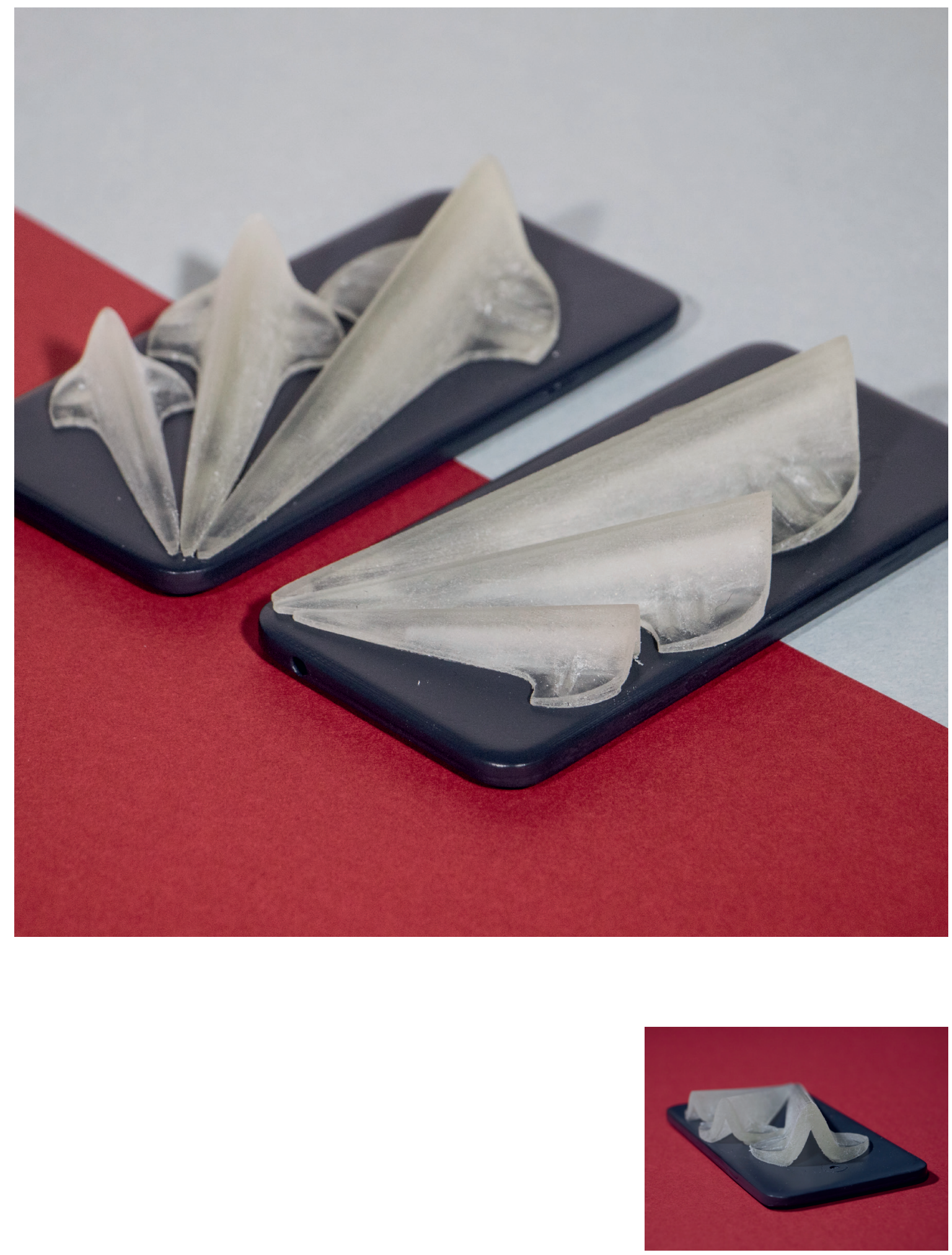

Left-handed $Q$ right-handed oientation of the persuasive design prototype. 


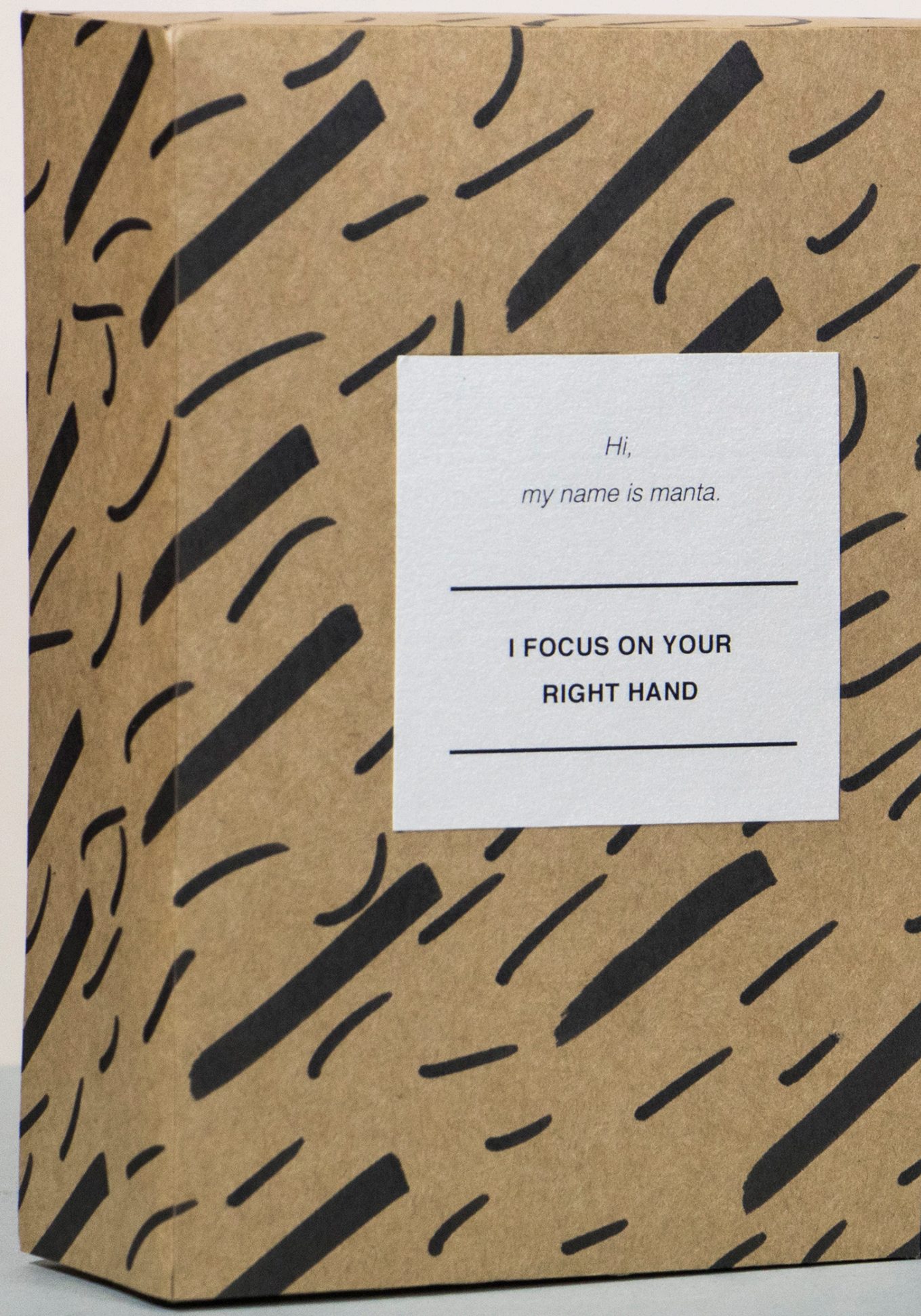




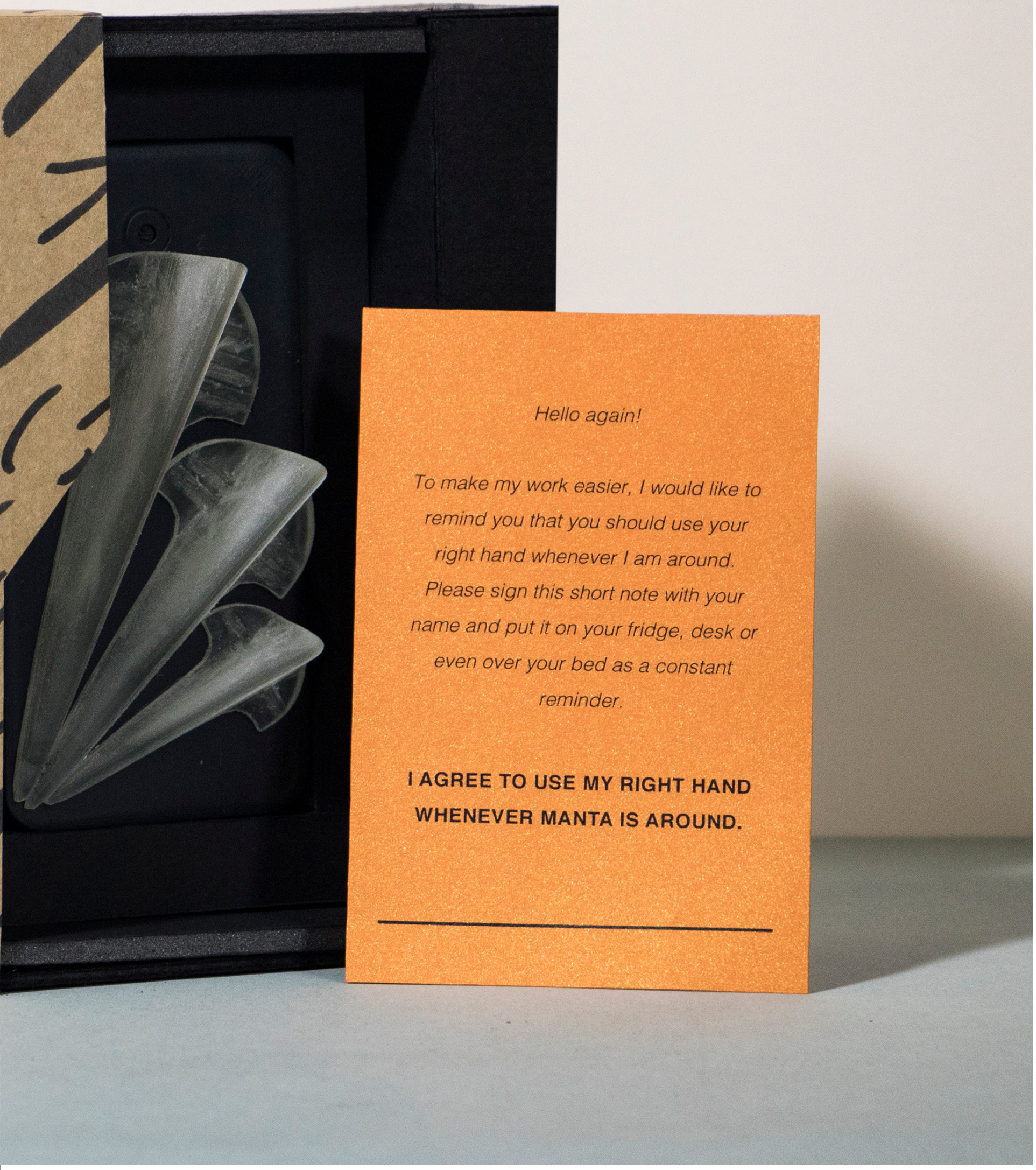




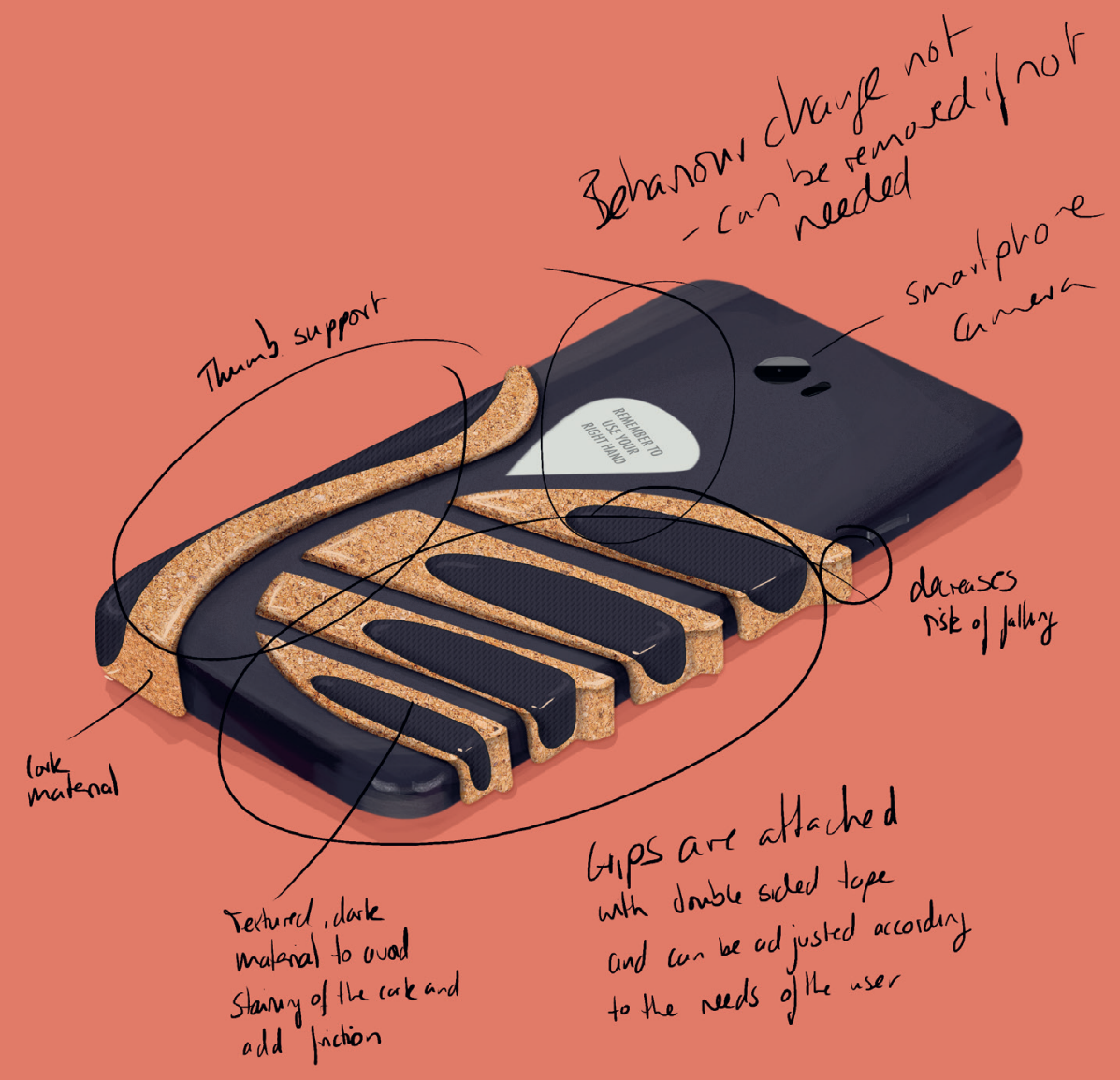

The formative euduation process revealed a number of usability issues in rigards of the use of the wo prototypes. I have shetched out how the persusive design, the most promising approuch, could be redefured Is increase the restraining effect. 


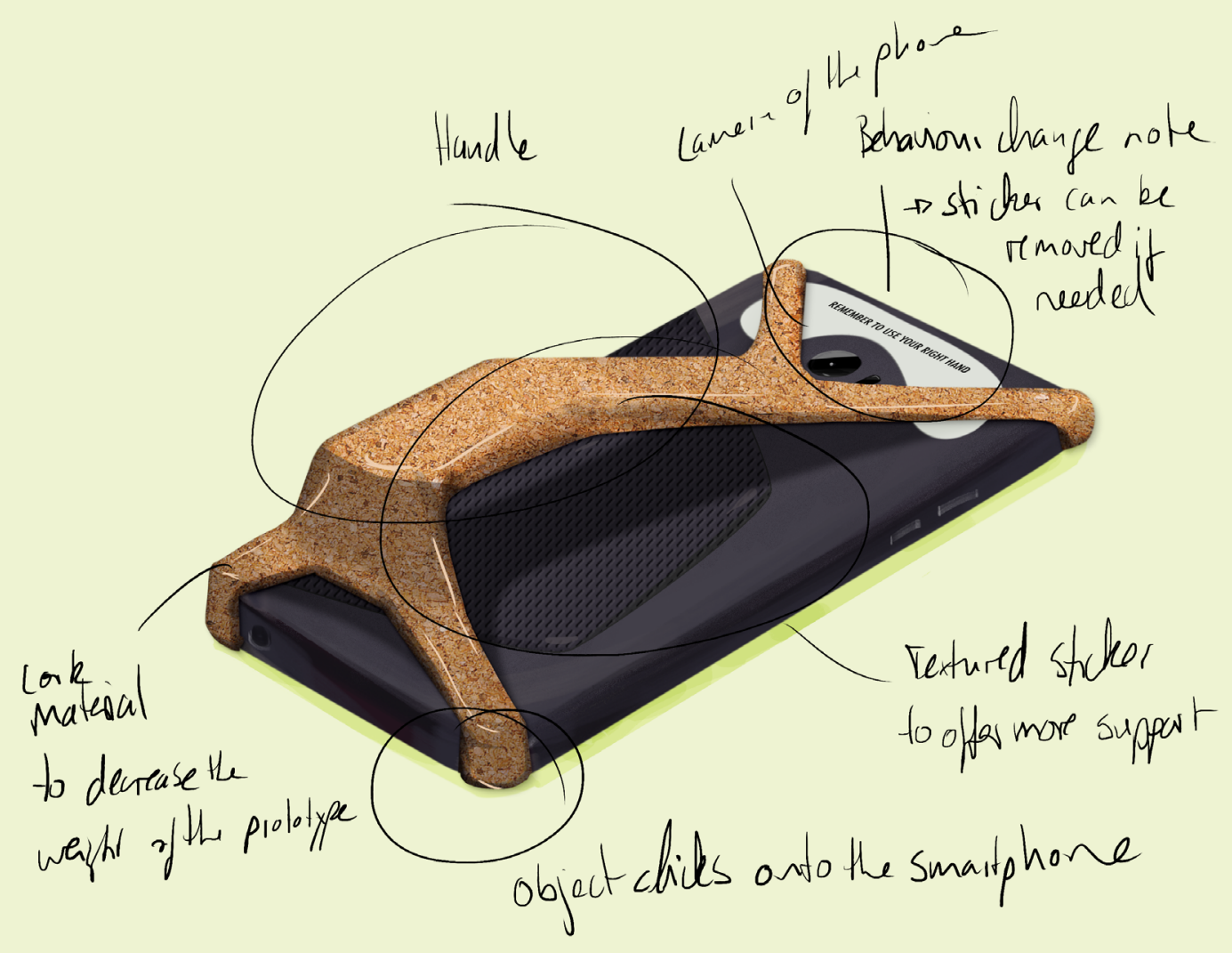


